\title{
CLIMATE CHANGE ADAPTATION IN A COASTAL HOTEL SECTOR: THE CASE OF SIHANOUKVILLE, CAMBODIA
}

\author{
BY \\ TINAT NHEP
}

A thesis submitted to the Victoria University of Wellington in fulfilment of the requirements for the degree of Doctor of Philosophy in Tourism Management

Victoria University of Wellington

2019 


\begin{abstract}
Tourism is one of the world's biggest and fastest-growing economic sectors with key contributions to gross domestic product (direct and total contribution), employment (direct and total contribution), visitor exports, and investment (UNWTO, 2018b; WTTC, 2018b). Cambodia is a post-conflict country, which has seen very rapid tourism development over the last decade, while also being identified as being very vulnerable to climate change impacts by several key international agencies. Along with the rapid growth of the country's tourism, UNWTO (2014) argues that Cambodia's coastal tourism is at the forefront of climate change impacts. Two tools widely used to respond to climate change are adaptation and mitigation (Parry, 2007). The overall effect of mitigation is 'global' while the positive effect of adaptation is 'local to regional' (Füssel \& Klein, 2006). Therefore, adaptation is most needed for tourism in developing nations (Scott, de Freitas, \& Matzarakis, 2009), especially for a rapidly developing coastal destination like Sihanoukville, Cambodia. Taking this into account, the thesis takes the form of a climate change-focused case study of the coastal hotel sector in Cambodia's Sihanoukville. The central concern of the thesis is to determine whether Sihanoukville's hotel sector adapts to climate change and critically examine the barriers and enabling factors that influence adaptation.
\end{abstract}

Adopting a postpositivist approach, the study conducted semi-structured interviews with 50 hotel respondents and field observations in Sihanoukville. Mixed method (qualitative \& quantitative) and single-case study were used. Prior to exploring the adaptation, the vulnerability of Sihanoukville's coastal tourism and its hotels were assessed through the perceptions on environmental and socio-economic factors, supported by the existing scientific evidence. The purpose is to identify the vulnerability, which is prerequisite before determining adaptation actions. The assessment is guided by key studies such as Moreno and Becken (2009), Smit and Wandel (2006), UNWTO (2014) and MOE, GEF and UNEP (2015) which explain that identifying key vulnerabilities is prerequisite knowledge before further identifying the adaptation responses for tourism. Adopted from WMO, UNEP, and WTO 
(2008) and Simpson, Gössling, Scott, Hall, and Gladin (2008), an adaptation framework that includes technical, managerial, policy, research and education was used to determine the hotels' adaptation. Within the context of hotels' adaptation, the study investigates the perceived criticality levels of barriers and enabling factors to adaptation because little research has been done to address the issue. The most critical (significant) barriers and enabling factors have been identified by measuring mean scores on a six-point rating scale with 0 being 'uninfluential' barrier and 5 being 'very major' barrier, and 0 being 'uninfluential' enabling factor and 5 being 'very important' enabling factor. In order to explore the underlying dimensions of hotel attributes (star rating, ownership etc.) and participants' backgrounds (level of education, experience etc.) with regard to the barriers and enabling factors to adaptation, descriptive statistics and independent sample tests was used to determine whether there is statistical evidence that the associated sub-groups of respondent means are significantly different.

Although Cambodia and Sihanoukville have been identified as very vulnerable by several key reports, the participants perceived that Sihanoukville's coastal tourism and its hotel sector are moderately and slightly vulnerable respectively, mainly owing to 'risk perception' or 'perception gap' that leads to a subjective judgement on the actual climate change impacts. These respondents were surrounded by uncertainty of climate change information. While some studies identified the hotel sector as possessing the lowest adaptive capacity that is relative to their fixed structures (buildings) (e.g. WMO et al., 2008), this study found that the hotel attributes led to considerable variation in the adaptations. Of all the five types of adaptation, the technical adaptation was most significantly employed in the hotel sector. In the context of the hotels' adaptation, the greatest barriers and enabling factors to climate change adaptation were also identified. Measured by mean scores, the findings further showed the most critical barriers to be 'limited resources', limited knowledge/perception of climate change, and 'lack of political will', and the most critical enabling factors to be 'sufficient resources', 'sufficient information' and 'good leadership and management structures'. Subsequently, the study critically examines the extent to which hotel attributes and participants' backgrounds influenced the barriers and enabling factors to adaptation. It was found that the barriers and enabling factors vary due to hotel attributes as well as 
participants' backgrounds. Finally, the study proposes a conceptual framework of coastal hotel sector adaptation to climate change in the context of developing countries. 


\section{DEDICATION}

To my beloved family, most importantly for my father, Chuch Tem

[Losing you is so painful, especially when I could not do anything from afar] 


\section{ACKNOWLEDGEMENTS}

Most of all, I must express my profound appreciation to my supervisors, Dr Christian Schott and Associate Professor Mondher Salhi for their constant and continued support, patience and tolerance. Their characters and working manners have influenced and formulated a part of my future academic and normal life. My gratitude is also extended to all professors/lecturers within the Tourism Management Group, and staff in School of Management (SoM), Victoria Business School, for their support throughout my study. Without your kind assistance, this thesis would not have been completed.

I must thank to the Victoria Doctoral Scholarship, the Victoria Doctoral Hardship Scholarships and the Victoria Doctoral Submission Scholarship of Victoria University of Wellington (VUW) for the awarding of the funding that enabled me to undertake this study. Without the funds, it would have been challenging and difficult for me to come to the end of my $\mathrm{PhD}$ journey.

I am very thankful to all my family members for their moral support, particularly my beloved wife, Boravy Rith, who accompanied me to New Zealand, which is new place she has had to challenge, especially during her two miscarriages. She has been very patient during my long academic journey. I am also so grateful to my mum. Although she spent most of her time in hospitals, she kept praying for me from afar. A big thanks to my older brother, Tinal Nhep, for giving good advice and never-exhausting encouragement. Thanks to my nephew Phourie Phann (4 years old) who quite often called me from home with funny questions and talks. I frequently heard his question: "when will you come home?" This is both highly emotional and funny and later I found that these have contributed to relieving my academic stress.

Special thanks to Lisa Wood, Victoria's Statistician Consultant, for her advice regarding my statistical analysis. She is brilliant.

I am so grateful to other staff members for their moral support, particularly Jackie Anderson, Dr Simin Nina Littschwager, Amanda Fraser and Catherine Guo, who listened to my 
problem during my daunting challenge after my father's passing and my wife's miscarriage. They provided useful advice to help me continue the journey.

Last but not least, my acknowledgements would not be complete without thanking all my research participants for their valuable participations and materials. I welcome this opportunity to thank colleagues and friends in the SoM: Sandra Goh Mei Ching, Qi Hongxia, Jharna Joshi, Solmaz Nazari and Tai Anh Vu. Without their friendship and suggestions, my journey would have been less enjoyable and applicable. Thanks for being a part of this project. 


\section{Table of Contents}

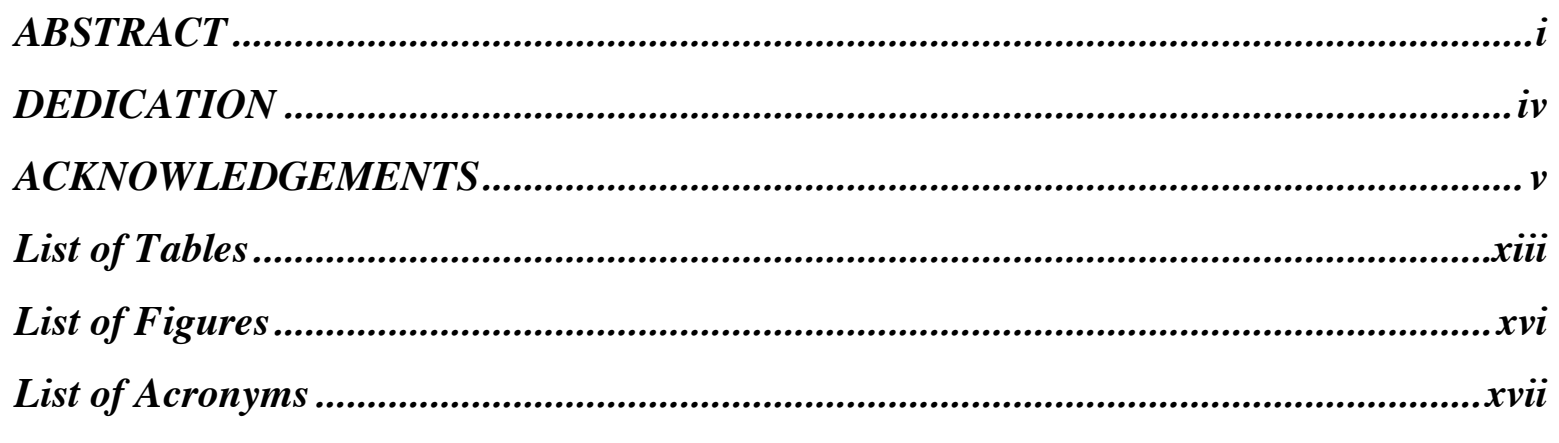

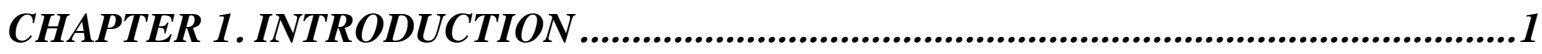

$1.1 \quad$ Background of the study ..............................................................................................1

$1.2 \quad$ Research context ....................................................................................................2

1.3 Motivation for the research ....................................................................................4

$1.4 \quad$ Research aims and questions ............................................................................................

$1.5 \quad$ Research methodology ........................................................................................................

$1.6 \quad$ Thesis outline .......................................................................................................................

CHAPTER 2: LITERATURE REVIEW ...............................................................11

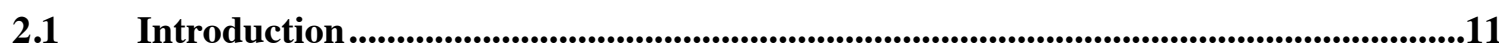

2.2 Significance of tourism and coastal tourism and accommodation and hotel sector $\mathbf{1 1}$

2.2.1 Tourism and coastal tourism.............................................................................................. 11

2.2.2 Accommodation and hotel sector ............................................................................... 12

2.3 Climate change, tourism, coastal tourism and accommodation sector .......................13

2.3.1 Climate change ………………………………………………………………….... 13

2.3.2 Climate change and tourism/coastal tourism.......................................................................... 14

2.3.3 Climate change and accommodation sector ........................................................................ 15

2.4 Climate change vulnerability ……….............................................................................16

2.4.1 Vulnerability assessment through environmental and socio-economic factor ....................... 16

2.4.2 Vulnerability assessment through combined scientific and local knowledge ....................... 19

2.5 Climate change adaptation ...........................................................................20

2.5.1 Different types of climate change impacts .......................................................................2 21

2.5.2 Different types of adaptation ......................................................................................... 22

2.5.3 The limit of adaptation .................................................................................................2 22

2.6 Climate change adaptation, tourism/coastal tourism and the accommodation sector 24

2.7 Adaptation studies in tourism/coastal tourism and the accommodation sector ........27 


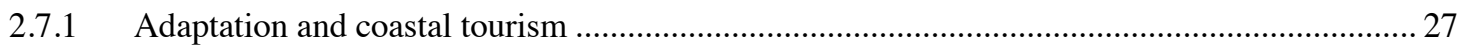

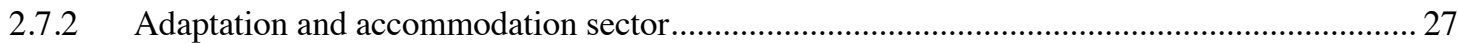

$2.8 \quad$ Adaptation frameworks .............................................................................................................28

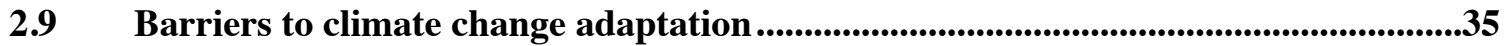

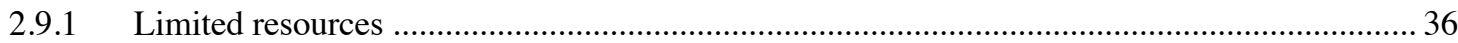

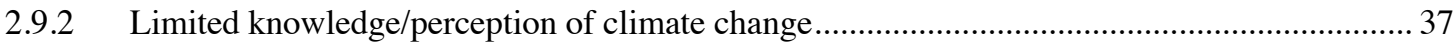

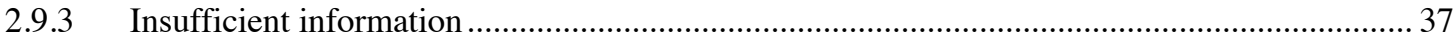

2.9.4 Institutional barriers and uncertain responsibilities for adaptation .......................................... 38

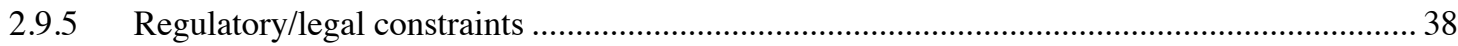

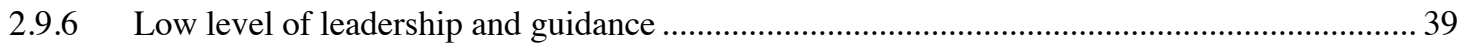

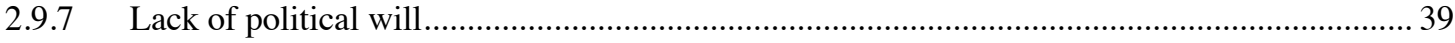

2.9.8 Insufficient engagement and interaction with other stakeholders ........................................... 39

2.10 Critical barriers in implementation of climate change adaptation ...............................42

2.11 Barriers to climate change adaptation related to tourism/tourism business .............43

2.12 Enabling factors for climate change adaptation ............................................................46

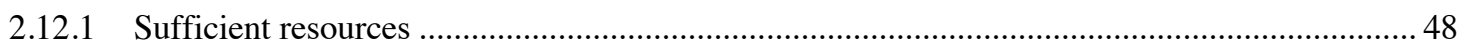

2.12.2 Previous and current adaptation experiences/ knowledge and climate change education ........ 48

2.12.3 Engagement/collaboration/communication with other stakeholders.......................................... 49

2.12.4 Individuals' motivation to respond (committed workforce) ..................................................... 49

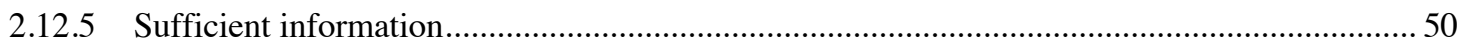

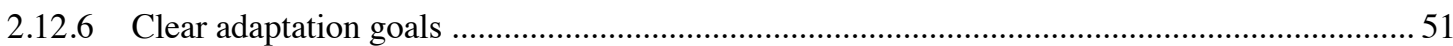

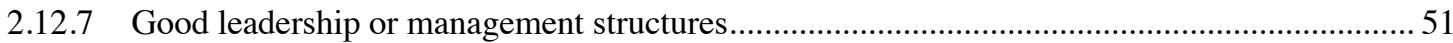

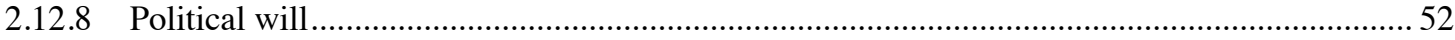

2.13 Critical enabling factor for climate change adaptation ................................................54

2.14 Conceptual framework ................................................................................................................55

2.15 Chapter summary …....................................................................................................................57

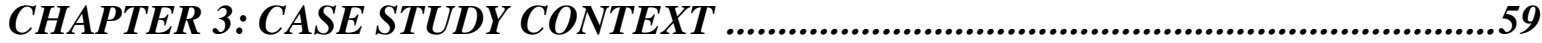

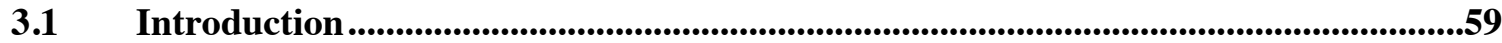

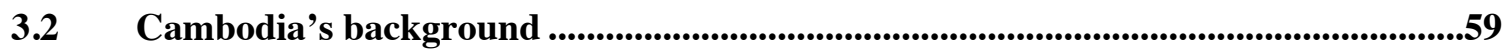

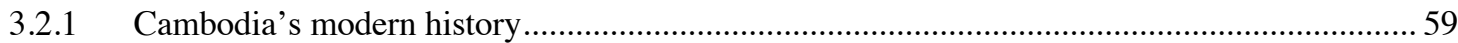

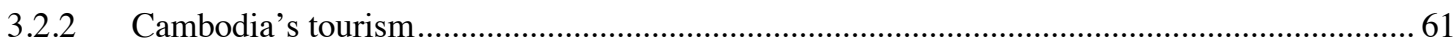

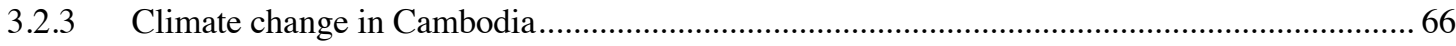

3.2.4 The efforts to respond to climate change in Cambodia ............................................................... 75

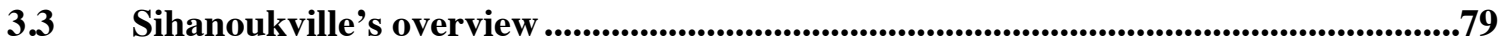

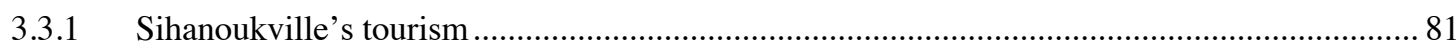

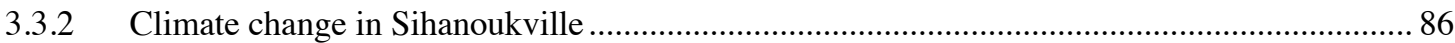


$3.4 \quad$ Chapter summary ......................................................................................................................91

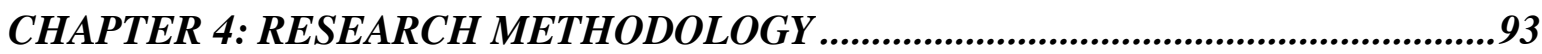

4.1 Introduction ...................................................................................................................................93

4.2 Research paradigm ..........................................................................................................93

4.3 Research approach .............................................................................................96

4.4 Case study ...........................................................................................................................97

4.5 Recruiting respondents.......................................................................................................98

4.6 Data Collection ...................................................................................................................101

4.6.1 Pilot interviews and adjustments ................................................................................ 102

4.6.2 Before the interviews or preparation stage ..................................................................... 102

4.6.3 In-depth semi-structured interviews ................................................................................. 103

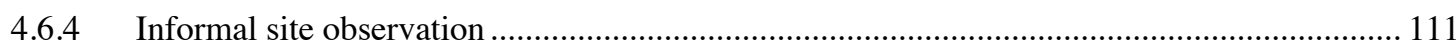

4.6.5 Document analysis ................................................................................................... 112

$4.7 \quad$ Data preparation .........................................................................................................112

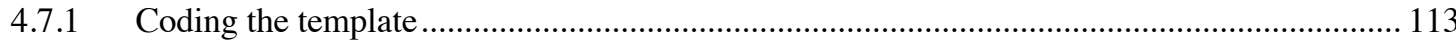

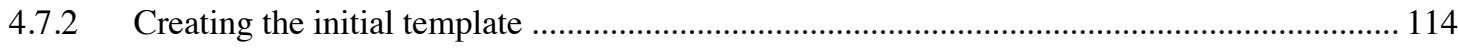

4.7.3 Revising the template …………………………………………………………….... 115

4.7.4 Finalising the template ....................................................................................................... 116

4.7.5 Interpreting and presenting template analysis .................................................................. 116

4.8 Analytical framework .............................................................................................117

4.9 Data Analysis .....................................................................................................118

$4.10 \quad$ Validity and Reliability ..................................................................................................122

4.11 Ethical Considerations .....................................................................................................128

4.12 Limitations of the study ........................................................................................128

4.13 Chapter summary ..................................................................................................129

CHAPTER 5: VULNERABILITY AND ADAPTATION ...........................................131

$5.1 \quad$ Introduction .............................................................................................................................131

5.2 Vulnerability assessment of Sihanoukville's coastal tourism ......................................133

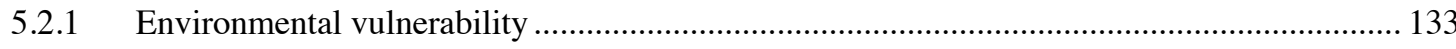

5.2.2 Socio-economic vulnerabilities .................................................................................... 139

5.3 Vulnerability assessment of Sihanoukville's hotel sector ..............................................144

5.3.1 Environmental vulnerabilities......................................................................................... 145

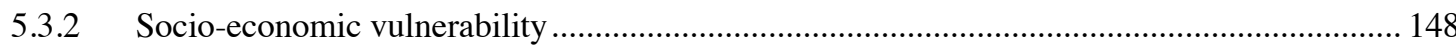

5.4 The adaptation framework in SHV's hotel sector .....................................................151

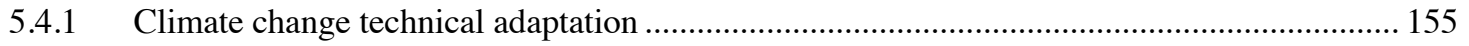




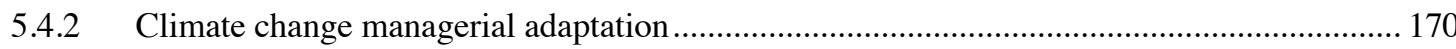

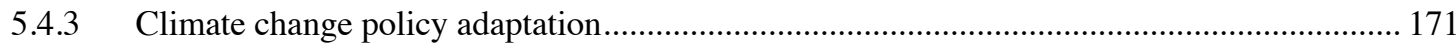

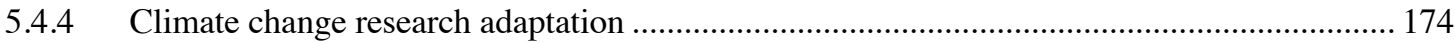

5.4.5 Climate change education adaptation ............................................................................... 175

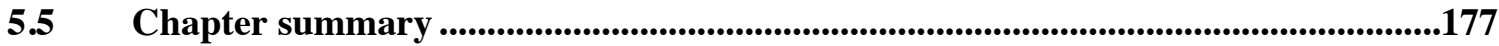

CHAPTER 6: BARRIERS AND ENABLING FACTORS IN CLIMATE CHANGE

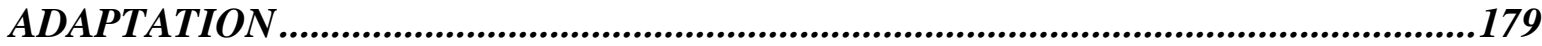

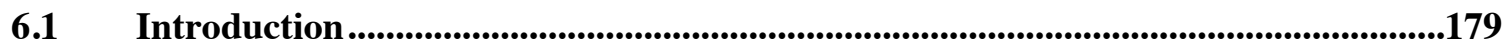

6.2 The perceived ratings of adaptation barriers in the hotel sector .............................181

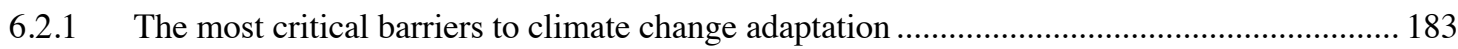

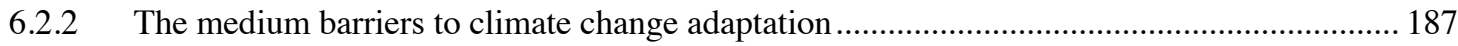

6.2.3 The least critical barriers to climate change adaptation …........................................................ 191

6.3 Additional adaptation barriers to climate change adaptation....................................197

6.4 The perceived ratings of enabling factors for adaptation in the hotel sector..........199

6.4.1 The most critical enabling factors for climate change adaptation ............................................ 201

6.4.2 The medium enabling factors for climate change adaptation .............................................. 204

6.4.3 The least critical enabling factors for climate change adaptation ........................................... 209

6.5 Additional enabling factors for climate change adaptation .....................................214

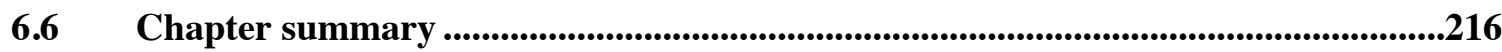

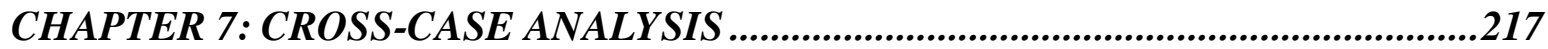

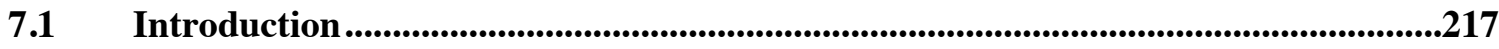

7.2 Relationships between adaptation barriers and hotel attributes ..............................221

7.3 Relationships between adaptation barriers to CCA and participants' backgrounds 225

7.4 Relationships between enabling factors for adaptation and hotel attributes..........228

7.5 Relationships between enabling factors for adaptation and participants'

backgrounds .......................................................................................................................................................233

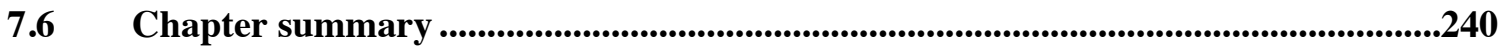

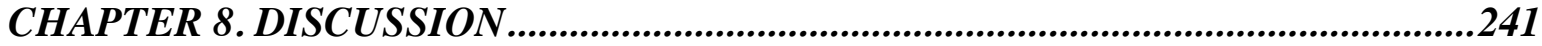

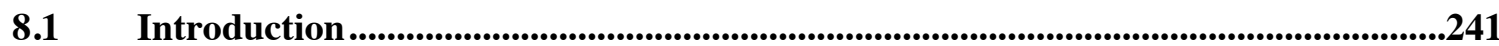

8.2 Reintroduction of the research aims/questions ..........................................................241

8.3 Discussion on aim 1: Vulnerability assessment of Sihanoukville's coastal tourism242

8.4 Discussion on aim 2: Vulnerability assessment of Sihanoukville's hotel sector ......248

8.5 Discussion on aim 3: Climate change adaptation in Sihanoukville's coastal hotel sector 253 
8.6 Discussion on aim 4: Hotel sector's barriers and enabling factors to adaptation ..258

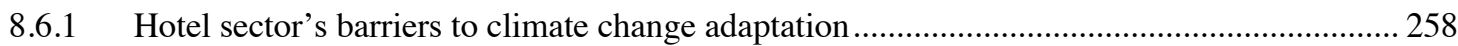

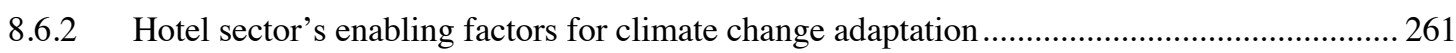

8.7 Discussion on aim 5: To critically examine the barriers and enabling factors to CCA according to hotel attributes and participants' backgrounds.............................................266

8.7.1 The perceived barriers and enabling factors influencing adaptation between hotel sizes ....... 266

8.7.2 The perceived enabling factors for adaptation between participants' nationalities.................. 270

8.7.3 The perceived barriers and enabling factors to adaptation between length of residence ........ 273

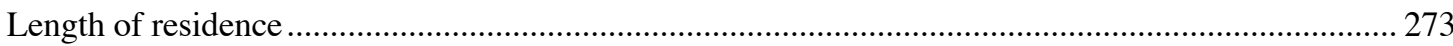

8.7.4 The perceived enabling factors for adaptation between participants' experiences .................. 276

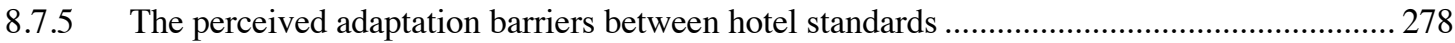

8.7.6 The perceived enabling factors influencing adaptation and hotel ownerships ......................... 279

8.7.7 The perceived enabling factors for adaptation between hotel locations .................................... 281

8.7.8 The perceived barriers and enabling factors influencing adaptation between business formats (owned vs. leased).

8.7.9 The perceived barriers and enabling factors affecting adaptation between business age (years of trading)......

8.7.10 The perceived barriers and enabling factors affecting adaptation between participants' education

8.7.11 The perceived barriers and enabling factors affecting adaptation between participants' age groups 285

8.8 Climate change adaptation in hotel sector: A revised conceptual framework ........286

8.9 Chapter summary ......................................................................................................................293

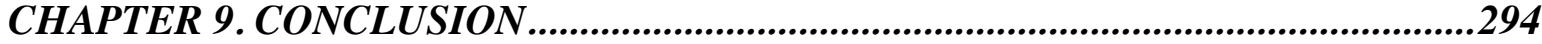

9.1 Introduction ................................................................................................................................294

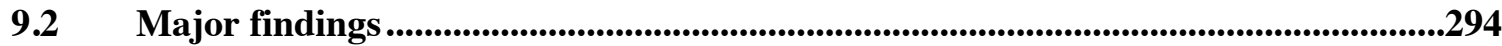

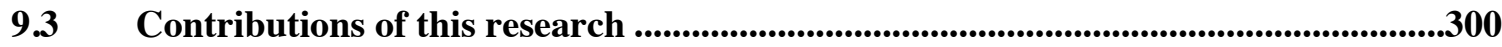

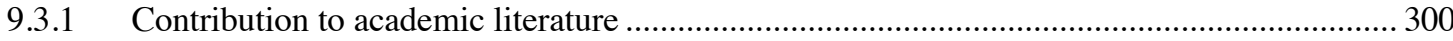

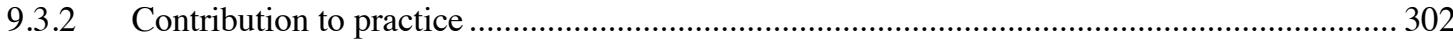

9.4 Research limitations ...........................................................................................................306

$9.5 \quad$ Future research ............................................................................................................................308

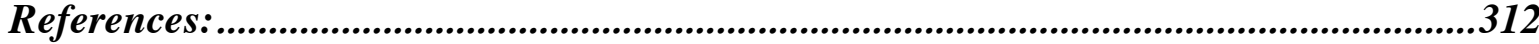

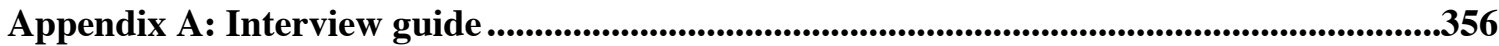

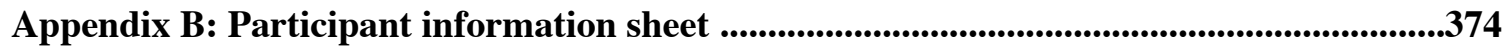

Appendix C: Participant consent form ...........................................................................................376

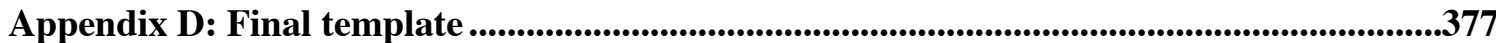


Appendix E: HEC Approval for interviews .................................................................................380

Appendix F: Descriptive test/independent sample test...............................................................381 


\section{List of Tables}

Table 2.1: Portfolio of climate change adaptation by tourism stakeholders ......................30

Table 2.2: Summary of the adaptation barriers ...................................................... 41

Table 2.3: Indirect and direct barriers of (small) hotels concerning climate change

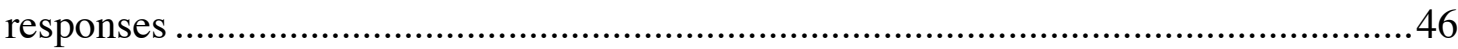

Table 2.4: Summary of enabling factors for CCA ..................................................53

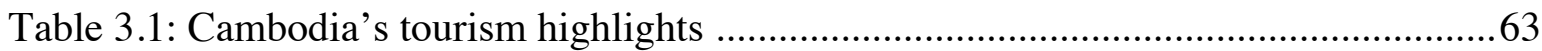

Table 3.2: Number of hotels and guesthouses in Cambodia from 1997-2016 ..................64

Table 3.3: Actual and projected change of Cambodia's annual rainfall ...........................67

Table 3.4: Actual and projected change of Cambodia's annual mean temperature ............ 70

Table 3. 5: Key reports documenting and analysing climate change in Cambodia.............78

Table 3. 6: Domestic/international tourism statistics report in 2012 - 2016 .....................83

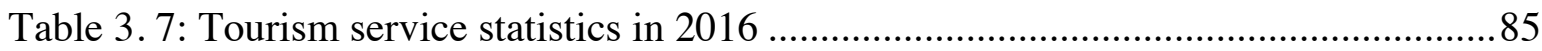

Table 4. 1: Number of interviewees categorised in hotel attributes and participants'

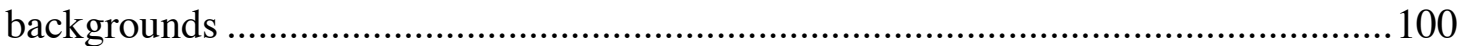

Table 4. 2: Key questions using the Likert scale in the study ......................................106

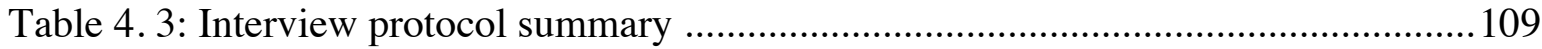

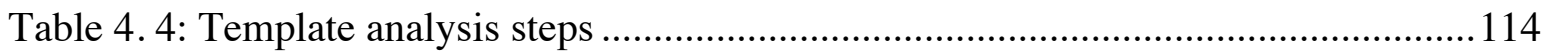

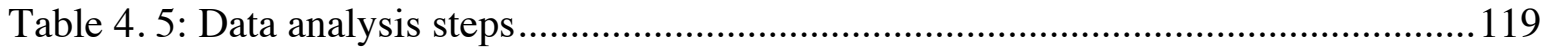

Table 4. 6: Rigour in the design phase, adapted from Dubé and Paré (2003), following the styles of Strode (2012) and Abeysekara (2017) ...................................................125

Table 4. 7: Rigour in the data collection phase, adapted from Dubé and Paré (2003), following the styles of Strode (2012) and Abeysekara (2017) .................................126

Table 4. 8: Rigour in the data analysis phase, adapted from Dubé and Paré (2003), following the styles of Strode (2012) and Abeysekara (2017)

Table 5. 1: Vulnerability assessment on SHV's coastal tourism 133

Table 5. 2: The extent to which SHV's coastal tourism is vulnerable to climate change ..143 
Table 5. 3: Vulnerability assessment on SHV's hotel sector 144

Table 5. 4: The extent to which the hotel sector is vulnerable to climate change 151

Table 5. 5: Adaptation framework of the hotel sector 152

Table 5. 6: Adaptation measures for excessive rainfall. 155

Table 5. 7: Technical adaptation to storm 157

Table 5. 8: Technical adaptation to rising temperature 159

Table 5. 9: Technical adaptation to SLR/beach erosion/storm surges 164

Table 5. 10: Technical adaptation to prolonged drought 165

Table 5. 11: Enabling access to early warning systems about climate change impacts .....169

Table 5. 12: Managerial adaptation employed by the hotel sector ..... 170

Table 5. 13: Policy adaptation employed by the hotel sector. 172

Table 5. 14: Research adaptation used by the hotel sector 174

Table 5. 15: Education adaptation employed by the hotel sector 175

Table 6. 1: Rating results of adaptation barriers in the hotel sector 182

Table 6. 2: Rating results of enabling factors for CCA for the hotel sector

Table 7.1: Overall test results between barriers and enabling factors to CCA and hotel attributes and participants' backgrounds

Table 7.2: Test results of the relationship between adaptation barriers and hotel attributes (North West quadrant) 221

Table 7.3: Test results of the relationship between adaptation barriers and participants' backgrounds (North East quadrant) .225

Table 7.4: Test results of the relationship between enabling factors for adaptation and hotel attributes (South West quadrant) 228

Table 7.5: Test results of the relationship between enabling factors for adaptation and participants' backgrounds (South East quadrant) .234

Table 8. 1: Reasons why the participants perceived the vulnerability of SHV's coastal tourism lower than early findings .243 
Table 8. 2: Reasons why the participants perceived the vulnerability of SHV's hotel sector

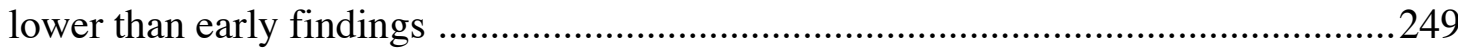

Table 8.3: Reasons why the SHV's hotel sector has adapted to climate change ...............254

Table 8.4: Comparison of the adaptation barriers between the findings and the literature 259

Table 8.5: Comparison of the enabling factors for adaptation between the findings and literature .263

Table 8.6: The perceived barriers and enabling factors affecting adaptation between hotel sizes 266

Table 8. 7: The perceived enabling factors affecting adaptation between participants' nationalities

Table 8. 8: The perceived barriers and enabling factors to adaptation between length of residence in SHV 273

Table 8.9: The perceived enabling factors for adaptation between participants' experiences .276

Table 8.10: The perceived adaptation barriers between hotel standards .........................278

Table 8.11: The perceived enabling factors influencing adaptation between hotel ownerships .279

Table 8.12: The perceived enabling factors for adaptation between hotel locations .281

Table 8. 13: Comparison of the initial and revised conceptual framework 290 


\section{List of Figures}

Figure 2. 1: Conceptual framework for CCA in the coastal hotel sector

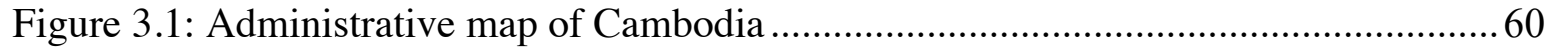

Figure 3. 2: Cambodia: Visitor exports and international tourist arrivals .........................6 62

Figure 3.3: Organisational structure of the Ministry of Tourism ....................................66

Figure 3.4: Average Temperature from 1960-2099 ......................................................... 70

Figure 3.5: Timeline of flooding from 1996 to 2013 ............................................. 72

Figure 3.6: Cambodian institutional framework for addressing climate change................ 76

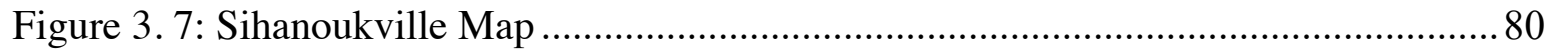

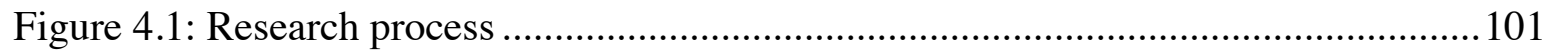

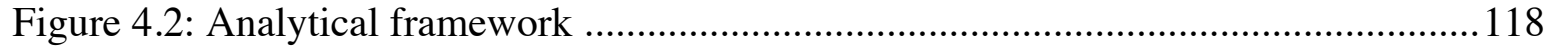

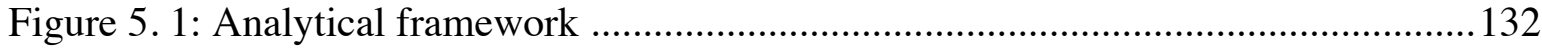

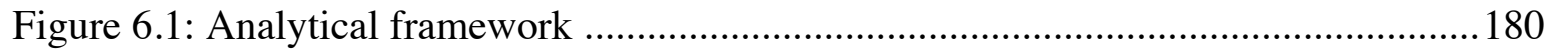

Figure 7. 1: Analytical framework .....................................................................219

Figure 8. 1: A revised conceptual framework for hotel sector's adaptation.....................288 


\section{List of Acronyms}

\begin{tabular}{ll} 
AC & Air Conditioner \\
ADB & Asian Development Bank \\
CCA & Climate change adaptation \\
CCCA & Cambodia Climate Change Alliance \\
CSIRO & Commonwealth Scientific Industrial Research Organization \\
DANIDA & Denmark's development cooperation \\
DOE & Department of Environment \\
DOT & Department of Tourism \\
DP & Development Partners \\
EU & European Union \\
GDP & Gross Domestic Product \\
GEF & Global Environment Facility \\
GM & General Manager \\
H1 & Hotel 1 (First participant) \\
H2 & Hotel 2 (Second participant) \\
HR & Human resource \\
IPCC & Intergovernmental Panel on Climate Change \\
JICA & Japan International Cooperation Agency \\
LDC & Least Developed Countries \\
MEF & Ministry of Economy and Finance (Cambodia) \\
MOE & Ministry of Environment (Cambodia) \\
MOT & Ministry of Tourism (Cambodia) \\
MoWRAM & Ministry of Water Resources and Meteorology \\
NAPA & National Adaptation Programme of Action to Climate Change \\
NCSC & National Coastal Steering Committee \\
NTP & National Tourism Policy \\
RGC & The Royal Government of Cambodia \\
SD & Standard Deviation \\
SDGs & Sustainable Development Goals \\
SHV & Sihanoukville \\
SLR & Sea Level Rise \\
UN-Habitat & United Nations Human Settlements Programme \\
UNDP & United Nations Development Programme \\
UNEP & United Nations Environment Programme \\
UNFCCC & United Nations Framework Convention on Climate Change \\
UNTAC & United Nations Transitional Authority in Cambodia \\
UNWTO & United Nations World Tourism Organization \\
USD & United States dollar \\
WMO & World Meteorological Organization \\
WTO & World Tourism Organization \\
WTTC & World Travel \& Tourism Council \\
\hline &
\end{tabular}




\section{CHAPTER 1. INTRODUCTION}

\subsection{Background of the study}

Tourism is one of the world's biggest and fastest-growing economic sectors with the international tourist arrivals growing 6\% in January-April 2018 compared to the same period in 2017 (UNWTO ${ }^{1}, 2018$ b). Whilst tourism has grown significantly, coastal tourism is growing as the fastest sub sector because the world's population mostly live in coastal areas, which tourists visit the most (Lakshmi \& Shaji, 2016). Of all the world's tourism attractions, about $30 \%$ are on the coast or within the coastal zone and the economy of that area depends on coastal tourism (Ghosh, 2012). Hall and Page (2014) explain that "coastal tourism embraces the full range of tourism, leisure, and recreationally oriented activities that take place in the coastal zone and the offshore coastal waters. These include coastal tourism development (accommodation, restaurants, food industry, and second homes) and the infrastructure supporting coastal development (e.g. retail businesses, marinas, and activity suppliers)" (p. 291). Coastal tourism creates an interdependence between the coastal resources and the individuals who live and earn in the area (Ghosh, 2011). Coastal tourism is important to the global economy and its sustainable impacts on coastal communities are mainly through the social, economic and environmental factors (Sharpley, 2006). The economic significance of coastal tourism is unquestionable. It has become one of the main economic sources for many countries and regions (Lakshmi \& Shaji, 2016; Moreno \& Amelung, 2009; Moreno \& Becken, 2009). For example, coastal tourism establishes a source of employment for many communities, countries and regions globally (Moreno \& Amelung, 2009).

However, climate change is the greatest challenge for tourism in the 21 st century (WMO ${ }^{2}$ et al., 2008). Climatic changes affect a whole range of environmental resources that are a great attraction for tourism such as environmental quality, which can deter tourist flows (Cabrini, 2009; Schott, Milfont, \& Reisinger, 2010). This situation becomes worse if tourism is considered through the lens of socio-economic factors of climate change (Grasso, Moneo,

\footnotetext{
${ }^{1}$ United Nations World Tourism Organization

${ }^{2}$ World Meteorological Organization
} 
\& Arena, 2014). Climate change has also threatened coastal tourism (Amelung, Moreno, \& Scott, 2008; Moreno \& Becken, 2009) through storms and severe climatic incidents, damage to infrastructure, coastline erosion, SLR, flooding, water contamination and water shortfalls (Simpson et al., 2008). Despite challenges caused by climate change, the interrelationships between tourism/coastal tourism and climate change have not been sufficiently studied (Amelung et al., 2008; Schott et al., 2010).

Two tools widely used to respond to climate change are adaptation and mitigation (Parry, 2007). The overall effect of mitigation to climate change is 'global' while the positive effect of adaptation is 'local to regional' (Füssel \& Klein, 2006). Adaptation is most needed for tourism in developing nations (Scott et al., 2009), especially for a rapidly developing tourism destination like Cambodia and its coastal zone.

\subsection{Research context}

After the Khmer Rouge regime collapsed in 1979, Cambodia's political situation remained unsteady due to political and rebellious movements of Khmer Rouge troops in the western part of the country. It was not until 1998, when the Khmer Rouge top leader, Pol Pot passed away, that the people could enjoy real peace. Since then, Cambodia's economy has grown slowly but steadily. The country's economy has depended on four main sectors: agriculture, tourism, garment manufacturing and construction. Cambodia's tourism has grown rapidly over the last decade, with a near doubling in visitor arrivals over a seven-year period from 2.8 million in 2011 to 5.5 million in 2017 . It is expected to grow further by $3.2 \%$, with $5,552,000$ international tourist arrivals in 2018 (WTTC, 2018a). In 2028, the projection is 7,969,000 international tourist arrivals that will generate USD 5 billion and 80,000 jobs (RGC ${ }^{3}, 2012 ;$ WTTC, 2018a).

Cambodia's Sihanoukville (SHV) is considered a rising star in the south east and one of the newly attractive coastal destinations in Asia (Ong \& Smith, 2014). Located on the Gulf of Thailand, SHV is $230 \mathrm{~km}$ southwest of Phnom Penh, the Cambodian capital. SHV is the hub

\footnotetext{
3 The Royal Government of Cambodia
} 
of coastal tourism and beaches, including sea activities along the major beaches, with fishing, scuba diving and snorkelling on the island areas as the main tourism activities (Ong \& Smith, 2014).

While enjoying rapid tourism growth, Cambodia has faced the challenge of climate change and developing countries are more vulnerable and have weak adaptive capacities for climate change responses (Smit \& Wandel, 2006). The UN (2017) has classified Cambodia as one the least developed countries (LCDs) and the UN's (2017) criteria for LCDs identifies that structural vulnerability to rapid economic and environmental changes is high for these countries. Cambodia was also among the world's top 10 countries with the highest exposure to natural hazards and climate change (World Risk Report, 2012) and the most vulnerable to climate change in the South East Asian region (Yusuf \& Francisco, 2009). Cambodia lost $3.1 \%$ of its annual GDP to extreme events (Harmeling \& Eckstein, 2013). Climate change threatens its coastal zone in particular, through SLR, floods, drought, increased storm frequency, increased temperature and erratic rainfall (MOE et al., 2015). All of these have already affected the living conditions, agricultural activities, other production systems, infrastructure, coastline areas, and ecological system in the coastal provinces (MOE et al., 2015).

In order to respond to climate change, Cambodia has applied 'no-regrets' adaptation options at the national level (RGC, 2006). This approach places an emphasis on maximising positive and minimising negative impacts of nature-based adaptation strategies and options (Ali Raza Rizvi et al., 2014). Cambodia's 'no-regrets' adaptation measures can be separated into three distinctions: (i) infrastructure development, (ii) capacity building/training, and (iii) awareness raising/education (RGC, 2006). Meanwhile, the country has enacted various legal documents related to climate change. For example, in 2015 the government initiated the first tourism-climate change document entitled Climate Change Action Plan in Tourism Sector 2015-. There are three goals/objectives within the plan: First, it intends to reduce climate change vulnerability of people and tourism sector, and in particular the most vulnerable and significant systems (societal and natural), and cultural heritage. Second, it aims to shift towards a green development pathway by encouraging low-carbon technologies and 
development. Third, it is to promote public participation and awareness in climate change response activities in the sector. Within the plan, MOT is expected to receive a total budget estimation of USD2,900,000 for implementing the actions from 2015 to 2018.

The efforts in climate change responses contributed to the identification of important actions. For example, due to high vulnerability, the existing studies have suggested SHV to implement appropriate and urgent adaptation (MOE et al., 2015). It is also recommended that the coastal hotels in the area need to develop strategies to deal with climate vulnerability so as to sustain the many economic benefits brought about by tourism (also see Dzoga \& Koske, 2014).

\subsection{Motivation for the research}

Cambodia is a post-conflict country, which has seen very rapid tourism development over the last decade. While Cambodia is reaping benefits from tourism, several key international agencies anticipated future challenges, which include climate change impacts. Climate change has mostly threatened the country's coastal zone (MOE, 2015; UNWTO, 2014). This has increased my personal concern over the issue. My experience of working in the Ministry of Tourism (MOT) Cambodia, in the coastal tourism sector is the strongest motivation for the research. The work allowed me to frequently travel back and forth from my home in Phnom Penh, the capital, to SHV, and to familiarise myself with the place and make connections with people. In the meantime, I feel that SHV is not only an attractive place but also an environmentally sensitive area. In addition, my academic work also contributes to the intrinsic motivation. In my master's thesis at a Japanese university, I worked on the National Tourism Policy (NTP) in Cambodia and SHV's coastal tourism. I began to wonder why the NTP is too broad and less focused on environmental issues, especially at the coastal zone, which is one of the country's most attractive tourist areas. What about more important issues such as the interrelationship between the coastal tourism and climate change?

In early 2015, prior to the commencement of this study, I had planned to conduct my research on tourism sustainability. After reading a book entitled Tourism and the Implications of Climate Change: Issues and Actions by my primary supervisor, Christian Schott, it strongly 
influenced and motivated me. I began to reconnect the concept of the book with the context of Cambodia's tourism. With curiosity and concern, I became committed to conduct a study in the area. Fink (2000) argues that this allows a researcher to feel very personally involved in the steps of the research process. The working and research experience and relationship with the study site also enabled the researcher to gain access to selective groups of participants in order to conduct the interviews and field observations.

\subsection{Research aims and questions}

Considering the importance of motivations stated in the above section, this thesis takes the form of a climate change-focused case study of the coastal hotel sector in SHV. The central concern of the thesis is to determine whether Sihanoukville's hotel sector has adapted to climate change and to critically examine the barriers and enabling factors that are influencing adaptation. This overarching topic is divided into five sequential sub-aims:

\footnotetext{
Aim 1: To assess the vulnerability of Sihanoukville's coastal tourism to climate change The first aim assesses the climate change vulnerability of SHV's coastal tourism (destination level) through perception-based data on environmental and socio-economic factors, supported by existing scientific evidence. The purpose is to identify the vulnerability, which is prerequisite before determining adaptation actions. The assessment is guided by key studies such as Moreno \& Becken, (2009), Smit \& Wandel (2006), UNWTO (2014) and MOE et al. (2015) in explaining that identifying key vulnerabilities is prerequisite knowledge before further identifying the adaptation responses for tourism. Without understanding the vulnerability of a destination, it is not known as to what extent the adaptation is suitable and effective (Moreno \& Becken, 2009; Walmsley, 2012).
}

\section{Aim 2: To assess the vulnerability of Sihanoukville's hotel sector to climate change}

Similar to the first aim, the second aim assesses the climate change vulnerability of SHV's hotel sector (sector level) through the perceptions on environmental and socio-economic factors, supported by existing scientific data. The purpose is to identify the vulnerability of 
the hotel sector, which is prerequisite before determining the hotel sector's adaptation actions. The assessment is also guided by key studies as outlined above.

\section{Aim 3: To determine whether/how the hotel sector adapts to climate change impacts}

After the vulnerability was assessed in aims 1 and 2 respectively, this third aim addresses the key question whether the hotel sector has adapted to climate change. As guided by key studies outlined in aims 1 and 2, this aim also determines the adaptation measures being adopted by appreciation of an adaptation framework by WMO et al. (2008) and Simpson et al. (2008), which comprises technical, managerial, policy, research and education.

\section{Aim 4: To identify the most significant barriers and enabling factors in the context of the hotel sector's adaptation}

After the vital relationship between vulnerability and adaptation was established in aims 1, 2 and 3 respectively, the fourth aim investigates the perceived criticality levels of the barriers to and enabling factors for adaptation because little research has been done to address the issue. Previous research has focused on the status of adaptation measures and barriers and enabling factors but ignored the perceived differences in importance of these barriers and

enabling factors. The perception of barriers and enabling factors to CCA (informed by the literature in CCA in the context of developing countries) will be investigated by asking respondents to rate each potential barrier and enabling factor.

\section{Aim 5: To critically examine the barriers and enabling factors to adaptation according to the hotel attributes and participants' backgrounds}

The main goal of the fifth aim is to explore the extent to which hotel attributes (star rating, ownership, hotel size, hotel location, hotel age and business format) and participants' backgrounds (level of education, age, experience, nationality and length of residence in SHV) in the hotel sector in SHV have impacted on their perceptions of barriers and enabling factors in CCA. Previous studies have not investigated the role of hotel attributes and respondents' backgrounds in their perceptions of barriers and enabling factors in regard to CCA in tourism but arguably differences will exist between hotels and owners/managers (e.g. Su, Hall, \& Ozanne, 2013; Su, 2014). 


\subsection{Research methodology}

Adopting a postpositivist approach, the study conducted semi-structured interviews with 50 hotel respondents and field observations in SHV. To have flexibility in data collection, be able to gather 'rich' data and explain complex phenomenon, mixed method (qualitative \& quantitative) and a single-case study were employed. To guide the research process, both a conceptual framework and a research process diagram were developed.

Prior to exploring adaptation, the vulnerability of SHV's coastal tourism (destination level) and its hotels (sector level) were assessed through the perceptions on environmental and socio-economic factors, supported by existing scientific evidence (see Hiwasaki, Luna, Syamsidik, \& Marçal, 2015; Kelly \& Adger, 2000; Moreno \& Becken, 2009; Vulturius \& Gerger Swartling, 2015). Adopted from WMO et al. (2008) and Simpson et al. (2008), an adaptation framework that includes technical, managerial, policy, research and education was subsequently used to determine the hotels' adaptation actions. Within the hotels' adaptation, the greatest barriers and enabling factors in CCA were then examined by measuring mean scores on a six-point rating scale, with 0 being 'uninfluential' barrier and 5 being 'very major' barrier, and 0 being 'uninfluential' enabling factor and 5 being 'very important' enabling factor. Guided by the analytical framework, the study initially analysed data from the whole case and subsequently cross-case analysis was employed for descriptive test and nonparametric test to explore the extent to which hotel attributes and participants' backgrounds in the hotel sector (in SHV) have impacted on their perceptions of barriers and enabling factors in CCA.

\subsection{Thesis outline}

The thesis has 9 chapters. Chapter 1 has briefly introduced the background information of the study, research problem, motivation of the research and the research aims as well as an overview of the research methods. The remaining chapters of the thesis are organised as follows:

Chapter 2 contextualises the study in respect to the relevant literature by reviewing previous research on the topic. While the chapter presents the socio-economic importance of 
tourism/coastal tourism and the accommodation sector, it also outlines one of tourism's greatest challenges, which is climate change. The chapter summarises the existing body of knowledge on vulnerability assessment through the environmental and socio-economic factors and perception-based data, supported by existing scientific evidence. After summarising the approach in vulnerability assessment, it identifies the gap between CCA and tourism/coastal tourism/accommodation sector and introduces an adaptation framework, which includes technical, managerial, policy, research, education and behavioural adaptation. In the last part, informed by literature, the chapter presents the barriers and enabling factors to CCA associated with tourism/ hotel sector in the context of developing countries. It also highlights the merits of distinguishing between the criticality levels (significance/importance levels) of the barriers (e.g. limited resources) and enabling factors (e.g. sufficient resources). The chapter concludes by presenting the observed barriers and enabling factors in CCA that could vary according to hotel attributes (e.g. hotel size) and participants' backgrounds (e.g. level of education and level of experience) (e.g. Su et al., 2013; Su, 2014).

Chapter 3 sets the context for the study by introducing the research site in SHV, Cambodia by focusing on two issues: tourism and climate change. The first half of the chapter describes Cambodia's modern history, tourism and climate change. It also highlights the tourism growth in Cambodia. Meanwhile, it also highlights past and future climate change records using existing scientific data. In a similar pattern, the second part focuses on SHV with a particular concentration on tourism and climate change.

Once the previous research on the topic is analysed, chapter 4 describes and justifies the data collection method used. The chapter introduces the adopted research paradigm and explains the research approach, which consists of mixed methods (qualitative \& quantitative) using a case study. The chapter features data collection techniques detailing how data collection was conducted using semi-structured interviews, informal site visits and document analysis. It introduces the research participants of the study. It details the data preparation, starting from coding of the template, creation of the initial template, revision of the template, finalising of the template, and interpretation and presentation of template analysis. The chapter presents the analytical framework to guide the analysis and the analytical methods using overall case 
and subsequently cross-case analysis. It presents the ethical considerations of the study and acknowledges the limitations of the study.

The findings are presented in three chapters. Based on the analytical framework, chapter $\mathbf{5}$ introduces vulnerability assessment outcomes through environmental and socioeconomic factors perceived by the hotel participants in SHV; their perceptions are subsequently supported/validated by the existing scientific data in the area. It proceeds to identify the actual adaptation measures employed by the hotel sector by adopting an adaptation framework. As such this chapter addresses research aims 1,2 and 3. After the vital link between vulnerability and adaptation has been established, chapter 6 outlines the perceived adaptation barriers and enabling factors (informed by the literature in CCA in the context of developing countries). This finding leads to the identification of the criticality level of each adaptation barrier or enabling factor or the classifications of adaptation barriers and enabling factors to CCA in the hotel sector into the most critical, medium and the least critical barriers and enabling factors. This addresses research aim 4. Chapter 7, the final part of the findings, critically examines the perceived barriers and enabling factors affecting adaptation according to the hotel attributes and participants' backgrounds (the cross-case analysis). Using descriptive tests and independent sample tests, the findings show that the score means of respondents regarding the perceived barriers and enabling factors to CCA vary according to hotel attributes (star rating, ownership, hotel size, hotel location, hotel age and business format) (or/and) and participant's background (level of education, age, experience, nationality and length of residence in SHV). This answers research aim 5.

Integrating the research findings in chapter 5,6 and 7, chapter $\mathbf{8}$ discusses the findings in relation to the theoretical body of knowledge introduced in the literature review. Prior to that, the chapter reintroduces the major findings of the thesis. The main purpose of this chapter is to determine to what extent the findings are different or similar to the broader literature. The chapter revisits the initial conceptual framework in light of the key findings and proposes a revised conceptual framework for adaptation by the coastal hotel sector of developing country. 
Finally, drawing together the preceding chapters, chapter 9 concludes by synthesising the findings and revisiting the research aims. This chapter emphasises the key findings and contributions to theory and practices. The major contribution of the findings will be of interest to academics in the fields of CCA and of help to relevant stakeholders such as the public sector, private sector (tourism industry, hotels and other accommodation sectors), and IOs \& NGOs ${ }^{4}$. The study has put vulnerability, adaptation and its perceived barriers and enabling factors into a more detailed and coherent context, particularly towards the hotel sector. At the same time, the chapter acknowledged the limitations of the study. The thesis concludes with an indication of directions for future research areas.

\footnotetext{
${ }^{4}$ In Cambodia, a range of organisations are referred to by many designations such as IOs and NGOs. They are different in terms of their missions (who they work with and where they work) and budgets (amount and source of budget: bilateral or multilateral). NGOs are local and Cambodia-based. They are operated by either foreigners or Cambodians with a couple of people assisting in the development of domestic and family-based sectors. IOs are operated by development partners. They are the United Nations and all its family agencies such as the World Food Programme (WFP), UN Development Programme (UNDP), Food and Agriculture Organisation (FAO), etc. Some are labelled as IOs including International Labour Organisation (ILO), the World Trade Organisation (WTO) and several others.
} 


\section{CHAPTER 2: LITERATURE REVIEW}

\subsection{Introduction}

The previous chapter outlined the importance of tourism, its challenges such as climate change, and identified the problems/questions that must be robustly addressed to explore this issue further. This chapter reviews literature integrating concepts and topics that provide the theoretical background for this research. The major purpose of this chapter is to review literature on two main interdependent themes: vulnerability and adaptation relative to tourism in the context of developing countries. To reflect this purpose, the chapter first presents the importance of tourism/coastal tourism and the accommodation sector. Second, it outlines the climate change effects on tourism/coastal tourism and accommodation sector by reviewing combined scientific and local knowledge on environmental and socio-economic vulnerabilities. Third, it proceeds to outline adaptation options relative to tourism/coastal tourism and the accommodation sector. Subsequently, it presents an adaptation framework necessary for tourism businesses (e.g. hotel). Finally, potential barriers and enabling factors to adaptation are reviewed, focusing on the context of developing countries. All information will be synthesised in a conceptual framework.

\subsection{Significance of tourism and coastal tourism and accommodation and hotel sector}

\subsubsection{Tourism and coastal tourism}

Tourism has become one of the world's biggest and fastest-growing economic sectors with international tourist arrivals, growing 6\% in January-April 2018 compared to the same period in 2017 (UNWTO, 2018a). Whilst tourism has grown significantly, coastal tourism is growing as the fastest subsector (Lakshmi \& Shaji, 2016) because of all the world's tourism

attractions, about $30 \%$ are on the coast or within the coastal zone (Ghosh, 2012). Hall and Page (2014) explain that, "coastal tourism embraces the full range of tourism, leisure, and recreationally oriented activities that take place in the coastal zone and the offshore coastal waters. These include coastal tourism development (accommodation, restaurants, food industry, and second homes) and the infrastructure supporting coastal development (e.g. retail businesses, marinas, and activity suppliers)" (p. 291). 
In 2010, approximately 2.7 billion people lived in coastal areas - over $40 \%$ of the world's population (Tobey et al., 2010). Coastal tourism creates an interdependence between the coastal resources and those who live and earn in the area (Ghosh, 2011). Coastal tourism is important to the global economy, and its sustainable impacts on coastal communities are mainly through the social, economic and environmental factors (Sharpley, 2006). The economic significance of coastal tourism is unquestionable. It has become one of the main economic sources for many countries and regions (Lakshmi \& Shaji, 2016; Moreno \& Amelung, 2009; Moreno \& Becken, 2009). For example, coastal tourism establishes sources

of employment for many communities, countries and regions globally (Moreno \& Amelung, 2009). It is undeniable that growth of tourism is largely due to its many subsectors such as the accommodation/hotel sector. The next section highlights significant contributions of the accommodation/hotel sector to the tourism sector.

\subsubsection{Accommodation and hotel sector}

The accommodation sector is a fundamental part of the total tourism product and industry (Aminian, 2012; Poudel, 2013). It is "the largest and most ubiquitous subsector within the tourism economy" (Cooper et al., 1998, p. 313). Accommodation includes hotels, motels, vacation homes, vacation villages, and caravans amongst others (Walmsley, 2012). Accommodations in the form of small to medium-sized hotels and budget to luxury hotels also play a role in tourism attraction (Ekinci, Prokopaki, \& Cobanoglu, 2003).

The hotel industry has seen very rapid growth in recent years (Xia, Quan Vu, Lan, Law, \& $\mathrm{Li}, 2018$ ). Hotels and other lodging businesses are one of the significant areas of the hospitality sector (Hall et al., 2016; Poudel, 2013) and the accommodation sector (Poudel, 2013). The hotel's mechanisms are well formulated for tourism (Dzoga \& Koske, 2014). The hotel business promotes economic outcomes, attracts investments and creates employment (Humair, 2011; Lado-Sestayo, Vivel-Búa, \& Otero-González, 2017). The hotel sector plays a fundamental role in attracting visitors to a destination by providing comforts and conveniences for guests (Poudel, 2013). Hotels often employ crucial different ways to fill the needs of the various customers in the tourism market (O’Connor \& Frew, 2002). 
The hotel sector is not only a housing modernisation, but is also a dynamic sector that can affect various key economic sectors such as the banking and building/construction sectors. The hotel sector revitalises energy, transport, and communication networks (Humair, 2011). For example, it is undeniable that hotel development leads to the creation of employment; this is the most powerful impact of the hotel sector. Its further impact is that the arrival of tourists is connected to the massive purchasing power that includes medical care, entertainments, souvenirs, excursions and other luxury objects (Humair, 2011). Wang, Dai, $\& \mathrm{Xu}$ (2018) argue that through the hotels' generated income, hotel investment potentially increases firm value, land opportunism and real estate appreciation. This hugely increases market value.

The hotel sector is a competitive industry that requires high management goals (LadoSestayo et al., 2017; Rhee \& Yang, 2015), especially in regard to its environmental performance (Page, 2009). Hotels that respect the principles of sustainable development and are environmentally friendly to reflect guests' concerns (Page, 2009) may be even more attractive to stay at.

\subsection{Climate change, tourism, coastal tourism and accommodation sector}

\subsubsection{Climate change}

In the IPCC 5 synthesis report (2007), climate change is defined as "a change in the state of the climate that can be identified (e.g. using statistical tests) by changes in the mean and/or the variability of its properties, and that persists for an extended period, typically decades or longer. It refers to any change in climate over time, whether due to natural variability or as a result of human activity" (IPCC, 2007, p. 30). Recently, emissions are recorded as the highest in history (IPCC, 2014). Because human activities have influenced the climate system, recent climate change in turn has led to an extensive impacts on the natural systems and humans (IPCC, 2014). By the end of the 21st century (expecting between 2081-2100) compared to 1986-2005, the rising global average surface temperature is projected to be $0.3^{\circ} \mathrm{C}$ to $1.7^{\circ} \mathrm{C}$ (the lowest projection) or $2.6^{\circ} \mathrm{C}$ to $4.8^{\circ} \mathrm{C}$ (the highest projection) (IPCC, 2014). On average,

\footnotetext{
${ }^{5}$ Intergovernmental Panel on Climate Change
} 
global sea level rose by $0.19 \mathrm{~m}$ (or between 0.17- 0.21m) from 1901-2010 (IPCC, 2013). Abdi, Creighton, and Nahavandi (2013) argue that climate change consequences threaten lives, particularly for the next generations. Over the period 2001-2010, over 370,000 people lost their lives due to severe climate conditions such as cold spells, heat waves, storms, floods, and drought that have increased by $20 \%$ compared to the past decade (WMO, 2013).

Developing countries have been heavily affected and will be affected by extreme weather events more than developed nations (Fankhauser \& McDermott, 2014). There are three main reasons: First, these countries have higher exposure to climate risk due to a semi-arid climate (Fankhauser \& McDermott, 2014). Second, the populations of low-income countries live in hazard zones (Fankhauser \& McDermott, 2014). Third, these countries have lower adaptation measures or higher adaptation deficit (Barr, Fankhauser, \& Hamilton, 2010).

Accumulating evidence suggests that climate change is recognised as the greatest challenge to many main economic sectors including (coastal) tourism of developing countries. The section below continues to present the key challenges (coastal) tourism has faced in changing climate.

\subsubsection{Climate change and tourism/coastal tourism}

Despite its economic importance (see section 2.2.1), tourism growth is facing a number of challenges, one of which is climate change impact. Climate change must be regarded as the most critical challenge to the tourism sustainability in the 21 st century (WMO, UNEP, \& WTO, 2008). Climatic changes affect a whole range of environmental resources that are a great attraction for tourism such as environmental quality, which can deter tourist flows (Cabrini, 2009; Schott et al., 2010). This situation becomes worse if tourism is considered through the lens of socio-economic factors (Grasso et al., 2014).

Climate change has highly threatened coastal and marine conditions (IPCC, 2007; Moreno \& Amelung, 2009; Walmsley, 2012) especially coastal and island destinations (Moreno \& Becken, 2009). The climate change impacts include increased variability in precipitation storms and severe climatic conditions, beach erosion/SLR, damage to infrastructure, 
flooding, water shortfalls and water contamination (Simpson et al., 2008). These have threatened sustainability of coastal tourist destinations (Moreno \& Amelung, 2009; Shakeela \& Becken, 2014) because the impacts are not only on the environmental, physical and social resources essential for tourism but also the perceptions, convenience and safety of populations (Moreno \& Amelung, 2009).

Coastal communities worldwide have experienced social and environmental changes that are becoming progressively unpredictable and complex, principally because of the combined and individual effects of climate change (Bennett \& Dearden, 2013). Most effects are seen in developing countries, which have led to a number of environmental and socio-economic problems (Smit \& Wandel, 2006) such as environmental risks (drinking water loss, draught, extreme temperature pattern, SLR, etc.) and economic risks (beach loss, property damage from hurricanes, tourism declines, etc.) (Carlton \& Jacobson, 2013).

Identifying key vulnerabilities is prerequisite knowledge before further understanding on adaptation responses for coastal tourism (Smit \& Wandel, 2006). For example, coastal tourism destination managers are recommended to understand their susceptibility to climatic changes and then to formulate suitable adaptation (Moreno \& Becken, 2009). It is not until vulnerability is assessed that adaptation can be formulated and effectively implemented. Without understanding vulnerability of a destination, we cannot know to what extent the adaptation was, is, and will be implemented, and whether it is suitable and effective (Moreno \& Becken, 2009; Walmsley, 2012). To achieve sustainable coastal tourism, these impacts on coastal communities, along with their socio-economic and environmental issues, must be managed efficiently (Ghosh, 2012). Climate change also significantly affects tourism and its subsectors such as accommodation. The effects of climate change on accommodation are elaborated in the following section.

\subsubsection{Climate change and accommodation sector}

The accommodation sector is also highly exposed to environmental impacts (Aminian, 2012; Poudel, 2013). Due to the inability to simply relocate from one location to another, hotels' adaptive capacity is lower than other tourism businesses or tourists (Simpson et al., 2008; Su 
et al., 2013; Walmsley, 2012; WMO et al., 2008). Global warming has direct effects on the accommodation sector, for instance increasing cooling costs for responding to rising temperatures (Becken, 2010; Poudel, 2013). Climate change constitutes the leading cause of temperature drops, for example, in low-temperature countries (Poudel, 2013). This has made tourism businesses such as accommodation sector face mounting pressure to improve their environmental performance in a greater urgency (Tzschentke, Kirk, \& Lynch, 2008).

\subsection{Climate change vulnerability}

In the $4^{\text {th }}$ Assessment Report of IPCC 2007, vulnerability is defined as "the degree to which a system is susceptible to, and unable to cope with, adverse effects of climate change, including climate variability and extremes. Vulnerability is a function of the character, magnitude, and rate of climate change and variation to which a system is exposed, the sensitivity and adaptive capacity of that system" (IPCC, 2007, p. 6). Vulnerability is conceptualised differently by various scholarly communities (Füssel, 2007; Fussel \& PIK, 2010; Hiwasaki et al., 2015). For example, Adger, Arnell, and Tompkins (2005) and Ford et al. (2015) recommend that vulnerability assessments need to consider exposure, sensitivity, and adaptive capacity. Maiti et al. (2015) evaluate that the net effect of exposure and sensitivity (potential impact) on the adaptive capacity was calculated as the result of vulnerability assessment. However, Schröter, Polsky, and Patt (2005) argue that there is no globally best standard to assess vulnerability. Vulnerability assessment should be 'placebased', which means a study area that consists a group or village/villages that are not a group or country/countries. To assess vulnerability, this study therefore takes one approach, assessing through the perceptions on environmental and socio-economic factors, supported by the combined scientific data and local knowledge. These assessment approaches are elaborated as follows:

\subsubsection{Vulnerability assessment through environmental and socio-economic factor}

As indicated above, vulnerability is conceptualised differently by various scholars. Kelly and Adger (2000) argue that developing countries face the most impact of climate change, which has led to a number of environmental and socio-economic problems. In tourism, Kelly and Adger (2000) and Moreno and Becken (2009) conduct the assessment through environmental 
and social, economic (or socio-economic) factors since these factors occur at local through to national scales. Taking this into account, the next section discusses the assessment of environmental and socio-economic vulnerability.

\subsubsection{Environmental vulnerability}

Yoo et al. (2014) define environmental vulnerability as "a function of environmental exposure, sensitivity, and adaptive capacity" (p. 170). Due to the increasing global environmental change in recent years, research on the concept of vulnerability has attracted greater attention (Schröter et al., 2005). Schröter et al. (2005) argue that for minimising the significant harm concerned with global climate change, societies and people are required to have an accurate evaluation of the vulnerability of the environmental systems they are related to, and associated adaptation obstacles and opportunities. Consequently, environmental vulnerability assessment serves as a good foundation for environmental management evaluations as it provides an objective standard to prioritise implementation (Yoo et al., 2014). Managing environmental vulnerability is a precondition for global sustainable development (Yoo et al., 2014).

In coastal areas, Bennett et al. (2014) explain that coastal communities globally are experiencing a broad array of changes, which include environmental, climatic and economic factors. The coastal zone, an area associated with relative high likelihood, gets impacted owing to a set of environmental dimensions (Sahoo, Dhar, \& Kar, 2016). Zou and Yoshino (2017) explain that environmental vulnerability is relevant to the risk of damage to the natural environment, including a specific ecosystem type. Environmental vulnerabilities include the effects of SLR, and other relative events including hurricanes, cyclones, and seismic activity (Scheyvens \& Momsen, 2008). Therefore, identifying environmental vulnerability is a crucial step for a protection framework of environmental sustainability (Sahoo et al., 2016).

There is a need for different assessment approaches towards local environmental vulnerability because most of the existing environmental vulnerabilities have been assessed through historical records of natural disasters or pollution (Yoo et al., 2014). For this reason, Sahoo et al. (2016) argue that assessing environmental vulnerability also requires denoting 
data that are associated with a particular location, especially socio-economic data, which are outlined in the next section.

\subsubsection{Socio-economic vulnerability}

Socio-economic vulnerability predominantly contributes to climate change (Santos-Lacueva, Clavé, \& Saladié, 2017). For example, climate change impacts affect income-generating activities at both the community and national levels (Grasso et al., 2014). Socio-economic and climate scenarios are often combined and geared towards the increase of the climate change impacts and vulnerabilities within different sectors (Berkhout et al., 2013). Social and economic factors have a significant effect on the adaptive capacity and susceptibility of different social systems (Berkhout et al., 2002). Social and economic losses result from current climate change and variability conditions have completely pressurised the strategic range of the community systems (Zuka, 2014).

Assessing vulnerability through socio-economic factors could reap many benefits. Socioeconomic scenarios comprise a vital tool for exploring the long-term effects of anthropogenic climate change and available response choices (Kriegler et al., 2012). Assessing climate impact needs to consider two interrelated processes: Climatic changes and socio-economic changes. Combining these two is possible in order to assess the exposure of future ecosystems and societies harmed by climate change (Berkhout et al., 2002). Bele et al. (2014) argue that the analysis of social and economic vulnerabilities strengthens information processes for identifying priorities for adaptation.

Nevertheless, Lorenzoni et al. (2000) argue that feedback between climatic and socioeconomic systems, particularly adaptation measures, tends to be ignored or analysed in an extremely basic way. Berkhout et al. (2002) also claim that prospective change in socioeconomic systems has not been well considered with a critical analysis of climate change effects. The above authors also notice that only few studies have been conducted to address the issue systematically. This may result from the insufficiency of current information about social and economic change (Metcalf, van Putten, Frusher, Tull, \& Marshall, 2013). 


\subsubsection{Vulnerability assessment through combined scientific and local knowledge}

Climate change evidence is documented by scientific records, but to what extent these data are reliable/trusted depends on people's knowledge, understanding and perception. As indicated above, vulnerability has been assessed differently. More recent literature explains that the increasing number of research studies and activities that combine or compare science with local knowledge recognise that climate change-related challenges cannot be solved by science or local knowledge alone (Hiwasaki et al., 2015). Instead, research has now given increasing attention to combining both local and scientific knowledge (Leary, Conde, Kulkarni, Nyong, \& Pulhin, 2008). Combining outside knowledge (science) with local knowledge has helped communities to manage several crises and conflicts (Scaglion, 2010).

In climate change responses, it is true that knowledge of scientific information likely improves individual and collective responses to issues affected; this would seem to be widely trusted not only by scientists themselves but also by the news media, educators, policymakers, managers, and participants of the public (Cone et al., 2013). In interaction with climate change scientific information, the sender (scientists) is generally conceived of as an expert while the receiver (e.g. normal people) is generally considered to be non-expert (Cone et al., 2013). To make communities and individuals understand and adapt to climate change, it is not just simply providing facts about climate change science and its conclusions; it requires something more (Cone et al., 2013), such as the local acceptance, by simplifying information and making it trustworthy. Käkönen et al. (2014) continue that because climate change is considered a complicated issue domain, it requires scientific knowledge to interpret such complexity (Käkönen et al., 2014). Yet often the scientific climate change information is not specific for local decision makers and implementers (Cone et al., 2013) especially in many developing countries (Bangay \& Blum, 2010).

Literature indicates that a majority of individuals have a tendency to respond to concerns or risks only if they perceive them as immediate impacts and personally relevant (Moser \& Dilling, 2004). People's beliefs and perceptions affect implementation of CCA (Simonet \& Fatorić, 2016). If climate science opposes one's personal experience, it can be a substantial cognitive obstacle to engage with climate change (Lorenzoni, Nicholson-Cole, \& Whitmarsh, 
2007; Vulturius \& Gerger Swartling, 2015). If people perceive climate change risks as an insignificant issue, they are unwilling to begin adaptive action (Dow, Berkhout, \& Preston, 2013). In line with these, Marshall et al. (2009) and Vulturius \& Gerger Swartling (2015) found that one's personal beliefs in climate change and experiences with climate change hazards and perception of risk were strong motivators for adaptive action. Likewise, Amundsen (2015) supports that people are encouraged to respond when their emotions are connected with place, and the authors explain that place attachment serves the best starting point for climate change adaptation rather than emphasising climate change impacts straight away (Amundsen, 2015).

In relation to tourism, Schott et al. (2010) argue that understanding local responses and options to encourage local tourism activities to act on climate change impacts will need strong place-specific studies carried out by researchers that are properly required to understand these place-based conditions. Through an inclusive process matching the climate change issues with the technical capabilities of the institutions and community stakeholders of the area, coastal adaptations must be tailored to the local context (Tobey et al., 2010).

In this thesis, a similar approach to vulnerability assessment will be used by combining both scientific and local knowledge as outlined above, and environmental and socio-economic factors as argued below:

As argued above, vulnerability assessment is prerequisite knowledge to understand adaptation. The next sections review the literature on climate change adaptation (CCA).

\subsection{Climate change adaptation}

In the $4^{\text {th }}$ Assessment Report of IPCC 2007 report, CCA is defined as "adjustment in natural or human systems in response to actual or expected climatic stimuli or their effects, which moderates harms or exploits beneficial opportunities" (IPCC, 2007, p. 6). Two tools widely used to respond to climate change are adaptation and mitigation (IPCC, 2007). In IPCC report, mitigation is defined as "an anthropogenic intervention to reduce the sources or enhance the sinks of greenhouse gases" (IPCC, 2007). The purpose of mitigation is to reduce 
emission (IPCC, 2007), especially in industrialised countries, but developing countries are the victims of climate-related impacts (Hess, 2012). To deal with climate change consequences, adaptation has gained popularity in climate agenda (Afroz \& Naser, 2014). Therefore, adaptation is most needed in developing countries, especially for tourism (Scott et al., 2009).

The term 'adaptation' is widely used in climate change arena (Linnerooth-Bayer \& Mechler, 2006). Effective adaptation not only takes into account the many different changes that are happening simultaneously but also multiple socio-economic factors (Bennett et al., 2016; Bunce, Rosendo, \& Brown, 2010; Tuler et al., 2008). Therefore, constant adaptation is not only essential but also compulsory (Berrang-Ford, Ford, \& Paterson, 2011). Adaptation to these continuous changes might be difficult, but it might provide communities with an opportunity to determine and attain desired future environmental and social outcomes (Bennett et al., 2015).

\subsubsection{Different types of climate change impacts}

As this study clearly discusses adaptation effects, the different types of climate change impacts need to be raised. For instance, SLR and increasing heat are continuous, ongoing impacts. Other effects, such as rainfall and drought, are more likely to happen as events that are short-lived but occur frequently and on an increasing scale. Forster (2015) adds that severe weather events, including cyclones and heat waves, do not occur frequently, but their consequences can be very disruptive and destructive. Some climate change impacts need a long time (decades) for observation and identification such as drought in the tropics and subtropics (Trenberth et al., 2007; Linnenluecke, Griffiths, \& Winn, 2012). Organisations (e.g. in the tourism sector) tend to have a long-lived effect and exposure by climatic sensitivity due to their various capital assets such as their fixed infrastructures (Linnenluecke et al., 2012). Due to different types of exposure and impacts, sometimes with extended period, organisations need different types of adaptation. 


\subsubsection{Different types of adaptation}

As outlined above, there are different types of climate change effects. Some impacts are continuous and ongoing, while others are short-lived events but occurring on an increasing and more frequent scale. This means that adaptations will be of a different character for some types of impacts (e.g. SLR and increasing heat) and their consequences will be quite different for the hotel sector. For instance, wind is identified as having direction, strength and timing, so tourism operators (including hotel operators) have different sensitivities to these identifications (Becken \& Wilson, 2016), which leads to different adaptations employed by hotel operators.

In examining anticipatory adaptation to extreme weather events, Linnenluecke, Griffiths, and Winn (2012) classify the anticipatory adjustments as the long-term adaptation. Nevertheless, Lewin, Weigelt, and Emery (2004) contend that adaptation is a progressive or gradual adjustment and optimisation in order to obtain a better performance of an institution fitting with its business modes. Smithers and Smit (1997) distinguish that reactive adaptation depends on experience of operation under the weather extremes, while proactive adaptation depends on environmental perception and risk assessment of future weather extremes. Lewin, Weigelt, and Emery (2004) suggests that organisational actors should deploy anticipatory adaptation to the weather extremes that happen as single or recurring events.

Wilbanks et al. (2007) explain that extreme weather stresses tend to exceed the certain thresholds of adaptation when organisational actors aim to undertake short-term adaptive responses immediately prior to their organisation's exposure, such as reaction to prior warnings, evacuation of personnel, or relocation of non-stationary components. Because of the nature of the impact, different types of adaptation are employed, but some adaptations have limits, which are detailed in the sections below.

\subsubsection{The limit of adaptation}

The nature of the climate change impacts leads to different types of adaptation, but some adaptation actions encounter different limitations. The discussion on limits to adaptation frequently focuses on three dimensions: Ecological and physical limits, economic limits, and 
technological limits (Adger et al., 2009). Both the hazards and risk literatures have not yet provided a sufficient indication of how mutable and constructed limits may be overcome in the context of limits to adaptation. This is because of the complex interactions between social and individual characteristics, with a view to securing sustainable societal adaptation in the long run (Adger et al., 2009).

Adaptation limits may also happen due to the consideration of the economic costs of adaptation (Agrawala \& Fankhauser, 2008) or due to technological innovation for adaptation (Adger et al., 2009). The other implication is that even though enough information is provided about how to adapt, people who do not believe adaptation is necessary are highly unlikely to keep or act on this information (Adger et al., 2009). Adger et al. (2009) explain that adaptation action is also limited by social and individual factors. Factors including habit, social status and age as well as perception of risk constrain both individual and collective adaptation action.

Adger et al.'s (2009) further argue that limits to adaptation result from 'inside' society and are endogenous. The limits to adaptation vary, depending on goals, values, risk and social choice. The operative question is whether limits to adaptation are constructed, rather than how they become discovered. The goals of adaptation will differ between institutions and different generations and vary within a sector and society (Adger et al., 2009). Adaptation goals differ between nation states. Wealthier nations or individuals might focus on maintaining standards of living through adaptation, but developing nations may seek to continue the development and enhancement of their citizens' standards of living (Adger et al., 2009).

There are variations in risk perceptions, the incapability to shorten gap between current experiences and future projections, the distance nature of climatic change, and limits to identifying and implementing adaptation actions which indicate that little response to climate change is taking place (Adger et al., 2009). There are similar identified areas of concern at the organisational level, which limit adaptation (Frans Berkhout, Hertin, \& Gann, 2006) including limited knowledge of climate change effects and challenges to assessing and 
implementing adaptation measures (Adger et al., 2009). Adger et al. (2009) contend that risk perception contributes to adaptation decision. If the society or organisation does not believe the risk is major enough to take action, it is a limiting factor.

In coping with the problem of limits to adaptation, Adger et al. (2009) have suggested start at looking at the way societies are constructed, the values they have, the knowledge they organise, and the relationships between the state, entities and individuals. O'Brien (2009) adds that social values and institutional arrangements tend to vary significantly within and between societies over time. Weather events that occur with greater intensity, magnitude, frequency, persistence and abruptness that exceed thresholds for adaptation may lead to harmful impacts. To adapt to these impacts, it is suggested that organisations use sets of resources and capacities to cope with discontinuities as they occur, and align them with the crisis response (Lewin, Weigelt, \& Emery, 2004). In a positive perspective, uncertainty of knowledge of future climate change adaptation does not limit adaptation (Adger et al., 2009) because society is still able to make adaptation decisions despite the absence of accurate and precise climate predictions (Dessai, Hulme, Lempert, \& Pielke, 2009).

However, Adger and his colleagues (2009) argue that a limit or failure of adaptation may in fact be an adaptation potential for another agent or actor, because of the different priorities and values held within society. Adger et al. (2009) continue that although CCA is constrained by power structures, processes, values and perceptions, the limit depends on ethical stance, places and cultures valued, the society's risk perceptions and scientific projections. CCA limits in one society might not be in another.

Turning attention now to CCA and (coastal) tourism, the next sections illustrate a dearth of research on the how (coastal) tourism and its subsector (accommodation) involve CCA.

\subsection{Climate change adaptation, tourism/coastal tourism and the accommodation sector}

Research on climate change adaptation in tourism has gradually increased. Adaptation has been a vital research need in tourism and climate change studies recently (Kaján \& Saarinen, 
2013). Adaptation is recommended for tourism sector in order to soften the climate change impacts and to obtain sustainable tourism development in the longer term (Pham, Simmons, \& Spurr, 2010). For instance, because climate change directly impacts on the physical, environmental and social resources important for tourism (Moreno \& Amelung, 2009), tourist destinations must adapt to climate change so that potential risks are reduced and potential opportunities are exploited if it wants to remain economically, environmentally and socially sustainable (Jopp, DeLacy, Mair, \& Fluker, 2013; Pham et al., 2010). Jopp, DeLacy, \& Mair (2010) conclude that adaptation is a very suitable strategy for combating climate change impacts for tourism. As developing countries are the victims of climate-related impacts (Hess, 2012), adaptation is also considered a vital research requirement in tourism and climate change (Kaján \& Saarinen, 2013; Schipper, 2006).

As stated above, adaptation follows vulnerability. For instance, coastal tourism destination managers should first advance their understanding of the vulnerability to climatic changes in order to subsequently formulate proper adaptation strategies and measures (Moreno \& Becken, 2009). This point is later underscored by Walmsley (2012), who states that potential impacts need to be mentioned when discussing vulnerability to make sense of the need of adaptation.

In the existing literature, only a few publications have examined CCA in the accommodation sector in the context of developing countries. Authors such as Su et al. (2013) and Walmsley (2012) suggest that all hotels are in a need to adapt to climate change. Adaptation is significantly related to how tourism businesses cope with the effects, or potential impacts of climate change (Walmsley, 2012). Another study conducted by Dzoga and Koske (2014) undertook a study on beach hotels' CCA actions such as on-shore vegetation cover, rainwater harnessing, and establishment of seawall barriers. Dzoga and Koske (2014) found that the measures initiated by beach hotels were not effective in addressing climate unpredictability and are unable to maintain tourism economy during climate variability era.

However, the level of implementation of hotels' adaptations is dependent on hotel attributes such as star rating, size, ownership, location, business age (year of trading), business format 
(owned vs. leased). For instance, the study conducted by Su et al. (2013) suggests that hotel attributes such as size, ownership, and location have influence on the application of green practices. The large and affiliated hotel chains tend to have better environmental knowledge and implementation of green practice (Chan, 2011; Kasim, 2009) than the small and independent hotels. Additionally, the accommodation facilities in less-developed areas are likely be greener than those in big cities and coastal destinations (McNamara \& Gibson, 2008). Larger firms likely have more expertise in climate change efforts and more resources to implement projects to respond to climate change (Amran, Ooi, Nejati, Zulkafli, \& Lim, 2012). Based on Nguyen's (2015) systematic literature review and research findings about a small number of privately owned hotels in Vietnam, it was found that the smaller ones are likely to have less environmental practices because large hotels were believed to have more capital resources with greater motivation to integrate environmental issues into their business operation than the small and medium hotels.

In addition, the level of hotels' adaptations is influenced by people or hoteliers' backgrounds, such as their level of education, experience, nationality and age. For example, education is associated with high risk awareness; it could contribute to vulnerability reduction behaviours (Muttarak \& Lutz, 2014). For instance, Ainuddin et al. (2014) found that highly educated people know earthquake risks better and are likely become more ready for disaster than those having low education level (Paul \& Bhuiyan, 2010). Highly qualified and educated individuals appear to access diversified communication linkages and have received useful information better (Muttarak \& Lutz, 2014; Neuenschwander, Abbott, \& Mobley, 2012). Education increases socio-economic resources, facilitates access to information and enhances social capital, thereby promoting vulnerability reduction and adaptive capacity (Muttarak \& Lutz, 2014).

The sections below expand the literature on adaptation studies in (coastal) tourism and the accommodation sector, which emerge as knowledge gaps in a forward-looking aspect of study. 


\subsection{Adaptation studies in tourism/coastal tourism and the accommodation sector}

\subsubsection{Adaptation and coastal tourism}

The interrelationship between climate change and tourism is insufficiently studied (Matasci et al., 2014; Perch-Nielsen, 2010; Schott et al., 2010). Additional evidence found by other authors. First, Berrang-Ford et al. (2011) reviewed 1,741 adaptation research studies carried out between 2006 and 2009 and found that only 9\% considered the tourism sector. Second, Becken (2013) reviewed 459 publications with 539 authors and finally found that $50 \%$ of publications on climate change and tourism were related to adaptation. Yet the focus of publications (48 publications) remains on the case of winter tourism (Becken, 2013). In terms

of geographic locations, studies on tourism and climate change were mainly dispersed in a limited number of Western tourist areas such as Australia (38), UK (30), Canada (40 studies), USA (33), and New Zealand (24) (Becken, 2013). Third, other authors such as Scott and Becken (2010) and Scott (2011) claim that there are limited studies on how climate change affects the tourism industry and how the sector responds to it. Adaptation of tourism industry has received little focus from researchers compared to other sectors such as agriculture, water resources and construction (Berrang-Ford et al., 2011; Kaján \& Saarinen, 2013; Scott \& Becken, 2010; Scott et al., 2009). In sum, research on tourism is 5-7 years behind other sectors that are connected to climate change (Scott et al., 2009).

Although coastal communities have faced a wide range of environmental and social changes (Bennett \& Dearden, 2013), there are fewer choices for adaptation at the coastal communities (Nandy, Ahammad, Alam, \& Islam, 2013). Although coastal adaptation is a complex system (van den Bergh \& Nijkamp, 1998), efforts that directly target coastal adaptation are only at the initial stage (Tobey et al., 2010).

\subsubsection{Adaptation and accommodation sector}

Hotels' responses to environmental issues have been piecemeal (Tzschentke et al., 2008), especially when related to climate change responses (Arcodia \& Dickson, 2008; Su et al., 2013). Chiang (2010) and Jarvis and Ortega (2010) found that there has been little research into the management of change in the hotel industry, specifically in the way it relates to the challenges caused by climate change, especially from the perspectives of small firms (e.g. 
hotels) (Jarvis \& Ortega, 2010). This likely aligns with Tzschentke et al. (2008) who argue that while environmental management standard is theoretically appropriate to all sizes and types of businesses, it is perceived as irrelevant by small tourism firms. The adoption of this standard within a small-sized tourism business context is limited (Tzschentke et al., 2008). This is because of the insufficient resources to consider climate change adaptation and priority in those small tourism enterprises (Marshall et al., 2011; Tervo-Kankare, 2011). Their deficits, which include insufficient time and capacity as well as incompetent expertise, have been variously described as reasons for low levels of involvement with environmental issues (Sampaio, Thomas, \& Font, 2012; Tzschentke, Kirk, \& Lynch, 2008).

After the vital relationship between vulnerability and adaptation is built and relevant literature is expanded, the next section presents an adaptation framework for this study.

\subsection{Adaptation frameworks}

As climate change keeps changing, adaptation in tourism has become more challenging because tourism mainly depends on the resources and activities of other economic sectors that do not serve tourism purposes solidly (Brown et al., 2011; Csete \& Szécsi, 2012). Climate change affects the whole tourism system by generating changes (Leiper, 1995); for example, even transport systems of a tourism destination should adapt to these changes (Kaján \& Saarinen, 2013).

In order to adapt to climate change impacts more effectively, many authors discussed adaptation frameworks such as Cross et al. (2012) and Benedikter et al. (2013). However, what they proposed was insufficient for this research that aims to explore adaptation in tourism business. In contrast, WMO, UNEP and WTO (2008) and Simpson et al. (2008) provided an adaptation framework for tourism interrelating with climate change, which strongly influences this study. Table 2.1 indicates a wide range of technical, managerial, policy, research, education and behavioural adaptation that are now used by tourism stakeholders to respond to climate effects at the destination level. In relation to the proposed adaptation framework, WMO et al. (2008) explain that CCA is rarely implemented alone. The adaptations normally involve various actions contextual to the destination climate and 
its tourism subsectors. A complex mix of adaptations being practised in the tourism sector across the globe will be eventually created that are particular to a location (WMO et al., 2008). Tourism is less discussed than other economic sectors and is not openly involved in various adaptation frameworks such as the energy, agriculture, water resources, health, and biodiversity (Simpson et al., 2008). Njoroge (2014) also argues that the existing adaptation frameworks to help advise tourism managers and policy makers in achieving sustainable adaptation might not be available. Yet there is no single 'right' framework to implement CCA in tourism (Simpson et al., 2008). This study adopts the adaptation framework, which is based on Table 2.1. 
Table 2.1: Portfolio of climate change adaptation by tourism stakeholders

\begin{tabular}{|c|c|c|c|c|}
\hline $\begin{array}{c}\text { Type of } \\
\text { adaptation }\end{array}$ & $\begin{array}{l}\text { (a) Tourism } \\
\text { operators/businesses }\end{array}$ & $\begin{array}{l}\text { (b) Tourism industry } \\
\text { associations }\end{array}$ & $\begin{array}{l}\text { (c) Governments and } \\
\text { communities }\end{array}$ & $\begin{array}{l}\text { (d) Financial sector } \\
\text { (investors/insurances) }\end{array}$ \\
\hline Technical & $\begin{array}{l}\text {-Rainwater collection and water } \\
\text { recycling systems (see example } \\
\text { below) } \\
\text {-Cyclone-proof building design } \\
\text { and structure } \\
\text {-Snowmaking } \\
\text {-Slope contouring }\end{array}$ & $\begin{array}{l}\text {-Enable access to early warning } \\
\text { equipment (e.g. radios) to tourism } \\
\text { operators } \\
\text { - Develop websites with practical } \\
\text { information on adaptation } \\
\text { measures }\end{array}$ & $\begin{array}{l}\text {-Reservoirs, and desalination } \\
\text { plants } \\
\text { - Fee structures for water } \\
\text { consumption } \\
\text {-Weather forecasting and early } \\
\text { warning systems }\end{array}$ & $\begin{array}{l}\text {-Require advanced } \\
\text { building design or material } \\
\text { (fire resistance) standards } \\
\text { for insurance } \\
\text {-Provide information } \\
\text { material to customers }\end{array}$ \\
\hline Managerial & $\begin{array}{l}\text {-Product and market } \\
\text { diversification (see example } \\
\text { below) } \\
\text {-Water conservation plans } \\
\text {-Low season closures } \\
\text {-Regional diversification in } \\
\text { business operations } \\
\text {-Redirect clients away from } \\
\text { impacted destinations }\end{array}$ & $\begin{array}{l}\text {-Snow condition reports through } \\
\text { the media } \\
\text { - Use of short-term seasonal } \\
\text { forecasts for the planning of } \\
\text { marketing activities } \\
\text { - Training programmes on climate } \\
\text { change adaptation } \\
\text { - Encourage environmental } \\
\text { management with firms (e.g. via } \\
\text { certification) }\end{array}$ & $\begin{array}{l}\text {-Impact management plans (e.g., } \\
\text { 'Coral Bleaching Response } \\
\text { Plan') } \\
\text {-Convention/ event interruption } \\
\text { insurance } \\
\text {-Business subsidies (e.g., } \\
\text { insurance or energy costs) }\end{array}$ & $\begin{array}{l}\text {-Adjust insurance } \\
\text { premiums or not renew } \\
\text { insurance policies } \\
\text {-Restrict lending to high } \\
\text { risk business operations }\end{array}$ \\
\hline Policy & $\begin{array}{l}\text {-Comply with regulation (e.g. } \\
\text { building code) (see example } \\
\text { below) } \\
\text {-Hurricane interruption } \\
\text { guarantees }\end{array}$ & $\begin{array}{l}\text {-Coordinated political lobbying } \\
\text { for GHG emission reductions and } \\
\text { adaptation mainstreaming } \\
\text { - Seek funding to implement } \\
\text { adaptation projects }\end{array}$ & $\begin{array}{l}\text {-Coastal management plans and } \\
\text { set back requirements } \\
\text {-Building design standards (e.g., } \\
\text { for hurricane force winds) }\end{array}$ & $\begin{array}{l}\text {-Consideration of climate } \\
\text { change in credit risk and } \\
\text { project finance } \\
\text { assessments }\end{array}$ \\
\hline Research & $\begin{array}{l}\text {-Site location (e.g., north facing } \\
\text { slopes, higher elevations for ski } \\
\text { areas, high snow fall areas. (see } \\
\text { example below) }\end{array}$ & $\begin{array}{l}\text { - Assess awareness of businesses } \\
\text { and tourists and knowledge gaps }\end{array}$ & $\begin{array}{l}\text {-Monitoring programmes (e.g., } \\
\text { predict bleaching or avalanche } \\
\text { risk, beach water quality) }\end{array}$ & $\begin{array}{l}\text {-Extreme event risk } \\
\text { exposure }\end{array}$ \\
\hline Education & $\begin{array}{l}\text {-Water conservation education } \\
\text { for employees and guests (see } \\
\text { example below) }\end{array}$ & $\begin{array}{l}\text {-Public education campaign (e.g., } \\
\text { 'Keep Winter Cool') }\end{array}$ & $\begin{array}{l}\text {-Water conservation campaigns } \\
\text {-Campaigns on the dangers of } \\
\text { UV radiation }\end{array}$ & $\begin{array}{l}\text {-Educate/inform potential } \\
\text { and existing customers }\end{array}$ \\
\hline Behavioural & $\begin{array}{l}\text {-GHG emission offset } \\
\text { programmes (see example } \\
\text { below) } \\
\text {-Real-time webcams of snow } \\
\text { conditions }\end{array}$ & $\begin{array}{l}\text {-GHG emission offset } \\
\text { programmes } \\
\text {-Water conservation initiatives }\end{array}$ & $\begin{array}{l}\text {-Extreme event recovery } \\
\text { marketing }\end{array}$ & - Good practice in-house \\
\hline
\end{tabular}

Sources: WMO, UNEP \& WTO (2008) and Simpson et al. (2008) 
WMO et al. (2008) and Simpson et al. (2008) endeavour to provide wider understanding to the framework by illustrating an explanation of each type: technical, managerial, policy, research, education and behavioural adaptation. Since there are many stakeholders involved, the attempt to provide illustrations covering all stakeholders are impossible. Instead, some examples are provided in relation to category (a) tourism operators/businesses, which are closely relevant to this study's aims.

\section{- Technical}

This involves utilising technology and innovations in order to identify approaches of dealing with climate change and vulnerability (Jopp et al., 2010, 2013). Here is an example:

Rainwater collection and water recycling systems: to respond to an existing seasonal water shortage caused by rainfall variability (and projected to be intensified by future climate change) in the Island of Phuket, Thailand. The Tourism Authority of Thailand (TAT), coupled with other local governments and national entities, initiated supporting structures of the multi-year water supply plan including the building of new dams as water sources, water recycling systems and expansion of water transmission. Additionally, TAT also initiated nonstructural adaptations, such as an adjusted fee structure for water conservation projects and water usages (Simpson, et al., 2008; WMO et al., 2008).

\section{- Managerial}

Tourism investors, entrepreneurs and businesses can improve their climate change management by approaching the problem in different ways, and at different levels. These include vulnerabilities to direct impacts from climate change and other vulnerabilities such as changes in the resources or customer bases (Becken \& Hay, 2007). Normally, the tendency of these actions are based on good communication procedures as well as a certain level of reliability among users (Lim et al., 2005). Here is the example:

Product and market diversification: to respond to expanded summer temperature intensities, variations in demand seasonality, and intensities of storms in the Caribbean Region and Gulf of Mexico. Tourism organization and each member state in the Caribbean have commenced 
to promote themselves as destinations visited by tourists in all seasons. The organisation invested in multi-million-dollar dynamically marketing campaigns aiming at the honeymoon advertisement and budget-constraint families. In addition, marketing provides messages that downplay the destination's summer heat are newly installed with air-conditioning techniques, reduced-fee room rates, and new disruption (e.g. storms) policies at different companies in the resort. The strategy has been profitable as summer occupancy rates at the coastal resorts are increasing or equalling winter season in several destinations (Simpson et al., 2008; WMO et al., 2008).

\section{- Policy}

Concerning policy needs, strategies are designed to minimise susceptibility to climate effects and the uncertainty of economic conditions in the short term. The designed strategies also contribute to minimising vulnerability to climate change and socio-economic tendencies over the longer-term period (Lim et al., 2005). Here is an example:

Comply with regulation (e.g. building code): to respond to severe wind forces (e.g. cyclones) and storm surges causing damage to structures and coastal erosion in Coastal Tourist Resorts Fiji. Various government departments and tourism businesses initiated a building compliance that structures are required to remain firm with wind speeds of $60 \mathrm{~km}$ per hour. The review of the building compliance is a continuous process. In addition, each business, including the larger resorts, has emergency plans, insurance procedures and cover prior to the onset of the cyclone/storm occurrence. The policy adaptation should include first aid materials, water and food storage, trimming of tree branches and a direct connection (e.g. phones) to the Meteorological Service for immediate warnings (Simpson et al., 2008; WMO et al., 2008).

\section{- Research}

Research and innovations are necessary to respond to climate change, which include the economic assessment of adaptations and other adaptations. Research may also be needed to investigate the adaptation of existing technologies in order to understand the adaptation demands e.g. the research to explore the development of solutions for more power-efficient air conditioning techniques (Lim et al., 2005). Here is an example: 
Site location of a ski area in ski regions of Western Canada (the Canadian Rocky Mountains): to respond to further decline in natural snowfall, and alteration of ski season. Despite the negative impact of climate change on ski areas, the ski resort companies and local government have conducted research to seek for an opportunity to promote an altered marketplace of the future. They have raised the investments over 15 years to develop a new ski resort in the area. This critical investment in research is an illustration of the opportunities that climate change will make for forward-looking destinations and tourism enterprises, when the impacts change the competitiveness within the ski sector over a period of time (Simpson et al., 2008; WMO et al., 2008).

\section{- Education}

Education and raising awareness enable the minimisation of the external climate change stresses, and increase the profile and knowledge foundation of stakeholders of a destination that struggles to cope with the climate change impacts (Simpson et al., 2008). Education and training introduce climate change issues at different levels. This can contribute to building capacity among stakeholders to improve adaptation in the future, and helping the development of appropriate research activities and a wider understanding among citizens (Lim et al., 2005). Here is an example:

Water conservation education for employees and guests: to respond to water limitation caused by the incidence of extended droughts in Tobago, the Caribbean and Fiji, South Pacific, for accommodation operators and tour operators. Individual accommodation providers and tour operators conducted adaptation measures, including water conservation such as the instalment of devices/containers for water saving and educating guest and staff, revising landscaping practices and restricting use of pools. Education adaptation promotes sustainability planning, including long-term weather forecasts, water source management, monitoring health and environmental safeguards (e.g. water quality), and recycling (e.g. use of treated water for irrigation) were also initiated (Simpson, et al., 2008; WMO et al., 2008). 


\section{- Behavioural ${ }^{6}$}

Climate change mitigation leads to emission reductions through socio-economic and technological changes as well as replacements. However, when technological restructuring alone cannot reduce crucial emissions, mitigation meets challenges, thus requiring behavioural and structural changes (WMO et al., 2008). Here is an example:

Behavioural adaptation is "the process by which certain features of behavioural actions or strategies become more prevalent among individuals because those features enable better outcomes under changing climatic conditions." (Hamilton et al., 2018, p. 3). In responding to climate change-induced wildfire (largely shaped by weather patterns), behavioural adaptation by individuals include the modification of home structures and limitation of flammable vegetation (Hamilton et al., 2018). A further example of behavioural adaptation associated with tourists is given in the paragraph below.

In their studies, Scott et al. (2009) and Jopp et al. (2010, 2013) adopted the framework for their tourism-climate change studies. They considered only three types: technical, managerial and behavioural adaptation. For technical adaptation, Jopp et al. (2010, 2013) explained that it involves employing technology and innovations in order to identify approaches of dealing with climate change and susceptibility. They further indicated that the adaptation often needs specialised equipment or/and the employment of encouraging innovations and new technologies. They illustrated snowmaking machines or desalination plants as examples of technical adaptation. For managerial adaptation, they explained that it is associated with techniques employed by local/regional governments, tourism operators and tourism industry associations to minimise vulnerability to climate change. They further indicated that the adaptation might instruct destination managers to adjust their advertising strategies in order to try and lengthen or shorten travel during certain periods and/or guide/redirect tourists to alterative areas/locations or encourage them to continue with different touristic activities. They illustrated advertising techniques such as new pricing promotions, market segmentation or/and product diversification, which can all be used as examples of managerial adaptation.

\footnotetext{
6 'Behavioural' adaptation is associated with tourists (see Jopp et al., 2010; Jopp et al., 2013; Scott et al., 2009).
} 
Behavioural adaptation is used by tourists as they have the capability to decide whether to carry on the touristic activities they are involved in, and when and where they do so. The adaptation provides them with the ability for temporal, spatial and alternative activities that afterward provide tourists with fundamental adaptive capacity. They illustrated that the adjustment of the type of clothing used, change of the activities of tourists, adjustment of the scheduling of the visits, and change of the destination as examples of behavioural adaptation.

However, work by WMO et al. (2008) and Simpson et al. (2008) discussed skiing destinations without sufficient application to a particular tourism region (Jopp et al., 2013) and accommodation sector. Geographically, their work discusses the adaptation application in developed countries. Taking the existing framework into consideration, the study adopted the adaptation framework by WMO et al. (2008) and Simpson et al. (2008) by contextualising and conceptualising it into a developing country's' accommodation context, which will be detailed in chapter 5: Vulnerability and Adaptation.

Several lines of evidence in the following sections suggest that while adapting to climate change, adaptation actors are confronted by a number of challenges. These are detailed below.

\subsection{Barriers to climate change adaptation}

CCA is not new because humans have lived on Earth with climatic variability for a long time and have automatically started coping with this variability (Burton et al., 2002; Smit \& Wandel, 2006). Yet adaptation actors have faced challenges in responding to the climatic variability. It has been hard to define what a barrier to adaptation is (Biesbroek et al., 2013) but Huang et al. (2011) refer to it as "any condition that makes it difficult to achieve progress towards adaptation" (p. 185). The concept of 'barriers' is often used interchangeably with other terms such as 'constraint', 'hindrance' or 'obstacle' (Biesbroek et al., 2013). For example, barriers and limits are often conceptualised interchangeably while other researchers may distinguish between them (Moser \& Ekstrom, 2010). 
Based on literature on CCA, it is concluded that there are a large number of barriers that obstruct the development and implementation of CCA actions (Biesbroek et al., 2011). Developing countries face more barriers because earlier research on barriers and limits to climate change adaptation may be more studies examining the developed countries' adaptive capacity, with less focus on adaptation shortfall in developing countries (Burton, 2009; Moser \& Ekstrom, 2010). Since developing nations are more vulnerable than developed countries, great financial investments will be required for adaptation in developing nations within the next few decades (Bele et al., 2014). External finance is a significant additional resource (Hartzell-Nichols, 2011). In addition, Bele et al. (2014) found (in Africa) that barriers to adaptation include, among others, high poverty levels, lack of information on climate change impacts and adaptation options, and lack of infrastructure.

Based on a thorough review of the literature, the following barriers have emerged, which have particular relevance to developing countries.

\subsubsection{Limited resources}

Amundsen et al. (2010) explain that lack of resources, or the inaccessibility of resources, can be an intense adaptation barrier. According to Biesbroek et al. (2011), essential tangible and intangible resources include human capital (availability of staff, time to become informed, managerial support, and skilful and qualified individuals), financial resources (budget for implementing adaptations), and natural resources (availability of land).

Budget constraints have been considered the most predominant element in implementing adaptive measures (Suraje Dessai, Lu, \& Risbey, 2005; Lorenzoni et al., 2000). Budget deficits are becoming a severe danger to the development and advancement of the adaptation process (Matasci et al., 2014). For instance, when there is the shortage of funds from the central government, it often leads to having no institutions that act as financing facilitator, limited resources to monitor the progress, or no political will to mobilise financial resources (Adger et al., 2007). Because climate change impact will not stop in the short term, the world needs to respond to the unfavourable impacts of the changing climate. Therefore, responses to climate change will need large financial investment in adaptation (Fankhauser, 2010; 
Narain, Margulis, \& Essam, 2011). Many obstacles such as financial limitations reduce the efficiency or effectiveness of adaptation, and sometimes require high cost to repair (Moser \& Ekstrom, 2010).

\subsubsection{Limited knowledge/perception of climate change}

One of the most easily identifiable barriers to engaging with climate change is a low of understanding about causes, effects and solutions to climate change (Lorenzoni et al., 2007). Limited trust in climate science and unclear perceptions of scientific knowledge about climate change and adaptive strategies can also pose hindrance to adaptation (Vulturius \& Gerger Swartling, 2015). Insufficient knowledge may be a contribution to a sense of uncertainty about climate change (Lorenzoni et al., 2007). Another study by Amundsen et al. (2010) found that four identified key barriers to adaptation: i) a lack of knowledge with existing data on climate change; ii) lack of solid data; iii) unfamiliarity of local expertise for responding to climate change risks; and iv) an uncertain role for local authorities' governments associated with adaptation measures and policies. The first two barriers take root in the limited knowledge/perception of climate change.

\subsubsection{Insufficient information}

The main barriers to the adoption of an environmental policy as well as climate change are not only budget and time constraint but also insufficient information (Jarvis \& Ortega, 2010). The information and awareness are also identified as factors in climate change response ( Dessai et al., 2005) and its limitation has been recognised as one of several barriers to CCA (Harriet, 2000; Norgaard, 2006). This is because the access to information relevant to vulnerability to climate change effects has been insufficient either in urban or rural locations (Crabbé \& Robin, 2006; Mukheibir \& Ziervogel, 2007). The lack of awareness and communication is a true impediment for the implementation of CCA (Biesbroek et al., 2011). Without communication, the public or stakeholders remain uninformed and unheard about climate change impacts and the collective efforts on climate change adaptation might not be

formulated promptly (Biesbroek et al., 2011). If there is insufficient information on the effects and the potential adaptation measures, if stakeholders do not trust information sources 
or if they cannot communicate and collaborate, then the planning and the implementation phases will not be achieved (Matasci et al., 2014).

\subsubsection{Institutional barriers and uncertain responsibilities for adaptation}

Institutions (e.g. tourism businesses) become role players in ensuring adaptation responses to an increasing and unpredictable change (Cuevas, 2015). The inflexibility of existing institutional arrangements and unclear responsibilities are a significant adaptation barrier (Huang et al., 2011). Schröter et al. (2005) call it 'institutional barriers' that hamper people's actions towards adaptation initiatives. At the local level, the incompetence of local institutions to adapt to climate change through planning matters institutionally. In most cases, local authorities do not have constitutional standing and lack institutional independence (Measham et al., 2011). Grasso et al. (2014) argued that mostly in developed countries, proper institutional structures allow widespread access to technology, the responsibility for adaptation, climate information and equitably allocated resources. It can be inferred that developing countries have limited proper institutional structures or uncertain institutional responsibilities, especially at local level.

\subsubsection{Regulatory/legal constraints}

Another constraint in climate change adaptation is that policies at national level limit the ability of local level to respond to impacts. Formulating adaptation policy is sometimes conflicting if viewed horizontally and vertically (Urwin \& Jordan, 2008). Local levels should organise their own planning that is their territory of expertise, and the national government should prioritise the policy efforts (Urwin \& Jordan, 2008). Prutsch et al. (2010) suggest that adaptation that is conflicting or overlapping between present policies is not performed well through funding systems, legislation, processes (e.g. in decision making) and management structures (e.g. networks). There is likely a reason behind this, which is explained by Measham et al. (2011), who inform that local municipalities are very low in financial capacity. Integration of adaptation through the examination and modification of existing regulations/mechanisms should not be limited to the public authority and to the environmental sector; they are also meaningful for other economic sectors and private institutions such as businesses (Prutsch et al., 2010). Prutsch et al. (2010) further argue that 
there are sufficient regulations/rules and policies for environmental sectors but there are gaps for regulations/rules and policies for CCA, which thereafter can create barriers for CCA.

\subsubsection{Low level of leadership and guidance}

Low levels of leadership and guidance lead to the impediment of the willingness and capacity to make adaptation decisions (Tribbia \& Moser, 2008). Therefore, it is suggested that good leadership, resourcefulness, strategic thinking, creativity, effective communication and collaboration are resources for overcoming adaptation barriers (Moser \& Ekstrom, 2010). An effective leadership can help overcome adaptation barriers (Moser \& Ekstrom, 2010). At the societal level, different leaderships may be able to mobilise social engagements from one sequence of the adaptation phase to another (Vignola, Leclerc, Morales, \& Gonzalez, 2017).

\subsubsection{Lack of political will}

Government is the biggest institution to adapt to climate change. Government plays a key role that can intervene and overcome existing barriers through changes in legislation or delivering potential resources (Ford \& Pearce, 2010; Measham et al., 2011). Adger et al. (2005) also support many other papers throughout the world, which have come to similar conclusions that national governments could support climate adaptation by including principles of statutory ordering, delivering frameworks for action, inspiring or applying adaptive practice, offering resources and capacities, distributing powers and responsibilities, and addressing issues of fairness and equity at all levels of governance. However, Biesbroek et al. (2011) argue that the highest ranked barriers to adaptation are the imbalance between long-term impacts of climate change and short-term consideration/thinking of politicians. Local bottom-up adaptation initiatives may be obstructed by the government's lack of political will (Amundsen et al., 2010; McNeeley, 2012).

\subsubsection{Insufficient engagement and interaction with other stakeholders}

CCA is a cross-level and cross-sector issue that requires involvement from different stakeholders (Prutsch et al., 2010). Adaptation planning can be well achieved through stakeholders' collaboration and communication (Matasci et al., 2014). CCA practice can be improved through learning by doing, engaging with stakeholders, and sharing best practices 
(Bierbaum et al., 2013). Without sufficient engagement and interaction with other stakeholders it could result in a number of adaptation barriers. Huang et al. (2011) argue that barriers that hamper social feasibility are difficulties of working together among all stakeholders or limited social funds that prevent collective adaptation initiatives.

Based on the literature above, the adaptation barriers/obstacles have been summarised in Table 2.2 below. 
Table 2.2: Summary of the adaptation barriers

\begin{tabular}{|c|c|c|}
\hline No. & Adaptation barriers & Sources \\
\hline 1 & $\begin{array}{l}\text { Limited resources (e.g. economic limits or } \\
\text { budget limits/constraint, lack of technical } \\
\text { skills/ technological limits or human resources) }\end{array}$ & $\begin{array}{l}\text { (Amundsen, Berglund, \& Westskog, 2010) } \\
\text { (Biesbroek et al., 2011) } \\
\text { (Dessai, Lu, \& Risbey, 2005) } \\
\text { (Lorenzoni et al., 2000) } \\
\text { (Matasci et al., 2014) } \\
\text { (Adger et al., 2007) } \\
\text { (Fankhauser, 2010) } \\
\text { (Narain, Margulis, \& Essam, 2011) } \\
\text { (Moser \& Ekstrom, 2010) }\end{array}$ \\
\hline 2 & $\begin{array}{l}\text { Limited knowledge/perception of climate } \\
\text { change (e.g. limited basic knowledge about } \\
\text { causes, effects and solutions to climate change) }\end{array}$ & $\begin{array}{l}\text { (Lorenzoni et al., 2007) } \\
\text { (Amundsen et al., 2010) } \\
\text { (Vulturius \& Gerger Swartling, 2015) }\end{array}$ \\
\hline 3 & $\begin{array}{l}\text { Insufficient information (about climate } \\
\text { vulnerabilities, or absence of reliable climate } \\
\text { data) }\end{array}$ & $\begin{array}{l}\text { (Jarvis \& Ortega, 2010) } \\
\text { (Dessai et al., 2005) } \\
\text { (Harriet, 2000) } \\
\text { (Norgaard, 2006). } \\
\text { (Crabbé \& Robin, 2006) } \\
\text { Mukheibir \& Ziervogel, 2007) } \\
\text { (Biesbroek et al., 2011) } \\
\text { (Matasci et al., 2014) }\end{array}$ \\
\hline 4 & $\begin{array}{l}\text { Institutional barriers (e.g. lack of institutional } \\
\text { flexibility, unclear or contradictory goal within } \\
\text { institution, restriction of individual capacity to } \\
\text { adapt etc.) and uncertain responsibilities for } \\
\text { adaptation }\end{array}$ & $\begin{array}{l}\text { (Cuevas, 2015) } \\
\text { (Schröter et al., 2005) } \\
\text { (Huang et al., 2011) } \\
\text { (Measham et al., 2011) } \\
\text { (Grasso et al., 2014) } \\
\end{array}$ \\
\hline 5 & $\begin{array}{l}\text { Regulatory/legal constraints (e.g. policies at } \\
\text { national level that limit the ability of local level } \\
\text { to response to climate change) }\end{array}$ & $\begin{array}{l}\text { (Urwin \& Jordan, 2008) } \\
\text { (Prutsch et al., 2010) } \\
\text { (Measham et al., 2011) }\end{array}$ \\
\hline 6 & Low level of leadership and guidance & $\begin{array}{l}\text { (Moser \& Ekstrom, 2010) } \\
\text { (Tribbia \& Moser, 2008) } \\
\text { (Vignola, Leclerc, Morales, \& Gonzalez, } \\
\text { 2017) }\end{array}$ \\
\hline 7 & Lack of political will & $\begin{array}{l}\text { (Ford \& Pearce, 2010) } \\
\text { (Amundsen et al., 2010) } \\
\text { (Measham et al., 2011) } \\
\text { (Biesbroek et al., 2011) } \\
\text { (McNeeley, 2012) } \\
\text { (Adger et al., 2005) }\end{array}$ \\
\hline
\end{tabular}

Even though data collection from prior studies about barriers to CCA exists, the hierarchy of criticality is in doubt, especially when questions arise about which barrier is the most major. The following section presents the level of criticality of adaptation barriers. 


\subsection{Critical barriers in implementation of climate change adaptation}

Conducting a study of CCA in agriculture (in Malaysia), Masud et al. (2017) found that 'high cost' or 'lack of budget' is the most critical barrier to CCA. This likely aligns with McNamara, Westoby, and Smithers (2017) who argue that insufficient and protracted provision of funding and resources can be critical adaptation barriers. In research on adaptation to external stressors in coastal communities, Fischer (2018) exemplifies that resource scarcity likely becomes a critical element in how coastal communities address rising sea levels and erosion as well storm surges. In the context of adaptation planning, McClure and Baker (2018) further argued that scientists and community experts consider 'planning' a critical component in facilitating CCA. This likely suggests that a 'lack of planning' is in turn a significant barrier to CCA. Castells-Quintana, Lopez-Uribe, and McDermott (2018) continue that 'inadequate infrastructure' is one of the most critical barriers for maximising adaptation, especially in the poor countries where the infrastructures are currently basic. Cuevas (2015) regards 'lack of leader' as a 'critical barrier'. He explains that a leader can create a significant opportunity (Cuevas, 2015; Simões et al., 2017), while the absence of a champion leadership would lead to the failure to the climate change endeavour and be a critical barrier to the action. Simões et al. (2017) provide a list of emerging critical barriers and/or opportunities for CCA that include perceived signal, available and accessed information, existing management structures, and leadership.

Although existing literature outlines potential barriers in section 2.9 and critical barriers to CCA (this section), additional challenges have been identified. The efforts to define what it means by significant barrier or the most significant barrier to CCA remain a challenge. Berrang-Ford et al. (2011) argue that knowing the greatness of adaptation challenges is not enough. Who is adapting, to what, and how? Although there is a need for adaptation to climate change, adaptation processes are not easily formulated and implemented (Amundsen et al., 2010). There are insufficient studies that have systematically examined how adaptation is taking place (Berrang-Ford et al., 2011). Studying barriers to adaptation is quite young and much more needs to be studied about their nature and influence on the governance of adaptation to climate change (Biesbroek et al., 2011). The current academic depth on adaptation barriers is highly fragmented and low (Biesbroek et al., 2013). Various studies 
have used traditional literature reviews to trace adaptation barriers (Biesbroek et al., 2011; Ekstrom, Moser, \& Tom, 2011). These previous studies are believed to have intended and unintended bias in selecting, interpreting and organising content (Biesbroek et al., 2013). Likely, the adaptation barriers have been narrowly focused. To date, the research has tended to focus on normal adaptation barriers rather than critical adaptation barriers.

Despite extensive literature review about barriers to CCA outlined in section 2.9, it is found that papers that directly and indirectly discuss the barriers to CCA in tourism or coastal tourism are few, especially within the accommodation sector in developing countries. This remains a large gap and this study attempts to fill this gap. This is further outlined in the next section. To be more specific, the next section aims to discuss the linkage between adaptation barriers and tourism and tourism-related businesses.

\subsection{Barriers to climate change adaptation related to tourism/tourism business}

Despite concerted effort, it appears challenging to find literature that clearly identifies the barriers to CCA in tourism. Due to this gap, this section engages the barriers to environmental performances or responses to environmental stresses (Linnenluecke et al., 2012) in tourism businesses or/and accommodation sector. Yet, a link between CCA and environmental performance needs to be strengthened. The link allows to identify barriers while tourism businesses respond to environmental stresses. Investigating the link, the barriers to CCA related to tourism and tourism-related businesses (e.g. accommodation sector) may be subsequently identified.

According to Oliver-Smith (1991), all societies are imbedded with different ties between environment, communities and individuals. The changes in the environment influence collective and individual formations of reality. The link between adaptation and environment is that adaptation initiatives today might produce unintended consequences on environmental and social impacts in the future (Adger et al., 2009). Gössling et al. (2012) further explain that climate change affects tourism in a number of ways, one of which is the indirect effects of environmental change. This includes the increase of operational costs (Becken \& Hay, 2012). According to Clark and Crawford (2012) who reviewed previous research (e.g. 
Rowley \& Berman, 2000; Strike, Gao, \& Bansal, 2006), social performance, starting from community to environment, and which emphasises exclusively on strengths and concerns in the environment, is all related to climate change. The environmental performance of a firm is its involvement in proactive actions to soften climate change or to initiate relevant measures to climate change (Clark \& Crawford, 2012). Firm (including the hotel business) is an environmental performer in enhancing its political engagement in climate change (Clark \& Crawford, 2012). Building on the above arguments, climate change is related to environmental change, which may include CCA or environmental performances.

Tzschentke et al. (2008) propose an argument that adaptation barriers to environmental management practices have widely gained popularity in the academic interest but the studies are partly related to tourism with a focus on small firms. Local tourism operators seem to have relatively low awareness of CCA (Elsasser \& Bürki, 2002). For example, Berry and Ladkin (1997) have noted that small tourism firms have low awareness of their environmental footprint. A lack of understanding of the commercial benefits of environmental management is recognised as a barrier leading to low adoption levels in tourism firms (Tzschentke et al., 2008). There is a strong need to communicate effectively between the climate change science community and tourism operators regionally and locally (WMO et al., 2008). Yet limited knowledge or awareness of environmental strategies and performance, in addition to limited support and information, has been recognised as further barriers, specifically in SmallMedium Tourist Enterprises (SMTEs) that normally have insufficient resources such as the necessary expertise to perform environmental management systems (Tzschentke et al., 2008). For SMTEs, the impediment of environmental management actions may result from an uncertainty of government policy coupled with an over-dependence on government regulation as the expected mechanism for change (Tilley, 2000). Frey and George (2010) found (in South Africa) that the majority of tourism businesses do not believe that government assistance is helpful for putting responsible tourism practices in place and only a small percentage see the government's efforts as positive.

In the hotel sector, a lack of understanding and awareness is seen as a significant barrier to respond to the environmental issues (Hobson \& Essex, 2001). Information regarding 
environmental impacts is a constraint for small hotels (Tzschentke et al., 2008), especially in remote areas or island destinations where basic infrastructures and recycling facilities are often absent (Tzschentke et al., 2008). Meanwhile, inadequate resources (e.g. funds) for hotels are also seen as a great barrier (Alvarez Gil, Burgos, \& Lorente, 2001). Because of limited funds, it becomes clear as to why the hotel sector is unable to or slowly responds to environmental problems (Chan, 2005; Frey \& George, 2010) and only larger hotel businesses could afford it (Alvarez Gil et al., 2001). Inadequate infrastructures, coupled with a limited support and limited interest from local and national authorities, are also considered main barriers to action (Tzschentke et al., 2008). Hotel managers resisting the implementation of sustainable strategies is one of the fundamental barriers (Doody, 2010); this is called 'a lack of motivation to respond'. At the operational level, a limited number of staff members who understand the sustainable practice of hotels could also pose a barrier (Alonso-Almeida et al., 2017) . For further example, Jarvis and Ortega (2010) argue that the smaller-sized hotels may not be proactive due to limited information concerning environmental problems and climate change. Jarvis and Ortega (2010) distinguished hotel's barriers to responses to climate change to direct and indirect barriers, which are outlined in Table 2.3 below: 
Table 2.3: Indirect and direct barriers of (small) hotels concerning climate change responses

\begin{tabular}{|c|c|}
\hline Direct barriers to action & Indirect barriers to action \\
\hline $\begin{array}{l}\text { 1. Time } \\
\text { - The economic imperative } \\
\text { 2. Effort } \\
\text { - Practicalities } \\
\text { - Lack of infrastructure } \\
\text { - Lack of support } \\
\text { - Regulations } \\
\text { 3. Fonstration } \\
\text { - Costs } \\
\text { 5. Lack of resources } \\
\text { 6. Lack of customer demand } \\
\text { 7. The business practices of suppliers } \\
\text { 8. The availability of local produce and labour } \\
\text { 9. Lack of knowledge } \\
\text { - Lack of information } \\
\text { - Misinformation } \\
\text { - Lack of time } \\
\text { 10. Image Lack of expertise } \\
\text { 11. Distanced responsibility }\end{array}$ & $\begin{array}{l}\text { 1. Infrastructure barriers } \\
\text { - The public transport system } \\
\text { - The road system } \\
\text { - Saste and recycling facilities } \\
\text { 2. Economic barriers } \\
\text { - Concentration of the season } \\
\text { - Totel rating system } \\
\text { - The availability of finance } \\
\text { 3. Political barriers } \\
\text { - Hospitality associations } \\
\text { - Administrative boundaries } \\
\text { 4. Strategy formation } \\
\text { 5. Public awareness of environmental issues and } \\
\text { public confusion about policies } \\
\text { 6. Lack of enforcement of environmental regulations } \\
\text { 7. Understanding of the hotel sector } \\
\text { 8. Investment in the hotel sector } \\
\text { 9. Control of the built environment }\end{array}$ \\
\hline
\end{tabular}

Source: (Jarvis \& Ortega, 2010)

Table 2.3 highlights some barriers that can be seen in general case and tourism/hotel's adaptation.

In order to illustrate both the barriers and enabling factors in adaptation in the same context of study, the above sections have presented the adaptation barriers while the next section provides a thorough review of the existing literature about the enabling factors.

\subsection{Enabling factors for climate change adaptation}

"Enabling factors are defined as factors that make it possible for individuals to change their behaviour or their environment. Enabling factors include resources, conditions of living, societal supports, and skills that facilitate a behaviour's occurrence." (Pipithirankarn, n.d., p. 4). Concerning CCA, these factors are believed to have a positive influence on CCA or 
produce a good adaptation outcome. Various attempts have been made to define the desirable outcomes of CCA due to varying and different criteria in assessment (e.g. Adger, Arnell, \& Tompkins, 2005; and Doria et al., 2009). According to Adger et al. (2005), there are limited studies on commonly consensual agreement of 'good' adaptation. Broadly, good adaptation is whenever the adaptation goals are achieved without causing negative effects on others. Papers defining 'enabling factor' remain fragmented. The definitions are in multiple interpretations, sometimes competing each other and in non-CCA context (e.g. in health sector). Adaptation potential is conceptualised differently both in practice and research ( $\mathrm{J}$. Ford, Berrang-Ford, Lesnikowski, Barrera, \& Heymann, 2013). The literature for this study shows that more substantive efforts are required to define what is meant by conditions, environment or factors that encourage or influence adaptation behaviour. There are different terms used to show factors or conditions affecting adaptation. One is 'adaptation opportunities', used by Biesbroek et al. (2013), Murthy, Tiwari, \& Ravindranath (2011), Klein et al. (2014), Ioris, Irigaray, and Girard (2014), Lehmann et al. (2015), and Nguyen et al. (2017). Second is 'adaptation prospect', used by Zuka (2014). Third is 'factors influencing adaptation', 'influencing factors' or 'positive influence for adaptation' (Magness, Lovecraft, \& Morton, 2012; Bryan, Deressa, Gbetibouo, \& Ringler, 2009). Fourth is 'facilitating factors' or 'facilitating adaptation' (Flugman, Mozumder, \& Randhir, 2012; Glaas et al., 2015). Fifth is 'adaptation readiness' (Ford \& King, 2015) and (Tilleard \& Ford, 2016). Sixth is 'enabling adaptation' (Ayesha \& Heather, 2014). Finally, there is 'enabling environment for adaptation' (Zuka, 2014).

In CCA, while Biesbroek et al. (2013) argue that the concepts of 'opportunity', 'stimulus' and 'driver' are often used interchangeably, the literature reviewed for this study reveals that there are similar concepts in the same context of CCA. As the universal concept or term defining what is meant by condition or factor-stimulating adaptation is not available, this research uses the term 'enabling factor' by building on the initial research and its own context of study (also see Jones, Chesterman, Cramer, \& Crane, 2015; Jones, Champalle, Chesterman, Cramer, \& Crane, 2017; Pasquini, Ziervogel, Cowling, \& Shearing, 2014) which is perceived or reported to facilitate, help or influence the hotel sector's adaptation. The term 'enabling factor' seems to be the most prominent in the literature and commonly 
used in similar context of this study. An understanding of the factors that enable CCA investments or actions is important for development or improvement of CCA in the developing countries' coastal hotel sectors.

A considerable amount of literature has been published on CCA. Based on these studies, the conditions or factors that help or facilitate adaptation have been determined. While some factors are generalisable to other countries, this study particularly focuses on those of developing countries. These factors are now reported to be the enabling factors for CCA, which is outlined below.

\subsubsection{Sufficient resources}

Firms having sufficient capital to compensate for the consequences of disasters seem less vulnerable than those with inadequate resources (Orhan, 2016). In process of adaptation, factors such as 'funding' allow having economic resources reserved for the detailed implementation plan and also competent human resource (HR) are taken into consideration in the 'reliability' element while adapting (Olazabal, Galarraga, Ford, Lesnikowski, \& Sainz de Murieta, 2017). Abundant or sufficient resources particularly prove essential in every stage of adaptation, especially implementation and monitoring; resources here refer to financial capacities, staff expertise, technology, information/technical resources and sufficient time (Lowe, Foster, \& Winkelman, 2009; Tribbia \& Moser, 2008). While HR strongly influences the quality of adaptation options, what is important in adaptation are the available financial resources (Simpson et al., 2008). In a broader perspective, effectiveness of community adaptation to climate change needs local resources to be allocated fairly and shared among their members (Zuka, 2014). Financial resources boost adaptive capacity, making local communities raise investments in environmental controls and have independence to select suitable climate change choices (Zuka, 2014).

\subsubsection{Previous and current adaptation experiences/ knowledge and climate change education}

"Experience is the best teacher, it is said." (Akerlof et al., 2013, p. 81). In some accounts, people can accurately identify climatic changes of a decade or more through direct experience 
(Akerlof et al., 2013; Marin, 2010). Previous and current experience of adaptation and adaptation initiatives in organisations, sectors, and communities/regions can offer a valuable knowledge foundation when adaptation actors evaluate adaptive capacity and select adaptation measures (Prutsch et al., 2010). Widespread understanding of climate change affects the implementation of CCA (Simonet \& Fatorić, 2016). In line with this, it is found that people's awareness of and experiences with climate change hazards were strong motivators for adaptive action. Having knowledge of adaptation dimension is also crucial for pioneering ways of tracking progress toward the final goal of helping vulnerable people and businesses productively adapt to a changing climate (Ayesha \& Heather, 2014).

\subsubsection{Engagement/collaboration/communication with other stakeholders}

Adapting to climate change often requires multiple collaboration and coordination between various levels of government from local, to national and to international (Prutsch et al., 2010). This has to be done to guarantee that adapting to climate change takes advantage of stakeholder knowledge to avoid contradiction, identify collaborations, and compromises between different adaptation activities (Prutsch et al., 2010). Key people and stakeholders from governmental decision levels, non-governmental organisations (NGOs) \& International Organisations (IOs), and different business/private sectors should participate in the adaptation processes (Prutsch et al., 2010). There have been increasing trends in efforts to encourage participations in CCA among key people/stakeholder groups (Petersen et al., 2013). Support documents, tools, workshops, and websites are being made at all levels of government and in NGOs as well as in the private sector (Bierbaum et al., 2013). The

guidance, instruments, and information that will have the greatest benefits to help stakeholders to advance further concerning adaptation could vary from place to place (Petersen et al., 2013) but different multiple stakeholders need to work together to deal with the problem of climate change and environmental security to develop place-based measures at community level (Rao, 2013).

\subsubsection{Individuals' motivation to respond (committed workforce)}

Effectiveness of adaptation will depend largely on individuals' motivation to respond. For example, individuals with limited self-efficacy are pessimistic with themselves as being able 
to respond to perceived threats comprehensively (Bandura, 1977, \& Hines et al., 1987, as cited in Adger et al., 2009). Because adaptation tends to be neglected or given a low priority in various sectors, regions and organisations, the starting point of adaptation very much relies on individuals to activate and drive such processes (Prutsch et al., 2010). Literature indicates that most individuals have a tendency to respond to negative impacts or concerns, only if they face an immediate impact and are personally relevant (Moser \& Dilling, 2004; Paton, Millar, \& Johnston, 2001).

While resources have become available, the level of adaptation significantly depends on individuals who could take part in adaptation activities without political and socio-economic barriers (Zuka, 2014). The effects of adaptation are ultimately based on the individuals' actions and flexibility in their social structures that support them (Kalaugher et al., 2013). In responding to environmental impacts, including climate change, Tzschentke et al. (2008) argue that in tourism firms, the personal commitment and value credited are in fact essential motivators. In particular, if the action is considered to be morally or financially satisfying or worthwhile, commitment is likely greater.

\subsubsection{Sufficient information}

Relevant information is a valuable resource in adapting to climate change. Any institutions or businesses that have access to place-based information have been reported as more dynamic in both planning and implementation of adaptation (Archie et al., 2014). This includes the predicted climate change impacts that could facilitate and prepare adaptive responses to mitigate risks (Glaas et al., 2015). Dessai et al. (2005) continue that accessible information to the existing legal, social, and organisational arrangements is an important element for the adaptation process. At the regional level, the existence of sound and timely information detailing the persistent uncertainty can help enhance the adaptive capacity of a particular region. This becomes more effective when combined with communication that engages the public in climate change on a regular basis (Moser et al., 2008). The adaptive capacity of destinations can be increased through consistent and effective interaction with

and engagement of the public (Moser et al., 2008) but this must be appropriately supported by suitable, accurate and punctual information (Moser et al., 2008). 


\subsubsection{Clear adaptation goals}

In order to benefit from environmental performances, more dedicated and clear guidance is needed for accommodation businesses (Coles, Dinan, \& Warren, 2016). Although adaptation actions can be undertaken with diverse objectives, its effectiveness is simply defined by meeting those objectives and how they influence the ability of others to achieve their objectives of adaptation (Adger et al., 2005). Good and effective adaptation goals are the first to identify climate-related risks at current levels and to minimise impacts from current points (Adger et al., 2005). Second, the good adaptation goals minimise exposure of the most vulnerable groups or other populations (Adger et al., 2005). Yet the goals of adaptation could differ and vary within a sector, a community, a society, and between different generations (Adger et al., 2009).

\subsubsection{Good leadership or management structures}

Leadership becomes an essential condition at any stage of the adaptation process (Cuevas, 2015). Strong leadership in CCA ensures that the outcomes meet the needs of beneficiaries (Rissik \& Reis, 2013). Leadership plays a further role in contributing to the benefits of climate change responses through the power of explicit policy directives articulating climate change adaptation as a municipal priority (Burch, 2010). Another finding in Zhang et al. (2013) states that the right kind of employees and management team is the most essential factor for the achievement in the service industry.

Studying the business recovery from disaster-induced problems, Orhan (2016) argues that generally a good manager is embedded with experience, foresight, vision, and leadership. The outcome of responses is determined by these factors. Lozano (2015) distinguishes between external and internal motivators for environmental response for greening the hotel sector. Lozano also states that while the regulations and stakeholder demands are classified as the primary external motivators, the internal motivation include the management's opinions and the promise of improved efficiency. In a broader understanding, planning for climate adaptation strongly needs a political leadership by cooperating with authority (e.g. mayors and city engineers) (Anguelovski, Chu, \& Carmin, 2014). 


\subsubsection{Political will}

Political awareness is regarded as a key condition in the literature on CCA (Biesbroek et al., 2011). Adapting to climate change requires participations and responses of the government, as government cooperation at all levels (national and local) is the key requirement for CCA. Clear political signals are needed, especially the climate-related messages from the national to local government and vice versa. The local governments are definitely challenged to develop effective adaptation policies and implement them without clear political message from the national authorities (Amundsen et al., 2010; Measham et al., 2011). Clear political will means that there is a responsibility for CCA by financing it (Amundsen et al., 2010). "Climate change is a political issue with which political leaders from all political parties and localities are concerned" (Amundsen et al., 2010, p. 281). However, Biesbroek et al. (2011) are concerned that climate change is in competition with other issues for political attention. Politics have little influence on people living in the remote, rural communities off the road system, which are communities with comparatively limited infrastructure (McNeeley, 2012).

Informed by the literature above, the enabling factors affecting adaptation have been summarised in Table 2.4 below. 
Table 2.4: Summary of enabling factors for CCA

\begin{tabular}{|c|c|c|}
\hline No. & Enabling factors & Sources \\
\hline 1 & $\begin{array}{l}\text { Sufficient resources (e.g. finance/budget, human } \\
\text { resource, and available technology) }\end{array}$ & $\begin{array}{l}\text { (Orhan, 2016) } \\
\text { (Olazabal, Galarraga, Ford, Lesnikowski, } \\
\text { \& Sainz de Murieta, 2017) } \\
\text { (Lowe, Foster, \& Winkelman, 2009) } \\
\text { (Tribbia \& Moser, 2008) } \\
\text { (Zuka, 2014) }\end{array}$ \\
\hline 2 & $\begin{array}{l}\text { Previous and current adaptation } \\
\text { experiences/awareness/knowledge and climate } \\
\text { change education }\end{array}$ & $\begin{array}{l}\text { (Akerlof et al., 2013) } \\
\text { (Marin, 2010) } \\
\text { (Prutsch, Grothmann, Schauser, Otto, \& } \\
\text { McCallum, 2010) } \\
\text { (Simonet \& Fatorić, 2016) } \\
\text { (Vulturius \& Gerger Swartling, 2015) } \\
\text { (Ayesha \& Heather, 2014) }\end{array}$ \\
\hline 3 & $\begin{array}{l}\text { Engagement/collaboration/communication with } \\
\text { other stakeholders }\end{array}$ & $\begin{array}{l}\text { (Prutsch et al., 2010) } \\
\text { (Bierbaum et al., 2013) } \\
\text { (Petersen, Hall, Kahl, \& Doran, 2013) } \\
\text { (Rao, 2013) }\end{array}$ \\
\hline 4 & $\begin{array}{l}\text { Individuals' motivation to respond (committed } \\
\text { workforce) }\end{array}$ & $\begin{array}{l}\text { (Bandura, } 1977 \text { \& Hines et al., } 1987 \text { cited } \\
\text { in Adger, Lorenzoni \& O'Brien, 2009) } \\
\text { (Prutsch et al., 2010) } \\
\text { (Moser \& Dilling, 2004) } \\
\text { (Paton, Millar, \& Johnston, 2001) } \\
\text { (Zuka, 2014) } \\
\text { (Kalaugher et al., 2013) } \\
\text { Tzschentke et al. (2008) }\end{array}$ \\
\hline 5 & Sufficient information (e.g. about vulnerabilities...) & $\begin{array}{l}\text { (Archie et al, 2014) } \\
\text { (Glaas et al., 2015) } \\
\text { (Dessai et al. (2005) } \\
\text { (Moser et al., 2008) }\end{array}$ \\
\hline 6 & Clear adaptation goals & $\begin{array}{l}\text { (Coles, Dinan, \& Warren, 2016) } \\
\text { (Adger et al., 2005) } \\
\text { (Adger, et al., 2009) } \\
\end{array}$ \\
\hline 7 & $\begin{array}{l}\text { Good leadership or management structures (e.g. } \\
\text { strengthen institutions to implement CCA } \\
\text { programmes) }\end{array}$ & $\begin{array}{l}\text { (Cuevas, 2015) } \\
\text { (Rissik \& Reis, 2013) } \\
\text { (Burch, 2010) } \\
\text { (Zhang et al., 2013) } \\
\text { (Orhan, 2016) } \\
\text { (Lozano, 2015) } \\
\text { (Anguelovski, Chu, \& Carmin, 2014) }\end{array}$ \\
\hline 8 & Political will & $\begin{array}{l}\text { (Ford \& Pearce, 2010) } \\
\text { (Amundsen, Berglund, \& Westskog, } \\
2010) \\
\text { (Measham et al., 2011) } \\
\text { (Biesbroek et al., 2011) } \\
\text { (McNeeley, 2012) }\end{array}$ \\
\hline
\end{tabular}


Even when substantial CCA literature about enabling factors is reviewed, there are still knowledge gaps about the hierarchy or criticality level of the enabling factors, especially when questioning which enabling factor is the most important. The following section presents the level of criticality of enabling factors that affect adaptation.

\subsection{Critical enabling factor for climate change adaptation}

Key components and concepts encouraging adaptation implementation were presented in section 2.12 and the term 'enabling factor' has been determined for this study. It is common to see it used with 'important' or 'unimportant' enabling factors to show the level of its positive effects or the level of criticality within the adaptation process. The authors below show higher or stronger hierarchy levels by using the term 'critical' enabling factor for CCA initiatives.

Bowen et al. (2013) examine to what extent there are enabling factors to enhance the development of adaptation activities in the health sector. They identify some 'critical' components as enabling adaptiveness, such as political recognition of climate change. Vaughan et al. (2017) add that the usage of climate information for societal decision-making is regarded as the 'critical' component to improve the capability of individuals, businesses, and governments to respond to climate change and variability. Vaughan et al. (2017) ascertain that in the field of climate services, knowledge about the planning and investment approaches of local, national, and international actors is the 'critical' component in climate change responses. Dinku et al. (2014) argue that in climate services, capacity development is another 'critical' aspect. Hurford et al. (2017) present a set of five 'critical' factors that increase the climate resilience of water infrastructures, which are as follows:

- simple and effective communication of climate risks and uncertainty,

- involving the right stakeholders at every stage,

- capitalising on entry points (gaining a thorough understanding of the entry points into decision making and planning processes to maximise the opportunity for the effective change),

- going beyond the project (adjusting infrastructure design to improve resilience), and 
- building institutional capacity for assessment, design and financing.

However, it has been rare to see the term 'critical enabling factor' engaged in the CCA studies. The studies that were reviewed appear to have limited connection between 'critical' enabling factor and CCA, and there is a clear need to deepen an understanding of the criticality of enabling factors in tourism-CCA studies. In order to fill the gap or be motivated by these studies, this research has shown that the 'enabling factor' could be used in the same hierarchy or three-level hierarchy such as the most, medium and the least critical enabling factors for CCA in the hotel sector which is outlined in chapter 6 .

Building on the existing literature, the following section incorporates a conceptual framework suitable for the coastal hotel's adaptation. The framework might provide crosscutting benefits to other stakeholders such as the public and private sectors (e.g. other accommodation sectors), and IOs \& NGOs concerning CCA.

\subsection{Conceptual framework}

Conceptual frameworks play a crucial role in identifying and combining existing knowledge and in identifying future research directions by merging fragmented or broad themes (Pearce, 2012). Furthermore, they help to shape the research design by assisting with identifying, defining and operationalising the concepts to be used and the relationships to be studied (Veal, 2006). From the literature, it is often easy to see conceptual framework on tourism on one hand and conceptual framework on CCA on the other hand. Due to lack of combination of a conceptual framework between tourism and adaptation, Figure 2.1 combines themes/variables of coastal tourism hotel sector and CCA from the literature into a comprehensive framework. 
Figure 2. 1: Conceptual framework for CCA in the coastal hotel sector

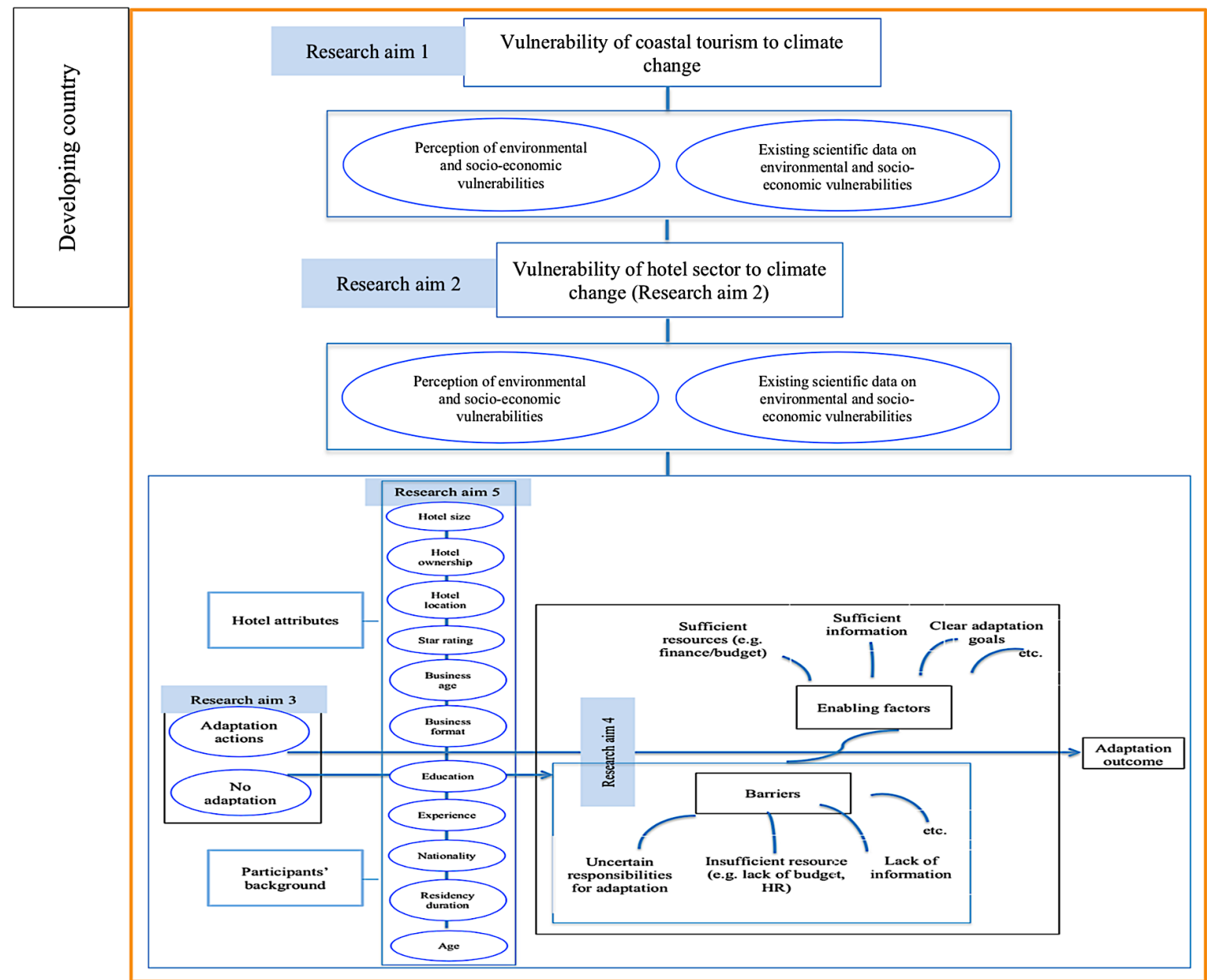

First, the importance and necessity of vulnerability assessment are included in the framework (see Jopp et al., 2010; Moreno \& Becken, 2009). The vulnerability of SHV's coastal tourism and the hotel sector to climate change is assessed using the perceptions of environmental and socio-economic vulnerabilities, supported by the existing scientific data. This assessment is influenced/recommended by a number of researchers (see section 2.4). Vulnerability assessment is a precondition for identifying adaptation because the assessment results provide a summary of key information for adaptation. Vulnerability assessment encompasses knowledge and other benefits that may facilitate adaptation (Hamilton, Fischer, Guikema, \& Keppel-Aleks, 2018). This corresponds with the research aims 1 and 2 as outlined in the previous chapter and Figure 2.1. 
Second, under the effects of climatic changes, the starting assumption is that some hotels have initiated adaptation actions while some do not have such initiatives. Those that have the initiatives will have both barriers and enabling factors to adaptation. Those that do not have initiatives will neither experience the fruitful lessons nor produce the remarkable adaptation outcome, but they will exhibit adaptation barriers. The initiatives provide a comprehensive map to visualise the key determinants of whether/how the hotel sector adapts to climate change, which informs research aim 3.

Third, after reviewing the literature, many barriers and enabling factors have been identified. Barriers to CCA include limited resources (e.g. lack of HR and budget), uncertain responsibilities of adaptation, lack of information, etc. While enabling factors include sufficient resources (e.g. HR and budget), sufficient information and clear adaptation goals. These two distinct groups are expected to be influenced by the hotel attributes and participants' backgrounds as highlighted in above section. This remains open to the question as to whether which barriers are more major or the most major and which enabling factors are more or the most important. This is relevant to research aim 4, which aims to identify the most significant/critical barriers and enabling factors in the context of the hotel sector's adaptation to CCA.

Finally, as outlined in section 2.6, in adapting to climate change, the outcome of the hotel sector's adaptation appears to be influenced by hotel attributes (hotel size, hotel ownership, hotel location, star rating etc.) and hoteliers' backgrounds (education, nationality, experience, age etc.). This may reflect the reality that barriers and enabling factors are influenced by the same variables (hotel attributes and hoteliers' backgrounds). This aligns with research aim 5, which is positioned to critically examine the barriers and enabling factors to adaptation according to hotel attributes and participants' backgrounds.

\subsection{Chapter summary}

Accommodation/hotel sector is considered one of the world's most important subsectors in tourism. However, within tourism, it is considered to have the lowest adaptive capacity due to fixed structures (buildings). Meanwhile, the accommodation/hotel sector is often limitedly 
discussed in tourism and climate change literature, especially in CCA context. Instead, it has been examined in the context of environmental performance and management.

After a vital relationship between vulnerability and adaptation was developed, an adaptation framework was proposed for the hotel sector. At the same time, potential barriers and enabling factors to adaptation were also identified. While the barriers and enabling factors to adaptation in other sectors are widely discussed in the literature, there is limited engagement the discussion with the barriers and enabling factors relevant to the accommodation in the hotel sector's adaptation of developing countries. 


\section{CHAPTER 3: CASE STUDY CONTEXT}

\subsection{Introduction}

This chapter contributes to an extensive understanding of the research context, the coastal hotel sector of Cambodia's SHV. This chapter presents the background, tourism and climate change of both Cambodia and SHV. SHV has been selected as the case study because of its high vulnerability to climate change and low adaptive capacity. Development Partners (DPs) and individual researchers have increasingly paid attention to the issue of climate change. The Royal Government of Cambodia (RGC) has also identified its coastal area as one of the country's most vulnerable places and suggested urgent adaptations, especially in the tourism sector. This aligns with the world's commitment to the sustainability of tourism. SHV's coastal tourism offers a good example of a climate change-focused study because its tourism is strongly dependent on the climate but highly vulnerable to climate change. In addition, the CCA in coastal hotel sector has not been sufficiently studied either in Cambodia or in the literature more broadly.

The chapter is divided into two main sections. First, it introduces Cambodia's background, such as its modern history, tourism and climate change (section 3.2). The second section presents the research site, SHV with a particular focus on its background, tourism and climate change (section 3.3).

\subsection{Cambodia's background}

\subsubsection{Cambodia's modern history}

Geographically, Cambodia is located in Southeast Asia (see figure 3.1). Cambodia comprises of $90 \%$ Khmer, 5\% Vietnamese, 2\% Cham, 1\% Chinese, and 1\% hill tribes (Poole, 2009), and $90 \%$ of them live in rural areas (ADB, 2014). The capital city is Phnom Penh, which is connected by roads, waterways (rivers) and railways to other provinces and by main roads, airways and seaports to other countries. With the density of 84 people per square kilometre (ADB, 2017b), Cambodia covers an area of 181,040 square kilometres bordering with Laos, Vietnam, and Thailand with a coastal territory on the Gulf of Thailand. Its coastal line is 440 
$\mathrm{km}$ and constituted by four coastal provinces, the $4 \mathrm{Ks}$ : Kampot, Kep, Koh Kong and Kompong Soam $/ \mathrm{SHV}^{7}$.

Figure 3.1: Administrative map of Cambodia

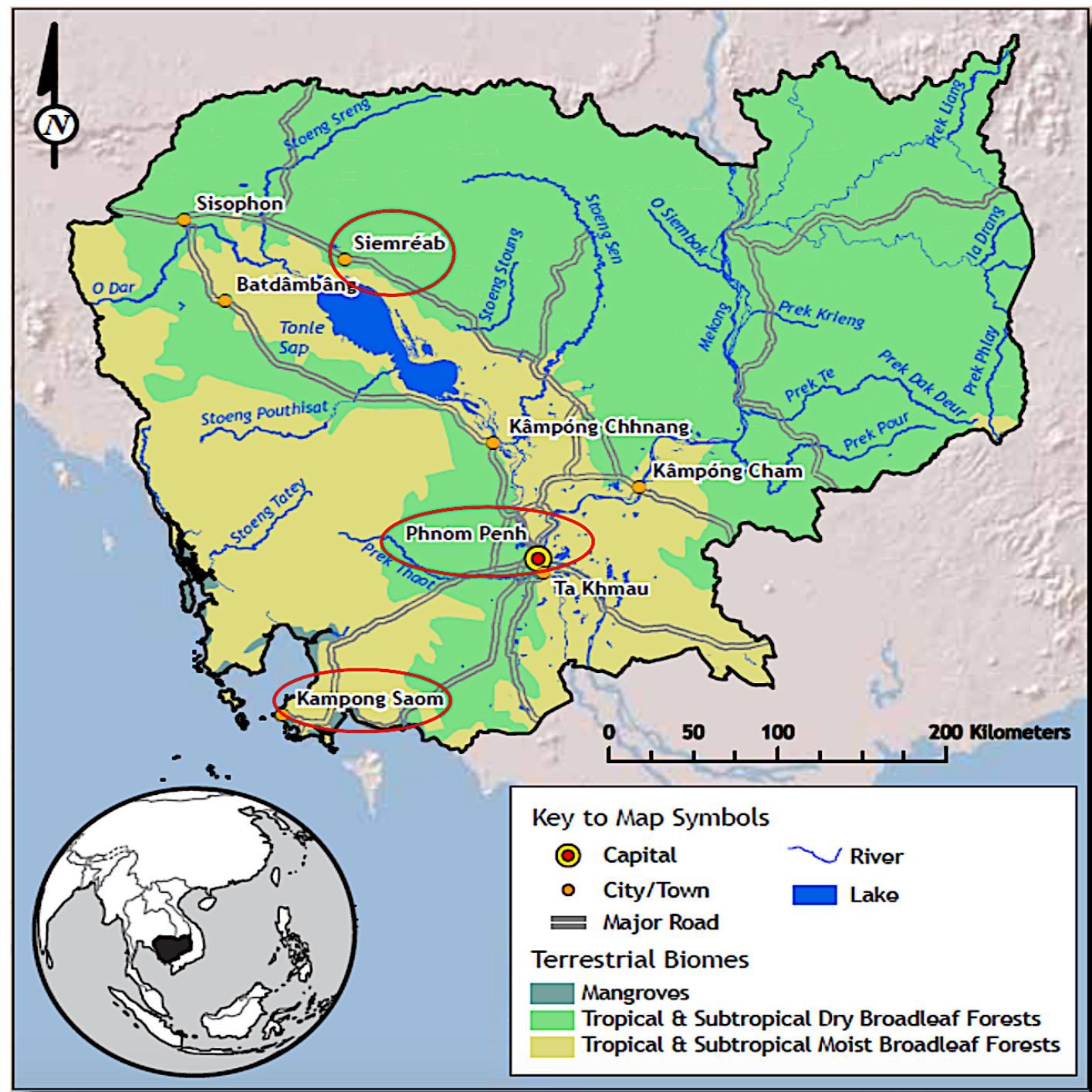

Source: Adapted from Botkosal, Kong, \& Chanthou (2012)

After the Khmer Rouge regime collapsed in 1979 followed by continued fighting and ultimately through the 1991 Paris Peace Accord, Cambodia has opened a new chapter for its

${ }^{7}$ It is known by local people as Kompong Soam or Preah Sihanouk province and internationally known as Sihanoukville, which is named after the King Norodom Sihanouk. 
population and economic growth. As of 2016, Cambodia had a population of 15.76 million with 20.02 billion USD of GDP and 1,269.91 USD GDP per capita (World Bank, 2018) ${ }^{8}$.

Since 1993, the first national election supported by the United National Transitional Authority in Cambodia (UNTAC), the economy began to grow slowly but steadily. Cambodia's economy has marked impressive growth with $7.1 \%, 7 \%, 7 \%$ and $7.1 \%$ in 2014 , 2015, 2016 and 2017 respectively, and it is projected to reach 7.1\% in 2018 (ADB, 2018) ${ }^{9}$. This steady economic growth reflects Cambodia's efforts towards poverty reduction. For instance, the poverty rate decreased from $47.8 \%$ in 2007 to $22.9 \%$ in $2009,19.8 \%$ in 2011 , and $18.9 \%$ in 2012 (ADB, 2014). Despite efforts, over 30\% of the labour force make below USD1.25 per day and 53\% of the Cambodian population earns below USD2.00 per day (ADB , 2017a). Cambodia is still classified as a lower middle income country (World Bank, 2018) or the Least Developed Countries $\left(\operatorname{LDC}^{10}\right)$ (UN, 2017b).

The power is highly centralised at the national level. However, decentralisation has emerged since the involvement of DPs after 1993, the first national election backed by UNTAC. The office of the Council of Ministers administers 25 ministries in Cambodia and a number of separate secretariats governing the country's development. Under each ministry there are provincial departments/offices, which oversee all activities relevant to their assigned responsibilities. For instance, the MOT governs all tourism-related actions nationwide through its sub-national departments/offices located in each province. The Ministry of Environment (MOE) oversees all climate change-related and other actions nationwide through its sub-national departments/offices located in each province.

\subsubsection{Cambodia's tourism}

For many years, Cambodia's economy has depended on four main sectors: agriculture, tourism, garment manufacturing, and construction. Cambodia's tourism has grown rapidly over the last decade, with a near doubling in visitor arrivals over a seven-year period from

\footnotetext{
${ }^{8}$ Available at https://data.worldbank.org/indicator/SP.POP.TOTL, accessed on March 17, 2018.

${ }^{9}$ Available at https://www.adb.org/countries/cambodia/economy accessed on March 17, 2018.

10 The Least Developed Country
} 
2.8 million in 2011 to 5.5 million in 2017 . This is expected to grow by $3.2 \%$, with $5,552,000$ international tourist arrivals in 2018 (WTTC, 2018a). In 2028, the projection is 7,969,000 international tourist arrivals that will generate USD 5 billion and 80,000 jobs (RGC, 2012; WTTC, 2018a) (see Figure 3.2 and Table 3.1). The average tourist expenditure is USD120 per day across all international overnight markets (ADB, 2017b).

Figure 3. 2: Cambodia: Visitor exports and international tourist arrivals ${ }^{11}$

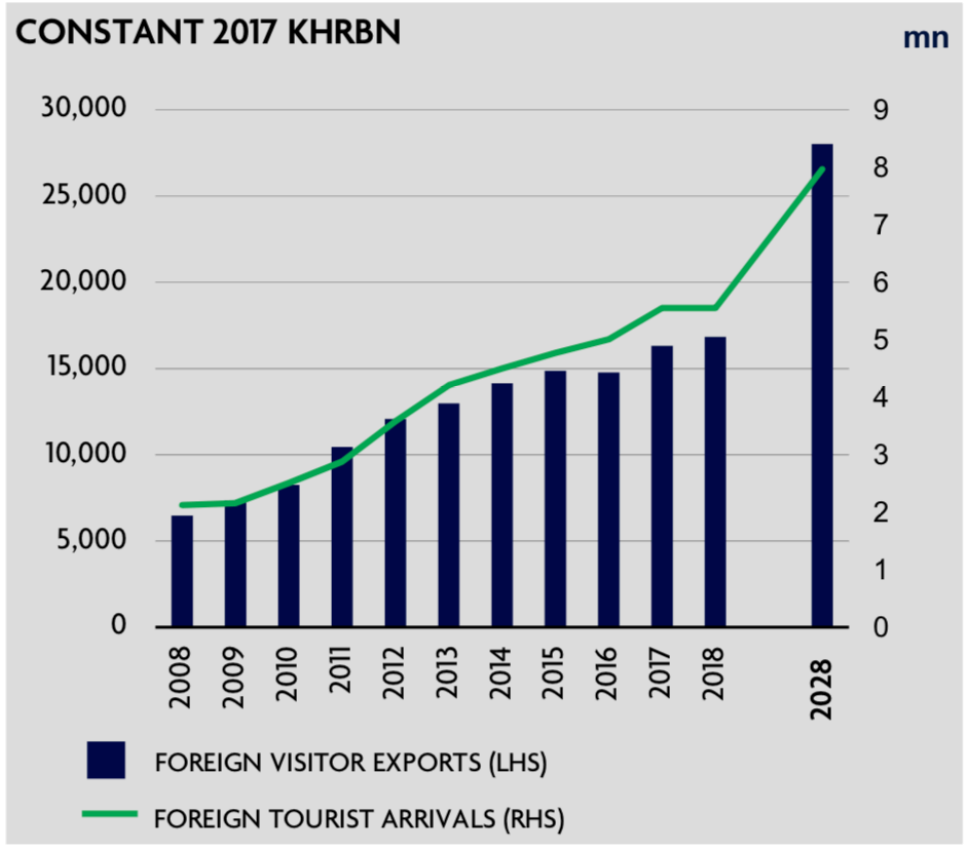

Source: (WTTC, 2018a)

\footnotetext{
${ }^{11} \mathrm{KHRBN}=$ Khmer/Cambodian billion Riel
} 
Table 3.1: Cambodia's tourism highlights ${ }^{12}$

\begin{tabular}{|c|c|c|c|c|c|}
\hline \multirow{2}{*}{ Years } & \multicolumn{2}{|c|}{ Int'| Tourist Arrivals } & \multirow{2}{*}{$\begin{array}{l}\text { Average Length } \\
\text { of Stays (Days) }\end{array}$} & \multirow{2}{*}{$\begin{array}{c}\text { Hotel Occupancy } \\
(\%)\end{array}$} & \multirow{2}{*}{$\begin{array}{c}\text { Tourism Receipts } \\
\text { (Million US\$) }\end{array}$} \\
\hline & Number & Change (\%) & & & \\
\hline 1993 & 118,183 & - & N/A & N/A & $N / A$ \\
\hline 1994 & 176,617 & 49.4 & $\mathrm{~N} / \mathrm{A}$ & $\mathrm{N} / \mathrm{A}$ & N/A \\
\hline 1995 & 219,680 & 24.4 & 8.00 & 37.0 & 100 \\
\hline 1996 & 260,489 & 18.6 & 7.50 & 40.0 & 118 \\
\hline 1997 & 218,843 & -16.0 & 6.40 & 30.0 & 103 \\
\hline 1998 & 286,524 & 30.9 & 5.20 & 40.0 & 166 \\
\hline 1999 & 367,743 & 28.3 & 5.50 & 44.0 & 190 \\
\hline 2000 & 466,365 & 26.8 & 5.50 & 45.0 & 228 \\
\hline 2001 & 604,919 & 29.7 & 5.50 & 48.0 & 304 \\
\hline 2002 & 786,524 & 30.0 & 5.80 & 50.0 & 379 \\
\hline 2003 & 701,014 & -10.9 & 5.50 & 50.0 & 347 \\
\hline 2004 & $1,055,202$ & 50.5 & 6.30 & 52.0 & 578 \\
\hline 2005 & $1,421,615$ & 34.7 & 6.30 & 52.0 & 832 \\
\hline 2006 & $1,700,041$ & 19.6 & 6.50 & 54.8 & 1,049 \\
\hline 2007 & $2,015,128$ & 18.5 & 6.50 & 54.8 & 1,400 \\
\hline 2008 & $2,125,465$ & 5.5 & 6.65 & 62.7 & 1,595 \\
\hline 2009 & $2,161,577$ & 1.7 & 6.45 & 63.6 & 1,561 \\
\hline 2010 & $2,508,289$ & 16.0 & 6.45 & 65.7 & 1,786 \\
\hline 2011 & $2,881,862$ & 14.9 & 6.50 & 66.2 & 1,912 \\
\hline 2012 & $3,584,307$ & 24.4 & 6.30 & 68.5 & 2,210 \\
\hline 2013 & $4,210,165$ & 17.5 & 6.75 & 69.5 & 2,547 \\
\hline 2014 & $4,502,775$ & 7.0 & 6.50 & 67.6 & 2,736 \\
\hline 2015 & $4,775,231$ & 6.1 & 6.80 & 70.2 & 3,012 \\
\hline 2016 & $5,011,712$ & 5.0 & 6.30 & 68.9 & 3,212 \\
\hline
\end{tabular}

Source: (MOT, 2016)

In Cambodia, tourism businesses are private-owned enterprises. There are no state-owned tourism enterprises. Table 3.1 illustrates that from 1993-2016 the hotel occupancy rate has an increasing trend. For example, in eight years from 2008-2016, the average occupancy rate was $66.98 \%$, higher than the previous decade from 1998-2007 with 54.06\%. Table 3.2 below also shows that the number of hotels has grown from 1997 to 2016. For example, in the period from 2008-2016, the number of hotels was 4,782, higher than the previous decade from 1998-2007 with 3,024 hotels.

Most hotels are located in the most popular tourist areas: in Siem Reap, 417 hotels (with 17,000 rooms), in Phnom Penh, 317 hotels (with 15,000 rooms) (The Phnom Penh Post, 2015), and SHV 75 hotels (with 3,666 rooms) (the researcher's interviews). Accommodation

\footnotetext{
${ }^{12}$ Int'l = International
} 
options comprise various ranges from small family-owned businesses to five-star internationally luxurious standard (ADB, 2017b; Chankoulika \& Bopha, 2017).

Table 3.2: Number of hotels and guesthouses in Cambodia from 1997-2016

\begin{tabular}{|c|c|c|c|c|}
\hline \multirow{2}{*}{ Years } & \multicolumn{4}{|c|}{ Hotels } \\
\hline & Number & Change (\%) & Rooms & Change (\%) \\
\hline 1997 & 179 & & 6,989 & \\
\hline 1998 & 216 & 20.7 & 8,247 & 18.0 \\
\hline 1999 & 221 & 2.3 & 9,115 & 10.5 \\
\hline 2000 & 240 & 8.6 & 9,673 & 6.1 \\
\hline 2001 & 247 & 2.9 & 10,804 & 11.7 \\
\hline 2002 & 267 & 8.1 & 11,426 & 5.8 \\
\hline 2003 & 292 & 9.4 & 13,201 & 15.5 \\
\hline 2004 & 299 & 2.4 & 14,271 & 8.1 \\
\hline 2005 & 317 & 6.0 & 15,465 & 8.4 \\
\hline 2006 & 351 & 10.7 & 17,914 & 15.8 \\
\hline 2007 & 395 & 12.5 & 20,470 & 14.3 \\
\hline 2008 & 398 & 0.8 & 20,678 & 1.0 \\
\hline 2009 & 451 & 13.3 & 23,010 & 11.3 \\
\hline 2010 & 440 & -2.4 & 24,393 & 6.0 \\
\hline 2011 & 476 & 8.2 & 26,484 & 8.6 \\
\hline 2012 & 490 & 2.9 & 27,117 & 2.4 \\
\hline 2013 & 545 & 11.2 & 29,937 & 10.4 \\
\hline 2014 & 554 & 1.7 & 29,729 & -0.7 \\
\hline 2015 & 668 & 20.6 & 34,619 & 16.4 \\
\hline 2016 & 760 & 13.8 & 40,160 & 16.0 \\
\hline
\end{tabular}

Source: (MOT, 2016)

Various laws, policies and strategic plans have been initiated for the country's tourism development. The National Tourism Policy 2008 was expanded to the Law on Tourism $2009^{13}$. Both identify and place emphasis on the need for diversifying tourism destinations, products and services, and in return Cambodians can inclusively reap direct benefits. Along with the potential growth of tourism, the Tourism Development Strategic Plan 2012-2020 (TDSP 2012-2020) ${ }^{14}$ reemphasises Cambodia's determination to achieve sustainable

\footnotetext{
${ }^{13}$ Available at http://www.cncc.gov.kh/userfiles/image/download/Laws-A11\%20Law\%20on\%20TourismEn.pdf accessed on March 19, 2018.

${ }^{14}$ Available at

http://www.tourismcambodia.org/images/mot/legal_documents/tourism_development_stategic_plan_2012_20 20 english.pdf, accessed on March 19, 2018
} 
tourism and its capacity in elevating the socio-economic improvement. The TDSP 20122020 strengthens the strategic goal and direction stated in the Strategic Plan and National Eco-tourism Policy to promote tourism based on cultural and natural attractions, including the marine and seaside potentials in the coastal southwest. The country's National Strategic Development Plan Update 2014-2023 ${ }^{15}$ likewise identifies the role of tourism, including heritage, cultural and eco-tourism in the economy of the country's development.

Figure 3.3 shows the organisational structure of MOT. There are a number of responsible general departments, departments, offices and units. The Department of Tourism Planning and Development is responsible for tourism promotion and communication with other Ministries, particularly MOE regarding climate change issues. This Department is also responsible for implementing tourism-climate change plans.

\footnotetext{
${ }^{15}$ Available at http://www.cambodiaip.gov.kh/DocResources/ab9455cf-9eea-4adcae9395d149c6d78c 007729c5-60a9-47f0-83ac-7f70420b9a34-en.pdf, accessed on March 18, 2018
} 


\section{Figure 3.3: Organisational structure of the Ministry of Tourism}

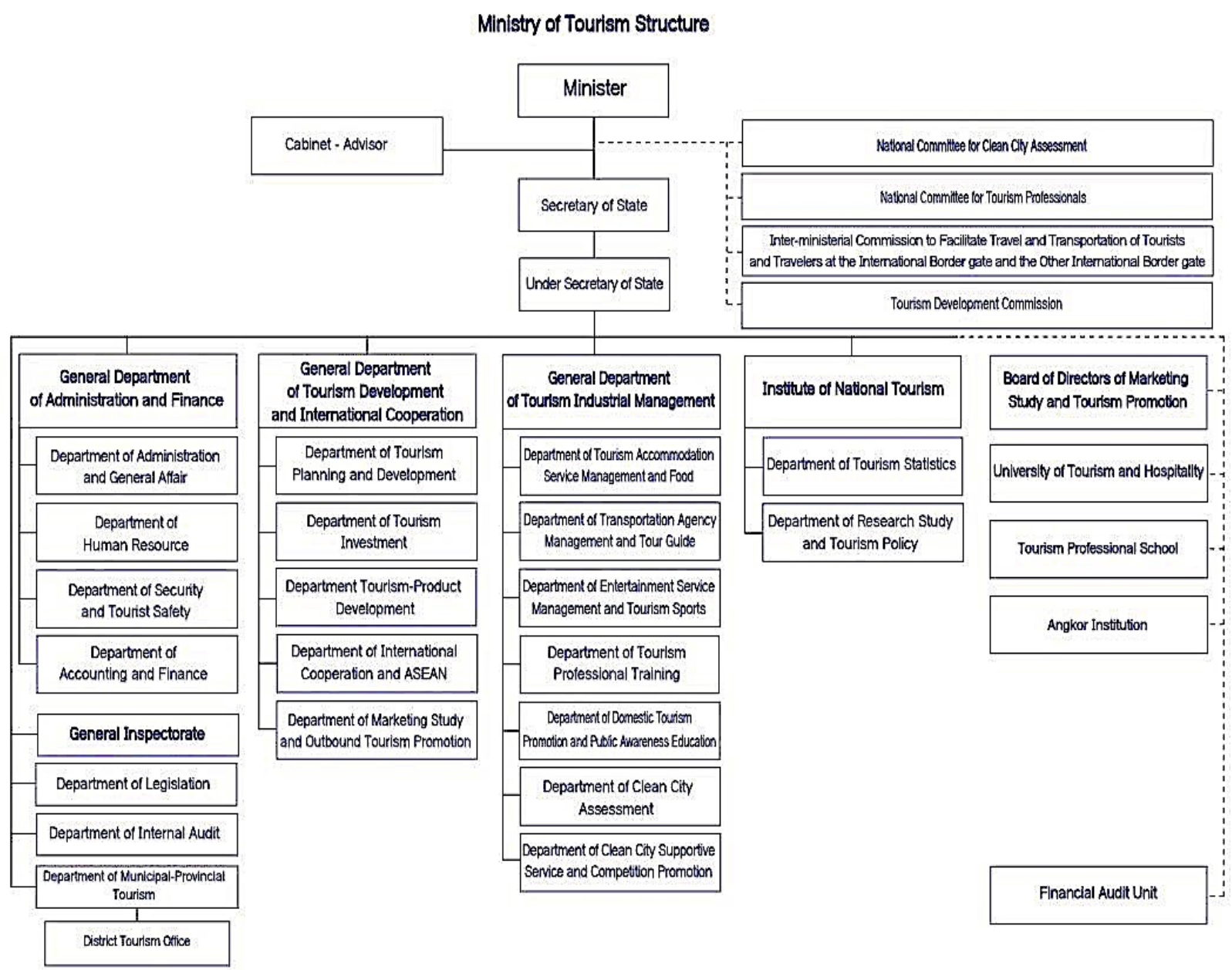

Source: (MOT, 2018)

\subsubsection{Climate change in Cambodia}

Climate change poses special risks and vulnerabilities to LDCs or developing countries (Halsnæs, Kaspersen, \& Trærup, 2016). Climate change effects threaten Cambodia which was classified in 2011 among the world's 15 most vulnerable countries (World Risk Report, 2012), with a loss of GDP $3.1 \%-4.3 \%$ caused by severe climate change incidents annually (Harmeling \& Eckstein, 2013; RGC, 2013). At the international stage, Cambodia is defining herself as a global victim of climate change (Käkönen et al., 2014). The coastal zone is vulnerable to excessive rainfall, increased temperatures, storm/storm surges, SLR/beach 
erosion, and drought and flooding (MOE, GEF ${ }^{16}$, \& UNEP, 2015a; UNWTO, 2014) and these will now be discussed in turn to contextualise the research.

\subsubsection{Vulnerability to excessive rainfall}

As outlined above, Cambodia is a highly vulnerable country in the context of climate change. A report by RGC (2013) shows that variations in precipitation in Cambodia have begun since the second half of the 20th Century. Based on $\mathrm{CSIRO}^{17}$, the MOE (2002) indicates that nationally the rainfall increases over the baseline ${ }^{18}$ are expected to be between 5-15\%, 5$23 \%$ and $3-35 \%$ for 2025, 2050 and 2100. Validated by Thoeun (2015) who found that the central low land regions receive the annual mean rainfall of $1400 \mathrm{~mm}$ while the coastal zones or highland areas obtain $4000 \mathrm{~mm}$. Thoeun (2015) further projects that the annual mean precipitation in the country tend to rise between $3 \%$ and $35 \%$ by the year 2100 , with the degree of change based on time and location. Low-lying areas are expected to have a higher trend in precipitation than plateaus and highlands.

Table 3.3 presents the past-recorded and predicted changes of rain patterns in major areas with different time periods. Excessive rainfall resulting from climate change causes a number of effects that are especially damaging to infrastructure and leading to food shortages (see Table 3.3 below). These are likely to negatively affect the country's tourism growth, particularly at the coastal zone.

Table 3.3: Actual and projected change of Cambodia's annual rainfall

\begin{tabular}{|c|c|c|c|c|}
\hline \multirow{2}{*}{ Area } & \multicolumn{2}{|r|}{ Year } & \multicolumn{2}{|c|}{ Future projection } \\
\hline & 2010 & 2013-2015 & 2050 & 2100 \\
\hline Low-lying area & $\begin{array}{l}1,000 \mathrm{~mm} \text { to } \\
1,700 \mathrm{~mm}\end{array}$ & Increase $4-8 \%$ & Increase $8-12 \%$ & \multirow{3}{*}{ Increase $3-35 \%$} \\
\hline Highland area & $\begin{array}{l}1,000 \mathrm{~mm} \text { to } \\
2,700 \mathrm{~mm}\end{array}$ & Increase $0-4 \%$ & Increase $2-6 \%$ & \\
\hline Coastal zone & $\begin{array}{l}1,000 \mathrm{~mm} \text { to } \\
3,000 \mathrm{~mm}\end{array}$ & $\begin{array}{l}\text { Increase } 0-4 \% \text { or } 3,800 \mathrm{~mm} \\
\text { to } 4000 \mathrm{~mm} \text { or } 5000 \mathrm{~mm}\end{array}$ & Increase $2-6 \%$ & \\
\hline
\end{tabular}

Sources: (Country Water Partnership Southeast Asia, 2010; GSSD, 2015; Phirun, 2013; UNEP, 2015 a and

Thoeun, 2015)

\footnotetext{
16 Global Environment Facility

17 The Commonwealth Scientific Industrial Research Organization, Australia

18 The baseline refers to the mid to late $1990 \mathrm{~s}$
} 


\subsubsection{Vulnerability to storm}

In Cambodia, storms happen more commonly from August to November, with the highest intensity in October (GSSD, 2015). For example, Cambodia was hit by Typhoon Ketsana on September 29-30, 2009. The storm caused significant damage and loss, affected about 50,000 families, and resulted in the deaths of 43 people and 67 severely injured (RGC, 2010a). From 1996 to 2014, it was recorded that 1,213 severe storm events throughout Cambodia with the average mean 76 storms/year (Halsnæs, Kaspersen \& Trærup, 2016). From this baseline data, they project that Cambodia is forecast to be more at risk from heavy storms in the future.

In the coastal zone, in the rainy season, strong winds or wind contraction sweep from the sea or from the west and can accumulate to storms continuing up to one week. The southern and western region of Cambodia's coastal area is threatened by increasing wind speeds in the future. Worse, the southern region of the coast is where a great number of vulnerable communities are located (GSSD, 2015). During storms and strong winds, wave height could be 2-3.5 m, thus making sea travel difficult (GSSD, 2015). SLR, unusual variations in high and low tides, storm, rising temperatures and surges have been recorded (MOE \& Mangrove for the Future, 2013). Although winds may cause little potential damage to infrastructure, they can significantly affect crops including maize, where there are no windbreaks. In this connection, the vulnerability to elevated sea waves is also high due to storm surges on top of SLR (GSSD, 2015).

In Cambodia, the effects of climate change on tourism are significant. While coastal areas are of economic importance because of their fiscal revenue contributed by tourism, fishing, services, agricultural production and navigation, they would be influenced by tropical typhoons from the Pacific (RGC, 2013). Becken (2010) argues that storms threaten tourism facilities, interrupt businesses and increase insurance costs/loss of insurability. SHV's tourism is strongly dependent on the climate as its attractions are beaches and island areas. The increasing great storm incidents lead to greater tourist and community health risks (UNWTO, 2014). 


\subsubsection{Vulnerability to rising temperature}

Recent findings suggest that in Cambodia variations in temperature have already happened since the second half of the $20^{\text {th }}$ century (RGC, 2013). World Bank (2011) claims that since 1960, data in Cambodia have marked an increasing trend in average annual temperature, which has been rising by around $0.18^{\circ} \mathrm{C}$ per decade with $0.20-0.23^{\circ} \mathrm{C}$ per decade in drier season and $0.13-0.16^{\circ} \mathrm{C}$ per decade in wet season (World Bank, 2011). This finding was later validated by Thoeun (2015). Since then, the prevalence of 'hot' days has increased considerably (+46, with the highest temperature in noted September-November), and so has the recurrence of 'hot' nights ( +63 , with greatest increases in December-February) (World Bank, 2011).

In the future, these trends are expected to continue, with the annual mean of $0.7-2.7^{\circ} \mathrm{C}$ by the 2060 s and $1.4-4.3^{\circ} \mathrm{C}$ by the 2090s throughout the year; the varying degrees based on the amount of greenhouse gas and the climate models (McSweeney, New, Lizcano, \& Lu, 2010). The temperature change is forecasted to be higher in the lower altitude areas in Cambodia, such as the central plains, but lower in mountainous areas (MOE, MEF \& UNDP, 2011). Using the General Circulation Models (GCMs), Thoeun (2015) further projected that average annual temperatures should rise between $0.3-0.6^{\circ} \mathrm{C}$ by 2025 and between $1.6-2.0^{\circ} \mathrm{C}$ by 2100 .

Table 3.4 and Figure 3.4 show the actual recorded temperature, starting from 1960, and present the projected increase of the temperature in the country. Rising temperature also has both a direct and indirect impact on the country's tourism growth. First, it would lead to a fall in tourist arrivals to hot-climate countries, particularly Asia. Tourists will take serious consideration before visiting the country in the hot season. During hot temperatures, visiting the country is often uncertain for non-Asian tourists as well because non-Asian tourists could not well adapt to the temperature. Second, Becken (2010) also explains that the extreme heat period poses limitations on tourists and recreationists. For example, tourists are not allowed to trek in forests due to the risk of fire (Becken, 2010) but trekking is becoming increasingly popular for tourists in Cambodia. Increased temperature will put pressure on tourism businesses as well, for example, tourists need increasing cooling in hot temperatures, thus increasing costs for air conditioning (Becken, 2010) especially for accommodation operators. 
Increasing surface sea-water temperatures, for instance, may cause coral bleaching and the migration of marine species toward higher lands (GSSD, 2015); these have implication for tourism.

Table 3.4: Actual and projected change of Cambodia's annual mean temperature

\begin{tabular}{|l|l|l|l|l|l|}
\hline \multicolumn{3}{|c|}{ Year } & \multicolumn{3}{c|}{ Future projection } \\
\hline $\mathbf{1 9 6 0 - 1 9 8 9}$ & \multicolumn{1}{|c|}{$\mathbf{2 0 1 1}$} & \multicolumn{1}{|c|}{$\mathbf{2 0 1 5}$} & \multicolumn{1}{c|}{$\mathbf{2 0 2 5}$} & \multicolumn{1}{c|}{$\mathbf{2 0 6 0}$} & \multicolumn{2}{c|}{} \\
\hline $27.5^{\circ} \mathrm{C}$ & Increase & Increase $0.8^{\circ} \mathrm{C}$ & $\begin{array}{l}\text { Increase } 0.3 \text { and } \\
0.6^{\circ} \mathrm{C}\end{array}$ & $\begin{array}{l}\text { Increase } 0.7- \\
2.7^{\circ} \mathrm{C}\end{array}$ & $\begin{array}{l}\text { Increase } 1.4- \\
4.3^{\circ} \mathrm{C}\end{array}$ \\
\hline
\end{tabular}

Sources: (McSweeney et al., 2010; Thoeun, 2015; World Bank, 2011)

Figure 3.4: Average Temperature from 1960-2099

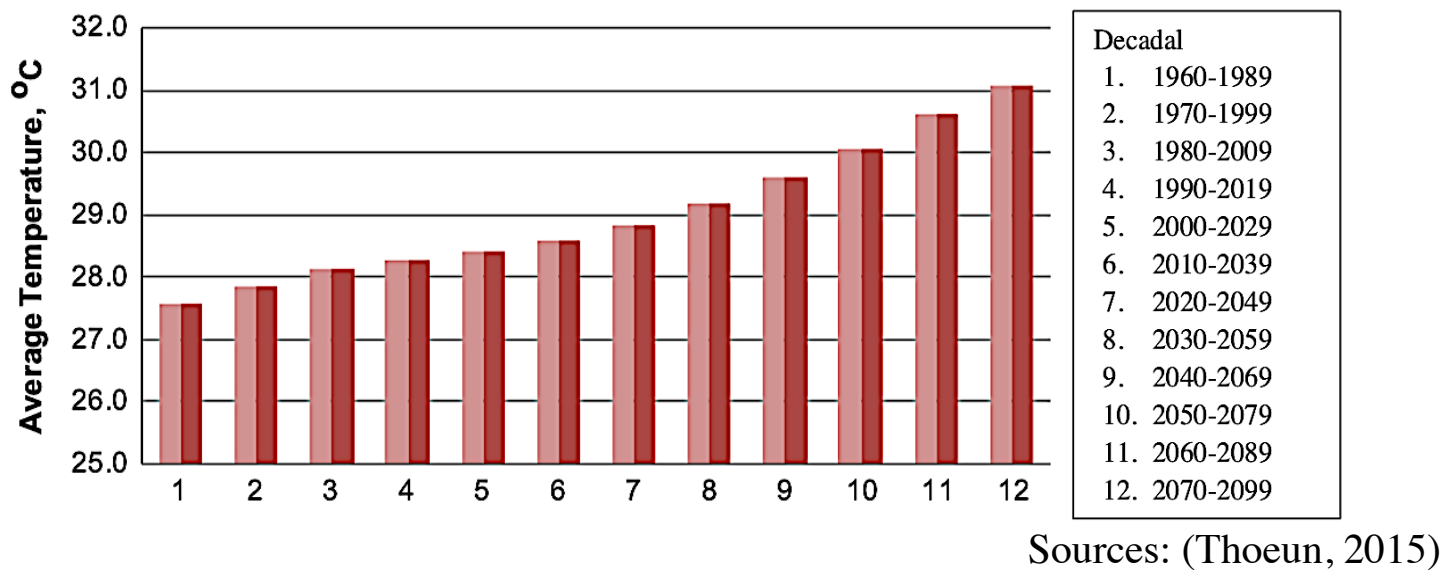

\subsubsection{Vulnerability to sea level rise/beach erosion/storm surge}

UNEP (2015a) found that climate change long-term records in the Gulf of Thailand indicate a SLR in the area of 3-5 mm/year over the last several decades. This fits well with NOAA ${ }^{19}$ observations of a global SLR of $3 \mathrm{~mm} /$ year from 1993 to 2012.

In addition, many reports predict future SLR continue to rise. Ung et al. (2017) argue that with the projection of global mean SLR, many coastal areas in Cambodia would be under water. At the high rate of $0.56 \mathrm{~m}$, it would trigger permanent flooding of about 25,000 ha of Cambodia's coastal zones within 90 years (RGC, 2013). UNEP (2015a) claims that SHV and Koh Kong, the two coastal provinces in Cambodia, are most vulnerable to SLR and extreme

\footnotetext{
${ }^{19}$ National Oceanic and Atmospheric Administration (in the US)
} 
weather events. GSSD (2015) also projected that Cambodian coastal area of approximately 25,000 ha will be permanently flooded by $1 \mathrm{~m}$ rise and this would increase to 38,000 ha with $2 \mathrm{~m}$ rise. If this scenario were to result, of the 25,000 ha, about $11 \%$ of the submerged area is in SHV and $80 \%$ in Koh Kong Province.

At the coastal zone, storm patterns and variations in rainfall will worsen flood risk and the scale of storm damage. The bio-geophysical impacts will ultimately have indirect and direct socio-economic effects on tourism, freshwater supply and quality, fisheries, financial services, human settlements, agriculture, and people's health (Nicholls \& Small, 2002). Under future climate projections, the detrimental impacts of more frequent typhoons could cause coastal erosion, damage settlements in coastal areas and affect tourism (RGC, 2013). Rising sea temperature and ocean acidification have a direct erosion and interruption of marine systems of the shallow East Gulf of Thailand, which would also influence both the tourism and the fisheries sectors (UNWTO, 2014). The incidents can pressurise hotel sector due to seafood shortages if the fishermen could not collect sufficient food due to the storms.

\subsubsection{Vulnerability to floods and droughts}

In 2009 World Bank classified Cambodia as among the 12 countries that are most at risk from flooding worldwide (World Bank, 2009). While Bangay \& Blum (2010) projected that due to climate change $2.5-10 \%$ reduction in crop production and yields in $2020 \mathrm{~s}$ and about 49 million will experience hunger in Asia, drought and flood heavily affect Cambodia's rice production annually (Phirun, 2013). The short-lived dry spell or the small dry season as it is called locally, which usually starts in the middle of the wet season (July to August), often develops into extended drought. Flooding commonly damages agriculture and human systems at the end of the wet season (mid-October to mid-November) (Phirun, 2013). Previously, between 2004 and 2010, DanChurch Aid/Christian Aid (2011) found that droughts and floods are liable for the most significant damages in Cambodia. It is estimated that 100 Cambodian lives are lost due to flood incidents annually, and the floods cause agricultural damages and losses of USD 100-170 million annually, and affect important infrastructures along the floodplain (UNWTO, 2014). MOE (2016) found that of Cambodia's 24 provinces and autonomous municipalities, 12 have experienced at least eight years of both 
droughts and floods over a decade period already. Droughts and flooding are predicted to increase in severity, frequency and duration (Thoeun, 2015).

In Asia, Bangay \& Blum (2010) projected that between 120 million-1.2 billion population will be vulnerable to water shortages by 2020 and 2.5 million people will be flooded by 2050 , predominantly in Cambodia's Mekong mega-deltas (Chea \& Sharp, 2015). In Cambodia, the annual river flooding along with the two major watersheds, Tonle Sap Lake and Mekong River, have already affected 18 out of 24 provinces (Chea \& Sharp, 2015). Resurreccion, Sajor \& Fajber (2008) argue that "two third of Cambodia is prone to flooding and drought" (p. 58). During the last 10 years, the average precipitation level in September was $24 \%$ higher than other months. This was a main cause of flooding throughout Cambodia (Chea \& Sharp, 2015).

Figure 3.5 indicates a series of flooding events, increasingly more severe and extensive from 1996-2013 (Chea \& Sharp, 2015). There is a trend in increase of floods to the present.

Figure 3.5: Timeline of flooding from 1996 to 2013

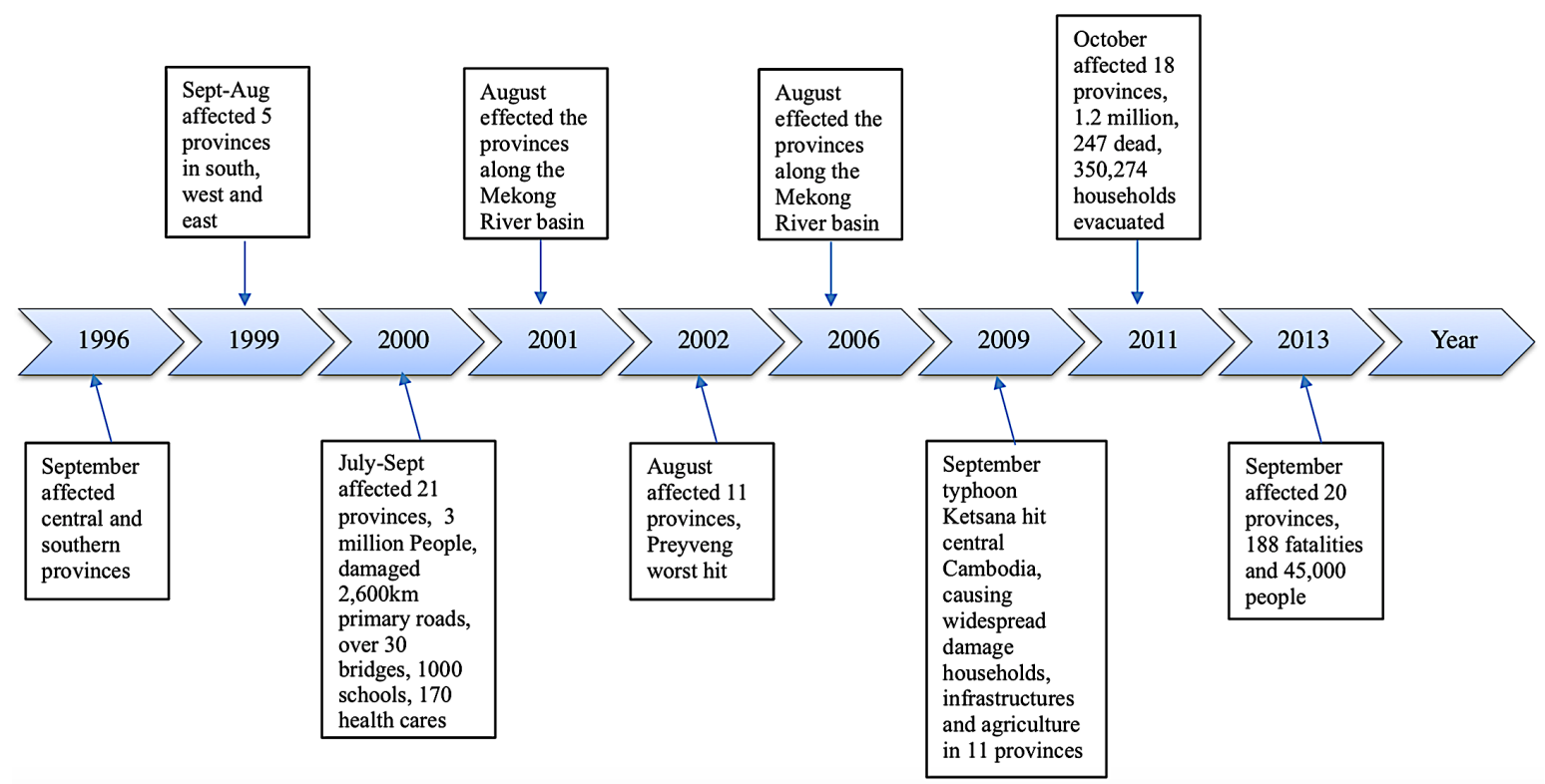

Source: (Chea \& Sharp, 2015) 
According to UNEP (2014a), Cambodia's coastal areas are exposed to different types of floods. First, they are coastal floods that are caused by a high sea level brought about by storm surge and the tide and/or by high rainfall and insufficient drainage systems. Second, inland floods, which include flash floods, are generated by direct erratic rainfalls. Floods damage people's daily livelihoods such as agriculture (e.g. productivities, land use for agricultural activities), infrastructures (e.g. roads, bridges, canals, buildings, irrigations), industry (SME, agroindustry plantations), commerce (e.g. businesses, services) (Chea \& Sharp, 2015). This has strong implications for tourism, which depends on and is relevant to these resources. King et al. (2009) argue that flash and widespread flooding, and longer wet seasons may not only directly affect tourism infrastructure, disrupt access to rural ecotourism areas but also downgrade the popularity of Cambodia as a 'sunny' destination (UNWTO, 2014). Droughts and floods have negative impacts on food security for both local populations and tourists. The limited availability of drinking water is an interruption to creating tourist services and attractions in most parts of Cambodia. Prolonged drought would add pressure to the water shortfall and impact either tourists or local populations (UNWTO, 2014).

The following section will discuss other contributing factors to increasing vulnerability in Cambodia. These factors are largely induced by human-related activities.

\subsubsection{Other factors increasing Cambodia's vulnerability}

In Cambodia, the lack of available information and measures increase the vulnerable populations to climate change. The use of existing meteorological information is low. If it is available, it is specific to some agencies (World Bank, 2011). The Department of Meteorology has a few field stations and their services are not largely available for the use of agriculture and fisheries and other important sectors. A majority of the vulnerability assessments have been initiated by DPs and are heavily dependent on international experts (Käkönen et al., 2014) because there are not many Cambodian experts on climate change issues at the national level. Vulnerability assessments conducted by local authorities face various constraints, which include the deficit of historical climate information, the limitation

of local expertise and facilities in conducting climate projection studies, the low capacity to 
employ assessment methods, and the limited financial resources for research (Käkönen et al., 2014). The low quality and limited vulnerability assessments have been a concern (Bowen et al., 2013). The local officials' capacity to access, understand and utilise the results of vulnerability assessments in English-language publications is also a major concern (Dany et al., 2015).

There is a limited political will regarding climate risks (Resurreccion et al., 2008). Most of the country's national policies, strategies, plans and projects, particularly budget, did not sufficiently take climate change into consideration (RGC, 2010b). It is widely known that ministerial capacities are influenced by low, late and lost wages, nepotism and corruption which include the purchasing of promotions or positions (Ek, 2013). The absence of implementation of existing regulations and policies is the greatest challenge to environmental governance (Kato et al., 2000). Cambodia has enacted a series of good relations and policies for most of the main economic sectors but there is a desperate lack of translation of these works into reality. This has led to a low will in climate change response by Cambodian people as well.

Evidence indicates that the RGC does not control its environment and natural resources in a sustainable way evidenced by improper developments, which has led to over-exploitation and resource depletion (Ek, 2013). The deforestation rate of 1.2\% (World Bank, 2012) is among the highest in the world and is strongly indicative of other key environmental problems. These include land degradation and grabbing, extinction of wildlife, soil erosion, decreased water tables and general reduction of biodiversity and ecosystem services, resulting in reduced resilience to disasters and increased climate change impacts (Ek, 2013). The rapid depletion of forests, coastal areas, soil and fishing areas are being witnessed in current development, which shows no thorough consideration of the long-term effects coupled with the expected effects of climate change, increasing the vulnerability of the country's ecosystem $(\mathrm{Ek}, 2013)$.

Other contributing factors that exacerbate climate change effects, often restraining the opportunities for the poor to adapt to climate change, is that Cambodia is coping with 
widespread poverty, socio-economic marginalisation, and gender inequalities (Solar, 2010). Contributed by the impact of nature disaster, one out of five Cambodian households stays below the poverty line (Chea \& Sharp, 2015). At the coastal zone, the country's poverty levels are high. With few livelihood opportunities, and many coastal resources are already under pressure. The expansion, industrialisation, and tourism development will intensify this concern (MOE, MEF, \& UNDP, 2011).

For the interrelationships between climate change and tourism, Cambodia's tourism laws, policies, and plans/strategies that genuinely target the risks of climate change on tourism are in the initial stage [researcher's document reviews and also see UNWTO, (2014)]. Climate change impacts on tourism are yet to be considered sufficiently because there is limited information on the climate change impacts on the country's tourism systems (UNWTO, 2014). The knowledge, awareness and capacity in the MOT to deliver essential leadership to adapt to climate change issues is currently limited (UNWTO, 2014). As a response to climate change, the tourism system has two main options: mitigation and adaptation. As Cambodia is a developing country, it is advised that Cambodia choose adaptation over mitigation (UNWTO, 2014), including study on the tourism sector.

Due to its high vulnerability to climate change, as outlined above, Cambodia is making considerable efforts to combat climate change impacts, which are shown in the next section.

\subsubsection{The efforts to respond to climate change in Cambodia}

The Climate Change Department, under supervision of MOE, is working collaboratively with various units (e.g. committees, other ministries and DPs) to address climate change in Cambodia (see Figure 3.6). The national response began in 1995 when the UNFCCC was ratified, which took effect in 1999. In the same year, the implementation of Phase I of its Climate Change Enabling Project (CCEP) was started (UNWTO, 2014). Secondly, endorsement of the Kyoto Protocol and Phase 2 (CCEPII) followed in 2002. Thirdly, the National Adaptation Programme of Action to Climate Change (NAPA) in Cambodia was formulated in 2006, providing the framework for addressing climate change issues. 
Subsequently, the institutional arrangements for managing climate change and adaptation interventions were established.

Figure 3.6: Cambodian institutional framework for addressing climate change ${ }^{20}$

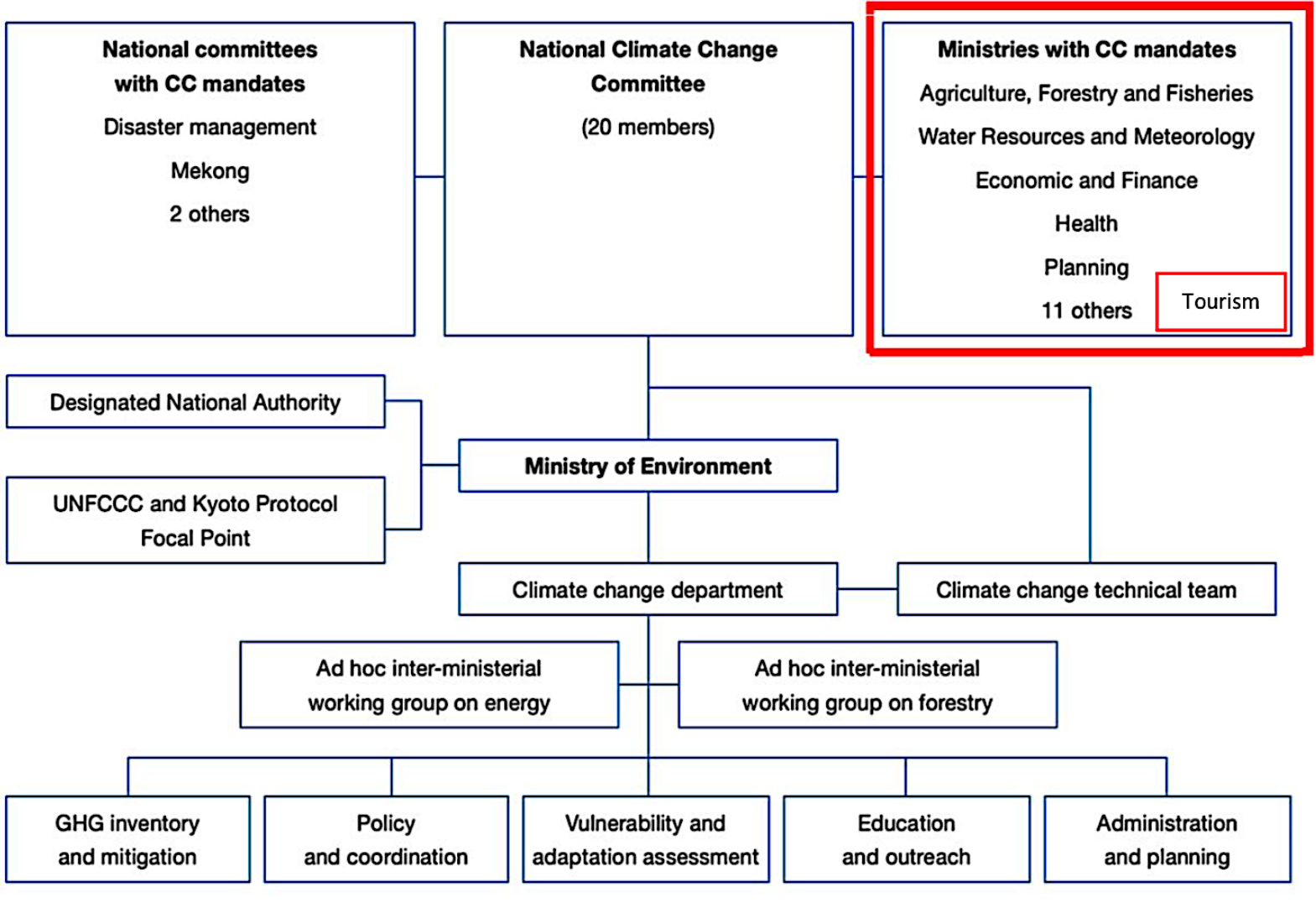

Source: (UNWTO, 2014)

In response to climate change, at the national level, Cambodia is taking 'no-regrets' adaptation options (RGC, 2006). The approach places an emphasis on maximising positive and minimising negative impacts of nature-based adaptation strategies (Rizvi et al., 2014). No-regret adaptation includes measures taken by communities and/or collaborated by organisations, which reduce vulnerability to climate change or which enhance the capacities and measures to adapt that will always have a positive impact on livelihoods and ecosystems (Rizvi et al., 2014). Cambodia's 'no-regrets' adaptation options can be divided into three

${ }^{20}$ Tourism fits in the box 'the Ministries with CC mandates. 
classifications: (i) raising awareness/education, (ii) building capacity and training, and (iii) developing infrastructures (RGC, 2006).

In an effort to combat climate change, Cambodia has received a significant amount of funding from DPs: $90 \%$ of the budget is covered by DPs/donors (Dany et al., 2015), 24\% of the funds is grant and the remaining 36\% is concessional loan (Enrich Institute, 2014). Several key donors have supported Cambodia including bilateral donors such as the Governments of Australia and multilateral institutions such as UNDP. For example, Cambodia is among the nine countries worldwide that was chosen in a Pilot Programme for Climate Resilience (PPCR), which was jointly supported by the World Bank Group and ADB, with involvement by International Finance Cooperation (IFC) and UNDP (CWPR, 2010; RGC, 2010b); not to mention NGOs that have implemented small-scale projects. The PPCR was initiated to pilot nine developing countries by integrating climate vulnerability and resilience into their fundamental planning and development policies (RGC, 2010b). The PPCR is split into two stages. Phase 1 is to design a Strategic Programme for Climate Resilience (SPCR) while phase 2 is to implement it (Wedderburn, 2009). The objective of SPCR is to mainstream climate resilience into national and local development plans, projects, and policies (RGC, 2010b). It was approved for USD1.5 million for the support of preparing Cambodia's Strategic Pilot Programme for phase 1 (RGC, 2010b). Nevertheless, experts from UNDP think that Cambodia needs more funding (Hess, 2012) because climate change impacts have become more obvious in main economic sectors while tourism receives more and more tourists.

In relation to climate change, apart from reports published by MOE, there are a number of key documents that have been published by the DPs and individual researchers. Table 3.5 below presents some reports, among many of which are related to the country's climate change issues. However, based on literature reviews specific to Cambodia, the reports discussing climate change issues in tourism/hotel sector are unavailable. 
Table 3. 5: Key reports documenting and analysing climate change in Cambodia

\begin{tabular}{|c|c|c|}
\hline Year & Document title & Summary \\
\hline 2009 & $\begin{array}{l}\text { Public perceptions of Climate Change in } \\
\text { Cambodia }\end{array}$ & $\begin{array}{l}\text { This document was compiled by an NGO called geres Cambodia. This study reports } \\
\text { Cambodian people's insights about climate change issues and the coping mechanisms they } \\
\text { have implemented to deal with the issues. geres Cambodia documented that "as an LDC, } \\
\text { Cambodia is immediately highly vulnerable to climate change, due to its limited capacity to } \\
\text { invest resources in adaptation; and with an economy that is based largely on agriculture - an } \\
\text { industry that fundamentally relies on climate patterns - its vulnerability is further intensified." }\end{array}$ \\
\hline 2013 & $\begin{array}{l}\text { Strategic guidelines for sustainable } \\
\text { tourism on the Khmer coast }\end{array}$ & $\begin{array}{l}\text { This is report was done by the University of the Sunshine Coast, Australia to report to the } \\
\text { Ministry of Tourism, Cambodia. The report summarized a number of climate change impacts } \\
\text { especially at the Cambodian coastal zone. For example, the authors reported that "coastal } \\
\text { communities and small islands with infrastructure that has not included consideration of extreme } \\
\text { weather, early warning systems and information and knowledge of appropriate community } \\
\text { adaptation behavior are at high risk from the impacts of climate change." }\end{array}$ \\
\hline 2014 & $\begin{array}{l}\text { Responding to Climate Change: } \\
\text { Tourism Initiatives in Asia and the } \\
\text { Pacific (pp. 115-124) }\end{array}$ & $\begin{array}{l}\text { This report was published by UNWTO. The report gives insights how tourism of each nation in } \\
\text { Asia and the Pacific respond to climate change. Among countries, Cambodia was reportedly as } \\
\text { one of the highest vulnerable. In the report, UNWTO stated that "sea levels along the Cambodian } \\
\text { coast are projected to rise by about } 15 \text { centimeters by } 2030 \text { and up to centimeters by } 2070 \text {. Rising } \\
\text { sea levels pose a threat to coastal areas, which are already experiencing storm surges, higher tides, } \\
\text { beach erosion, and seawater intrusion. Low-lying areas, including settlements, beach resorts, } \\
\text { seaports, coastal fisheries, and mangrove forests are particularly vulnerable." }\end{array}$ \\
\hline 2014 & $\begin{array}{l}\text { Vulnerability Assessment and } \\
\text { Adaptation Programme for Climate } \\
\text { Change within the Coastal Zone of } \\
\text { Cambodia Considering Livelihood } \\
\text { Improvement and Ecosystems }\end{array}$ & $\begin{array}{l}\text { This was a project that was conducted by UNEP as the guidance for the Least Developed } \\
\text { Countries Fund (LDCF) like Cambodia. This proposal seeks LDCF funding for a Medium- } \\
\text { Size Project in Cambodia to implement the adaptation priorities 'rehabilitation of coastal } \\
\text { protection infrastructure' and 'community mangrove restoration and sustainable use of natural } \\
\text { resources', and contribute to adaptation priority 'assessment of needs for setbacks, vegetation } \\
\text { buffers and protection structures in coastal zones', all of which were identified during the } \\
\text { Cambodian National Adaptation Programme of Action (UNEP, 2013a). } \\
\text { The project documented that "climate change is likely to adversely affect the natural ecosystems, } \\
\text { infrastructure, agriculture and, indeed, community livelihoods within the coastal zone by } \\
\text { resulting in: i) an increase in mean annual rainfall and rainfall intensity and a concomitant } \\
\text { increase in episodes of flooding; ii) an increase in mean annual temperature; and iii) sea level } \\
\text { rise. Sea level rise, for example, will increase the impact of cyclonic activity and storm surges } \\
\text { and result in greater incidences of saline intrusion." }\end{array}$ \\
\hline
\end{tabular}

Sources: (Carter et al., 2013; geres Cambodia, 2009; UNEP, 2013a; UNWTO, 2014) 
In 2015 the government initiated the first tourism document, 'Climate change action plan in tourism sector 2015-2018'. There are three goals/objectives within the plan: first, to reduce climate change vulnerability of people and tourism sector, and particularly the most critical and vulnerable systems (societal and natural), and cultural heritage. Second, it aims to shift towards a green development pathway by fostering low-carbon development and technologies. Third, it is to promote public participation and awareness in climate change response activities in the sector. Within the plan, MOT is expected to receive a total budget estimation of USD2,900,000 for implementing the actions from 2015 to 2018. MOT has identified seven prioritised actions to respond to climate change. Promoting Green Standard hotels in Cambodia is one of the prioritised actions. Promoting Green Standard hotels involves hotels being evaluated for green hotel awards, events/campaigns for hotel associations and hotel owners, and joint campaigns or advertisement on Green Hotel in Cambodia for tourists.

The next sections will contextualise the research site by providing an overview of SHV's tourism development and its vulnerability to climate change.

\subsection{Sihanoukville's overview}

Sihanoukville $^{21}$ (see Figure 3.7) is located $230 \mathrm{~km}$ southwest from Phnom Penh, the country's capital. There was a total population of 199,902 in 2008 in SHV (NIS, 2008). SHV has the longest coast, with $128 \mathrm{~km}$ out of the $440 \mathrm{~km}$ of Cambodian coast covering $30 \%$ of the total length of the Cambodian coastline. Ream National Park covering $210 \mathrm{~km}^{2}$, with about 30\% marine park, is also located in SHV (Carter et al., 2013; Ong \& Smith, 2014).

${ }^{21}$ It is known as 'Kompong Soam' in local language. 
Figure 3. 7: Sihanoukville Map

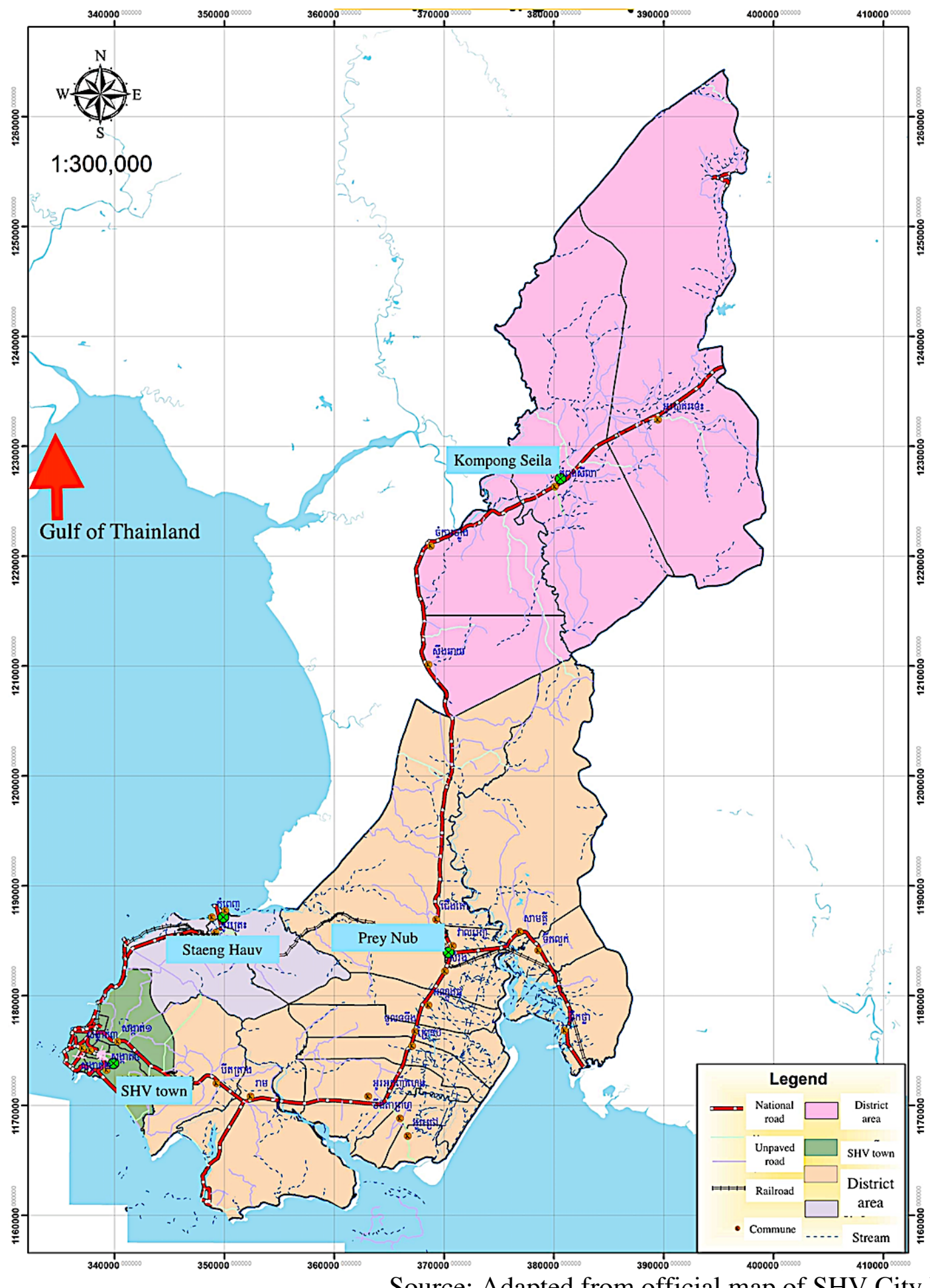

Source: Adapted from official map of SHV City Hall

With a total land area of $868 \mathrm{~km}^{2}$, SHV consists of three districts, 23 communes and 82 villages (Rizvi \& Singer, 2011). The SHV municipality is responsible for managing the daily 
administrative affairs. It is composed of four steering groups at local level and 11 other public offices (located in each province but under the government's ministries of Phnom Penh). Its role is to protect, manage and regulate environmental resources (MOE \& Danida, 2006). In this multi-institutional and multilevel arrangement, there are duplications of tasks and overlapping responsibilities, so managing coastal tourism is complicated and challenging, particularly as each group's priority differs (Kim, 2003).

\subsubsection{Sihanoukville's tourism}

SHV is considered a rising star in the south east and one of the newly attractive coastal destinations in Asia (Ong \& Smith, 2014) that is gradually gaining fame internationally. Cambodia's SHV is the country's most matured coastal destination and is ranked $1^{\text {st }}$ as the country's most attractive coastal tourism. It has a long history, starting from French colonialism. SHV is the hub of the Cambodian coast for tourism and commerce as well as the main catalyst of job generators. The international deep-water port is central to the local and national economy. SHV has an international airport and road (and rail) connections to Phnom Penh, Siem Reap, and the adjoining coastal provinces (Carter et al., 2013).

There are 22 islands in the jurisdiction of SHV (Carter et al., 2013). They are natural areas with considerable tourism potential. Most of them remain undeveloped. The quality of the beaches and water are the major tourism attractions of the islands. The activities on the islands include snorkelling, diving, fishing and boat trips, and now trekking is a new trend. Speed and slow wooden boats play the main roles in transporting the tourists and residents

back and forth. Yet the infrastructure at the islands remains weak and scarce. Most areas of the islands are inaccessible. Despite limitations, the islands provide a range of accommodations and activities for tourists and many more are being constructed.

Tourist activities along the main beaches, fishing, snorkelling, and scuba diving from the coastal islands of SHV are the major attractions (Ong \& Smith, 2014). The concentration of SHV's touristic activities and amenities are at the beach lines within approximate 1-2 km of the city centre. This downtown hub comprises of a number of business commerce and facilities, such as markets, souvenirs, medical and clinics hospitals, banking, and travel 
agencies. From south to north, there are beaches close to SHV City Centre (Ong \& Smith, 2014; Tinat, 2011). These beaches are good in quality, especially the quality of white sand. The Australian Travel Magazine awarded the SHV's Ochheuteul Beach as one of the 'Top Ten Asia Beaches' (Charlotte, 2016), and the Cambodian coast has been included in the Club of the World's Most Beautiful Bays (Carter et al., 2013). 
Table 3. 6: Domestic/international tourism statistics report in $2012-\mathbf{2 0 1 6}^{22}$

\begin{tabular}{|c|c|c|c|c|c|c|c|c|c|c|c|c|c|c|c|c|}
\hline \multirow{3}{*}{\multicolumn{2}{|c|}{ No. }} & \multirow{3}{*}{ Destinations } & \multicolumn{2}{|c|}{2012} & \multicolumn{2}{|c|}{2013} & \multicolumn{2}{|c|}{2014} & \multicolumn{2}{|c|}{2015} & \multicolumn{2}{|c|}{2016} & \multirow{2}{*}{\multicolumn{2}{|c|}{$\begin{array}{r}\text { Share }(\%) \\
2016^{*}\end{array}$}} & \multirow{2}{*}{\multicolumn{2}{|c|}{$\begin{array}{r}\text { Change }(\%) \\
2016^{*} / 15\end{array}$}} \\
\hline & & & \multirow[t]{2}{*}{ KHM } & \multirow[t]{2}{*}{ INT } & \multirow[t]{2}{*}{ KHM } & \multirow[t]{2}{*}{ INT } & \multirow[t]{2}{*}{ KHM } & \multirow[t]{2}{*}{ INT } & \multirow[t]{2}{*}{ KHM } & \multirow[t]{2}{*}{ INT } & \multirow[t]{2}{*}{ KHM } & \multirow[t]{2}{*}{ INT } & & & & \\
\hline & & & & & & & & & & & & & KHM & INT & KHM & INT \\
\hline I & 1 & Phnom Penh & $1,984,612$ & $1,520,971$ & $2,204,573$ & $1,972,879$ & $2,257,498$ & $2,151,838$ & $2,273,150$ & $2,650,368$ & $2,538,157$ & $2,806,438$ & 23.9 & 46.6 & 11.7 & 5.9 \\
\hline \multirow{5}{*}{\multicolumn{2}{|c|}{ II }} & Cultural Zone & $2,125,322$ & $2,107,982$ & $2,345,567$ & $2,290,778$ & $2,865,457$ & $2,414,737$ & $3,106,482$ & $2,198,107$ & $3,253,955$ & $2,296,096$ & 30.6 & 38.1 & 4.7 & 4.5 \\
\hline & & Kampong Thom & 75,505 & 22,272 & 71,538 & 27,179 & 83,389 & 23,765 & 86,296 & 27,235 & 73,825 & 31,313 & 0.7 & 0.5 & -14.5 & 15.0 \\
\hline & 3 & Oddar Meanchey & 43,598 & 3,186 & 34,272 & 1,763 & 48,331 & 2,360 & 47,633 & 3,851 & 62,496 & 2,702 & 0.6 & 0.0 & 31.2 & -29.8 \\
\hline & 4 & Preah Vihear & 48,361 & 19,188 & 46,757 & 24,550 & 60,787 & 37,675 & 86,100 & 42,158 & 109,260 & 56,807 & 1.0 & 0.9 & 26.9 & 34.7 \\
\hline & 5 & Siem Reap & $1,957,858$ & $2,063,336$ & $2,193,000$ & $2,237,286$ & $2,672,950$ & $2,350,937$ & $2,886,453$ & $2,124,863$ & $3,008,374$ & $2,205,274$ & 28.3 & 36.6 & 4.2 & 3.8 \\
\hline \multirow{5}{*}{\multicolumn{2}{|c|}{ III }} & Coastal Zone & $1,827,927$ & 280,658 & $2,080,790$ & 513,891 & $2,115,489$ & 600,367 & $2,403,570$ & 610,458 & $3,044,451$ & 643,289 & 28.6 & 10.7 & 26.7 & 5.4 \\
\hline & & Kampong Som & 657,821 & 212,643 & 731,604 & 302,325 & 969,109 & 358,639 & $1,156,813$ & 362,061 & $1,648,099$ & 404,939 & 15.5 & 6.7 & 42.5 & 11.8 \\
\hline & 7 & Kampot & 401,528 & 24,972 & 614,610 & 117,280 & 679,933 & 131,468 & 683,027 & 176,435 & 717,178 & 156,596 & 6.7 & 2.6 & 5.0 & -11.2 \\
\hline & & Kep & 685,780 & 35,316 & 638,496 & 46,840 & 300,599 & 61,982 & 428,485 & 51,008 & 516,442 & 53,733 & 4.9 & 0.9 & 20.5 & 5.3 \\
\hline & 9 & Koh Kong & 82,798 & 7,727 & 96,080 & 47,446 & 165,848 & 48,278 & 135,245 & 20,954 & 162,732 & 28,021 & 1.5 & 0.5 & 20.3 & 33.7 \\
\hline \multirow{5}{*}{\multicolumn{2}{|c|}{ IV }} & Eco-tourism & 371,127 & 53,388 & 407,551 & 60,501 & 444,918 & 60,031 & 720,463 & 63,261 & 510,381 & 66,349 & 4.8 & 1.1 & -29.2 & 4.9 \\
\hline & & Kratie & 48,412 & 19,714 & 46,546 & 22,766 & 67,757 & 23,662 & 316,482 & 24,304 & 67,669 & 24,350 & 0.6 & 0.4 & -78.6 & 0.2 \\
\hline & 11 & Mondul Kiri & 78,784 & 5,138 & 95,059 & 5,215 & 108,716 & 6,498 & 129,712 & 7,253 & 142,104 & 8,679 & 1.3 & 0.1 & 9.6 & 19.7 \\
\hline & 12 & Rattanak Kiri & 134,965 & 19,163 & 145,633 & 20,280 & 153,978 & 21,126 & 156,716 & 22,596 & 165,977 & 23,692 & 1.6 & 0.4 & 5.9 & 4.9 \\
\hline & 13 & Stung Treng & 108,966 & 9,373 & 120,313 & 12,240 & 114,467 & 8,745 & 117,553 & 9,108 & 134,631 & 9,628 & 1.3 & 0.2 & 14.5 & 5.7 \\
\hline \multirow{5}{*}{\multicolumn{2}{|c|}{$\begin{array}{r}\mathbf{v} \\
1 \\
1 \\
1 \\
1\end{array}$}} & Battambang Zone & 595,328 & 228,130 & 687,848 & 191,899 & 772,769 & 171,156 & 646,003 & 162,893 & 777,635 & 119,964 & 7.3 & 2.0 & 20.4 & -26.4 \\
\hline & & Banteay Meanchey & 80,276 & 15,378 & 79,604 & 1,902 & 97,888 & 2,303 & 92,943 & 4,533 & 106,007 & 4,116 & 1.0 & 0.1 & 14.1 & -9.2 \\
\hline & & Battambang & 317,010 & 72,314 & 392,583 & 83,403 & 437,345 & 89,152 & 501,260 & 94,448 & 513,300 & 96,460 & 4.8 & 1.6 & 2.4 & 2.1 \\
\hline & & Pailin & 188,240 & 136,586 & 197,225 & 101,919 & 201,998 & 73,410 & 21,913 & 55,279 & 129,477 & 12,749 & 1.2 & 0.2 & 490.9 & -76.9 \\
\hline & & Pursat & 9,802 & 3,852 & 18,436 & 4,675 & 35,538 & 6,291 & 29,887 & 8,633 & 28,851 & 6,639 & 0.3 & 0.1 & -3.5 & -23.1 \\
\hline \multirow{10}{*}{\multicolumn{2}{|c|}{$\begin{array}{r}\text { VI } \\
1 \\
1 \\
2 \\
2 \\
2 \\
2 \\
2 \\
2 \\
2\end{array}$}} & Others & $1,349,544$ & 49,581 & 792,424 & 97,619 & 548,306 & 214,138 & 529,324 & 114,340 & 517,502 & 93,607 & 4.9 & 1.6 & -2.2 & -18.1 \\
\hline & & Kampong Cham & 276,194 & 10,976 & 403,684 & 54,865 & 347,869 & 31,554 & 264,064 & 28,794 & 232,620 & 25,615 & 2.2 & 0.4 & -11.9 & -11.0 \\
\hline & & Kampong Chhnang & 14,484 & 3,428 & 24,162 & 3,417 & 26,459 & 3,622 & 22,158 & 3,394 & 36,339 & 4,959 & 0.3 & 0.1 & 64.0 & 46.1 \\
\hline & & Kampong Speu & 22,701 & 13,511 & 22,632 & 12,545 & 23,391 & 12,628 & 22,600 & 11,937 & 27,284 & 13,019 & 0.3 & 0.2 & 20.7 & 9.1 \\
\hline & & Kandal & 963,128 & 7,552 & 253,784 & 12,564 & 54,266 & 9,536 & 49,116 & 10,307 & 35,573 & 8,251 & 0.3 & 0.1 & -27.6 & -19.9 \\
\hline & & Prey Veng & 9,682 & 3,046 & 8,634 & 2,730 & 7,783 & 2,208 & 8,019 & 2,175 & 15,115 & 1,617 & 0.1 & 0.0 & 88.5 & -25.7 \\
\hline & & Svay Rieng & 20,679 & 1,042 & 22,830 & 1,711 & 30,381 & 585 & 42,742 & 614 & 42,955 & 891 & 0.4 & 0.0 & 0.5 & 45.1 \\
\hline & 24 & Takeo & 42,676 & 10,026 & 56,698 & 9,787 & 42,706 & 9,224 & 38,994 & 10,949 & 58,627 & 13,932 & 0.6 & 0.2 & 50.3 & 27.2 \\
\hline & 25 & Thbong Khmum & & & & & 15,451 & 144,781 & 81,631 & 46,170 & 68,989 & 25,323 & 0.6 & 0.4 & -15.5 & -45.2 \\
\hline & & TOTAL & $8,253,860$ & $4,240,710$ & $8,518,753$ & $5,127,567$ & $9,004,437$ & $5,612,267$ & $9,678,992$ & $5,799,427$ & $10,642,081$ & $6,025,743$ & 100.0 & 100.0 & 10.0 & 3.9 \\
\hline
\end{tabular}

Source: (MOT, 2016)

${ }^{22}$ KHM=Cambodian Visitors, INT=Foreign Visitors and Kompong Soam=Sihanoukville 
Table 3.6 shows that SHV is ranked third in Cambodia for tourist attractions after Phnom Penh and Siem Reap. The number of tourists has remarkably increased. For example, in 2015 SHV accommodated approximately 1,156,813 domestic visitors and 362,016 international tourists. In 2016, 1,648,099 domestic and 404,939 international visited SHV with 42.5\% and $11.8 \%$ marked as increase if compared to the figure in 2015 (MOT, 2016). The average length of stay in SHV is unavailable.

Ong \& Smith (2014) found that approximately $30 \%$ of the business establishments were foreigner-owned. Ponna (2009) found that 59.9\% of SHV's labour forces were employed in hotel, guesthouse, restaurant, tour-guide, taxi-boat, souvenir-shop, water-sport, massage, and other tourism and hospitality sector occupations. 
Table 3. 7: Tourism service statistics in 2016

\begin{tabular}{|c|c|c|c|c|c|c|c|c|c|}
\hline \multirow{2}{*}{ No. } & & \multirow{2}{*}{ Destinations } & \multicolumn{2}{|c|}{ Hotels } & \multicolumn{2}{|c|}{ Guesthouses } & \multirow[t]{2}{*}{ Restaurants } & \multirow[t]{2}{*}{ Massage } & \multirow[t]{2}{*}{ Karaoke } \\
\hline & & & No. & Rooms & No. & Rooms & & & \\
\hline I & 1 & Phnom Penh & 291 & 14,634 & 513 & 9,792 & 442 & 66 & 93 \\
\hline \multirow[t]{5}{*}{ II } & . & Cultural Zone & 226 & 14,003 & 417 & 6,505 & 435 & 107 & 95 \\
\hline & 2 & Kampong Thom & 8 & 320 & 52 & 737 & 78 & 8 & 33 \\
\hline & 3 & Oddar Meanchey & 4 & 244 & 34 & 516 & 22 & 17 & 24 \\
\hline & 4 & Preah Vihear & 3 & 50 & 40 & 523 & 67 & 7 & 18 \\
\hline & 5 & Siem Reap & 211 & 13,389 & 291 & 4,729 & 268 & 75 & 20 \\
\hline \multirow[t]{5}{*}{ III } & & Coastal Zone & 86 & 4,637 & 438 & 5,694 & 197 & 42 & 33 \\
\hline & 6 & Kampong Som & 64 & 3,405 & 253 & 3,808 & 82 & 19 & 9 \\
\hline & 7 & Kampot & 10 & 805 & 181 & 1,727 & 114 & 23 & 24 \\
\hline & 8 & Kep & 8 & 228 & & & 1 & & \\
\hline & 9 & Koh Kong & 4 & 199 & 4 & 159 & & & \\
\hline \multirow[t]{5}{*}{ IV } & & Eco-tourism Zone & 45 & 1,945 & 163 & 2,065 & 153 & 12 & 63 \\
\hline & 10 & Kratie & 18 & 751 & 46 & 376 & 40 & 3 & 19 \\
\hline & 11 & Mondul Kiri & 6 & 303 & 45 & 623 & 40 & & 18 \\
\hline & 12 & Rattanak Kiri & 14 & 625 & 36 & 504 & 39 & 3 & 13 \\
\hline & 13 & Stung Treng & 7 & 266 & 36 & 562 & 34 & 6 & 13 \\
\hline \multirow[t]{5}{*}{$\mathbf{v}$} & & Battambang Zone & 68 & 3,154 & 177 & 2,895 & 267 & 43 & 77 \\
\hline & 14 & Banteay Meanchey & 20 & 648 & 63 & 1,163 & 26 & 21 & 19 \\
\hline & 15 & Battambang & 39 & 1,797 & 60 & 873 & 62 & 4 & 23 \\
\hline & 16 & Pailin & 5 & 360 & 20 & 498 & 95 & 3 & 16 \\
\hline & 17 & Pursat & 4 & 349 & 34 & 361 & 84 & 15 & 19 \\
\hline \multirow[t]{10}{*}{ VI } & & Others & 44 & 1,787 & 462 & 5,512 & 488 & 37 & 265 \\
\hline & 18 & Kampong Cham & 13 & 673 & 117 & 1,188 & 129 & 20 & 110 \\
\hline & 19 & Kampong Chhnang & 6 & 180 & 30 & 381 & 46 & 2 & 10 \\
\hline & 20 & Kampong Speu & 7 & 244 & 60 & 493 & 48 & & 22 \\
\hline & 21 & Kandal & 1 & 29 & 51 & 663 & 27 & 1 & 12 \\
\hline & 22 & Prey Veng & 7 & 154 & 48 & 453 & 93 & 2 & 46 \\
\hline & 23 & Svay Rieng & 3 & 253 & 42 & 745 & 51 & 9 & 1 \\
\hline & 24 & Takeo & 1 & 15 & 69 & 820 & 87 & 3 & 44 \\
\hline & 25 & Tboung Khmum & 6 & 239 & 45 & 769 & 7 & & 20 \\
\hline & & TOTAL & 760 & 40,160 & 2,170 & 32,463 & 1,982 & 307 & 626 \\
\hline
\end{tabular}

Source: (MOT, 2016)

The published statistics on SHV's hotel occupancy rate are not available, receipt/revenue data or average length of stay (Ong \& Smith, 2014). Table 3.7 shows that in 2016 there were 64 hotels with 3,405 rooms and 253 guesthouses with 3,808 rooms, 82 restaurants, 19 massage parlours and nine Karaoke clubs. Based on the tourist statistics provided by the SHV Department of Tourism (DOT), dated January 2017, there were 77 hotels (3,666 rooms); 74\% was Khmer-owned and 25\% was foreign-owned (Chinese 3.9\%, Japanese 1.3\%, Korean $2.6 \%$ and other $18.18 \%$ ). However, after the fieldwork the researcher further updated that there were 75 hotels being operated (two went bankrupt), 301 guesthouses (4,016 rooms), $69 \%$ of which was Khmer-owned and 30\% was foreign-owned, 198 restaurants and canteens, nine Karaoke clubs, three disco clubs, 36 massage parlours, 36 tourist boats, 15 tourist sport 
parlours, 33 travel agencies and five resorts, and this number keep increasing, especially guesthouses.

\subsubsection{Climate change in Sihanoukville}

As outlined above, Cambodia and its coastal zone as well as SHV are vulnerable to excessive rainfall, rising temperatures, storms, SLR/beach erosion/storm surge and prolonged drought/flooding. These will now be discussed in turn to contextualise the research.

\subsubsection{Vulnerability to excessive rainfall}

As indicated in section 3.2.3.1, at the national level, rainfall in Cambodia has been increasing and is expected to increase in the future. Due to its location on the coast and closeness to the Cardamom Mountains as well as the influence of an inter-tropical convergence zone, SHV receives significantly higher rainfall than many other parts of the country (UN-Habitat ${ }^{23}$, 2012). In SHV an average annual rainfall in the period 1983-2011 was 3,198 mm, more than double the national average. The outcome of this is that SHV receives heavier rainfall than much of the rest of Cambodia (UN-Habitat, 2012). This has put pressures on the city's infrastructure and residents, which are currently weak in adaptive capacity. Flooding, drainage issues and wastewater management are additional concerns (UN-Habitat, 2012). SHV's unpaved roads are susceptible to rainstorms and flooding (UN-Habitat, 2012).

Based on the national data, the amount of annual rainfall was 3,198 mm between 1983-2011 and it increased to $4000 \mathrm{~mm}$ or $5000 \mathrm{~mm}$ at the coastal zone in 2015 (Thoeun, 2015; UNEP, 2015a), which suggests that SHV's rainfall tends to increase. The increasing trend is due to the increase of annual temperature and storm intensity. Heavy precipitation would lead to the rainfall-induced flooding. Specific to tourism, Becken (2010) argues that in national parks and other mountainous areas, heavy rainfall will require for frequent track maintenance. This reflects to SHV's touristic activities.

\footnotetext{
${ }^{23}$ United Nations Human Settlements Programme
} 


\subsubsection{Vulnerability to storms}

In SHV, storms are a real concern. Almost every year, storms occur from mid-October through December $\left(\mathrm{CCCA}^{24}, 2015\right.$; UNEP, 2015a). For instance, fruit and rice crops are knocked down and destroyed by storms in SHV mainly from October-December (MOE, GEF \& UNEP, 2015a). It can be estimated that the high tide is over $0.5 \mathrm{~m}$ to $0.75 \mathrm{~m}$ from mean sea level. Therefore, the tidal altitude will affect low-lying areas with an elevation of around $0.5 \mathrm{~m}$ to $0.75 \mathrm{~m}$ (MOE, GEF \& UNEP, 2015a). In November 1997, Typhoon Linda swept the Gulf of Thailand. Within five days, $400 \mathrm{~mm}$ of precipitation was recorded in coastal zone. At that time, the wind speed of $60 \mathrm{~m} / \mathrm{second}$ in SHV was recorded. After the sweep, Typhoon Linda damaged 81 fishing boats, killing and injuring 100 people and damaging most of the paddy rice fields in SHV (MOE, 2016). Ecosystem damages have been informed and recorded. For SHV's marine habitats and ecosystems, it is of particular concern that coral reefs and seagrass beds are damaged by storm surge and strong waves (MOE, GEF \& UNEP, 2015a).

Storm and strong winds have both indirect and direct effects on the province's tourism. The direct impacts include the challenges of tourists' travels from mainland to the islands and vice versa. At the mainland, some touristic activities are disturbed, such as swimming at sea and visiting the beaches. Some infrastructures important for tourism might be damaged. The indirect impacts include the shortages of food supplies. While fishermen are unable to go to the sea, marine resources drop whereas prices increase; this indirectly influences tourism, especially domestic tourists who come for both touristic activities and fresh seafood. In addition, rice and vegetable productions are also affected. Transportation lines may be disconnected. Tourists might reduce their visits to the place.

\subsubsection{Vulnerability to increased temperature}

As indicated in section 3.2.3.3, at the national level, the average temperature has been recorded as getting higher and higher. In the coastal zone, climate change adversely results in an increase in mean annual temperature affecting crop growth cycles and health (MOE, GEF \& UNEP, 2015a; UNEP, 2015a).

\footnotetext{
${ }^{24}$ Cambodia Climate Change Alliance
} 
In SHV, although foreign tourists from cold countries tend to like hot weather, the rising temperature has strong effects on domestic tourists and Asian tourists. They might be vulnerable to increasing vector-borne diseases including diarrhoea and malaria, which are in high prevalence rates in the country. They are also exposed to dehydration and common skin problems caused by sunburn. The increased temperature might contribute to severe droughts resulting in water shortages; this leads to a fierce competition over water demand between tourism and other economic sectors (also see Becken, 2010) and among tourism businesses of the same types (e.g. hotel sector). In the meantime, the public infrastructures and private businesses might be affected. As Becken (2010) explains, increased temperature will put pressure on tourism businesses as well such as increasing costs for air conditioning, especially for accommodation operators. Becken (2010) is further concerned that warmer temperatures will lead to heat stress for tourists, a change of seasonality, changes in populations and distribution of plant, wildlife and insect, increased cooling costs, and infectious diseases.

\subsubsection{Vulnerability to sea level rise/beach erosion/storm surge}

In SHV, using Google Earth and other technical tools to measure beach erosions in SHV, Fernando, Yvainne, \& Maria (2013) in collaboration with the SHV city hall conducted a scientific research and claimed that beach erosion has occurred, especially at the most popular beaches, Ochheauteal and O'tress. A SLR of 5 mm per year from 1992- 2012 may account for $10 \%$ and $25 \%$ of the average rate of shoreline retreat respectively for the developed and undeveloped segments of Ochheauteal Beach (Fernando, Yvainne, \& Maria, 2013). Assessing the community vulnerability and risks from climate change in Prey Nup district in SHV (see Figure 3.7), UNEP (2017) claims that SHV is vulnerable to SLR, flooding and increased temperature. Based on the monitoring data from MoWRAM ${ }^{25}$, the dykes of Prey Nub are sinking into water by about $2 \mathrm{~cm}$ annually. This resulted from the consequences SLR. In the next 20 years, this could assume that the dykes would be $50 \mathrm{~cm}$ under seawater (CCCA, 2012a). Several beaches in SHV are eroding, particularly inside the

\footnotetext{
${ }^{25}$ The Ministry of Water Resources and Meteorology
} 
canal Tum Noprolok, the northern end of Tum Noprolok and Hun Sen Beach (MOE, GEF \& UNEP, 2015a).

In SHV, the sensitivity of tourism to climate change is mixed and unique from other industries. SLR presents a serious threat to the existence of the industry primarily due to beach erosion (UN-Habitat, 2012a). Tourism in SHV is increasingly vulnerable to climate change owing to its dependency on climate sensitive attractions - primarily its beaches, but also water sports including diving (Ung et al., 2015; Ung et al., 2017). Climate change, such as SLR and storm surges, can disrupt tourism development through climate-related events (MOE, 2014a). A 1-metre rise in sea level would submerge over half of Koh Kong (MOE, GEF \& UNEP, 2015a), as well as much of the coastal tourism infrastructure of SHV (King et al., 2009). In further relation to tourism, SLR would lead to coastline erosion, loss of beach area, and increasing costs to protect and maintain seafronts (Becken, 2010). In SHV's Ream National Park, MOE and DANIDA (2006) also reported severe beach erosion, where several structures were threatened and needed protection.

\subsubsection{Vulnerability to prolonged drought and flood}

According to UNEP (2014b), Cambodia's coastal parts are exposed to different types of floods. First, there are coastal floods, caused by a high sea level brought about by storm surge and the tide and/or by high rainfall and insufficient drainage systems. Second, there are inland floods, which include flash floods generated by erratic rainfall. These are sudden inland floods caused by tropical depressions or local rainstorms in mountainous areas. CCCA (2012c) adds that along the Cambodian coast, consequences and combinations of floods and droughts have already caused a substantial number of fatalities and great economic losses. The coastal zone people have access to fundamental sources of fresh water from nearby rivers, streams and lakes that flow in the area. Despite the availability of these sources of fresh water, the limited freshwater is a problem in the area (CCCA, 2012a).

Assessing vulnerability throughout SHV, MOE GEF, and UNEP (2015a) found that drought has occurred, especially in Prey Nub. Examples of damage caused by drought include crops, erosion of soil and degradation of water quality and loss of farming land (CCCA, 2013). Over 
$80 \%$ of households in SHV have narrow access to safe drinking water (MOE, GEF, \& UNEP, 2015a). As safe water is inaccessible, households have high sensitivity to climate change impacts, including drought (MOE GEF, \& UNEP, 2015a). During the rainy season, streams, rivers and lakes flood, caused by extreme rainfall that results in damage of crops in low lying areas (CCCA, 2013). The excessive rainfalls compound the pressures on the city's infrastructure and residents. Rainfall-induced flooding, drainage issues and wastewater management remain a particular concern (UN-Habitat, 2012a). Furthermore, coastal tourism has already been affected by the changing climate as Cambodia has already had more severe and frequent storms, droughts and floods in recent years (Ung et al., 2015; Ung et al., 2017).

\subsubsection{Other contributing factors to vulnerability}

High density and poverty were other contributing factors increasing the province's vulnerability. The higher the density, the more vulnerability there is to climate change. The provincial town, SHV City, is observed as the most populated area, especially in Sangkat Lekh Pir, with a density of 4,390 persons/sq. km (MOE GEF \& UNEP, 2015a) and most of accommodations are located in that area.

Information limitation is another pressing concern. According to the Department of Meteorology, MoWRAM, Cambodia did not have a Doppler radar. This means that it is not able to forecast weather as accurately as that of neighbouring countries (UN-Habitat, 2012a). Both the ministry and the provincial department have no equipment for marine monitoring of weather or oceanic conditions (UN-Habitat, 2012a). In particular, information about climate change is not extensively distributed to Cambodian people in the coastal zone (UNEP, 2013b). According to a study in SHV by CCCA (2012a), information regarding weather hazards shared by the local government to communities is surprisingly low. CCCA (2012b) still found that Cambodian and SHV people have low understanding relevant to 'climate change'. They have little knowledge about the government work on CCA (CCCA, 2012b).

As SHV is a rapid developing area, huge pressure in SHV's carrying capacity begins to take shape. While infrastructures are necessary for economic sectors, most are currently weak and 
the need to use public structures and facilities (e.g. roads, power supplies) increases. For example, out of 39 Special Economic Zones (SEZs) in Cambodia, there are eight (8) SEZs in SHV, with the total developed areas of 2,356 ha $\left(\mathrm{JICA}^{26}, 2017\right)$ that need to use the infrastructures. Meanwhile, new developments have been mushrooming, especially in the accommodation sector.

Furthermore, a number of issues still challenge SHV . According to National Coastal Steering Committee (NCSC), SHV's forest cover reduced from 63\% in 1992 to $55 \%$ in 2002, which critically affected the natural environment (NCSC, 2005). Mangrove forests also encountered a great loss from 146 ha in 1997 to 45 ha in 2002 (NCSC, 2005). Carter et al. (2013) argue that the SHV's uncontrolled development in the environmentally sensitive areas has threatened the long-term sustainability of tourism. For instance, pollution from insufficiently treated sewage, littering and dumping of wastes is the threat to the social and environmental values of the province (als see Carter et al., 2013; Tinat, 2011). Because SHV's coastal resources are the core elements of socio-economic well-being of the people, expanded economic activities and population growth especially more tourists, will increasingly pressurise the management of the significant natural environment (Ong \& Smith, 2014).

In conclusion, SHV is highly vulnerable to climate change, which will cause increasingly serious challenges in the future. Additionally, when linked to the environmental, social and economic problems in the province, climate change poses a more serious risk of exacerbating current problems (UN-Habitat, 2012a).

\subsection{Chapter summary}

Due to its richness of natural and cultural legacy, especially after the Peace Accord, along with tourism, Cambodia's coastal tourism has grown significantly. However, various challenges have come along, one of which are climate change issues, which have interrupted tourism growth. Much evidence by the government bodies, DPs and individuals has identified that Cambodia is a highly vulnerable place whereas its adaptive capacity is low.

\footnotetext{
26 Japan International Cooperation Agency
} 
The coastal zone is identified as one of the most vulnerable areas. SHV, one of the coastal provinces, is also reported as being vulnerable. The existing data tend to report that $\mathrm{SHV}$ is highly vulnerable to excessive rainfall, rising temperature, storm, SLR/beach erosion/storm surge and prolonged drought, which clearly interrupt its tourism growth. In the meantime, other contributing factors, including the rapid development of the area, leads to environmental degradation and infrastructural constraints. Since SHV is a poor-data region, on one hand, this has been a challenge for the study. On the other hand, there is a large gap for the study to contribute to the existing knowledge about climate change data in SHV. The study aims to fill this knowledge gap in an appropriate context. 


\section{CHAPTER 4: RESEARCH METHODOLOGY}

\subsection{Introduction}

The preceding chapters provided valuable background information for this study. This chapter provides the methodological approaches used for this study. Section 4.2 presents the research paradigm. Sections 4.3 and 4.4 outline the research approach which is mixed research method and case study adopted for this study. Section 4.5 outlines the recruitment of respondents and introduces their backgrounds. Section 4.6 highlights the data collection techniques detailing semi-structured interviews, informal site visits and document analysis. It highlights the method for collecting data in order to acquire the information necessary to address the research aims/questions. Section 4.7 presents how data is prepared prior to data analysis. Sections 4.8 introduces the analytical framework. Section 4.9 presents the analytical methods using overall case analysis and subsequently cross-case analysis. Section 4.10, 4.11 and 4.12 present the validity and reliability, ethical considerations of the study and limitations of the study.

\subsection{Research paradigm}

A number of studies have argued that post-positivism is closely relevant to 'reality' or 'truth' of a particular research phenomenon. The post-positivistic norms involve believing in the reality, knowledge, and value in research (Bisel \& Adame, 2017). To the meaning of the 'scientific approach', the post-positivist doctrine considers one objective, 'truth', with confirmable patterns that can be projected with certainty (Littlejohn, 2007). In postpositivism, researchers acknowledge that their research approaches and collected data reflect reality (Ryan, 2006). The truth is created through a dialogue. Researchers never question themselves with 'is this the reality?' Rather, all discuss the problems raised in the interviews, the reactions of the participants, and the explanations of these intertwined opinions to share our thoughts and how the perspectives might be utilised (Richie \& Rigano, 2001, as cited in Ryan, 2006). In post-positivism, research attempts to uncover the meaning of the reality that is a social construction, which is understood by an individual or a group (Sharma, 2010). Parallel to the arguments, by adopting a case study, this research chooses to seek the 'reality' of climate change vulnerability through the combination of perception and scientific 
evidence and the 'actual' adaptation actions taking place in the site. Within the adaptation process, the study further identifies and critically examines the 'real' barriers and enabling factors that impact adaptation.

Post-positivist research is related to work in the natural sciences (Ryan, 2006). Postpositivism places emphasis on the concept of meaning and seeks to explain social concerns (Henderson, 2011). Within post-positivism, research usually takes place in local communities and in everyday lives of people - in natural settings. It attempts to seek for meanings in definite cultural and social settings rather than in general laws applicable to everything and everyone (McGregor \& Murnane, 2010). Henderson (2011) argues that postpositivism places emphasis on the meanings and seeks to explain social concerns. Samdahl (1999) considers post-positivism as one of the numerous paradigms that are likely to be used in the research on human behaviour. Corresponding with these previous research studies dealing with climate change issues, this research seeks to explain social concerns. This study takes account of work that is much related to natural sciences such as coastal tourism. Furthermore, for CCA in the local community of a developing country, this research tries to determine the vulnerability level of a destination and see how this has affected people's lives, especially in the tourism businesses. Finally, the study attempts to uncover the behaviours of the tourism businesses in response to climate change, which is closely relevant to human behaviours as noted above.

According to Creswell (2007), post-positivist researchers will tend to perceive inquiry as a series of logically connected steps, and believe in several opinions from participants rather than a single reality. Post-positivism provides a practical approach to gather data with mixed methods (Henderson, 2011). Within the post-positivist standpoint, this research commences with logically connected steps by first identifying the vulnerability and building vital link towards adaptation actions through collecting various evidence such as the interviews, information site visits and documents analysis. Post-positivist research has characteristics with the idea that research is associated with the methods to collect and classify information is insufficient (Schratz \& Walker, 1995, as cited in Ryan, 2006). Post-positivism provides flexibility to researchers about their stance that is relevant to a topic that they find challenging 
(Dupuis, 1999). As the information about climate change responses in tourism businesses is currently lacking, the study challenges to fill this gap.

Henderson (2011) notes that post-positivism is applicable to both qualitative and quantitative approaches. McGregor and Murnane (2010) explain that while quantitative (measuring what, where and when) is often related to positivism, the qualitative (interpreting the why and how of human behaviour) is also connected to post-positivism. Case study investigation can be conducted with any of the following research paradigms: critical, interpretive, positivist or post-positivist, (Myers, 1997). Yin (2003) explains that case research has been identified as an appropriate study approach to exploring phenomenon asking "How" or "Why" questions for investigating studies concerned with their natural settings. By focusing on the research question of 'how', the hotel sector has adapted to the climate change impacts in coastal tourism (RQ 3) and by analysing reasons for 'how' and 'why' the barriers and enabling factors to CCA have happened in the adaptation processes (RQ4 \& 5). This research aligns with both conditions explained by Yin. This study adopts the mixed methods and case study approach seeking perceptions/opinions from several participants rather than a single reality. Meanwhile, the research used more than one method (semi-structured interviews and informal site observation as well as document analysis) to gather data. The researcher also involved himself in conducting the study by learning with the participants rather than researching on them. Wolcott (1990) as cited in Ryan (2006) argues that in post-positivism, researchers involve themselves as people who carry out studies among other participants and learn with them, rather than conduct studies on them.

In conclusion, post-positivism is the most appropriate paradigm for this research. Firstly, post-positivism is suitable for identifying the climate vulnerability at the coastal zone because it is as much about human adaptation behaviours as it is about natural science (Ryan, 2006) and it seeks to explain social concerns (Henderson, 2011) such as climate change problems. Secondly, post-positivism is suitable for qualitative and quantitative approach (Henderson, 2011) and more than one method (Henderson, 2011) for data collection. Thirdly, the anticipated findings of this study will mirror the 'truth' in vulnerability assessment, adaptation and barriers and enabling factors that affect adaptation, as acknowledged by Ryan 
(2006), who argues that in post-positivism, research methods and data reflect reality. Fourthly, post-positivism is undertaken in leisure studies with quantitative or qualitative data, or a mix of them (Henderson, 2011). In conclusion, post-positivism supports both the focus of this study and the methodological approach applied in order to address the research questions/aims.

\subsection{Research approach}

In social sciences, in order to investigate problems, researchers adopt quantitative or qualitative approaches or mixed methods (Tuan, 2016). Collecting, analysing and mixing data by combing the qualitative and quantitative approaches is recognised as the mixed methods mainly to provide a wider understanding of the problem (Creswell, 2014). By combing both of them, this method aims at reducing the limitations of specific types of data and increasing the strengths of data (Tuan, 2016).

While there are discussions about which method is better (Davies, 2003; Gelo, Braakmann, \& Benetka, 2008), this study adopts mixed method. While Creswell (2003) and Guba and Lincoln (1994) argue that qualitative research can be used to unearth participants' views, quantitative study usually is associated with systematic and empirical examination of phenomena through statistics and the processing of numerical values (Basias \& Pollalis, 2018). This study first unearths participants' perceptions on a particular phenomenon and second, involves an investigation of the phenomenon through statistical and numerical data to critically examine their answers. There are no specific criteria as to whether mixed method is selected because Creswell (2014) explains that selecting mixed method depends on factors such as the timing of the data gathering, type of study design most suited for a field, and the selection based on a single researcher or team.

Basias and Pollalis (2018) explain that the common characteristics of the quantitative research usually involves the use of closed questions, and its advantages involve fact-based, measurable and observable numeric data, and allowing researchers to compare numerical data easily. Basias and Pollalis (2018) continue that the question form of qualitative research is open question. Its advantages enable researchers to understand the nature and complexity 
of the phenomenon under consideration, support the examination of a phenomenon in its natural environment, and involves in-depth research. Interviews, which are one of the data collection methods of this thesis, contain both open and closed questions. The closed questions are Likert scale, which involve numeric values, and the open questions involve indepth questions to examine a phenomenon in its natural environment. The quantitative research usually involves a systematic investigation of phenomena through statistical data while the qualitative approach examines phenomena by analysing experiences, behaviours and interactions without the involvement of statistics (Basias \& Pollalis, 2018). Combing both approaches best characterises the study's aims, which use Likert scale to obtain statistical data and open questions to examine phenomena through investigating experiences, behaviours and interactions of the interviewees.

\subsection{Case study}

Using an instrumental case study in order to explore a specific phenomenon or theory, Pickard (2007) argues that the importance of case study is not actually the case itself but rather how the case can be used as a vehicle for investigation. A case study can use qualitative, quantitative, or triangulation data collection techniques (Pickard, 2007) and be applicable to post-positivism (Myers, 1997). A case study is a method that is employed to investigate a particular fact within a real-life situation, often with a very specific purpose (Pickard, 2007). The major purposes of case-based investigation set out to deepen, elaborate, and impassion the investigator or researcher (Hyde, Ryan, \& Woodside, 2012).

Hyde et al. (2012) categorise the objectives of case studies into four types: complexity/coverage, value/impact, accuracy, and generalisability. The 'complexity/coverage' allows the depth and breadth of the processes, occurrence of antecedents, and outcomes emerged from a case study investigation. The 'value/impact' refers to the usefulness and utilisation of a case study outcome to advance understanding, help solve complex issues, and/or help to formulate/design and carry out strategies, policies and actions. The 'accuracy' involves reporting the 'correct' answer to objective knowledge and subjective perspectives. The 'generalisation' comprises three forms: context generalisation that generalises from a single case to alternative theories and generalises 
across cases. This work adopted case study as it allows deep investigation of the phenomena and issues of this underlying topic and cover the objective and subjective perceptions. In turn, its results could be used to solve perplexing problems and help devise strategies.

Similar to this thesis, other scholars have also used single case study. For example, Warrick (2011) studied community-based adaptation to climate change (CBA) in a rural Pacific Islands context. Hopkins (2013) conducted research in Climate Change Contextual Vulnerability, Risk Perception and Adaptation in the Ski Industry of Queenstown, New Zealand. Jopp (2012) studied CCA in Tourism: A Case Study of the Victorian Surf Coast Region. In assessing climate change vulnerability and resilience of ecotourism, Jamaliah's (2015) thesis also employed a case study approach.

Prior to data collection, preparations such as the interview guide (including types of questions), pilot tests, revision of the interview guide, and other materials (e.g. camera) are necessary. These steps and processes will now be discussed.

\subsection{Recruiting respondents}

A range of industry sectors are operating in SHV, including guesthouse, travel agency, and transport sector, which serve the needs of tourism growth. Among these subsectors, the hotel sector hugely contributes to the development of SHV's tourism. As outlined in Chapter 3, SHV is the country's third most attractive tourist site. The number of tourists has remarkably increased with 1,648,099 domestic and 404,939 international tourists in 2016 (MOT, 2016), 2,000,000 domestic and 470,000 foreign tourists in 2017 (Hakim, 2018) (Hakim, 2018) and 2,600,000 domestic and 581,564 foreign visitors in 2018 (The Phnom Penh Post, 2018). This remarkable growth has led to the increase in the number of hotels: 75 hotels in early 2017 (confirmed during field research) to 156 hotels by mid-2019 (The Phnom Penh Post, 2019), and this is expected to increase by $138 \%$ or 7,391 rooms by 2020 , which are both under construction and in the pipeline (Hakim, 2018). In return, hotel establishments have become the main economic generator/driver in the study site. In SHV, the hotel sector is also considered the most important element among tourism subsectors, if a study is to explore the link between tourism/coastal tourism and climate change because the initiatives and 
outcomes of adaptations are influenced by hotel attributes (e.g. star rating, size, ownership, and location) and people's or hoteliers' backgrounds (e.g. level of education, experience, nationality and age) (see section 2.6 of Chapter 2). Building on existing literature, studying the hotel sector's adaptation may produce more interesting findings than other subsectors (e.g. guesthouse, travel agencies), particularly in SHV where areas are developing rapidly. The study considered two primary rationales in selecting the hotel sector. First, the importance of the hotel sector linked to growth and business profitability for the whole region is the rationale behind the selection (see Briggs, Sutherland, \& Drummond, 2007). Second, the high vulnerability of the hotel sector to climate change due to its fixed structures has provided rationale for this research (Simpson et al., 2008; Su et al., 2013; Su, 2014; WMO et al., 2008).

The hotel sector was the target group for interviews. Prior to the interviews, there were a number of visits to SHV, including the SHV DOT and Department of Environment (DOE) office. The DOT is SHV-based governmental organisation, which manages all tourism activities while SHV DOE manages all climate change-related activities in the area. The purpose of the visits was to understand overall issues relevant to tourism, particularly about managing the hotel sector in relation to climate change issues. A number of documents were obtained from the visits. A list of registered hotels in the area was given. The list provided the exact number of hotels, contacts, ownerships and specific addresses. At the same time, there was a visit to the SHV Municipality because of its responsibility for managing the dayto-day administrative functions such as coastal management plans.

Based on the hotel list provided by the SHV DOT, there were 77 hotels in total in SHV. However, after the researcher contacted each hotel, there were 75 hotels operating because two hotels had gone bankrupt. 50 participants representing 50 hotels in SHV were recruited and interviewed. The 50 hotels or respondents are categorised based on the hotel attributes and their personal backgrounds in Table 4.1 . 
Table 4. 1: Number of interviewees categorised in hotel attributes and participants' backgrounds

\begin{tabular}{|c|c|c|c|c|c|c|c|}
\hline \multicolumn{2}{|c|}{ Hotel attributes } & \multirow{2}{*}{$\begin{array}{c}\text { Number } \\
11\end{array}$} & Total & \multicolumn{2}{|c|}{ Participants' background } & \multirow{2}{*}{$\begin{array}{c}\text { Number } \\
9\end{array}$} & \multirow{2}{*}{ Total } \\
\hline \multirow{3}{*}{ Star rating } & Budget & & \multirow{14}{*}{50} & \multirow{2}{*}{ Education } & Low (< bachelor) & & \\
\hline & Midscale & 17 & & & High ( $\geq$ bachelor) & 41 & \multirow{13}{*}{50} \\
\hline & Upscale & 22 & & \multirow{3}{*}{ Experience } & Short $(0-5$ years $)$ & 33 & \\
\hline \multirow{2}{*}{ Ownership } & Khmer-owned & 31 & & & $\begin{array}{l}\text { Medium (6-10 } \\
\text { years) }\end{array}$ & 8 & \\
\hline & Foreign-owned & 19 & & & $\begin{array}{l}\text { Long (over } 10 \\
\text { years) }\end{array}$ & 9 & \\
\hline \multirow{3}{*}{ Hotel size } & $\begin{array}{l}\text { Small (1-45 } \\
\text { rooms) }\end{array}$ & 30 & & \multirow{2}{*}{ Nationality } & Khmer & 38 & \\
\hline & $\begin{array}{l}\text { Medium (46-100 } \\
\text { rooms) }\end{array}$ & 13 & & & Foreign & 12 & \\
\hline & $\begin{array}{l}\text { Large (over } 100 \\
\text { rooms) }\end{array}$ & 7 & & \multirow{2}{*}{$\begin{array}{l}\text { Length of } \\
\text { residence }\end{array}$} & Short $(<5$ years $)$ & 27 & \\
\hline \multirow{2}{*}{ Location } & Mainland & 34 & & & Long ( $\geq 5$ years) & $\begin{array}{c}19 \\
(4 \text { missing } \\
\text { data })\end{array}$ & \\
\hline & Front beach & 16 & & \multirow{3}{*}{ Age } & $\begin{array}{l}\text { Young (20-29 } \\
\text { years) }\end{array}$ & 12 & \\
\hline \multirow{2}{*}{$\begin{array}{l}\text { Business } \\
\text { age }\end{array}$} & Short $(<3$ years $)$ & 30 & & & $\begin{array}{l}\text { Medium }(30-39 \\
\text { years }\end{array}$ & 30 & \\
\hline & Long ( $\geq 3$ years) & 20 & & & $\begin{array}{l}\text { Senior (over } 39 \\
\text { years) }\end{array}$ & 8 & \\
\hline \multirow{2}{*}{$\begin{array}{l}\text { Business } \\
\text { format }\end{array}$} & Owned hotel & 16 & & - & - & - & \\
\hline & Leased hotel & 34 & & - & - & - & \\
\hline
\end{tabular}




\subsection{Data Collection}

In this research, the data collection process started from July 2016 to January 2017. The indepth semi-structured interviews, informal site investigations and document analysis were used as approaches to collect the data. The data collection process is detailed in Figure 4.1.

\section{Figure 4.1: Research process}

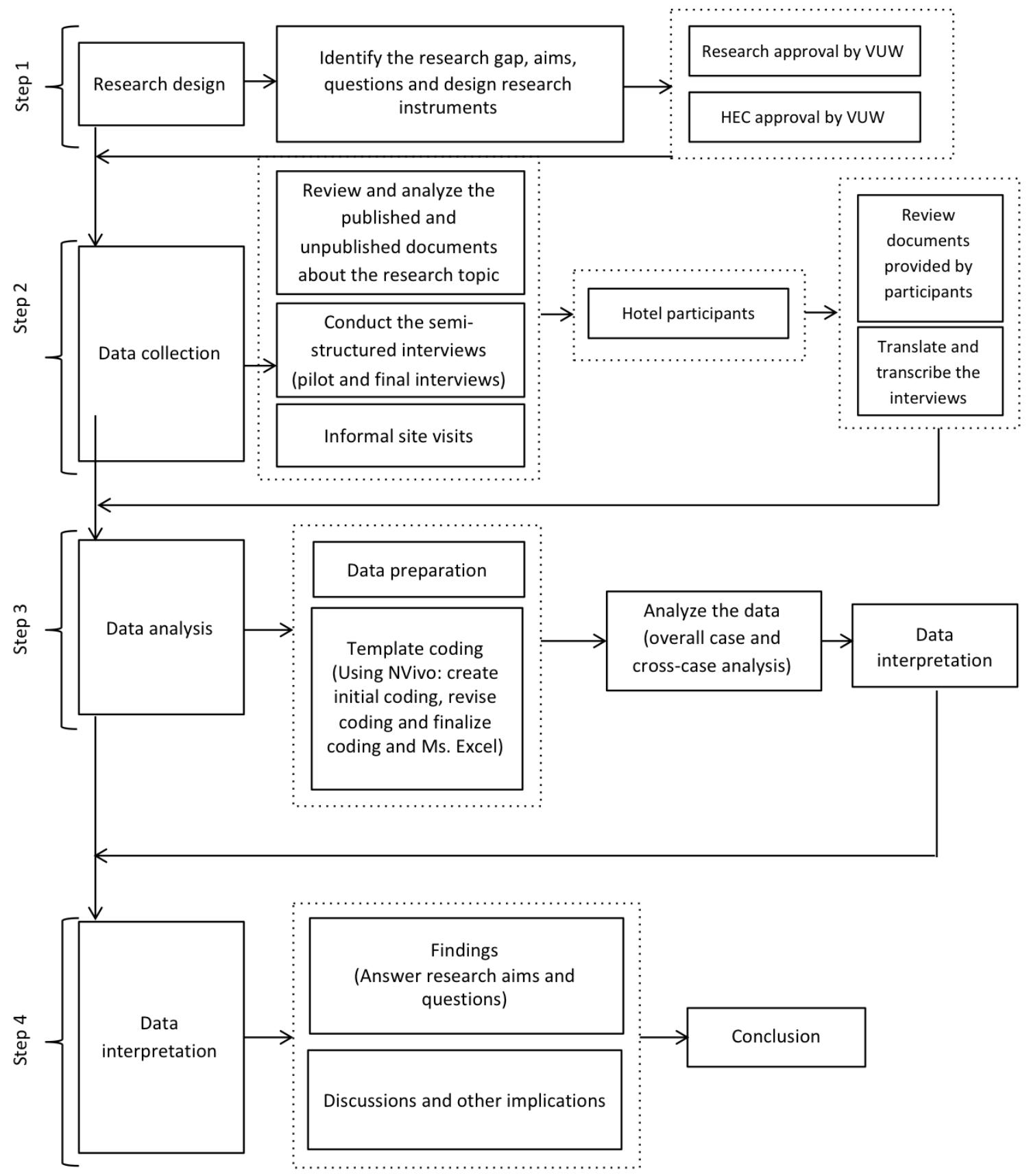




\subsubsection{Pilot interviews and adjustments}

Before the actual interviews, pilot interviews were done. For some research, this may form a crucial part prior to the main data collection. Veal (1997) explains that pilot interviews may eliminate the uncertainty and, in return, improve the wording and sequencing of questions. According to Veal (1997), there are a number of ways to conduct pilot test. It allows the testing of the wording and sequence of the questions, estimate responses and interview time, and test analysis procedures. The pilot tests in this study allowed the researcher to anticipate challenges and advantages such as the appropriate length of time, the effectiveness of each question and the wording. The tests may be helpful in identifying key people (e.g. hotel owners, GMs, supervisors) as this research anticipated answers from higher management. This significantly contributed to both revision/improvement of the interview guide and other preparations for the final interviews. For this study, there were two pilot interview phases were conducted. The first pilot was done from August 6 to 11, 2016. The second one was from November 3 to 5 2016. Seven hotel participants were recruited.

\subsubsection{Before the interviews or preparation stage}

The hotels were identified from the list given by the SHV DOT. All hotels in SHV were first contacted by phone. In order to confirm and update, numerous visits were made to the hotels as well. Once all such contacts and addresses were updated and confirmed, the appointments were made. Direct visits, phone calls and emails were used to recruit the respondents. The introduction of the topic was made in both phone calls and direct visits as well as via emails. Once the participants agreed to participate, an email was sent with the re-introduction of the topic and the full attachments such as the Information Sheet, Consent Forms and the Interview Guide. Follow-up phone calls and direct visits were made soon after the emails were sent. One or two days before the interviews, as always, a full set of hard copies of necessary documents was brought to the reception desks of the hotels for the target participants. On the interview days, each participant was given an extra complete set of the hard copies in case they read neither the email nor hard copies previously sent through the reception desks. 


\subsubsection{In-depth semi-structured interviews}

There are three primary kinds of research interviews: unstructured, semi-structured and structured (Gill, Stewart, Treasure, \& Chadwick, 2008). Taking the research questions/aims into consideration, this study adopted the in-depth semi-structured interview, which is detailed here. In-depth semi-structured interviews were used as the main data collector for this study because they allowed researcher to directly collect information from the participants. Veal (2006) explains that in-depth interviews are often used in circumstances "where the information is likely to be obtained from each subject vary considerably, and in complex ways" (p. 257). Veal and Darcy (2014) add that a semi-structured interview is the more usual format that enables the investigator to obtain the facts and the participants' perception. A semi-structured interview is less formal but allows the researcher to take control over the essence of questioning by using the sequence of questions (Creswell, 2003). This approach is useful because it enables participants to make a contribution in their own words, it offers more flexibility in the way it is undertaken than surveys, and it allows complex questions to be probed (Sarantakos, 1993) or probe an issue or reaction in more elaborated way (Britten, 2006).

While tightly structured interview/approach has a tendency to draw out short responses, the semi-structured interview has a tendency to bring out more open-ended, inconsequential responses (Campbell et al., 2013). Normally these are carried out for a group or an individual. Within the semi-structured interview, the research frequently reveals substantive clues about the codes and thematic clusters or categories emerging from the data (Galletta, 2013a). Time spent on the semi-structured interview can be half an hour to many hours (DiCicco-Bloom $\&$ Crabtree, 2006). The in-depth interview individually enables the researcher to probe the personal and social issues in depth (DiCicco-Bloom \& Crabtree, 2006). The semi-structured interview provides researchers with a way to focus on lived experience and pursue questions from existing theory (Galletta, 2013b). This allows more space for participants and investigators by engaging in a significant reflection of the collected evidence (Galletta, 2013a). 
Galletta (2013b) highlights a number of advantages of semi-structured interviews:

- $\quad$ They establish a level of convenience and ensure understanding of the rights of the participants.

- $\quad$ They can move into broad questioning by creating openings for a participant to talk based on her/his experience.

- $\quad$ They can probe for clarification, when necessary.

- They enable mental noting of significant junctures in a participant's story to which the researcher can return later in the conversation for greater depth and exploration.

- They support the flow of the interview with investigation, which guides its direction concerning the studied area.

There are a number of authors who studied climate change and tourism by using semistructured interviews. For instance, Jarvis and Ortega (2010) conducted a study on climate change responses in hotel sector, and argue that semi-structured interviews are best for the knowledge and management experience of the hoteliers and how they are responding to climate change impacts. The semi-structured interviews allow both the researcher and the participants to deepen understandings of particular social phenomenon during the research process (Goodson \& Phillimore, 2002). Davidson and Sahli (2015) also conducted semistructured interviews in relation to a study of hotel sector.

\section{Likert scale questions}

In this research, in order to elicit the opinions from participants about the topic, some questions used Likert scale. It is believed that using Likert scale questions with combination of other open-ended questions create data-rich results that are important for the findings. Wadgave and Khairnar (2016) define that "Likert scale is a psychometric response scale primarily used in questionnaires to assess subject's perception” (p. 67). Likert-scale items are normally employed to examine the attitudes of participants towards a series of written or verbal statements or items (Dittrich et al., 2007). Normally, in the statements with sets of questions, participants are asked to explain their perceptions on a typical ordinal scale. The response rating scales are often labelled with five-point categories from 1 to 5 , indicating the endpoints including 'not at all serious' to 'very serious' or 'very unimportant' to 'very 
important' (Dittrich et al., 2007). A simple and often-used technique is to interpret each Likert scale question using a mean and standard deviation (SD); the items can then be ranked depending on the means (Dittrich et al., 2007).

Likert scale questions become crucial to measure responses on a continuum (Leedy \& Ormrod, 2013). Therefore, rating scales, including the Likert scale, are used to gather information about opinions (Drake, 2010) related to major environmental concerns by asking the respondents' perception of environmental dangers (Dittrich et al., 2007). Table 4.2 shows the Likert scale questions in this study. 
Table 4. 2: Key questions using the Likert scale in the study

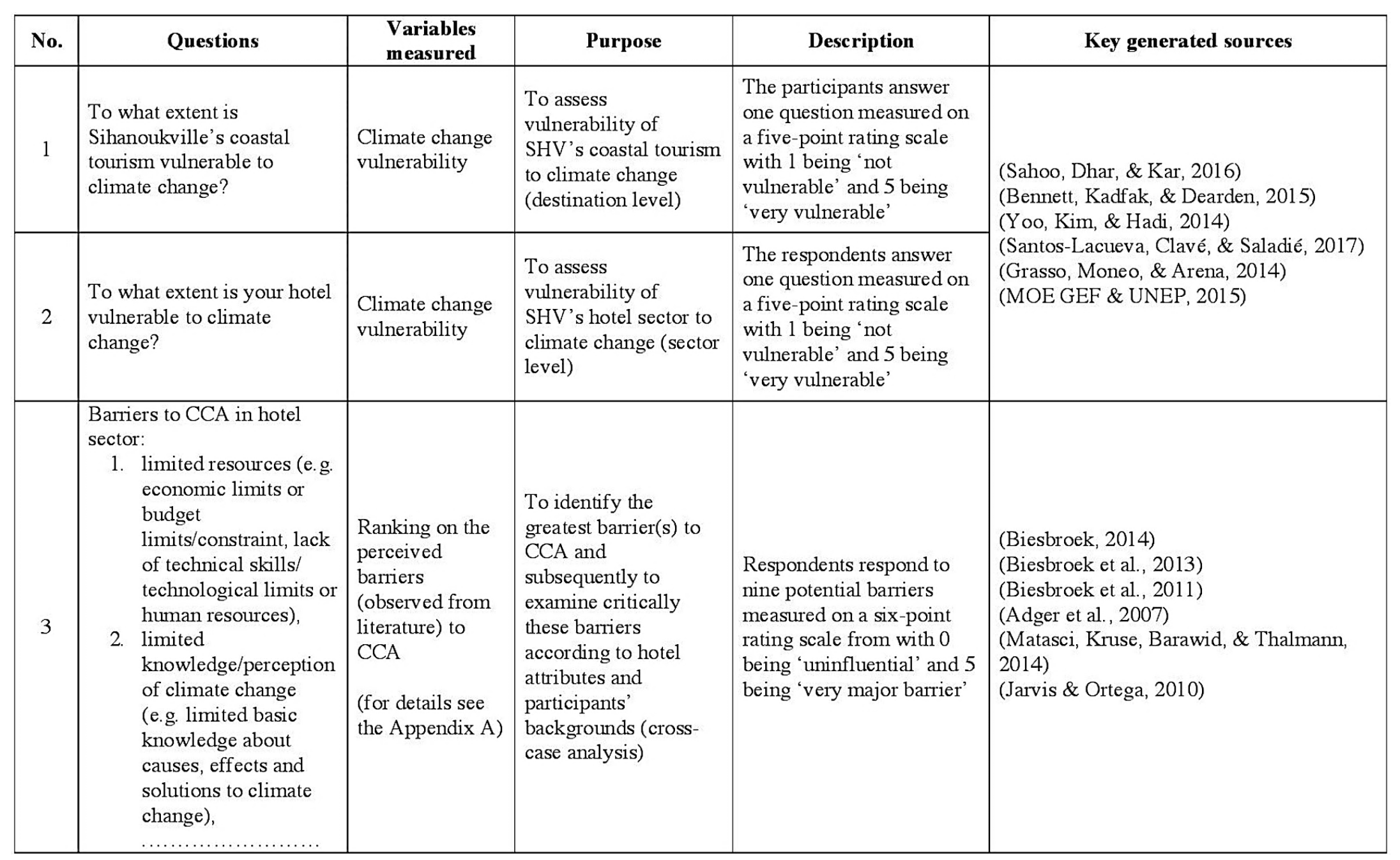




\begin{tabular}{|c|c|c|c|c|c|c|}
\hline & 9. & $\begin{array}{r}\text { (for details see the } \\
\text { Appendix A) }\end{array}$ & & & & \\
\hline 4 & $\begin{array}{c}\text { Enal } \\
\text { hote } \\
1 . \\
\\
\\
2 .\end{array}$ & 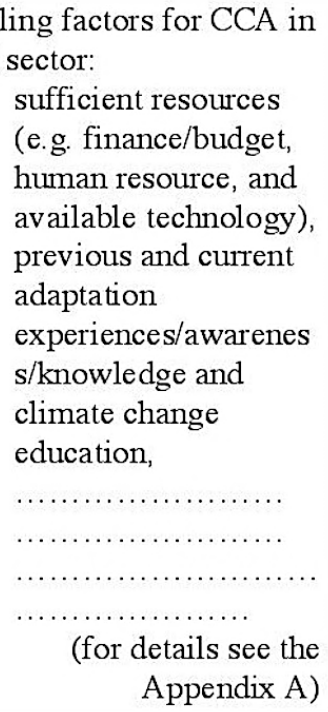 & $\begin{array}{l}\text { Ranking on the } \\
\text { perceived } \\
\text { enabling factors } \\
\text { (informed by } \\
\text { literature) to } \\
\text { CCA } \\
\text { (for details see } \\
\text { the Appendix A) }\end{array}$ & $\begin{array}{l}\text { To identify the most } \\
\text { significant enabling } \\
\text { factor(s) to CCA and } \\
\text { subsequently to } \\
\text { critically examine } \\
\text { these enabling factors } \\
\text { according to hotel } \\
\text { attributes and } \\
\text { participants' } \\
\text { backgrounds (cross- } \\
\text { case analysis) }\end{array}$ & $\begin{array}{l}\text { Respondents respond to } \\
\text { nine potential enabling } \\
\text { factors measured on a six- } \\
\text { point rating scale from } \\
\text { with } 0 \text { being } \\
\text { 'uninfluential' and } 5 \text { being } \\
\text { 'very important' }\end{array}$ & $\begin{array}{l}\text { (Simonet \& Fatorić, 2016) } \\
\text { (Vulturius \& Gerger Swartling, 2015) } \\
\text { (Tribbia \& Moser, 2008) } \\
\text { (Glaas et al., 2015) } \\
\text { (Coles, Dinan, \& Warren, 2016) } \\
\text { (Zuka, 2014) }\end{array}$ \\
\hline
\end{tabular}


During the interviews, all questions that were asked followed the interview guide. Table 4.3 indicates the main questions of the interviews. 
Table 4. 3: Interview protocol summary

\begin{tabular}{|c|c|c|c|c|c|c|c|}
\hline $\begin{array}{l}\text { Section of the } \\
\text { interview }\end{array}$ & \multicolumn{6}{|c|}{ Main questions in the interview } & Purpose \\
\hline Introduction & \multicolumn{6}{|c|}{$\begin{array}{l}\text { - Questions about the participants' profiles/backgrounds } \\
\text { - Questions about the hotel attributes }\end{array}$} & $\begin{array}{l}\text { To obtain background information } \\
\text { of the participants and the hotels. }\end{array}$ \\
\hline \multirow{2}{*}{$\begin{array}{l}\text { Vulnerability } \\
\text { assessment }\end{array}$} & \multicolumn{6}{|c|}{$\begin{array}{l}\text { - To what extent is Sihanoukville's coastal tourism vulnerable to climate change? } \\
\text { What environmental vulnerabilities do you see in Sihanoukville? } \\
\quad>\text { What socio-economic vulnerabilities do you see in Sihanoukville? }\end{array}$} & $\begin{array}{l}\text { Focus on first research aim: to } \\
\text { assess the vulnerability of SHV's } \\
\text { coastal tourism to climate change } \\
\text { (destination level) }\end{array}$ \\
\hline & \multicolumn{6}{|c|}{$\begin{array}{l}\text { - To what extent is your hotel vulnerable to climate change? } \\
\text { D What environmental vulnerabilities do you see on your hotel? } \\
\quad>\text { What socio-economic vulnerabilities do you see on your hotel? }\end{array}$} & $\begin{array}{l}\text { Focus on second research aim: to } \\
\text { assess the vulnerability of SHV's } \\
\text { hotels to climate change (sector } \\
\text { level) }\end{array}$ \\
\hline $\begin{array}{l}\text { Climate change } \\
\text { adaptation (CCA) }\end{array}$ & \multicolumn{6}{|c|}{ - Has your hotel adapted to climate change? If so, how? } & $\begin{array}{l}\text { Focus on third research aim: to } \\
\text { determine whether the hotel sector } \\
\text { adapts to the climate change } \\
\text { impacts. And how? }\end{array}$ \\
\hline \multirow{3}{*}{$\begin{array}{l}\text { Ratings of } \\
\text { adaptation barriers } \\
\text { and enabling } \\
\text { factors and } \\
\text { identification of } \\
\text { additional } \\
\text { adaptation barriers } \\
\text { and enabling } \\
\text { factors in SHV }\end{array}$} & - Can you provi & le rating & f the followi & ing barric & ers? & & \multirow{3}{*}{$\begin{array}{l}\text { Focus on fourth \& fifth research } \\
\text { aim: To identify the most } \\
\text { significant barriers and enabling } \\
\text { factors for CCA in the context of } \\
\text { hotel sector's adaptation and } \\
\text { subsequently critically examine } \\
\text { these barriers and enabling factors } \\
\text { according to the hotel attributes } \\
\text { and participants' backgrounds }\end{array}$} \\
\hline & $\begin{array}{c}\square \\
5 \\
\text { (very major) }\end{array}$ & $\begin{array}{c} \\
4 \\
\text { (major) }\end{array}$ & $\begin{array}{c} \\
3 \\
\text { (moderate) }\end{array}$ & $\begin{array}{c}\square \\
2 \\
\text { (small) }\end{array}$ & \begin{tabular}{|c|}
$\square$ \\
1 \\
(very small)
\end{tabular} & $\begin{array}{c} \\
0 \\
\text { Uninfluential } \\
\text { (not a barrier) }\end{array}$ & \\
\hline & \multicolumn{6}{|c|}{ 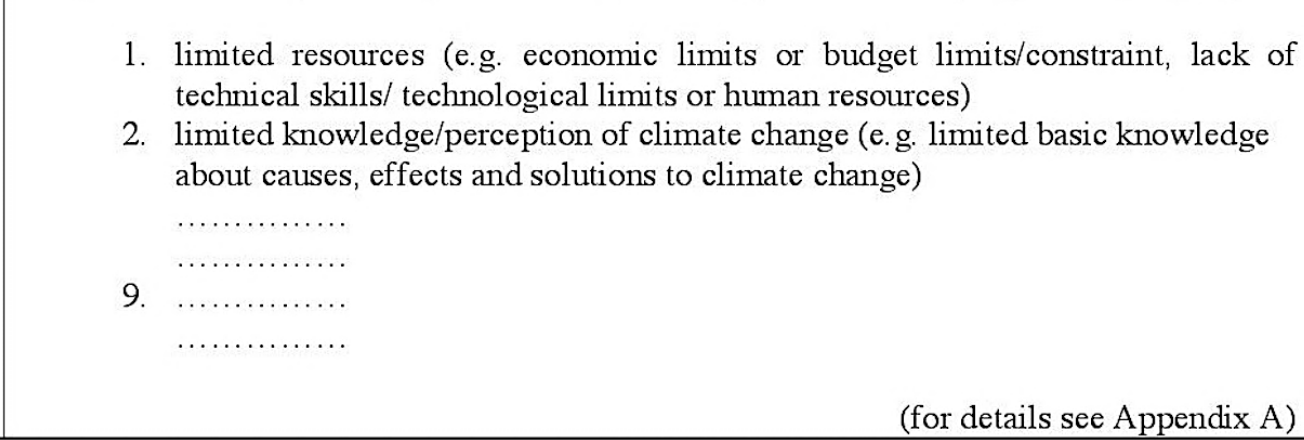 } & \\
\hline
\end{tabular}




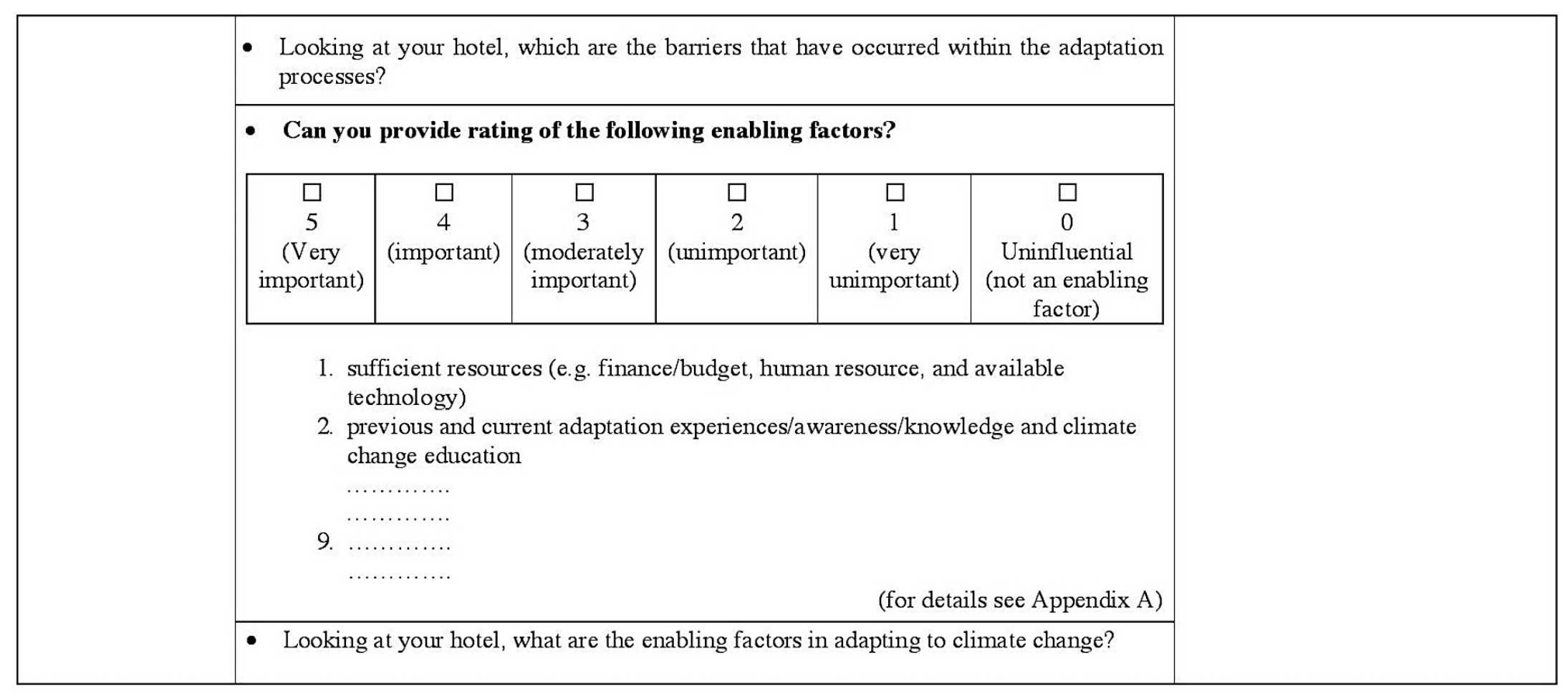


The duration of interviews varied, depending on the participant, between 50mn and $90 \mathrm{mn}$. As stated in the Information Sheet and Consent Forms sent to participants prior to the interviews, the in-depth interviews would be either voice-recorded and/or notes would be taken. Only one interview was note-taken. The remainders were audio-recorded. In terms of interviewing language, 12 interviews were conducted in English; the remainders were all in Khmer. All interviews took place at the interviewees' offices.

\subsubsection{Informal site observation}

Informal site observation is another method for accessing information by doing several visits to the area (Longjit, 2010; Yin, 2003). This allows the investigator to have a direct experience and to document information as it unfolds (Creswell, 2003). Cameras, both photo and video, were used to take photographs and video clips at the site for visual evidence. It had to be done for additional contextual evidence concerning tourism and climate change characteristics and issues in coastal areas (see Longjit, 2010). At the same time, additional notes were taken. Using the official map of SHV, the informal site visits were conducted in six distinct temporal phases targeting three different locations: at the mainland, along the beach lines and at the islands by keeping in mind two dimensions: environmental and socio-economic vulnerability due to the complexity and uncertainty concerning environmental, social and economic change (Frans Berkhout \& Hertin, 2000) towards climate change. The collected information was compared and contrasted with the data informed by the participants and the published reports. This was important for critical analysis and ultimately interpretation.

Firstly, extensive travel in the mainland in SHV was done to familiarise the researcher with the research site and observe the hotel locations, whether they are far or near resources needed (e.g. information and water availability). Secondly, a visit was made on nearly all the main beach lines in SHV by observing the coastal adaptations. Out of town, the visits to the coastal dams, mangrove forest areas and reservoir lakes were also made. Thirdly, the final places of travel were islands. Many island visits target the adaptations such as mangrove forest areas and anti-drought measures (e.g. water storage and humandug wells). A number of nights were spent staying in the community houses, travelling around island communities, and participating in the community meetings. The informal site visits were either done alone or facilitated by SHV DOE official and community chiefs. 
In summary, the informal site visits allowed an investigation of historic patterns of climate impacts and the CCA initiatives in the area. The information from these site visits was integrated and analysed. The informal site visits contributed to strengthening the data collection methods by supplementing the information from actual visualisation in the area, in addition to in-depth semi-structured interviews and document analysis.

\subsubsection{Document analysis}

In addition to the interviews and informal site visits, a wide range of archival records, and printed and electronic documents were reviewed and compiled. This had to be done to double-check and support the collected data in the interviews and informal site visits. The online secondary sources of documents from websites of the MOT, MOE, IOs, NGOs and hotels were retrieved (including information on the master plan of Cambodian tourism development, hotels and climate change-related issues). In this case, online documents have been used as attracting attention for information to enable the researcher to retrieve at any time period (Creswell, 2014). Publicly available documents of the Cambodian government, solutions on tourism and climate change development, laws on service sector, directives, circulars, decisions of government on service development, and commitments of Cambodia relative to tourism and climate change were also examined and collected to complement the interviews. These documents and sources were reviewed to make sure that the researcher gained enough information for the analysis.

\subsection{Data preparation}

To help the analysis, the data collected through interviews was transcribed so that they are refined into text (Miles, Huberman, \& Saldaña, 2013). Of all 50 interviews, the researcher transcribed recordings of 49 while one was note-taken. Thirty-eight were interviewed in the Khmer language and later translated into English by the researcher for interpretation. Transcriptions and collected data from all other sources were organised and kept securely. As stated in the Information Sheet, the raw data (interview tapes, transcripts, notes and documents) were securely kept in a locked cabinet and passwordprotected computer. In the computer, a separate folder was created with a subfolder for each group storing the electronic records such as website addresses, transcripts, audio files, and contact information. Once polished and finalised, the transcripts were uploaded to the data analysis software NVivo, version 11. Based on the analytical framework, an 
initial template (coding) was made. Although initial coding (template) was done using the NVivo software, some data analysis needed additional use of the Microsoft Excel. For convenience, easier visualisation of the data and cross-tabular format in the cross-case displays, and some elements of the data could be better addressed by combining the two software programs for either overall analysis or cross-case analysis.

\subsubsection{Coding the template}

Brooks et al. (2015) say that "Template Analysis is a form of thematic analysis which emphasises the use of hierarchical coding but balances a relatively high degree of structure in the process of analysing textual data with the flexibility to adapt it to the needs of a particular study" (p. 203), while coding is the process to identify themes in accounts and attach labels (codes) to index them (Brooks \& King, 2014).

Developing a coding template is the central technique, usually starting with the basis of a subset of data that initially is applied to the data but can be refined and revised, and then coded and finalised later (Brooks et al., 2015). This approach can be adopted to be suitable for different research areas and the obtainable resources of a specific research case (Brooks et al., 2015). Template analysis is not only usable with the data in the format of interview transcripts but can also be used with any type of textual data such as diary entries, focus group data, or open-ended question responses (Brooks \& King, 2014). King (2008) argues that template analysis can be applicable to both small sample size such as qualitative data and larger data sets (quantitative).

The data collected through textual data such as focus groups (Brooks, 2014; KirkbyGeddes, King, \& Bravington, 2013), interview transcripts (Lockett et al., 2012; Thompson, Clarke, Newell, \& Gawkrodger, 2010), open-ended question feedbacks on a written questionnaire (Dornan, Carroll, \& Parboosingh, 2002; Kent, 2000), and diary entries (Waddington \& Fletcher, 2005), can be used in the template analysis. To produce answers to the research questions, the analytical process extends further beyond the scope of sorting, coding and sifting (Chowdhury, 2015). According to King (2012), there are six sequential steps to conducting the template analysis (see Table 4.4): 
Table 4. 4: Template analysis steps

\begin{tabular}{|c|l|l|}
\hline No. & \multicolumn{1}{|c|}{ Step } & \multicolumn{1}{c|}{ Actions } \\
\hline 1 & Step 1 & Become familiar with the contents to be analyzed \\
\hline 2 & Step 2 & Conduct preliminary/initial coding of the data \\
\hline 3 & Step 3 & $\begin{array}{l}\text { Organize the emerging themes into meaningful clusters, and begin to } \\
\text { define how they relate to each other within and between these groupings }\end{array}$ \\
\hline 4 & Step 4 & Define a preliminary/initial coding template \\
\hline 5 & Step 5 & $\begin{array}{l}\text { Apply the preliminary/initial template to further data and revise as } \\
\text { necessary }\end{array}$ \\
\hline 6 & Step 6 & Finalize the template and apply it to the full data set \\
\hline
\end{tabular}

Source: (King, 2012)

Template development tends to be a way to making sense of data, and does not aim for the analysis in and of itself (Brooks et al., 2015). Template analysis shares common goals with the analytical framework because the involvement of template analysis is in parallel to tackling many of the same needs (Brooks et al., 2015). The difference is that template analysis plays a crucial role in providing thorough guidance, the development of the structure of coding than the analytical framework, and is less associated with outlining approaches as assistance to interpreting the data when fully coded (Brooks et al., 2015).

\subsubsection{Creating the initial template}

Theron (2015) explains that a code is a descriptive concept that is allocated by the researcher for capturing the significant content or essence of the data, and a code template contains a set of codes (Stein, Lauer, \& Kharbili, 2009). Initial coding is the process of splitting the data down into different distinct parts (Theron, 2015). Conducting the preliminary/initial template after the continual process of coding allows the method to use less time than other methods to analyse data (Brooks et al., 2015). The ongoing use of the template supports thorough examination of how themes are identified and how they are relevant to one another (Brooks et al., 2015).

The literature does not provide much guidance that is relevant to the establishment of reliable coding of transcripts of in-depth semi-structured interviews (Campbell et al., 2013). However, in the initial template coding, King (2008) argues that there should not be too many initial codes as this may limit the analysis and impede exploration of more relevant problems. However, having few codes may turn into a limitation of the clear 
direction and create an extremely rich and complex data. The better approach is to start the beginning template by using an interview topic guide (King, 2008).

In this research, the interview questions were following the analytical framework. Therefore, initial codes for the template were produced from the variables of the analytical framework. The key questions from the interview guide served as the higherorder codes. Higher-order codes were split into many levels of lower-order codes, depending on their relative significance to the study and thereafter contributing to the analysis into the greatest depth. Theron (2015) explains that it should be kept in mind that these initial codes and categories are ongoing and may be revised as the analysis process develops. Likewise, the next subsection explains the revision of the template.

\subsubsection{Revising the template}

While coding, Saldaña (2009) suggests that researchers should pay particular attention to their research questions and aims of the study and the researcher makes adjustments of the initial codes based on the particular attention. The revision of the template is based on the initial codes. Once the initial template is built, the researcher must double check to seek for any insufficiency. After finding the deficiency, the codes of the initial template are added or deleted.

King (1998) provides a guideline for the modification process leading to the development of the final template. Adjustments of the template involve inserting new codes, deleting irrelevant codes, changing the scope of codes, and changing higher-order categorisation as outlined below.

Inserting: When some views and concepts were not included in any initial codes in the template, those items were inserted as a new code.

Deleting: At the end of the template coding some prior codes were deleted because they were irrelevant or considerably overlapped.

Changing scope: By keeping in mind the research questions/aims, some codes were reidentified at a higher or lower level if the initial codes were seen to be poorly, broadly or narrowly built.

Changing higher-lower order categorisation: Once some initial codes were later noticed to fit as a subcategory of a different higher-order code, then their higher-order classification was changed. This was also applied to the lower-order codes. When some 
initially defined codes were later noticed to fit as the main category, and then their lowerorder classification was changed (to higher-order classification).

\subsubsection{Finalising the template}

Once sufficient time and efforts were given in the initial and revising stage, the template was finally built. There are no specific arguments to explain whether the template is finalised; this is differently unique to a specific investigation and the researcher (King, 1998). The codes should not be either too many or too few. Saldaña (2013) argues that the number of codes is dependent on the context, the nature of the data, and to what extent of suitability the researcher needs to examine the detail. Data can be 'grouped' together with a single code or can be 'split' into numerous smaller parts, each bearing its own code. In this research, the goal of coding is to keep in mind the research questions/aims and maintain the quality of the answers/findings. Coding must guarantee that all data are thoroughly considered and included. Therefore, once all the relevant data collected through interviews were coded and inserted in the template, the template was considered 'final'.

In the final template of this study, first the transcripts were printed out and perused through several times. Second, the transcribed documents were uploaded in the NVivo. Third, the finalised template codes were crosschecked to ensure the consistency and reliability. This is to ensure that all the relevant information was coded. Finally, it was confirmed that no significant changes were suggested. The 'final' template can play a role as both the basis for interpreting data set and usefully guiding and structuring the writingup of research outcomes (Brooks \& King, 2014). Once the template is finalised the data interpretation is done, which is detailed in the next subsection.

\subsubsection{Interpreting and presenting template analysis}

King (1998) explains that there are no general rules for how the coded data is interpreted by a researcher. The tactics for interpretation should be developed and align with the aims and content of a specific study. King (1998) adds that it is impossible for the researcher to interpret everything about the themes identified in the set of data. Instead, the researcher must attempt to identify the themes that are most fundamentally relevant to understanding the phenomena under the investigation. 
King (1998) and Brooks and King (2014) further provided approaches for interpretation and presentation of the final template:

- Within a set of individual case studies, researchers discuss the differences and similarities between cases,

- Once an account structured regarding the major features was identified, researchers draw indicative examples from each transcript when necessary, and

- With a thematic presentation of the findings, researchers use a different individual case study to indicate each of the main features.

The guidelines for analysing data in case studies by Creswell (2014) in collaboration with the approach recommended by King (1998) and Brooks and King (2014) have influenced this study in analysing and interpreting the template and other relevant data. Data analysis is detailed in the next section.

\subsection{Analytical framework}

The analytical framework, Figure 4.2 illustrates the chronological and logical steps for analysing the data into two phases: overall case analysis and subsequently cross-case analysis. 
Figure 4.2: Analytical framework

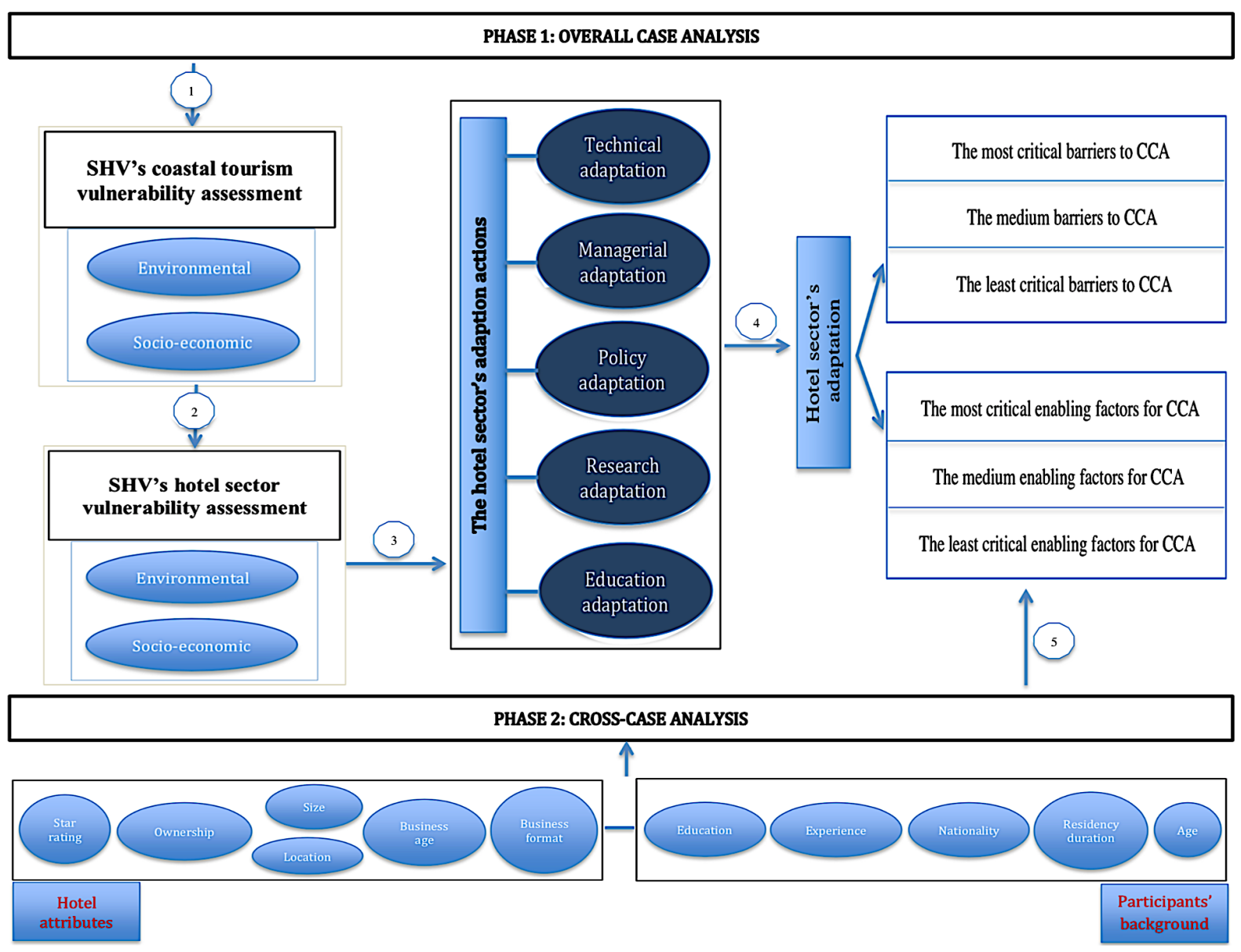

\subsection{Data Analysis}

Data analysis involves the process to arrange and reduce the raw data for the researcher to produce essence to their work (Miles et al., 2013). In data analysis, researchers transfer the data collected into some forms to explain, understand, or interpret people and situations that were investigated (Gibbs, 2002). From raw data to coding, sorting and examining of data, the researcher studies and interprets participants' attitudes, desires, environment, behaviours, needs, routines, and a range of other information that is critical to deeply investigating a research topic (Chowdhury, 2015).

Although many ways for data analysis have been suggested, Creswell (2014) provides the most comprehensive steps in analysing data. The researcher deals with the following processes (see Table 4.5): 
Table 4. 5: Data analysis steps

\begin{tabular}{|c|c|c|}
\hline No. & Step & Actions \\
\hline 1 & Step 1 & $\begin{array}{l}\text { Organizing and preparing the data for analysis: Once the data is } \\
\text { available, the next step is to deal with the transcriptions of the } \\
\text { interviews and the sorting and arranging of the data with different } \\
\text { sources of information }\end{array}$ \\
\hline 2 & Step 2 & $\begin{array}{l}\text { Reading through all the data: By reading through all the transcribed } \\
\text { interviews, the researcher can capture a general sense of the } \\
\text { information and the researcher can take additional notes by writing } \\
\text { down general ideas emerged from the data }\end{array}$ \\
\hline 3 & Step 3 & $\begin{array}{l}\text { Coding the data: It is the process of breaking down into parts of } \\
\text { information and writing a word symbolizing a category in the margin }\end{array}$ \\
\hline 4 & Step 4 & $\begin{array}{l}\text { Describing the setting or people and categories or themes for analysis: } \\
\text { In the coding process, the researcher gives detailed descriptions of the } \\
\text { context or the people involved as well as descriptions of the categories } \\
\text { or themes or even refining to be ready for analysis }\end{array}$ \\
\hline 5 & Step 5 & $\begin{array}{l}\text { Presenting the results of the analysis: This is often done in a format of } \\
\text { a descriptive passage to be well ready for data analysis. The narrative } \\
\text { information at this stage includes chronological orders of events, a } \\
\text { thorough discussion of numerous themes or a discussion of } \\
\text { interrelating themes }\end{array}$ \\
\hline 6 & Step 6 & $\begin{array}{l}\text { Interpreting the results of the analysis: The interpretation can take } \\
\text { various forms. The interpretation can be adapted for different types of } \\
\text { research designs. The interpretation can be flexible to convey personal } \\
\text { and research-based meanings. The overall aim of this step is to } \\
\text { research questions or reach the aims of the study. }\end{array}$ \\
\hline
\end{tabular}

Source: (Creswell, 2014)

Guided by the analytical framework (see Figure 4.2), the next section details the analytical methods.

\section{Analytical methods}

The template was significantly built based on the analytical framework, and each single element of the framework was used to guide the analysis and present the findings. To create the robustness of the study, the analysis is conducted in two main stages: overall case analysis and subsequently cross-case analysis (see Abeysekara, 2017; Strode, 2012) using descriptive statistics and nonparametric test (see Phan, 2017).

\section{Overall case analysis}

Collier (2012) explains that the terms 'case within a case' and 'overall case' are used interchangeably. Collier (2012) further adds that a case study is primarily a sustained 
analysis of a single case. In a case study, the researcher seeks to explain why a specific case has a particular outcome (Collier, 2012). A single case is broken down into a subset of smaller cases to allow researchers to identify the similarities and differences within the subcases (Gondo, Amis, \& Vardaman, 2012). Collier, Brady, and Seawright (2010) imply that causal inference in research using the causal process observation requires the withincase analysis.

As this study is composed of 50 cases, analysing within subcase or each individual case (one hotel) was impossible. Based on the research questions/aims, this analysis was only feasible for the whole region (SHV) to explain. Therefore, the data were analysed by adopting the components of the analytical framework built for the study. Description of the overall analysis begins by detailing an overall case profile. Then, using the finalised coding template, data were analysed adopting the structure of the analytical framework and with the integration of the themes that emerged from the research data. In order to have stronger grounds for the participants' claims, direct and indirect quotes from interviews/transcripts were used where appropriate. In short, the findings were analysed across the region, the study site. Collier (2012) argues that by practice, most research significantly depends on either within case or cross case analysis or the combination of the two tactics. This study adopted Collier's argument by combining overall case and cross-case analysis. Thus, the next subsection details the cross-case analysis process of this study.

\section{Cross-case analysis using descriptive test/independent sample test}

Miles and Huberman (1994) explain that researchers have had a tendency of using multiple individual cases because there has been a significant increase in studies of complex settings. At a greater depth, this is a need to have an understanding on how they are competent in local contexts and thus develop more sophisticated descriptions and more influential explanations (Miles \& Huberman, 1994). There are two reasons for using multiple cases analysis: one reason is to enhance generalisability and a second reason is to deepen understanding and explanation (Miles \& Huberman, 1994). Miles et al. (2013) and Yin (2009) add that this leads to the comparison, contrasting, or combination of data from all cases to improve the applicability, generalisability, and robustness of findings in different contexts. McGuiggan and Lee (2008) continue that using the cross-tabular design compares various classifications and allows graphing of continuous measurement 
scales and continual comparison of theory to data to validate a concept. Cross-case analysis does not go alone; its patterns can be sought and identified from the constructs or dimensions of the literature, and then the researcher looks for inter-group differences and within-group similarities (Eisenhardt, 1989).

Silverman (2010) supports that techniques in analysis remain effective in the identification of intangible factors, including socioeconomic status, ethnicity, social norms, gender roles, and religion. Hyde et al. (2012) continue that most research methods in tourism, leisure and hospitality studies use inter-individual analyses. Chowdhury (2015) notes that, in addition to the coding, sifting and classifying of data, the results could be influenced by investigators' interpretation and awareness on participants' behaviour desires, attitudes, needs, environment, routines, and a wide range of other information that is important to deeply investigating a research topic. The findings produced from the coding, classifying and sifting of data might often be generalised to groups of people with backgrounds similar to those in the study (Mack, Woodsong, MacQueen, Guest, \& Namey, 2005). Such generalization increases importance of the study's contribution.

For this thesis, the hotel attributes and participants' backgrounds (see Table 4.1) were used for cross-case analysis. The main goal is to explore the extent to which hotel attributes and participants' backgrounds in the hotel sector (in SHV) have impacted on their perceptions of barriers and enabling factors to CCA. It assesses how hotel attributes and respondents' characteristics influence the participants' perceptions towards the barriers and enabling factors to CCA. The starting assumption is that the score means of respondents regarding the perceived barriers to CCA and perceived enabling factors to CCA could vary according to hotel attributes (star rating, ownership, hotel size, hotel location, hotel age and business format) (or/and) participant's background (level of education, age, experience, nationality and length of residence in SHV) (e.g. Su et al., 2013; Su, 2014).

In order to explore the underlying dimension of hotel attributes and participants' backgrounds on the mean scores of perceived barriers to CCA and enabling factors for CCA, descriptive statistics and independent sample tests have been used to determine whether there is statistical evidence that these characteristics influence the participants' 
perceptions towards the rating of the barriers and enabling factors for CCA. To undertake ANOVA tests, it is expected that the dependent variable is normally distributed and there is approximately equal variance on the scores across groups, which is not the case with the collected data. Since the dependent variables of this study are ordinals, but not normally distributed to conduct one-way ANOVA tests, nonparametric (distribution free) tests are used to compare the means of the respondents. The Mann Whitney U test (MW test) was used to compare the means of the respondents' perceived barriers to CCA and enabling factors for $\mathrm{CCA}$ and determine any significant differences between two independent groups. In the case of more than two independent groups, the Kruskal Wallis test (K-W test) was conducted for the assessment of any significant differences between the means of the respondents' perceived barriers to and enabling factors for CCA. The data were processed with the statistical package SPSS version 23 .

\subsection{Validity and Reliability}

Validity and reliability are closely related to the quality of the research (Yin, 2003). Yin (2014) provides four tests that have been commonly employed to establish the quality of any empirical social research. The tests have been summarised as follows:

Construct validity: identifying the right operational measures for the concepts being researched (Yin, 2003). Yin (2014) and Dubé and Paré (2003) make these recommendations for achieving construct validity in postpositivist case study research:

- use multiple sources of evidence (also called triangulation)

- establish a chain of evidence (e.g. use a research protocol)

- have key informants review draft case study reports.

In line with Dubé and Paré's (2003) recommendations, in this study 'triangulation' is obtained by using multiple sources of evidence as outlined in section 6.4 (Data Collection). Establishing a 'chain of evidence' that is commonly used, is done with case studies: documentation, archival records, interviews, direct observations, participation observations and physical artefacts (Yin, 2003, 2014). Key informants were requested to review transcribed interviews to confirm the validity.

Internal validity: (only applicable for explanatory or causal studies). This is to look for 
establishing a causal relationship, whereby specific conditions are said to lead other conditions, as distinguished from spurious relationships. Firstly, Yin (2003, 2014) explains that internal validity is significantly concerned with the explanatory case studies, especially when a researcher attempts to explain how and why event $x$ led to event $y$. In this study, in order to investigate that relationship, an analysis of the participant interviews was undertaken to identify specific statements that link the cause and effect (causes of climate change and effect on the coastal tourism and hotels).

External validity: outlining the domain to which a study's findings can be generalised. The presentation of the findings allows the audiences to assess their potential applicability to other contexts or similar situations (Miles \& Huberman, 1994; Miles et al., 2013). There is a way to ensure the generalisability in case studies as suggested by Yin (2003, 2014): the use of the theory of single-case studies. By having a precise explanation of the research context and explanation of the data analysis process, audiences can decide about the applicability (external validity) of the findings to other similar settings.

Hyde et al. (2012) provide three forms of generalisation that apply in case-based research:

- Context generalisation: That is generalisation across observations within a case that includes observations (and touch points) for multiple contexts and time periods.

- Generalising from a single case to alternative theories: Case study research is theoretically insights (also see Campbell, 1975).

- Generalising across cases: This was detailed in section 4.9 (Data Analysis).

Reliability: proving that the operations of the study - such as the data collection mechanisms - can be repeated with the same results (Yin, 2003, 2014). To ensure the reliability, Paré (2004) argues that other researchers could repeat the findings of a study within the same or similar results. According to Yin $(2003,2014)$, using a case study protocol and a data repository to ensure reliability are the major ways of increasing the reliability. A case study protocol should consist of the following sections:

- an overview of the case study (project objectives, case study issues and relevant literature)

- field procedures (presentation of credentials, access to the study site as the case 
study, and sources of data)

- case study questions (the specific questions being investigated and kept in mind during data collection, and the expected sources of information to answer each question)

- the guide for the case study report (outline or format for the data and referencing information).

Dubé and Paré (2003) explain that a data repository is composed of the raw data such as interview transcripts, researcher's field notes, documents collected during data collection, survey material, coded data, coding scheme, memos and other analytic material, and data displays.

To ensure the reliability of the data repository in this study, this chapter has provided details of the Research Process (Figure 4.1). It defined protocols for data collection and how transcripts were verified with a range of participants. The data analysis was explained using the analytical framework (Figure 4.2) and coding was double-checked and obtained high reliability. A data repository was maintained throughout the research process as an electronic database. All records related to particular informants (the hotels) were with provided codes/labels with a unique identifying code and stored in a structured file system for convenient access.

The following tables contain attributes showing how this study's reliability is addressed in postpositivist case studies. These attributes are adapted from Dubé and Paré's (2003) practices. Taking the study aims into consideration, only relevant attributes are included. Table 4.5 shows how rigour was achieved in Research Design. Table 4.6 shows how rigour was achieved in Data Collection. Table 4.7 shows how rigour was achieved in Data Analysis. Yin's $(2003,2014)$ recommendations for obtaining the validity are also added in the tables. 
Table 4. 6: Rigour in the design phase, adapted from Dubé and Paré (2003), following the styles of Strode (2012) and Abeysekara (2017)

\begin{tabular}{|c|c|c|}
\hline Attributes & Purpose & How this attribute was met in this study \\
\hline $\begin{array}{l}\text { Clear research } \\
\text { questions }\end{array}$ & $\begin{array}{l}\text { - Provide a particular focus } \\
\text { for the study and indicate } \\
\text { where the practical and } \\
\text { theoretical contributions are } \\
\text { likely. } \\
\text { - Address reliability (Miles } \\
\text { \& Huberman, 1994). }\end{array}$ & $\begin{array}{l}\text { - There are five sequential research } \\
\text { aims/questions. }\end{array}$ \\
\hline $\begin{array}{l}\text { A priori } \\
\text { specification of } \\
\text { constructs }\end{array}$ & $\begin{array}{l}\text { - Assist in the initial research } \\
\text { design by providing initial } \\
\text { concepts of interest. }\end{array}$ & $\begin{array}{l}\text { An initial conceptual framework was } \\
\text { developed based on the perception of } \\
\text { environmental and socio-economic } \\
\text { vulnerability and existing scientific data, the } \\
\text { adaptation measures (including the barriers } \\
\text { and enabling factors) in relations to the } \\
\text { coastal hotel sector. }\end{array}$ \\
\hline $\begin{array}{l}\text { Clean theoretical } \\
\text { slate }\end{array}$ & $\begin{array}{l}\text { - Reduces bias in the study } \\
\text { analysis. } \\
\text { - Reduces the chance of the } \\
\text { researcher placing } \\
\text { limitations on the findings. }\end{array}$ & $\begin{array}{l}\text { - Sufficient sample size was selected to } \\
\text { conclude a valid research result coupled with } \\
\text { using statistical test. } \\
\text { - Previous research on the topic was } \\
\text { extensively reviewed. This provides } \\
\text { foundation to reduce limitations of the } \\
\text { findings. }\end{array}$ \\
\hline $\begin{array}{l}\text { Nature of single- } \\
\text { case design }\end{array}$ & $\begin{array}{l}\text { Provides specific instances } \\
\text { such as being critical, } \\
\text { unique and revelatory (Yin, } \\
\text { 1994). }\end{array}$ & $\begin{array}{l}\text { Qualitative and quantitative single-case was } \\
\text { designed to have critical and unique } \\
\text { investigations on the climate environmental } \\
\text { and socio-economic vulnerability, and } \\
\text { revelatory findings of the adaptation } \\
\text { measures (including the barriers and enabling } \\
\text { factors) in relations to the coastal hotel sector }\end{array}$ \\
\hline $\begin{array}{l}\text { Unit of analysis } \\
\text { (UoA) }\end{array}$ & $\begin{array}{l}\text { - Relates the case to a } \\
\text { broader body of knowledge. } \\
\text { - Sets a boundary on both the } \\
\text { data to collect and the } \\
\text { applicability of research } \\
\text { conclusions. } \\
\end{array}$ & $\begin{array}{l}\text { - UoA is CCA actions of the coastal hotels } \\
\text { representing similar types of other } \\
\text { accommodations. } \\
\text { - Applicability of the framework is at the } \\
\text { similar level of the coastal hotel sector. }\end{array}$ \\
\hline Pilot case & $\begin{array}{l}\text { - A case selected to } \\
\text { determine the } \\
\text { appropriateness of the UoA } \\
\text { and refine the case protocol } \\
\text { and any instruments used. }\end{array}$ & $\begin{array}{l}\text { The pilot tests were used to trial the case } \\
\text { study protocol and interview schedule which } \\
\text { made modifications to the interview schedule } \\
\text { by adjusting the questions. }\end{array}$ \\
\hline $\begin{array}{l}\text { Context of the } \\
\text { case study }\end{array}$ & $\begin{array}{l}\text { - Clarifies the context in } \\
\text { which the new theory is } \\
\text { applicable. } \\
\text { - Improves case creditability. }\end{array}$ & $\begin{array}{l}\text { - The case is described in full using an initial } \\
\text { and revised conceptual framework which } \\
\text { serve as base for introducing new theories. }\end{array}$ \\
\hline
\end{tabular}


Table 4. 7: Rigour in the data collection phase, adapted from Dubé and Paré (2003), following the styles of Strode (2012) and Abeysekara (2017)

\begin{tabular}{|c|c|c|}
\hline Attributes & Purpose & $\begin{array}{l}\text { How this attribute was met in this } \\
\text { study }\end{array}$ \\
\hline $\begin{array}{l}\text { Elucidation of the } \\
\text { data collection } \\
\text { process }\end{array}$ & $\begin{array}{l}\text { - Ensures external parties can } \\
\text { understand how data were } \\
\text { accumulated, what sources } \\
\text { were used and why, and } \\
\text { how each source } \\
\text { contributed to the findings } \\
\text { - Addresses reliability (Miles } \\
\text { \& Huberman, 1994) by } \\
\text { ensuring another person } \\
\text { could follow the same } \\
\text { procedures and arrive at the } \\
\text { same conclusions. }\end{array}$ & $\begin{array}{l}\text { The methodology chapter fully } \\
\text { describes the data sources, data } \\
\text { collection procedures, and how } \\
\text { each source contributed to the } \\
\text { findings (also see Figure } 4.1 \\
\text { Research Process). }\end{array}$ \\
\hline $\begin{array}{l}\text { Multiple data } \\
\text { collection } \\
\text { methods }\end{array}$ & $\begin{array}{l}\text { - Enable convergence of } \\
\text { evidence by providing more } \\
\text { than one source of evidence } \\
\text { for a phenomenon. } \\
\text { - Address construct validity } \\
\text { (Y in, 2003, 2014). }\end{array}$ & $\begin{array}{l}\text { Multiple data sources include: in- } \\
\text { depth interviews, observation with } \\
\text { field notes, photographs \& videos, } \\
\text { organizational websites, and } \\
\text { secondary data from government } \\
\text { authorities. }\end{array}$ \\
\hline Data triangulation & $\begin{array}{l}\text { - Strengthens evidence for } \\
\text { findings as they are based } \\
\text { on more than one sources of } \\
\text { evidences. } \\
\text { - Addresses construct validity } \\
\text { (Y in, 2003, 2014). }\end{array}$ & $\begin{array}{l}\text { Whenever possible evidences from } \\
\text { more than one sources are used to } \\
\text { confirm the findings. }\end{array}$ \\
\hline $\begin{array}{l}\text { Case study } \\
\text { protocol }\end{array}$ & $\begin{array}{l}\text { Ensures external parties can } \\
\text { understand what procedures } \\
\text { were followed, how data } \\
\text { were accumulated, what } \\
\text { sources were used and why, } \\
\text { and how each source } \\
\text { contributed to the findings. } \\
\text { - Contributes to both validity } \\
\text { and reliability of the study } \\
\text { (Y in, 2003, 2014). }\end{array}$ & $\begin{array}{l}\text { - The case protocol includes these } \\
\text { documents: } \\
\circ \text { Participant Information Sheet } \\
\circ \text { Participant Consent Form } \\
\circ \text { Interview schedule for the } \\
\text { owner-manager } \\
\text { - Detailed explanation of the research } \\
\text { process (Figure } 4.1 \text { Research } \\
\text { Process). }\end{array}$ \\
\hline $\begin{array}{l}\text { Case study } \\
\text { database }\end{array}$ & $\begin{array}{l}\text { - Contributes to the reliability } \\
\text { of a study as findings can be } \\
\text { traced to original data } \\
\text { sources (Y in, 2003, 2014). }\end{array}$ & $\begin{array}{l}\text { Data pertaining to each case is } \\
\text { stored electronically in an easily } \\
\text { searchable form and the hard copies } \\
\text { of documents given to the } \\
\text { researcher were filed manually for } \\
\text { individual cases (promotional } \\
\text { flyers, name cards etc.). }\end{array}$ \\
\hline
\end{tabular}


Table 4. 8: Rigour in the data analysis phase, adapted from Dubé and Paré (2003),

following the styles of Strode (2012) and Abeysekara (2017)

\begin{tabular}{|c|c|c|}
\hline Attributes & Purpose & How this attribute was met in this study \\
\hline $\begin{array}{l}\text { Elucidation of the } \\
\text { data analysis } \\
\text { process }\end{array}$ & $\begin{array}{l}\text { - Contributes to an auditable } \\
\text { trail of evidence from data to } \\
\text { research conclusions; } \\
\text { improves reliability and } \\
\text { reduces bias. }\end{array}$ & $\begin{array}{l}\text { - The data analysis process is fully } \\
\text { described with the analytical framework } \\
\text { (Figure 4.2). }\end{array}$ \\
\hline Field notes & $\begin{array}{l}\text { Provide evidence to confirm } \\
\text { or extend interview data. }\end{array}$ & $\begin{array}{l}\text { - Notes and sketches on climate } \\
\text { vulnerabilities and CCA actions were } \\
\text { made during interviews. } \\
\text { - Observations of expressions to emphasize } \\
\text { some facts were written down during or } \\
\text { shortly after the interviews. }\end{array}$ \\
\hline $\begin{array}{l}\text { Coding and } \\
\text { reliability check }\end{array}$ & $\begin{array}{l}\text { - Contribute to an auditable } \\
\text { trail of evidence from data to } \\
\text { final theory; improve } \\
\text { reliability and reduces bias. }\end{array}$ & $\begin{array}{l}\text { - Analytical codes were used in the NVivo } \\
\text { application to categorize source data into } \\
\text { concepts. } \\
\text { - A reliability check was performed using } \\
\text { proof-reader. } \\
\text { - Translations and transcripts were verified. }\end{array}$ \\
\hline Data displays & $\begin{array}{l}\text { - Contribute to an auditable } \\
\text { trail of evidence from data to } \\
\text { final theory; improves } \\
\text { reliability. }\end{array}$ & $\begin{array}{l}\text { - Tables were used to display quantified } \\
\text { data out of the interview participants. } \\
\text { - Several charts and graphs were created to } \\
\text { support the analysis of single case and } \\
\text { subsequently across cases. }\end{array}$ \\
\hline $\begin{array}{l}\text { Logical chain of } \\
\text { evidence }\end{array}$ & $\begin{array}{l}\text { - Improves reliability of } \\
\text { information presented. } \\
\text { - Addresses construct validity } \\
\text { (Y in, 2003, 2014). }\end{array}$ & $\begin{array}{l}\text { - Interviews are transcribed and } \\
\text { organization ID codes attached to quotes } \\
\text { so findings can be traced to their sources. } \\
\text { - All written materials are kept in a } \\
\text { research database and ID codes identify } \\
\text { the case. } \\
\text { - Participants' claims were verified against } \\
\text { statistical evidences from government } \\
\text { authorities and IOs \& NGOs where } \\
\text { possible. }\end{array}$ \\
\hline $\begin{array}{l}\text { Flexible and } \\
\text { opportunistic } \\
\text { process }\end{array}$ & $\begin{array}{l}\text { - Allow for emerging concepts } \\
\text { and relationships to be } \\
\text { further investigated either } \\
\text { with new cases or with } \\
\text { further evidence from a } \\
\text { single case. }\end{array}$ & $\begin{array}{l}\text { Project documents were collected, } \\
\text { photographs taken, and participant } \\
\text { observation was carried out when the } \\
\text { opportunity arose (for on-going projects). }\end{array}$ \\
\hline $\begin{array}{l}\text { Mode of analysis/ } \\
\text { Explanation } \\
\text { building }\end{array}$ & $\begin{array}{l}\text { - How data is analysed and } \\
\text { interpreted is described } \\
\text { - Be more important in the } \\
\text { context of exploratory } \\
\text { studies. }\end{array}$ & $\begin{array}{l}\text { - The method of explanation building from } \\
\text { pattern-matching and textual explanation } \\
\text { was carried out relying on the propositions } \\
\text { of the initial/final conceptual framework } \\
\text { developed for the study. }\end{array}$ \\
\hline $\begin{array}{l}\text { Searching for } \\
\text { cross-case } \\
\text { patterns }\end{array}$ & $\begin{array}{l}\text { - Searches for similarities and } \\
\text { differences between cases by } \\
\text { selecting categories or } \\
\text { dimensions for comparison. } \\
\text { - Addresses internal validity } \\
\text { (Y in, 2003, 2014). }\end{array}$ & $\begin{array}{l}\text { - Cross-case analysis is used to compare } \\
\text { the findings from each participant (which } \\
\text { is justified as the basis for clustering). }\end{array}$ \\
\hline
\end{tabular}




\begin{tabular}{|c|c|c|}
\hline Quotes (evidence) & $\begin{array}{l}\text { Provides evidence for the } \\
\text { development of theore tical } \\
\text { concepts and their } \\
\text { relationships. }\end{array}$ & $\begin{array}{l}\text { - Quotes are used throughout the overall } \\
\text { case, and across-case analysis to support } \\
\text { findings. } \\
\text { - Contribution to theoretical concept was } \\
\text { presented. }\end{array}$ \\
\hline Project reviews & $\begin{array}{l}\text { - Validate factual information } \\
\text { and improves reliability. } \\
\text { - Address construct validity. }\end{array}$ & $\begin{array}{l}\text { - For each case, the interview transcript was } \\
\text { thoroughly checked to verify its accuracy. }\end{array}$ \\
\hline $\begin{array}{l}\text { Comparison with } \\
\text { extant literature }\end{array}$ & $\begin{array}{l}\text { - Contributes to internal } \\
\text { validity. } \\
\text { - Strengthens the findings of a } \\
\text { study because other studies } \\
\text { either support the findings, or } \\
\text { do not support the findings } \\
\text { indicating the theory that } \\
\text { explains more than previous } \\
\text { theories. }\end{array}$ & $\begin{array}{l}\text { - Extensive discussion was presented to } \\
\text { indicate whether other studies align or do } \\
\text { not align with the findings. } \\
\text { - Findings from extant research studies } \\
\text { might be used to extend the theory in } \\
\text { conclusion chapter. }\end{array}$ \\
\hline
\end{tabular}

\subsection{Ethical Considerations}

Ethics play an essential role in this thesis. Victoria University of Wellington requires that studies concerning human subjects must obtain approval by the human ethics approval process. Prior to data collection, the research received approval by the Victoria University Pipitea Human Ethics Committee (HEC) (see Appendix E). Upon approval, all sections stipulated in HEC request were respected. Pseudonyms were used in the thesis. Names were confidential, but other identifying characteristics were gathered and discussed, such as for the hotel: star rating, ownership, business size, hotel location, business age and business format (owned vs. leased), and for the participants: education, experience, nationality, length of residence and age group.

\subsection{Limitations of the study}

This research might be affected by some limitations often originating from the time and financial restraints. Firstly, as the study sought to interview various participants from different backgrounds, there was no absolute guarantee that those who participated answered the questions accurately, especially the perceived level of climate change vulnerability on SHV's CT and their hotels. It was challenging to measure the reliability of their answers and the way they understood and interpreted the vulnerability to climate change. Secondly, while the focus was on one coastal tourist destination, the findings of this research are not necessarily generalised to other non-coastal regions. The restriction in location affects the overall sample in a different way, as it is not clear whether hotel businesses in non-coastal destinations face the same climate change vulnerabilities. 
Finally, the study recruited the representatives of only a sub-sector (hotel) in the tourism. It was impossible to study all tourism stakeholders such as guesthouses, travel agencies and tourism transport companies. Furthermore, the two main stakeholder groups such as the government (national and local) and IOs and NGOs were not recruited; or interviews with only the hotel sector not giving full understanding of climate change-related issues as this requires perceptions of relevant stakeholders. Meanwhile, the hotel sector's adaptation is a cross-cutting issue that is closely relevant to other stakeholders. CCA, especially barriers and enabling factors, requires more comprehensive data to confirm as it is influenced by other stakeholders as well, especially the government. For example, strong infrastructures built by the government contribute to be the enabling factor of hotels' adaptations (e.g. in terms of storms) while weak infrastructures may contribute to barriers to hotels' adaptations.

\subsection{Chapter summary}

This chapter covers the research methodology that was used to gather data and subsequently addresses the research aims/questions. The research paradigm adopted in this study is the post-positivism. The paradigm first reflects the reality of the research nature. Second, it is concerned with 'social concern' and 'natural science'. Third, it is supportive to the research that employs more than one method using single case study. The methodological approach used in this research is mixed method and single-case study with several benefits, which allows researchers to have flexibility in data collection, gather 'rich' data, and explain the complex phenomenon. These are reflected in a number of studies in climate change and tourism using the case studies given as an example. The detailed descriptions of the data collection start with the preparation stage, which was detailed with a diagram to indicate each phase of the research. Prior to data analysis, template coding, creating the initial template, revising the template, finalising the template and interpreting the template are required for the research. The data analysis was indicated in the analytical framework with detailed steps. Guided by the analytical framework, the overall analysis and subsequently cross-case analysis using descriptive test and non-parametric test to explore the extent to which hotel attributes and participants' backgrounds in the hotel sector (in SHV) have impacted on their perceptions of barriers and enabling factors to CCA. Ethical considerations and the concurrence for 
data collection were clearly outlined and respected. Ultimately, the study limitations were highlighted. 


\section{CHAPTER 5: VULNERABILITY AND ADAPTATION}

\section{$5.1 \quad$ Introduction}

This chapter develops the relationship between vulnerability and adaptation. Guided by the analytical framework (see the red colour block of Figure 5.1), this chapter first assesses the vulnerability of SHV's coastal tourism and its hotel sector and then determines whether and how the hotel sector adapts to climate change impacts. The vulnerability is assessed in two layers: SHV's coastal tourism (destination level) and its hotel sector (sector level). This is done by examining respondents' perceptions about environmental and socio-economic vulnerabilities, supported by existing scientific data. This assessment was guided by key studies (e.g. Grasso, Moneo, \& Arena, 2014; Jopp, DeLacy, \& Mair, 2010; MOE GEF, \& UNEP, 2015; Moreno \& Becken, 2009; Sharma et al., 2013) who recommended that (coastal) tourism should be assessed through the environmental and socio-economic vulnerabilities indicated above. After identifying vulnerability, the second part provides insights into hotels' adaptations, including technical, managerial, policy, research and education adopted from an adaptation framework by WMO, UNEP, \& WTO (2008) and Simpson et al. (2008). The purpose of this chapter then is to identify the vulnerability following the coastal hotels' adaptation actions. The chapter answers research aim 1,2 and 3. 
Figure 5. 1: Analytical framework

PHASE 1: OVERALL CASE ANALYSIS
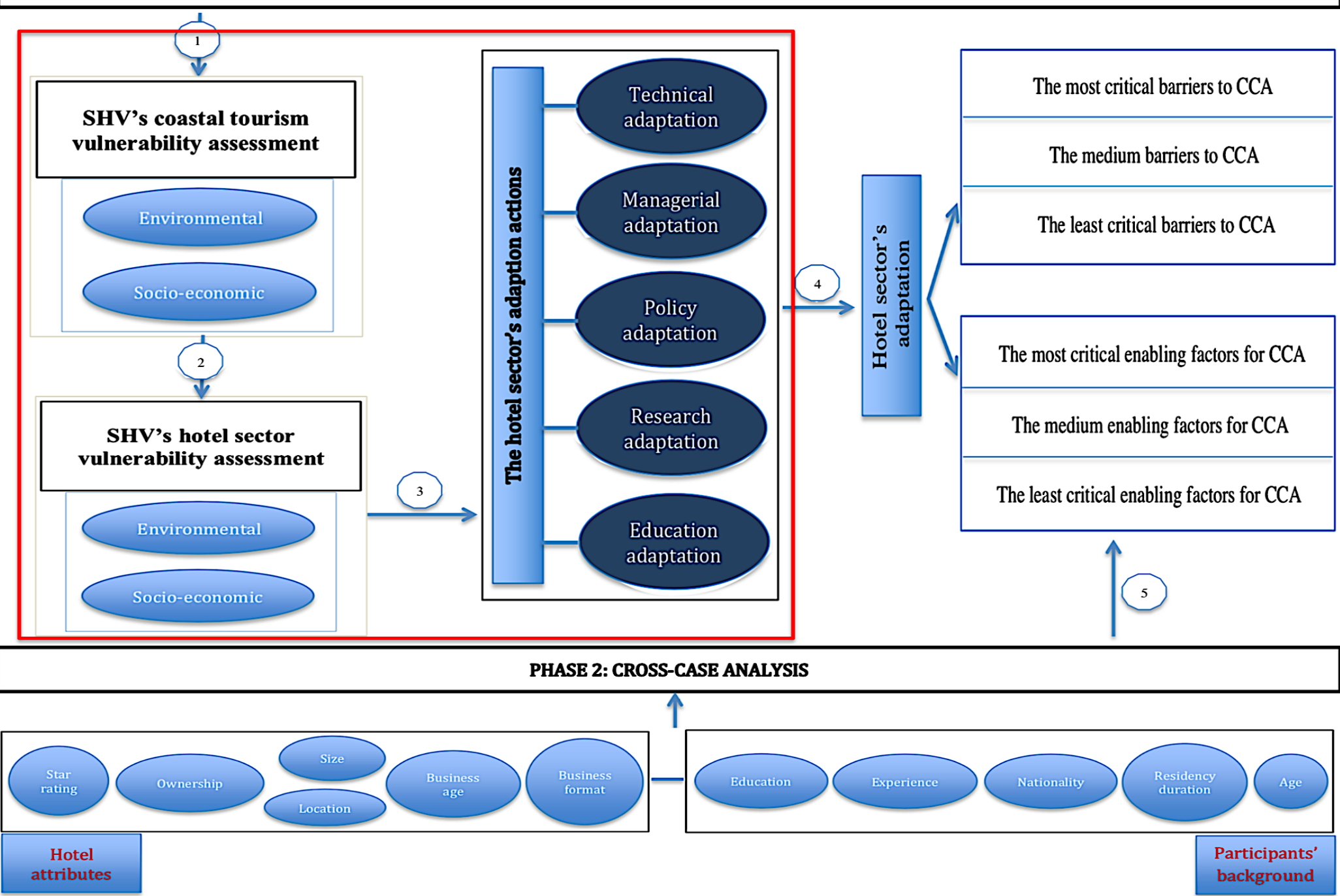


\subsection{Vulnerability assessment of Sihanoukville's coastal tourism}

Guided by the literature outlined in chapters 2 and 3, the interview guide was designed for the participants to assess vulnerability in terms of environmental and socio-economic dimensions. The findings in Table 5.1 below show that SHV's coastal tourism was environmentally vulnerable to excessive rainfall/rainfall-induced flooding, storm, rising temperature, SLR/beach erosion/storm surge and prolonged drought, and socioeconomically vulnerable due to damage of infrastructures (from excessive rainfall and storm/storm surges), spread of diseases (from the increased temperature and exposure to rain), and economic pressure (e.g. income loss).

Table 5. 1: Vulnerability assessment on SHV's coastal tourism

\begin{tabular}{|l|l|}
\hline Vulnerability factors & \multicolumn{1}{c|}{$\begin{array}{c}\text { Findings of climate change vulnerability on SHV's } \\
\text { coastal tourism }\end{array}$} \\
\hline $\begin{array}{l}\text { Environmental } \\
\text { vulnerability }\end{array}$ & $\begin{array}{l}\text { Excessive rainfall/rainfall-induced flooding } \\
\text { - Storm }\end{array}$ \\
& - Rising temperature \\
& - SLR/beach erosion/storm surges \\
\hline $\begin{array}{l}\text { Socio-economic } \\
\text { vulnerability }\end{array}$ & $\begin{array}{l}\text { Damages of infrastructures (from excessive rainfall } \\
\text { and storm/storm surges) (e.g. roads, beaches) }\end{array}$ \\
& $\begin{array}{l}\text { Widespread of diseases (resulted from the increased } \\
\text { temperature and exposure to rain) and }\end{array}$ \\
& - Economic pressure (e.g. income loss) \\
\hline
\end{tabular}

\subsubsection{Environmental vulnerability}

The findings indicate that environmental vulnerabilities such as the excessive rainfall and rainfall-induced flooding, storms, rising temperature, SLR/beach erosion/storm surges, and prolonged drought have threatened SHV's coastal tourism. The next section will outline the first environmental vulnerability, which is the excessive rainfall and rainfallinduced flooding. All the remaining of the environmental vulnerabilities will also be presented subsequently.

\subsubsection{Environmental vulnerability to excessive rainfall and rainfall-induced flooding}

According to the participants, the excessive rainfall and rainfall-induced flooding has threatened SHV's coastal tourism. The heavy rainfall has become a predominant 
environmental effect on SHV and its tourism. Due to its location at the coastal zone, SHV has increasingly become a rain-fed province. The participants further reported that during the rainy season, the most intensive rainfall is around September when rain consecutively falls up to two weeks or one month. This has sometimes extended to a longer period during the year into a normally dry season. They called it irregular or unseasonal rainfalls. One added, "I think it's massively changing. First of all, it feels like when it's rainy season, it continues it doesn't stop so it's getting later, later and later, still raining." H39.

They continued to say that the increased rainfall poses problems not only to the businesses but also the people and tourists, especially in July, August and September when rainfall is heavy. The most vulnerable places are low-lying areas, including beaches that are submerged during and after heavy rains. During the excessive rainfalls, the participants complained that half of the tourists/guests do not come to SHV because rainfall limits their travels. Flows of tourists' travels are being disturbed. Tourists were struggling to visit some places in SHV. The sea is not attractive for tourists while it is in rainy season with strong winds. The more it rains, the more disturbances there are for the tourists.

Rainfall-induced flooding was reported as being caused by excessive rainfall. Although the heavy rainfall is one of the main reasons for flooding, the causes of flooding can be exacerbated by rubbish that blocks the public drainage systems, which are already poorly designed. This puts additional pressures on weak capacity infrastructures such as road damages. It reflects Cambodia society where environmental education remains low. This is why rubbish disposal remains an issue for most Cambodians. Since there are no sufficient drainages for water to flow, sea, beaches and other low-lying area receive enormous amounts rainwater, which causes flash flooding along the beaches. This environmental problem is caused by both climate change and the existing weak capacity of SHV.

As stated in section 3.2.3.1 and section 3.3.2.1, based on the scientific data and its prediction both nationally and regionally (at the coastal zone), SHV's annual rainfall has increased and will most likely increase further, and SHV remains the leading place to receive rainfall nationwide. 


\subsubsection{Environmental vulnerability to storm}

The informants indicated storm or strong wind as another form of environmental vulnerability. They felt that this vulnerability is getting stronger, especially when it rains; it is more unpredictable. One respondent added, "Not only me but also everyone knows that SHV is vulnerable to storms especially once it rains." H12. Storm intensities vary from year to year and place to place. For example, one participant explained, "This year there is likely no storm but there was last year." H13. In their view, storm caused three main effects. First, it affects tourism because it disrupts touristic activities, especially at the islands. Second, storm is thought to be contributing to cause of death such as drowning, especially for tourists. Third, it poses challenges for fishermen who collect marine resources significant for hotels' restaurants and domestic tourists' demands.

First, storm is the disruption of touristic activities, especially at the islands. According to the participants, the mainland of SHV had been the main tourist site for years, but recently the island destinations have gained popularity and attracted more tourists; more and more tourists visit there. Yet climate change appears to be a constraint for the island visitors, especially during storm surges. The storms threaten tourists who go back and forth. For example, during storm surges, the island visitors have no choice but force themselves to stay there for several more nights. It was reported that a tourist boat capsized in heavy storms. Yet in their view, there were two possible main causes: the overload and storm surges. Because tourists either cannot go to the island or are unable to walk or travel out to the mainland, they must reduce their stays and change the destinations of visitation. Galas or party dinners at the beaches were also interrupted. Storms damaging public and private infrastructures were also reported.

Second, drowning incidents at sea were reported and storm surge was thought to be a contributing factor. The highest height of wave mentioned by an interviewee was 3 metres during the strongest wind. While some participants quoted local news, some directly informed that at least one death caused by drowning is reported annually. Beach erosion/storm surges have changed the beaches; the waves wash ashore the beaches and made the drop more sudden. The participants continued to say that most tourists, both foreign and local, did not come to SHV during storm surges. 
Third, storms pose challenges for fishermen. The participants further commented that the fisherman groups who like going fishing at night (due to less competition and collect more marine resources at nights) are also vulnerable to storms. Although this is not a direct effect on tourism, the incidents can put pressure on the tourism and hotel sector due to seafood shortages if the fishermen could not collect sufficient food due to the storms.

As described in sections 3.2.3.2 and 3.3.2.2, storm surge is a real concern in SHV. Storms occur almost every year from mid-October and through December. The occurrences of strong wind are defined as being higher than $40 \mathrm{kph}$, and it is projected that it will become more intense in the next 30 years.

\subsubsection{Environmental vulnerability to increased temperature}

Results of the interviews also suggested that the increased temperature also affects SHV's coastal tourism. Some SHV natives explained that the climate was getting hotter and hotter compared to when they were young, particularly in the dry season. Others said that the rising temperature causing bothersome and scruffy environments in SHV. One participant asserted that the hottest month is April and the highest recorded temperature was 40 degrees. This participant said, "Last year in SHV, from December to March, April, it should rain at least 1-2 times but last year there were no rains. This is strange, this made the climate hot. We brought temperature measurement and saw that the weather became $40^{\circ} \mathrm{C}$ so it was quite hot." $\mathrm{H} 14$.

In their views, rising temperature caused 'heat stress', especially for local and international Asian tourists. One added that the weather was too hot, leading to a loss of some tourist activities. For instance, one respondent said, "It is being affected ... first before the European tourists who like sunbathing, they came and stayed at the pool exposing their bodies under the sunshine. Now this activity has been lost. Now they just come and play in the pool then after drying themselves at the pool, they come back to the rooms. They don't have sunbathing like before. At the beach, there is similar trend." $\mathrm{H} 37$. Due to hot weather, people as well as tourists over-consumed electricity. Due to incapable supplies from the state, the power/electricity is usually cut off during the hottest season. One participant viewed that outside temperature comes to a point where it gets too hot for the air-conditioners (ACs) to function anymore. Rising temperature also threatens 
water catchments and agriculture. This also puts pressure on tourism businesses, particularly the hotel sector.

As stated in sections 3.2.3.3 and 3.3.2.3, the annual mean of temperature in Cambodia and its coastal zone has increased since 1960 and this trend is expected to continue in the future.

\subsubsection{Environmental vulnerability to SLR/beach erosion/storm surge}

They believed that SHV's coastal tourism is under threat due to SLR/beach erosion/storm surge. It has been a long-term concern that the water tends to rise, and it will have significant effects on SHV because they are aware it through media ${ }^{27}$, which has indicated that the water at the Cambodian coast could rise by 1 metre. Reacting to the news, one participant iterated, "I heard of Im SLR in here (SHV). Yes, I wouldn't be surprised since it's about right to me. We can tell the sea has already changed in 5 years, quite a lot." $\mathrm{H} 28$.

Some SHV natives did a comparison. They compared the current size of the beaches to the far past and reported that the present beaches are shrinking. One participant explained, "What's happening is we have two different currents in the gulf of Thailand. At some stage in some parts of the year the water takes the sands away. Now it brings the sand back again but there is no planning from the government to control it." H10. The islands follow the same trend. They believed that the seawater is seen as rising up slowly. At some stages, the sands have been washed away. The rising seawater causes beach losses at seashore areas and other nearby areas along the coast. These climate change problems would affect SHV visitors, as well as the hotels and people locating and dwelling near the beaches. Ultimately, SHV's coastal tourism mainly depends on pristine beaches while those beaches are being strongly affected by climate change.

However, climate change may not be the only cause of beach erosion. The interviewees explained that beaches are shorter because on one hand the seawater is rising. On the other hand, new hoteliers have encroached the beach areas with new constructions. They

\footnotetext{
${ }^{27272727}$ Media continues to release information about SLR in the country. Available at: https://www.khmertimeskh.com/50114119/world-bank-says-cambodia-vulnerable-to-rising-sea-levels/, accessed on March 162018
} 
constructed the building as close as 5 metres from the high tide line. Meanwhile, sand dredging is an issue. This is in line with the field observation. Sand dredging ships could be seen offshore. One interviewee explained that for ship navigation, the navigational channel should be as deep as 9.5 metres while others said the sand is dredged and commercially sold to Singapore.

\subsubsection{Environmental vulnerability to prolonged drought}

Interviews revealed that prolonged drought endangers the development of SHV's coastal tourism. Influenced by tropical monsoon, Cambodia/SHV has two seasons: rainy season and dry season. However, they thought this has changed. It has become hotter and drought is extending. Under this threat, there were frequent water disconnections in tourism businesses (due to water evaporation). For businesses, the most vulnerable period is April-May. One respondent asserted, "Water at Kbal Chhay (a reservoir lake) is in shortage in dry seasons sometimes. The water is disconnected at households due to the insufficient supplies. The water is mainly connected from there to tourist areas; other less priority areas face water shortages; it takes 1-2 days to get the water connected." $\mathrm{H} 22$. They perceived that the droughts have been more severe. While travelling to many islands, the researcher observed that the island areas followed the same trend.

Although water shortage resulting from climate change is very common during the interviews, non-climatic problems such as population growth (people, business, tourists, etc.) are other reasons. At the same time, water mismanagement by the government/authority exacerbates the problem. Unregulated developments such as filling up lakes and streams for land encroachment by the elite have been happening. These uncontrollable actions combined with climate change effects may cause water deficit.

As outlined in sections 3.2.3.5 and 3.3.2.5, existing reports have suggested that being a predominantly agrarian country, Cambodia is vulnerable to floods and droughts. The impact of drought is severe in some regions in the country. SHV's households have a high sensitivity to climate change impacts, including drought, because over $80 \%$ of its households have limited access to safe drinking water. The tourism sector is also affected by this limitation. 


\subsubsection{Socio-economic vulnerabilities}

The findings indicate that socio-economic vulnerabilities such as damage of infrastructures, spread of diseases and economic pressure (see Table 5.1) have threatened SHV's coastal tourism. The next section will outline the first socio-economic vulnerability, which is the damage of infrastructures. All the remaining socio-economic vulnerabilities will also be presented subsequently.

\subsubsection{Socio-economic vulnerability due to damage of infrastructures (e.g. roads and beaches)}

The interviews indicated that excessive rainfalls (causing flash floods) and storms contribute to the damage of infrastructures in SHV. The damage includes roads, bridges, households and beach erosion as well as crops, making food supply insufficient. The first said, "SLR and flash flood effects crops, making the crops grow slowly. I've known this from a workshop." The second continued with: "Effects on tourism and hotels, yes it affects stronger on coastal areas because when the water rises, we could lose the beaches, natural sands as well as at the high land areas and the nearby areas along the coast. If the prediction of 1-metre SLR (in 2050) is correct, the businesses along the coast will face difficulty and disappear." H4 and H6. Roads and bridges of this province need more permanent repair and maintenance than other provinces. At the islands, a similar trend can be observed (through site observations). In addition, most island hotel participants mentioned that beach erosion, especially during the high tides, has occurred. The rainfallinduced flooding has overwhelmingly contributed to those damages.

Storm was also reported as affecting the economy of SHV's coastal tourism, both directly and indirectly. The respondents identified that its direct impacts include the damage to the businesses along the beaches, including the hotel structures and residents' houses. In some worst cases, the winds blew houses apart while the hotels were less vulnerable than houses due to their better-constructed buildings. Additionally, some small business structures and trees along the coastline were torn apart. As described in the previous section, section 5.2.2.2, storms have predominantly threatened deep-sea fishing.

The damage poses challenges for the people and tourists. People' livelihoods were interrupted because they could not properly run their businesses. Tourists' travels (within SHV) are also disturbed. In another sequence, the tourists' vacations were also disrupted. 
The damaged roads and bridges as well as heavy rainfalls hampered the tourists' travels. Tourists could not access some hotels where access roads were under lengthy repair and maintenance. Disturbing/annoying noise (from maintenance/repairing) to tourists or the guests was also reported. The limited accessibility to SHV's hotels due to the road maintenance reduced tourists' visitation satisfaction. Meanwhile, repairing and maintaining infrastructures consumed much of the budget of the local government.

SHV's infrastructures have also been weakened by non-climatic factors. The participants criticised that limitation of traffic regulation is contributing to road damages. For example, while roads are limited in number, big trucks are allowed to run over the roads where they should not. Road damages are a combination of the rains and big trucks' heavy pressures. The lack of new roads creates the same outcome. The existing roads are under constant pressure. Another reason is that the current infrastructures are currently weak, especially the drainage systems. One said, "But the thing is the public infrastructures are very weak so like the roads are very weak, so the climate change can damage easily, I think the roads are vulnerable because they are bad anyway.” H30. Others exemplified that they (the government) built the roads but paid little attention to underground structures such as the drainage systems. Roads were built under standard. Fake products (e.g. low-quality asphalt concrete) are assumed to have been used in road constructions.

As indicated in section 3.3.2.6, SHV's vulnerability has been worsened by both climate change impacts and non-climatic factors such as poor infrastructure.

\subsubsection{Socio-economic vulnerability due to economic pressure (e.g. the loss of income)}

The interviewees explained that climate change such as excessive rainfalls, rising temperature, drought, and drowning incidents affected the socio-economy of SHV's coastal tourism, causing a great loss of income for the businesses as well as people.

First, they noted that once rain excessively falls, tourists cancel all their bookings and shift their visitations to other places. One explained, "Commonly, in SHV you can ask those who run hotel and guesthouse business. If there is constant rainfall, you can hear them complain about having no guests." H17. Visitors who plan to go to the islands have also been disturbed; seawater tends to rise once it rains. The participants reported that 
after inconveniently experiencing this, tourists might go back and tell either their TripAdvisor or their friends as well as the travel agencies to stop sending tourists to SHV. The concern is that due to heavy rainfall, the high seasons do not last long enough; this will cause an economic downturn in the tourism businesses, both in the short and long term.

Second, the interviews illustrated that rising temperature has annoyed tourists, especially Asian guests. They became stressed, especially those who had planned to visit for a longer period (up to 3-4 months). In a hot environment, the hoteliers require high-tech equipment or facilities to reduce the heat for their guests. The high-tech equipment is often very expensive, which makes the hotels' expenses soar. In the meantime, as part of their responses to guests, some hoteliers have no choice but use the ACs, which increase overall cost. One hotel owner explained, "Surely, rising temperature will negatively impact our business, absolutely and negatively affects the tourism." $\mathrm{H} 28$. According to the respondents, the increased temperature produced another undesirable consequence, which is the fire incident. In SHV, there been reports of fire incidents almost every year. Some businesses burned down. The scope of the damage varies, depending on their prior preventive measures. One participant informed, "When it is hot, there is fire. When there is an increased temperature, we must take care in maximum. The most dangerous months for fire accidents are March, April and May." H7. Yet it was observed that the causes are many, which could be unorganised electricity cables and the increased temperature. Presumably, very dry vegetation and other nearby trees/forests play a role due to hot and dry weather. WMO, UNEP, and WTO (2008) support that fire incident is an indirect effect of climate change that can have substantial effects on tourism activities and the capacity of firms to do business.

Third, as mentioned in earlier findings, drought affects SHV's coastal tourism. In a broader affect, droughts also caused water shortages for both people's daily usage and rice or crop farming. The lack of irrigation system aggravates the situation. This might suggest that the agricultural products necessary for tourism sector are insufficient. One participant said, "Outside tourism, we can see it affects our family members of our staff and friends here that rely on the agriculture to live during the drought. Here is a familyoriented society, the family-based society. If our staffs have families who are farmers around the village in SHV then, they're all getting hungry and getting poorer so then a 
lot more pressures on our staff to try to support them." $\mathrm{H} 28$. To the best of the researcher's knowledge, SHV's coastal tourism has become more vulnerable in a situation where most SHV's land is left unfarmed. Most food, such as meat, fruit and vegetables are imported from other countries (e.g. China, Thailand and Vietnam). The imported amounts and prices are unstable, making SHV's coastal tourism economy unpredictable. At the islands, drought or insufficient water is common. Tourism depends on uncertain sources of water, and sources such as wells and rainwater collection tanks face shortages in high seasons.

Fourth, the findings presented that rain and storm surges might contribute to drowning. Drowning is a new phenomenon that is bad for the image of SHV's coastal tourism. It appeared that tourists hesitated to come due to the incidents. One respondent added, "When they played/swam, it was no wind. Yet they walked to the area as deep as their waists, the air started to be more furious and they didn't swim well... they got drown. They drown and died at Ochheuteal Beach and here." H22. In the area, local news had also reported a number of drowning cases, usually killing at least one person annually. The incidents might stem from other factors such as being unable to swim well rather than the climate change effect.

\subsubsection{Socio-economic vulnerability due to spread of illnesses}

In a broader context, the findings revealed that due to climate change such as rising temperature, disease was spread around SHV. The participants illustrated that, in a family, if a person gets sick, the whole family does too. This has spread to more and more SHV people; the children were the most vulnerable. One respondent argued, "Mostly whenever climate changes, people get cold. The kids get sick strangely. During the last one-year, most diseases are sore throat. Yet I am unsure it is from the climate change or what. I just see that the kids' illnesses are increasingly stronger and stronger” H12. They informed about spread of insects. Spread of biting insects such as mosquitos was based on season and time. For example, if the sea is calm, without air current, there are more biting insects/mosquitos. The mosquitos can result in the spread of diseases in SHV. Cold, fever, sore throat, skin itchiness and temperature were common while dengue fever and malaria were occasionally reported during the interviews. 
At the islands, the SHV natives reported that tourists got sick, especially foreign visitors. Tourists were reported sick not just one year but every year. A participant explained, "Yes, the tourists here get sick when climate changes. Not only this year, every year it is likely in January, February... people get sick.” H47. A notable example was last year; a few tourists reported that they could not breathe after falling sick. The participants emphasised that the domestic tourists get used to climate change-borne illness while the foreign tourists who are new to the area get sick easily. The latter groups are more vulnerable or prone to climate change-borne disease.

McIver et al. (2014) argue that one of climate change's most significant impacts, particularly in developing countries, is on human health. Cambodia is geographically located in an area vulnerable to vector and water-borne diseases (e.g. malaria and dengue fever). Thus, changes in climatic conditions will lead to a significant influence on the epidemic diseases (Country Water Partnership Reports, 2010).

After reporting their perceptions on the environmental and socio-economic vulnerability on SHV's coastal tourism, one key question was given to the participants: "To what extent is Sihanoukville's coastal tourism vulnerable to climate change?" The perception on this question was investigated by asking respondents to rate the vulnerability of SHV's coastal tourism on a five-point rating scale with 1 being 'not vulnerable' and 5 being 'very vulnerable' (see Appendix A). As a result, Table 5.2 shows the Likert-scale results (in $\%$ ), mean ratings, and standard deviation (SD).

Table 5. 2: The extent to which SHV's coastal tourism is vulnerable to climate change

\begin{tabular}{|c|c|c|}
\hline \multirow{2}{*}{$\begin{array}{c}\text { SHV's coastal tourism } \\
\text { vulnerability to climate change }\end{array}$} & \multicolumn{2}{|c|}{ Responses } \\
\hline & $N$ & Percent \\
\hline Not vulnerable & 1 & 2.0 \\
\hline Slightly vulnerable & 15 & 31.0 \\
\hline Moderately vulnerable & 18 & 37.0 \\
\hline Vulnerable & 9 & 18.0 \\
\hline Very vulnerable & 6 & 12.0 \\
\hline Total & $49^{28}$ & 100.0 \\
\hline
\end{tabular}

Mean $=3.08$ and $\mathrm{SD}=1.038$

\footnotetext{
${ }^{28} 1$ missing
} 
As a result, Table 5.2 shows that of 50 respondents, around two thirds $(68 \%)$ felt that it is either slightly or moderately vulnerable, while nearly one third (30\%) reported that it is either vulnerable or very vulnerable.

As indicated in previous chapters, this study seeks to assess vulnerability in two layers: destination level (SHV's coastal tourism) and sector level (SHV's hotel sector). After vulnerability of SHV's coastal tourism is assessed, the next section details the vulnerability assessment of SHV's hotel sector.

\subsection{Vulnerability assessment of Sihanoukville's hotel sector}

Guided by the literature outlined in chapters 2 and 3, the interview guide was designed for the participants to assess vulnerability into environmental and socio-economic dimensions. The findings in Table 5.3 below shows that SHV's hotel sector was environmentally vulnerable to excessive rainfall/rainfall-induced flooding, storm, rising temperature, SLR/beach erosion/storm surge and prolonged drought, and has socioeconomic vulnerabilities due to damages of infrastructures (from excessive rainfall and storm/storm surges), spread of diseases (from the increased temperature and exposure to rain) and economic pressure (e.g. income loss).

Table 5. 3: Vulnerability assessment on SHV's hotel sector

\begin{tabular}{|c|c|}
\hline Vulnerability factors & Findings of vulnerability on SHV's hotel sector \\
\hline Environmental vulnerability & $\begin{array}{l}\text { - } \text { Excessive rainfall/rainfall-induced flooding } \\
\text { - Storm } \\
\text { - } \text { Rising temperature } \\
\text { - SLR/beach erosion/storm surges } \\
\text { - Prolonged drought }\end{array}$ \\
\hline Socio-economic vulnerability & $\begin{array}{l}\text { - Damages of infrastructures (from excessive rainfall } \\
\text { and storm/storm surges) (e.g. roads, beaches) } \\
\text { - Widespread of diseases (resulted from the increased } \\
\text { temperature and exposure to rain) and } \\
\text { - Economic pressure (e.g. income loss) }\end{array}$ \\
\hline
\end{tabular}




\subsubsection{Environmental vulnerabilities}

Similar to environmental vulnerabilities on SHV's coastal tourism, the findings indicate that environmental vulnerabilities such as the excessive rainfall and rainfall-induced flooding, storms, rising temperature, SLR/beach erosion/storm surges and prolonged drought have threatened SHV's hotels. The next section will outline the first environmental vulnerability, which is the excessive rainfall and rainfall-induced flooding. All the remaining environmental vulnerabilities will also be presented subsequently.

\subsubsection{Environmental vulnerability to excessive rainfall}

According to the interviews, excessive rainfall was the most challenging issue perceived by the participants. They indicated hotels' vulnerability in a variety of ways. First, excessive rainfalls were inconvenient for guests and limited their travels. If disturbed by rainfalls, guests mostly stayed in hotels for a short time and then checked out. One interviewee explained that at around November, starting from the Water Festival (annually, Cambodia celebrates the Festival, which is one of main public holidays), the rooms are fully booked at weekends. The hotel sector always expects more guests because guests come and extend their stays by going further to the islands and other attractive areas. However, extended rainfall reduces the number of guests. Hotels with restaurants (50\% of SHV's hotels have restaurants) were even more vulnerable. The sale volumes of their restaurants decreased as well. Another adverse consequence was that guests who had experienced unpleasant amounts of rain, sometimes commented negatively against the hotels through online reviews, thereby making the hotels receive low rates in online bookings.

Second, rainfall-induced humidity has had two invisible unwanted consequences for the hotels: decaying hotel buildings/materials/amenities and humid environment. Rainfall, coupled with salty air as well as many other unknown chemical elements, has caused hotel buildings to decay. Paint on hotel buildings often fades away. This requires highly technical maintenance. Strong rainfalls and rainstorm have made rooms wet and caused other materials/amenities left uncovered to decay. One added, "In this area, whether hotel or general materials made of metals are easily rusty and decayed.” H18. Another added, "We're on the beach, anything that ever lasts 4 years, it only lasts 2 years" H34. Rain causes humidity. For example, one respondent said, "You know, when it rains all the time, 
you have very humid things inside the rooms so everything gets smelly and you have... everywhere becomes humid." $\mathrm{H} 27$.

Third, some participants criticised that the weak infrastructures such as public drainage system have made SHV and their hotels more vulnerable to rainfall-induced flooding. The rainwater remained on the roads or surrounded hotels for hours, especially those that are at low-lying areas. When rainwater flowed strongly all at once, its force damaged the hotel drainage systems. Flash flood often had another unwanted consequence for the hotels: dirtiness/mud in front of and surrounding the hotels. This created inconveniences for guests and the staff (for working). One participant explained, "There are no guests to stay here when it strongly rains. Anyway, if it strongly rains, it floods." H35. The second complained, "We have some heavy rains, the rainfall also like a lot, then the water was coming in the middle of the hotel which normally must not happen, so we have to put a lot of sand bags all around..." H38. Staff were required to clean up and repair the damages more frequently, which increased their overall expenses.

\subsubsection{Environmental vulnerability to storms}

The participants also indicated that storms or wind contractions occur frequently at the coast. Their intensities caused many other unwanted consequences for the hotels. For example, the storms either damaged the hotels' buildings or brought debris/rubbish/tree leaves to their hotel premises and beach areas. Debris/rubbish/tree leaves reduce the beach beauty and quality (both the beaches that belong to the hotels and public beaches).

One mainland participant commented, "Generally, we have problems resulted from climate change during these months (pointing to a calendar) like storms, wind contractions.” H1.

\subsubsection{Environmental vulnerability to rising temperature}

The participants asserted that the increased temperature also affects their hotels in two ways: the damages to hotel buildings/materials/amenities and effects on staff/guests' health.

First, in climate conditions that changed from being too hot (dry season) to being too wet (rainy season), the hotel buildings start to crack. One explained, "We did on one occasion in this room here on the top on the right and I watch the security camera to watch if 
someone enter the room, no one has entered the room. The huge glass window to the bathroom has smashed.I believed it expanded due to the heat." H39. The most vulnerable area is at the rooftop. It made other hotel materials such as swimming pools crack. Laundry beddings decayed more easily. Hot temperature also resulted in the deaths of small plants. In hot weather, more water is used to spray plants in the hotel complex and the gardens (to cool down the hot environment), but more water usages increased costs. Due to the increased temperature, the electricity was cut off, quite often due to overconsumption. Likewise, nothing could function for the businesses. In more severe cases, ACs could not function well. One hotel owner said, "We come to the point where AC just can't function anymore because it's too hot, outside the temperature." H28. Increasing power to operate the ACs made the hotels' expenses soar as well.

Second, many held the view that the increased temperature caused the staff to get sick. When they were getting sick or heat stress, they could not provide good services. This was affecting the whole service system in hotels. Another undesirable consequence was that some places in the hotels where staff could stay and work was no longer bearable due to the increased temperature. So, the layout of working places must be restructured or removed to more convenient places. In hotels where spaces are not available, the working places of staff must be equipped with high power ACs, which increase hoteliers' expenses. Rising temperature also puts their guests under compounding pressure such as heat stress.

\subsubsection{Environmental vulnerability to SLR/beach erosion/storm surges}

The respondents reported that the SLR/beach erosion/storm surge threatens the hotels. They observed that the water is getting higher and higher. For instance, one informant commented, "... the sea brings the sands away, when the water rises it brings the sands away. Sometimes, we have no places to prepare dinners (dinner events for the hotel guests at the beaches) ..." H50. Another added "Yes, it becomes serious you can tell that this is beach erosion." H15.

They observed that after the storm surges, when the water receded, beaches turned to normal but with some loss of sands. This happened twice a year. The first added, "Yes we can see that at the beginning of the rainy seasons, sands level really move away I think at least 1 metre down revealing some tree roots which we've never seen before. We're 
really scared at that moment." $\mathrm{H} 34$. The second continued, "Our hotel obviously lies on the beach here and we've already seen the tides coming up much higher in the last couple of years, almost enters our restaurant and so we can see the next couples of years that it gonna be vulnerable." H28. Some reported that there are no spacious beaches for big events such as gala dinners. At the back of some hotels, the beaches have been eroded. Due to this forward-looking aspect of vulnerability, the SLR/beach erosion will affect businesses at low-lying areas and then there will be no beaches left for touristic activities.

As outlined in sections 3.2.3.4 and 3.3.2.4, historical data show that the sea level has risen in recent years and beaches have been eroded, particularly in SHV. There is also a projection for further SLR in the future.

\subsubsection{Environmental vulnerability to prolonged drought}

The respondents also believed that the hotel business is also a drought-prone sector. They reiterated that their hotels become most vulnerable during the peak time of dry season in March, April and May. Drought could cause water shortfall up to consecutively 2-4 days or up to 2 weeks in the worst case. This happened 4-5 times annually or 10-15 times per year in the worst case. Without alternative sources of water, the drought or water deficit could lead to the closure of some hotels because these tourism enterprises need plenty of water to supply to their customers. One argued, "The hotels should have to close down, you know anyone who has the hotels and restaurants has to close because you can't function without water." H28. This also caused a shortfall of local products such as vegetables, which is already declining in the area. Therefore, a variety of produce has been imported from other countries. At the islands where clean water was already scarce; the hotels' vulnerability becomes more severe.

Nevertheless, as argued above, other factors such as uncontrolled development of SHV and the increase of hotel businesses and tourists as well as population growth additionally contribute to water shortage.

\subsubsection{Socio-economic vulnerability}

Similar to socio-economic vulnerabilities of SHV's coastal tourism, the findings indicate that socio-economic vulnerabilities such as the damage of infrastructures, spread of diseases and economic pressure (see Table 5.3) have threatened SHV's hotels. The next 
section will outline the first socio-economic vulnerability, which is the damage of infrastructures. All the remaining will also be presented subsequently.

\subsubsection{Socio-economic vulnerability due to damage of hotel buildings, infrastructures and materials}

Responding to the question, "Has your hotel experienced or suffered any loss or damages as a result of any of climate change impacts?", the interviews revealed that almost two thirds of the participants (66\%) reported that their hotels have suffered losses and damages caused by climate change. The purpose of question was to gain more insightful understanding of the actual and physical direct socio-economic impacts on the hotel sector.

Based on the interviews, the loss and damages resulted from excessive rainfalls/rainfallinduced flooding and the salty air (which contains chemical elements formed at sea) (see section 5.3.11), SLR/beach erosion/storm/storm surge (see section 5.3.1.4) and increased temperature (see section 5.3.1.3). In addition, the respondents projected that these effects will threaten their hotels in the future. This requires constant and thorough maintenance of the buildings, which is costly. This will continue to put hotels under economic pressure.

\subsubsection{Socio-economic vulnerability due to economic pressure (e.g. loss of income)}

Of the 50 participants, some reported that as rainfall is unevenly distributed throughout the country, SHV is affected and so is the hotel sector. Their businesses face loss of income and increased expenses due to climatic changes. As outlined above, the guests will not visit SHV when anticipating heavy rainfalls. Those who were already in SHV cancelled their bookings and moved out, making the hotel business lose significant income. For some hotels, the owners must repair the buildings after the rainy season, which makes some parts of the buildings non-functional. One respondent said, "The expenses are much like the concrete ceilings. Sometimes when there are constant rains for many days, the water leaks.... We repaired and spent USD1,000 and next rainy seasons it leaks again.” H16.

When it was hot, hotels increased their expenses because the staff as well as guests consumed much water and electricity. When the increased temperature annoys their 
guests, they require high-tech equipment or facilities to respond to the needs that will reduce the heat stress for their guests. The high-tech equipment often costs more. In the meantime, as part of their responses, some hoteliers have no choice but to use the ACs.

\subsubsection{Socio-economic vulnerability due to spread of illnesses}

Broadly in SHV, climate change increasingly contributes to common illnesses such as colds and dehydration or heat-related illnesses in tourists. The participants explained that this might be caused by the fluctuating climate (being suddenly hot and cold). Right in their hotels, many held the views that the increased temperature caused the guests and staff to get sick as well. Being unhealthy, the staff could not maintain service quality. This was affecting the whole services in the hotel sector, which put compounding pressure on their hotels. The first added, "Since my staff drive motorbikes to work, they are directly exposed to rain. This makes them sick" H2. During the interviews a few participants also mentioned malaria. Their guests and staff were likely to be vulnerable to it because Cambodia has a high prevalence of malaria annually. One participant complained, "If there's a lot of flooding, there's an increase of malaria, chances of increased sickness in the hotel and put stress on the staff to cover other people” H15.

After reporting their perceptions on the environmental and socio-economic vulnerability on their hotels, one key question "To what extent is your hotel vulnerable to climate change?" was given to the participants. The perception on this question was investigated by asking respondents to rate the vulnerability of their hotels on a five-point rating scale with 1 being 'not vulnerable' and 5 being 'very vulnerable' (see the Appendix A). As a result, Table 5.4 shows the Likert-scale results (in \%), mean ratings, and standard deviation (SD). 
Table 5. 4: The extent to which the hotel sector is vulnerable to climate change

\begin{tabular}{|c|c|c|}
\hline \multirow{2}{*}{$\begin{array}{l}\text { SHV's hotel sector vulnerability } \\
\text { to climate change }\end{array}$} & \multicolumn{2}{|c|}{ Responses } \\
\hline & $N$ & Percent \\
\hline Not vulnerable & 1 & 2.0 \\
\hline Slightly vulnerable & 25 & 50.0 \\
\hline Moderately vulnerable & 16 & 32.0 \\
\hline Vulnerable & 5 & 10.0 \\
\hline Very vulnerable & 2 & 4.0 \\
\hline Total & $49^{29}$ & 100.0 \\
\hline
\end{tabular}

Mean $=2.63$ and $\mathrm{SD}=0.859$

Table 5.4 indicates that half $(50 \%)$ of the respondents perceived that their hotels were slightly vulnerable while about one third (32\%) were moderately vulnerable.

As argued in previous chapters, adaptation follows vulnerability. The next section details the adaptation framework employed by SHV's hotel sector.

\subsection{The adaptation framework in SHV's hotel sector}

After the vulnerability was assessed in sections 5.2 and 5.3 respectively (aim 1 and 2), this section addresses research aim 3, which sought to determine the extent to which the hotel sector has adapted to climate change. This section presents adaptation measures being adopted according to the adaptation framework by WMO et al. (2008) and Simpson et al. (2008) which comprises technical, managerial, policy, research, and education adaptation (see section 2.8). The overall purpose is to establish the relationships between vulnerability and adaptation that are consistent with several lines of literature as outlined earlier.

Interviews indicated that the participants initiated various adaptation actions for their hotels. Table 5.5 summarises all five types of adaptation: technical, managerial, policy, research and education, which were mentioned during the interviews.

\footnotetext{
291 missing data
} 
Table 5. 5: Adaptation framework of the hotel sector

\begin{tabular}{|c|c|c|c|}
\hline \multirow{2}{*}{$\begin{array}{c}\text { Type of } \\
\text { adaptation }\end{array}$} & \multirow{2}{*}{ Vulnerability } & \multicolumn{2}{|l|}{ Adaptation in hotel sector } \\
\hline & & Adaptation & Purpose \\
\hline \multirow{10}{*}{$\begin{array}{l}\text { Technical } \\
\text { (see Table } 5.6 \text { to } \\
\text { 5.11) }\end{array}$} & \multirow{2}{*}{$\begin{array}{l}\text { Excessive } \\
\text { rainfall/rainfall- } \\
\text { induced flooding }\end{array}$} & $\begin{array}{l}\text { Check hotel structures and buildings and redesign and reconstruct } \\
\text { their buildings such as roofs, doors, windows and enlarge their } \\
\text { connecting drainage systems for better rainwater flows. }\end{array}$ & $\begin{array}{l}\text { To make hotel buildings and drainage } \\
\text { areas strong and adaptable to rainfall } \\
\text { and rainfall-induced flooding }\end{array}$ \\
\hline & & Prepare materials for guests (e.g. first aid boxes, umbrellas) & $\begin{array}{l}\text { To cope with rain-exposed illness such } \\
\text { as colds }\end{array}$ \\
\hline & \multirow{2}{*}{ Storm } & $\begin{array}{l}\text { Check hotel structures and buildings and redesign and reconstruct } \\
\text { their buildings such as roof, door, window and others }\end{array}$ & $\begin{array}{l}\text { To make hotel buildings resistant to } \\
\text { storm }\end{array}$ \\
\hline & & $\begin{array}{l}\text { Use natural trees/forest such as mangrove and other species of } \\
\text { trees in and near hotel premises }\end{array}$ & $\begin{array}{l}\text { To balance strengths of storms and to } \\
\text { stabilize the beach areas }\end{array}$ \\
\hline & \multirow{6}{*}{$\begin{array}{l}\text { Increased } \\
\text { temperature }\end{array}$} & $\begin{array}{l}\text { Use air conditioners (ACs)/big chiller (large areas such as hotel } \\
\text { lobby) }\end{array}$ & \multirow{2}{*}{$\begin{array}{l}\text { To keep rooms and hotel environment } \\
\text { (e.g. lobby areas) cool for guests and } \\
\text { staff }\end{array}$} \\
\hline & & Maintain the gardens (e.g. vine trees and other species) & \\
\hline & & Construct and maintain swimming pools & $\begin{array}{l}\text { To adapt to the effects of higher } \\
\text { temperature }\end{array}$ \\
\hline & & $\begin{array}{l}\text { Check hotel structures and buildings, restructure their buildings } \\
\text { especially roof, building hotels with thatching roof, and installing } \\
\text { insulations }\end{array}$ & $\begin{array}{l}\text { To make hotel buildings resistant to } \\
\text { higher temperature }\end{array}$ \\
\hline & & $\begin{array}{l}\text { Install solar panel (e.g. for air-conditioning, solar hot/cold water } \\
\text { systems and light energy) }\end{array}$ & $\begin{array}{l}\text { To function ACs and solar cold/hot } \\
\text { water systems to minimise cost from } \\
\text { increased demand on ACs and cold } \\
\text { water }\end{array}$ \\
\hline & & Spray water on the ground and gardens at night time & To reduce higher temperature for \\
\hline
\end{tabular}




\begin{tabular}{|c|c|c|c|}
\hline & & Install special mirrors (heat reflecting mirror) & \multirow[t]{2}{*}{ guests/staff } \\
\hline & & $\begin{array}{l}\text { Provide free facilities/amenities (e.g. transport, water } \\
\text { dispensers...etc.) }\end{array}$ & \\
\hline & \multirow{6}{*}{$\begin{array}{l}\text { Prolonged } \\
\text { drought/deficit water }\end{array}$} & Build water storage/tanks & \multirow{6}{*}{$\begin{array}{l}\text { To collect/ensure sufficient water for } \\
\text { guests and staff }\end{array}$} \\
\hline & & Dig wells & \\
\hline & & Use underground water container & \\
\hline & & Own truck water & \\
\hline & & Connect pipe to a nearby lake & \\
\hline & & Harness rainwater & \\
\hline & \multirow{4}{*}{$\begin{array}{l}\text { SLR/beach erosion } \\
\text { and storm surges }\end{array}$} & $\begin{array}{l}\text { Use human labour, particularly their staff to bring back dozens of } \\
\text { sand gabions to strengthen to the affected areas }\end{array}$ & \multirow[t]{2}{*}{ To stabilize beach areas } \\
\hline & & Institute seawall barriers & \\
\hline & & Remove infrastructures away from the eroded beach areas & $\begin{array}{l}\text { To avoid damages caused by beach } \\
\text { erosion }\end{array}$ \\
\hline & & $\begin{array}{l}\text { Plant mangrove (and others of its species) in and near their hotel } \\
\text { premises }\end{array}$ & $\begin{array}{l}\text { To balance strengths of storm surges } \\
\text { and stabilize the beach areas }\end{array}$ \\
\hline & All vulnerabilities & Enable access to early warning equipment (e.g. radios, TV...) & $\begin{array}{l}\text { To access climate change-related } \\
\text { information }\end{array}$ \\
\hline \multirow{2}{*}{$\begin{array}{l}\text { Managerial } \\
\text { (see Table 5.12) }\end{array}$} & $\begin{array}{l}\text { All vulnerabilities } \\
\text { especially extreme } \\
\text { weather }\end{array}$ & $\begin{array}{l}\text { Suggest other touristic activities to clients to be away from } \\
\text { impacted areas (to alternative destination or from the islands to } \\
\text { mainland or vice versa) }\end{array}$ & $\begin{array}{l}\text { To maintain guests' touristic activities, } \\
\text { being safe from climate change }\end{array}$ \\
\hline & All vulnerabilities & Create short and long-term plan for better adaptation & $\begin{array}{l}\text { To initiate adaptation plans/programs } \\
\text { for their business operations }\end{array}$ \\
\hline
\end{tabular}




\begin{tabular}{|c|c|c|c|}
\hline & $\begin{array}{l}\text { Excessive rainfall, } \\
\text { rising temperature } \\
\text { and storm }\end{array}$ & Provide free transports for the staff & $\begin{array}{l}\text { To avoid rain/heat-exposed illness } \\
\text { such as cold, heat exhaustion and } \\
\text { possible injuries caused by storm }\end{array}$ \\
\hline $\begin{array}{c}\text { Policy } \\
\text { (see Table 5.13) }\end{array}$ & $\begin{array}{l}\text { All vulnerabilities } \\
\text { especially storm } \\
\text { surges, rain and } \\
\text { increased temperature }\end{array}$ & $\begin{array}{l}\text { Comply with self-regulation (e.g. building design standards for } \\
\text { force winds) }\end{array}$ & $\begin{array}{l}\text { To make hotel buildings strong and } \\
\text { adaptable to climate change risks }\end{array}$ \\
\hline $\begin{array}{c}\text { Research } \\
\text { (see Table 5.14) }\end{array}$ & All vulnerabilities & $\begin{array}{l}\text { Assess business operations and awareness of staff's knowledge } \\
\text { gaps }\end{array}$ & $\begin{array}{l}\text { To evaluate business risks of climate } \\
\text { change and staff's existing knowledge } \\
\text { to create adaptation programs }\end{array}$ \\
\hline \multirow{4}{*}{$\begin{array}{c}\text { Education } \\
\text { (see Table 5.15) }\end{array}$} & \multirow{4}{*}{ All vulnerabilities } & Inform guests and staff & $\begin{array}{l}\text { To make guests and staff well- } \\
\text { prepared for potential climate change } \\
\text { impacts }\end{array}$ \\
\hline & & $\begin{array}{l}\text { Receive climate change trainings from other parties esp. the } \\
\text { government }\end{array}$ & \multirow{3}{*}{$\begin{array}{l}\text { To obtain climate change-related } \\
\text { information and increase awareness of } \\
\text { climate change issues }\end{array}$} \\
\hline & & Join public education campaign (e.g. climate change awareness) & \\
\hline & & Provide climate change-related trainings to the staff & \\
\hline
\end{tabular}

Source: Adopted from WMO, UNEP, and \& WTO (2008) and Simpson et al. (2008) 


\subsubsection{Climate change technical adaptation}

\subsubsection{Technical adaptation to excessive rainfall and rainfall-induced flooding}

Table 5. 6: Adaptation measures for excessive rainfall

\begin{tabular}{|c|l|c|c|}
\hline \multicolumn{2}{|c|}{ Excessive Rainfall } & \multicolumn{2}{|c|}{ Response } \\
\cline { 3 - 4 } \multirow{2}{*}{ Technical } & $\begin{array}{l}\text { Checking and redesigning hotel structures } \\
\text { and surrounding (e.g. drainage system) }\end{array}$ & 18 & 66.7 \\
\cline { 2 - 4 } & $\begin{array}{l}\text { Preparing materials for guests (e.g. first aid } \\
\text { boxes, umbrellas) }\end{array}$ & 9 & 33.3 \\
\hline Total & 27 & 100.0 \\
\hline \multicolumn{2}{|c|}{}
\end{tabular}

Excessive rainfall is the most challenging effect perceived by the participants. First, Table 5.6 represents that $66.7 \%$ of them adapted by checking the hotel structures and surroundings. The participants explained that their adaptations included redesign and reconstruction of buildings such as roofs, guttering, doors, windows and other building features exposed to rain. Most paid more attention to roof structures. One said, "This year (rain pattern) has changed much; it extremely increases until we make new roof two layers." H32. One more respondent reported that connecting the roof from one place (e.g. restaurant) to the main hotel building was built to enable the guests to walk from place to place (inside the hotel premise) without exposure to the rains which become more frequent.

Learning from peers, prior to running the businesses, a few new business managers/owners constructed their buildings to be adaptable to this effect. They built structures (e.g. roofs, doors and windows) in such a way as to reduce exposure to rain. One informant explained, "It is not an equipment. In fact, it is the way that we design the hotel, we know it that rain is excessive in one season. It comes from one side; most of the time it has come like this. So, we built our building like this." H44. Upscale hotel businessmen tended to make significant changes to the hotel buildings such as reconstructing their connecting drainage systems for better rainwater flows. Hallegatte (2009) supports that new infrastructure that enables hotels to deal with a wide range of changing climate effects is more difficult and expensive in the design and construction. 
Others contended that they must instruct their technical staff to prevent or repair the ceiling damages resulting from rainwater leakages. Some further pointed out that they had formed a technical group that could prevent, maintain and repair damage to the buildings. One participant continued, "We have the rainwater leakage on the ceiling. We have more staffs to repair; we have CTV, engineering, maintenance team, AC repairmen, water system repairmen and electricians. This allows us to adapt/respond faster..." H17. A similar thought noted by WMO, UNEP, and WTO (2008) was that the modification of the poor infrastructure may have to be improved if existing performance standards are inconsistent with the changing climatic conditions.

The most striking concern caused by heavy rainfall was the quality of connecting rainwater drainage. The participants said that they adapted by frequently checking the areas, clearing rubbish or materials that block the water flow. In the meantime, prior to rainy season necessary materials such as water pumps, spades and large rubber brooms were prepared. During flash flooding, while some participants informed that they must pay for a private truck equipped with some materials to pump the water out with the price of USD60 per time, others replaced drainage pipes for bigger size ones so that the rainwater could flow easily. The participants' adaptation measures likely align with Coles, Dinan, and Warren (2016) who explain that newly designed buildings, technologies and construction techniques increase opportunities for companies to adapt to changing climate, which leads to decreasing their environmental load.

Second, Table 5.6 shows that $33.3 \%$ adapted by providing necessary materials to their guests. Inside each room, boxes of medicine as first aid were available to combat rain-exposed illnesses. Becken and Wilson (2013) noted a similar point: "climate change adaptation starts at home" (p. 636) for tourists planning for their trip and are knowledgeable about the climate of their next destination. In this study, hotel operators who know about and have experience with SHV's climate must initiate such adaptation for their guests. 
Additionally, while raining prior to going out to nearby places, some guests often came and asked if the supporting materials such as medicines and umbrellas were available. To fill their needs, the hotel managers/owners provided free medicines, transport such as buggies and cars, and materials such as umbrellas. Without supporting materials such as the first aid, they mentioned that the guests might feel inconvenient. Aall and Høyer (2005) call this a "personal adaptation" by explaining that companies can do various simple things to help visitors in cases of unexpected weather such as excessive rainfall.

\subsubsection{Technical adaptation to storm}

Table 5. 7: Technical adaptation to storm

\begin{tabular}{|c|c|c|c|}
\hline \multicolumn{2}{|r|}{ Technical adaptation to storm } & \multicolumn{2}{|c|}{ Responses } \\
\hline & & $N$ & Percent \\
\hline \multirow[t]{2}{*}{ Technical } & $\begin{array}{l}\text { Check and readjust hotel structures and } \\
\text { surroundings } 30\end{array}$ & 10 & 40.0 \\
\hline & Use/maintain natural trees/forest & 15 & 60.0 \\
\hline \multicolumn{2}{|r|}{ Total } & 25 & 100.0 \\
\hline
\end{tabular}

In their views, there are frequent occurrences of strong winds and wind contractions as well as storms. Table 5.7 shows that $40 \%$ of them technically adapted by checking and readjusting hotel structures and the surrounding areas to make sure they were strong enough to withstand the strong winds and storms. They believed that strong structures could prevent storms while the storms threaten the weak buildings, especially old ones. The respondents paid more attentions to the roofs and windows. One respondent added, "Previously, we had problem on the roof. In our recent repairing, I told the repairmen to add storm-resistant materials. For example, we before had one hook on the roof, we now have two hooks" H3. This indicated that the strength of wind is stronger.

\footnotetext{
30 Table 5.6 above shows that checking and redesigning the hotel structures/buildings and the surrounding areas was used by $66.7 \%$ to adapt to excessive rainfall but by only $40 \%$ (see Table 5.7) to adapt to storms. This was likely because excessive rainfall was a more concerning issue than storm in SHV and the adaptation measures were slightly different. For this instance, whilst drainage systems must be checked to adapt to high rainfall, they are not done in response to storms (coming without rain).
} 
Other respondents whose hotel structures are weaker, continued, "We have made/cut the bamboo $^{31}$ to cover on the roofs. You see we put these to withstand the strong winds and rains." H30. Some mentioned that they could take reactive adaptation actions only after storms by repairing the damages or affected areas. For example, one said, "We repair it (after storms). We cannot do more than that." H22. The second said, "Now we have experience of storms damaging leave (thatching) roofs. Next year we will change to brick roofs.” H35. What could be clearly seen in these arguments that this was the lack of proactivity. Proactive preventions seemed to be beyond the capacity of these participants, due to their limited resources, which are seen in developing countries.

$60 \%$ of them used another way of adaptation, which is keeping natural forest $/$ trees $^{32}$. They believed that keeping densely forests/trees around their hotel compounds could provide many benefits, including lessening the intensity of strong winds or storms towards their hotels. One island participant explained, "Trees help, they help to withstand the salty moisture (salty air blown from the sea). They help they help a lot, the trees; they help to withstand the wind blow that bring the salty air like the salt..." H47. Although trees/forest are also vulnerable to climate change (Andrei \& Roger, 2007; Wan et al., 2018), they also serve as an essential component for adaptation strategies, for example protecting soil and land against harmful impacts of flooding and having the potential to protect people from some of the detrimental effects of rising global temperatures. Forests and trees of other species provide a wide range of benefits beyond those relevant to climate change by laying a foundation for better sustainable economic and social development (UN, 2007). However, they might not have been aware that some trees could cause damage such as collapsing on their hotels during the storm turbulences. Yet, based on the observations they chose trees and other species that were environmentally friendly to their hotels.

\footnotetext{
${ }^{31}$ Bamboo is used for countryside households that are fastened with nails on thatching roof so that the roof becomes more resistant to strong winds. A few SHV's hotels were built with strong concrete foundations topped with thatching roof. There are two reasons: first, for adaptation to rising temperature and second, for beautification purpose.

${ }^{32}$ In SHV, some hotels have large compounds where various trees, including mangroves, can be planted, while others have no spaces where a few trees can be planted in large vases. This indicates different ranges of hotel sizes in SHV.
} 


\subsubsection{Technical adaptation to increased temperature}

As described in the previous sections 5.2 and 5.3 respectively, temperature has been recorded and is projected to become higher. All hotels were reported to be vulnerable to it. Table 5.8 shows various technical adaptation measures initiated by the respondents.

Table 5. 8: Technical adaptation to rising temperature

\begin{tabular}{|c|l|c|c|}
\hline \multicolumn{2}{|c|}{ Technical adaptation rising temperature } & \multicolumn{2}{c|}{ Responses } \\
\cline { 2 - 3 } & \multicolumn{1}{|c|}{$N$} & Percent \\
\hline \multirow{5}{*}{ Technical } & Using/installing ACs & 50 & 49.0 \\
\cline { 2 - 3 } & Using big chiller & 2 & 1.9 \\
\cline { 2 - 3 } & Maintaining gardens (including growing vines) & 16 & 15.6 \\
\cline { 2 - 3 } & Constructing new and maintaining existing swimming pool & 9 & 8.8 \\
\cline { 2 - 4 } & $\begin{array}{l}\text { Adjusting structures and adding technical materials } \\
\text { Installing solar panel (e.g. for air-conditioning, solar } \\
\text { hot/cold water systems and light energy) }\end{array}$ & 9 & 8.8 \\
\cline { 2 - 4 } & Spraying water on hotels' compounds and roof structures & 5 & 5.8 \\
\cline { 2 - 4 } & $\begin{array}{l}\text { Providing free materials (e.g. transport, water } \\
\text { dispensers...etc.) }\end{array}$ & 3 & 2.9 \\
\cline { 2 - 4 } & Installing heat reflecting mirror & 2 & 1.9 \\
\hline
\end{tabular}

Table 5.8 shows the greatest percentage, $49 \%$ of the respondents said they simply used/installed (new) ACs. In a rare condition, electric fans were supplemented in addition to the functioned ACs while room climate is still hot. Likewise, only $1.9 \%$ used similar adaptation techniques. They mentioned that big chillers ${ }^{33}$ were used in the hotel lobby to cool down the hot environment.

While in hot weather, $15.6 \%$ of respondents said that maintaining the forest/trees/gardens contributes to cooling down their hotel environment. Besides beautification purposes, it was believed that trees could provide shades and oxygen and bring fresh air. One said, "We're lucky with the design; it's filled with the plants and we're trying to put more plants as we can.” H39. Among this group, three used uniquely different adaptations. They mentioned that a vine tree was planted on roof areas. The vines bear leaves and provide shades as well

\footnotetext{
${ }^{33}$ The participants referred to air-conditioning like machines emitting cool air. The standing machine is mobile and can be moved closer or further to the guests. Usually they are put in a big lobby room.
} 
as absorb heat. The first informant pointed out, "Yes, we plant the vines on the top; they really help a lot to decrease the heat. Naturally, it makes the room environment cooler." $\mathrm{H} 21$. The second added, "Since coconut trees did not provide sufficient shades, we planted the vines to reduce the heat." H41. A similar method explained by Alonso-Almeida et al. (2017) (in Chilean hotel context) that in the summer hotel used vertical vines covering the building's walls to mitigate heat by generating shade while in winter they reduce heating costs by allowing sunlight to warm up the building when the vine leaves become dried up in winter. Furthermore, in broader perspective general perceptions throughout Cambodia as well as in SHV revealed that trees (afforestation) were believed to be a contributing factor to CCA while deforestation is perceived as the major cause of climate change.

$8.8 \%$ of the respondents said that as the climate has become increasingly hotter, they began constructing (for those that do not have) and maintained (for those that already constructed) swimming pools. Regardless of other criteria such as room rates, interviews and observations found that hotels with swimming pools receive more bookings, especially in hot periods. In hot weather, some guests, especially children could adapt by swimming in the pools because going to the sea takes time (for mainland hotels) and swimming in it may be dangerous. While in hot weather or adverse climate (including strong winds), the guests increasingly like swimming in the pools. One participant demonstrated that special consideration was taken at the swimming pool. The pool attendants were on standby to help guests who like both swimming and sunbathing from drowning. All materials were prepared and fully ready for the guests' needs. For example, big umbrellas were given to provide more shades to the guests. From tourist side, Becken and Wilson (2013) argue that the detrimental effects of weather can be adapted by behavioural responses by changing touristic activities to match with weather conditions, by utilising structural or mechanical supports, including umbrellas and modification of the thermal insulation of the body by changing clothing. In the destination level, Pröbstl-Haider and Haider (2013) argue that visitors' motivations and behaviours are certainly influenced by climate change. Tourism destinations try to influence visitors' behaviours with the implementation of adaptation measures and provision of new products. 
$8.8 \%$ of participants acted by adjusting the hotel structures and buildings as a measure to adapt to rising temperature. They believed that rising temperature was quite related to the hotel structures and buildings. For instance, they constructed new extra roof to reduce hotels' exposure to hot environment. Extra roofing could reduce heating effects significantly. Two high standard hotel participants (4 and 5 stars) adapted by constructing thatching roof. One respondent said, “...it's our (thatching) roof, yes it's good for keeping customers cooler ...” $\mathrm{H} 28$. Yet these respondents reported damages caused by storm on their thatching roof as well. They are foreign participants who may not be aware of climate change-related issues in SHV. Another said that for the purpose of reducing temperature at the ceilings, special aluminium panels were put up during the construction. It is believed that the panels would absorb heat, preventing it going through into the rooms.

Of this group, a few participants from new hotels explained that they had known rising temperature was a challenge prior to constructions. They designed and constructed the hotels/rooms adaptable to it. Then there was not much requirement for them to redesign and readjust periodically. They just made a slight change to hotel materials such as window shades and room curtains to reduce sunlight coming directly to the rooms. One mentioned, “... we must only make adjustments on the curtains in the rooms. If we use the thin-layer curtains, they cannot be resistant to the sunlight/heat. If we use the good quality curtains, there is no problems; they can be resistant to the sunlight/heat." H16. According to Coles et al. (2016), new buildings perform environmental actions better than the old ones, because new buildings often benefit from cutting edge in building technology, construction systems and environmental management.

$5.8 \%$ of informants reported that in the high temperatures, especially with full sunshine, they used solar systems to exploit the opportunity. Nevertheless, due to its high cost, they could not afford large-scale solar systems (for the whole hotels). Full instalments of solar systems were impossible due to their high price. The solar energy for some ACs, hot/cold water systems and light energy. One explained, "We have well prepared. I have already installed solar panels for hot/cold water I don't use electricity, I use solar. We have 1,000L; we use $60 \%$ of solar in dry seasons, so we only use $40 \%$ of the city electricity.” $\mathrm{H} 7$. Nevertheless, 
installing solar systems for their hotels may not be a direct adaptation to high temperature. They beneficially exploit the opportunity for the businesses because solar power minimises cost from increased demand on ACs and cold/hot water. This likely aligns with the adaptation definition as indicated in section 2.5 by IPCC (2007), which defines that on one hand adaptation is adjusting to natural or human systems to respond to actual or expected climatic impacts, and on the other hand it is to exploit the beneficial opportunities.

It is generally known that Cambodia's electrification remains low and the price is high. This challenges the businesses. The electricity price in some places where electricity is available is one of the highest in the world (San, 2015). In hotels, financial benefits, mainly through cost reductions, is one of the motives that stimulate the actions to reduce the environmental impact (Jarvis \& Ortega, 2010). In relation to technological innovation, Niang-Diop and Bosch (2005) argue that it may be needed to adapt current technologies well-matched to the adaptation demands, for instance the improvement of higher energy-efficient air conditioning techniques. This is likely to be the main reason why the hotel sector in SHV endeavours to adapt to rising temperature by using solar-powered facilities to meet the rising demand on air-conditioning due to climate change.

It is important to note that there are three main sources of power for SHV's hotel sector: the electricity supplied by the government, the power generators (private possession of hotel owners or other suppliers) and solar systems. The first source is unreliable due to frequent power cuts. The second adds extra costs to the hotel sector. The third is high in investment cost but relatively cheap in operation and maintenance and simply promising in the near future when investment costs declines.

$4.9 \%$ of interviewees asserted that during an extremely hot environment, they must cool down their hotel compounds by spraying water on all surrounding land areas, gardens and roof structures. They believed that this could reduce the heat or high temperature. It is more beneficial if they water at night-time because of low evaporation at nights. The minute they water, the water remains and plays roles like an environment coolant to reduce heat. Once the surrounding environment is not so hot, further benefits could be reaped. For instance, the 
guests would not use much electricity (e.g. ACs and cold/hot water system). The hotel operators could decrease their expenses this way as well.

$2.9 \%$ of respondents provided free materials/amenities such as transport and cold-water dispensers. The guests were transported to the nearby beaches during the hot days. They used environmentally friendly transports such as electric buggies. This was an additional assistance for their guests. One interviewee said, "One more solution for increased temperature, we have buggies (electric cars). We must provide proper ridings to the nearby areas for free of charge." H1. Becken and Wilson (2013) address that the extent to which tourists adjusted in response to weather conditions was facilitated by transport mode and length of residence. At the same time, others provided free drinking water and made water dispensers accessible to the guests to avoid dehydration.

$1.9 \%$ explained that special mirrors or large glass panels (heat reflecting mirror) were used. The mirrors or large glass panels were installed in front of each room or somewhere facing to excessive sunlight to reflect the heat back out of the rooms, thereby contributing to reducing heating effect and cooling down the room environment.

It is important to inform that most of them used multiple adaptation measures such as using ACs, provision of free transports, and spraying the hotel premises with water at the same time, while quite a few used ACs as the only measure. These could be done, depending on the hotel resources and availability of hotel spaces. For example, while some hotels have spaces for maintaining large gardens, others do not.

\subsubsection{Technical adaptation to SLR/beach erosion and storm surges}

The hotels that were located on the mainland were not directly vulnerable to SLR/beach erosion/storm surges while those that were front beach have experienced it. Only $32 \%$ were front beach hotels. Although both front-beach and mainland hotel participants were able to provide their views, this study obtained answers mainly from the front-beach hotel respondents. 
Table 5. 9: Technical adaptation to SLR/beach erosion/storm surges

\begin{tabular}{|c|c|c|c|}
\hline \multirow{2}{*}{\multicolumn{2}{|c|}{$\begin{array}{c}\text { Technical adaptation to } S L R / \text { beach erosion/ } \\
\text { storm surges }\end{array}$}} & \multicolumn{2}{|c|}{ Responses } \\
\hline & & $N$ & Percent \\
\hline \multirow{4}{*}{ Technical } & Use sand gabion & 5 & 41.7 \\
\hline & Institute seawalls & 3 & 25.0 \\
\hline & $\begin{array}{l}\text { Remove infrastructures away from } \\
\text { the eroded beach areas }\end{array}$ & 2 & 16.7 \\
\hline & Plant mangrove and trees & 2 & 16.7 \\
\hline \multicolumn{2}{|r|}{ Total } & 12 & 100.0 \\
\hline
\end{tabular}

Table 5.9 indicates that $41.7 \%$ of them used sand gabions as a tool to adapt to SRL/beach erosion/storm surges. After sands were washed away, they used human labour, particularly their staff, to bring back dozens of sand gabions to refill the affected areas. They repeated such action from year to year. One hotel owner said, “For beach erosion, we haven't many ways to prevent. What we can do is once the water recedes, we must go and fill the beach with the extra sand." H30. Another added, "For beach erosion ... we just have to react, we have to quickly rebuild the beach and all of us bring sand bags to strengthen the affected areas.” H39.

The interviews and field observation found that $25 \%$ of them instituted seawall barriers as part of the adaptation measure. All the $25 \%$ were high standard hotels (5-star). The seawall barriers looked strong and firm. However, it was assumed that it must have been costly to build the seawalls. Cooper and Pile (2014) argue that the construction of defences is usually seen in coastal urban environments to respond to increased flood risk from storm surges.

$16.7 \%$ said that some hotel structures were slightly removed away from the eroded beach areas due to SLR/beach erosion/storm surges. This was a huge structure removal and an expensive measure. Most structures are restaurants.

$16.7 \%$ initiated a programme to reforest mangroves and other species that act as wave protection whilst the other maintained trees at the beaches to protect against erosion. At one island one said, "By having a lot of trees, we have roots that keep the sands, the soil blocked 
so we have no intention to cut down the trees and that's the most important thing on these properties.” H46. Cambers (2009) supports that coastal forest can back wide beach and protect a healthy coral reef to withstand SLR and future high wave. This is better than concrete infrastructures that are likely to confine beach on the landward and degrade coral reef on the seaward side (Cambers, 2009). Tobey et al. (2010) call it "nature-based adaptation".

\subsubsection{Technical adaptation to prolonged drought}

Table 5. 10: Technical adaptation to prolonged drought

\begin{tabular}{|c|l|c|c|}
\hline \multicolumn{2}{|c|}{ Technical adaptation to prolonged drought } & \multicolumn{2}{|c|}{ Responses } \\
\cline { 3 - 4 } & Building water tank/storage & $N$ & Percent \\
\hline \multirow{5}{*}{ Technical } & 33 & 50.0 \\
\cline { 2 - 4 } & Digging wells & 25 & 37.9 \\
\cline { 2 - 4 } & Using underground water container & 5 & 7.6 \\
\cline { 2 - 4 } & Owning truck water & 1 & 1.5 \\
\cline { 2 - 4 } & Connecting pipe to a nearby lake & 1 & 1.5 \\
\cline { 2 - 4 } & Collecting rainwater & 1 & 1.5 \\
\hline \multicolumn{2}{|c|}{ Total } & 66 & 100.0 \\
\hline
\end{tabular}

According to the interviews, during drought period, all hotels significantly depend on the water supplied by SHV's city hall. Although SHV people called it 'state water', the water was actually managed and distributed by a local private company. The government licensed the company, namely ANCO, to treat water and supply it as much as possible throughout SHV. This indicates that the local company managed and distributes the water while the local government plays a minimum role as a monitor. A site visit to the company shows that the company is located close to SHV's largest lake reservoir, Kbal Chhay, $25 \mathrm{~km}$ from SHV downtown. However, because of the lack of reliability (e.g. high price, low pressure) in the city water supply, Table 5.10 indicates that the respondents adapted in a number of ways, by installing water tanks or building water storage facilities (50\%), digging wells (37.9\%), and building underground water containers (7.6\%). A few other options were having their own truck water $(1.5 \%)$, connecting water pipe to a nearby lake $(1.5 \%)$ and harnessing rainwater 
(1.5\%). In the most severe drought, when all sources of water run out, the hotel managers/owners bought water from private mobile trucks.

The greatest percentage, $50 \%$, used the water storage tanks as a measure to adapt to prolonged drought and water shortages. The interviews and site observations found that the water storage tanks used by hotel operators include the small and big water tanks (up to 20,000 cubic metre) elevated on the top or higher places of the hotels. Some reported that they have more than one water storage tank while others have many, putting them at the ground of hotel premise, at the side of hotel and at the top of the hotel roof. This had to be done to ensure sufficient water for the guests/staff in the event of water disconnection or extended drought. When the city water was available, they always pumped into the tanks. With tank waters, they could have water available from 1-2 days up to 1 week, depending on the number of guests and sizes of tanks. One explained, “Yes. You see in other countries; people don't care of building water storage tanks because they never have water shortages. To be frank, in SHV there are water tanks in all households, just look at their rooftops because they don't trust the water state supplies.” H4. WMO, UNEP, and WTO (2008) argue that alleviation of water shortfall requires fundamental investments in storage facilities. Zhou and Tol (2005) explain the most common features of technical adaptations such as making water supply available for tourism operations by installing water transfers through pipelines or tankers, water reservoirs and desalination plants.

At the islands, households and hotels must depend on different private water suppliers who connect water pipes to the tourism businesses and households. The suppliers usually possess plenty of greater and deeper wells, pump up the water into their gigantic-sized elevated water tanks and subsequently through connecting pipes, distribute it to the hotels and households. The distributed water is not available 24 hours. Through connecting pipes, the suppliers pour the water, and all hotel operators as well as households must collect it as much as they could in the morning and store it in their own tanks or whatever containers. They must do this every morning, or they would not have sufficient water for later times during the day. As always, the suppliers do not distribute water at noon and night. In this scenario, if the water is disconnected or becomes suddenly unavailable, the island hotels are quite vulnerable because 
they do not have their own wells, and in the islands, it is costly to dig their own wells. The price could be USD20,000, depending on the distance of islands (measured from the mainland to transport construction materials to the islands) and soil conditions.

$37.9 \%$ used human-dug wells as adaptation measures. The wells had almost the same sizes and shapes, but the depth was different, depending on the places (e.g. at the mainland vs. islands) and soil conditions. The costs spent on digging wells contribute to the depth level as well. The more they spend, the deeper the wells they have. It could be as deep as 50 metres. While they have to spend USD10,000 per one well on the mainland, they must spend USD20,000 at the islands. Although interviews evidence that nearly half of them had at least one well, one reported that there were up to five wells in the hotel compound. The availability of water is dependent on first, the depth, and second, the location of wells.

Only $7.6 \%$ of them had large underground water containers. The containers were usually possessed by a richer business owners/managers or old containers (but later renovated) left over by the previous constructions. Generally, it was believed that these containers had a huge capacity to store water. Although the large containers were available underground, they had to pump water up to transfer to other smaller empty tanks elevated at higher places. Therefore, these hotels must have water pumps in place. In the meantime, they must also pump both the city water and water stored underground into the elevated tanks due to low pressure to distribute to each room. However, doing so suggests that they are racing against and pressurise each other for the water availability for the hotels. This shows there are unclear/conflicting rules how water is distributed (by the local government) and used (by hotels).

There was one water truck $(1.5 \%)$ on standby in front of a hotel. Interviewing this hotel representative found that the truck was used to transport water to the hotel during drought period.

Only $1.5 \%$ said that a water pipe was connected directly to a lake near the hotel. It is important to note that this measure only could be done if the hotel location is close to water 
reservoirs such as lakes, rivers or streams. This also suggests that location of the hotel influences the adaptation measures.

At another island, one high standard hotel participant (1.5\%) said a rainwater collection programme had been initiated to collect rainwater. There were rainwater collection tanks sponsored by DPs such as ADB. The informant said, "Because it rains more, let's find ways to collect more water that was something apparently, nobody thought about before. We've started our first project, so we try to use rainwater as much as possible...” H46. However, the water might contain parasites and various water-borne diseases if not properly treated. WMO, UNEP, and WTO (2008) explain that the hotel sector responds to drought through assessment of water needs and preparation of alternative sources of supply. For hotels under the detrimental effect of extended drought during peak-time demand, conserving water is even more essential (Su, 2014).

It is important to note that all 50 hotels were reported to adapt to drought; some used both water tanks and wells together for their hotels while others had only water tanks or wells.

In the most severe drought, when all sources of water run out, almost all hotel managers/owners bought water from private mobile trucks. The cost depends on the location but usually was USD80 per truck. One informant said the price is three times higher than market price during the most severe drought. However, the truck-in water is low in quality and often delivered late. This suggests that the water might be brought from far locations (outside SHV) without quality check and the number of suppliers is quite few whereas the demands are very high. The truck-in water supply is likewise not completely clean. 


\subsubsection{Other technical adaptations (e.g. enable access to early warning systems)}

Table 5. 11: Enabling access to early warning systems about climate change impacts

\begin{tabular}{|c|c|c|}
\hline \multirow{2}{*}{$\begin{array}{c}\text { Enabling access to early } \\
\text { warning equipment }\end{array}$} & \multicolumn{2}{|c|}{ Responses } \\
\hline & $N$ & Percent \\
\hline Computer/smart phone/tablet & 36 & 59.3 \\
\hline TV & 18 & 30.5 \\
\hline Radio & 6 & 10.2 \\
\hline Total & 60 & 100.0 \\
\hline
\end{tabular}

Table 5.11 shows that $59.3 \%$ of them used computer/smart phone/tablet to access climate change information. To receive the information, the businessmen and individuals must 'Like' the important Pages of the government agencies concerning the climate change, for example MORWAM and MOT. They believed that the Internet is one of the most reliable sources currently. This method is believed to be fast, convenient and accurate. For example, while Khmer participants like checking websites of local online news, Facebook has become increasingly popular for obtaining climate change information. They said this social network allows faster information delivery. The information is posted by the national government and subsequently shared by the local government. The information was indicative of possible extreme climate such as storm and storm surges, flash floods and excessive rainfall.

$30.5 \%$ of them received climate change information through watching TV. While Khmer participants watch national broadcast channels, the foreigners watched international channels such as $\mathrm{CNN}$ and BBC. They reported that TV remains one of the most effective methods. However, the international news channels rarely informed directly about climate change in Cambodia as well as SHV. One foreign owner said, "I don't know how well the Khmer news report climate change problem. I'm not sure because I can't read Khmer. CNN, BBC rarely talks about Cambodia and SHV's climate but neighbouring places. It's global problem." $\mathrm{H} 28$.

10.2\% obtained climate change-information through radio. In Cambodia, there are many radio channels based at both national and local stations. However, most of them are 
broadcasted using Khmer language. The foreign participants appear challenging to access to information disseminated through radio.

Early warning systems (e.g. radio) play a crucial role in CCA (WMO et al., 2008). These systems provide prior warning relevant to severe wind events, storm surges, flooding and heat waves (WMO et al., 2008). Media, including the Internet, television and newspapers, are the main sources from which the public obtains information about climate change (Wei et al., 2014), thereby influencing the public perceptions of climate change (Brulle, Carmichael, \& Jenkins, 2012). Warning systems that are accessed early allow people to prepare their businesses and escape to a safer place before extreme weather, if needed.

\subsubsection{Climate change managerial adaptation}

Table 5. 12: Managerial adaptation employed by the hotel sector

\begin{tabular}{|c|l|c|c|}
\hline \multicolumn{2}{|c|}{$\begin{array}{c}\text { Adapting to excessive rainfall, rising temperature, storm and } \\
\text { SLR/beach erosion/storm surge and drought }\end{array}$} & \multicolumn{2}{c|}{ Responses } \\
\cline { 2 - 4 } Managerial & $\begin{array}{l}\text { Suggest other touristic activities to clients to be } \\
\text { away from impacted areas (to alternative destination } \\
\text { or from the islands to mainland or vice versa) }\end{array}$ & 43 & 82.7 \\
\cline { 2 - 4 } & Create short and long term for CCA & 6 & 11.5 \\
\cline { 2 - 4 } & Provide free transports for the staff & 3 & 5.8 \\
\hline Total & 52 & 100.0 \\
\hline
\end{tabular}

First, results in Table 5.12 show that $82.7 \%$ of them suggested that touristic activities for clients to be away from impacted areas (to alternative destinations or from the islands to mainland or vice versa). During climate change turbulence, most participants suggested that their guests not to visit the islands on day excursions and remain on the mainland. Some guided their guests to visit nearby places with activities such as cycling or walking instead. In a similar vein, at one island some suggested their guests visit the nearby mountain by trekking and have a community tour in a nearby village instead of choosing activities at the beaches and sea (e.g. fishing). A point noted by Becken and Wilson (2013) is that it is reasonable and common for tourists to change their activities due to bad weather. Tourists often tend to change an original destination with an alternative choice once the original 
destination becomes unavailable or they are unhappy with their experience and expectations (Han, Noh, \& Oh, 2015).

Second, $11.5 \%$ of them have created short and long-term plans for better adaptation, the creation that initiates a programme (e.g. Green Programme) to be self-sustaining in changing climate. For instance, one said that maintaining and expanding gardens was needed in the hotel management plan while others were currently unclear about what to do concerning their plans but would definitely formulate adaptation initiatives. This is a subject for further discussions with relevant people in their businesses, especially other members of their management team.

Third, in a few cases $(5.8 \%)$, the participants provided their staff free transportation to commute back and forth to work during bad weather such as strong storms. The purpose was to enable the staff to continue working or the hotels would have labour shortages. At the beaches, they must be more careful about the storm surges than those on the mainland. In the meantime, they kept encouraging their staff to work dynamically with flexibility. Olazabal et al. (2017) agree that flexibility allows preparedness for unpredicted climate events and efficient use of existing resources.

\subsubsection{Climate change policy adaptation}

Data reveal that the hotel sector employed policy adaptation for their hotels' adaptations. Based on the interviews, they adopted a compliance of (self-) regulations ${ }^{34}$ in building design standards for CCA in response to climate change impacts such as wind forces.

\footnotetext{
${ }^{34}$ Literature review and interviews revealed that the government and authority issued many policies and regulations in the construction sector. Yet due to the high requirement and cost, almost all hotels do not obey regulations imposed by the government. Instead, individual hoteliers adapt self-regulation in their constructions with minimum considerations for the government's regulations. This (self-regulation) contributes to CCA in their hotels.
} 
Table 5. 13: Policy adaptation employed by the hotel sector

\begin{tabular}{|c|c|c|}
\hline \multirow{2}{*}{$\begin{array}{c}\text { Compliance with (self-) regulation } \\
\text { (e.g. building design...) }\end{array}$} & \multicolumn{2}{|c|}{ Responses } \\
\hline & $N$ & Percent \\
\hline Yes & 16 & 36.3 \\
\hline Not sure & 14 & 31.8 \\
\hline No & 14 & 31.8 \\
\hline Total & $44^{35}$ & 100.0 \\
\hline
\end{tabular}

First, Table 5.13 indicates that nearly one third $(36.3 \%)$ of them complied with selfregulation in hotel constructions (e.g. building design). Some explained that their hotels were built with strong attention paid to the current and future climatic change, particularly on the rooftops. The rooftop was changed from plastic to metal roofing and the hotel structure was designed to allow sufficient airflow and sunshine by bringing in architects from overseas. The hotels were built to allow sufficient airflow in and out of the hotel through particular structures such as many holes of brick wall, thus making the hotel less vulnerable to strong wind. Land subdivisions and the balance of the building were clearly taken into consideration and analysed during the construction. One of them explained, "Cambodia is different from other countries we must have our own techniques. We build, and we think of the hot weather that is why we don't build the ceilings high. We must comply the standard of $2.70 \mathrm{~m}$ or 2.80m.” H7.

At the islands, they reported that they took the hotel structures into consideration, especially during the construction. One of them said, "I think we build this place with all of these in mind because once the owners arrived in 2005. We're aware of this problem and when they built this place, they built it knowing what the problem especially on the climate change or environmental issues.” H46.

Second, almost one third (31.8\%) of them pinpointed that they likely complied with selfregulations in their hotel buildings for climate change response. All of them informed that the constructions were somehow built with moderate consideration of CCA. One foreign informant said, "Not enough, they could have been much better, I'll give you an example.

${ }^{35} 6$ data missing 
Although insulation is known and available in Cambodia, in our roof we've got no insulation." H10. A few representatives from newly built hotels either agreed or disagreed that their hotel constructions were adaptable to climate change. They referred the questions to the owners and contactors. One hotel participant said, "Concerning the building and climate change, I don't know what he (the boss) did." H14. A second added, "All of these were the works of owner and architect." H19. Other respondents explained that the current hotel buildings had been renovated (mainly the leased hotels). It is unsure as to whether the construction complied with policy adaptation.

Third, almost one third (31.8\%) of them confirmed that they did not comply with policy adaptation (e.g. building design) for climate change responses. They said the constructions had been built based on minimum experience of the designers and owners. One participant indicated, "Yes I understand, no, I think they don't think about it..., just build it." H23. The second said, "Basically we have this place built by ourselves, we designed it by ourselves. We're not expert in it so we make quite a lot of mistakes you know, we know it. You know, we just learn it's experience, sort of kicking out our mistakes." H28. Others explained that due to the unresisting hotel buildings or lack of defence mechanism, there were a number of consequences such as leakages from the rooftops to the hotel rooms. The whole hotel buildings pose serious weaknesses in climate change responses.

It is crucial to note that in Cambodia and SHV, there are many policies and rules available for hotel operators to comply with, such as the building standard for wind forces. However, in practice few business operators have complied with the CCA policies or regulations imposed by the government/authority. While the government/authority themselves do not have capacity to enforce the policies, the private sector only minimally respects the policy standards, such as only obtaining construction permits. Other major climate change construction policies such as instituting seawalls are not fully respected. The discussed policies for CCA above were mainly not the ones imposed by the government/authority. They are the individual-based policy standards. In private sectors like the hotel business, there are no policies currently available for evaluating CCA practices such as building standards for wind forces. 


\subsubsection{Climate change research adaptation}

Table 5. 14: Research adaptation used by the hotel sector

\begin{tabular}{|c|c|c|}
\hline \multirow{2}{*}{$\begin{array}{l}\text { Research to assess the business operations } \\
\text { and awareness of staff knowledge gaps }\end{array}$} & \multicolumn{2}{|c|}{ Response } \\
\hline & $N$ & Percent \\
\hline Yes & 5 & 11.1 \\
\hline $\mathrm{No}$ & 40 & 88.8 \\
\hline Total & $45^{36}$ & 100.0 \\
\hline
\end{tabular}

Table 5.14 shows that only $11.1 \%$ of them conducted research to assess staff awareness and knowledge gaps on climate change. One said that there was a question of high expense in the hotel towards the energy consumption. A research team conducted closer research into the issue. The second studied why the hotel was vulnerable to climate change such as high rainfall. As a result, it was found that it was the contractor's mistake. The participant complained, "We assessed but all blames are put towards the climate change impacts. The techniques of the contractors are main causes. They're local/provincial contractors." H43. For another example, a higher standard hotel representative claimed that there was a research and programme for climate change actions. The participant said, "Yes that would be very good, and we definitely do more research and now that this program is actually kicked off. That's what we take care of this (climate change)." H46. According to Niang-Diop and Bosch, (2005), research, development and innovation are necessary as responses to climate change. These could propose specific measures to climate change vulnerability, for example the economic assessment of adaptations, technological adaptations and examination of new alternative sources of water and high-quality resource management.

However, up to $88.8 \%$ of participants said that they have not researched to assess awareness and staff and knowledge gaps on climate change. Instead, they just orally advised their staff to provide good services and take care of guests' conveniences without clear or accurate assessments on climate change issues. Some expressed that the assessments were only done to evaluate the working performances of staff while research to assess climate change issue

${ }^{36} 5$ data missing 
is several steps away. They reported that there are a few barriers in assessing awareness and staff and knowledge gaps on climate change. First, it is the frequent staff turnover and the low educational background of the staff. Sometimes employees resigned after working just 3-4 months. Second, it is the time constraint. Time should be spent on other commitments rather than the research. The climate change was of little concern in terms of research. One added, "As I mentioned we have little concern on the climate change on our hotel, we have not had many surveys. We should not be busy on that climate change plan (research), but we rather physically prevent it." $\mathrm{H} 21$. Third, a few respondents shifted this research obligation to the government/authority. They argued that this should be the task of the government/authority to conduct such research for the hotel sector.

\subsubsection{Climate change education adaptation}

Table 5. 15: Education adaptation employed by the hotel sector

\begin{tabular}{|c|l|c|c|}
\hline \multirow{2}{*}{$\begin{array}{c}\text { Adapting to drought, rising temperature, excessive } \\
\text { rainfall, storm, storm surges and beach erosion }\end{array}$} & \multicolumn{2}{|c|}{ Responses } \\
\cline { 2 - 4 } & Inform/advise guests and staffs & $N$ & Percent \\
\cline { 2 - 4 } \multirow{4}{*}{$\begin{array}{c}\text { Education } \\
\text { adaptation }\end{array}$} & Obtain climate change trainings & 7 & 32.0 \\
\cline { 2 - 4 } & $\begin{array}{l}\text { Attend climate change-related public } \\
\text { campaigns or events }\end{array}$ & 5 & 28.0 \\
\cline { 2 - 4 } & Train staff about climate change & 5 & 20.0 \\
\hline Total & 25 & 100.0 \\
\hline
\end{tabular}

Based on Table 5.15, first, nearly one third (32\%) of them took actions by informing/advising their staffs and guests. They informed their guests to be careful about possible impacts, especially for those who want to go to the islands. Prior to informing their guests, they must check the weather situation with relevant parties, notably the boat companies about the possible impacts such as heavy rains. They must tell the guests to have necessary preventive measures such as life jackets if they ultimately risk going to the islands. Incredibly, although even when informed about possible adverse weather conditions, a minority of guests risked visiting the islands. They also informed their staff to proactively take care the hotel properties and watch out for vulnerable objects. One respondent said, "I tell the staffs to take care about 
the hotel properties to avoid getting affected by heavy rains. I tell them to find waterproof shelter to cover properties to avoid being wet." H31. In a similar vein, they adapted to storm by informing their staff and guests of the possible impacts. They basically warned their guests not to swim in the sea due to possible large wave occurrences whereas the staff were informed to collect all possible flying objects/inventory, including big umbrellas, to a safe place. One indicated, "First, we move all things for example the tables, glass, and table cover that can be blown up. We must prepare all these things especially at the rooftop, the most vulnerable place." H9. This had to be done for the safety of their own staff and so that their staff were fully ready to help the guests. Some of their staff were more experienced and had gotten used to the changing weather owing to their family background as the descendants of fishermen. Jeuring (2011) and Becken and Wilson (2013) provided an important finding that the use of information leads to better options and helps to protect tourists from extreme weather events. The hotel sector responds to cyclone or storm warnings by taking preventive actions by removing surplus branches from trees, particularly those close to buildings and electric and telephone equipment and servicing of the sewerage system (WMO et al., 2008).

Second, 28\% of respondents informed that there was training, or best practices related to CCA offered by the local government and an NGO partner. One who joined climate change training by the local government said, "Soft and hard adaptation is what I've learned from the workshops in relation to SLR.” H6. Another participant who received the training from an NGO partner explained, "I think that's why we have opened the dialogue with the government and we have a lot of members of the government that come and stay here and try to learn more about how we are sustainable, why we're sustainable, how the sustainability is determined?" H46. Niang-Diop and Bosch (2005) note that education and training about climate change issues at different levels would help to capacitate stakeholders so as to support adaptation and could formulate suitable research activities and a greater understanding among citizens.

Third, $20 \%$ of respondents said that they have attended climate change-related public campaigns or events. The campaigns were mainly initiated by the government/authority such as SHV DOT. The campaigns were mainly about the environmental awareness while climate 
change issues were limitedly included in the events. One explained that whenever there were requests/invitations from government/authority, the hotel representative always joined the events, the campaigns that inform the public about the benefits of planting trees. Yet they had to bear cost for buying the trees. Another informed that there was a public campaign awareness of drowning incidents as well. Niang-Diop and Bosch (2005) further explain that public awareness campaigns enable the raising of awareness and spreading information, which increases the concern and participation of a wide range of stakeholders. They also provide an opportunity for adaptation decision actors to better understand the perception and opinions of the public on CCA.

Fourth, $20 \%$ of them said that they have provided their staff with climate change-related training. The first said that the purpose of training was about environmental sustainability, with some syllabus about climate change issues. The participant claimed, "The training is ongoing all the time, all the time we're running a workshop, concentrating on sustainability.” H10. The second said the training programmes were about how to deal with leakage and selfpreparations for climate change issues in rainy seasons. A third added that training about

preventive and post-interventions on drowning incidents was given to the staff. Anderson (2012) argues that "education is a critical component of adaptive capacity" (p. 193). The approach is that people are educated and trained, and the syllabus of education and training can equip people with the knowledge and skills. This knowledge is necessary to make decisions about how to adapt to different ecological, social or economic systems in an environmental change (Anderson, 2012).

\subsection{Chapter summary}

Building the vital relationship between vulnerability and adaptation is the main purpose of this chapter. The first section determined to what extent SHV's coastal tourism (destination level) and its hotel sector (sector level) are vulnerable to climate change from the perceptions of the 50 hotel participants, with the support of the available scientific evidence of existing data. As a result of vulnerability assessment, it is found that both SHV's coastal tourism and its hotel sector are vulnerable to climate change. The finding also presents that SHV's coastal tourism and its hotel sector are environmentally, socio-economically vulnerable to climate 
change. SHV's coastal tourism is perceived as being more vulnerable than its hotel sector. The environmental vulnerabilities include excessive rainfalls, storms, rising temperature, SLR/beach erosion/storm surges and prolong drought. The economic vulnerabilities include the damage of infrastructures (from excessive rainfall and storm/storm surges) (e.g. roads, beaches), spread of diseases (from the increased temperature and exposure to rain), and economic pressure (e.g. income loss).

After the vulnerabilities were identified, SHV's hotel sector initiates adaptations. There are five adaptation types: technical, managerial, policy, research and education, which were mentioned during the interviews. Technical adaptation actions are most significantly employed, followed by managerial and education adaptation. Policy and research adaptations are the least used by SHV's hotels. 


\section{CHAPTER 6: BARRIERS AND ENABLING FACTORS IN CLIMATE CHANGE ADAPTATION}

\subsection{Introduction}

The previous chapter (chapter 5) developed the link between vulnerability and adaptation. Following the steps outlined in the analytical framework (see Figure 6.1), this chapter investigates the perceived barriers and enabling factors in climate change adaptation. The critical importance of each barrier and enabling factor closes the knowledge gap in research that has been carried out in similar areas because little research has been done to address the issue. Previous research has focused on the status of adaptation measures and barriers and enabling factors separately without bringing both conditions (barriers and enabling factors) in the same study. Prior research also largely ignored the perceived rankings of these barriers and enabling factors.

The chapter is divided into two main sections. The first part presents barriers to CCA (informed by the literature in CCA in the context of developing countries) and highlights the criticality level of those barriers through the perceptions of respondents through mean test and standard deviation (SD) by measuring on a six-point rating scale with 0 being 'uninfluential' barrier and 5 being 'very major' barrier. Additionally, the actual barriers to CCA of SHV's hotel sector are also presented.

The second part of this chapter outlines enabling factors to CCA (informed by the literature in CCA in the context of developing countries), highlighting the criticality level of those enabling factors through the perceptions of respondents or mean test and SD by measuring on a six-point rating scale, with 0 being 'uninfluential' enabling factor and 5 being 'very important' enabling factor. Additionally, the actual enabling factors to CCA of SHV's hotel sector are also outlined. 
Figure 6.1: Analytical framework

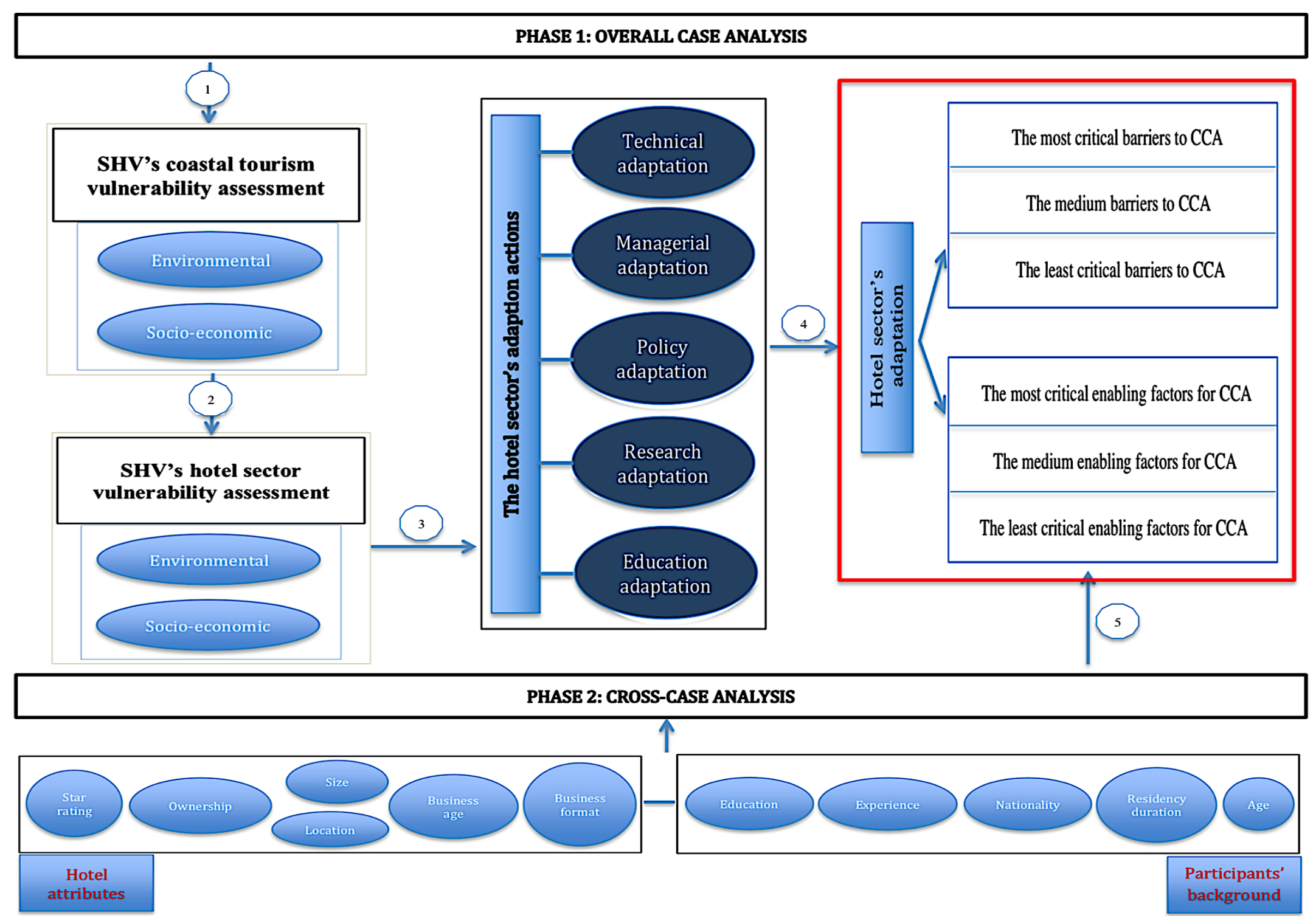




\subsection{The perceived ratings of adaptation barriers in the hotel sector}

As described in chapter 4, a list of barriers (informed by the literature in CCA in the context

of developing countries) was given to the participants. The perception of barriers to CCA was investigated by asking respondents to rate each of the nine potential barriers to CCA. Table 6.1 shows the Likert-scale results (in \%), the mean ratings, and the SD. The perceptions of barriers in the hotel sector in SHV were measured on a six-point rating scale with 0 being 'uninfluential' barrier and 5 being 'very major' barrier. Each distinction is detailed in the next sections: 
Table 6. 1: Rating results of adaptation barriers in the hotel sector

\begin{tabular}{|c|c|c|c|c|c|c|c|c|c|c|c|}
\hline \multirow[b]{2}{*}{ No. } & \multirow[b]{2}{*}{ Barriers } & \multicolumn{6}{|c|}{ Likert-scale results in \% } & \multirow[b]{2}{*}{$\begin{array}{c}\text { Total in } \\
(\%)\end{array}$} & \multirow[b]{2}{*}{ Mean } & \multirow[b]{2}{*}{ SD } & \multirow[b]{2}{*}{$\begin{array}{l}\text { Level of } \\
\text { criticality }\end{array}$} \\
\hline & & $\begin{array}{c}(0) \\
\text { Uninfluential }\end{array}$ & $\begin{array}{c}\text { (1) } \\
\text { Very small }\end{array}$ & $\begin{array}{c}(2) \\
\text { Small }\end{array}$ & $\begin{array}{c}(3) \\
\text { Moderate }\end{array}$ & $\begin{array}{c}(4) \\
\text { Major }\end{array}$ & $\begin{array}{c}(\mathbf{5}) \\
\text { Very } \\
\text { major }\end{array}$ & & & & \\
\hline 1 & Limited resources & 0 & 0 & 0 & 8 & 27 & 65 & 100 & 4.56 & 0.649 & \multirow{3}{*}{$\begin{array}{l}\text { The most } \\
\text { critical }\end{array}$} \\
\hline 2 & Limited knowledge/perception of climate change & 0 & 0 & 0 & 14 & 23 & 63 & 100 & 4.49 & 0.739 & \\
\hline 3 & Lack of political will & 0 & 0 & 0 & 4 & 44 & 52 & 100 & 4.48 & 0.586 & \\
\hline 4 & Insufficient information & 0 & 0 & 2 & 13 & 22 & 63 & 100 & 4.46 & 0.798 & \multirow{3}{*}{ Medium } \\
\hline 5 & Uncertain responsibilities for adaptation & 0 & 0 & 2 & 10 & 41 & 47 & 100 & 4.33 & 0.747 & \\
\hline 6 & Low level of leadership and guidance & 4 & 0 & 0 & 8 & 31 & 57 & 100 & 4.33 & 1.107 & \\
\hline 7 & $\begin{array}{l}\text { Insufficient engagement and interaction with } \\
\text { other stakeholders }\end{array}$ & 0 & 0 & 0 & 30 & 27 & 43 & 100 & 4.16 & 0.85 & \multirow{3}{*}{$\begin{array}{l}\text { The least } \\
\text { critical }\end{array}$} \\
\hline 8 & Regulatory/legal constraints & 4 & 0 & 2 & 23 & 27 & 44 & 100 & 4 & 1.203 & \\
\hline 9 & Institutional barriers & 2 & 0 & 10 & 28 & 28 & 32 & 100 & 3.78 & 1.141 & \\
\hline
\end{tabular}




\subsubsection{The most critical barriers to climate change adaptation}

The results in Table 6.1 show the three most critical barriers (limited resources, limited knowledge/perception of climate change and lack of political will) with the largest mean response on a 0 to 5 Likert scale (with means of $4.56,4.49$ and 4.48 respectively). Based on the mean rankings and frequency/percentage distribution of Likert point ( 0 being 'uninfluential' and 5 being 'very major'), it shows that the respondents from the hotel sector in SHV highly perceive these three obstacles as the greatest barriers to CCA. Each distinction is detailed in the next sections.

\section{Limited resources}

'Limited resource' refers to the lack of both tangible and intangible resources such as technical materials/budget (budget for implementing adaptations) and HR (skilful and qualified individuals). The 'resources' can be external (national/local government) and internal (hotel sector) capital that is available for adaptation actions and contributes to the achievement of adaptation in the hotel sector.

As can be seen from Table 6.1, the barrier entitled 'limited resources' has the highest mean rating of 4.56 (with $27 \%$ being 'major' and $65 \%$ being 'very major'). In the respondents' perception, it is the greatest barrier in hotels' adaptations. The participants explained that limited money and HR as well as technology and technical materials challenge hotel operators to initiate and facilitate prompt adaptations. In contrast, when money is reserved and allocated, hotel operators could bring technical skills (employing highly qualified staff) and technical materials (e.g. building insulators) to deal with climate change. While skilful staff can identify risks and diversify strategy to minimise the impacts, the technical materials such as insulators do not readily allow the passage of heat or sound. With 'limited resources', hotel operators cannot develop new projects such as installing water filtering systems (to adapt to rising temperature) because they could not afford them. In the meantime, the maintenance costs would deteriorate financial problems. Some mentioned that their expenses must be worthy and necessary spending concerning CCA because their businesses can only generate limited income or marginal benefits. In this case, some reserved budgets were available but not for the actions like climate change. One participant expressed, "Sometimes, 
the technical equipment is high in price and we don't have enough budget (to purchase them)." H31.

Only $8 \%$ of the remaining respondents rated it as a moderate barrier. They thought that their resources depend on seasons. While in low season there are fewer resources, and in high season there are more. One foreign respondent (who just resided in SHV) mentioned external resources that might be helpful for her/his hotel. She/he expressed that it was unknown how much budget the Cambodian government has allocated to the hotel sector. The participant further explained that in European Union (EU), the governments have provided numerous motivations such as electric power to private sectors such as hotels, which contribute to adaptation outcome.

\section{Limited knowledge/perception of climate change}

Table 6.1 shows the second greatest barrier to CCA, which is the second highest mean score of 4.49 (86\% being 'major' and 'very major'). As addressed in literature in section 2.9.2, 'limited knowledge/perception of climate change' is the lack of basic knowledge about causes, effects and solutions to climate change, the knowledge that allows hotel operators and/or relevant stakeholders to adapt to climate change productively.

One SHV-native participant explained that insufficiency of knowledge severely impedes all activities towards the solution of climate change problems. For instance, hotel operators might not know what to do regarding rising temperature. Others argued that although people have abundant resources such as technical materials in place, they might still face challenge without sufficient knowledge. Furthermore, if hotel managers do not have knowledge, they could not think of any innovative or creative solutions to advise their staff to reduce climate change impacts. Others recommended that to overcome this barrier, new people with high knowledge/perception of climate change should be hired in the hotel sector. One expressed, "We have difficulties; we cannot adapt because our staffs are low-educated, quite hard to explain them to have solutions." H13. 
$14 \%$ of informants rated this second greatest barrier as a moderate barrier. Two Khmer participants explained that knowledge/perception of climate change was not everything about CCA. Technical knowledge/skill/experience and technical materials play more important roles. As long as people have techniques and technical materials to respond to, basic knowledge/perception of climate change should be sufficient for CCA. One of respondents stated, "If someone has sufficient knowledge but limited materials, he/she cannot do anything. He/she knows the climate change will happen, knowing what to do, but he/she doesn't have materials, is impossible." H14. One more Khmer participant explained that while the hotel was having sufficient resources such as HR, technical knowledge/experience and technical materials, what they needed was a certain amount of time to get the knowledge/perception of climate change through training or reading reports. It is a matter of time. Available time was perceived as more essential than 'the knowledge/perception of climate change'.

\section{Lack of political will}

As can be seen in Table 6.1, the third most critical barrier is 'lack of political will', with the third highest mean rating of 4.88 with $52 \%$ being 5 'very major'. Referring back to the literature in section 2.9.7, the barrier entitled 'lack of political will' refers explicitly to the lack of the participation and motivation of both the national and sub-national government towards the SHV's hotel adaptation.

Of all 50 respondents, up to $96 \%$ of informants rated this third most critical barrier as being 'major and very major'. Nearly all of informants illustrated that politics influences everything. However, in developing countries, there is a frequent lack of political participation, including climate change issues. One stated, "I mean, there is will there is everything. If no will, even though we have knowledge, if we have no will to help develop or improve ourselves, it doesn't produce good result.” H1. While the hotel sector has not had the full capacity to adapt, the government people at national level often ignore climate change issues. This leads to a serious disadvantage such as having limited actual adaptation initiatives in place. Shortness of political will/leadership means that there are insufficient policies to respond to climate change. It lacks knowledge, goal and prior planning from the 
current government. For example, the knowledge on how to save water and power by exploiting from the country's resourceful sunshine (e.g. for solar-powered hot/cold water system) to minimise costs from increased demand of power/water is significantly unavailable due to climate change. The first participant added, "No political influence, no goals, nobody says we want to do. Nobody gives the knowledge to people on how to save water, how to keep power, how to use the sun...” H44.

Some mentioned that the government is a public body that surely has the highest capacity such as knowledge and technical skills as well as mechanisms to protect the hotel sector from climate change impacts. Another explained, "Yes, leaders are a steering wheel and engine to lead the country. If they don't believe this issue climate change, how can we have packages of budget or program to respond or adapt.” H6. Two foreign respondents thought that lack of political motivation of the current government might be due to their limited knowledge about climate change. One of informants argued, “I don't think they don't want to; I think they don't have the knowledge. There should be there should be an advisory board to the government...” H10. As outlined in chapter 2, Cambodia has formed an inter-ministerial committee (advisory board) to tackle climate change issues but the scale of effect of their work is at the national level. The positive impacts of their work may not reach the local level. That is why the respondent $(\mathrm{H} 10)$ did not feel the impacts.

$4 \%$ of the remaining respondents rated the third greatest barrier entitled 'lack of political will' as being moderate. They argued that CCA should be self-initiated measures, which generate their own organisational commitment to implement, rather than depending on external conditions. One of informants stated, "It is also a barrier but there must be other principles or ways to reduce (climate change impacts) rather than depending on the politicians.” H5. One Khmer participant reported that although the politics influence every problem, the government/authority either rarely helps or their assistance is not fully reliable. This is the case for developing countries like Cambodia. The participant illustrated that the hotel sector must additionally cover all deficiency needs in adapting to climate change. 


\subsubsection{The medium barriers to climate change adaptation}

Measured by mean ratings, Table 6.1 shows the second category of perceived medium barriers to CCA, which include 'insufficient information', 'uncertain responsibilities for adaptation', and 'low level of leadership and guidance' with mean response on 4.46, 4.33, and 4.33 respectively. In this second category, the frequency/percentage distribution of being 2 'small' and 3 'moderate' can be seen 15\%,12\% and $8 \%$ for the first, second and third medium barriers respectively. It is also noticed that $4 \%$ of the remaining respondents illustrated that the barrier of 'low level of leadership and guidance' is an 'uninfluential' barrier for their hotels' adaptation.

In these barriers, the distribution of respondents can be seen at the scale of being (2) small. This makes these barriers no longer the most critical (but medium barriers), although the mean differences between these barriers and the first three barriers are small.

\section{Insufficient information}

Mainly referring to the literature in section 2.9.3, this first medium barrier entitled 'insufficient information' singles out the limited information about climate vulnerabilities, or absence of reliable climate data. All respondents understood that information is from two sources: individually accessed information and information provided by external parties, especially the national and subnational government. For them, the information is not only closely related to their climate change adaptation initiatives but also determines the profitability or failure of their adaptation efforts.

It is revealing that in Table $6.1,23 \%$ and $63 \%$ rated this first medium barrier entitled 'insufficient information' as a (4) 'major' and (5) 'very major' barrier. In SHV, the respondents reported that the relevant stakeholders have a weak capacity (especially the local government) in providing (accurate) climate change information. Hotel managers must depend on themselves to access to climate change information (without external assistance). Sometimes in a normal climate, storm suddenly comes without prior notice from relevant parties and nothing could be prepared to respond to it. This is indicative of information shortfall. They continued that climate change is a phenomenon that sometimes happens 
quickly and is short-lived, but its intensity is unpredictable and presumably high. One of informants stated, "With information, yes, I can work with the information. Lack of information, I don't see the problem, it's hard sorting out the problem. This is a huge obstacle." H27. The second added, "Insufficient information is like we are completely blind, seeing nothing ahead (of time).” $\mathrm{H} 45$.

They continued that this barrier is a primary and persistent deficit for the adaptation of SHV's hotel sector. In another example, when storm was predicted as low, it actually came unexpectedly, and with strong intensity. The participants received contradictory messages from the ministries and other sources. One foreign participant explained that while information in Khmer might be available, the information in English is largely unavailable in SHV. One informant mentioned, "This is also a barrier. If we completely have no information how can we adapt? ... We have information but not enough. We will still face the serious problem. When the climate change occurs, it is serious.” H22. They illustrated that sufficient information basically allowed the participants to prepare necessary materials to brace for climate change.

$15 \%$ of the remaining respondents felt that it was a (3) 'moderate' and (2) 'small' barrier. One Khmer participant felt that information has already been exchanged between the government/authority and hotel sector. In whatever situation, $100 \%$ of information exchange was never guaranteed in the developing countries like Cambodia. It was not surprising, because insufficiency normally happens in the process of information exchange due to the weakness of information sharing arrangement/structures in Cambodia. The second respondent thought that SHV native people did not find themselves difficult to access to climate change information. The participant stated, "Because I live here, the information issue is not a big barrier because we are SHV people (natives)... Yes.” H40. Of this group, one participant from a 5-star hotel rated it as a small barrier. The participant stated that the most important condition is capable HR that could carry out communication and networking. Once the quality of HR is strong, information would come to the fore. It was believed that information has little effect on CCA. 


\section{Uncertain responsibilities for adaptation}

Concerning this second medium barrier entitled 'uncertain responsibilities for adaptation', which appeared in previous studies in section 2.9.4, all 50 respondents understood that an institution or organisation, especially the hotel sector, has not formulated responsible units or clear working arrangements that purely deal with climate change impacts. They tended to believe that the public sector rarely formulates responsible groups or experts for tackling climate change issues. Responsibilities for adaptation in the private sector such as their hotels vary, depending on the actual situations and available resources.

$41 \%$ and $47 \%$ of the respondents felt that 'uncertain responsibilities for adaptation' were 'a major' and 'very major' barrier. They explained that whenever climate change threatens their guests/tourists and if there is no responsible unit to advise them on what to do, this creates inconvenience for them. One Khmer respondent added, “Yes, it is. If we don't know who's responsible, although we have numerous ways (strategies), it's too late, too late for solutions." H14. The second added, "This is a big issue. True, when we cannot take our own stance, we don't know who does what for climate change responses." H6. They added that whenever individual staff were not aware of their own work, the outcome to respond to the impacts would not be secured. One participant added, "Because ... in general in working conditions, if there are uncertain responsibilities, we don't know what to do. We don't know what sections to be shared the information. This factor makes hotels difficult in CCA.” $\mathrm{H} 22$.

Limited cohesive governmental organisational structures, which they do not react quickly to climate change, are also a huge barrier for hotel operators. For example, because there are no clear responsibilities from the government/authority, the hotel sector's adaptation initiatives cannot be ensured. They stated that in Cambodia, the government is a major institution with more resources such as finance and HR, but sometimes they could not respond to climate change effectively. They, therefore, recommended clear/certain responsibilities for CCA in both the governmental institutions and hotel sector.

$12 \%$ of the remaining participants thought that it was a (3) moderate and (2) small barrier. One Khmer respondent thought that in Cambodia and SHV, after the climate change impacts, 
the local government might form a team to tackle the issues. They might involve helping people. They believed that the government would form a responsible unit and send it to the affected areas. This is a matter of time. It is also a bit time consuming. They believed that the hotel sector might also formulate their own teams to intervene. It is their businesses that must be taken care of. Hotel managers need a bit of time to think of the problems first before formulating responsible teams subsequently. Once the problems become bigger, the responsibilities for CCA might become greater as well. One participant rated this barrier as being small. He/she indicated that major responsibilities for CCA are really needed. Forming a good, responsible unit would take time and add complexities to the problem because climate change needs high flexibility and quick solutions. Simple responsibility should be enough. For example, whenever it rains, the guests are just advised to stay in the rooms and the staff are just encouraged to work harder.

\section{Low level of leadership and guidance}

Mainly observed in the prior studies in section 2.9.6, the smallest medium barrier of 'low level of leadership and guidance' is the inability of leaders and managers who are unable to guide the direction of adaptation in their own working arrangements specifically in their hotels. This may include the leadership and management of stakeholders, particularly the local and national government, who provide parental guidance on hotel sector's adaptation.

Results in Table 6.1 indicate that $31 \%$ and $57 \%$ of the participants believed that 'low level of leadership and guidance' was 'a major' and 'very major' barrier. They noticed that being unable to manage particular problems including climate change often causes a number of serious weaknesses. They added that low level of leadership and guidance often has its roots in improper plans and management. Company leaders are generally responsible for all issues, whether minor or major problems. If they do not care about climate change effects, they put their businesses at high risk. Although the lower level staff have an appropriate idea on how to adapt to the change of climate, leaders might not understand and listen to their staff. One

of informants indicated, "For me, whenever there are inconsistencies between management and staff, it is the biggest barrier." H12. The interviewees indicated that whenever the leaders become weak in adaptation decision and guidance, their staff have the same trend. For 
instance, leaders do not know the root cause of climate change, even a minor task like an adjustment of ACs for room environment; this would constitute a barrier.

They further explained that the top management are the key decision makers. They must make decisions on whichever choice is better for CCA. The management must be the most responsible unit, including for the information flow. One informant indicated, "It is clear, if the leadership is not standardized, there must be a problem because the leaders don't know how to advise or to prepare." H14. Outside the hotel sector, the local authorities are like bosses/leaders. The hotel adaptations need their leadership and management. If they were unaware of issues relevant to CCA, the hotel sector would experience difficulty, creating inconsistencies in adaptation efforts.

$12 \%$ of the respondents identified 'low level of leadership and guidance' as a moderate and uninfluential barrier. The participants perceived that whenever something wrong happens in their places/hotels, they must automatically react to the identified problem. They indicated that their management team must not be too passive but reactive in nature One of informants indicated, "In hotels if we're being affected, everyone must know their jobs. It's their jobs. If they lose guests, they lose money, that's simple.” H27.

\subsubsection{The least critical barriers to climate change adaptation}

The results in Table 6.1 show the three least critical barriers (insufficient engagement and interaction, regulatory/legal constraints, and institutional barriers) with the smallest mean response on a 0 to 5 Likert scale (with means 4.16, 4 and 3.78 respectively). This finding shows that respondents from the hotel sector in SHV have the lowest perception on these three obstacles as the least critical barriers to CCA. These perceived conditions do not constitute significant obstacles in their adaptation activities for their hotels. The frequency/percentage distribution of being (3) 'moderate', (2) 'small' and (0) 'uninfluential' has increasing trend with $30 \%$ being (3) moderate, $29 \%$ from being (3) moderate to (0) uninfluential, and $40 \%$ from being (3) moderate, to (0) uninfluential for the first, second and third critical barriers respectively. Each barrier is detailed below: 


\section{Insufficient engagement and interaction}

As mainly detailed in the literature in 2.8.8, it was understood that this first critical barrier, entitled 'insufficient engagement and interaction', is the inability of hotel operators to interact and communicate sufficiently between three layers: national, provincial and local or among other stakeholders as well as their peer group for the sake of their hotels' adaptations.

Up to $30 \%$ of the respondents stated that it is a just moderate barrier. They indicated that in running businesses, especially hotels, people tend to communicate to exchange information. The engagement and interactions with relevant stakeholders exist in their daily work. They explained that although engagement and interaction are not exchanged smoothly among the government/authority, this is not the case in the hotel sector. It is significantly strengthened and improved among hotel operators in SHV, especially concerning CCA. One of informants indicated, "For hotel and hotel (peer group), we have enough interactions, but we contact the district level or authorities, but they don't contact us back, yes. So, the problem is not from our side.” H50.

Table 6.1 indicates that only $27 \%$ and $43 \%$ rated 'insufficient engagement and interaction' as a major and very major barrier. They thought that in SHV, although engagement and interaction have significantly improved among hotel operators, it remains limited among government people themselves, especially between the government and hotel sector. One added, "True because due to this, they (the government) don't care about me as a small business.” H30. Insufficient engagement and interaction might lead to having different wills among hotels that create conflicting ideas, especially on how hotel sector should work together to respond to climate change. One of informants explained, “... why? Because if we lack engagement and interaction, and when the problems happen, we cannot solve them.... If we are weak at communication, that's our barrier.” $\mathrm{H} 21$.

They continued that this barrier significantly reduces information exchange. With this limitation, nothing could be heard about climate change and adaptations. Those who are working in the hotel sector should be interactive to each other so that prior adapting actions or preparation are made available. With limited engagement and interaction, the 
government/authority does not know well what the hotel sector is currently facing, for instance the shortage of water and power supplies. The government/authority might not be able to share climate change-related knowledge with the hotel sector. One participant stated, "Normally we don't know for sure what materials/facilities to take measure; only the government does. So, when there is a lack of communication to lower level, then this is a difficult condition.” $\mathrm{H} 22$.

\section{Regulatory/legal constraints}

Readers might only understand that 'regulatory/legal constraints' in here refers to the lack of limitations of regulations and rules on CCA in the hotel sector. Although this is the case for Cambodia, this barrier in this study also means that there are too many general (non-climatic) regulations and rules in Cambodia as well as SHV that contradict each other, thereafter complicating and limiting the ability of local levels such as the local authority and the hotel sector to respond to climate change. In Cambodia, many regulations and rules have been enacted. However, these also constitute a barrier. First, it is due to the shortage of actual implementations. Second, it is because of the bureaucracy and complications in the enforcement. Regulations or rules could contradict each other or were not well designed for implementation at the regional/local level. There is a lack of holistic coherence in regulations and rules in either formulations or implementations. Also, these also do not provide coherency for CCA. These in turn have hampered the ability of the private sector such as the hotel sector to follow, one of which are the climate change adaptation actions. The role of the hotel sector was largely neglected. Every interviewee in this study understood this.

Up to $29 \%$ of the respondents perceived that the barrier of 'regulatory/legal constraints' was a moderate, small and uninfluential barrier. They reported that in general, regulations and rules were vividly enacted. A respondent indicated, "Not so big because to approve laws/regulations is not be done by one person." H5. There are likely no laws, rules and regulations conflicting each other or limiting the ability of the hotel sector and local authority to adapt. They further exemplified that the national level does not support hotel sector with sufficient budget; this social norm or management/practice is not an issue. This is normal in developing countries like Cambodia. 
One respondent was an island participant (at Koh Rong Island). The respondent thought that the current laws, rules and regulations for the hotel sector's adaptation are much supportive. The participant exemplified that the local authority bans the construction of hotels higher than five floors (at the island). Using the current regulations, the ban is to reduce pressures of hotel business from the intensity of storm. The respondent explained that law is law; this is a norm in every country. This was not a huge restriction for the hotel and people must respect it.

Two respondents had a similar view that the 'regulatory/legal constraints' were not an impactful barrier to CCA. One explained that the approved laws/regulations/rules have overall purposes of helping people. From their practices of climate change adaptation, they thought that there was no way that regulations and rules constrained the ability of the national and local level and stakeholders to adapt to climate change.

$27 \%$ and $44 \%$ of the remaining respondents rated the second least critical barrier entitled 'regulatory/legal constraints' as major and very major. They explained that in other countries, the laws/regulations are helpful for their people and businesses while some laws/regulations in Cambodia do not. They were aware that laws or regulations at the local and national levels might be inconsistent with each other. For example, while the national level has lacked budget, the provincial/local level needs more budget for adaptation. They reported that these constraints have threatened and limited the hotel sector and local capacity to adapt. One indicated, "Yes, it is a barrier, because this is an unclear principle (way) and at the same time budget is not evenly allocated." H22. The second continued, "Yes, it is barrier because the hoteliers need to care about climate change, but the national level do not see this problem closely and clearly. The real victim could feel the pain. Those who just looks on, don't." H6. This factor might be resulting from serious weakness in formulating and implementing of those laws/rules, thus leading to poorly managing the problems. This was indicative of lacking in consistencies for CCA formulation nationally and locally. 


\section{Institutional barriers}

Gathered from the literature for section 2.9.4, the 'institutional barriers' was understood as the lack of institutional flexibility, unclear or contradictory goals within the institution, and restrictions in individual capacity to adapt, particularly with their hotels.

Up to $40 \%$ of the respondents felt that it is a moderate, small and uninfluential barrier. One respondent stated that institutional barrier is closely related to institutional arrangements. Whenever the institutions are poorly arranged, it leads to a lack of good flows of work. This leads to a barrier, the barrier that limits the staff's will and motivation to work, including actions on climate change, which can be significantly reduced through setting clear individual responsibility. When clear goals were set, and everyone was sufficiently told/advised what to do, this would contribute to reducing institutional barriers.

Among this (40\%), five respondents viewed it as a small barrier. One of informants explained that internal problems such as institutional barriers could be robustly addressed internally. For instance, after identifying areas of concern, all staff were called for a meeting or advised to read the archive data/records and learn from these. The second argued that this barrier was not so relevant to climate change issues. Others continued that every institution has their own working hierarchy, starting from top management to the bottom line. Whenever the low-level staff obeyed the rules of higher management, there were no significant institutional barriers. One foreign participant indicated that probably within the institution, people already have experiences of what is happening to them so institutional barriers would not be majorly affecting the CCA. The participant emphasised, "I think probably within our hotel, they (staff) most probably know what they are dealing with like an individual basis, probably alright.” $\mathrm{H} 28$.

Out of $40 \%$, one respondent reported that 'institutional barrier' was not impactful in CCA. The participant thought that in whatever institutions, especially in the hotel sector, whenever something affects the businesses, the businessmen tended to act promptly to the problems, including climate change problems. Institutional barriers would not happen in such situations. The informant pointed out, "I think in most of the hotels, if they see the effect on 
their business, they will be flexible to really do something about it in most cases. If it affects their finance, flow of the tourists, they aren't stupid. They must do, so does my hotel.” H27.

The smallest percentage, $28 \%$ and $32 \%$, rated the greatest barrier entitled institutional barriers' to adaptation as being major and very major. They explained that the institutional barriers could be relevant to the shortfall of information transfer and documentations/archive of data/records from one to another in a particular institution. Some perceived that institutional barriers create inconsistency in working, including the actions of adapting to climate change. One exemplar provided by one participant was limited institutional memory for instance the past climate change events on the hotels were not well documented, thus making the staff unable to know what happened in the past. They do not have institutional memories so they cannot learn from previous experience for current and future adaptation. Finally, one informant pointed out, "Right because if inside our place something remains unclear, we cannot have long vision. Therefore, it affects (our hotel) ... If we cannot manage our place, we can't advance further, and this is true for our CCA." $\mathrm{H} 21$. Institutional barriers also happen when there are staff turnovers, which were frequent in SHV. This would constitute impediments to working system and effective actions for climate change.

From their own experiences, some further exemplified that once there were requests from the low or medium level working staff, the requests were often rejected by the top management. This constituted barriers to CCA inside the hotel. The participant defended, "This is a big condition because we, at the lower level, are more practical. He (the boss) is not. He just waits for the information from us. Yet when we request, there is a rejection." $\mathrm{H} 22$. Institutional barriers or disorganised working arrangements reduce staff's awareness what to do and how to work concerning adaptation. An institution is like everyone on the same boat, journey and direction. Everyone must share failures and profitability. Institution arrangements need to be strengthened for problems such as climate change because it does not happen only once and disappear endlessly. It is a sequential occurrence.

This research, which has been well-established from a variety of previous research studies about adaptation barriers, adds to the existing stock of knowledge by analysing the actual 
barriers that affect the capacity of SHV's hoteliers to adapt to climate effects in the following section.

\subsection{Additional adaptation barriers to climate change adaptation}

After rating the nine barriers above (informed by the literature), through their adaptation activities for their hotels in SHV, some participants reported additional barriers. These adaptation barriers are the actual obstacles they have had while adapting to climate change in the area. The following barriers were reported:

First, of 50 participants, $10 \%$ of informants placed great emphasis on '(vulnerable) location of hotel'. They reported that location of hotel influences adaptation outcome. They exemplified that hotels located in an area (near mountain or mangrove forest areas acting as storm protection) that is less affected by storms adapt much better than those in a vulnerable area. Others pointed out that hotels located in a surrounding area where roads were bad, and lakes have been filled might be more vulnerable to rainfall-induced flooding and face more difficulty to adapt. One of informants pointed out, "Barrier is related to two things. First, it is money and second it is the location" $\mathrm{H} 29$. The second added, "While a hotel is located in the vulnerable zone, it's quite hard to adapt." H16.

Second, $4.6 \%$ of informants pointed out that 'bad design and quality of the buildings (e.g. limited space)' constitutes an obstacle for CCA for their hotels. The structures of hotels and buildings influenced the adaptation actions. The badly designed and constructed buildings reduce the flexibility in adaptation actions such as being unable to install building insulators. Some held views that they had little capacity to make a change to existing hotel buildings (e.g. building foundation) due to the already wrongly fixed structures. They had to work with what they basically had. One participant explained that the builders just followed the design and layout of the existing buildings (leased and renovated buildings) with minimum consideration of CCA. The hoteliers and hotel builders, especially the Khmer, had little experience of relationships between constructions and climate change. Because of their limited experiences, the constructions produced unintended consequences such as rainwater leakages that constitute a barrier for CCA. One respondent confessed, "Our hotel building 
was mismanaged since the beginning. Due to this mismanagement, the structures become under standard.” H25. A foreign participant pointed out, “... basically we have this place built by ourselves; we designed it by ourselves. We're not expert in it so we make quite a lot of mistakes.” H28. The quality of the building was also a determinant of CCA. It became critical barrier once it got older or if it was built with low quality. It was struggling to adapt to rainfall or storms. Furthermore, two respondents explained that due to the limited spaces in the hotels (maybe due to the wrong design and hotel structures), they could not install some technical equipment such as insulators/solar panels.

It is crucial to note that most SHV's hotel owners (34 hotels or 68\%) rented and renovated the existing buildings to run the businesses under leasing contracts. The existing fixed buildings provide little flexibility to adapt to climate change, thereafter increasing barriers to CCA. For example, one lower standard hotel participant further described that owing to the poorly designed and renovated hotel from the existing buildings, the hotel businesses must be allocated separately. The restaurant was far from the hotel rooms, thus making guests walk inconveniently under the sunshine and rain. Any adaptation for this situation, such as further restructuring, would experience major barrier. Due to the poorly designed buildings (e.g. restaurants), some parts were inundated with rainfall-induced flooding, thereafter, easily causing damage to the inside areas. The restaurant staff needed to work harder to adapt to the impacts, thus requiring the owners to make extra constructions such as building more windows and doors.

Third, $4.6 \%$ of the participants believed that 'limited research or studies available' makes adaptation difficult. They added that there were limitations on existing data, research and studies about CCA in Cambodia and SHV. One participant highlighted that no one comes and studies climate change issues and in return advises on what to do regarding the adaptation. This indicated a limitation of technical climate change experts. This has led to a barrier to CCA to SHV's coastal tourism as well as the hotel sector.

Finally, only $2 \%$ of all 50 respondents pointed out that 'being a new hotel and limited time' is also a difficult condition for CCA. The participant pointed out that the hotel being new and 
time constraints are also barriers. They said that while management and staff put priorities on hotel operations such as having guests, they do not have time to initiate CCA initiatives. One from a new hotel mentioned, “We're new. We don't have time. We've not experienced about CCA yet. We instead put priorities on other more important issues such as having more guests.” $\mathrm{H} 7$.

\subsection{The perceived ratings of enabling factors for adaptation in the hotel sector}

As described in chapter 4, a list of enabling factors (informed by the literature in CCA in the context of developing countries) was given to the participants. The perception of enabling factors to CCA was investigated by asking respondents to rate each of the nine-potential enabling factors to CCA. Table 6.2 shows the Likert-scale results (in \%), mean ratings, and SD. The perceptions of enabling factors in the hotel sector in SHV were measured on a sixpoint rating scale with 0 being 'uninfluential' enabling factor and 5 being 'very important' enabling factor. Each distinction is detailed in the next sections: 
Table 6. 2: Rating results of enabling factors for $\mathrm{CCA}$ for the hotel sector

\begin{tabular}{|c|c|c|c|c|c|c|c|c|c|c|c|}
\hline \multirow[b]{2}{*}{ No. } & \multirow[b]{2}{*}{ Enabling factors } & \multicolumn{6}{|c|}{ Likert-scale results in \% } & \multirow[b]{2}{*}{$\begin{array}{c}\text { Total in } \\
(\%)\end{array}$} & \multirow[b]{2}{*}{ Mean } & \multirow[b]{2}{*}{ SD } & \multirow[b]{2}{*}{$\begin{array}{l}\text { Level of } \\
\text { criticality }\end{array}$} \\
\hline & & $\begin{array}{c}(0) \\
\text { Uninfluential }\end{array}$ & \begin{tabular}{|c|}
$(1)$ \\
Very \\
unimportant \\
\end{tabular} & $\begin{array}{c}(2) \\
\text { Unimportant }\end{array}$ & $\begin{array}{c}\text { (3) } \\
\text { Moderately } \\
\text { important }\end{array}$ & $\begin{array}{c}(4) \\
\text { Important }\end{array}$ & \begin{tabular}{|c|} 
(5) \\
Very \\
important
\end{tabular} & & & & \\
\hline 1 & Sufficient resources & 0 & 0 & 0 & 2 & 24 & 74 & 100 & 4.72 & 0.497 & \multirow{3}{*}{$\begin{array}{l}\text { The most } \\
\text { critical }\end{array}$} \\
\hline 2 & Sufficient information & 0 & 0 & 0 & 13 & 30 & 57 & 100 & 4.45 & 0.709 & \\
\hline 3 & Good leadership or management structures & 0 & 0 & 4 & 9 & 30 & 57 & 100 & 4.45 & 0.738 & \\
\hline 4 & $\begin{array}{l}\text { Individuals' motivation to respond (committed } \\
\text { workforce) }\end{array}$ & 4 & 0 & 0 & 12 & 30 & 54 & 100 & 4.39 & 0.931 & \multirow{3}{*}{ Medium } \\
\hline 5 & Clear adaptation goals & 0 & 0 & 0 & 16 & 36 & 48 & 100 & 4.38 & 0.703 & \\
\hline 6 & Political will & 4 & 0 & 0 & 16 & 20 & 60 & 100 & 4.28 & 1.161 & \\
\hline 7 & $\begin{array}{l}\text { Previous and current adaptation } \\
\text { experiences/awareness/knowledge }\end{array}$ & 6 & 0 & 0 & 2 & 39 & 53 & 100 & 4.27 & 0.709 & \multirow[b]{2}{*}{$\begin{array}{l}\text { The least } \\
\text { critical }\end{array}$} \\
\hline 8 & $\begin{array}{l}\text { Sufficient engagement/ } \\
\text { collaboration/communication } \\
\text { with other stakeholders }\end{array}$ & 0 & 0 & 0 & 16 & 48 & 36 & 100 & 4.2 & 0.7 & \\
\hline 8 & A & & 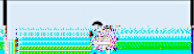 & & & & & & & & \\
\hline
\end{tabular}




\subsubsection{The most critical enabling factors for climate change adaptation}

The results in Table 6.2 show the three most critical enabling factors ('sufficient resources', 'sufficient information' and 'good leadership and management structures') with the largest mean response on a 0 to 5 Likert scale (with means of $4.72,4.45$, and 4.45 respectively). Based on the mean rankings and frequency/percentage distribution of Likert point ( 0 being 'uninfluential' and 5 being 'very important'), it shows that the respondents from hotel sector in SHV highly perceive these three conditions as the greatest enabling factors to CCA. Each critical enabling factor is detailed below:

\section{Sufficient resources}

Illustrated in the literature on enabling factors for CCA in section 2.12.1, the most significant enabling factor entitled 'sufficient resources' refers to available finance/budget, $\mathrm{HR}$ and technology that prove to be essential in every stage of adaptation, especially in implementation and monitoring. These resources strongly influence the adaptation quality for the hotel sector. This understanding sets the context for the interviewees to respond to the rating of Likert scale.

Table 6.2 indicates that the greatest percentage, nearly $100 \%$ (24\% and 74\%), believed that 'sufficient resources' was an important and very important enabling factor. They felt that this enabling factor is the most significant condition to enable hotel managers to fight climate change. Readily available resources would allow punctual and effective responses that in turn contribute to fruitful adaptation. In contrast with limited resources, it would not be possible to overcome adaptation barriers. They further reported that concerning either hotel operation or CCA, budget is an area of need, together with HR and technical materials. One participant asserted, "To adapt, we need resources especially the budget because when we have ideas and what we need most is budget. We have ideas to do it and have money. That is an enabling factor." H21. The second contended, "Resources are important. We have only knowledge and ideas but don't have money, so we cannot do anything." H9. They continued that available technology is a good illustration. For example, in order to adapt to a storm, resources such as technical materials and special technology are needed and competent HR to operate those technological devices are most needed. 


\section{Sufficient information (e.g. about vulnerabilities...)}

As reflected in the literature in section 2.12.5, this second most significant enabling factor of 'sufficient information (e.g. about vulnerabilities...)' mainly refers to the place-based or site-specific about previous, current and projected climate change-related information, especially about the environmental and socio-economic vulnerability for SHV's hotel sector. It sets the context that the information facilitates and prepares adaptive responses to mitigate risks in Cambodia, SHV and their hotels.

Table 6.2 also suggests high value, nearly $90 \%$ (30\% and 57\%) of the respondents rated sufficient information (e.g. about vulnerabilities...) as an important and very important enabling factor. They gave a reason that although in Cambodia information provided or obtained is not always 100\% true, the information allows hotel owners/managers to be aware of climate change impacts, providing indication as to what resources should be used and who is to be contacted to fit to a particular climate change problem. They explained that anticipating possible climate change impacts in advance allows for better preparation and prevention of any potential impacts. One foreign participant mentioned that because the government/authority has limitedly provided sufficient information, this enabling factor is quite important for the hotel sector. This participant revealed, "Yeah, I think so I'm really sure I never got any information from the government, so I feel like... yes that's very important." H39. One of informants also commented, "Yes, this is important because this helps us to plan." H36. Late and inaccurate information, especially through the media, would make hotel staff and guests fall into the trap of climate change troubles.

The frequency and percentage were 13\% of respondents believing that this enabling factor is moderately important. They compared and contrasted this enabling factor (sufficient information) to another enabling factor such as 'previous and current experience' and saw it as a less important one, thereby rating it as a 'moderately important' enabling factor. Others thought that starting the actual adaptation actions are more important than waiting for the information. Information just makes hotel operators prepared whilst a determination and actual responses will likely prove more evidence for fruitful adaptation. One added, "Even though we have enough information, if we don't do anything, we are still vulnerable." $\mathrm{H} 21$. 
Another revealed, "This one is not a determination; this is just information. This is prior information, that's why I rate number 3.” H35.

\section{Good leadership or management structures}

As discussed in the previous research in section 2.12.7, the critical enabling factor of 'good leadership or management structures' (with mean score of 4.45 equal to the second most significant enabling factor) enables the strengthening of institutions to implement CCA programmes. In short, there are good and capable leaders and managers, and working arrangements in adapting to climate change for their hotels. All 50 participants understood this before they began ratings.

Table 6.2 presents that the greatest percentage, nearly $90 \%$ (30\% and 57\%) of the respondents who rated this second/third (the same mean score of 4.45) most important enabling factor entitled 'good leadership or management structures' as an important and a very important

enabling factor respectively. They thought that logically, whenever there is good leadership and management, the entire teams tend to initiate adaptation activities, and this increases a high chance of dynamic adaptation. Good structures of leadership and management would enhance not only general hotel operations but also climate change adaptation actions specifically. One of respondents literally revealed, "I agree because there is the term "good". If they are good leaders, good management, it means all problems occur (all impacts) and they understand faster, then it is all done faster too. It means they are good and good people. If we talk about climate change impact, they accept it, right...?” $\mathrm{H} 9$. The second added, “Again, if you don't have good management, you don't have good decision, you don't make good decision ... this is important.” $\mathrm{H} 36$.

They added that leadership and management people are like a steering wheel. Whenever they are disorganised, a hotel would end up in trouble. If they are good, a hotel is heading toward a safe zone. Good leaders/managers could motivate everyone in a hotel to actively get involved in all necessary issues, including climate change kickbacks. Whenever the owners have proactively looked forwards to nature such as climate change, it would be great, as these people are in charge of money. The minute that these people care only about money, other 
issues like climate change would be largely ignored. One of participants mentioned, " $C C A$ needs flexibility, knowledge, and economic expenses. Sometimes we want to adapt and need money, and this needs approval from higher management. If the highest management doesn't approve, little can be done." H6. This enabling factor allows consistent exchange of ideas between the top and lower staff. Good leaders and managers would take the opinions of their staff into serious considerations. This would result in getting and synthesising the best ideas for climate change responses for a particular hotel.

$13 \%$ of the respondents thought of this enabling factor as being moderately important and unimportant. There were two reasons: First, it was their current hotels' management situations and second, their current hotels' standards. Firstly, they complained about the lack of leadership and management of their own hotels. They recalled that whenever there were requests to the top management, there were often uncooperative responses to the requests. Coupled with a lack of HR, budget and technology, they therefore rated this enabling factor a bit lower. Second, they complained about their disorganised leadership and management structures owing to their hotels' lower standards. One of participants explained, "For my scope of business, it is not right, but in the future, may be yes. Based on the scale/scope of my business which is family business, it is not right." H30. Only one respondent rated it as an unimportant enabling factor. This Khmer respondent recalled previous experience of working in other places in the hotel sector throughout SHV and stated that most investors/owners had generally low leadership and management. Some of them had low education and in serious cases, were illiterate. Therefore, this participant did not favour this condition much.

\subsubsection{The medium enabling factors for climate change adaptation}

Measured by mean ratings and SD, Table 6.2 shows the second category of perceived medium enabling factors to CCA, which include 'individuals' motivation to respond (to climate change)', 'clear adaptation goals', and 'political will' with mean response on 4.39, 4.38 , and 4.28 respectively. In this second category, the frequency/percentage distribution of being 'uninfluential' and 'moderately important' can be seen as $16 \%, 16 \%$ and $20 \%$ for the first, second and third medium enabling factors respectively. 


\section{Individuals' motivation to respond (committed workforce)}

Drawing on the previous literature in section 2.12.4, the first medium enabling factor of 'individuals' motivation to respond (committed workforce) refers to the personal and intrinsic motivation as well as the strong commitment of individual staff, managers and owners of a particular hotel in adapting to climate change. This enabling factor may be the starting point of adaptation that very much relies on individuals to activate and drive the initiatives. After sufficiently understanding this enabling factor, all 50 respondents began the ratings and subsequently provided their comments.

Table 6.2 shows that over $80 \%$ (30\% and 54\%) of respondents rated this first medium enabling factor as being an important and very important. They stated that this is a pioneering and enabling strategy/factor to commence CCA initiatives. It makes not only the management team but also each staff member committed to CCA. For instance, they are motivated to save water and power and seek alternative sources for power/water supply. 'Individuals' motivation to respond' encourage hotel operators to adjust themselves and increase flexibility to adapt, thereafter leading to the effective adaptation. With limited motivation, they might not try hard enough. Instead, they might put themselves too much at ease by depending on other stakeholders. The first explained, "Working in hotel sector, you must be very committed in both general business operations and CCA efforts." H32. The second reported, "Yes, it is, true because when we collaboratively work, we accept, we involve in solving the problems to prevent, protect the obstacles/barriers. Hence this collaborative works enables us to find solution for the adaptation." $\mathrm{H} 22$.

They further explained that whenever people are motivated in CCA, they have a high chance of effective adaptation outcome, almost $100 \%$ of achievement. When having this enabling factor, all the labour forces are motivated. It starts from one person, spreads out, consequently becomes a determination for many others, and becomes a working norm. This can start from an understanding from inside out, from the hotel to the surrounding area. It could pass on the important message to the area, thereby increasing people's awareness of and commitment to their community. This constitutes a collective power of workforce to adapt to climate change 
in the area, thus in turn benefiting both the hotel sector and community. One more participant from a new hotel took a more thorough consideration and explained that motivation is closely linked to knowledge. While one particular hotelier has strong motivation to adapt, he/she must find ways to increase knowledge concerning CCA.

In different opinions, the response rate was $15 \%$ of participants who said that this enabling factor was a moderately important and unimpactful enabling factor because it just partly influences adaptation. They added that adapting blindly even from a strong individual's motivation would not have considerable adaptation outcome. The respondents compared and contrasted between this enabling factor and others (the most critical enabling factors) and revealed that this factor might not be strongly needed. This factor alone does not provide a full picture of substantial adaptation effect. One wanted to see more determination from external parties. This informant revealed, "For me, normal (moderate)... because there are many problems concerning other issues in hotel operations. For me I have moderate commitment about CCA because I want to hear from the top leaders (the government).” H40.

\section{Clear adaptation goals}

In this second category, the enabling factor entitled 'clear adaptation goals' was perceived as the second medium barrier with the mean score of 4.38. This enabling factor refers to the specific and solid goal of adaptation that is formulated to target the adaptation in the hotel sector. The goal should not be mixed with the operational goal of hotel services but can be implemented together with other goals of hotel business (see section 2.12.6 for details).

Table 6.2 presents 'clear adaptation goals' with over $80 \%$ (36\% and $48 \%$ ) of rating as either important or very important enabling factor. They explained that setting a target is prerequisite before achieving considerable adaptation result. Since hotel operation requires enormous responsibilities, setting adaptation goals in advance could reduce working loads. This condition enables the anticipation of any problem that might occur before and during climate change responses. They further argued that although the goals are not easily set clearly, hotel operators must have them formulated because without good preparation, they might lead to failure in climate change efforts. One explained, "Because if we have clear 
adaptation goals, we will do it. If no clear adaptation goals, we cannot do it although we have materials, HR...” H14. They carried on by saying that setting clear adaptation goals is like having a guideline that could be used as an appropriate road map towards the positive effects of adaptation to climate change. When it is clear in people's opinions of what is going to be done, it increases chances of potential outcome of adaptation. One general manager (GM) revealed, "Important point, even though I'm not skilful of this (CCA), I must set clear goals on whatever I do. I (thoroughly) consider limitations, I set plans, yes, it's important." H9.

They continued that 'clear adaptation goals' offers a complete set of ideas to take care guests and advise the staff to be fully ready for all issues including climate change. Although in a case where all hotel operators cannot achieve $100 \%$ adaptation goals, it is still a good idea to have it set in place. The clearly set goals allow them to have indicative ideas of how much HR and money, especially time, would be needed. They exemplified that if the water is disconnected, hotel managers must have water reserved. When in storms, they must have preventive measures to respond. These are from clear adaptation goals. One supported, "Once we set clear adaptation goals, we likely could prevent climate change risks." H29.

In contrast, $16 \%$ of the respondents reported it as a moderately important enabling factor. All had similar ideas that setting clear adaptation goals is just part of the performance outcome while the actual adaptation responses would be a more realistic approach. One of respondents explained, "Just having the goals, it is not yet a substantial effect of adaptation. We need to implement it. I just rate it 3.” $\mathrm{H} 7$.

\section{Political will}

Based on background literature in section 2.12.8, 'political will' was understood as the political awareness from the government, especially the local authorities that directly influence the adaptation efforts in the private sector/hotel sector. The hotel sector is expected to obtain clear political messages, motivation and participation that may bring sufficient resources such as technical experts and materials to help, especially in rural adaptation (e.g. at islands). 
Table 6.2 indicates that the last medium enabling factor entitled 'political will' obtained $80 \%$ (20\% and $60 \%$ ) of rating as being important and very important. The participants perceived that whenever there is political will, the government might allocate money for helping hotel sector concerning CCA. One of the argued, "Political will... yes absolutely if the government can't be bothered, no one else would be ...” H39. They added that this enabling factor is about the involvements from the government/politicians to enthusiastically tackle the changing climate for all sectors, including the hotel industry, and having good policy for CCA. Furthermore, the political motivation could not only be providing HR and budget but also the laws, regulations and rules to help the hotel sector more conveniently adapt to climate change. The 'political will' truly influences every sector of a country. The government knows the intensity of the impacts much better than others. One of participants asserted, “... this is the most important thing. Without this nothing here ... all start from the head. We say when the head's not good, all the body suffers." H45. Another added, "Good, good it's also 5, absolutely necessary. All the governments of the world must involve themselves in their own countries and their own communities." H10.

$20 \%$ of the respondents ranked that 'political will' as a moderately important and unimpactful enabling factor. They asserted that politics is a bit broad in relation to CCA and slightly influences the adaptation actions in the hotel sector. They argued that the hotel sector should bear responsibility for the positive effect and failure by itself rather than depending on politics/politicians. They asserted that in the hotel sector the people themselves should be the main players. In most cases, the Cambodian government has not paid sufficient attention to the hotel sector, especially where relevant to climate change issues. The first said, "Sometimes, politics is just one part. If we are individuals working cooperatively it is better than the politicians." H31. The second continued, "I don't believe that the government would anyway bother to help us because we are foreigners, pretty much look after ourselves, we pay our taxes, but you don't expect anything in exchange of those." $\mathrm{H} 28$. Two respondents stated that 'political will' was uninfluential for the hotel sector's CCA. They contended that as the climate change impacts in SHV at the moderate level, the hotel sector could sufficiently help itself. They have sufficient capability to guide/explain their staff to adapt to climate 
change. It is unimportant as to whether or not the external parties such as the government give a hand.

\subsubsection{The least critical enabling factors for climate change adaptation}

The results in Table 6.2 show the three least critical enabling factors ('previous and current adaptation experiences/awareness/knowledge', 'sufficient engagement/collaboration/ communication with other stakeholders', and 'invulnerable location of hotel') with the smallest mean response on a 0 to 5 Likert scale (with means 4.27, 4.2 and 3.65 respectively).

In these enabling factors, the distribution of respondents can be seen increasing at the scale of being (0) uninfluential. This makes these enabling factors no longer medium (but the least critical enabling factors), although the mean differences between these enabling factors and the second three enabling factors are small.

This finding shows that respondents from the hotel sector in SHV have the lowest perception in these three conditions as the least critical enabling factors to CCA. That or these perceived conditions do not constitute significant outcomes in their adaptation activities for their hotels. The frequency/percentage distribution of being 'moderately important', 'unimportant', 'very unimportant' and 'uninfluential' has increasing trend with $8 \%$ being 'moderate' and very unimportant', 16\% being 'moderately important' and 36\% from being moderately important' through 'uninfluential' for the first, second and third critical barriers respectively. Each barrier is detailed below:

\section{Previous and current adaptation experiences/awareness/knowledge}

Table 6.2 shows that the third least critical enabling factor to CCA with the mean score of 4.27. As outlined in section 2.12.2, 'previous and current adaptation experience/ awareness/knowledge' is having high experience and knowledge about causes, effects and solutions to climate change, and the experience/knowledge allows hotel operators to adapt to climate change productively. The 50 respondents were mainly aware that this enabling factor is from adaptation experience/knowledge/perception in previous workplaces, particularly in hotel working arrangements. 
Up to $8 \%$ of the respondents rated 'previous and current adaptation experiences' as a moderately important and uninfluential enabling factor because one participant stated that the vulnerability levels of SHV and its hotels were at a moderate level as well. This enabling factor is not much needed. This Khmer participant was from an upscale hotel. Three participants thought that 'previous and current adaptation experiences/awareness/ knowledge' is an uninfluential enabling factor for CCA in the hotel sector. They argued that climate change is a natural phenomenon that happens continuously with different intensities and crises. Factors such as 'previous experience' would influence hotel operators in having the same or repetitive adaptation actions. Climate change requires evolving human diversities to respond. One of participants argued, "This (enabling factor) is not really right because climate change impacts have been changing and their forms (of impacts) are increasingly new and different." H6.

The respondents perceived 'previous and current adaptation experiences' as the third least significant enabling factor of being important (39\%) and very important (53\%). They reported that it allows hotel managers to access researched documents and learning from the previous experience of others. They thought that access to these documents is like having or bringing a consultant/expert/knowledge about adaptation to their hotels. This factor provides both knowledge and flexibility. For instance, while storms have come with different intensities and crises, experience and knowledge allow hotel managers to adjust appropriate adaptation measures responsive to the impacts. One Khmer respondent exemplified that knowledge and experience enabled him/her to have water filters and reservoirs properly installed and built to respond to extended drought, especially during hotel constructions. By doing so, the guests never face water deficit. They further added that this factor helps hotel managers to familiarise themselves in adapting to climate change and not feel panic and makes them project climate change effects more accurately in the future or anticipate climate change problems in advance. Whenever hotel managers do not have experience, they might not hear of climate change impacts and blindly adapt. The first explained, “... without this one, you will never have that one (CCA)." H36. The second said, "Very important, everyone needs experience and knowledge." $\mathrm{H} 5$. 


\section{Sufficient engagement/collaboration/communication with other stakeholders}

As mainly discussed in previous studies in 2.10 .3 , it was understood that this second least critical enabling factor entitled 'sufficient engagement and interaction with other stakeholders' is the ability of hotel operators to interact and communicate sufficiently between three layers: national, provincial and local, or among other stakeholders for the sake of their hotels' adaptations.

The most interesting aspect of this is that up to $16 \%$ of the participants perceived it as a moderately important enabling factor. They stated that the communication has been sufficiently exchanged either among hotel sector or between the hotel sector and other stakeholders. Almost all stakeholders have worked cooperatively and had mutual understanding in CCA. For example, there have been weekly meetings at both either the hotel sector or between the hotel sector and other stakeholders. This factor does not add critical value to the current hotels' adaptation. Others compared it to another enabling factors above and argued that this factor just partly contributes to CCA in the hotel sector.

Table 6.2 indicates that only $48 \%$ and $36 \%$ of the respondents rated this enabling factor as an important and very important. They asserted that communication and interaction enable participants to learn from each other or a particular group such as the government. For instance, they might learn how to make water storage or dig wells for adapting to extended drought. One of respondents asserted, "Important because if they face the problems, they can give us ideas, sharing to us about adaptation techniques." H32. They added that this condition contributes to obtaining key information from other parties as well. The first said, "It can be true because we have ability to adapt and if we know others well, different opinions, we can borrow their ideas for our hotel." H21. The other explained, "Because when we've interaction, we get information from each other. Then we share." $\mathrm{H} 22$. They suggested that communication and interaction with other stakeholders should be diversified into many forms, face-to-face meetings or public forums. The forums include the discussion with several organisations such as NGOs on various topics, including climate change. 
This enabling factor provides many additional benefits. In case of climate change emergency, this allows having strong cooperation that contributes to saving people's lives in time. For instance, this could allow participants to have hotlines of important stakeholders such as professional medical centres. For another example, the wider network internationally allows the hotel sector to receive a number of helps in adapting to climate change. One of respondents pointed out, "This is true, it does not confine only to our region but also other countries, which have experiences and meet the same problem. For example, they first encounter the impacts, they inform us, then we can brace for the climate change impacts and at least we can learn from these experiences." H6. One more asserted, "We've been picked by TUI (a big travel and tourism company) in Germany. They have provided good message into the industry including CCA measures, it contributes to positive effect of CCA." $\mathrm{H} 10$.

\section{(Invulnerable) Location of hotel}

The greatest percentage, $36 \%$, rated this first least important enabling factor as from being 'moderately important' through 'uninfluential'. They stated that this condition (location of hotel) is debatable. It depends on the types of climate change impacts. Heavy storms and rains are different in terms of locations while rising temperature or spread of illness caused by climate change would equally affect hotels regardless of locations. The first one explained, “In general, CCA doesn't regulate or set which location is adaptable, this is about the strategy. If we have the strategy, we can withstand climate change impacts even though we're in adverse location." H31. The second one added, "Because climate change is not influencing only us, it is affecting everyone, location does not help anything.” H5.

Two respondents viewed this enabling factor as a very unimportant and unimportant enabling factor equally. They viewed this factor as a controversial evidence/condition. The first one argued that it is common for those located at coastal zones to be often threatened by climate change. The second said, "Because location of our hotel is slightly affecting our CCA actions, is not important factor for CCA.” H30. This participant further argued that climate change impacts such as severe drought threatens hotels regardless of their locations. 
Amongst this (36\%), six participants thought that this conduction was not an enabling factor for adaptation. They otherwise argued that stand-alone conditions like location could not make hotel managers adapt effectively. The result must be achieved by initiating the actual actions that require everyone to be involved. One of respondents explained, "There is a good location, but the hotel doesn't initiate any CCA actions, it is not a point for substantial adaptation." H21. Locations could be good for running businesses but for CCA actions they would not be a logical or impactful reason. The respondents illustrated that location is not closely related to CCA. Climate change intensities increase and affect all hotels regardless of locations, especially at the coastal zone. At last, one local respondent compared and contrasted this condition to other enabling factors such as good leadership and management structure and ultimately argued, "We sometimes have good location, but our management is bad. We don't spread the information widely. It is not $100 \%$ true." $\mathrm{H} 22$.

As can be seen in Table 6.2 , only $22 \%$ and $42 \%$ of respondents perceived this least significant enabling factor as being either important or very important. They reported that location influences both vulnerability and adaptation outcome of the hotels. They illustrated that $\mathrm{SLR} /$ beach erosion/storm surges threaten hotels at beach areas more than those at the mainland. They illustrated that invulnerable location not only provides a good condition for CCA but also trustful feelings for the guests (being unheard of climate change) who tend to stay in a convenient place. They further explained that hotels in vulnerable places would be firstly and strongly hit by climate change while hotels at less vulnerable areas (e.g. close to mountains acting as storm protection) have sufficient time to prepare and adapt. At one island (A Ma Sour Island), one participant asserted that the location plays the most essential role for CCA. This informant explained, "(In A Ma Sour island) some locations have been affected for 6 months, some locations have been affected for just 1.5 months like mine. When we can have a right location, very good location, there are little impacts." H45. The second expressed, "... the hill and it has many trees, even now we have jogging track by riding bicycles, the guests are increasingly interested, and it isn't being affected..." H50.

Building on the growing number of works by previous scholars about enabling factors for adaptation, this study adds to the existing stock of knowledge by analysing the actual 
enabling factors that are helping or influencing the SHV's hoteliers in adapting to climate effects in the following section.

\subsection{Additional enabling factors for climate change adaptation}

After rating the nine enabling factors above (informed by the literature), through their adaptation activities for their hotels in SHV, some participants reported additional enabling factors. These enabling factors for adaptation are the actual helpful conditions they have had while adapting to climate change in the area. The following enabling factors were reported:

First, $8 \%$ of the informants perceived that 'good structure/building (providing easy maintenance)' is an enabling factor for hoteliers' adaptation behaviour. They, including one new participant, otherwise explained that their hotel structures and buildings were resistant enough for climate change impacts. Their hotels were built with good standards and were not easily tilted or damaged while being affected by climate change. They exemplified that ever since the hotels had been constructed, there has been no history of damage and significant impacts. The firm and reliable constructions allowed the participants to adapt easily and, in the meantime, this puts maintenance work at ease. One of respondents literally explained, "If comparing the building to others, maybe mine (my building) is better. $\mathrm{H} 40$. One foreign participant (from a 5-star hotel) additionally highlighted that the knowledgeable and experienced builders from own country (Israel) constructed the hotel with sound technology.

They further perceived that 'renovation' of hotel buildings would be also a favourable condition. This participant continued that in SHV many old buildings limits the flexibility in adapting to climate change. The buildings should be carefully and technically renovated before being allowed to run businesses in. In the meantime, hotels that were built recently (a few years back) need to be renovated as well. Of all 50 hotels, 68\% (34 hotels) hotel businesses are run by renting the buildings and/or the land. Most leaseholders did not build strong structures. They just made the buildings fairly strong just to run the businesses.

Second, $6 \%$ perceived that 'innovative thinking' would be a key condition for hotel sector's adaptation. One foreign participant exemplified that definitely reinforcing the beaches should 
be one of the strategic missions. Reinforcing the beaches means keeping the water back. Breaking the water away from the beaches would likely prevent beach erosions. The measure is inexpensive as concrete, and rocks could be used to break water. This participant further explained that it is more regretful to lose sands which leads to having no beaches. Another added, "Enabling factors could be creative ideas, just create ideas, that they can put but just can think of like big thing, how to prevent their hotels in an innovative way." H46.

Third, $6 \%$ of informants explained that 'forestation/maintaining forest/gardens' would be a key condition for remarkable adaptation result. They thought that while lacking forest would contribute to irregular rainfall, forestation provides many benefits. First, it could act as storm and beach erosion protection (e.g. mangroves). Second, it stabilises the surrounding soil to prevent beach erosion and landslide (some hotels are located on high hills). Other participants rationally explained that large and tall trees cast a shade over the guests and staff, thus making them less affected by rising temperature. One indicated, "In adapting to rising temperature we already have like big trees. We have techniques by advising our gardeners who take care of the trees." H21. A second added, "The garden is helpful with the rains and other stuffs such as rising temperature." H28. As argued in chapter 5 (section 5.4.3.1), some hotels used vertical vines to cover the building's walls and roof in order to adapt to rising temperature.

Finally, 4\% explained that 'good service' would be a key condition for the hotel sector's adaptation. Although 'good service' is bit broad in relation to CCA, these participants challenged to argue that although there is bad climate, guests might keep staying because hotel managers could provide good services such as providing convenient transport during hot days. In a broader picture, they illustrated that good service is a core task that influences all hotel operations, including CCA. One of informants emphasised, "Whenever there are possible climate risks, we must try to contact the guests and ask if they need helps". H48. Rhee and Yang (2015) argue that "In any business, everything starts with service and end[s] with it." (p. 582). 


\subsection{Chapter summary}

After vital links between vulnerability and adaptation were developed, this chapter coherently captures the fundamental features in these relationships by identifying both conditions (barriers and enabling factors) in the same research. After a number of arguments were proposed, the following statements are summarised:

First, according to the interviews, among the nine potential barriers (informed by the literature in CCA in the context of developing countries), the findings show that the most critical barriers are 'sufficient resources', 'low level of leadership and guidance', and 'lack of political will'. These provide important clues for the hotel sector while adapting to climate change for their businesses. This study adds to the existing stock of knowledge on the growing number of works in previous tourism and climate change research that are particular to the context of developing countries. In the meantime, the participants reported additional barriers. The barriers include '(vulnerable) location of hotel', 'bad design and quality of the buildings (e.g. limited space)', 'limited research or studies available' and 'being a new hotel and limited time'. These additional barriers are the actual obstacles that SHV's hotel sector has faced in their adaptation activities in the area.

Second, among the nine potential enabling factors (informed by the literature in CCA in the context of developing countries), the findings show that the greatest enabling factors are 'sufficient resources', 'previous and current adaptation experiences/awareness/knowledge', and 'individuals' motivation to respond'. These provide important indications for the hotel sector in adapting to climate change for their businesses. The perceived rankings of criticality levels significantly contribute to related literature. In the meantime, the participants reported of additional enabling factors. These enabling factors include 'good structures and renovation of hotel buildings', 'innovative thinking', 'forestation/maintaining garden', and 'good service'. These are the actual barriers that SHV's hotel sector has determined from their adaptation activities. 


\section{CHAPTER 7: CROSS-CASE ANALYSIS}

\subsection{Introduction}

In previous chapters, the assessment of possible relationships between participants' backgrounds, hotel attributes and their perceptions of barriers and enabling factors were not examined, The starting assumption of this chapter 7 is that the mean scores of respondents regarding the perceived barriers and enabling factors to CCA could vary according to hotel attributes (star rating, ownership, hotel size, hotel location, hotel age and business format) (or/and) participant's background (level of education, age, experience, nationality and length of residency in SHV).

Guided by the analytical framework (see the red colour block of Figure 7.1), the main goal of this chapter is to explore the extent to which hotel attributes and participants' backgrounds in the hotel sector in SHV impact on their perceptions of barriers and enabling factors to CCA.

In order to explore the underlying dimension of hotel attributes and participants' backgrounds on the mean scores of perceived barriers and enabling factors to CCA, descriptive statistics and independent sample tests have been used to determine whether there is statistical evidence that these characteristics influence the participants' perceptions towards the rating of the barriers and enabling factors to CCA. To undertake ANOVA tests, it is expected that the dependent variable is normally distributed and there is approximately equal variance on the scores across groups, which is not the case with the collected data. Since the dependent variables of this study are ordinals, but not normally distributed to conduct one-way ANOVA tests, nonparametric (distribution free) tests are used to compare the means of the respondents. The Mann Whitney $\mathrm{U}$ test (MW test) was used to compare the means of the respondents' perceived barriers and enabling factors to CCA and determine any significant differences between two independent groups. In the case of more than two independent groups, the Kruskal Wallis test (K-W test) was conducted for the assessment of any significant differences between the means of the respondents' perceived barriers and enabling factors to CCA. Finally, this cross-case analysis is expected to answer the fifth research aim 
of this thesis, which critically examines the barriers and enabling factors to adaptation according to hotel attributes and participants' backgrounds.

In this context, the chapter has the following structure. The main results based on the relationships between adaptation barriers and hotel attributes and participants' backgrounds are discussed in the first two sections (7.2 and 7.3). Then, the findings of the enabling factors for adaptation in regard to hotel attributes and participants' backgrounds are presented in section 7.4 and 7.5. The data were processed with the statistical package SPSS version 23. The four quadrants in Table 7.1 present the statistical results of this explorative empirical study, considering the following relationships.

- Relationship between adaptation barriers and hotel attributes (North West quadrant)

- Relationship between adaptation barriers and participants' backgrounds (North East quadrant)

- $\quad$ Relationship between enabling factors for adaptation and hotel attributes (South West quadrant)

- Relationship between enabling factors for adaptation and participants' backgrounds (South East quadrant)

The empirical results regarding descriptive statistics and independent sample tests between barriers and enabling factors to CCA and hotel attributes and participants' backgrounds are summarised in three crucial distinctions: statistically significant difference (when p. value < 0.05 ) (highlighted in red), non-significant difference (when p. value $>0.1$ ) (empty cells), and trending towards statistical significance (when $0.05<\mathrm{p}$. value $<0.1$ ) (highlighted in green).

In the four quadrants, relationships between nine barriers and nine enabling factors to adaptation with six hotel attributes and five participants' backgrounds are tested by means of Mann-Whitney U test (two groups) or Kruskal-Wallis Test (more than two groups). Each individual quadrant is examined in detail in the following sections: 
Figure 7. 1: Analytical framework

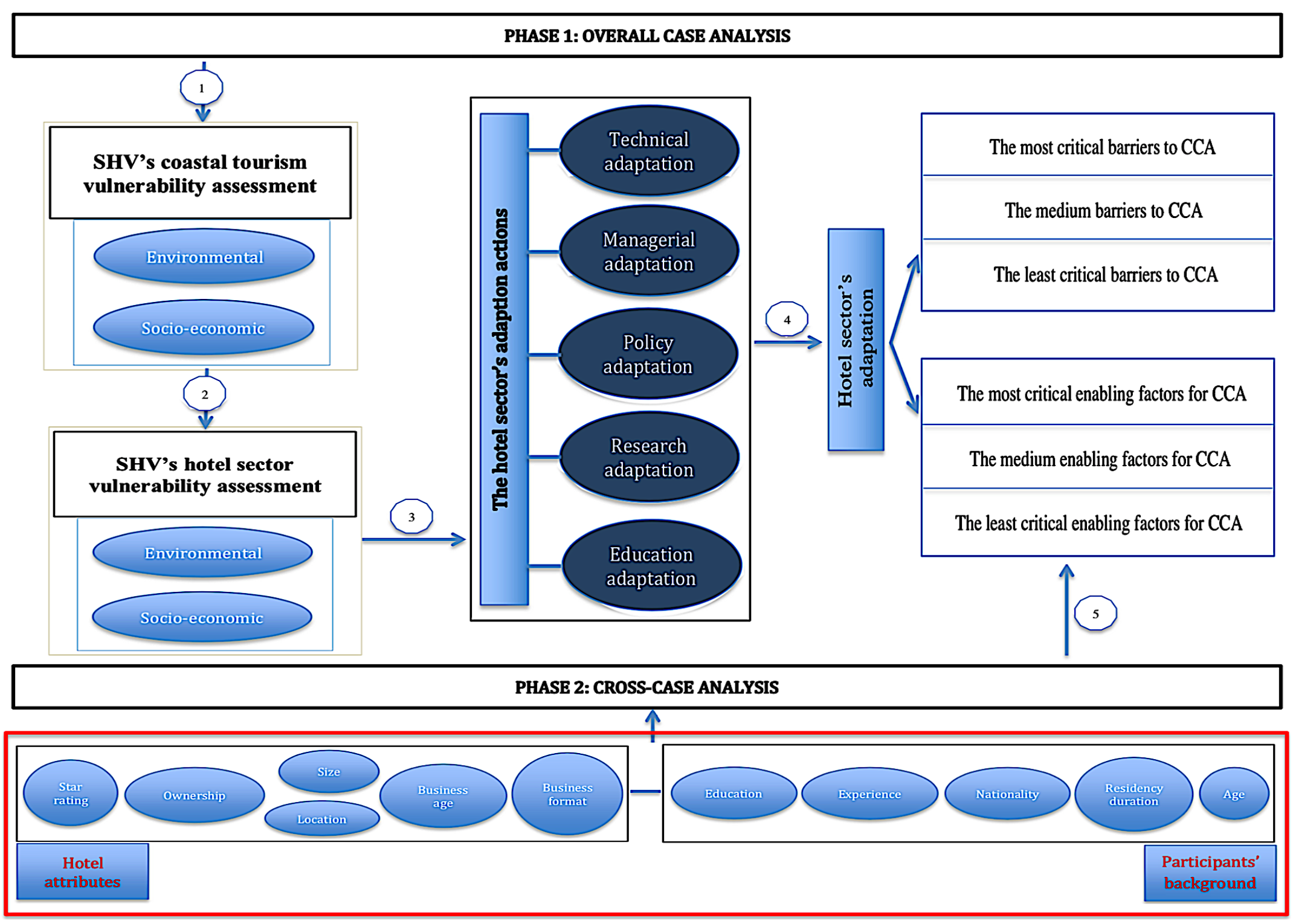


Table 7.1: Overall test results between barriers and enabling factors to CCA and hotel attributes and participants' backgrounds

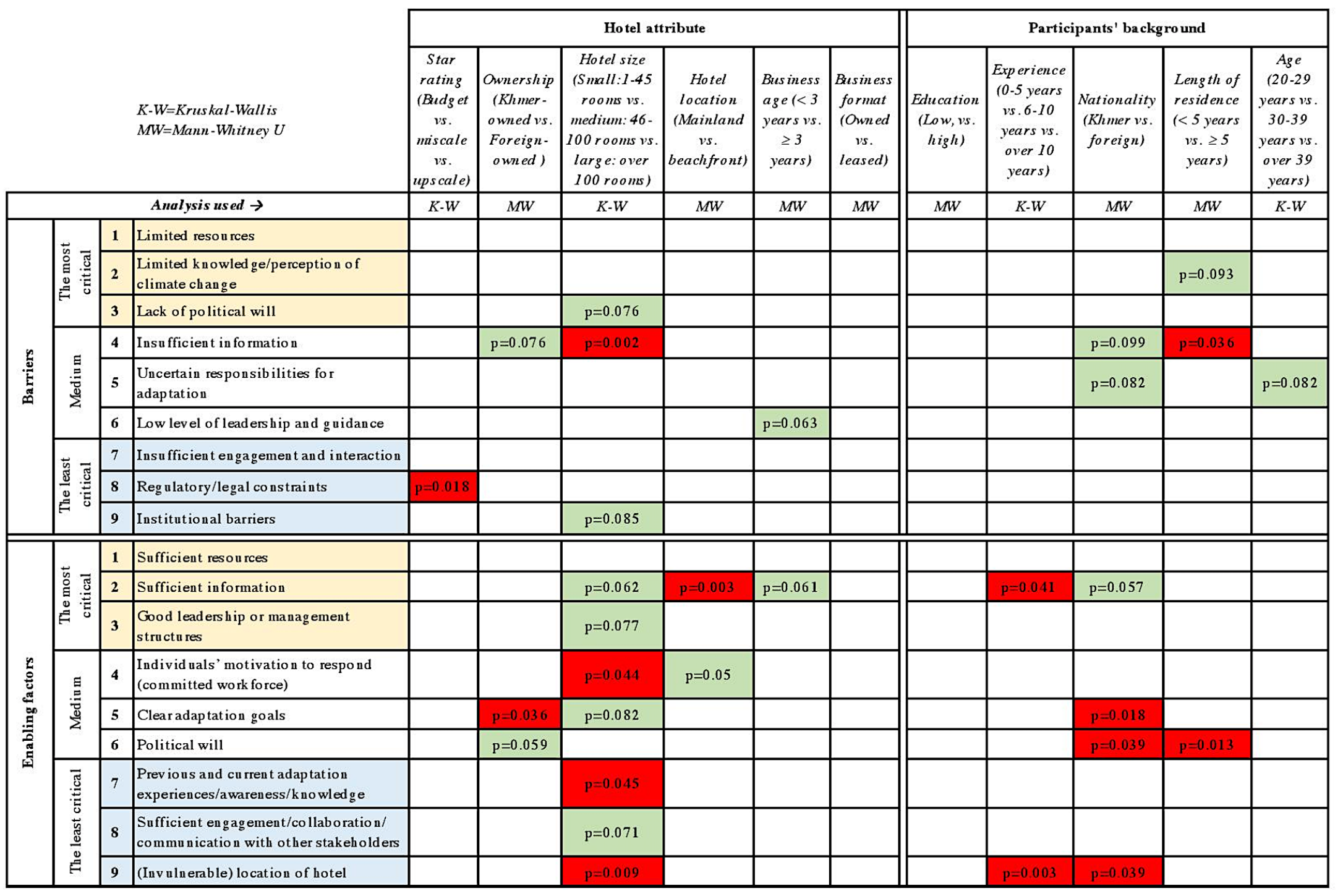




\subsection{Relationships between adaptation barriers and hotel attributes}

Table 7.2 below summarises the findings regarding the relationships between the nine enabling factors affecting adaptation and hotel attributes. This latter corresponds to the North West quadrant of table 7.1.

Table 7.2: Test results of the relationship between adaptation barriers and hotel attributes (North West quadrant)

\begin{tabular}{|c|c|c|c|c|c|c|c|c|c|}
\hline & & & & & & Hotel att & tribute & & \\
\hline & & & $\begin{array}{l}K-W=\text { Kruskal } \text { - Wall is } \\
M W=\text { Mann }- \text { Whitney } U\end{array}$ & $\begin{array}{c}\text { Star } \\
\text { rating } \\
\text { (Budget } \\
\text { vs. } \\
\text { miscale } \\
\text { vs. } \\
\text { upscale) }\end{array}$ & $\begin{array}{l}\text { Ownership } \\
\text { (Khlmer- } \\
\text { owned vs. } \\
\text { Foreign- } \\
\text { owned) }\end{array}$ & $\begin{array}{c}\text { Hotel size } \\
\text { (Small:1-45 } \\
\text { rooms vs. } \\
\text { medium: } 46- \\
100 \text { rooms vs. } \\
\text { large: over } \\
100 \text { rooms) }\end{array}$ & $\begin{array}{c}\text { Hotel } \\
\text { location } \\
\text { (Mainland } \\
\text { vs. } \\
\text { beachfront) }\end{array}$ & $\begin{array}{c}\text { Business } \\
\text { age }(<3 \\
\text { years vs. } \\
\geq 3 \\
\text { years })\end{array}$ & $\begin{array}{c}\text { Business } \\
\text { format } \\
\text { (Owned } \\
\text { vs. } \\
\text { leased) }\end{array}$ \\
\hline & & & Analysis used $\rightarrow$ & $K-W$ & $M W$ & $K-W$ & $M W$ & $M W$ & $M W$ \\
\hline & & 1 & Limited resou rces & & & & & & \\
\hline & 焉 & 2 & $\begin{array}{l}\text { Limited knowled ge/perception of } \\
\text { climate change }\end{array}$ & & & & & & \\
\hline & & 3 & Lack of political will & & & $\mathrm{p}=0.076$ & & & \\
\hline & & 4 & Insu fficient in formation & & $\mathrm{p}=0.076$ & $p=0.002$ & & & \\
\hline ڤ્d & 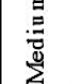 & 5 & $\begin{array}{l}\text { Uncertain responsibilities for } \\
\text { adaptation }\end{array}$ & & & & & & \\
\hline & & 6 & Low level of leaders hip and guidance & & & & & $\mathrm{p}=0.063$ & \\
\hline & & 7 & Insu fficient engagement $t$ and interaction & & & & & & \\
\hline & $\frac{\mathscr{S}}{0 .}$ & 8 & Reg ulatory/legal constraints & $\mathrm{p}=0.018$ & & & & & \\
\hline & & 9 & Institutional barriers & & & $\mathrm{p}=0.085$ & & & \\
\hline
\end{tabular}

First, in the North West quadrant, relationships between nine barriers to CCA with six hotel attributes are tested by means of Mann-Whitney U (MW) test (two groups) or Kruskal-Wallis (K-W) Test (more than two groups). As discussed in chapter 6, the three most critical barriers with the largest mean response on a 0 to 5 Likert scale are: 'limited resources', 'limited knowledge/perception of climate change' and 'lack of political will' with means of 4.56, 4.49 and 4.48 respectively, see Table 6.1). The results in Table 7.2 show that there were no significant differences found at the 0.05 (or 5\%) significance level with respect to the above three most critical barriers.

This finding shows that respondents from the hotel sector in SHV highly perceive these three barriers as the most critical ones to CCA regardless of the category of hotel 
attributes they belong to. In brief, this result reflects a common perception from the respondents of the hierarchy among the three greatest barriers to CCA.

For instance, the perception of those from budget, midscale and upscale hotels scored the barrier entitled, 'limited resources' as the most critical barrier to CCA. They, from budget (e.g. H7, H5), midscale (e.g. H6, H10) and upscale (e.g. H19, H4) hotels, stated that without budget and technical skills, hotel operators cannot make further investments concerning climate-proofing and adaptation activities such as purchasing water containers and digging wells for adapting to prolong drought, while lack of competent HR and technical skills present difficulty in adaptation as well.

In another example, the respondents also perceived the barrier of 'limited knowledge/perception of climate change' as the second greatest barrier to CCA regardless of the category of hotel attributes they belong to. For instance, the MW test indicated no significant differences between Khmer and foreign owned hotels in terms of comparison of the means of respondents. This revealed that there was no significant difference of opinions regarding the perceived importance of this barrier 'limited knowledge/perception of climate change' when it comes to the nature of the ownership of hotels (Khmer/local/domestic vs. foreign ownership). Most hoteliers believed that knowledge plays an important role in substantial adaptation outcome. Knowledge of climate change is prerequisite for adaptation initiatives. Without sufficient knowledge, hotel managers as well as their staff cannot think of any new strategies for adapting to constantly changing climate. 'Limited knowledge' does not allow managers and staffs to have even basic solutions to the problems of climate change. This might lead them to blindly adapting to climate change, which cannot reach considerable adaptation results.

Among the three most critical barriers in this North West quadrant, the only noticeable observed result that trended towards statistical significance (but did not meet the selected significance threshold level 0.05) is found between the barrier of 'lack of political will' and the size of hotels $(\mathrm{H}=5.15$, degrees of freedom or $\mathrm{df}=2, \mathrm{p}=0.076)$ (see Appendix $\mathrm{F}$ ). On the basis of the K-W comparison test, this finding suggests that it might be a perceptual difference of the barrier entitled 'lack of political will' between respondents belonging to hotels of different sizes in SHV. This finding also suggests that further empirical research is needed in the future with a larger sample of respondents. 
Second, regarding the findings of the second category of perceived medium barriers to the implementation of CCA, the analysis showed that statistically significant differences across the six hotel attributes are only found for the perceived barrier entitled 'insufficient information' to CCA with respect to hotel size $(\mathrm{H}=12, \mathrm{df}=2, \mathrm{p}=0.002)$. The pairwise comparison test suggests that respondents from small hotels (1-45 rooms) and medium hotels (46-100 rooms) provided higher mean-score for this barrier than those from large hotels ( $>100$ rooms) with $p$-values of $p=0.021$ and $p=0.02$ respectively (see the Appendix E-1). This finding indicates that participants from small and medium size hotels were in greater agreement and were more cautious than those from large hotels about the lack of information in SHV when it comes to CCA.

This could be also explained by the fact that in this study, large hotels in SHV are predominantly upscale hotels. Highly star-rated hotels often have sufficient financial and human resources, especially through their regional and international networking. The extensive resources increase their adaptive capacity specially to reduce barrier of 'limited/insufficient information'. For instance, having broader networking allows them to fully access sensitive information such as climate change, thus making them perceive 'limited/insufficient information' as a less major barrier. By contrast, small and medium hotels in the study are majorly budget and midscale. They often have limited resources, particularly in networking. With low adaptive capacity, especially without strong networking to access to climate change information, they are rather passive and wait for climate change information provided by other parties such as the government, and IOs and NGOs. They therefore showed greater consideration with respect to this barrier.

All the remaining results show that there are no significant differences (at the 5\% significance level) in terms of mean ratings between medium barriers to CCA and the other hotel attributes. The only detectible findings that trended towards statistical significance (but did not meet the selected significance threshold level 0.05) are found between the barrier 'insufficient information' and hotel ownership (U=3334,5, Z=1.777, $\mathrm{p}=0076$ ) and the barrier 'low level of leadership and guidance' with the business age $(\mathrm{U}=371.000, \mathrm{Z}=186, \mathrm{p}=0.063$ ). As outlined above, this finding also suggests that further empirical research is needed in the future with larger samples of respondents. 
Finally, among the least critical perceived barriers to CCA (insufficient engagement and interaction, regulatory/legal constraints, and institutional barriers), the only significant variability in responses to these barriers was found between 'regulatory/legal constraints' and the star rating $(\mathrm{H}=8.003, \mathrm{df}=2, \mathrm{p}=0.018)$. The $\mathrm{K}-\mathrm{W}$ comparison test shows that there is at least one sub-group of respondents that has different perception from the other two remaining groups. The pairwise comparison test suggests that respondents from upscale hotels show a greater consideration of the barrier 'regulatory/legal constraints' to CCA than those that belong to midscale hotels $(\mathrm{p}=0.029)$ (see Appendix F-2).

This could be explained by the reality that while the government is aware that imposing laws, regulations and rules of environment-related issues such as CCA on the lower ranked hotels is impossible (including midscale hotels in this study). These lower standard hotels are often family businesses and depend on daily income and have insufficient resources such as budget and HR to respond to climate change. Strict law/rule enforcement could lead to a closure of those low standard hotels. The government have changed their focus to higher standard hotels. The higher ranked hotels (upscale hotels in this study) are more pressurised to perform environmental issues, especially CCA, because of their available resources in place. Furthermore, it is known that the high standard hotels themselves are more potentially contributing to environmental problems. For example, one high standard hotel participant confessed, "Likely we are a big industry (hotel). We have produced waste and consume much power such as big chiller; these can contribute to climate change.” H1. However, Cambodia's laws, regulations and rules are insufficiently supportive of the adaptation actions. Meanwhile, the lower ranked hotels are not more significantly demanding in environmental performance at the current priority of the country's development. This is a reason why higher standard hotel participants felt that 'regulatory and legal constraints' is a greater barrier.

All the remaining results show that there are no significant differences (at the 5\% significance level) in terms of mean scores between the least critical perceived barriers to CCA and the remaining hotel attributes. The only observable finding that trended towards statistical significance is related to the relationship between 'institutional barriers' and the size of hotels $(\mathrm{H}=4.941, \mathrm{df}=2, \mathrm{p}=0.085)$. 


\subsection{Relationships between adaptation barriers to CCA and participants' backgrounds}

Table 7.3: Test results of the relationship between adaptation barriers and participants' backgrounds (North East quadrant)

\begin{tabular}{|c|c|c|c|c|c|c|c|c|}
\hline & & & \multirow[b]{2}{*}{$\begin{array}{l}K-W=\text { Kruskal } \text {-Wall is } \\
M W=\text { Mann }- \text { Whitney } U\end{array}$} & \multicolumn{5}{|c|}{ Participants' background } \\
\hline & & & & $\begin{array}{c}\text { Education } \\
\text { (Low, vs. } \\
\text { high) }\end{array}$ & $\begin{array}{c}\text { Experience } \\
(0-5 \text { years } \\
\text { vs. } 6-10 \\
\text { years vs. } \\
\text { over } 10 \\
\text { years })\end{array}$ & $\begin{array}{c}\text { Nationality } \\
\text { (Khmer vs. } \\
\text { foreign) }\end{array}$ & $\begin{array}{c}\text { Length of } \\
\text { residence } \\
(<5 \text { years } \\
\text { vs. } \geq 5 \\
\text { years })\end{array}$ & $\begin{array}{c}\text { Age } \\
(20-29 \\
\text { years vs. } \\
30-39 \\
\text { years vs. } \\
\text { over } 39 \\
\text { years })\end{array}$ \\
\hline & & & Analysis used $\rightarrow$ & $M W$ & $K-W$ & $M W$ & $M W$ & $K-W$ \\
\hline \multirow{9}{*}{$\stackrel{\mathscr{n}}{\mathscr{E}}$} & \multirow{3}{*}{ 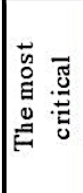 } & 1 & Limited resou rces & & & & & \\
\hline & & 2 & $\begin{array}{l}\text { Limited knowled ge/perception of } \\
\text { climate change }\end{array}$ & & & & $\mathrm{p}=0.093$ & \\
\hline & & 3 & Lack of political will & & & & & \\
\hline & \multirow{3}{*}{ 景 } & 4 & Insu fficien $t$ in fo rmation & & & $\mathrm{p}=0.099$ & $\mathrm{p}=0.036$ & \\
\hline & & 5 & $\begin{array}{l}\text { Uncertain responsibilities for } \\
\text { adaptation }\end{array}$ & & & $\mathrm{p}=0.082$ & & $\mathrm{p}=0.082$ \\
\hline & & 6 & Low level of leaders hip and guidance & & & & & \\
\hline & \multirow{3}{*}{ 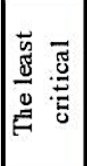 } & 7 & Insu fficient en gagemen $t$ and interaction & & & & & \\
\hline & & 8 & Regulatory/legal constraints & & & & & \\
\hline & & 9 & Institutional barriers & & & & & \\
\hline
\end{tabular}

The findings regarding the relationships between the nine adaptation barriers and participants' background are presented in this section.

Firstly, the results in Table 7.3 show that there are no significant differences found at the 0.05 (or 5\%) significance level in respect to the three most critical perceived barriers ('limited resources', 'limited knowledge/perception of climate change' and 'lack of political will') and participants' backgrounds.

This finding suggests that respondents from the hotel sector in SHV highly perceive these three obstacles as the most critical barriers to CCAs regardless of the five backgrounds' criteria that were used in this study. 
For example, both Khmer (e.g. H1, H6) and foreign (e.g. H10, H44) participants raised similar concerns regarding the perceived third most critical barrier (lack of political will) to CCA of hotels in SHV. They had common perception that working collaboratively between individual hotel and other stakeholders regarding CCA needs involvement from the politics. Using an example of metaphor, they argued that the politicians are the head while the hotel sector is the body. These two (politicians and hoteliers) must be collaborative and consistent to each other. Without 'political will', there are insufficient resources such as budget, HR and technology allocated by the government for the hotel sector's adaptation. Without those resources, the hotel sector is extremely challenged to put further investments in climate change-related actions or just raises minimal investments in adaptations. This might lead to an ineffective outcome.

All the remaining results in this North East quadrant show that there are no significant differences (at the 5\% significance level) in terms of mean scores between the most critical barriers to CCA and participants' background. The only noticeable observed result that trended towards statistical significance (but did not meet the selected significances threshold level 0.05) is found for the barrier of 'limited knowledge/perception of climate change' with respect to the length of residence of the participants $(\mathrm{U}=183.000, \mathrm{Z}=-1.680, \mathrm{p}=0.093)$.

Secondly, based on the empirical results on the relationship between medium perceived barriers to CCA ('insufficient information', 'uncertain responsibilities for adaptation', and 'low level of leadership and guidance') with participants' backgrounds, significant difference has been found in the mean-scores of respondents to the barrier 'insufficient information' with respect to the length of residence in SHV. Moreover, the MW test suggests that hotel respondents who have lived longer in SHV ( $>5$ years) provided lower mean-score to this barrier than those who have lived less than 5 years in $(U=156.500, Z=-$ 2.100, $\mathrm{p}=0.036$ ) (see the Appendix F-3).

This result indicates that the shorter the participants lived in SHV the more concerned they were about 'limited information' or the more they lived in the area, the less cautious they were about 'limited information'. This finding suggests that those who had resided longer might have had sufficient time to adjust themselves in the local context and perhaps access to climate change-related information through their own local network. In 
contrast, those who have had a shorter length of residence duration often did not sufficiently familiarise themselves with the place and found it difficult to access information. They show greater consideration with respect to the barrier 'limited information'.

All the remaining results indicate that there are no significant differences (at the 5\% significance level) in terms of mean scores between the medium perceived barriers to CCAs with respect to participants' background.

The only noticeable observed results that trended towards statistical significance (but did not meet the selected significance threshold level 0.05) are found between the barrier of 'insufficient information' and 'participants' nationality' ( $U=261.500, Z=1.651, p=0.099)$, and the barrier 'uncertain responsibilities for adaptation' in respect to participants' nationality $(\mathrm{U}=275.000, \mathrm{Z}=1.738, \mathrm{p}=0.082)$ as well as participants' age groups $(\mathrm{H} 5.008=$, $\mathrm{df}=2, \mathrm{p}=0.082)$.

Finally, among the least critical perceived barriers to CCAs ('insufficient engagement and interaction', 'regulatory/legal constraints', and 'institutional barriers') with the smallest mean response on a 0 to 5 Likert scale (with means of $4.16,4$ and 3.78 respectively, see Table 6.1), there are no significant differences between these least critical barriers and participants' backgrounds at the 0.05 (or 5\%) significance level. Most likely, this result reflects a common perception from the hotel respondents of the relatively low level of importance of these barriers to CCAs, even though the mean scores are still relatively high.

For example, all hotel respondents (with short, medium and long experiences) ranked the barrier 'insufficient interaction and engagement' as being the first major factor among the three least critical perceived barrier to CCA. The short (e.g. H20, H22), medium (e.g. H5, H13, H7) and long (e.g. H48, H15) experience respondents thought that a lack of interaction and engagement may hamper their businesses from getting key information about climate change vulnerabilities and adaptation. In contrast, they had common perceptions that hoteliers need to learn from each other and other key stakeholders. The lessons learned allow effective information for acting promptly towards climate change impacts. Working collaboratively is often better than working individually in terms of 
adaptation. A medium-experienced informant asserted, "It's 100\% true that it (insufficient interaction and engagement) is a huge impediment. Adaptation requires comprehensive involvement and participation from other stakeholders.” $\mathrm{H} 7$.

Likewise, differences in perception based on participants' backgrounds and the barrier of 'regulatory and legal constraints' were not found. For instance, the MW test indicated no significant differences between Khmer and foreign participants in terms of mean-scores between respondents. This revealed that there was no significant difference of opinions regarding the perceived importance of this barrier 'regulatory and legal constraints' when it comes to the nationalities of respondents (Khmer vs. foreign). All participants, both Khmer (e.g. H8, H9, H50) and foreign (e.g. H28, H34), explained that due to unclear rules from the government, they do not know what rules help and what do not in terms of CCA. They exemplified that forestation is one way to adapt to climate change (e.g. for erosion from heavy rainfall) but there are unclear rules on where hoteliers should plant the trees and what species are helpful in protecting hotels from climate change impacts.

\subsection{Relationships between enabling factors for adaptation and hotel attributes}

Table 7.4: Test results of the relationship between enabling factors for adaptation and hotel attributes (South West quadrant)

\begin{tabular}{|c|c|c|c|c|c|c|c|c|c|}
\hline & & & \multirow[b]{2}{*}{$\begin{array}{l}K-W=\text { Kruskal }- \text { Wallis } \\
M W=\text { Mann }- \text { Whitney } U\end{array}$} & \multicolumn{6}{|c|}{ Hotel attribute } \\
\hline & & & & $\begin{array}{c}\text { Star } \\
\text { rating } \\
(\text { Budget } \\
\text { vs. } \\
\text { miscale } \\
\text { vs. } \\
\text { upscale) }\end{array}$ & $\begin{array}{c}\text { Ownership } \\
\text { (Khmer- } \\
\text { owned vs. } \\
\text { Foreign- } \\
\text { owned) }\end{array}$ & $\begin{array}{c}\text { Hotel size } \\
\text { (Small:1-45 } \\
\text { rooms vs. } \\
\text { medium: } 46- \\
\text { 100 rooms vs. } \\
\text { large: over } \\
100 \text { rooms) }\end{array}$ & $\begin{array}{c}\text { Hotel } \\
\text { location } \\
\text { (Mainland } \\
\text { vs. } \\
\text { beachfront) }\end{array}$ & $\begin{array}{c}\text { Business } \\
\text { age }(<3 \\
\text { years vs. } \\
\geq 3 \\
\text { years })\end{array}$ & $\begin{array}{c}\text { Business } \\
\text { format } \\
\text { (Owned } \\
\text { vs. } \\
\text { leased) }\end{array}$ \\
\hline & & & Analysis used $\rightarrow$ & $K-W$ & $M W$ & $K-W$ & $M W$ & $M W$ & $M W$ \\
\hline & & 1 & Sufficient resources & & & & & & \\
\hline & बृ. & 2 & Sufficient information & & & $\mathrm{p}=0.062$ & $\mathrm{p}=0.003$ & $\mathrm{p}=0.061$ & \\
\hline & 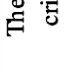 & 3 & $\begin{array}{l}\text { Good leadership or management } \\
\text { structures }\end{array}$ & & & $\mathrm{p}=0.077$ & & & \\
\hline 离 & $\Xi$ & 4 & $\begin{array}{l}\text { Individuals' motivation to respond } \\
\text { (committed workforce) }\end{array}$ & & & $\mathrm{p}=0.044$ & $\mathrm{p}=0.05$ & & \\
\hline .00 & $\sum_{\Sigma}^{\bar{d}}$ & 5 & Clear adaptation goals & & $\mathrm{p}=0.036$ & $\mathrm{p}=0.082$ & & & \\
\hline 可 & & 6 & Political will & & $\mathrm{p}=0.059$ & & & & \\
\hline & 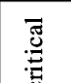 & 7 & $\begin{array}{l}\text { Previous and current adaptation } \\
\text { experiences/awareness/knowledge }\end{array}$ & & & $\mathrm{p}=0.045$ & & & \\
\hline & $\begin{array}{l}\frac{0}{\tilde{J}} \\
\frac{\mathbb{J}}{0}\end{array}$ & 8 & $\begin{array}{l}\text { Sufficient engagement/collaboration/ } \\
\text { communication with other stakeholders }\end{array}$ & & & $\mathrm{p}=0.071$ & & & \\
\hline & Е & 9 & (Invulnerable) location of hotel & & & $\mathrm{p}=0.009$ & & & \\
\hline
\end{tabular}


Table 7.4 summarises the findings regarding the relationships between the nine enabling factors for adaptation and hotel attributes. This latter corresponds to the South West quadrant of table 7.1.

First, the results regarding the three most critical perceived enabling factors ('sufficient resources', 'sufficient information' and 'good leadership and management structures') with respect to hotel attributes show that there is significant difference in terms of mean scores between the enabling factor "sufficient information (about climate change issues)' and (invulnerable) location of hotels. The MW test suggests that hotel respondents from beachfront hotels in SHV provided higher scores to this enabling factor than those from mainland hotels in the area $(\mathrm{U}=397.500, \mathrm{Z}=2.967, \mathrm{p}=0.003$ ) (see Appendix F-4). This result indicates that hotel respondents located around the beach seem to be more cautious of the importance of sufficient information and show greater consideration to climate change issues than those located in the mainland.

According the findings, due to their farther locations, the beachfront hotels (including those on the islands), do not sufficiently interact with the government (both national and local) and other stakeholders such IOs and NGOs. They (the participants) are often put in isolation. Moreover, the early warning systems for climate change are often not available in those places, especially at the islands. Contrary to the preceding argument, most hotels (66\% in the study) are located on the mainland close to downtown where the participants conveniently have access to information. In the meantime, the early warning systems for climate change (e.g. SHV radio and TV stations) are located on the mainland. All local government offices, including SHV DOT and DOE (providing information relevant to tourism and climate change), are located on the mainland as well. Therefore, the former group (beachfront) viewed 'sufficient information' as more important than the latter group.

All the remaining results show that there are no significant differences (at the 5\% significance level) in terms of mean scores between the most critical perceived enabling factors for CCA and the remaining hotel attributes. The first detectible finding that trended towards statistical significance (but did not meet the selected significance threshold level 0.05) are found between the enabling factor 'sufficient information' and 
hotel size $(\mathrm{H}=5.546, \mathrm{df}=2, \mathrm{p}=0.062)$ as well as with business age or year of trading $(\mathrm{U}=371.500, \mathrm{Z}=1.873, \mathrm{p}=0.061)$. The second detectible findings that also trended towards statistical significance is found between the enabling factor 'good leadership and management structures' and the hotel size $(\mathrm{H}=5.138, \mathrm{df}=2, \mathrm{p}=0.077)$.

The relationships between the medium perceived enabling factors for CCA ('individuals' motivation to respond', 'clear adaptation goals', and 'political will') and hotel attributes also provided some interesting findings. Table 7.4 shows that there is significant difference in the mean scores between hotel respondents with respect to the enabling factor 'individuals' motivation to respond' and the size of hotel $(\mathrm{H}=6.240, \mathrm{df}=2$ and $\mathrm{p}=0.044)$. Pairwise comparison test suggests that respondents from small hotels (145 rooms) have higher perception of this enabling factor than those from large hotels ( $>$ 100 rooms) with $\mathrm{p}$-values of $\mathrm{p}=0.046$. The test also indicates that there was no statistically significant difference between [medium (46-100 rooms) and large hotels] (see the Appendix F-5).

One implication of this is that while small hotels lack other resources such as budget, technical materials and HR, they likely depend on other non-budgeted factors such as motivation. Although the government has not sufficiently helped the hotel sector in the area, the interviews showed that the respondents (including those from small-sized hotels) are particularly persistent and resilient; they tried their best to employ a wide range of adaptation actions. Each participant looked dynamic and motivated in adapting to climate change for the sake of their businesses. In additional argument, this is likely explained by the fact that in this study, the large hotels are all upscale and have sufficient resources such as budget and HR as well as international and regional networking. While they have all resources, they see other enabling factors such as 'individuals' motivation to respond' as a small part of contribution to their positive adaptation results.

The result from table 7.4 regarding the relationship between the enabling factor 'clear adaptation goal' and hotel ownership shows a significant difference between the respondents in terms of perceptions of the importance of this medium enabling factor for CCA. Moreover, the MW test suggests that respondents from foreign-owned hotels provided higher scores or perception to this enabling factor than those from the Khmerowned hotels in the area $(U=365.50, Z=2.094$, $p=0.036)$ (see Appendix F-6). 
The interviews and observations indicated that local hotel business owners tend to be family-orientated and have poor business goals and plans, including CCA-related goals. Particularly in this study, some Khmer-owned hotel participants further reported that they do not have clear goals or plans concerning future adaptations. For instance, participants from H24 said, "We haven't thought of it (adaptation in the future)". Another participant from H11 added, "I'm unsure of this. This needs to be decided by my management teams." At the national level, there is only a study entitled Climate change action plan in tourism sector 2015-2018 (see chapter 3 for details). For a long time, Cambodia and its tourism have been affected by CCA but the climate change action plan that directly engages tourism and climate change in the country just came into existence in 2015 and its implementation is at an early stage. MOT, Department of Tourism Planning and Development (DTPD) are the executing agencies while funds are provided by the MOE, Department of Climate Change (DCC). Nevertheless, based on the informal talks with Director of DTPD and DCC as well as the own working experience in the ministry, goals set in these documents might not be fully achieved due to the lack of resources such as budget and HR. This indicates that adaptation goals and plans remain poorly formulated both locally and nationally within the hotel sector.

In contrast, most foreign-owned hotel participants tended to have better goal setting and planning for their future adaptations, thereafter, feeling that 'clear adaptation goals' is more important. Based on the interviews, a respondent from $\mathrm{H} 10$ indicated, "We're now considering installing more solar panel (to meet increasing demand on air-conditioning due to climate change) so that we provide our own electricity. We're also the first hotel that is environmentally conscious and looking for sustainability." The second stated, "We will improve our buildings (adaptable to climate change). In the rooms, we make new paintings, prepare the beds, change old to new, prepare enough facilities/amenities. We must plant more trees at the garden as well." H37. The third asserted, "Yes, obviously in the future, we will specific equipment that can tell something (weather forecast) in advance." $\mathrm{H} 28$.

All the remaining results show that there are no significant differences (at the 5\% significance level) in terms of mean scores between the medium perceived enabling factors for CCAs and the other hotel attributes. The only detectible findings that trended 
towards statistical significance (but did not meet the selected significance threshold level $0.05)$ are found between the enabling factor 'individuals' motivation to respond' and hotel location $(\mathrm{U}=354.500, \mathrm{Z}=1.957, \mathrm{p}=0.05)$, the enabling factor 'clear adaptation goal' with the hotel size $(\mathrm{H}=4.995, \mathrm{df}=2, \mathrm{p}=0.082)$, and the enabling factor 'political will' with respect to hotel ownership ( $\mathrm{U}=377.500, \mathrm{Z}=1.888$, $\mathrm{p}=0.059)$.

Finally, the empirical results on the relationship between the least critical perceived enabling factors for CCA ('previous and current adaptation experience/awareness/ knowledge', 'sufficient engagement/collaboration/communication with other stakeholders', and 'location of hotel') with hotel attributes show that there is significant difference in the mean scores between the enabling factor "previous and current adaptation experience/awareness/knowledge' and size of hotels $(\mathrm{H}=6.212, \mathrm{df}=2$ and $\mathrm{p}=0.045)$. However, the pairwise comparison suggests that the finding trended towards statistical significance (but did not meet the selected significance threshold level 0.05). The pairwise further suggests that concerning this enabling factor, the finding trended towards statistical significance between the small-sized and medium-sized hotels with $\mathrm{p}=0.072$. There is no statistically significant difference between other groups [i) small and large hotels and ii) medium and large hotels]. As outlined above, this finding also suggests that further empirical research is needed in the future with larger samples of respondents (see Appendix F-7).

The findings also indicate that there is significant difference in the mean scores between the least critical barrier '(invulnerable) location of hotel' and size of hotels ( $H=9.449$, $\mathrm{df}=2, \mathrm{p}=0.009$ ). The pairwise comparison test suggests that respondents from small hotels (1-45 rooms) and large hotels (> 100 rooms) have higher perceptions of this enabling factor than those from medium hotels (46-100 rooms) with $\mathrm{p}$-values of $\mathrm{p}=0.043$ and $\mathrm{p}=0.016$ respectively (see Appendix F-8).

It is argued that most small-sized hotels in SHV are family-oriented businesses and operate in leased properties. Due to their shortage of resources, the small hotels owned older buildings and did not have contemporary building designs, which put their owners, employees, and inventory as well their guests (e.g. hotels) at potential risk. With common perception on the enabling factor of '(invulnerable) location of hotel', large hotels likely have many wider and more complicated structures. Large hotels may have franchises that 
are being operated in different places. Because larger hotels may have more than one location, they are increasingly located in climate change-prone areas where needed resources are available. By contrast, the medium hotels are likely either invulnerable or easy to adapt due to their sizes/structures and scope of operations being fit to the area. This study further showed the medium hotels had high commitment to adapt to climate change rather than depending on indirect factors such as location to adapt to climate change. They said that adaptation is about commitment to initiate strategy. When there are strategies available, hoteliers can withstand and reduce the climate change impacts. One respondent from medium-sized hotels said, "I think it is not true. If there is a good location, the hotel doesn't initiate any CCA actions, it is not a point for substantial adaptation level. $\mathrm{H} 21$.

All the remaining results show that there are no significant differences (at the 5\% significance level) in terms of mean scores between the least critical perceived enabling factors for CCA and the remaining hotel attributes. The only detectible findings that trended towards statistical significance (but did not meet the selected significance threshold level 0.05) are found between the enabling factor 'sufficient engagement/collaboration/ communication with other stakeholders' and hotel size $(\mathrm{H}=5.302, \mathrm{df}=2, \mathrm{p}=0.071)$.

\subsection{Relationships between enabling factors for adaptation and participants' backgrounds}

Table 7.5 below summarises the findings regarding the relationships between the nine enabling factors for adaptation and participants' backgrounds. This latter corresponds to the South East quadrant of Table 7.1. 
Table 7.5: Test results of the relationship between enabling factors for adaptation and participants' backgrounds (South East quadrant)

\begin{tabular}{|c|c|c|c|c|c|c|c|c|}
\hline & & & \multirow[b]{2}{*}{$\begin{array}{l}K-W=\text { Kruskal }- \text { Wallis } \\
M W=\text { Mann }- \text { Whitney } U\end{array}$} & \multicolumn{5}{|c|}{ Participants' background } \\
\hline & & & & $\begin{array}{c}\text { Education } \\
\text { (Low,vs. } \\
\text { high) }\end{array}$ & $\begin{array}{c}\text { Experience } \\
(0-5 \text { years } \\
\text { vs. } 6-10 \\
\text { years } v s . \\
\text { over } 10 \\
\text { years })\end{array}$ & $\begin{array}{c}\text { Nationality } \\
\text { (Khmer vs. } \\
\text { foreign) }\end{array}$ & $\begin{array}{c}\text { Length of } \\
\text { residence } \\
(<5 \text { years } \\
\text { vs. } \geq 5 \\
\text { years })\end{array}$ & $\begin{array}{c}\text { Age } \\
(20-29 \\
\text { years vs. } \\
30-39 \\
\text { years } v s . \\
\text { over } 39 \\
\text { years })\end{array}$ \\
\hline & & & Analysis used $\rightarrow$ & $M W$ & $K-W$ & $M W$ & $M W$ & $K-W$ \\
\hline & & 1 & Sufficient resources & & & & & \\
\hline & 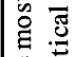 & 2 & Sufficient information & & $\mathrm{p}=0.041$ & $\mathrm{p}=0.057$ & & \\
\hline & $\Xi$ & 3 & $\begin{array}{l}\text { Good leadership or management } \\
\text { structures }\end{array}$ & & & & & \\
\hline है & $\Xi$ & 4 & $\begin{array}{l}\text { Individuals' motivation to respond } \\
\text { (committed workforce) }\end{array}$ & & & & & \\
\hline 80 & $\bar{d}$ & 5 & Clear adaptation goals & & & $\mathrm{p}=0.018$ & & \\
\hline 可 & & 6 & Political will & & & $\mathrm{p}=0.039$ & $\mathrm{p}=0.013$ & \\
\hline & 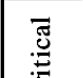 & 7 & $\begin{array}{l}\text { Previous and current adaptation } \\
\text { experiences/awareness/knowledge }\end{array}$ & & & & & \\
\hline & 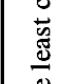 & 8 & $\begin{array}{l}\text { Sufficient engagement/collaboration/ } \\
\text { communication with other stakeholders }\end{array}$ & & & & & \\
\hline & $E$ & 9 & (Invulnerable) location of hotel & & $\mathrm{p}=0.003$ & $\mathrm{p}=0.039$ & & \\
\hline
\end{tabular}

Table 7.5 illustrates the findings regarding the relationships between the three most critical perceived enabling factors (sufficient resources, sufficient information and good leadership and management structures) with participants' backgrounds.

Firstly, the findings show that there is significant difference in the mean scores of the enabling factor of 'sufficient information' and experience of participants ( $\mathrm{H}=6.398, \mathrm{df}=2$ and $\mathrm{p}=0.041$ ). However, pairwise comparison suggests that the finding trended towards statistical significance (and did not meet the selected significance threshold level 0.05). The pairwise comparison further suggests that in regard to this enabling factor, the findings trended towards statistical significance between the long-experienced and the short-experienced respondents with $\mathrm{p}=0.084$. There was also no statistically significant difference between other groups [i) short and medium-experienced respondents, and ii) medium and long-experienced participants]. As outlined above, this finding also suggests that further empirical research is needed in the future with larger samples of respondents (see Appendix F-9). 
All the remaining results show that there are no significant differences (at the 5\% significance level) in terms of mean scores between the most critical perceived enabling factors for CCAs and the other hotel attributes. The only detectible findings that trended towards statistical significance (but did not meet the selected significance threshold level $0.05)$ are found between the enabling factor 'sufficient information' and participants' nationality $(\mathrm{U}=294.500, \mathrm{Z}=1.905, \mathrm{p}=0.057)$.

Secondly, the empirical results of the medium enabling factors for CCA with participants' backgrounds indicate a significant difference in the mean scores of the enabling factor 'clear adaptation goals' with respect to nationality of respondents. The MW test suggests that foreign hotel participants rated this enabling factor higher by comparison with Khmer respondents ( $\mathrm{U}=306, \mathrm{Z}=2.365$, $\mathrm{p}=0.018)$ (see Appendix F-10).

It is argued that it is generally observed that local hotel respondents tend to be familyoriented and are incapable of formulating future goals and plans in their businesses, including climate change-related goals and plans. Thus, Khmer hotel respondents the showed lower consideration to company goals than foreign hotel respondents.

A significant difference was also found in the scores of respondents of the medium perceived enabling factor 'political will' in terms of the nationality of respondents. The MW test suggests that foreign hotel participants rated a higher score to this enabling factor than Khmer respondents ( $U=308, Z=2.068$, p=0.039) (see Appendix F-11).

A possible explanation for this might be that foreign participants compared and contrasted the Cambodian governments' support of climate change-related activities in Cambodia and their countries and felt that SHV's coastal tourism gets little help from the government. They, therefore, reported that enabling factor such as 'political will' is really important for SHV and their hotels. The interviews with participants revealed that most foreign participants are originally from developed countries where the governments' support on climate change-related issues is largely available. One foreign participant mentioned, "I don't know how much a budget we need especially here without the government's support for this. For example, in EU, governments provide some funds that's kind of motivation for people you know to get into this. In Lithuania I think we get financial supports in places." H27. By contrast, the Khmer get used to a situation where 
there is lack of support from their government. It is generally known that expectations from the government are not responsive. This is evidenced in several interviews that the government has serious limitations in climate change-related responses as well. Respondent from H6 said, "The government (both national and local) lacks HR, economic resources. They cannot help anything." Another from $\mathrm{H} 3$ asserted, "They lack techniques, no labour forces to involve in the adaptation actions to reduce the impacts.". In short, they rather depend on themselves in climate change-related responses, thereby providing low ranking for 'political will'.

A significant difference was also found in the mean scores of the medium perceived enabling factor 'political will' with respect to respondents' length of residence in SHV. The MW test also indicates that hotel respondents who have lived in SHV longer ( $>5$ years) rated lower score) to this enabling factor than those who have lived less than 5 years in SHV (U=150.000, Z=-2.489, p=0.013) (see Appendix F-12).

The result suggests that hotel respondents who are new residents expected a lot more from the government (both national and local). They indicated that the hotel sector in SHV requires a lot more assistance from the government. However, participants who resided longer in the area seem to be more aware of the fact that the government has not sufficiently helped; this is the general pattern in developing countries like Cambodia. In the meantime, they have increasingly become aware that the government has a number of barriers to make SHV' tourism adapt to climate change as well. One said, "Currently, concerning CCA there are various limitations in this area. There are insufficient human resources and budget, technical materials and inadequate infrastructures necessary for CCA." H49. A second added, "In this area, there are limited communications/networking nationally and overseas concerning climate change issues.” H35. Finally, they felt that waiting for the government's assistance is nonsense and further makes them disheartened and disappointed regarding their adaptations. From this lesson, the longer-lengthresidence participants tended to feel that the 'political will' is not a greater enabling factor.

All the remaining results show that there are no significant differences (at the 5\% significance level) in terms of mean scores between the medium enabling factors for CCA and the other criteria of participants' backgrounds. 
For instance, the enabling factor of 'individuals' motivation to respond (committed workforce)' was perceived by most of the hotel respondents as the first medium enabling factor for CCA regardless of the category of participants' personal backgrounds. For instance, the K-W test indicated no significant differences in terms of mean-scores between short, medium and long experience participants. This revealed that there was no significant difference of opinions regarding the perceived importance of the enabling factor 'individuals' motivation to respond (committed workforce)' when it comes to the nature of the personal experience (short vs. medium vs. long experience). Participants with both short, medium and long tourism-related work experience perceived that this enabling factor makes hoteliers more proactive to adapt; being passive which cannot combat climate change for the sake of hotels' business. 'Individuals' motivation to respond' means that everyone, including management teams and owners, wakes up, works collaboratively, and strongly commits to reducing vulnerability and adapting to climate change.

For another example, the perception of those with short and long length of residence duration (length of stay in SHV) scored the enabling factor 'clear adaptation goals' as the second medium enabling factor for CCA. Participants with both short and long length of residence in SHV thought that 'clear adaptation goals' allow hoteliers to have a clear plan, which is the plan that initiates study and research on a particular vulnerability. In the meantime, needed resources are identified and allocated. This makes them fully ready for adaptation, thereafter, contributing to substantial adaptation. In short, 'clear adaptation goals' also provide hoteliers with strategic ideas of what to do regarding adaptation needs. One short-length-of-residence respondent said, “'Clear adaptation goals' is quite essential. Once we have it, we can walk towards a right direction concerning adaptations.” $\mathrm{H} 45$.

Finally, based on the empirical results on the relationship between the least critical enabling factors for CCAs (previous and current adaptation experience/awareness/ knowledge, sufficient engagement/collaboration/communication with other stakeholders, and location of hotel) with respondents' backgrounds, we found that there is significant difference in the mean scores between the enabling factor 'location of hotel' and participants' experience $(\mathrm{H}=11.545, \mathrm{df}=2, \mathrm{p}=0.003)$. Pairwise comparison test suggests 
that the long-experienced participants ( $>10$ years) rated a higher score (or provided a higher score) to this enabling factor than the short-experienced participants $(0-5$ years) with $p$-values of $p=0.002$. There was no statistically significant difference between other groups [i) short and medium experienced (6-10 years) participants, and ii) medium and long experienced (over 10 years) participants] (see Appendix F-13).

This finding can be explained by the fact that hotel respondents who have shorter working experience have faced fewer climate change problems. These participants have not sufficiently involved challenges in climate change-related response actions. They cannot think of enabling factors, including '(invulnerable) location of hotel', that would help them adapt to climate change more substantially. In contrast with less experienced hotel participants, those who have longer working experience might have faced more climate change problems. They might have become increasingly aware of the need to have key factors such as location for adaptation. A long-experienced representative said about CCA, "I've lived and worked on an island in the Pacific: Fiji, Vanuatu, a lot of it (vulnerability) within the islands; the islands have been submerged." H15.

The findings also show that there is significant difference in the mean scores between the least critical enabling factor 'location hotel' and nationality of respondents. The MW test reveals that foreign participants rated relatively higher this enabling factor than Khmer respondents ( $U=306.50, Z=2.068, p=0.039)$ (see Appendix F-14).

The implication for this finding is that foreign participants may have worked in different locations both locally and overseas. Working in different places might have allowed them to compare and contrast between different locations. They might have found that location of hotel contributes to the positive adaptation effect. On the contrary, Khmer respondents may have never worked outside the country or even outside of SHV. Consequently, they are not able to compare and between their hotel location and other areas outside of Cambodia. They might not be aware of the importance of hotel location with respect to CCA. In addition, there are most likely other factors influencing their perceptions. There are two possible explanations. First, in this study, participants from the beachfront (and also from the island) tended to consider that '(invulnerable) location of hotel' is an important enabling factor while those whose hotels on the mainland likely feel that 'location of hotel' is a less important enabling factor. Second, as argued above, it is 
relevant to work experiences. Those having shorter working experience were less cautious while those with longer working experience showed a greater consideration to this enabling factor.

All the remaining results presented the South East quadrant show that there are no significant differences (at the 5\% significance level) in terms of mean scores between the least critical enabling factors for CCA and the other participants' backgrounds.

For example, the respondents perceived the enabling factor of 'previous and current adaptation experiences/awareness/knowledge' as the first least critical enabling factor for CCA regardless of the level of education. The MW test indicated no significant differences of the mean scores between low and highly educated participants regarding the enabling factor of 'previous and current adaptation experiences/awareness/ knowledge'. This revealed that there was no significant difference of opinion regarding the perceived importance of this enabling factor 'previous and current adaptation experiences/awareness/knowledge' when it comes to the level of education of respondents (low vs. high education). Participants with both low and high education believed that this enabling factor allows hoteliers to become fully aware of what their key weaknesses are and how to improve the shortcomings concerning their adaptation initiatives. They further exemplified that in order to adapt to prolong drought, what hoteliers could learn from previous experience was whether the water catchments sufficiently provide water and water reservoirs are readily available. One highly educated respondent explained, 'Yes, 'knowledge, previous and current adaptation experiences' is essential. We need researched documents from the previous experiences, they can bring new knowledge for our adaptations.” H1.

For another example, the perception of those with short, medium and long experience scored enabling factor of "sufficient engagement/collaboration/communication with other stakeholders' as the second least critical enabling factor for CCA. Respondents with both short, medium and long experience thought that this enabling factor is closely relevant to having adequate and useful information exchange regarding climate change responses. This can be shared through a forum or workshop organised by the government or/and OIs and NGOs. Sufficient communication allows every hotel to work collaboratively in CCA. They can help each other among peer groups. Also, there is a high chance that the 
government and IOs and NGOs provide help with their adaptations. One shortexperienced respondent argued, "It must be true because if we know others well. We can have different opinions. We can borrow each other ideas to implement adaptations." $\mathrm{H} 21$.

\subsection{Chapter summary}

Overall, the findings in Table 7.1 show that most hotel participants had similar perceptions in terms of rating of the barriers to CCA and enabling factors for CCA. The influence of hotel attributes and participants' backgrounds on the perceptions of the barriers to CCA and enabling factors for CCA seems limited to certain cases. Table 7.1 also shows that among all hotel attributes, the most differences were found with respect to hotel size and among all participants' backgrounds, the most differences were found with regard to participants' nationality. With regard to hotel size, the common differences include the barrier of 'insufficient information', the enabling factors of 'individual motivation to respond', 'previous and current adaptation experience/knowledge' and '(invulnerable) location of hotel'. With respect to the participants' nationality, the common differences are the enabling factors entitled, 'individual motivation to respond', 'political will' and '(invulnerable) location of hotel'.

In relation to the existing statistical differences at $5 \%$ level between hotel attributes and participants' backgrounds, this finding suggests that further empirical research is needed in the future with larger samples of respondents. 


\section{CHAPTER 8. DISCUSSION}

\subsection{Introduction}

The purpose of this chapter is to bring together the findings from the overall case and cross case analysis presented in chapter 5, 6 and 7 to address the research aims. To successfully bring this about, the findings are discussed in relation to the literature, on vulnerability, CCA and the barriers and enabling factors to adaptation relative to the hotel sector in the context of developing countries. Combining the key findings of this research, the initial conceptual framework has been revised to reflect the new insights gained from this research.

First, this chapter re-introduces the main research aim of this study and the five-sub research aims. In the second section, it discusses the finding presented in the first part of chapter 5 about the vulnerability of the SHV's coastal tourism to climate change and why their perceptions are moderately different from the existing studies. In a similar vein, the third section provides a discussion in the mid part of chapter 5 about the vulnerability of SHV's hotel sector to climate change and why their perceptions are overwhelmingly different from prior research. The fourth section further discusses the last part of chapter 5 to determine whether SHV's hotels adapt to climate change, considering adaptation types that are technical, managerial, policy, research and education. The fifth section summarises the barriers and enabling factors to CCA in chapter 6 and discusses the crosscase analysis presented in chapter 7 , which discusses the statistical evidence that hotel attributes and participants' characteristics influence the participants' perceptions towards the barriers and enabling factors to CCA. Finally, after integrating the key insights of this study into the initial conceptual framework, the revised framework of the hotel sector's adaptation is presented with an explanation of the variables in the new framework.

\subsection{Reintroduction of the research aims/questions}

The central concern of this thesis is to determine whether Sihanoukville's hotel sector adapts to climate change and to critically examine the barriers and enabling factors to adaptation. This overarching topic is divided into five sequential sub-aims:

Aim 1: To assess the vulnerability of Sihanoukville's coastal tourism to climate change.

Aim 2: To assess the vulnerability of Sihanoukville's hotel sector to climate change. 
Aim 3: To determine whether/how the hotel sector adapts to climate change impacts.

Aim 4: To identify the most significant barriers and enabling factors to climate change adaptation in the context of hotel sector.

Aim 5: To critically examine the barriers and enabling factors to climate change adaptation according to the hotel attributes and participants' backgrounds.

With reference to the research aims, the next section starts with the discussion of aim 1 about how the thesis findings relate to the literature.

\subsection{Discussion on aim 1: Vulnerability assessment of Sihanoukville's coastal tourism}

It was found that of 50 respondents, two thirds (68\%) felt that SHV's coastal tourism is either slightly or moderately vulnerable, while nearly one third (30\%) reported that it is either vulnerable or very vulnerable (see Table 5.2). As reflected by its mean of 3.08, it clearly shows that most participants perceived that SHV's coastal tourism is 'moderately vulnerable'. Their opinions do not align with earlier findings, which showed that developing countries like Cambodia exhibit various forms of climate change vulnerabilities, due to their high structural vulnerability to environmental and socioeconomic shocks outlined in chapters 2 and 3. Chapter 2 and 3 further indicate that coastal tourism is not only one of the important sectors of Cambodia's recent economic development, but also highly vulnerable to climate change. For example, both the lack as well as the oversupply of rain is one of the most pronounced challenges highlighted by respondents. This may be true for most tropical countries like Cambodia where it receives six-month rains annually. This indicates that the participants' perceptions are moderately different from early findings. Table 8.1 outlines why most participants perceived vulnerability lower than those in previous studies. 
Table 8. 1: Reasons why the participants perceived the vulnerability of SHV's coastal tourism lower than early findings

\begin{tabular}{|c|c|c|c|}
\hline $\begin{array}{l}\text { Existing studies about } \\
\text { SHV's vulnerability }\end{array}$ & \multirow{6}{*}{ vs. } & $\begin{array}{l}\text { The hotel participants' perception on the } \\
\text { vulnerability of SHV's coastal tourism }\end{array}$ & \multirow{2}{*}{ Discussion } \\
\hline Very vulnerable & & Moderately & \\
\hline \multirow{4}{*}{$\begin{array}{l}\text { Climate change } \\
\text { vulnerability in } \\
\text { Cambodia } \\
\text { (see section } 3.2 .3 \text { ) } \\
\text { Climate change } \\
\text { vulnerability in coastal } \\
\text { zone/SHV (see section } \\
\text { 3.3.2) }\end{array}$} & & \multirow{4}{*}{$\begin{array}{l}\text { Research question } 1 \text { : To what extent is } \\
\text { Sihanoukville's coastal tourism vulnerable } \\
\text { to climate change? } \\
\text { Answer/Finding: Moderately vulnerable } \\
\text { with its mean of } 3.08\end{array}$} & $\begin{array}{l}\text { First, human resilience to hardship suggests that Cambodian } \\
\text { people (especially the Khmer participants) could endure the } \\
\text { unwelcome climate change consequences. This resilience may } \\
\text { result from their endurance during post-war conflict and on- } \\
\text { going unrest through the } 1980 \text { s and 1990s. }\end{array}$ \\
\hline & & & $\begin{array}{l}\text { Second, it is because the private sector, including hotel sector, } \\
\text { has initiated adaptations more than expected. This study also } \\
\text { found that hotel adaptive capacity varies, depending on the } \\
\text { hotel attributes. The higher standard hotels tend to initiate more } \\
\text { adaptations. Meanwhile, in Cambodia/SHV, existing studies } \\
\text { that evaluated the hotel sector's adaptive capacity is } \\
\text { unavailable. }\end{array}$ \\
\hline & & & $\begin{array}{l}\text { Third, the perceived low vulnerability is relevant to risk } \\
\text { perception or perception gap that leads to a subjective } \\
\text { judgement on the actual vulnerability to climate change. }\end{array}$ \\
\hline & & & $\begin{array}{l}\text { Fourth, vulnerability was compared to neighboring countries, } \\
\text { which were perceived to have more vulnerabilities in some } \\
\text { cases. }\end{array}$ \\
\hline
\end{tabular}


The points highlighted in Table 8.1 will now be discussed in turn to contextualise the research.

First, the perceived low vulnerability may be related to the concept of human resilience. Despite the same hardships being caused by external stresses, including climate change, resilient people might feel less vulnerable. It is common that rich countries are more resilient than poor countries in the foreground of natural disasters (Barr et al., 2010; Fankhauser \& McDermott, 2014). Yet resilience varies predominantly across countries, depending on various other factors such as inequality and safety nets (Hallegatte et al., 2017) especially across population. Although Cambodia is not a resilient country in the face of climate change, this may not be the case for her people. Civil war, especially Khmer Rouge, devastated the country. Yet these hardships have taught the people various lessons. They are 'resilient' towards various hardships, one of which is external risks, including climate change.

Previous research has investigated resilience among Khmer children in the aftershock of war such as Coles (1986), who claimed that in spite of the shocking political bloodshed under the Khmer Rouge regime, "I have never seen a group of children, in all the years of my work, who are more resilient and perceptive." (p. 266). This reflects this study's finding that there were $38 \mathrm{Khmer}$ participants (out of 50) with an average age of 32.26. All those children are now working in the tourism/hotel industry today. While being highly resilient, Cambodians may have familiarised themselves with environmental hardship, including climate change, thus making them perceive the current vulnerability as low. This may be true in the case of SHV. As also supported by CCCA (2012a) is that although information regarding weather hazards from the local government is surprisingly low, only $2 \%$ and Cambodians and SHV people have low understanding relevant to 'climate change' CCCA (2012b); these people show high commitment to cope with climate change impacts such as weather hazards (CCCA, 2012b).

Second, the private sector, including the hotel sector, has put huge investments in CCA. This most probably increases their overall adaptive capacity, making them perceive climate change vulnerability as low. UNFCCC (2010) reported that in the Copenhagen Accord, USD 30 billion over the period 2010-2012 and around USD 100 billion per year 
from 2020 onwards was committed by the developed nations to provide financial resources to help developing nations with CCA and mitigation. It is undeniable that a part of this is supposed to come from the private sector $\left(\mathrm{UNFCCC}^{37}, 2010\right)$. The private sector plays a potential role in (financing) adaptation (Pauw \& Pegels, 2013) because private sector investment makes up $86 \%$ of the worldwide investment and financing flows (UNFCCC, 2009), and in developing nations $90 \%$ of the population is involved in the private sector to generate their income (Pauw \& Pegels, 2013). The above arguments may align with the findings. The above-mentioned findings indicate that SHV's hoteliers have put remarkable investments for CCA, especially higher standard hotels. The potential climatic adaptations include institutions of seawall barriers, redesigns and reconstructions of their hotel buildings and buildings of water tanks/storage and digging of human-dug wells. Nguyen (2015) also argues that (in Vietnam), the private sector, such as the hotel sector, has financial potential, technological know-how and managing standards to enhance sustainable development and environmental performance. Although there is limited information on CCA investments by private sector, particularly for the hotel sector in Cambodia, it can be assumed that in this sector financial capacity varies, depending on hotel attributes. The higher standard they are, the higher financial capacity they have; this will be discussed in more detail under aim 5 .

Although Cambodia has low adaptive capacity, this may not be the case of SHV's hotel sector; the hotel sector's adaptive capacity varies, depending on hotel attributes. The generalisability of published research on this issue is problematic. Reviewing literature for this study found that although some papers argue that the private sector, such as hotel sector, has the lowest adaptive capacity relative to their fixed structures (e.g. WMO, UNEP, \& WTO, 2008), this study found that hotels have capacity that is especially relative to technical adaptation. Although some interviewees reported that their hotels were affected by climate change, existing technical reports by the government, IOs and NGOs evaluated similar incidents that were not available to confirm the level of damage or vulnerability due to climate change. Meanwhile, in Cambodia and SHV, information about whether hotel adaptive capacity is low or high is unavailable. The information about climate change vulnerability and the hotel sector remains a large gap. Crick et al. (2016) argue that while numerous CCA studies in developing countries have targeted households

${ }^{37}$ United Nations Framework Convention on Climate Change 
and communities, especially in rural environments, very little attention has focused on the private sector. The private sector not only contributes to developing countries' development efforts but also helps society to productively adapt to climate change and increase climate resilience. Crick et al. (2016) continue that while it is important to increase understanding on how to provide an enabling environment to support their adaptation to climate change, as of now much of the literature on private sector's adaptation focuses on the larger companies and those based in developed nations. They add that very little research examines how to promote and facilitate private sectors' adaptation, especially for private sectors in developing nations.

Private sectors such as the hotel sector have already increased their adaptive capacity. What the sector needs is the help from other stakeholders, especially from the government. This study found that despite some help by the government, this is not sufficient for the hotel sector. The government has little emphasis on enabling condition and supportive environments to stimulate and incentivise local private sector's adaptation (Crick et al., 2016). This study argues that the assumption that the hotel sector has the lowest adaptive capacity remains questionable. Their adaptive capacity varies, depending on their available resources to adapt to the climatic changes. Therefore, the quick assumption that hotels of all types have the lowest adaptive capacity could be challenged. The gap in the hotels' adaptation literature remains an issue, especially the disconnection with the hotel sector's adaptation.

Third, the low perception is closely related to risk perception or perception gap for instance the gap in processing information and the knowledge gap in analysing the information. Risk perceptions are related to information processing and sensemaking towards climate change as an external threat, phenomenon, or situation (Reser et al., 2012). SHV's hoteliers likely have two ways to receive climate change information. First, in a well-ordered hierarchy, they obtain it from the national level, local level and peer groups (e.g. other hotels and boat companies). Second, they access information on an individual basis by different methods (see Table 5.11). In the first option, it consumes time to transfer messages. In the second option, it requires them to have sufficient knowledge to make sense of the information. Due the lack of technical equipment to detect accurate information (see section 3.3.2.6), the national government is reliant on 
information from international experts (see Käkönen et al., 2014) and other neighbouring countries (e.g. Vietnam, Thailand and Laos) to make sure the climate change information is correct especially about the time and place of occurrence before transferring this message to local governments. Due to lack of knowledge, hoteliers may perceive the information differently. While Ainuddin, Routray, and Ainuddin (2014) call risk perception a subjective judgment, Mahon (2014) calls it 'incomplete understanding' of climate change. Mahon (2014) further explains the uncertainty of perceptions relevant to the dynamics of climate for hoteliers. The hoteliers reported a lack of understanding about 1) the nature of climate change; 2) the dynamics of the phenomenon relevant to a change in climate; 3) the progress of change; 4) whether climate change can be escaped from; and 5) how climatic changes will impact the tourism sector.

Due to bureaucracy and a time delay from the national government's message to the hotel sector, the information may be delivered late. Interviews in this study finds that the participants felt that there was a severe lack of climate change information, making the actual climate change occurrences and their perceptions uncertain. Peterson (2006) explains that lay people are surrounded by much uncertainty concerning the magnitude, timing and location of climate change, thus making it difficult to comprehend its impacts. In Cambodia, Chean (2017) also found that although climate change is increasingly considered the greatest challenge to development in every country around the world, including Cambodia, about half participants in his/her study were not aware of the climate change causes because they do not have access to much information or literature about climate change, such as academic articles, policy, public debates and discussions. This aligns with Resurreccion, Sajor, and Fajber (2008) who found in 2008 that very few Cambodian people believe that climate change is an issue to the country - this observation may still have relevance today as reflected by Chean (2017) who found similar trend.

Fourth, it is relevant to the perceptions of vulnerabilities climate change on neighbouring countries. Table 5.2 indicates that $2 \%$ and $31 \%$ of the participants perceived SHV's coastal tourism as slightly vulnerable and not vulnerable. They felt that SHV might not be the only place to have experienced serious climate change impacts compared to other neighbouring countries or their countries of origins (24\% were foreigners). The Khmer respondents often compared SHV's vulnerability to those of other neighbouring countries 
such as the Philippines, Thailand and Vietnam where they are more or less affected. One of them mentioned, "Generally, we have problems of climate change during these months like storms, wind contractions, and being under the climate influences from other countries...” H1. Shrestha (2014) found that Myanmar and Vietnam, the lowest areas in the Mekong basin, are the most vulnerable countries to intense floods recently in the region due to the tropical and monsoonal influence. For another example, World Risk Report (2012) also classified the Philippines along with Cambodia as the two of the top ten countries most vulnerable to climate change in the world. Marks (2011) also found that climate change impacts in Thailand include extended droughts, reduced agricultural and fishery products, severe flooding, SLR and health-related problems that are already severe and will probably create or intensify many additional problems during the next few decades.

\subsection{Discussion on aim 2: Vulnerability assessment of Sihanoukville's hotel sector}

In respect to the second research aim, it was found that half (50\%) of the respondents explained that their hotels are slightly vulnerable while about one third (32\%) reported that their hotels are moderately vulnerable (see Table 5.4). As reflected by its mean of 2.63 , it clearly shows that most participants perceived that SHV's coastal tourism hotel sector is 'either slightly' or 'moderately vulnerable'. Nevertheless, literature indicated that Cambodia as well as SHV is very vulnerable to climate change. This clearly indicates that the participants' perceptions are overwhelmingly different from early findings. Table 8.2 presents why the vulnerability is perceived lower than the early research. 
Table 8. 2: Reasons why the participants perceived the vulnerability of SHV's hotel sector lower than early findings

\begin{tabular}{|c|c|c|c|}
\hline $\begin{array}{l}\text { Existing studies about } \\
\text { SHV's vulnerability }\end{array}$ & & $\begin{array}{l}\text { The hotel participants' perception } \\
\text { on the vulnerability of SHV's hotels }\end{array}$ & Discussion \\
\hline Very vulnerable & & Slightly/Moderately & \\
\hline \multirow{6}{*}{$\begin{array}{l}\text { Climate change } \\
\text { vulnerability in } \\
\text { Cambodia } \\
\text { (see section } 3.2 .3 \text { ) } \\
\text { Climate change } \\
\text { vulnerability in coastal } \\
\text { zone/SHV } \\
\text { (see section } 3.3 .2 \text { ) }\end{array}$} & \multirow{6}{*}{ vs. } & \multirow{6}{*}{$\begin{array}{l}\text { Research question } 2 \text { : To what extent } \\
\text { is your hotel vulnerable to climate } \\
\text { change? } \\
\text { Answer/Finding: Slightly/moderately } \\
\text { vulnerable with its mean of } 2.63\end{array}$} & $\begin{array}{l}\text { First, the perceived low vulnerability is relevant to risk perception or } \\
\text { perception gap (e.g. underestimate the climate risk or perceive that } \\
\text { they are safer than others) that leads to a subjective judgement on the } \\
\text { actual vulnerability to climate change. }\end{array}$ \\
\hline & & & $\begin{array}{l}\text { Second, it is related to the uncertainty of the perceived climate } \\
\text { change-related information. The hoteliers are surrounded by the } \\
\text { uncertainty and not able to judge the information. }\end{array}$ \\
\hline & & & $\begin{array}{l}\text { Third, it is because the SHV's hotel sector has initiated adaptations } \\
\text { more than expected for their businesses, especially the high star } \\
\text { hotels. }\end{array}$ \\
\hline & & & $\begin{array}{l}\text { Fourth, vulnerability was compared to neighboring countries, which } \\
\text { were perceived to have more vulnerabilities in some cases. }\end{array}$ \\
\hline & & & $\begin{array}{l}\text { Fifth, it is because some had perceived rankings based on the } \\
\text { occupancy rate rather than the actuality of climate change. }\end{array}$ \\
\hline & & & $\begin{array}{l}\text { Sixth, human resilience to hardship suggests that Cambodian people } \\
\text { (especially the Khmer participants) could endure the unwelcome } \\
\text { climate change consequences. This resilience may result from their } \\
\text { endurance during post-war conflict and on- going unrest through the } \\
\text { 1980s and 1990s. }\end{array}$ \\
\hline
\end{tabular}


Based on Table 8.2, these will now be discussed in turn to contextualise the research.

First as argued above in section 8.3, the low perception of vulnerability, also the context of hotel sector, could be related to risk perception or perception gap (e.g. underestimate the climate risk or perceive that they are safer than others (Gifford et al., 2009). The perception of risk is also limited by social and individual factors (Adger et al., 2009). The individual factors include controllability, perception of self-efficacy, preferences, beliefs, knowledge, experience, habitual behaviour, and perception of risk, while the social factors comprise of values and norms (Adger et al., 2009). Adger et al. (2009) argue that perceptions of risk, experience and knowledge are the significant factors at the individual and societal level that influence whether and how adaptation happens. The adaptation initiative is relevant to the knowledge gap and low of perception on climate changerelated information. There are variations in risk perceptions: the limit to shortening gaps between current experiences and future projections, the distance of the nature of climatic change, and the limit to identifying and implementing adaptation actions (Adger et al., 2009). For instance, due to the low level of perceived important information, particularly about the relationship between climate change and hotel businesses in the area, the hotel participants seemed to underestimate the vulnerability towards their businesses. Surprisingly, Mahon (2014) found that not only hoteliers but also tourism policy-makers tend to underestimate future climate risk. Previous findings by Kunreuther et al. (2002) also found that experts such as policy-makers are prone to misunderstandings about climate change issues as non-experts. In SHV, the interviewed participants, who are nonpolicy makers, may have the same bias. Shakeela and Becken (2014) explain that there are insufficient studies on psychological, social, and cultural aspects of climate change risk perceptions amongst tourism practitioners. Zhang, Lindell, and Prater (2009) suggest that more research about climate change impacts on business is required so that communities can have better preparation for, responses to and recovery from environmental disasters. In Botswana, Saarinen et al. (2012) found that only a few tourism operators (including accommodation) perceived higher vulnerability of tourism; none thought that climate change had affected their own operations and activities.

Second, the low perception is related to the uncertainty of the obtained climate changerelated information. Hoteliers were uncertain to choose between the reality and 
uncertainty of climate change or they were surrounded by uncertainty and not able to use the information. Linnenluecke, Griffiths, and Mumby (2015) explain that there is a question on the relationships between the information that hoteliers obtain on climate change and how they assess their businesses' vulnerability to climate change. For instance, Campos (2009), studying the relationships of climate change and hotels in Phuket (in Thailand), found that climate change is not the only concern of hoteliers. They are more concerned about other more important issues such as economic crisis, political instability, oil prices and competitors. Su (2014) found that in Taiwan $99 \%$ of hoteliers had access to climate change-related information and over half of them obtained information from their 'customers'. Therefore, the perception of information did not provide them with sufficient insights in respect to climate change ( $\mathrm{Su}, 2014)$. $\mathrm{Su}$, Hall, and Ozanne (2013) found that through media reports, hoteliers are aware of natural disasters, but they did not know how to relate hotels' environmental and business approaches directly to climate change concerns.

The above arguments reflect the perceived vulnerability of respondents. At the national level, tourism-related climate change plans just emerged in 2015 (see section 3.2.4), meaning that little information has signalled the relationship between their hotels and climate change. They were uncertain as to how the information relates to their daily business operations. Decision-makers have little choice but to depend on their own sensemaking to rationalise climate information (Linnenluecke et al., 2015).

Third, SHV's hotel sector has already initiated adaptations to a greater degree (see Table 5.10). For example, almost all hotels constructed water storage and had human-dug wells (digging a well cost around USD10,000 at the mainland and USD20,000 at the islands). For some luxury hotels, they instituted seawalls and initiated particular programmes for CCA. Although previous studies considered Cambodia's adaptive capacity low, the site visits found that there were a number of adaptations initiated by the DPs in place. The initiatives included capacity building, planting, conserving and re-planting mangroves in large areas and constructing coastal dams in SHV. These could be because SHV is vulnerable and has potential for Cambodia's economy. The government and IOs and NGOs have paid more attention to the area. UN SDGs ${ }^{38}$ (2017) also indicate that

${ }^{38}$ Sustainable Development Goals 
Cambodia (as one of the LCDs) is receiving financial support, including finance, technology and capacity building for climate change-related planning and management. In addition to the observations, supporting informal interviews with government officials and some DPs, confirmed that despite insufficiency, support was given to the MOE at the national level, which later decentralised climate change-related projects at the subnational level. For example, at the national level, along with other LCDs, Cambodia was invited to formulate NAPAs in 2001 to prioritise urgent activities to address climate change. NAPA is a well-organised document that was produced to secure international public funding for adaptation (see section 3.2.4). These initiatives may indirectly benefit the adaptations of SHV's hotels.

Fourth, it is relevant to the comparison of perceived climate change impacts to neighbouring countries and their countries of origins as discussed above in section 8.3. The participants believed that Cambodia's SHV is not the only place which is threatened by climate change. Some places in the neighbouring countries (e.g. Thailand, Myanmar and Vietnam) also experience similar or more serious climate change effects.

Fifth, some participants rated the vulnerability based on the effects of occupancy rate. As long as the guests keep coming and hoteliers generate sufficient income, they think that their hotels have no climate change effects and are safe from climate change impacts. This leads to their rating their hotel's vulnerability as low. In some hotels, some guests came and sat in casinos. As a result, the hotels did not feel affected by climate change. One participant said, "It's hard one to say, you know a lot of tourists come at this time to sit in the casinos, they don't go to the beach ...” H15. The second added, "I think also it is based on the kind of tourism because I think there is a lot of gambling tourists. I think they don't care about it as long as they have casinos." H34. This does not reflect the actual vulnerability of climate change while they have self-favouring bias on room occupancy figures.

Sixth, as argued above human resilience to hardship suggests that Cambodian people (especially the Khmer participants) could endure the unwelcome climate change consequences. This resilience may result from their endurance during post-war conflict and on-going political turbulence through the 1980s and 1990s. They are now working as 
hotel owners, managers or supervisors in SHV. These people tend to perceive low vulnerability.

\subsection{Discussion on aim 3: Climate change adaptation in Sihanoukville's coastal hotel sector}

On the third aim, existing studies such as Su (2014), Walmsley (2012), Simpson et al. (2008), Su et al. (2013) and WMO UNEP and WTO (2008) indicated that within tourism, the hotel sector has the lowest adaptive capacity especially relative to their fixed structures (buildings). In Cambodia, many reports (see chapter 3) indicated that Cambodia and its coastal zone as well as SHV exhibit many vulnerabilities and its adaptive capacity is low. However, these prior research studies do not fully correspond to the findings of this study. The points highlighted in Table 8.3 will now be discussed in turn to contextualise the research. 
Table 8.3: Reasons why the SHV's hotel sector has adapted to climate change

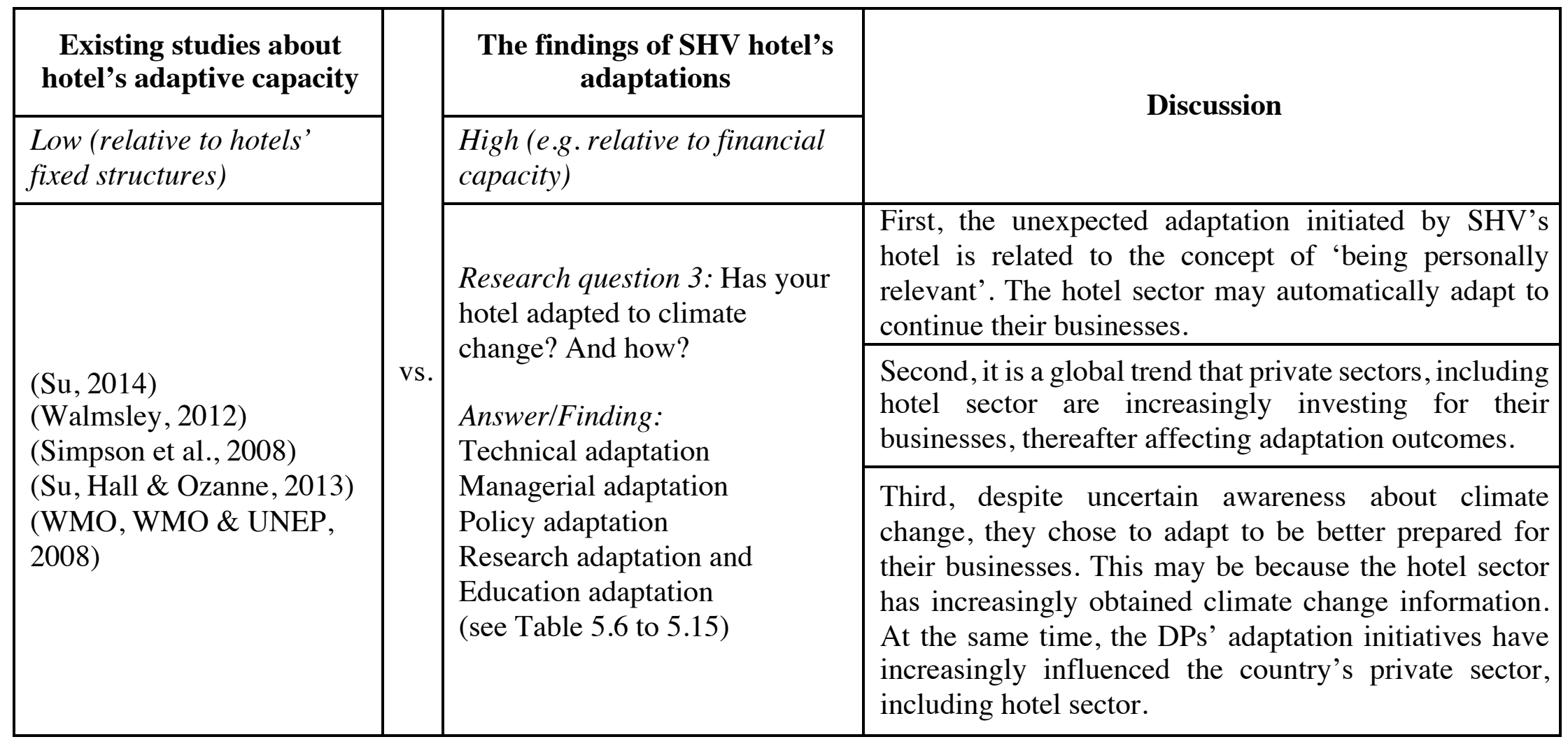


First, the adaptation in SHV's hotel may be related to the concept of being personally relevant (see Moser \& Dilling, 2004) to their businesses. Scannell and Gifford (2013) explain that personally relevant information or messages enhance the motivation and capacity to act. Lewin, Weigelt, and Emery (2004) call it "fit perspective" (p. 20) with the business environment. Although each adaptation (e.g. adapting to beach erosion) by SHV's hotel sector has its specific intention (e.g. to stabilise beach areas), the overall purpose is to facilitate income generation or cost reduction, which is the most central concern in their business operations. Business survival is the enabling condition for their efforts. Many private businesses may automatically adapt their operations to maintain profit generation under climatic change (Pauw \& Pegels, 2013). Levina and Tirpak (2006) called it 'private adaptation', which is initiated and executed by private companies, households or individuals. It is usually triggered by the actor's rational personal interest. Every investment (including CCA) by hotel operators must obtain financial gain; investments outside this are not motivated by hoteliers because every one of them wants to quickly generate income (Campos, 2009).

Cost reduction is another key factor to encourage hoteliers to adapt. Findings in section 5.4 shows that adaptation such as installing solar panels to meet increasing demand on air-conditioning due to climate change would beneficially exploit the opportunity for the businesses because it contributes to the cost reduction of increased demand of water energy. One explained, "At the back we have one truck on standby. If the water is not enough, the truck can fill up. More water is spent on gardening especially when there is high evaporation at midday due to excessive heat." $\mathrm{H} 21$. This corresponds to argument by Jarvis and Ortega (2010) explaining that in hotels, financial gain, primarily through a reduction in costs, is one of the motives that stimulate the actions to reduce the environmental impact and climate change. Based on economic theory, this would provide them with advantage over others (Walmsley, 2012). To sustain the business, hoteliers become aware of the financial implications, increase the resilience of their performances, and readjust their main business approach. For instance, companies are beginning to take solutions to water scarcity induced by drought (Chrysostomidis \& Constable, 2015).

Second, as stated above in section 8.5, due to the global trend, the hotel sector has increasingly put investments into climate change-related responses especially CCA. 
Meanwhile, SHV's hotels have adapted to climate change to a greater degree especially by high standard hotels. As outlined above, Nguyen (2015) supports that (in Vietnam), private sectors such as the hotel sector have financial potential, technological know-how and managing standards to enhance sustainable development and environmental performance.

Third, the increasing trend of hotels' adaptation is influenced by the perceptual information from media and involvement and influence of developed countries, IOs and NGOs. Table 5.11 shows that the participants have access to early warning systems about climate change impacts. They obtained climate change-related information through computer/smart phone/tablet (59.3\%), TV (30.5\%) and radio (10.2\%). Begum and Pereira (2015) explain that businesses begin to become aware of the risks and opportunities from climate change. They perceive risks dependent on information they obtain from media and may be due to peer influence (Wachinger et al., 2013). Su et al. (2013) argue that through media reports, hoteliers are aware of natural disasters. Su et al. (2013) found in their study (in Taiwan) that up to $95 \%$ of hoteliers are aware of climate change existence. They obtain information from media, such as newspaper, TV, radio, and the Internet. Campos (2009), studying the relationships between climate change and hotels in Phuket (Thailand), also found that Phuket's hotels were also affected by climate change, $61.1 \%$ by water shortage, $38.5 \%$ by rising temperature, and $26.5 \%$ by spread of diseases. Such research by Campos contributed to information sources for hotel operators.

As indicated in section 6.4.1 and Table 6.2, information is one of the most critical enabling factors in hotels' adaptation. Although SHV's hotels appear challenging to obtain and judge information provided by the national/local government, they have to take actions on climate change for the sake of their business survival. Linnenluecke et al. (2015) explain that in business operation, in order to ensure its continued survival, decision-makers must adapt their company by assessing the information on their company's business condition and formulating strategic solutions to changed circumstances. They continue that in perception-based strategic choices, the decisionmakers as adaptation actors take actions, depending on the information obtained rather than the objectively considered 'best' information available concerning business risks and opportunities. 
As indicated in section 3.2.4, because Cambodia's adaptive capacity is low, various projects have been approved and implemented. Joint climate change-supported DPs, namely CCCA, were initiated. The CCCA is jointly funded by UNDP, Swedish International Development Cooperation Agency (Sida), EU, and Danish International Development Agency (Danida). CCCA has its purpose in enhancing the capacity of the $\mathrm{NCCC}^{39}$ to realise its commitment to address climate change and to facilitate line ministries and NGOs to implement the most important climate change actions. Site visits and informal conversations with SHV DOE found that the DPs had initiated a number of adaptations for example, planting conserving and re-planting mangroves, constructing coastal dams and capacity building. Interviews also indicated that some hotel managers participated in DP-initiated workshops. Some were aware of projected 1m SLR in the coastal zone and other important climate change-related information. UN SDGs (2017) also indicates that a number of LCDs are receiving amount of (financial) support, including finance, technology and capacity building for climate change-related planning and management. These initiatives may influence and be supportive to SHV and the hotel sector's adaptations. The government itself is taking concerted efforts to combat climate change (see section 3.2.4). For instance, in 2015 the government created a document, namely Climate Change Action Plan in Tourism Sector 2015-2018. Although little is known about the implementation of the plan, it shows that the government, together with other DPs, is paying increasing attention to climate change-tourism issues. This has directly influenced local government and indirectly and gradually impacted the private sector.

Within the adaptation in the context of SHV's hotel sector, Tables 6.1 and 6.2 show the critical levels of the barriers and enabling factors to adaptation as perceived by the 50 hotel respondents. To contextualise the research, the next section will now discuss these results relative to the existing literature.

\footnotetext{
${ }^{39}$ National climate change committee (Cambodia)
} 


\subsection{Discussion on aim 4: Hotel sector's barriers and enabling factors to adaptation}

\subsubsection{Hotel sector's barriers to climate change adaptation}

Table 8.4 compares and contrasts the findings of the perceived rankings of the adaptation barriers to the existing literature. Comparing and contrasting all nine barriers to early studies is impossible. This study discusses the key adaptation barriers. Although the literature has highlighted several adaptation barriers in section 2.7, there is a relatively small body of literature that directly discusses them in regard to the hotel sector, especially in developing countries. This study is one of the first studies to present the relative rankings (the most critical, medium and the least critical) and identify barriers to adaptation in the hotel sector (see section 6.2 and 6.3). 
Table 8.4: Comparison of the adaptation barriers between the findings and the literature

\begin{tabular}{|c|c|c|c|}
\hline No. & Adaptation barriers & The findings & Literature \\
\hline 1 & $\begin{array}{l}\text { Limited resources (e.g. economic limits or budget } \\
\text { limits/constraint, lack of technical skills/ } \\
\text { technological limits or human resources) }\end{array}$ & \multirow{3}{*}{ The most critical } & $\begin{array}{l}\text { (Amundsen, Berglund, \& Westskog, 2010) } \\
\text { (Biesbroek et al., 2011) } \\
\text { (Dessai, Lu, \& Risbey, 2005) } \\
\text { (Lorenzoni et al., 2000) } \\
\text { (Matasci, Kruse, Barawid, \& Thalmann, 2014) } \\
\text { (Adger et al., 2007) } \\
\text { (Fankhauser, 2010) } \\
\text { (Narain, Margulis, \& Essam, 2011) } \\
\text { (Moser \& Ekstrom, 2010) }\end{array}$ \\
\hline 2 & $\begin{array}{l}\text { Limited knowledge/perception of climate change } \\
\text { (e.g. limited basic knowledge about causes, effects } \\
\text { and solutions to climate change) }\end{array}$ & & $\begin{array}{l}\text { (Lorenzoni, Nicholson-Cole, \& Whitmarsh, 2007) } \\
\text { (Amundsen et al., 2010) } \\
\text { (Vulturius \& Gerger Swartling, 2015) }\end{array}$ \\
\hline 3 & Lack of political will & & $\begin{array}{l}\text { (Ford \& Pearce, 2010) } \\
\text { (Amundsen et al., 2010) } \\
\text { (Measham et al., 2011) } \\
\text { (Biesbroek et al., 2011) } \\
\text { (McNeeley, 2012) } \\
\text { (Adger et al., 2005) }\end{array}$ \\
\hline 4 & $\begin{array}{l}\text { Insufficient information (about climate } \\
\text { vulnerabilities, or absence of reliable climate data) }\end{array}$ & Medium & $\begin{array}{l}\text { (Jarvis \& Ortega, 2010) } \\
\text { (Dessai et al., 2005) } \\
\text { (Harriet, 2000) } \\
\text { (Norgaard, 2006). } \\
\text { (Crabbé \& Robin, 2006) } \\
\text { Mukheibir \& Ziervogel, 2007) } \\
\text { (Biesbroek et al., 2011) } \\
\text { (Matasci et al., 2014) }\end{array}$ \\
\hline
\end{tabular}




\begin{tabular}{|c|c|c|c|}
\hline 5 & Uncertain responsibilities for adaptation & & $\begin{array}{l}\text { (Cuevas, 2015) } \\
\text { (Schröter, Polsky, \& Patt, 2005) } \\
\text { (Huang et al., 2011) } \\
\text { (Measham et al., 2011) } \\
\text { (Grasso, Moneo, \& Arena, 2014) }\end{array}$ \\
\hline 6 & Low level of leadership and guidance & & $\begin{array}{l}\text { (Moser \& Ekstrom, 2010) } \\
\text { (Tribbia \& Moser, 2008) }\end{array}$ \\
\hline 7 & $\begin{array}{l}\text { Insufficient engagement and interaction with other } \\
\text { stakeholders }\end{array}$ & \multirow{3}{*}{ The least critical } & $\begin{array}{l}\text { (Prutsch et al., 2010) } \\
\text { (Matasci et al., 2014) } \\
\text { (Bierbaum et al., 2013) } \\
\text { (Huang et al., 2011) }\end{array}$ \\
\hline 8 & $\begin{array}{l}\text { Regulatory/legal constraints (e.g. policies at } \\
\text { national level that limit the ability of local level to } \\
\text { response to climate change) }\end{array}$ & & $\begin{array}{l}\text { (Urwin \& Jordan, 2008) } \\
\text { (Prutsch et al., 2010) } \\
\text { (Measham et al., 2011) }\end{array}$ \\
\hline 9 & $\begin{array}{l}\text { Institutional barriers (e.g. lack of institutional } \\
\text { flexibility, unclear or contradictory goals within } \\
\text { institution, restriction of individual capacity to } \\
\text { adapt etc.) }\end{array}$ & & $\begin{array}{l}\text { (Schröter et al., 2005) } \\
\text { (Huang et al., 2011) } \\
\text { (Measham et al., 2011) } \\
\text { (Grasso et al., 2014) }\end{array}$ \\
\hline
\end{tabular}


As outlined in Table 6.1, 'limited resources', including budget and HR, is the most critical barrier. The finding overwhelmingly accords with prior research. In a study on adaptation to external stressors in coastal communities, Fischer (2018) found that resource scarcity likely becomes a critical element in how coastal communities address rising sea levels and erosion as well storm surges. Conducting a study of CCA in agriculture (in Malaysia), Masud et al. (2017) found that 'high cost' or 'lack of budget' is the most critical obstacle to CCA. This likely aligns with McNamara, Westoby, and Smithers (2017) who argue that insufficient and protracted provision of funding and resources can be critical barriers to adaptation. This is similar across other two most critical barriers such as 'limited knowledge/perception of climate change' and 'lack of political will' because a large volume of literature explains that knowledge is a key factor for potential adaptation and 'political will' from the government is key, as it can take action and confront the current barriers to change/amend legislation or provide additional resources (Ford \& Pearce, 2010; Measham et al., 2011).

This study found that the barrier of 'low level of leadership and guidance' is medium barrier. Yet this is inconsistent with previous studies. Cuevas (2015) regards 'lack of leader' as an 'important barrier'. A leader can create a significant opportunity but the absence of a champion leader would lead to the failure to the climate change endeavour and be a critical impediment to the action (Cuevas, 2015; Simões et al., 2017). Simões et al. (2017) argue that poor management context and leadership are the emerging critical barriers for CCA. This may be applicable to the barrier of 'insufficient information'. Although there is a large amount of literature arguing that lack of information presents a significant obstacle for CCA, the participants believed that this barrier is a medium barrier. This may be due to risk perception or perception gap or because most participants remained surrounded by uncertainty of climate change information (see Table 8.2).

\subsubsection{Hotel sector's enabling factors for climate change adaptation}

Table 8.5 compares and contrasts the findings relative to the perceived rankings of the adaptation of enabling factors to the existing literature. Comparing and contrasting all nine enabling factors to previous studies is impossible. This study discusses the key enabling factors for adaptation. Although the literature has highlighted several enabling factors in section 2.12 , there is a relatively small body of literature that directly discusses 
them in the hotel sector, especially in developing countries. The discussion of the critical enabling factor follows the same trend. This study is also one of the first studies to present the relative rankings (the most critical, medium and the least critical) and identify enabling factors for adaptation in the hotel sector (see section 6.4 and 6.5). 
Table 8.5: Comparison of the enabling factors for adaptation between the findings and literature

\begin{tabular}{|c|c|c|c|}
\hline No. & Enabling factors & The findings & Literature \\
\hline $\mathbf{1}$ & $\begin{array}{l}\text { Sufficient resources (e.g. finance/budget, human } \\
\text { resource, and available technology) }\end{array}$ & \multirow{3}{*}{ The most critical } & $\begin{array}{l}\text { (Orhan, 2016) } \\
\text { (Olazabal, Galarraga, Ford, } \\
\text { Lesnikowski, \& Sainz de Murieta, } \\
\text { 2017) } \\
\text { (Lowe, Foster, \& Winkelman, 2009) } \\
\text { (Tribbia \& Moser, 2008) } \\
\text { (Zuka, 2014) }\end{array}$ \\
\hline 2 & Sufficient information (e.g. about vulnerabilities...) & & $\begin{array}{l}\text { (Archie et al, 2014) } \\
\text { (Glaas et al., 2015) } \\
\text { (Dessai et al. (2005) } \\
\text { (Moser et al., 2008) }\end{array}$ \\
\hline 3 & $\begin{array}{l}\text { Good leadership or management structures (e.g. } \\
\text { strengthen institutions to implement CCA } \\
\text { programmes) }\end{array}$ & & $\begin{array}{l}\text { (Cuevas, 2015) } \\
\text { (Rissik \& Reis, 2013) } \\
\text { (Burch, 2010) } \\
\text { (Zhang et al., 2013) } \\
\text { (Orhan, 2016) } \\
\text { (Lozano, 2015) } \\
\text { (Anguelovski, Chu, \& Carmin, 2014) }\end{array}$ \\
\hline 4 & $\begin{array}{l}\text { Individuals' motivation to respond (committed } \\
\text { workforce) }\end{array}$ & \multirow[t]{2}{*}{ Medium } & $\begin{array}{l}\text { (Bandura, } 1977 \text { \& Hines et al., } 1987 \text { as } \\
\text { cited in Adger, Lorenzoni \& O'Brien, } \\
\text { 2009) } \\
\text { (Prutsch et al., 2010) } \\
\text { (Moser \& Dilling, 2004) } \\
\text { (Paton, Millar, \& Johnston, 2001) } \\
\text { (Zuka, 2014) } \\
\text { (Kalaugher et al., 2013) } \\
\text { Tzschentke et al. (2008) }\end{array}$ \\
\hline 5 & Clear adaptation goals & & $\begin{array}{l}\text { Coles, Dinan, \& Warren, 2016) } \\
\text { (Adger et al., 2005) } \\
\text { (Adger, et al., 2009) }\end{array}$ \\
\hline
\end{tabular}




\begin{tabular}{|c|c|c|c|}
\hline 6 & Political will & & $\begin{array}{l}\text { (Ford \& Pearce, 2010) } \\
\text { (Amundsen et al., 2010) } \\
\text { (Measham et al., 2011) } \\
\text { (Biesbroek et al., 2011) } \\
\text { (McNeeley, 2012) } \\
\end{array}$ \\
\hline 7 & $\begin{array}{l}\text { Previous and current adaptation } \\
\text { experiences/awareness/knowledge and climate change } \\
\text { education }\end{array}$ & \multirow{3}{*}{ The least critical } & $\begin{array}{l}\text { (Akerlof et al., 2013) } \\
\text { (Marin, 2010) } \\
\text { (Prutsch et al., 2010) } \\
\text { (Simonet \& Fatorić, 2016) } \\
\text { (Vulturius \& Gerger Swartling, 2015) } \\
\text { (Ayesha \& Heather, 2014) }\end{array}$ \\
\hline 8 & $\begin{array}{l}\text { Engagement/collaboration/communication with other } \\
\text { stakeholders }\end{array}$ & & $\begin{array}{l}\text { (Prutsch et al., 2010) } \\
\text { (Bierbaum et al., 2013) } \\
\text { (Petersen, Hall, Kahl, \& Doran, 2013) } \\
\text { (Rao, 2013) }\end{array}$ \\
\hline 9 & Location of hotel & & (Tierney, 2007) \\
\hline
\end{tabular}


Table 6.2 indicates that 'sufficient resources', including budget and HR is the most critical enabling factor. The finding overwhelmingly corresponds to prior research. Mazhar, Bull, and Lemon (2017), Zuka (2014), Lowe, Foster, and Winkelman (2009), Simpson et al. (2008) and Tribbia and Moser (2008) made similar observations. For example, Simpson et al. (2008) argue that HR strongly influences the quality of implementation of adaptation options. What is important in adaptation are the financial resources that must be available to support it. This may be closely similar to the enabling factor of 'sufficient information' and 'good leadership or management structures', which are also identified as the most critical. A growing body of literature in Table 8.5 shows that both 'sufficient information' and 'good leadership or management structures' are an indispensable component in the positive effect of hotels' adaptation.

Table 6.2 indicates that the enabling factor of "engagement/collaboration/ communication with other stakeholders' is the least critical. This finding seems to be inconsistent to existing literature. For example, Mannke (2011) argues that for local adaptation to climate change, it is critical to involve stakeholders, especially local participants; this can be done through networking, gathering valuable feedbacks and input. Mazhar et al. (2017) argue that stakeholder engagement is one of the critical components. It may be argued that the majority of SHV's hotels tended to deal with climate change individually. Working collaboratively with their peers could be seen such as forming a committee for better environmental performance but working together with external parties such as IOs and NGOs and the national/local government is largely absent. There are two possible explanations. First, as highlighted in section 3.2.2, all tourism businesses are privateowned. As there are no state-owned hotels, the government, especially local authorities, has either insufficient experience or too large a gap in knowledge to deal with climate change in private sector's hotels. Second, the IOs and NGOs often focus on communitybased adaptations and are goal-oriented by their funders. This largely reflects the current climate change issue in SHV where the communities are more vulnerable than the private sector such as hotels.

After discussing the criticality of barriers and enabling factors to adaptation, the study examines the extent to which hotel attributes and participants' backgrounds influence the barriers and enabling factors to adaptation from the responses of 50 hotel respondents. 


\subsection{Discussion on aim 5: To critically examine the barriers and enabling factors to CCA according to hotel attributes and participants' backgrounds}

Table 7.1 above shows that among all hotel attributes, the most differences were found with the hotel attribute of 'hotel size' (small vs. medium vs. large) while among all participants' backgrounds, the most differences were found with the background of 'participants' nationalities' (Khmer vs. foreign). Therefore, the discussion will begin with these findings as indicated in Table 8.6 below.

\subsubsection{The perceived barriers and enabling factors influencing adaptation between hotel sizes}

Table 8.6: The perceived barriers and enabling factors affecting adaptation between hotel sizes ${ }^{40}$

\begin{tabular}{|c|c|c|c|c|c|}
\hline \multirow{2}{*}{\multicolumn{2}{|c|}{ Description }} & \multirow{2}{*}{$\begin{array}{c}\text { K-W } \\
\text { test }\end{array}$} & \multicolumn{3}{|c|}{ Hotel size } \\
\hline & & & & Modi & I \\
\hline 党 & Insufficient information & $\mathrm{p}=0.002$ & \multicolumn{2}{|c|}{$\begin{array}{c}\Uparrow \\
\text { Higher }\end{array}$} & $\begin{array}{c}\text { Lower } \\
\Downarrow\end{array}$ \\
\hline \multirow{3}{*}{ 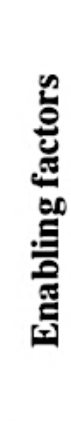 } & $\begin{array}{l}\text { Individuals' motivation to respond } \\
\text { (committed workforce) }\end{array}$ & $\mathrm{p}=0.044$ & $\begin{array}{c}\Uparrow \\
\text { Higher }\end{array}$ & & $\begin{array}{c}\text { Lower } \\
\Downarrow\end{array}$ \\
\hline & $\begin{array}{l}\text { Previous and current adaptation } \\
\text { experiences/awareness/knowledge }\end{array}$ & $\mathrm{p}=0.045$ & \multicolumn{3}{|c|}{$\begin{array}{l}\text { Pairwise comparison shows that } \\
\text { there was trending towards } \\
\text { statistical significance. This sets } \\
\text { avenue for future research. }\end{array}$} \\
\hline & $\begin{array}{l}\text { Location of hotel (relative to } \\
\text { adaptation) }\end{array}$ & $\mathrm{p}=0.009$ & $\begin{array}{c}\Uparrow \\
\text { Higher }\end{array}$ & $\begin{array}{c}\text { Lower } \\
\Downarrow\end{array}$ & $\begin{array}{c}\Uparrow \\
\text { Higher }\end{array}$ \\
\hline
\end{tabular}

First, it is revealing in Table 8.6 that the small and medium-sized hotels have a perceived barrier of 'insufficient information' that is greater than large hotels. Findings show that the small and medium-sized hotels appear to find it difficult to access climate changerelated information while the large hotels have sufficient capacity to access important information, which helps them to formulate better CCA measures. Because the large hotels in this study are all high star hotels, it is argued that they have sufficient resources

${ }^{40}$ See Table 7.1 for details 
such as budget and HR, which increases their adaptive capacity, especially their regional and international networking. In contrast, the small and medium-sized hotels often have limited resources such as budget and HR, particularly social networking tools.

The argument from this finding largely corresponds to prior research. Amran et al. (2012) argue that in climate change responses, a network vitally contributes to the great outcome of a business because it is a platform for sharing information. Results from a study by Orhan (2016) show that firms having regional networks may be better at recovering than those having only neighbourhood or narrow market range. Muttarak and Lutz (2014) further exemplify that individuals who are involved in large and well-created social networks and friendship groups have a larger chance to obtain warnings that are likely to engage them to respond. In SHV, while small hotels mostly obtained information from national/local government and on an individual basis, large hotels had two additional options, which are uniquely different. First, they formed a committee whose role was to share instant and crucial messages to each other in their particular area, Otress Beach II. The second option is international and regional networking. This allows them to receive further assistance, including important information for adapting to climate change. One participant indicated, "We've been picked by TUI (a big travel and tourism company) in Germany. They have provided good message into the industry including CCA measures, it contributes to our substantial CCA." H10 (for more see section 6.4.3). In SHV, due to their sufficient resources, large hotels appear to be more advantageous over small and medium-sized hotels, particularly in accessing the climate change information.

Most of SHV's small hotels often complained that while they have limited individual capacity to access to information, little information is shared by the local authorities, and if there is, the information is inaccurate (also see argument section 8.3). Due to this limitation, they are not fully motivated to involve the issues of adaptation more strongly. This corresponds to Su et al. (2013), who explain that 'climate change concern' was the least essential aspect of small and medium lodging facilities. Nguyen (2015) continue that the smaller hotels are likely to have less environmental practices with less motivation to integrate environmental issues into their business operation. This finding accords with the past studies that small hotels had low perception of climate change phenomenon (Bicknell \& Mcmanus, 2006; Jarvis \& Ortega, 2010). In the context of Vietnam, there are 
two main barriers in performing environmental issues: cost-related issues and lack of information and knowledge (Nguyen, 2015).

When obtaining sufficient information and having higher financial capacity, SHV's large hotels tend to engage in CCA for their businesses. For instance, instituting seawall barriers and planting mangroves were conducted by all upscale or large hotels (see Table 5.9). This widely accords with Su et al.'s (2013) finding that large-sized hotels, especially ones with more than 150 rooms, have more positively responded to climate change more than small and medium-sized hotels. Su (2014) found that large hotels had higher awareness of negative impacts of climate change than small and medium-sized hotels. Due to higher financial security, large hotels tend to experiment with environmental strategy, technology and product, even at the risk of long term profits from these investments (Shah, 2011; Su, 2014). Nguyen (2015) found similar results in Vietnam where large hotels have a greater motivation to integrate environmental issues into their business operation than the small and medium-sized hotels.

Second, Table 8.6 shows that small hotels have a higher mean rating for the enabling factor of 'individuals' motivation to respond (committed workforce)' than the large hotels. Small hotels believed that the 'motivation' shows a commitment of working together towards the reduction of climate change impacts. However, the large hotels believed that hotels' adaptations are about having sufficient information, knowledge and money to act and invest while the 'motivation', perceived as an indirect factor, plays a secondary role. This study argues that while small hotels have a lack of resources such as budget, technical materials and HR, they are likely to depend on other factors such as motivation. To maintain the continuity of their businesses, they tried their best to employ a wide range of adaptation actions. They must be dynamic, persistent and resilient as well as being constantly motivated or their businesses cannot grow in the face of a changing climate and competitive market environment. As in the above argument, the large hotels are all upscale (in this study) and have sufficient resources such as budget and HR as well as international and regional networking. While the large hotels have all the resources, they likely see the enabling factor entitled 'individuals' motivation to respond' as a small part of contribution to their potential adaptation result. 
Third, Table 8.6 further indicates that small and large hotels have higher mean score for the enabling factor of 'location of hotel' (relative to CCA) than medium-sized hotels. The small and large hotels believed that 'location of hotel', such as having spacious beach areas, allows hoteliers to respond to rainfall-induced coastal flooding smoothly. Yet the medium-sized hotels thought that as climate keeps changing, the actual adaptation is the solution while 'location' is not impactful on the adaptation.

Due to resource shortages, small businesses often own older buildings that rarely meet contemporary requirements for seismic protection and have predominantly no seismic design features, which puts their owners, employees and inventory as well their guests (e.g. hotels) at potential climate risk (Alesch et al., 2001). This tends to reflect on SHV's small hotels, especially the small and 'leased' hotels that are normally operated in renovated buildings with weak and temporary constructions. A majority of SHV's hotels are on leasing contracts (34 hotels or 68\%). When operated in tightly structured locations (e.g. already fixed and renovated buildings), small hotels had little choice/flexibility to adapt to climate change.

Dahlhamer and Tierney (1998) and Zhang et al. (2009) found that small firms face more barriers than large businesses and chains in recovering the operations after disasters due to their locations on non-engineered buildings, and lacking the design capacity. In addition, they have limited capacity to carry out hazard management work owing to the limited financial resources necessary for recovery. They also have limitedly access to governmental recovery agendas. Large businesses with an international or regional market have tended to recover more quickly than those having local market segmentations (Webb, Tierney, \& Dahlhamer, 2002a; Zhang et al., 2009). The interviews and existing studies (see section 3.3.2.6) show that a lack of (external) involvement from the government, 'lack of information' was the first medium barrier (see section 6.2.2) and vulnerable location (10\%) (see section 6.3) were the adaptation barriers, and it was mostly smaller hotels facing those challenges. This is predominantly consistent with the existing literature that due to many disadvantages; small hotels have no choices but to run their businesses in climate change-prone areas, thus emphasizing for some that 'location' is impactful condition for CCA. 
Although literature is insufficient to confirm why large hotels have a higher ranking on the enabling factor of 'location of hotel' than the medium hotels, it may be assumed that large hotels have many wider franchises, which are being operated in different places with different markets regionally and internationally. Because larger hotels may have more than one location, they are increasingly located in climate change-prone areas. Tierney (2007) argues that although the owners of large businesses know that some locations are vulnerable areas, they choose to use them because their businesses are near needed resources, including raw materials and skilled staff, to take advantage of synergies and transportation routes (Tierney, 2007). These large businesses later update their facilities to meet disaster resistance (Tierney, 2007). By contrast, due to their sizes/structures and scope of operations fit to the area, the medium hotels are likely either invulnerable or easy to adapt. This study further showed the medium hotels had high commitment to adapt to climate change rather than depending on indirect factors such as location to adapt to climate change.

\subsubsection{The perceived enabling factors for adaptation between participants' nationalities}

Table 8. 7: The perceived enabling factors affecting adaptation between participants' nationalities ${ }^{41}$

\begin{tabular}{|c|c|c|c|c|}
\hline \multirow{2}{*}{\multicolumn{2}{|c|}{ Description }} & \multirow{3}{*}{$\begin{array}{l}\text { MW test } \\
\mathrm{p}=0.018\end{array}$} & \multicolumn{2}{|c|}{ Nationality } \\
\hline & & & \multirow{2}{*}{$\begin{array}{c}\begin{array}{c}\text { Local } \\
\text { (Khmer) }\end{array} \\
\begin{array}{c}\text { Lower } \\
\Downarrow\end{array} \\
\end{array}$} & \multirow{2}{*}{$\begin{array}{c}\text { Foreign } \\
\Uparrow \\
\text { Higher }\end{array}$} \\
\hline$\frac{2}{2}$ & Clear adaptation goals & & & \\
\hline 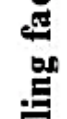 & Political will & $\mathrm{p}=0.039$ & $\begin{array}{c}\text { Lower } \\
\Downarrow\end{array}$ & $\begin{array}{c}\Uparrow \\
\text { Higher }\end{array}$ \\
\hline 氙 & Location of hotel & $\mathrm{p}=0.039$ & $\begin{array}{c}\text { Lower } \\
\Downarrow\end{array}$ & $\begin{array}{c}\Uparrow \\
\text { Higher }\end{array}$ \\
\hline
\end{tabular}

First, Table 8.7 illustrates that the foreign participants perceived higher ranking for the enabling factor of 'clear adaptation goals' than the Khmer. The foreign participants reported that this factor provides consistency and clarity for CCA, allowing hotel

\footnotetext{
${ }^{41}$ See Table 7.1 for details
} 
operators to allocate sufficient resources such as budget, HR and technical equipment for climate change problems. The local/Khmer participants perceived that experience, together with relevant knowledge, allows hoteliers to access to the existing documents about CCA, and this later contributes to formulating adaptation goals. Therefore, 'clear adaptation goals' might not be needed while 'experience' is strongly needed. This research argues that Cambodian businessmen, especially family-oriented hotel businesses or budget hotels, tend to ignore the formulation of goals and plans in their businesses, including climate change-related goals and plans. Fünfgeld (2012) argues that in order to adapt to climate change, local individuals have responsibilities in developing and refining community maps and explaining to the outsiders how they view their community, as opposed to simply answering questions. Yet, although findings in this study show that both local/Khmer and foreign participants reported significant adaptation actions, the local/Khmer did not reveal significant adaptation goals or plans. Gray, Owen, and Adams (1996) found that foreign companies or staff are influenced by the environmental norms and culture practice that originated in the parent company; this includes the budget allocation behaviour in environmental protection. As reflected to SHV, foreign participants' parent companies, which are mostly high star hotels, likely influenced the perception of foreign participants that in climate change responses. This requires 'clear adaptation goals' which may also be required by their parent companies.

Second, Table 8.7 further determines that the foreign participants perceived greater ranking for the enabling factor entitled 'political will' than the local/Khmer. The foreign respondents felt that the 'political will' allows the government to allocate sufficient resources such as budget, which would help both the hotel sector and local government to have higher commitments to initiate adaptation programmes and subsequently obtain remarkable adaptation outcome. Nevertheless, the local/Khmer respondents viewed that it is no use waiting for politicians to help in this case. The private sector, such as hotel sector, should work cooperatively (across hotel sectors) or individually to facilitate prompt adaptations that in turn contributes to adaptation effect. This study argues that foreign participants compared and contrasted the governments' supports of climate change-related activities in Cambodia and their countries of origins (all interviewed foreign participants are from developed countries) and felt that SHV's hotel sector gets little help from the Cambodian government. The Khmer get used to a situation where 
there is a lack of support from the government while the foreign participants were demanding better help from the Cambodian government. As argued above (in chapters 2 and 3), the governments of developing countries often have limited adaptive capacities. Their adaptations largely depend on external sources such as funds from developed countries and DPs. Cambodian government remains challenging in its efforts for climate change responses. However, most participants, both Khmer and foreign, might not be aware that some CCA actions have been initiated at the national level and in SHV, and the actions might be invisible if they are initiated in the form of education and training. Informal talks with the SHV DOE found that there are a series of CCA such as training and capacity building conducted at the grassroot level in SHV by CCCA.

Third, Table 8.7 further shows that the foreign participants have higher mean score rating for the enabling factor of 'location of hotel' than the Khmer. The foreign participants were concerned about the SLR/beach erosion for those that are located on the beachfront. In the meantime, locations with spacious beaches (with sufficient space to organise events) are quite useful for both adaptation preparation and touristic activities. Nevertheless, the local/Khmer respondents thought that the concerted efforts are more important than having a good location, which is perceived as an indirect factor that affects adaptation. It is argued that the foreign respondents had experiences working in other foreign countries such as Fiji and Vanuatu, which are notably vulnerable. While the local/Khmer participants mostly do not have overseas working experience, they cannot compare and contrast their current working places with other more or less vulnerable locations.

Although the hotel attribute such as location is the least essential to hotel guests as they would not care about using whatever available vehicles commute to their already-booked hotel of their favourite location (Rhee \& Yang, 2015), this study argues that location is crucial for the hotel operators who initiate CCAs for their hotels and the guests. Tierney (2007) supports that business susceptibility to disasters results from several interrelated factors such as physical location, the circumstances under which businesses are run, and business backgrounds such as the business types and characteristics of community where they are located. 


\subsubsection{The perceived barriers and enabling factors to adaptation between length of residence}

Table 8. 8: The perceived barriers and enabling factors to adaptation between length of residence in SHV

\begin{tabular}{|c|c|c|c|c|}
\hline \multirow{2}{*}{\multicolumn{2}{|c|}{ Description }} & \multirow{2}{*}{$\begin{array}{l}\text { MW } \\
\text { test }\end{array}$} & \multicolumn{2}{|c|}{ Length of residence } \\
\hline & & & Short & Long \\
\hline 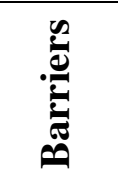 & $\begin{array}{l}\text { Insufficient } \\
\text { information }\end{array}$ & $\mathrm{p}=0.036$ & $\begin{array}{c}\Uparrow \\
\text { Higher }\end{array}$ & $\begin{array}{c}\text { Lower } \\
\Downarrow\end{array}$ \\
\hline 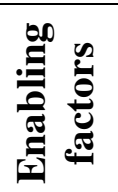 & Political will & $\mathrm{p}=0.013$ & $\begin{array}{c}\Uparrow \\
\text { Higher }\end{array}$ & $\begin{array}{c}\text { Lower } \\
\Downarrow\end{array}$ \\
\hline
\end{tabular}

Table 8.8 indicates that the short-length-of-residence participants have a significant higher mean rating for the barrier of 'insufficient information' than those who have lived in SHV longer. Those who have lived in SHV for a shorter time believed that 'insufficient information' poses numerous difficulties. For instance, climate change impacts such as storm would not be well informed in advance, thus making adaptation actions unprepared and ineffective. In different views, the long-residence participants had a perceptual acceptance that it is very normal in the area that the information is insufficiently shared among stakeholders, especially from the governments of developing countries like Cambodia. This is possibly argued that the shorter they lived in SHV, the more concerned they were, or the more they lived in the place the less concerned they were about 'limited information' because those who had resided longer could have sufficient time to adjust themselves to local context and found it more convenient to access the climate changerelated information. In contrast, those who have had shorter length of residence often did not sufficiently familiarise themselves with the place and found it difficult to access information. Time is required to observe and detect climate change information in a particular area such as SHV. Then they see 'limited information' as the more major obstacle.

In addition, the 'information' provided in SHV tends to narrowly focus on local context that is specifically available in local language (Khmer) while most of the short-length- 
residence participants were foreigners. Rudiak-Gould (2013) explains that those having a combination of both observation and gathering of local climate information tend to be familiar with the environment and have better understanding of climate change than those who do not. Nalau, Becken, Noakes, and Mackey (2017) further argue that some people show a great understanding of place-based local knowledge of weather conditions and patterns that they themselves have accumulated over time. Fernández-Llamazares et al. (2015) argue that local knowledge of climate change comes from spreading climatic information. They further explain that media coverage of the topic is usually too general or not sufficiently contextualised, which is unlikely to be superior to local observations of climate change. Media coverage often fails to shorten the wide epistemological gap between the information provided and the one perceived (Fernández-Llamazares et al., 2015). Because media failed to provide sufficient information in a foreign language (English), the information is largely absent from the perception of foreign participants who are mostly short-term residents in the study site. If there is information, it is decontextualized about SHV, which leads to their receiving climate change information that is less relevant to the area. As expressed by one foreign participant, "I don't know how well the Khmer news report of the climate change problem. I'm not sure because I can't read Khmer." H28. "This is because much of weather and climate knowledge is localized.” (Nalau, Becken, Noakes, \& Mackey, 2017, p. 388). Scannell and Gifford (2013) also suggest that in order to reduce perceived distance to the problem of information exchange, messages should be geographically and locally focused on current images rather than future or global images.

Living in a particular place longer might make someone familiarise themselves with and gain experience about the area. These people may believe their perceptual experience rather than outside knowledge. An individuals' connection to a particular place or landscape should facilitate a recognition of environmental change (Akerlof et al., 2013). Fernández-Llamazares et al. (2015) determine information that is derived from daily observation as the experiential knowledge, while information gained through the uptake of scientific information is the descriptive knowledge. They argue that the experiential knowledge is often superior to the descriptive knowledge. This aligns with those of previous studies (Patt \& Weber, 2014; Zaval et al., 2014). The interviews showed that foreign participants often obtained climate change information from delocalised media 
such as $\mathrm{BBC}$ and $\mathrm{CNN}$ and other international sources, which are usually irrelevant to local context. Marin and Berkes (2013) argue that there are mismatches between globally oriented media broadcast and locally particularised understandings of climate change. In a different approach, the local/Khmer tended to combine two methods to observe climate change in the area: the scientific evidence given by national/local and peer groups and their own observations and note taking from generation to generation. Some of them had backgrounds as fishermen or at least the descendants or relatives are fishermen, which allows them to have 'place-based' knowledge about climate change.

Based on Table 8.8, those who had resided for a shorter time in SHV perceived greater mean rating for the enabling factor of 'political will' than those who had resided longer. The short length residence participants explained that a strong 'political will' from the government means that the government accepts that climate change is increasingly challenging and reviews what sectors are more vulnerable. It allows for having some goals, better leadership, more information, funds and education from the government. These largely influence the adaptation in the private sector, including the hotel sector. In contrast, the long-residence duration participants had perceptual acceptance that the government is not willing enough to help the hotels' adaptations. The national and local government do not often work collaboratively for the sake of hotel sector's adaptation benefits. It is argued that those who are new residents (mostly foreign participants) expected a lot from the government (both national and local). They felt that the government should give more assistance. However, the longer they reside in the area, the more they know about government insufficiency; this is the general pattern in developing countries like Cambodia. Tierney (2007) argues that the fortune of each business is also dependent on local ability to operate, including the access to sources of aids for businesses and taking advantage of experienced professionals, both from outside and within the community.

In addition, while most of the long residency participants were local/Khmer, they overwhelmingly acknowledge that the government has limited capacity to help all issues in general and climate change in particular. Studying the local perception of water governance performance (in Thailand), Cookey, Darnswasdi, and Ratanachai (2016) also found that the (local) government's performance was ranked below average, with some 
pertinent barriers including institutional and agency fragmentation, weak coordination, integration and enforcement/compliance. All participants would only report positively about political will if the government's assistance helped their daily business operations. Meissner et al. (2018) call it a context-specific perception. Individuals report on waterrelated risk or/and opportunities and how it impacts them only when this influences their ordinary lives, including household water supply or the water availability for livestock (Meissner et al., 2018). Those who had just lived in the area (SHV) for a short time expected the impacts of the government's assistance. This was the reverse perception to those who had lived longer in the area, who were well aware that the government's assistance was several steps away.

Akerlof et al. (2013) explain that climate change beliefs, constructed with cultural differences, can strongly influence human perceptions, including experience relevant to climate change. This is the case in SHV because only those residing longer (mostly local/Khmer participants) understood the cultural practices of their own government while those residing shorter (mostly foreign participants) require more time for cultural acceptance that uncooperative behaviours of the government are common in developing countries.

\subsubsection{The perceived enabling factors for adaptation between participants' experiences}

Table 8.9: The perceived enabling factors for adaptation between participants' experiences

\begin{tabular}{|c|c|c|c|c|c|}
\hline \multirow{2}{*}{\multicolumn{2}{|c|}{ Description }} & \multirow{2}{*}{$\begin{array}{r}\text { K-W } \\
\text { Test }\end{array}$} & \multicolumn{3}{|c|}{ Experience } \\
\hline & & & Short & Medium & Long \\
\hline \multirow{2}{*}{ 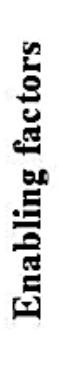 } & $\begin{array}{l}\text { Sufficient } \\
\text { information }\end{array}$ & $\mathrm{p}=0.041$ & \multicolumn{3}{|c|}{$\begin{array}{l}\text { Pairwise comparison indicates that the } \\
\text { enabling factor of 'sufficient information' is } \\
\text { trending towards statistical significance } \\
\text { between the participants' experiences. The } \\
\text { finding suggests future research. }\end{array}$} \\
\hline & $\begin{array}{l}\text { (Invulnerable) } \\
\text { location of hotel }\end{array}$ & $\mathrm{p}=0.003$ & $\begin{array}{c}\text { Lower } \\
\Downarrow\end{array}$ & & $\begin{array}{c}\Uparrow \\
\text { Higher }\end{array}$ \\
\hline
\end{tabular}


Results in Table 8.9 present that the long-experienced participants had higher perception of the enabling factor of 'location of hotel' than the short-experienced participants. Those having long working experience believed that 'location of hotel' means that a hotel is located in an area that is less vulnerable or better protected from climate change impacts and puts them at ease to adapt. By contrast, those with short working experience perceived that 'location' does not determine positive outcome or failure of a particular hotel in adapting to climate change, but effective strategies and real actions play a predominant role. It is argued that the shorter they work, the fewer problems they face, such as climate change. These people have not sufficiently experienced challenges in climate changerelated response actions. In the meantime, they cannot think of enabling factors, including 'location', to help them to adapt to climate change significantly. In a contrary argument, the longer they work the more problems they have experienced. They are increasingly aware of the need to have key factors such as good location for adaptation. This finding significantly aligns with previous studies. While Su (2014) found that hoteliers with experience of multiple extreme weather events, perceived higher negative climate change impacts on tourism industry and hotel's business in the past, this study argues that higher experienced hoteliers also perceive positive/negative factors for CCA on their businesses. They know what factors are supportive or constrain their hotels' adaptations. Mahon's (2014) research showed that hoteliers typically reported extensive and increasingly sophisticated ways where there was more experience with specific climate change-related hazards. Helgenberger (2011) also found that tourism operators having the experience of extreme weather events were more conscious about climate change impact on the industry. Lodging operators experiencing multiple climate change events had better understanding of the connection between climate change and tourism business ( $\mathrm{Su}, 2014)$.

Data from several studies suggest that experience also influences CCA practices. Su's (2014) research supported that the experience is the predominantly enabling factor in hotel response to environment and climate change. Su (2014) explains that hoteliers who have experienced multiple extreme weather events are likely to have stronger environmental awareness and show supportive attitudes towards government environmental policy and climate change concerns in their environmental performances (Hall \& Clayton, 2009). Hall and Gössling (2009) argue that hotels having experience 
with high-risk weather events are more likely to become involved in positive environmental practices.

\subsubsection{The perceived adaptation barriers between hotel standards}

Table 8.10: The perceived adaptation barriers between hotel standards

\begin{tabular}{|c|c|c|c|c|c|}
\hline \multicolumn{2}{|c|}{ Description } & \multirow{2}{*}{$\begin{array}{c}\text { K-W } \\
\text { test }\end{array}$} & \multicolumn{3}{c|}{ Hotel standard } \\
\cline { 4 - 6 } & & Budget & Midscale & Upscale \\
\hline \multirow{2}{2n}{} & Regulatory/legal constraints & $\mathrm{p}=0.018$ & & $\begin{array}{c}\text { Lower } \\
\Downarrow\end{array}$ & $\begin{array}{c}\Uparrow \\
\text { Higher }\end{array}$ \\
\hline
\end{tabular}

Table 8.10 reveals that upscale hotels have significantly higher mean scores for the barrier entitled 'regulatory and legal constraints' than midscale hotels. The higher standard hotels explained that sufficient laws, regulations and rules are crucially helpful for hotels' adaptations and were concerned that the current laws, regulations and rules are limited and conflicting with each other. They further reported that there are unclear laws, regulations and rules about CCA, which lead to the hotel sector using non-standardised rules and consequently, leading to wrong or conflicting adaptations. Contrary to this, the midscale hotels argued that Cambodia has sufficient laws, regulations and rules and these should not be too demanding for the hotel sector. The importance is actual implementation and rule enforcement. Furthermore, this study argues that since the government is aware that imposing laws, regulations and rules of environment-related issues such as CCA are impossible for the lower ranked hotels (budget and midscale in this study) because these businesses are often family businesses, the government has changed their focus to higher standard hotels. The strict law/rule enforcements could lead to the closure of lower standard hotels. Under the government's imposing strict rules, the more upscale the hotels, the more they feel that existing laws, regulations and rules are either insufficient or unclear. The Cambodian laws, rules and regulations are often conflicting to each other. In contrast, the midscale hotels (and probably including the budget) did not share this feeling; most likely because they have not sufficiently engaged with these rules. 
The finding aligns with existing literature. For instance, Su (2014) supports the notion that higher star hotels have a better implementation of environmental practices than low rated hotels and Nicholls and Kang (2012a, 2012b) had the same findings. Higher standard hotels (4-5 stars) implemented climate change measures $25 \%$ more than low standard hotels (1-3 stars) (Su, 2014). Using a similar test (One way ANOVA), Campos (2009) found that in Phuket, higher standard hotels are more active and responsive to climate change. It has been increasingly responsive for 4- and 5-star hotels. In a similar finding, Ali et al. (2008) found that one-star hotel managers are unwilling to make changes concerning the environmental responses such as saving energy while managers of 2-5 star hotels have shown greater willingness.

In other argument, this study shows that upscale hotels tend to be larger in size. As argued above, the government seems to focus on higher standard hotels or large hotels, which are more pressurised to conduct more environmentally friendly performances than small hotels (which are often family businesses or low income). Some prior research supports that large companies are often on the radar of stakeholders' attention (Amran et al., 2012; Deegan \& Gordon, 1996; Stanny \& Ely, 2008). They are both greatly pressurised and more likely to get involved in the climate change activities (Amran et al., 2012; Deegan \& Gordon, 1996) because they could afford subsiding the cost (Freedman \& Jaggi, 2005). Mensah (2006) argues (in the context of Ghana) that managers of the smaller hotels believe that the larger hotels should have more obligations about environmental management.

\subsubsection{The perceived enabling factors influencing adaptation and hotel ownerships}

Table 8.11: The perceived enabling factors influencing adaptation between hotel ownerships

\begin{tabular}{|c|c|c|c|c|}
\hline \multirow{2}{*}{\multicolumn{2}{|c|}{ Description }} & \multirow{2}{*}{$\begin{array}{c}\text { MW } \\
\text { test }\end{array}$} & \multicolumn{2}{|c|}{ Hotel ownership } \\
\hline & & & Local/ Khmer & Foreign \\
\hline 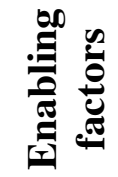 & Clear adaptation goals & $\mathrm{p}=0.036$ & $\begin{array}{c}\text { Lower } \\
\quad \Downarrow\end{array}$ & $\begin{array}{c}\Uparrow \\
\text { Higher }\end{array}$ \\
\hline
\end{tabular}


Table 8.11 presents that those from foreign-owned hotels have a greater ranking for the enabling factor of 'clear adaptation goals' than those from the Khmer-owned hotels. The foreign-owned hotel respondents believed that this condition is used to signal both management teams and staff to be fully ready to reduce climate stress for their guests. It also reflects the hotel's existing resources and the goals to be achieved. In different views, the Khmer-owned hotel participants believed that adaptations need something beyond the 'goals' on paper. Adaptation requires sufficient experience and knowledge and it is about the resources and implementation required to overcome climate change effects. As discussed above, Cambodian business owners, especially in the family-orientated businesses, generally have poor business goals and plans, including climate changerelated goals, thereby reporting that 'clear adaptation goals' are not more important. In particular in this study, Khmer-owned participants further reported that they do not have clear goals or plans concerning future adaptations. In contrast, most foreign-owned hotels or foreign participants tended to have better goal setting and planning for their future adaptations, thereafter feeling that 'clear adaptation goals' are more important.

Several existing studies have shown similar results. Fortanier and van Wijk (2010) explain that foreign hotels are likely to be larger and higher standard compared to locally owned hotels because they generally receive more foreign investment (Davidson \& Sahli, 2015). They tend to generate more income than local-owned hotels. While they have more profits, they could be more active in climate change efforts (Amran et al., 2012). This is consistent with Davidson and Sahli (2015), who argue that foreign-owned hotels have a desire to be involved in a greater community and environmental responsibility. This study argues that the more they are involved in CCA actions, the more they need to have 'clear adaptation goals', which are perceived as higher than the local-owned hotel respondents. 


\subsubsection{The perceived enabling factors for adaptation between hotel locations}

Table 8.12: The perceived enabling factors for adaptation between hotel locations

\begin{tabular}{|c|c|c|c|c|}
\hline \multirow{2}{*}{\multicolumn{2}{|c|}{ Description }} & \multirow{2}{*}{$\begin{array}{c}\text { MW } \\
\text { test }\end{array}$} & \multicolumn{2}{|c|}{ Hotel location } \\
\hline & & & Mainland & Beachfront \\
\hline 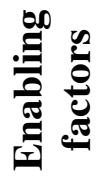 & $\begin{array}{l}\text { Sufficient } \\
\text { information }\end{array}$ & $\mathrm{p}=0.003$ & $\begin{array}{c}\text { Lower } \\
\quad \Downarrow\end{array}$ & $\begin{array}{c}\Uparrow \\
\text { Higher }\end{array}$ \\
\hline
\end{tabular}

The results in Table 8.12 highlight that the beachfront hotel participants perceived a significantly greater mean rating for the enabling factor of 'sufficient information' than those from the mainland hotels. The beachfront hotel respondents believed that information through media did not adequately reach the place and said that if it did, it would be greatly important for the hotel's adaptation initiatives. The mainland hotel participants acknowledged that in a developing country like Cambodia, climate change information has never been sufficiently shared. They accept this limitation and begin to think of appropriate adaptation techniques by examining the available information. They further argued that they still produce their adaptation result even with current (limited) information sharing platforms.

It is suggested that due to their farther locations, on the beachfront (including at the islands), the government (both national and local) and other stakeholders such IOs and NGOs do not sufficiently interact with these participants. The participants feel that they are often put in isolation. Moreover, the early warning systems for climate change are often not available at those places, especially at the islands. Most hotels $(66 \%$ in this study) are located at the mainland downtown where the participants conveniently have access to information. In the meantime, the early warning systems for climate change (e.g. SHV's radio and TV stations) are located at the mainland. All local government offices, including SHV, DOT and DOE (providing information relevant to tourism and climate change), are located at the mainland as well. In short, those at the mainland have access to information more conveniently while those on the beachfront, especially at the islands, face increasing challenges in obtaining information. Therefore, the former group viewed 'sufficient information' as more important than the latter group. 
As indicated in section 3.3.2.6, according to the Department of Meteorology, MoWRAM $^{42}$, Cambodia did not have a Doppler radar. This means that it is not able to forecast weather as accurately as that of neighbouring countries (UN-Habitat, 2012a). CCCA (2012b) found that Cambodian and SHV people have low understanding relevant to 'climate change'. This suggests that some people, especially those running businesses at islands and on the beachfront (more remote locations), face the most challenges to accessing either non-climatic or climatic information.

There is a similar trend in other developing countries. For example, Nidumolu et al. (2018) found (in southern India) that there are many barriers to the useful distribution, interaction and use of such information on farm and across the value chain. Specific to the hotel sector, Su (2014) found that coastal hotels performed lower environmental attitudes than hot spring and mountain hotels. The hot spring hotels are more involved in the promotion of green transportation and community conservation and use less material and air-conditioning than coast hotels.

The below sections show that there were no significant differences found at the 0.05 (or $5 \%$ ) significance level in the mean scores of the respondents' perceived barriers and enabling factors to adaptation between the hotel attributes such as business format (owned vs. leased) and business age and participants' backgrounds such as education and experience. The discussion below will extend whether the findings align with or contrast to prior research.

\subsubsection{The perceived barriers and enabling factors influencing adaptation between business formats (owned vs. leased)}

There were no significant differences found at the 0.05 (or 5\%) significance level in the mean scores of the respondents' perceived adaptation barriers and enabling factors between business format (owned vs. leased) (see Table 7.1). It may indicate that for all 50 participants, the hotel attribute of business format in SHV's hotel sector has not impacted on their perceptions of the barriers and enabling factors to CCA. This finding is likely opposed to existing research. Tierney (2007) argued that leasing a business

\footnotetext{
42 The Ministry of Water Resources and Meteorology, Cambodia
} 
property rather than owning the property can also be one of the factors in business vulnerability. A space rent for businesses commonly has fewer options to adapt to, such as loss-reduction measures (Tierney, 2007). For instance, the property renters cannot allocate budget to make their buildings more resistant to flood or wind by improving structures (Tierney, 2007). They tended to think that these actions should be the obligations of the building/land owners (Tierney, 2007). This overwhelmingly shows that leased hotels face more challenges in running their businesses than owned hotels that are especially relative to CCA.

Research by Alesch et al. (2001) and Tierney (2007) suggests that while making a lease agreement, the contracts often ignore the situations in which both leaseholders and landlords may play roles in the minute of a disaster. For instance, probably due to unclear clauses in the lease, when disasters strike, tenants tend to depend on landlords for repairs, especially when there is major damage to structures. If the building owner rejects or has financial limitation for repairs, the property renters or leasers may be forced to remove their business or run them under detrimental conditions (Tierney, 2007). While this study indicates that that a lack of resources and knowledge are obstacles for hotel's adaptations, McNamara and Gibson (2008) argue that barriers for hotels in 'going green' may also include existing building structures. In SHV, 68\% (or 34) of hotel businesses were on leasing contracts by renting either the building or land or both. It is explained by the fact that lease holders are more vulnerable than owners/landlords (Tierney, 2007; Wasileski, Rodríguez, \& Diaz, 2011) because the owners could take measures by obtaining loans more easily than renters of a business properties; they are in an advantageous position in business recovery (Dahlhamer \& Tierney, 1998).

\subsubsection{The perceived barriers and enabling factors affecting adaptation between business age (years of trading)}

The results show that there was no significant statistical difference of the mean rating between the hotel attribute of business age (short vs. long) and the perceived barriers and enabling factors (see Table 7.1). This may indicate that of 50 hotels, the hotel attribute of business age (short vs. long) has not influenced the perceived barriers and enabling factors. The finding is inconsistent with existing research. Su (2014) found that new hotels (short-run hotels) tend to act positively towards environment and climate change 
responsibility, including governmental regulation regarding climate change and voluntarily corporate actions. For example, newer hotels had more positive attitudes in adopting green building and low carbon practices, both for cost effectiveness and strategic concerns. In the disaster recovery, Webb et al. (2002b) argue that newly-established firms likely recover more easily than their older ones. Most importantly, new hotels decide to have the location at a lower risk of weather extremes and natural disasters. This could be explained by new hotels facing fewer challenges than their old counterparts, which are particularly relative to adaptations.

Based on prior studies, it is argued that the new businesses such as hotels learned lessons from others, especially from their peers. In the meantime, they obtain sufficient climate change information prior to commencement of their businesses. In the short term, it shows that the new hotels tend to have fewer barriers and more enabling factors in implementing CCA measures than the older businesses. However, these new counterparts might not have sufficient CCA experiences, which probably make them face more barriers than the enabling factors when they are operated for a longer period. The long-operated hotels tend to adjust themselves to changing climate change, gradually reducing barriers and enhancing enabling factors to CCA.

\subsubsection{The perceived barriers and enabling factors affecting adaptation between participants' education}

The mean scores of respondents regarding the perceived barriers and enabling factors do not vary according to participants' background of level of education (see Table 7.1). This is predominantly inconsistent with prior research. Muttarak and Lutz (2014) collected empirical studies from different levels (e.g. individual, household, village, community and societal level) and locations, mostly in developing countries (e.g. Thailand, Indonesia, Nepal and India) about the relationship between (formal) education and climate change vulnerability and adaptation. They found that risk perception is influenced and shaped by education. Formal education (higher level of education) has become a primary role for individuals to acquire knowledge, skills, and competencies; these influence their adaptive capacity. Scott, Hall, and Gössling (2012) argue that to promote behavioural change, education plays an essential part in increasing knowledge and awareness levels. Education is quite relevant to problem solving skills (Schnell-Anzola, 
Rowe, \& LeVine, 2005). While conducting a study in climate change-related topic in Cambodian coastal zone, Ung et al. (2017) found that those completing secondary education are more likely to report better health in terms of health risks of climate change compared to those having lower or no formal education.

While large amounts of literature have argued that the higher educated the people are, the less they are vulnerable and the more they adapt, this study did not find this correlation. Yet this study found that other backgrounds such as experience and nationalities are more impactful.

\subsubsection{The perceived barriers and enabling factors affecting adaptation between participants' age groups}

The mean scores of respondents regarding the perceived barriers and enabling factors do not vary according to participants' age groups (see Table 7.1). However, there are different perceptions of climate change susceptibility between young and old people observed in the literature (Linnenluecke et al., 2015). Linnenluecke et al. (2015) indicated that older participants perceived lower susceptibility and lower need for action. This aligns with other research, which found that younger people perceived a greater level of environmental concern (Hamilton, 2011). Yet these findings are rejected by Ainuddin et al (2014), who found that older people are more vulnerable in disasters and emergencies. Another research on the perceptions of elderly participants of heat wave risks found that these vulnerable people do not perceive its vulnerability and do little in response (Wolf, Lorenzoni, Few, Abrahmson, \& Raine, 2009). In the meantime, older people take more time to recover from natural hazards to original status (Ainuddin et al., 2014).

When reviewing literature for this thesis, and to the best knowledge of the author, studies that have examined the CCA (including barriers and enabling factors) between age groups are largely absent. Studying the barriers and enabling factors affecting climate change adaptation between young, medium and old people's perceptions is also insufficient. Therefore, it is best to further engage these perceived barriers and enabling factors to other backgrounds such as experience and nationalities. 
Although the results are one piece of many others, the findings raise new questions that set avenues for future exploration. It is emphasised that more work may be necessary to observe the differences. For example, there is speculation that an unexamined variable (e.g. positions and sexes of the respondents) that was not present in the study may provide alternative explanations for the results.

\subsection{Climate change adaptation in hotel sector: A revised conceptual framework}

The initial conceptual framework (see Figure 2.1) was based on literature. The discussion of the findings indicates a need to have more specific variables included in the framework in order to better reflect how the hotel sector has adapted to climate change and what the barriers and enabling factors affecting CCA are within their adaptation process. The core variables of the initial framework (coastal tourism and hotels' vulnerability assessment through the perception on environmental and socio-economic factors, supported by existing scientific evidence) remain applicable. Since these variables are contextualised in developing countries and hotel sector, they may be applicable to different accommodation sectors in the context of developing countries. The "no-adaptation actions' variable was deleted because all 50 hotels in SHV have adapted to climate change (although their adaptations varied depending on the hotel attributes and participants' backgrounds). The most common adaptation type is 'technical' (highlighted in green), followed by 'managerial' (highlighted in light blue) and 'education'. The least common adaptation employed is 'policy' and 'research'. The hierarchy of these adaptation types were added.

One of the key contributions of this research is first identifying/bringing both barriers and enabling factors to CCA in the same study of the tourism and hotel sector. Second, the most critical (the purple coloured blocks), medium (the orange coloured blocks) and the least critical (the blue coloured blocks) barriers and enabling factors to adaptation were added. The most critical barriers to CCA include 'limited resources', 'limited knowledge/perception of climate change' and 'lack of political will' (the purple coloured blocks, upper boxes). The most critical enabling factors for CCA are 'sufficient resources', 'sufficient information', and 'good leadership and management structures' (the purple coloured blocks, lower boxes). 
The barriers and enabling factors to adaptation that were exhibiting significant statistical differences between hotel attributes and participants' backgrounds were added (see the $\rightarrow$ relationships). The main goal is to explore the extent to which hotel attributes and participants' backgrounds in the hotel sector in SHV have impacted on their perceptions of barriers and enabling factors to CCA. The previous studies have not investigated the role of hotel attributes and residents' backgrounds in their perceptions of barriers and enabling factors to CCA in the tourism industry. Therefore, the initial conceptual framework has been revised to cover the new concepts that emerged from this thesis discussion (see Figure 8.1). 
Figure 8. 1: A revised conceptual framework for hotel sector's adaptation

The context of developing country

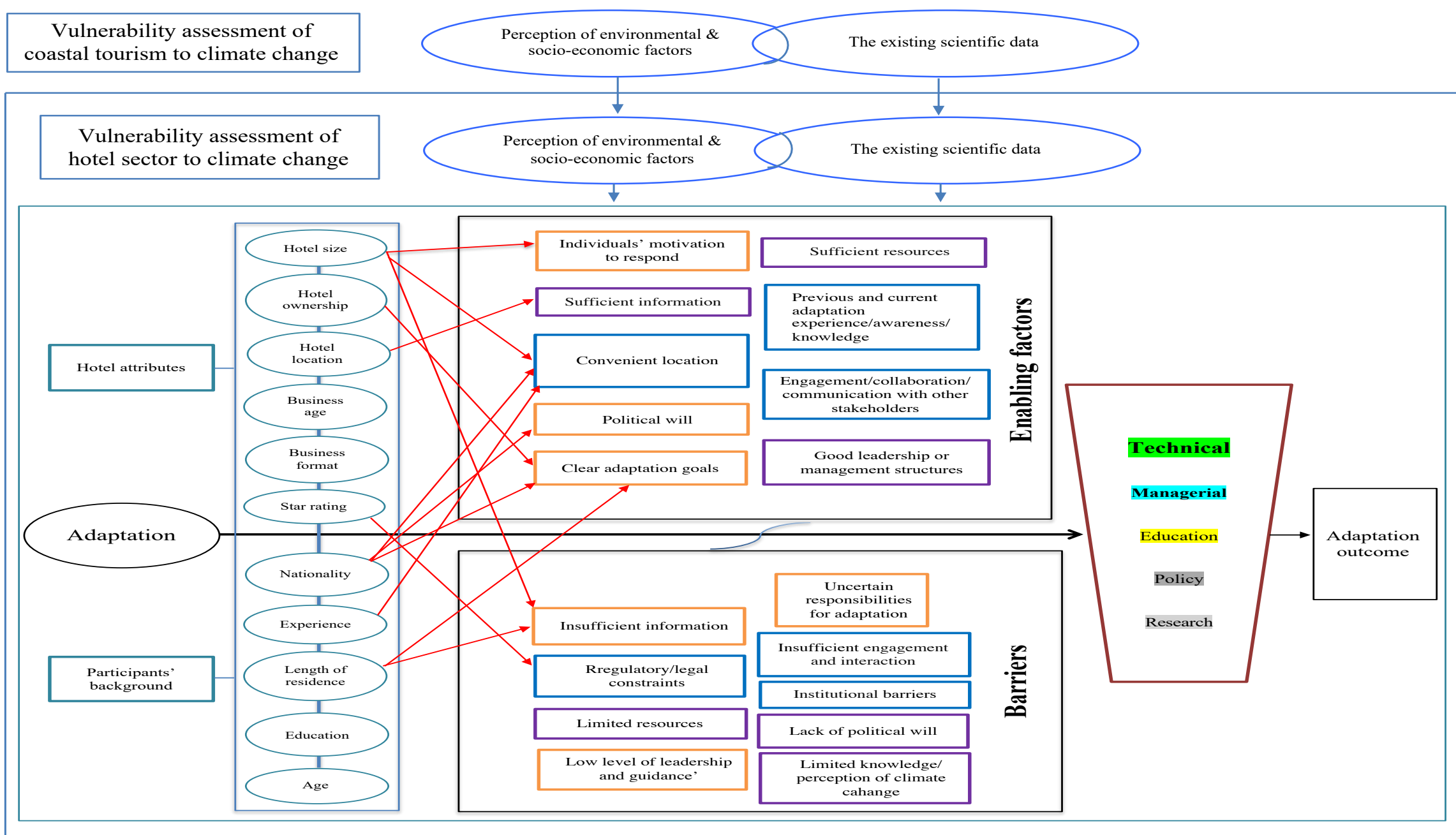


The next sections discuss each variable in the revised conceptual framework, starting with the vulnerability assessment.

First, the vulnerability of coastal tourism and hotel sector to climate change is assessed through the perception on environmental and socio-economic factors, supported by previous scientific data (see section 2.4). This assessment was influenced by key studies (e.g. Grasso, Moneo, \& Arena, 2014; Jopp, DeLacy, \& Mair, 2010; MOE, GEF \& UNEP, 2015; Moreno \& Becken, 2009; Sharma et al., 2013). Without vulnerability assessment, little is known about adaptation.

Second, under the effects of climatic changes, all hotels have initiated adaptation actions. The hotels' adaptation varies, depending on the hotel attributes (star rating, ownership, hotel size, hotel location, hotel age and business format) or/and participant's background (level of education, age, experience, nationality and length of residency in SHV). This research is one of the first studies to critically examine the barriers and enabling factors to adaptation according to the hotel attributes and participants' characteristics. Although there are a number of barriers that limit the implementation and development of CCA strategies (Biesbroek, Klostermann, Termeer, \& Kabat, (2011), there is limited understanding of relative importance of these barriers to climate change practices (Biesbroek et al., 2011). Moreover, studies on adaptation barriers are mainly carried out in developed countries (Biesbroek et al., 2013).

Third, in adapting to climate change, there are barriers and enabling factors affecting adaptation, which are observed across the existing literature in the context of developing countries. Using six-point rating scale with 5 being 'very major' and 0 being 'uninfluential' for barriers and 5 being 'very important' and 0 being 'uninfluential' for enabling factors, the perceived barriers and enabling factors affecting CCA are ranked/perceived to indicate the criticality levels (being the most critical, medium and the least critical). These rankings are important for existing research in the same discipline (tourism and climate change) such as Fischer (2018), Masud et al. (2017) and McNamara et al. (2017) who investigated the barriers of CCA but their work focused on coastal communities' adaptation to climate change. The 
findings of ranking of enabling factors follow the same trend, which contributes to the existing studies such as Bowen et al. (2013), Vaughan et al. (2017), Dinku et al. (2014), and Hurford et al. (2017). They used the term 'critical enabling factors' in climate change research but did not engage these factors with other key sectors such as tourism/hotel sectors.

The revised conceptual framework is formulated in Figure 8.1 followed by Table 8.13. It identifies the key factors, variables or constructs of the revised conceptual framework compared to the initial conceptual framework.

Table 8. 13: Comparison of the initial and revised conceptual framework

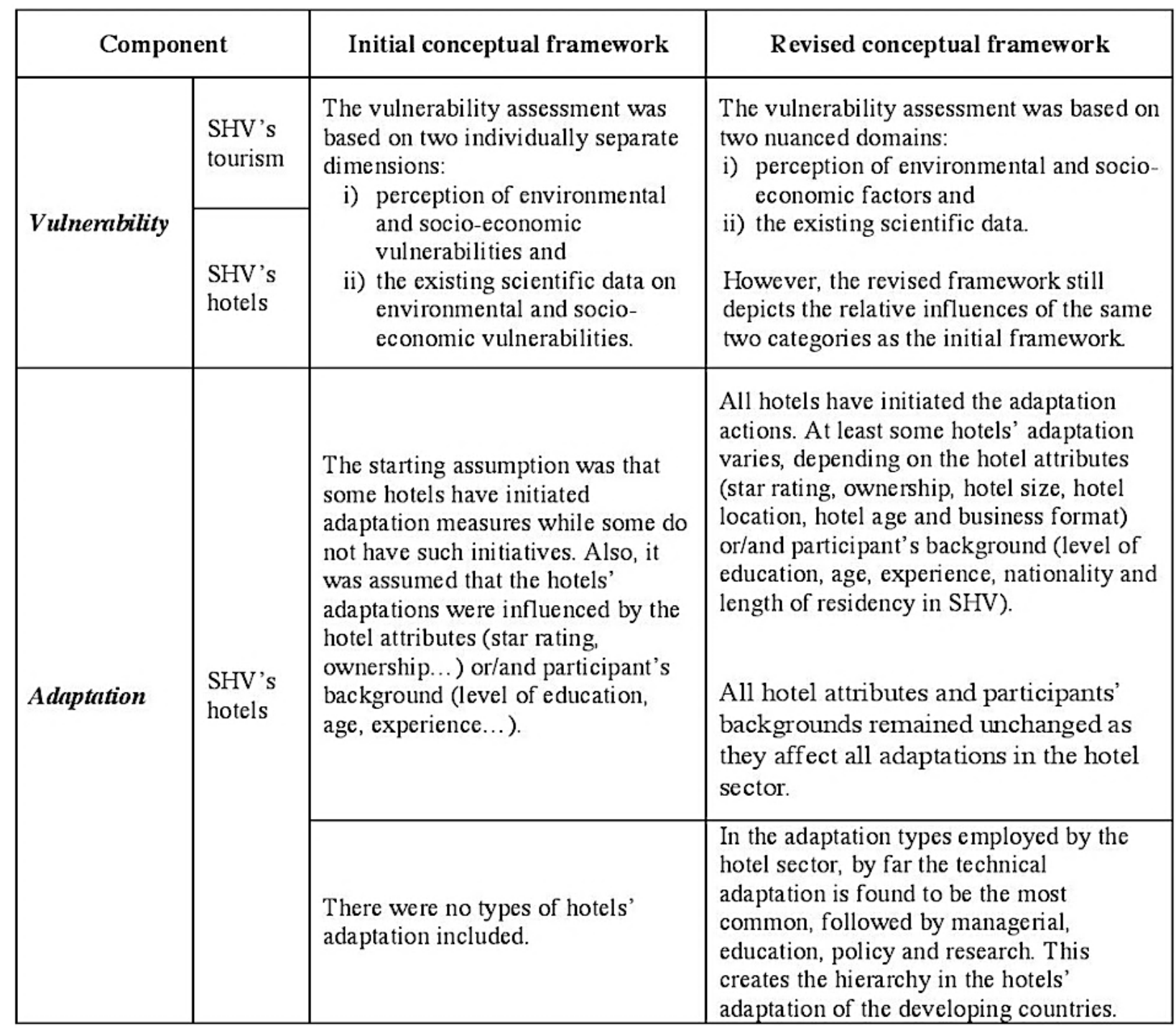




\begin{tabular}{|c|c|c|c|}
\hline $\begin{array}{l}\text { Barriers and } \\
\text { enabling } \\
\text { factors }\end{array}$ & $\begin{array}{l}\text { SHV's } \\
\text { hotels }\end{array}$ & $\begin{array}{l}\text { The literature identified many } \\
\text { barriers and enabling factors to } \\
\text { adaptation. The barriers include } \\
\text { 'insufficient resources (e.g. lack of } \\
\text { budget and HR)' and 'uncertain } \\
\text { responsibilities for adaptation'. The } \\
\text { enabling factors are 'sufficient } \\
\text { information' and 'clear adaptation } \\
\text { goals'. }\end{array}$ & $\begin{array}{l}\text { First, many barriers and enabling factors } \\
\text { to adaptation are identified. The barriers } \\
\text { include 'insufficient information', 'low } \\
\text { level of leadership and guidance' and } \\
\text { 'uncertain responsibilities for adaptation'. } \\
\text { The enabling factors are 'individuals' } \\
\text { motivation to respond', 'political will' and } \\
\text { 'clear adaptation goals'. } \\
\text { Second, the criticality of each barrier and } \\
\text { enabling factor is measured based on } \\
\text { varied views and classified into the most } \\
\text { critical (the purple coloured blocks), } \\
\text { medium (the orange coloured blocks) and } \\
\text { the least critical (the blue coloured } \\
\text { blocks). } \\
\text { Third, the barriers and enabling factors } \\
\text { that impact adaptation exhibiting } \\
\text { significant statistical differences between } \\
\text { hotel attributes and participants' } \\
\text { backgrounds are added (see the } \rightarrow \\
\text { relationships). The main goal is to explore } \\
\text { the extent to which hotel attributes and } \\
\text { participants' backgrounds in SHV's hotel } \\
\text { sector have influenced their perceptions of } \\
\text { barriers and enabling factors to CCA. }\end{array}$ \\
\hline
\end{tabular}

The revised conceptual framework helps to conceptualise the phenomenon of CCA in the developing country's hotel sector. For example, it helps situate and better understand the vulnerability assessment, followed by adaptation and its barriers and enabling factors in the hotel sector's adaptation in the developing country's context. In this study, this is of value for key stakeholders such as public sector, private sector (tourism and accommodation/hotel sector) and DPs (IOs and NGOs).

- For the public sector

○ National/central government: Through the revised framework, the government could identify barriers and enabling factors based on their cluster hierarchy. The highest hierarchy (the most critical levels) may inform that the government must overcome those obstacles if they wish to reduce vulnerability or enhance adaptive capacity for the sector or area. The government may be encouraged to adapt or assist other actors (e.g. hotel 
sector) to adapt by investing more in the most critical enabling factors because these factors are perceived to provide most effects. Broadly, the framework reflects the government to identify the strengths and limitations on their current vulnerability assessments and adaptation measure implementation. For instance, any assessment of vulnerability should be done by combining the scientific perspectives and adaptation actors' knowledge because this is a participatory process in which adaptation actors' purposes could be determined and subsequently implemented. In identifying and implementing adaptation, adaptation types or hierarchy levels should be considered. While technical adaptation is the most common or has the strengths in the context of developing country's adaptation, others (e.g. research and policy adaptation) may have their own limits in the same context (see Adger et al., 2009). The framework can guide the local authorities in building up the capacity of coastal tourism and hotel sectors to battle the climate change impacts.

- Local government: For destination managers, the revised conceptual framework, particularly the adaptation part, assists with identifying appropriate adaptation measures (e.g. between technical and managerial or both) that contribute to enhancing the destination's adaptive capacity. For instance, the framework may simply indicate that there is a need for early warning systems for extreme events or the construction of seawall barriers. The framework communicates how the destination managers, private tourism businesses and accommodation providers work together with the authorities in terms of CCA, especially technical adaptation, which is mainly a placespecific option. The framework also depicts the potential opportunities to increase the destination's competitiveness within the coastal zone, such as altering marketing efforts. In a broader significance, reducing vulnerability and enhancing adaptive capacity will promote the tourism sustainability.

- For practitioners such as accommodation (e.g. hotel sector): The framework is useful in helping accommodation providers initiate private adaptation (also see Levina \& Tirpak, 2006), consider the hierarchy of adaptation types, be cautious of which barriers are the most critical, and focus on which enabling factors are the most 
important. Additionally, the framework openly communicates with all accommodation operators to work together and partner with the government to initiate collective adaptation to climate change in the whole destination rather than being competitive between hotels and focusing on increasing business value. If tourists stop coming because of perceptions of destruction etc., nobody benefits.

- The framework opens up the opportunity for the IOs and NGOs to consider readjusting their funding mechanisms to the local government and private sectors (e.g. accommodation). Currently, all their financial aids go through public channels at the national level. The framework enhances the understanding of those DPs to urge the central government to review and rewrite laws and policies that shorten the gaps and address tourism-climate change issues, further fostering the private sector to deal with climate change. Also, the framework highlights enabling factors, which these agencies could support in a targeted fashion.

By highlighting key factors in the context of developing countries' CCA, this revised conceptual framework plays a crucial role in further identifying and bounding current knowledge and in setting avenues for future research (see Pearce, 2012) as a link to the conclusions.

\subsection{Chapter summary}

This chapter discussed the findings presented in chapters 5, 6 and 7 by engaging extant literature in order to address the main research aim, which is to determine whether Sihanoukville's hotel sector adapts to climate change and to critically examine the barriers and enabling factors influencing adaptation. Then a revised conceptual framework was developed that provides a nuanced understanding of CCA in hotel sector of developing country such as Cambodia. The next chapter presents the concluding statements of this thesis that includes the summary of major findings, contributions, limitations and recommendations for future research in this aspect. 


\section{CHAPTER 9. CONCLUSION}

\section{$9.1 \quad$ Introduction}

The central concern of this thesis is to determine whether Sihanoukville's hotel sector adapts to climate change and to critically examine the barriers and enabling factors influencing adaptation in the context of developing country such as Cambodia. To answer the overarching research aim, this study used two approaches: first, overall case analysis and second, cross-case analysis between the perceived barriers and enabling factors to adaptation and hotel attributes and participants' backgrounds using descriptive test and independent sample test. Mixed method research under the post-positivist paradigm was adopted. Using SHV as a case study, the research provides a comprehensive discussion in respect to climate change vulnerability on coastal tourism (destination) and hotel (sector), and an insight into the hotel's adaptation practices. In the context of the hotels' adaptation, the research further identifies the most significant barriers and enabling factors and critically examines each barrier and enabling factor according to the hotel attributes and participants' backgrounds.

To conclude the thesis, the next section revisits the major research findings (see section 9.2). Then, the research contributions (section 9.3), including the theoretical inferences for academics (contribution to academic literature) and the practical implications (contribution to practice), are presented. Finally, the research acknowledges the study limitations (section 9.4) and recommendations for future work are presented in section 9.5.

\subsection{Major findings}

The thesis takes the form of a climate change-focused case study of the coastal hotel sector's adaptation in Cambodia's SHV. While Cambodia, a post-conflict country, has seen very rapid tourism development over the last decade, several key international agencies have also identified it as being very vulnerable to climate change impacts. A similar trend can be observed in its coastal tourism such as SHV. Yet previous studies have so far largely neglected the relationships between tourism and climate change within Cambodia. This study is one of the first to examine whether coastal hotels in the context of developing countries respond to climate change impacts and how. 
The study's overarching topic is divided into five sequential sub-aims:

\section{Aim one: To assess the vulnerability of SHV's coastal tourism to climate change}

To answer this research aim, vulnerability was assessed through respondents' perceptions on environmental and socio-economic factors, supported by existing scientific evidence. This assessment was influenced by key studies (e.g. Grasso, Moneo, \& Arena, 2014; Jopp, DeLacy, \& Mair, 2010; MOE, GEF, \& UNEP, 2015; Moreno \& Becken, 2009; Sharma et al., 2013). Based on the interviews, both environmental and socio-economic vulnerabilities have threatened SHV's coastal tourism. The environmental vulnerabilities include excessive rainfalls, storms, rising temperature, SLR/beach erosion/storm surges and prolonged drought. The economic vulnerabilities include damage to infrastructure (from excessive rainfall and storm/storm surges) (e.g. roads and beaches), spread of diseases (resulted from the increased temperature and exposure to rain), and economic pressure (e.g. income loss).

However, most participants perceived that SHV's coastal tourism is 'moderately vulnerable' (reflected by its mean of 3.08). Their perceived vulnerability is lower than other studies suggest. Based on the discussion with the responses, there are several reasons behind their lower perceived vulnerability.

First, human resilience to hardship suggests that Cambodian people (especially the Khmer participants) could endure the unwelcome climate change consequences. This resilience may result from their endurance in post-war conflict time and on-going unrest in 1980s and 1990s. It is argued by the fact that their ability to endure difficult situations such as climate change may have lowered the perceived vulnerability. Second, it is because the private sector, including the hotel sector, has initiated adaptations to a greater level. This study further found that hotel adaptive capacity varies, depending on the hotel attributes. The higher standard hotels tend to have higher adaptive capacity. Their ability to adapt to climate change (especially high standard hotels) has lowered the perceived vulnerability. The assumption that hotels' adaptive capacity is low could be challenged, as in Cambodia/SHV the existing studies (e.g. MOE, GEF, \& UNEP, 2015; UNWTO, 2014) evaluating that hotel sector's 
adaptive capacity are unavailable. Third, the perceived low vulnerability is relevant to 'risk perception' or 'perception gap', which leads to a subjective judgement on the actual vulnerability to climate change. Fourth, vulnerability was compared to other neighbouring countries, which were perceived to have more vulnerabilities in some cases.

\section{Aim two: To assess the vulnerability of Sihanoukville's hotel sector to climate change}

In the same trend as the above aim, the vulnerability of SHV's hotel sector was also assessed. The vulnerability is again assessed through participants' perceptions on environmental and socio-economic factors, supported by existing scientific evidence. This assessment was also influenced by key studies (e.g. Grasso et al., 2014; Jopp et al., 2010; MOE, GEF, \& UNEP, 2015; Moreno \& Becken, 2009; Sharma et al., 2013). The environmental and socio-economic vulnerabilities have also threatened SHV's hotels. The threats are the same as those mentioned in the above aim (aim one).

However, most participants perceived that SHV's hotel sector is 'slightly vulnerable' (reflected by its mean of 2.63), which is lower than SHV's coastal tourism. Their perceived vulnerability is lower than that of early studies (e.g. Su, 2014; Walmsley, 2012; WMO, UNEP, \& WTO, 2008). Based on the discussion of findings, there are several reasons behind their lower perceived vulnerability. First, the risk perception or perception gap is again believed to be the factor. Second, it is related to the uncertainty of the perceived climate change-related information. The hoteliers are surrounded by uncertainty and not able to judge the information. Third, it is because the hotel sector has initiated adaptations more than expected for their businesses, especially the high star hotels. Fourth, it is because of the perceived comparison of vulnerability with other neighbouring countries that have similar or more vulnerabilities. Fifth, it is because they had rankings based on the occupancy rate rather than the actuality of climate change. Sixth, the human resilience to endure hardship is again believed to the factor. 


\section{Aim three: To determine whether/how Sihanoukville's hotel sector adapts to climate change impacts}

After the vulnerability was assessed, both at the destination level (coastal tourism) and sector level (hotel sector), the study sought to identify adaptation actions. Enlisting an adaptation framework by WMO et al. (2008) and Simpson, Gössling, Scott, Hall, and Gladin (2008), this research found that SHV's hotels employ all five adaptation types: technical, managerial, policy, research and education.

SHV's hotel sector most significantly employed technical adaptation, which aligns with previous studies (e.g. Dzoga \& Koske, 2014; Walmsley, 2012). Through this mechanism they adapt to excessive rainfall, storms, rising temperature, SLR/beach erosion/storm surges and prolonged drought. As discussed in detail in chapter 5, in order to adapt to heavy rainfalls, they checked and redesigned hotel structures and their surroundings (e.g. drainage system) and prepared materials for guests (e.g. first aid boxes, umbrellas). To adapt to stronger storms, they also checked and readjusted hotel structures and their surroundings and maintained natural trees/forest. A similar trend can be observed for their adaptations to increased temperature, SLR/beach erosion/storm surges, and prolonged drought.

SHV's hotel sector also employed managerial adaptation, which includes redirecting/suggesting or guiding customers' visits (to alternative, less affected areas), creating short and long-term plans for CCA, and providing free transport for the staff. The education adaptation includes informing/advising guests and staff, obtaining climate change training, attending climate change-related public campaigns or events, and training staff about climate change. The managerial and education adaptation is the second most commonly applied adaptation mechanism in SHV's hotel sector (also see Mahon, 2014; Su, Hall, \& Ozanne, 2013). Policy adaptation includes the compliance with (self-) regulation (e.g. building design), which is the most complicated mechanism as it involves many stakeholders, especially the government. Research adaptation was found to include conducting research to assess awareness of the businesses and staffs' knowledge gaps. Research adaptation is the least commonly used mechanism due to the limited capacity in research of SHV's hotel operators. This is a particular case in Cambodia. 
However, previous studies indicated that within tourism, the hotel sector has the lowest adaptive capacity especially relative to their fixed structures (being unable to relocate to other areas under climate change effects) (Simpson et al., 2008; Su et al., 2013; Walmsley, 2012; WMO et al., 2008; Su, 2014). This study found that hotels' adaptive capacity varies, depending on hotel attributes. Based on the findings, SHV's hotels adapted to climate change more than expected, especially the higher standard hotels. The discussion suggests that there are three reasons behind their adaptation initiatives, including, most importantly, the concept of 'personal relevance'. The hotel sector may automatically adapt in order to for their businesses to survive. The 'technical adaptation' is a particular case in the context of Cambodia's SHV. This technical mechanism is the most convenient for hotel operators. When they invest in/adapt to something, they want to see return benefits more quickly. Every one of hoteliers wants to quickly generate income (Campos, 2009). Investing in research or education adaptation takes time but they do not see quick return benefits. It is a global trend for the private sector, including hotels, to increasingly invest for their businesses. Their overall investments may contribute to increasing their adaptive capacity. Presumably, as opposed to waiting for government to take action or to provide financial support, the hotels make decisions and do it; this is called 'private adaptation' by Levina and Tirpak (2006). Another reason for hotels' adaptation is that despite uncertain awareness about climate change, they chose to adapt to be better prepared for their businesses rather than wait for the damage caused by the impacts. This may be because the hotel sector has increasingly obtained climate change information through the government's/DPs' adaptation initiatives and their individually accessed information.

\section{Aim four: To identify the most significant barriers and enabling factors affecting adaptation in the context of the hotel sector}

Fischer (2018), Masud et al. (2017), and McNamara, Westoby, and Smithers (2017) studied the critical barriers of CCA on the adaptation in coastal communities. Bowen et al. (2013) Vaughan et al. (2017), Dinku et al. (2014) and Hurford et al. (2017) identify some 'critical' environment as enabling adaptiveness and examining to which degree any enabling factors exist to boost the development of adaptation measures. Motivated by these authors, the aim 
of this study is to identify the most significant barriers and enabling factors (perceived rankings) and examine the levels of significance in terms of perception of barriers and enabling factors. The perception of barriers and enabling factors was investigated by asking the respondents to rate each of the nine potential barriers on a six-point rating scale with 5 being the greatest and 0 being the lowest. Previous research has focused on the importance of barriers and enabling factors separately without bringing both conditions (barriers and enabling factors) into the same study. Prior research also largely ignored the perceived rankings of these barriers and enabling factors. This study is one of the first research studies to present the relative rankings (the most critical, medium and the least critical) and to examine both barriers and enabling factors in the same study.

The findings show the perceived greatest barriers to be 'limited resources', limited knowledge/perception of climate change, and 'lack of political will'. The findings further show the most significant enabling factors to be 'sufficient resources', 'sufficient information' and 'good leadership and management structures'. These factors come up as the most significant effects in context of hotels' adaptation. For example, resources are most needed in both reducing barriers and advancing adaptation actions.

\section{Aim five: To critically examine the barriers and enabling factors affecting adaptation according to the hotel attributes and participants' backgrounds}

The fourth aim did not examine the comparative differences in terms of perception of barriers and enabling factors with hotel attributes and personal participants' backgrounds. Inspired by $\mathrm{Su}$ (2014) and Su et al. (2013) who looked into the impacts of hotels' characteristics on environmental performance, this aim instead explores the extent to which hotel attributes and participants' backgrounds in the hotel sector in SHV have impacted on their perceptions of the barriers and enabling factors to CCA. It assesses how the hotel attributes and respondents' characteristics influence the participants' perceptions towards the barriers and enabling factors to CCA. The result is that their perception could vary due to hotel attributes (star rating, ownership, hotel size, hotel location, hotel age and business format) and participant's backgrounds (level of education, age, experience, nationality and length of residence in SHV). For example, beachfront hotels may have more barriers in adapting to SLR/beach 
erosion/storm surges than mainland hotels. Table 7.1 presents the statistical results of this explorative empirical study by considering the above relationships

\subsection{Contributions of this research}

In summary, the findings make conceptual and practical contributions to knowledge. More specifically, the thesis contributes significantly to the literature on CCA as well as to coastal tourism and the hotel sector. In addition, the study's contributions to practice are relevant to a wide range of coastal tourism stakeholders, including the public sector, private sector (tourism business, hotel and the accommodation sector) and IOs and NGOs. The thesis will now discuss the most significant conceptual and practice-oriented contributions in turn.

\subsubsection{Contribution to academic literature}

Building on studies by or seeing limitations in studies by Su (2014) and Su et al. (2013) who limitedly engaged the role of hotel attributes, this study expands to fill the gaps by adding new knowledge with the study on the impacts of hotel attributes and respondents' backgrounds on the perceived barriers and enabling factors to CCA in the tourism industry. This research is one of the first studies to critically examine the barriers and enabling factors to adaptation according to the hotel attributes and participants' characteristics. Biesbroek, Klostermann, Termeer, and Kabat (2011) argue that although there are a number of barriers that limit the implementation and development of CCA strategies, there is limited understanding of relative importance of these barriers to climate change practices (Biesbroek et al., 2011). The studies on adaptation barriers are mainly carried out in developed countries (Biesbroek et al., 2013). This study found that the perceived barriers to CCA and perceived enabling factors for CCA could vary according to hotel attributes such as star rating, ownership, hotel size, hotel location, hotel age and business/hotel format. Additionally, it was found that the perceived barriers and enabling factors to CCA could vary according to the participant's background such as level of education, age, experience, nationality and length of residence in SHV.

While previous studies such as Su et al. (2013), Chan (2011), Kasim (2009), and Amran, Ooi, Nejati, Zulkafli, and Lim (2012) emphasised the importance of hotel attributes and green 
practices as well as sizes of firms and CCA practices, this thesis extends that research by exploring the relationships between a wide range of hotel attributes and the complex issue of CCA in the hotel sector. In a similar vein, while studies such as Muttarak and Lutz (2014), Neuenschwander, Abbott, and Mobley (2012) and Ainuddin, Routray, and Ainuddin (2014) investigated the impacts of natural disasters such as earthquakes on people with different levels of education, this study measured and assessed the barriers and enabling factors to CCA under the perceptions of hotel participants with various backgrounds. Taken together, these results add considerable new knowledge about obstacles of and enabling factors for CCA in related disciplines. The findings may also be of value in other disciplines (e.g. management and business studies).

Second, this study is one of the research studies to provide rankings (criticality level) of the barriers and enabling factors such as the most critical, medium and the least critical (adopted from the amount of literature outlined in sections 2.9 and 2.12). These rankings are important for existing research in the same discipline (tourism and climate change) such as Fischer (2018), Masud et al. (2017) and McNamara et al. (2017) who investigated the barriers of CCA but their work focused on coastal communities' adaptation to climate change. Their work did not engage CCA with a particular sector such as tourism or hotel sector. Nor did it indicate that certain barriers are greater than others (criticality level). This study fills the gap by adding a more nuanced understanding to the importance of adaptation challenge. The findings of the ranking of enabling factors follow the same trend, which contributes to existing studies such as Bowen et al. (2013), Vaughan et al. (2017), Dinku et al. (2014), and Hurford et al. (2017). They used the term 'critical' factors in climate change research but did not engage these factors with other key sectors such as tourism/hotel sectors. Their research did not indicate that certain enabling factors are more significant than others (criticality level). In this study, ranking of the enabling factors that impact adaptation may also add new knowledge to previous literature in other disciplines (e.g. management and business studies).

Third, as most previous adaptation frameworks were fragmented by focusing on a risk management or risk reduction process (Jopp, 2012), this study adds further new knowledge to the approach to identify adaptation actions engaged with private sector, including hotels' 
adaptation. Of all adaptation types such as technical, managerial, policy, research and education, technical adaptation is the most common and research adaptation is the least commonly used. This may be a particular case in developing countries. The literature and findings on the concept of adaptation expanded the framework by recognising the climate change issue as the real issue that requires multiple types of adaptation. After the identification of vulnerability, the framework also provides a central ground to inform the prioritisation of policy intervention and adaptation at the forefront of emerging climate change impacts and other multiple stressors. Findings in the adaptation framework add new knowledge to and/or contribute to the readjustments of the framework by WMO et al. (2008) and Simpson et al. (2008) as well as Jopp, DeLacy, Mair, and Fluker (2013) and Jopp (2012) to map onto the context of the hotel sector in developing countries.

Finally, a conceptual framework of hotel sector's adaptation in the context of developing countries was proposed by expanding from the initial framework built from literature. The revised framework was built on the vital link between vulnerability and adaptation, including the multiple barriers and enabling factors to adaptation. The framework highlights special requirements for hotels to critically examine their barriers and enabling factors while adapting to climate change. It highlights that certain barriers and enabling factor are more significant than others, which should help government and tourism operators prioritise certain adaptation actions over others. It also highlights the differences between hotel attributes (e.g. hotel ownership, hotel standard/star rating). Based on this revised framework, this study contributes to theory in both the CCA and tourism disciplines, with a particular focus on developing countries.

\subsubsection{Contribution to practice}

Practically, the findings of this research will be of value to all three key stakeholders, including public sector, private sector (tourism, accommodation/hotel sector) and IOs and NGOs. The study has put vulnerability, adaptation and its barriers, and enabling factors in a more holistic and detailed context, particularly in regard to the hotel sector. 


\section{For public sector}

- National/central government: To engage tourism businesses in CCA, the government, the vulnerability investigators and adaptation experts could focus their efforts on these findings. They could focus their cooperative efforts on testing, validating, and refining the findings, particularly in applying the vulnerability assessment and adaptation framework in the field. Furthermore, it is also necessary to encourage the development of guidance to help destination managers (through the MOT) translate the findings into practice, by indicating the strengths and limitations on the previous vulnerability assessments and adaptation options (e.g. MOE, GEF, \& UNEP, 2015; UNWTO, 2014). These actions are required if they are to build the capacity of coastal tourism and hotel sector to reduce the climate change impacts in the area. These findings provide information that is used to share awareness bridging the gap between private sector and the state level, which is currently large. The findings could clearly indicate the priority areas of climate change issues between the private-public partnership, thereby encouraging privatepublic adaptations. This could lead to the formulation of standalone tourism-climate change policy and implementation for the private sector of the country as the current adaptation policy is mixed with other sectors (e.g. agriculture and water sectors).

○ Local government: The findings are particularly important for local governments. While it was found that these local authorities have faced many adaptation barriers, these findings showed clear distinctions between the greatest barriers and the most significant enabling factors. They could prioritise their adaptation actions in the area or propose adaptation plans to strongly convince the central government with evidence from the research. All local governments are required to report their annual development plans to the central government so that resources such as funds and HR are allocated for CCA actions.

Furthermore, although these contributions are context-specific to Cambodia, they are transferable to similar regions with similar economic backgrounds and tourism attractions such as countries in Southeast Asia, as the literature has suggested that most of the barriers and enabling factors affecting CCA in the hotel sector in Cambodia are common in other 
developing countries in the region. Failure to reduce vulnerability and prevent hazards is seen as having negative consequences for the ability of those governments to compete for capital and investment for the destination (e.g. among ASEAN or Indochina) (see Campos, 2009; Nguyen, 2015).

Adaptation is an ongoing process and requires consistency that is particularly relevant in the national, regional and local decision-making, and as such, this study's framework provides a diagnostic tool for destination management and policymaking. Further investigation into the underlying barriers enables the destination managers to better understand the concerns of tourism, hotel sector and tourists. The greatest challenge is that non-climatic drivers of vulnerability are rarely considered by policy makers in both practice and policy (Jeffers, 2011). This study assessing vulnerability through non-climatic factors (through observation on the literature and site observations) also contributes to this gap.

\section{For the private sector}

○ Tourism sector: Tourism operators, including other private sectors (e.g. the Cambodian Hotel Association), need to recognise the importance of the hotel sector when planning CCA. The adaptation framework assists resort managers (e.g. private hotels/resorts owning whole islands offshore) in determining appropriate adaptation measures that will increase the island's adaptive capacity. The early warning systems for extreme events or the construction of seawall barriers may be provided. The application of the framework can be useful to other areas, private tourism businesses and accommodation providers. While applying the framework, the tourism sector's vulnerability will be reduced through adaptation, especially technical adaptation, thereafter, increasing its readiness. The sector will be best placed to exploit the potential opportunities such as altering marketing efforts to increase the tourism subsector's competitiveness within the coastal zone. In a broader contribution, reducing vulnerability and increasing adaptive capacity will improve the tourism sustainability. A change in climate is not only relevant to tourism's competitiveness but also sustainability. Changes in climate may transform tourism and its subsectors as losers or winners. Climate change has potential negative effects on a tourism's 
popularity but adaptation is used to reduce these concerns by reversing and compensating for climate change impacts (Mahon, 2014).

o For the hotel sector and other tourist accommodations: The hotel and other accommodation operators can use the research findings to identify private adaptation (also see Levina \& Tirpak, 2006) and find more effective ways to gain business value. Meanwhile, based on the findings, hoteliers may no longer underestimate risk factors caused by climate change. While the existing vulnerability assessments are in different quantitative formats that are not easily understood by accommodation operators, this research is more fundamental by integrating vulnerability into practice with more specific results.

The findings provide a great amount of climate change information for the accommodation sector. Although they have adapted to climate change more than expected (in SHV), they have limited awareness about climate science and incomplete understanding of tourism vulnerability to such changes. The information is useful for both existing conditions and future plans. As coastal tourism and hotels are sensitive to human and climate-induced environmental and socio-economic changes/impacts, they can use the information in the findings to minimise the risks induced by climate change or humans. Perhaps they can also work together and partner with the government to collectively lead adapt to climate change in the whole destination rather than being competitive between hotels. If tourists stop coming because of perceptions of destruction etc., nobody benefits.

\section{For IOs and NGOs}

While the findings suggest that views from all stakeholders regarding different adaptation options are important, DPs or IOs and NGOs (e.g. UNDP, ADB and World Bank) might need to consider private sectors for funding mechanisms. This finding enhances the understanding of DPs to review their funding mechanisms or to encourage the government to review and rewrite some laws and policies that link and address tourism-climate change issues, further fostering the private sector to deal with climate change. While the direct funding from DPs to private sector is sparse, the government's laws and policies should identify and implement 
adaptation measures for private sectors to reduce their vulnerability to climate change impacts. Due to the lack of commitment, the laws and policies have not fully been implemented. The findings will encourage the translation of on-the-paper laws and policies into practice. For example, while this finding showed that the low standard hotels (28 hotels in SHV) have a stronger need of financial help, the government and DPs should target the hotels through their funding mechanisms. Also, the findings highlight barriers and enabling factors. Here, these DPs could support CCA in a direct approach by providing funds or technical cooperation to the private sector (e.g. hotel sector) to reduce the barriers, especially the most critical ones, and enhance the enabling factors, particularly the most critical factors. Broadly, the role of DPs concerning the CCA is strengthened by modifying its mission towards cooperative work with the private sector. As the current role of DPs heavily involves the public sector, the findings provide strong reasons for private sector's (e.g. hotel sector) involvement in working directly with these DPs and financing such CCA projects.

\subsection{Research limitations}

Despite careful research design, this research has certain methodological limits, which affect the generalisability of its results. The first limitation is that this thesis takes the form of a climate change-focused case study, which means there are geographic and demographic limitations of this study. While this research was conducted in SHV, Cambodia, it is probably applicable to other Cambodia's coastal tourist provinces. Yet the data collected and analysed may not be generalisable to other non-coastal regions in Cambodia and other neighbouring countries.

Second, the study recruited the representatives of only a sub-sector (hotel) of the tourism industry. It was impossible to study all tourism stakeholders such as guesthouses, travel agencies, and tourism transport companies. Furthermore, the two main stakeholder groups such as the government (national and local) and IOs and NGOs were not recruited; however, it is acknowledged that the hotel sector's adaptation is a cross-cutting issue that is closely relevant to other stakeholders. CCA, especially barriers and enabling factors, requires more comprehensive data inputs as they are influenced by other stakeholders. For example, strong infrastructures built by the government contribute to positive effect of hotels' adaptations 
whereas the weak ones contribute to adaptation barriers. Jarvis and Ortega (2010) called this (weak infrastructure) external barrier or enabling factor, which contributes to increasing or weakening hotels' adaptive capacity to respond to climate change (see Table 2.3 for details).

The third limitation is that the study focused on an adaptation framework containing technical, managerial, policy, research and education. These do not fully represent other adaptation strategies such as behavioural adaptation. It is also acknowledged that the adaptation framework and the results may not be applied or generalised to all tourism destinations. This is mainly due to the selected methodology and the limitations constituted by a single case study. Because the research examined 'on the ground' adaptation efforts and asked for evidence of actual adaptations, it is impossible to report its consistency with those in other sectors at the national level. Adger et al. (2009) ahave pointed out earlier that adaptation has its own limits, which include three dimensions: ecological and physical limits, economic limits, and technological limits (Adger et al., 2009). For example, perhaps it is the complex interactions between social and individual backgrounds that attempt to secure sustainable societal adaptation in the long run (Adger et al., 2009) or the consideration of the economic costs of adaptation (Agrawala \& Fankhauser, 2008) or it is due to technological innovation for adaptation (Adger et al., 2009).

Fourth, the data collection for the research project was collected at various phases in 2016 and 2017; the analysis mainly captures the perceptions at the time of measurement while scientific evidence was collected from existing studies. As climate change keeps changing, a longitudinal research design that captures change in perceptions over time would be more effective because perceptions change based on the observed climate data (Alam, Alam, \& Mushtaq, 2017). A longer time-series data collection may be well matched to perceptionbased studies. For instance, climate change observed from the altered rainfall and temperatures is expected to take a longer time to study.

Research concerning perceptions contains uncertainty and this is enhanced when it involves the issues of a changing climate. The respondents may have limitations to contextualise, understand and interpret uncertainty around climate change, especially vulnerability. It 
appears challenging for them to comprehend the issues that are purely induced by climate change or nuanced by other non-climatic factors. As argued above, the hoteliers' perceptions of risk are also influenced by other stressors than climate change notions. They may not always interpret questions in the same manner as the scientist or researchers. It cannot be $100 \%$ guaranteed that the responses truly reflect reality, but the large sample sizes comparatively provide a high level of confidence in this interview-based study (see Archie, 2012).

Most of the limitations discussed above can be addressed through further research. Therefore, the following section will highlight several areas for future research.

\section{$9.5 \quad$ Future research}

Due to the nature of this work, which is exploratory and multi-disciplinary, the types of the theories recommended for future are related to perception such as social representation, psychological distance and construal level. Very little research has been carried out on the interaction of social representations of relevant phenomena (Sarrica \& Wachelke, 2010) and the in-depth exploration of the psychological distance of climate change (Spence, Poortinga, \& Pidgeon, 2012) and the associated role of construal level with climate action (Wang, Hurlstone, Leviston, Walker, \& Lawrence, 2019).

Through the lens of social representations theory, a wide range of studies have highlighted the complex social phenomena in people's everyday lives in order to make sense of expert knowledge and scientific concepts (Buijs, Arts, Elands, \& Lengkeek, 2011), including climate change and tourism. According to Bauer and Gaskell (1999), social representations represent a hybrid category of both life and scientific world knowledge; this is reflected in this study that explores the vulnerability assessment through local and scientific knowledge. The social representations theory reflects how the public perceive the complexities and to this extent it constitutes 'reality' more than the scientific understandings per se (Bauer \& Gaskell, 2008). As indicated earlier, due to the lack of in-depth exploration of the psychological distance of climate change (Spence et al., 2012), this study contributes to psychological distance theory because climate change is perceived as distant in regard to 
many different dimensions as argued in sections 8.3 and 8.4. The contribution of this research may be concerned with social representations and construal level theory. The perceptions of people have been influenced by their personal backgrounds such as experience and education (see Chapter 8). In the meantime, the climate change impact itself is characterised as psychologically distant for various people (Spence et al., 2012). Based on construal level theory (Trope \& Liberman, 2003), when information is at lengthy distance or things are in a weak or complex relationship, mental representations are more uncertain and abstract (Scannell \& Gifford, 2013). In this situation, psychological distance might obstruct climate change engagement. Awareness of climate change is insufficiently engaged due to the perception that it is a remote threat (Leiserowitz, 2005). Psychological closeness to climate change expected more involvement in pro-environmental behaviors (Wang et al., 2019); this may include adaptation behaviors. In obstruct construal level, an acceptance of the climate change problem and willingness to deal with it is low (Wang et al., 2019). Enhancing personal relevance as stated in section 8.5 through effective communications to reduce the distance because psychological distance is a common impediment to the perception of climate change (Scannell \& Gifford, 2013). Personally relevant information or message enhances motivation and the capacity to act (Scannell \& Gifford, 2013).

The research findings and these theories together could provide interesting avenues for future research. For instance, the lens of social representations could be set as a wider angel for further research. There are still many unanswered questions about complex social phenomena such as climate change vulnerability assessment. Vulnerability assessment remains fragmented and the assessment approaches remain unclear. Although vulnerability assessments have increasingly been on the agenda for research, how potentially vulnerable populations understand and interpret their own vulnerability is limited. Further research should be undertaken to investigate the following concerns:

- This research has demonstrated that although climate change has increasingly been recognised mainly as a physical impact, further study is required on how scientific knowledge is structured and understood. For example, with regard to vulnerability assessment outcome what is the perception gap or mismatch between the scientists and non-scientists? 
- The complexity of non-climatic factors such as rapid development (e.g. road development), and land use practice, which strongly influence vulnerability, have not been sufficiently explored.

- Given that most vulnerability assessments so far have focused on its spatial dimensions and ignored its temporal aspects, longstanding vulnerability assessments over several years or decades enable the addressing of some of these concerns (Jeffers, 2011).

- Future studies should include other stakeholders' perceptions from the national/local government and IOs and NGOs when investigating tourism's and hotels' vulnerability. Further exploration of various stakeholders' perceptions would add further value to this discipline. It would be better if the consultation is extended to the local communities (Jopp, 2012) because a more extensive community consultation process would provide a better overall picture of both the vulnerability assessment outcome and perceptual acceptance by host communities. Local messages are often more effective than global messages (Scannell \& Gifford, 2013).

As psychological closeness to climate change and concrete construal level enhance an acceptance of the climate change problem and willingness to deal with it (see Wang et al., 2019), Future studies should further develop or test the adaptation framework and revised conceptual framework. The contribution of both adaptation framework and revised conceptual framework should be tested either in hotel/accommodation sector or destination level. Additional research on its practical relevance is therefore needed to understand its effectiveness by using all underlining adaptation types. In the revised conceptual framework, the future research should focus on validating and exploring the certain components in more depth, especially the statistical data of trending towards significance findings (see Table 7.1) between the perceived barriers and enabling factors and hotel attributes and participants' backgrounds. This needs an empirical test with large sample size.

The concept of psychological distance could be applied in order to develop a deeper and more nuanced understanding of the barriers reported by the hotel managers. The next research may pay particular focus on the most critical barriers: limited resources, limited knowledge/ 
perception of climate change and lack of political will. While climate change is an obvious impact, why resources remain insufficient to fight climate change and why it is perceived as distant by the public and politicians. The gaps and distance between perceived barriers and the public and politicians' perceptions that obstruct climate change engagement and awareness may be uncovered. During the fieldwork, many respondents suggested that the government should increase the credibility of providing punctual information and sharing more accurate information. The local government should be more active in prioritising climate change issues for the private sector. This points to important implications for regional, national and global environmental change. The trends should also be applied in order to develop a deeper and more nuanced understanding of the enabling factors or their relative importance. Further work is to identify and reduce those gaps and enhance psychological distance and concrete construal level as these variables (barriers and enabling factors) will provide valuable insights into the vulnerability-adaptation dynamic, thereby contributing to increasing the destination's adaptive capacity. 


\section{References:}

Aall, C., \& Høyer, K. G. (2005). Adaptation and response managing the relationship between tourism, recreation and global climate change. In Tourism, recreation and climate change (1st ed., Vol. 22). Channel View Publications.

Abdi, H., Creighton, D., \& Nahavandi, S. (2013). A Sustainable energy saving method for hotels by green hotel deals. In A. Hakansson, M. Höjer, R. J. Howlett, \& L. C. Jain (Eds.), Sustainability in Energy and Buildings (Vol. 22, pp. 669-677). Berlin, Heidelberg: Springer Berlin Heidelberg.

Abeysekara, U. G. D. L. D. P. (2017). Business value of ICT for small tourism enterprises: The case of Sri Lanka. The Victoria University of Wellington, Wellington, New Zealand.

ADB. (2014). Cambodia: country poverty analysis 2014. Mandaluyong City, Philippines: Asian Development Bank. Retrieved from https://www.adb.org/sites/default/files/institutional-document/151706/cambodiacountry-poverty-analysis-2014.pdf

ADB. (2017a). Key indicators for Asia and the Pacific 2012. Mandaluyong City,

Philippines: Asian Development Bank. Retrieved from https://www.adb.org/sites/default/files/publication/29940/ki2012.pdf

ADB. (2017b). Tourism sector assessment, strategy, and road map for Cambodia, Lao

People’s Democratic Republic, Myanmar, and Viet Nam (2016-2018). Asian Development Bank. Retrieved from https://www.adb.org/documents/clmv-tourismsector-road-map-2016-2018

Adger, W N, Agrawala, S., Mirza, M. M., Conde, C., O’Brien, K., Pulhin, J., ... Takahashi, K. (2007). Assessment of adaptation practices, options, constraints and capacity. In Climate Change 2007: Impacts, Adaptation and Vulnerability (pp. 717-743). Cambridge, UK: Cambridge University Press.

Adger, W Neil, Arnell, N. W., \& Tompkins, E. L. (2005). Successful adaptation to climate change across scales. Global Environmental Change, 15(2), 77-86.

Adger, W Neil, Dessai, S., Goulden, M., Hulme, M., Lorenzoni, I., Nelson, D. R., ... Wreford, A. (2009). Are there social limits to adaptation to climate change? Climatic Change, 93(3-4), 335-354. 
Adger, W Neil, Lorenzoni, I., \& O’Brien, K. L. (2009). Adapting to climate change: Thresholds, values, governance. Cambridge University Press.

Afroz, T., \& Naser, M. M. (2014). Adaptation to climate change in the international climate change regime: challenges and responses. In Implementing Adaptation Strategies by Legal, Economic and Planning Instruments on Climate Change (pp. 1-11). Springer.

Agrawala, S., \& Fankhauser, S. (2008). Economic aspects of adaptation to climate change costs, benefits and policy instruments. Paris: OECD.

Ainuddin, S., Routray, J. K., \& Ainuddin, S. (2014). People's risk perception in earthquake prone Quetta city of Baluchistan. International Journal of Disaster Risk Reduction, 7, $165-175$.

Akerlof, K., Maibach, E. W., Fitzgerald, D., Cedeno, A. Y., \& Neuman, A. (2013). Do people "personally experience" global warming, and if so how, and does it matter? Global Environmental Change, 23(1), 81-91.

Alam, G. M. M., Alam, K., \& Mushtaq, S. (2017). Climate change perceptions and local adaptation strategies of hazard-prone rural households in Bangladesh. Climate Risk Management, 17, 52-63.

Alesch, D. J., Holly, J. N., Mittler, E., \& Nagy, R. (2001). Organizations at risk: What happens when small businesses and not-for-profits encounter natural disasters. Public Entity Risk Institute, (October), 105. Retrieved from http://www.ecocalltoaction.com/images/Organizations_at_Risk.pdf

Ali, Y., Mustafa, M., Al-Mashaqbah, S., Mashal, K., \& Mohsen, M. (2008). Potential of energy savings in the hotel sector in Jordan. Energy Conversion and Management, 49(11), 3391-3397.

Alonso-Almeida, M.-M., Fernández Robin, C., Celemín Pedroche, M. S., \& Astorga, P. S. (2017). Revisiting green practices in the hotel industry: A comparison between mature and emerging destinations. Journal of Cleaner Production, 140, 1415-1428.

Alvarez Gil, M. J., Burgos, J. B., \& Lorente, J. J. C. spedes. (2001). An analysis of environmental management, organizational context and performance of Spanish hotels. Omega, 29(6), 457-471.

Amelung, B., Moreno, A., \& Scott, D. (2008). The place of tourism in the IPCC fourth assessment report: A review. Tourism Review International, 12(1), 5-12. 
Aminian, A. (2012). Environmental performance measurement of tourism accommodations in the Pilgrimage Urban Areas: The case of the Holy City of Mashhad, Iran. Procedia - Social and Behavioral Sciences, 35, 514-522.

Amran, A., Ooi, S. K., Nejati, M., Zulkafli, A. H., \& Lim, B. A. (2012). Relationship of firm attributes, ownership structure and business network on climate change efforts: Evidence from Malaysia. International Journal of Sustainable Development \& World Ecology, 19(5), 406-414.

Amundsen, H. (2015). Place attachment as a driver of adaptation in coastal communities in Northern Norway. Local Environment, 20(3), 257-276.

Amundsen, H., Berglund, F., \& Westskog, H. (2010). Overcoming barriers to climate change adaptation - a question of multilevel governance? Environment and Planning C: Government and Policy, 28(2), 276-289.

Anderson, A. (2012). Climate change education for mitigation and adaptation. Journal of Education for Sustainable Development, 6(2), 191-206.

Andrei, P. K., \& Roger, A. S. (2007). Climate change impacts on forestry. The National Academy of Sciences of the USA, 104(50), 6.

Anguelovski, I., Chu, E., \& Carmin, J. (2014). Variations in approaches to urban climate adaptation: Experiences and experimentation from the global South. Global Environmental Change, 27, 156-167.

Archie, Kelli M, Dilling, L., Milford, J. B., \& Pampel, F. C. (2014). Unpacking the 'information barrier': Comparing perspectives on information as a barrier to climate change adaptation in the interior mountain West. Journal of Environmental Management, 133, 397-410.

Archie, Kelli Marie. (2012). Climate change adaptation in the Western U.S.: Examining barriers to planning, hurdles to implementation, and demand for information by Federal Public Lands Managers and Colorado Mountain Communities. University of Colorado at Boulder, Colorado, United States.

Arcodia, C., \& Dickson, C. (2008). Responding to climate change in Australian resort hotels: Setting a research agenda for water, energy and waste management.

Ayesha, D., \& Heather, M. (2014). A tailored view of successful adaptation to climate change: African and Latin American resilience to climate change (ARCC). USAID. 
Retrieved from http://community.eldis.org/.5b9bfce3/AAP 05 Successful Adaptation_CLEARED.pdf

Bangay, C., \& Blum, N. (2010). Education responses to climate change and quality: Two parts of the same agenda? International Journal of Educational Development, 30(4), $359-368$.

Barr, R., Fankhauser, S., \& Hamilton, K. (2010). Adaptation investments: A resource allocation framework. Mitigation and Adaptation Strategies for Global Change, 15(8), $843-858$.

Basias, N., \& Pollalis, Y. (2018). Quantitative and qualitative research in business \& technology: Justifying a suitable research methodology. Review of Integrative Business and Economics Research, 7(1), 91-105.

Bauer, M. W., \& Gaskell, G. (1999). Towards a paradigm for research on social representations. Journal for the Theory of Social Behaviour, 29(2), 163-186.

Bauer, M. W., \& Gaskell, G. (2008). Social representations theory: A progressive research programme for social psychology. Journal for the Theory of Social Behaviour, 38(4), 335-353.

Becken, S. (2010). The important of climate and weather for tourism. Retrieved from http://www.lincoln.ac.nz/PageFiles/6750/WeatherLitReview.pdf

Becken, S. (2013). A review of tourism and climate change as an evolving knowledge domain. Tourism Management Perspectives, 6, 53-62.

Becken, S., \& Hay, J. (2012). Climate Change and Tourism: From Policy to Practice. Routledge.

Becken, S., \& Hay, J. E. (2007). Tourism and climate change: Risks and opportunities (Vol. 1). Multilingual Matters.

Becken, S., \& Wilson, J. (2013). The impacts of weather on tourist travel. Tourism Geographies, 15(4), 620-639.

Becken, S., \& Wilson, J. (2016). Are tourism businesses' responses to weather variability a suitable precursor to climate change adaptation? Worldwide Hospitality and Tourism Themes, 8(5), 578-592.

Begum, R., \& Pereira, J. (2015). The awareness, perception and motivational analysis of climate change and business perspectives in Malaysia. An International Journal 
Devoted to Scientific, Engineering, Socio-Economic and Policy Responses to Environmental Change, 20(3), 361-370.

Bele, M. Y., Sonwa, D. J., \& Tiani, A. M. (2014). Local communities vulnerability to climate change and adaptation strategies in Bukavu in DR Congo. The Journal of Environment \& Development, 23(3), 331-357.

Benedikter, A., Läderach, P., Eitzinger, A., Cook, S., \& Bruni, M. (2013). Addressing adaptation to support disaster risk reduction: A framework for supply chain inclusive adaptation to climate change. In W. Leal Filho (Ed.), Climate Change and Disaster Risk Management (pp. 513-533). Berlin, Heidelberg: Springer Berlin Heidelberg.

Bennett, Nathan J, Dearden, P., Murray, G., \& Kadfak, A. (2014). The capacity to adapt?: Communities in a changing climate, environment, and economy on the northern Andaman coast of Thailand. Ecology and Society, 19(2).

Bennett, Nathan James, Blythe, J., Tyler, S., \& Ban, N. C. (2016). Communities and change in the anthropocene: Understanding social-ecological vulnerability and planning adaptations to multiple interacting exposures. Regional Environmental Change, 16(4), 907-926.

Bennett, Nathan James, \& Dearden, P. (2013). A picture of change: Using photovoice to explore social and environmental change in coastal communities on the Andaman Coast of Thailand. Local Environment, 18(9), 983-1001.

Bennett, Nathan James, Kadfak, A., \& Dearden, P. (2015). Community-based scenario planning: A process for vulnerability analysis and adaptation planning to socialecological change in coastal communities. Environment, Development and Sustainability, 1-29.

Berkhout, F, Hertin, J., \& Jordan, A. (2002). Socio-economic futures in climate change impact assessment: Using scenarios as 'learning machines'.' Global Environmental Change, 12(2), 83-95.

Berkhout, Frans, \& Hertin, J. (2000). Socio-economic scenarios for climate impact assessment. Global Environmental Change, 10(3), 165-168.

Berkhout, Frans, Hertin, J., \& Gann, D. M. (2006). Learning to Adapt: Organisational adaptation to climate change impacts. Climatic Change, 78(1), 135-156.

Berkhout, Frans, van den Hurk, B., Bessembinder, J., de Boer, J., Bregman, B., \& van 
Drunen, M. (2013). Framing climate uncertainty: Socio-economic and climate scenarios in vulnerability and adaptation assessments. Regional Environmental Change.

Berrang-Ford, L., Ford, J. D., \& Paterson, J. (2011). Are we adapting to climate change? Global Environmental Change, 21(1), 25-33.

Berry, S., \& Ladkin, A. (1997). Sustainable tourism: A regional perspective. Tourism Management, 18(7), 433-440.

Bicknell, S., \& Mcmanus, P. (2006). The canary in the coalmine: Australian ski resorts and their response to climate change. Geographical Research, 44(4), 386-400.

Bierbaum, R., Smith, J. B., Lee, A., Blair, M., Carter, L., Chapin, F. S., ... Verduzco, L. (2013). A comprehensive review of climate adaptation in the United States: More than before, but less than needed. Mitigation and Adaptation Strategies for Global Change, $18(3), 361-406$.

Biesbroek, G. R., Klostermann, J. E. M., Termeer, C. J. A. M., \& Kabat, P. (2013). On the nature of barriers to climate change adaptation. Regional Environmental Change, 13(5), 1119-1129.

Biesbroek, R., Klostermann, J., Termeer, C., \& Kabat, P. (2011). Barriers to climate change adaptation in the Netherlands. Climate Law, 2(2), 181-199.

Bisel, R. S., \& Adame, E. A. (2017). Post-positivist/functionalist approaches. In the International Encyclopedia of Organizational Communication. John Wiley \& Sons, Inc.

Botkosal, W., Kong, C., \& Chanthou, C. (2012). The state of climate change in Cambodia. In The 5th AUN/SEED-Net Regional Conference on Global Environment. AUN/SEED-Net Secretariat.

Bowen, K. J., Miller, F., Dany, V., McMichael, A. J., \& Friel, S. (2013). Enabling environments? Insights into the policy context for climate change and health adaptation decision-making in Cambodia. Climate and Development, 5(4), 277-287.

Briggs, S., Sutherland, J., \& Drummond, S. (2007). Are hotels serving quality? An exploratory study of service quality in the Scottish hotel sector. Tourism Management, 28, 1006-1019.

Britten, N. (2006). Qualitative Interviews. In C. Pope \& N. Mays (Eds.), Qualitative 
Research in Health Care (pp. 12-20). Blackwell Publishing Ltd.

Brooks, J. (2014, July 1). Young people with diabetes and their peers - an exploratory study of peer attitudes, beliefs, responses and influences. Retrieved from http://eprints.hud.ac.uk/20268/

Brooks, J., \& King, N. (2014). Doing template analysis: Evaluating an end-of-lfe care service. London: SAGE Publications, Ltd.

Brooks, J., McCluskey, S., Turley, E., \& King, N. (2015). The utility of template analysis in qualitative psychology research. Qualitative Research in Psychology, 12(2), 202222.

Brown, A., Gawith, M., Lonsdale, K., \& Pringle, P. (2011). Managing adaptation: linking theory and practice. UK Climate Impacts Programme, Oxford, UK.

Brulle, R., Carmichael, J., \& Jenkins, J. (2012). Shifting public opinion on climate change: an empirical assessment of factors influencing concern over climate change in the U.S., 2002-2010. An Interdisciplinary, International Journal Devoted to the Description, Causes and Implications of Climatic Change, 114(2), 169-188.

Bryan, E., Deressa, T. T., Gbetibouo, G. A., \& Ringler, C. (2009). Adaptation to climate change in Ethiopia and South Africa: Options and constraints. Environmental Science and Policy, 12(4), 413-426.

Buijs, A. E., Arts, B. J. M., Elands, B. H. M., \& Lengkeek, J. (2011). Beyond environmental frames: The social representation and cultural resonance of nature in conflicts over a Dutch woodland. Geoforum, 42(3), 329-341.

Bunce, M., Rosendo, S., \& Brown, K. (2010). Perceptions of climate change, multiple stressors and livelihoods on marginal African coasts. Environment, Development and Sustainability, 12(3), 407-440.

Burch, S. (2010). Transforming barriers into enablers of action on climate change: Insights from three municipal case studies in British Columbia, Canada. Global Environmental Change, 20(2), 287-297.

Burton, I. (2009). Climate change and the adaptation deficit. In The Earthscan reader on adaptation to climate change. London, Sterling.

Burton, I., Huq, S., Lim, B., Pilifosova, O., \& Schipper, E. L. (2002). From impacts assessment to adaptation priorities: The shaping of adaptation policy. Climate Policy, 
2(2-3), 145-159.

Cabrini, L. (2009). From Davos to Copenhagen and beyond: Advancing tourism's response to climate change: UNWTO background paper. World Tourism Organization.

Cambers, G. (2009). Caribbean beach changes and climate change adaptation. Aquatic Ecosystem Health \& Management, 12(2), 168-176.

Campbell, D. T. (1975). Degrees of freedom in the case study research. Comparative Political Studies, 8(2), 178-193.

Campbell, J. L., Quincy, C., Osserman, J., \& Pedersen, O. K. (2013). Coding in-depth semistructured interviews: Problems of unitization and intercoder reliability and agreement. Sociological Methods \& Research, 42(3), 294-320.

Campos, R. C. (2009). The awareness and responsiveness of the hotel industry in Phuket to climate change. Prince of Songkla University.

Carlton, S. J., \& Jacobson, S. K. (2013). Climate change and coastal environmental risk perceptions in Florida. Journal of Environmental Management, 130, 32-39.

Carter, R. W., O’Rourke, V., Livingstone, T., McKenzie, T., Lyell, M., Brown, K., ... Knight, J. (2013). Strategic guidelines for sustainable tourism on the Khmer coast. Report to the Ministry of Tourism, Royal Government of Cambodia.

Castells-Quintana, D., Lopez-Uribe, M. del P., \& McDermott, T. K. J. (2018). Adaptation to climate change: A review through a development economics lens. World Development, 104, 183-196.

CCCA. (2012a). Assessment of community vulnerability and risks from climate change in the coastal zone of Cambodia. Sihanoukville, Cambodia: Cambodia Climate Change Alliance (CCCA). Retrieved from http://country-profiles.geog.ox.ac.uk

CCCA. (2012b). Coastal adaptation and resilience planning component: Assessment of coping strategies in the coastal zone of Cambodia. Cambodia: Cambodia Climate Change Alliance (CCCA).

CCCA. (2012c). Coastal adaptation and resilience planning component - Final report: Analysis of costs \& benefits of modifying agricultural practices for climate change at the coast. Phnom Penh, Cambodia: Cambodia Climate Change Alliance (CCCA).

CCCA. (2013). Mainstreaming of climate change into the sub-national development planning in Cambodia. Phnom Penh, Cambodia: Cambodia Climate Change Alliance 
(CCCA).

Chan, E. S. (2011). Implementing environmental management systems in small-and medium-sized hotels: Obstacles. Journal of Hospitality \& Tourism Research, 35(1), 323.

Chan, W. W. (2005). Partial analysis of the environmental costs generated by hotels in Hong Kong. International Journal of Hospitality Management, 24(4), 517-531.

Chankoulika, B., \& Bopha, S. (2017). Hotel and guesthouse licensing. Phnom Penh, Cambodia. Retrieved from http://bnglegal.com/cn/wpcontent/uploads/2017/05/170502-Hotel-and-Guesthouse-Licensing-May.pdf

Charlotte, L. (2016, June 30). Top ten beaches in Asia. The Sunday Herald Sun. Retrieved from http://blog.paradizo.com/luxury-vacation/top-asia-beach-sunday-herald-sun/

Chea, S., \& Sharp, A. (2015). Flood management in Cambodia: Case studies of flood in 2009 and 2011. In 2015 International Conference on Environment And Civil Engineering (ICEACE’2015) (p. 6). Pattaya (Thailand): International Academy of Engineers. Retrieved from http://ia-e.org/siteadmin/upload/1886IAE0415413.pdf

Chean, R. (2017). Perceptions, attitudes and behaviour around climate change risks on livelihoods activities: A case study of a community in Takeo province, Cambodia. Victoria University of Wellington. Retrieved from https:/viewer.waireto.victoria.ac.nz/wairetostreams/get?dps_dvs=1525314921267 884\&dps_pid=FL989323

Chiang, C.-F. (2010). Perceived organizational change in the hotel industry: An implication of change schema. International Journal of Hospitality Management, 29(1), 157-167.

Chowdhury, M. F. (2015). Coding, sorting and sifting of qualitative data analysis: Debates and discussion. Quality \& Quantity, 49(3), 1135-1143.

Chrysostomidis, I., \& Constable, L. (2015). Understanding and managing climate change risks and adaptation opportunities in a business context - Handbook of climate change adaptation. In W. Leal Filho (Ed.) (pp. 565-587). Berlin, Heidelberg: Springer Berlin Heidelberg.

Clark, C. E., \& Crawford, E. P. (2012). Influencing Climate Change Policy: The Effect of Shareholder Pressure and Firm Environmental Performance. Business \& Society, 51(1), 148-175. 
Coles, R. (1986). The political life of children (1st ed.). Boston: Atlantic Monthly Press.

Coles, T., Dinan, C., \& Warren, N. (2016). Carbon villains? Climate change responses among accommodation providers in historic premises. Journal of Heritage Tourism, $11(1), 25-42$.

Collier, D. (2012). Within-case versus cross-case causal analysis. In A tale of two cultures : Qualitative and quantitative research in the social sciences. Princeton, New Jersey: Princeton University Press.

Collier, D., Brady, H. E., \& Seawright, J. (2010). Sources of leverage in causal inference: toward an alternative view of methodology. In Rethinking social inquiry: Diverse tools, shared standards (2nd ed). Lanham, MD: Rowman \& Littlefield Publishers.

Cone, J., Rowe, S., Borberg, J., Stancioff, E., Doore, B., \& Grant, K. (2013). Reframing engagement methods for climate change adaptation. Coastal Management, 41(4), 345360 .

Cookey, P. E., Darnswasdi, R., \& Ratanachai, C. (2016). Local people's perceptions of Lake Basin water governance performance in Thailand. Ocean \& Coastal Management, 120, 11-28.

Cooper, C., Fletcher, J., Gilbert, D., Shepherd, R., \& Wanhill, S. (1998). Tourism: Principles and practice (2nd editio). Harlow: Longman.

Cooper, J. A. G., \& Pile, J. (2014). The adaptation-resistance spectrum: A classification of contemporary adaptation approaches to climate-related coastal change. Ocean \& Coastal Management, 94, 90-98.

Crabbé, P., \& Robin, M. (2006). Institutional adaptation of water resource infrastructures to climate change in Eastern Ontario. Climatic Change, 78(1), 103-133.

Creswell, J. W. (2003). Research design: Qualitative, quantitative, and mixed method approaches (2nd ed). Thousand Oaks, Calif: Sage Publications.

Creswell, J. W. (2007). Qualitative inquiry \& research design: Choosing among five approaches (2nd ed). Thousand Oaks: Sage Publications.

Creswell, J. W. (2014). Research design: Qualitative, quantitative, and mixed methods approaches (4th ed.). California: SAGE Publications.

Crick, F., Diop, M., Sow, M., Diouf, B., Diouf, B., Muhwanga, J., \& Dajani, M. (2016). Enabling private sector adaptation in developing countries and their semi-arid 
regions - case studies of Senegal and Kenya.

Cross, M. S., Zavaleta, E. S., Bachelet, D., Brooks, M. L., Enquist, C. A. F., Fleishman, E., ... Tabor, G. M. (2012). The adaptation for conservation targets (ACT) framework: A tool for incorporating climate change into natural resource management. Environmental Management, 50(3), 341-351.

Csete, M., \& Szécsi, N. (2012). Tourism adaptation portfolio fostering regional sustainability. Periodica Oeconomica, 99-108.

Cuevas, S. C. (2015). Examining the challenges in mainstreaming climate change adaptation into local land-use planning: The case of Albay, Philippines. The University of Queensland.

CWPR. (2010). Status of climate change management in Southeast Asia. Retrieved from https://www.gwp.org/globalassets/global/gwp-sea_files/gwpsea_climate-changereport.pdf

Dahlhamer, J. M., \& Tierney, K. J. (1998). Rebounding from disruptive events: Business recovery following the Northridge earthquake. SOCIOLOGICAL SPECTRUM, 18, $121-141$.

DanChurchAid/Christian Aid. (2011). Climate change and disaster management policy mapping and analysis in Cambodia.

Dany, V., Bajracharya, B., Lebel, L., Regan, M., \& Taplin, R. (2015). Narrowing gaps between research and policy development in climate change adaptation work in the water resources and agriculture sectors of Cambodia. Climate Policy, (ahead-of-print), $1-16$.

Dany, V., Bowen, K. J., \& Miller, F. (2015). Assessing the institutional capacity to adapt to climate change: A case study in the Cambodian health and water sectors. Climate Policy, 15(3), 388-409.

Davidson, L., \& Sahli, M. (2015). Foreign direct investment in tourism, poverty alleviation, and sustainable development: A review of the Gambian hotel sector. Journal of Sustainable Tourism, 23(2), 167-187.

Davies, B. (2003). The role of quantitative and qualitative research in industrial studies of tourism. International Journal of Tourism Research, 5(2), 97-111.

Deegan, C., \& Gordon, B. (1996). A study of the environmental disclosure practices of 
Australian corporations. Accounting and Business Research, 26(3), 187-199.

Dessai, S, Hulme, M., Lempert, R., \& Pielke, R. J. (2009). Climate prediction: A limit to adaptation? In Adapting to climate change: Thresholds, values, governance (p. 64). Cambridge: Cambridge University Press.

Dessai, Suraje, Lu, X., \& Risbey, J. S. (2005). On the role of climate scenarios for adaptation planning. Global Environmental Change, 15(2), 87-97.

DiCicco-Bloom, B., \& Crabtree, B. F. (2006). The qualitative research interview. Medical Education, 40(4), 314-321.

Dinku, T., Block, P., Sharoff, J., Hailemariam, K., Osgood, D., del Corral, J., ... Thomson, M. (2014). Bridging critical gaps in climate services and applications in africa. Transdisciplinarity Enabled, 1(1), 1-13.

Dittrich, R., Francis, B., Hatzinger, R., \& Katzenbeisser, W. (2007). A paired comparison approach for the analysis of sets of Likert-scale responses. Statistical Modelling, 7(1), $3-28$.

Doody, H. (2010). What are the barriers to implementing environmental practices in the Irish Hospitality Industry? Retrieved from http://www.shannoncollege.com/wpcontent/uploads/2009/12/THRIC-2010-Full-Paper-H.-Doody.pdf

Doria, M. de F., Boyd, E., Tompkins, E. L., \& Adger, W. N. (2009). Using expert elicitation to define successful adaptation to climate change. Environmental Science \& Policy, 12(7), 810-819.

Dornan, T., Carroll, C., \& Parboosingh, J. (2002). An electronic learning portfolio for reflective continuing professional development. Medical Education, 36(8), 767-769.

Dow, K., Berkhout, F., \& Preston, B. L. (2013). Limits to adaptation to climate change: A risk approach. Current Opinion in Environmental Sustainability, 5(3), 384-391.

Drake, M. R. A. (2010). Student understanding of linear scale in mathematics: Exploring what year 7 and 8 students know. the Victoria University of Wellington. Retrieved from

http://researcharchive.vuw.ac.nz/bitstream/handle/10063/1225/thesis.pdf?sequence=1

Dubé, L., \& Paré, G. (2003). Rigor in information systems positivist case research: Current practices, trends, and recommendations. MIS Quarterly, 27(4), 597-636.

Dupuis, S. L. (1999). Naked truths: Towards a reflexive methodology in leisure research. 
Leisure Sciences, 21(1), 43-64.

Dzoga, M. N., \& Koske, J. K. A. (2014). Assessment of beach hotels adaptation capacity to climate variability: The case of Mombasa County, Kenya. International Journal of Humanities and Social Science, 4(3), 1-4.

Eisenhardt, K. M. (1989). Building theories from case study research. The Academy of Management Review, 14(4), 532-550.

Ek, G. (2013). Cambodia environmental and climate change: Policy brief. Phnom Penh, Cambodia: Sida.

Ekinci, Y., Prokopaki, P., \& Cobanoglu, C. (2003). Service quality in Cretan accommodations: Marketing strategies for the UK holiday market. International Journal of Hospitality Management, 22(1), 47-66.

Ekstrom, J. A., Moser, S. C., \& Tom, M. (2011). Barriers to climate change adaptation: A diagnostic framework: Final project report. California Energy Commission.

Elsasser, H., \& Bürki, R. (2002). Climate change as a threat to tourism in the Alps. Climate Research, 20(3), 253-257.

Enrich Institute. (2014, November 12). Climate change financing in Cambodia. NGO Forum. Retrieved from http://www.seachangecop.org/sites/default/files/documents/2014 01 NGOForum - CC Financing in Cambodia.pdf

Fankhauser, S. (2010). The costs of adaptation. Wiley Interdisciplinary Reviews: Climate Change, 1(1), 23-30.

Fankhauser, S., \& McDermott, T. K. J. (2014). Understanding the adaptation deficit: Why are poor countries more vulnerable to climate events than rich countries? Global Environmental Change, 27,9-18.

Fernández-Llamazares, Á., Méndez-López, M., Díaz-Reviriego, I., McBride, M., Pyhälä, A., Rosell-Melé, A., \& Reyes-García, V . (2015). Links between media communication and local perceptions of climate change in an indigenous society. An Interdisciplinary, International Journal Devoted to the Description, Causes and Implications of Climatic Change, 131(2), 307-320.

Fernando, P. S., Yvainne, M., \& Maria, Y. S. (2013). Coastal erosion assessment Ochheauteal Beach and Otress Beach in Preah Sihanouk province, Cambodia. 
Sihanoukville, Cambodia.

Fink, A. S. (2000). The role of the researcher in the qualitative research process. A potential barrier to archiving qualitative data. Forum Qualitative Sozialforschung / Forum: Qualitative Social Research, 1(3), 15.

Fischer, A. P. (2018). Pathways of adaptation to external stressors in coastal naturalresource- dependent communities: Implications for climate change. World Development, 108, 235-248.

Flugman, E., Mozumder, P., \& Randhir, T. (2012). Facilitating adaptation to global climate change: perspectives from experts and decision makers serving the Florida Keys. An Interdisciplinary, International Journal Devoted to the Description, Causes and Implications of Climatic Change, 112(3), 1015-1035.

Ford, J., Berrang-Ford, L., Lesnikowski, A., Barrera, M., \& Heymann, S. (2013). How to track adaptation to climate change: A typology of approaches for national-level application. Ecology and Society, 18(3), 1.

Ford, J. D., Champalle, C., Tudge, P., Riedlsperger, R., Bell, T., \& Sparling, E. (2015). Evaluating climate change vulnerability assessments: A case study of research focusing on the built environment in northern Canada. Mitigation and Adaptation Strategies for Global Change, 20(8), 1267-1288.

Ford, J. D., \& Pearce, T. (2010). What we know, do not know, and need to know about climate change vulnerability in the western Canadian Arctic: A systematic literature review. Environmental Research Letters, 5(1), 14008.

Ford, J., \& King, D. (2015). A framework for examining adaptation readiness. An International Journal Devoted to Scientific, Engineering, Socio-Economic and Policy Responses to Environmental Change, 20(4), 505-526.

Forster, M. (2015). Refining the definition of information literacy: the experience of contextual knowledge creation. Journal of Information Literacy, 9(1), 62.

Fortanier, F., \& van Wijk, J. (2010). Sustainable tourism industry development in subSaharan Africa: Consequences of foreign hotels for local employment. International Business Review, 19(2), 191-205.

Freedman, M., \& Jaggi, B. (2005). Global warming, commitment to the Kyoto protocol, and accounting disclosures by the largest global public firms from polluting industries. 
The International Journal of Accounting, 40, 215-232.

Frey, N., \& George, R. (2010). Responsible tourism management: The missing link between business owners' attitudes and behaviour in the Cape Town tourism industry. Tourism Management, 31(5), 621-628.

Fünfgeld, H. (2012). Local climate change adaptation planning: A guide for government policy and decision makers in Victoria. Melbourne.

Füssel, H.-M. (2007). Vulnerability: a generally applicable conceptual framework for climate change research. Global Environmental Change, 17(2), 155-167.

Füssel, H.-M., \& Klein, R. J. T. (2006). Climate change vulnerability assessments: An evolution of conceptual thinking. Climatic Change, 75(3), 301-329.

Fussel, H.-M., \& PIK. (2010). Review and quantitative analysis of indices of climate change exposure, adaptive capacity, sensitivity, and impacts. World Development Report, 35.

Galletta, A. (2013a). Conducting the interview: The role of reciprocity and reflexivity. In Mastering the semi-structured interview and beyond (pp. 75-118). NYU Press.

Galletta, A. (2013b). The semi-structured interview as a repertoire of possibilities. In Mastering the Semi-Structured Interview and Beyond (pp. 45-72). NYU Press.

Gelo, O., Braakmann, D., \& Benetka, G. (2008). Quantitative and qualitative research: Beyond the debate. Integrative Psychological and Behavioral Science, 42(3), 266290.

geres Cambodia. (2009). Public perceptions of climate change in Cambodia. DanChurch Aid/Christian Aid (DCA/CA).

Ghosh, T. (2011). Coastal tourism: Opportunity and sustainability. Journal of Sustainable Development, 4(6), 67.

Ghosh, T. (2012). Sustainable coastal tourism: Problems and management options. Journal of Geography and Geology, 4(1), 163.

Gibbs, G. (2002). Qualitative data analysis: Explorations with NVivo. Understanding social research. Buckingham: Open University.

Gifford, R., Scannell, L., Kormos, C., Smolova, L., Biel, A., Boncu, S., ... Uzzell, D. (2009). Temporal pessimism and spatial optimism in environmental assessments: An 18-nation study. Journal of Environmental Psychology, 29(1), 1-12. 
Gill, P., Stewart, K., Treasure, E., \& Chadwick, B. (2008). Methods of data collection in qualitative research: Interviews and focus groups. BDJ, 204(6), 291-295.

Glaas, E., Gammelgaard Ballantyne, A., Neset, T.-S., Linnér, B.-O., Navarra, C., Johansson, J., ... Goodsite, M. E. (2015). Facilitating climate change adaptation through communication: Insights from the development of a visualization tool. Energy Research \& Social Science, 10, 57-61.

Gondo, M., Amis, J., \& Vardaman, J. (2012). Case within a case. In Encyclopedia of Case Study Research (pp. 135-136). 2455 Teller Road, Thousand Oaks California 91320 United States: SAGE Publications, Inc.

Goodson, L., \& Phillimore, J. (2002). Qualitative research in tourism: Ontologies, epistemologies and methodologies. London, UK: Taylor \& Francis Group.

Gössling, S., Scott, D., Hall, C. M., Ceron, J.-P., \& Dubois, G. (2012). Consumer behaviour and demand response of tourists to climate change. Annals of Tourism Research, 39(1), 36-58.

Grasso, M., Moneo, M., \& Arena, M. (2014). Assessing social vulnerability to climate change in Samoa. Regional Environmental Change, 14(4), 1329-1341.

Gray, R., Owen, D., \& Adams, C. (1996). Accounting \& accountability: Changes and challenges in corporate social and environmental reporting. Financial Times/Prentice Hall.

GSSD. (2015). Cambodia's second national communication under the United Nations Framework Convention on climate change. Phnom Penh, Cambodia: General Secretariat, National Council for Sustainable Development/Ministry of Environment, Kingdom of Cambodia. Retrieved from http://unfccc.int/resource/docs/natc/khmnc2.pdf

Guba, E. G., \& Lincoln, Y. S. (1994). Competing paradigms in qualitative research. Handbook of Qualitative Research, 2(163-194).

Hakim, L. (2018). Economic snapshot: Cambodia real estate highlights (1st half 2018). Phnom Penh. Retrieved from https://content.knightfrank.com/research/1471/documents/en/cambodia-real-estatehighlights-h1-2018-5808.pdf

Hall, A. J., \& Clayton, A. (2009). How will climate change impact on the tourism industry? 
Worldwide Hospitality and Tourism Themes, 1(3), 269-273.

Hall, C Michael. (2006). New Zealand tourism entrepreneur attitudes and behaviours with respect to climate change adaptation and mitigation. International Journal of Innovation and Sustainable Development, 1(3), 229-237.

Hall, C Michael, \& Gössling, S. (2009). Global environmental change and tourism enterprise. In Tourism Enterprises and Sustainable Development: International Perspectives on Responses to the Sustainability Agenda. London, the UK: Routledge.

Hall, Colin Michael, Dayal, N., Majstorović, D., Mills, H., Paul-Andrews, L., Wallace, C., \& Truong, V. D. (2016). Accommodation consumers and providers' attitudes, behaviours and practices for sustainability: A systematic review. Sustainability, 8(7), 625.

Hall, Colin Michael, \& Page, S. (2014). The geography of tourism and recreation: environment, place and space (Vol. Fouth edit). New York: Routledge. Retrieved from http://helicon.vuw.ac.nz/login?url=http://search.ebscohost.com/login.aspx?direct=true $\& \mathrm{db}=$ nlebk $\& \mathrm{AN}=777629 \&$ site $=$ ehost-live

Hallegatte, Stéphane. (2009). Strategies to adapt to an uncertain climate change. Global Environmental Change, 19(2), 240-247.

Hallegatte, Stephane, Vogt-Schilb, A., Bangalore, M., \& Rozenberg, J. (2017). Building the resilience of the poor in the face of natural disasters climate change and development series. Washington, DC. Retrieved from https://openknowledge.worldbank.org/bitstream/handle/10986/25335/211003ovEN.pd f

Halsnæs, K., Kaspersen, P. S., \& Trærup, S. (2016). Climate change risks - Methodological framework and case study of damages from extreme events in Cambodia. In J. I. Uitto \& R. Shaw (Eds.), Sustainable Development and Disaster Risk Reduction (pp. 71-85). Tokyo: Springer Japan.

Hamilton, L. (2011). Education, politics and opinions about climate change evidence for interaction effects. An Interdisciplinary, International Journal Devoted to the Description, Causes and Implications of Climatic Change, 104(2), 231-242.

Hamilton, M., Fischer, A. P., Guikema, S. D., \& Keppel-Aleks, G. (2018). Behavioral 
adaptation to climate change in wildfire-prone forests. Wiley Interdisciplinary Reviews: Climate Change, 9(6), 17.

Han, J. H., Noh, E. J., \& Oh, C.-O. (2015). Applying the concept of site substitution to coastal tourism. Tourism Geographies, 1-15.

Harmeling, S., \& Eckstein, D. (2013). Global climate risk index 2013: Who suffers most from extreme weather events? Retrieved from https://germanwatch.org/en/download/7170.pdf

Harriet, B. (2000). Common knowledge? Public understanding of climate change in Newcastle, Australia. Public Understanding of Science, 9, 313-333.

Hartzell-Nichols, L. (2011). Responsibility for meeting the costs of adaptation. Wiley Interdisciplinary Reviews: Climate Change, 2(5), 687-700.

Helgenberger, S. (2011). The capacity of locally bound tourism firms to respond to climate variability and long-term change: Qualitative case studies on organizational learning in the Austrian winter tourism sector. Tourism Planning \& Development, 8(1), 69-86.

Henderson, K. A. (2011). Post-positivism and the pragmatics of leisure research. Leisure Sciences, 33(4), 341-346.

Hess, J. S. (2012). Adapting the Cambodian tourism sector to climate change: Needs and challenges. University of Applied Sciences Bremen.

Hiwasaki, L., Luna, E., Syamsidik, \& Marçal, J. A. (2015). Local and indigenous knowledge on climate-related hazards of coastal and small island communities in Southeast Asia. Climatic Change, 128(1-2), 35-56.

Hobson, K., \& Essex, S. (2001). Sustainable tourism: A view from accommodation businesses. Service Industries Journal, 21(4), 133-146.

Hopkins, D. M. (2013). The social phenomenon of climate change: contextual vulnerability, risk perception and adaptation in the ski industry of Queenstown, New Zealand.

Huang, C., Vaneckova, P., Wang, X., FitzGerald, G., Guo, Y., \& Tong, S. (2011). Constraints and barriers to public health adaptation to climate change: A review of the literature. American Journal of Preventive Medicine, 40(2), 183-190.

Humair, C. (2011). The hotel industry and its importance in the technical and economic development of a region: The Lake Geneva case (1852-1914). Journal of Tourism 
History.

Hurford, B. A. P., Moschini, F., \& Woolhouse, G. A. F. (2017). Critical success factors for resilient water infrastructure. Retrieved from

https://reliefweb.int/sites/reliefweb.int/files/resources/Working-Paper_Criticalsuccess-factors-resilient-water-infrastructure_CDKN.pdf

Hyde, K. F., Ryan, C., \& Woodside, A. G. (2012). Why case study research? Introduction to the field guide to case study research in tourism, hospitality, and leisure. In Field guide to case study research in tourism, hospitality and leisure (Vol. 6, pp. 1-10). Emerald Group Publishing Limited.

Ioris, A., Irigaray, C., \& Girard, P. (2014). Institutional responses to climate change: opportunities and barriers for adaptation in the Pantanal and the Upper Paraguay River Basin. An Interdisciplinary, International Journal Devoted to the Description, Causes and Implications of Climatic Change, 127(1), 139-151.

IPCC. (2007a). Climate Change 2007: Impacts, adaptation and vulnerability: Contribution of Working Group II to the fourth assessment report of the Intergovernmental Panel. (P. J. van der L. and C. E. H. M.L. Parry, O.F. Canziani, J.P. Palutikof, Ed.), Cambridge University Press. Cambridge, UK: Cambridge University Press.

IPCC. (2007b). Climate change 2007: Synthesis report contribution of working groups I, II and III to the fourth assessment report of the Intergovernmental Panel on Climate Change. Intergovernmental Panel on Climate Change [Core Writing Team, Pachauri, R.K and Reisinger, A. (eds.)]. Geneva, Switzerland.

IPCC. (2013). Climate change 2013 - The physical science basis: Working Group I Contribution to the fifth assessment report of the Intergovernmental Panel on Climate Change. Cambridge: Cambridge University Press.

IPCC. (2014). Climate Change 2014: Synthesis report. Contribution of Working Groups I, II and III to the Fifth Assessment Report of the Intergovernmental Panel on Climate Change [Core Writing Team, R.K. Pachauri and L.A. Meyer (eds.)]. Geneva, Switzerland: IPCC.

Jamaliah, M. M. (2015). The vulnerability and resilience assessments of ecotourism to climate change in the Dana Biosphere Reserve, Jordan. Clemson University . Retrieved from https://search-proquest- 
com.helicon.vuw.ac.nz/pqdtglobal/docview/1727773831/B92C34C16B14140PQ/1?ac countid=14782

Jarvis, N., \& Ortega, A. P. (2010). The Impact of climate change on small hotels in Granada, Spain. Tourism and Hospitality Planning \& Development, 7(3), 283-299.

Jeffers, J. M. (2011). Confronting climate: Linking knowledge, values and decision-making to vulnerability and adaptation in Ireland's coastal cities. The State University of New Jersey.

Jeuring, J. (2011). Intentions to seek severe weather information among travelers in New Zealand. Wageningen University \& Research Centre, Wageningen, The Netherlands.

JICA. (2017). Preparatory survey for Sihanoukville Port new container terminal development project. Phnom Penh. Retrieved from http://open_jicareport.jica.go.jp/pdf/12283990_01.pdf

Jones, L., C, C., Chesterman, S., Cramer, L., \& Crane, T. (2015). Identifying constraining and enabling factors to the uptake of medium- and long-term climate information in decision making. Copenhagen, Denmark: University of Copenhagen.

Jones, L., Champalle, C., Chesterman, S., Cramer, L., \& Crane, T. A. (2017). Constraining and enabling factors to using long-term climate information in decision-making. Climate Policy, 17(5), 551-572.

Jopp, R. (2012). Linking climate change, tourist destination adaptation and tourist attitudes: A case study of the Victorian Surf Coast region. Victoria University, Melbourne, Australia.

Jopp, R., DeLacy, T., \& Mair, J. (2010). Developing a framework for regional destination adaptation to climate change. Current Issues in Tourism, 13(6), 591-605.

Jopp, R., DeLacy, T., Mair, J., \& Fluker, M. (2013). Using a regional tourism adaptation framework to determine climate change adaptation options for Victoria's Surf Coast. Asia Pacific Journal of Tourism Research, 18(1-2), 144-164.

Kaján, E., \& Saarinen, J. (2013). Tourism, climate change and adaptation: A review. Current Issues in Tourism, 16(2), 167-195.

Käkönen, M., Lebel, L., Karhunmaa, K., Dany, V., \& Try, T. (2014). Rendering climate change governable in the least-developed countries: Policy narratives and expert technologies in Cambodia. Forum for Development Studies, 41(3), 351-376. 
Kalaugher, E., Bornman, J. F., Clark, A., \& Beukes, P. (2013). An integrated biophysical and socio-economic framework for analysis of climate change adaptation strategies: The case of a New Zealand dairy farming system. Environmental Modelling \& Software, 39, 176-187.

Kasim, A. (2009). Managerial attitudes towards environmental management among small and medium hotels in Kuala Lumpur. Journal of Sustainable Tourism, 17(6), 709-725.

Kato, T., Kaplan, J. A., Sophal, C., \& Sopheap, R. (2000). Cambodia: Enhancing governance for sustainable development. Phnom Penh, Cambodia: Cambodia Development Resource Institute. Retrieved from http://citeseerx.ist.psu.edu/viewdoc/download?doi=10.1.1.541.7032\&rep=rep1\&type= pdf

Kelly, P. M., \& Adger, W. N. (2000). Theory and practice in assessing vulnerability to climate change andFacilitating adaptation. Climatic Change, 47(4), 325-352.

Kent, G. (2000). Understanding the experiences of people with disfigurements: An integration of four models of social and psychological functioning. Psychology, Health \& Medicine, 5(2), 117-129.

Kim, J. D. (2003). Strategy for sustainable coastal tourism development under the integrated coastal management: Case study of Sihanoukville city, Kingdom of Cambodia. Kaiyo Kogaku Shinpoji-umu, 17, 405-410.

King, N. (1998). Template analysis. In Qualitative methods and analysis in organizational research: A practical guide (pp. 119-134). London: SAGE Publications.

King, N. (2008). What will hatch? A constructivist autobiographical account of writing poetry. Journal of Constructivist Psychology, 21(4), 274-287.

King, N. (2012). Doing template analysis. In Qualitative Organizational Research: Core Methods and Current Challenges (eds., pp. 50-426). London: SAGE.

King, P., Basiuk, R., Bou, S. C., \& Yem, D. (2009). Strengthening sustainable tourism: Strategic environmental assessment of the tourism industry in Cambodia. Bangkok: Asian Development Bank and Greater Mekong Sub-region Environment Operations Centre. Retrieved from http://www.gmseoc.org/uploads/resources/30/attachment/Strengthening Sustainable Tourism - SEA of the Tourism Sector in Cambodia.pdf 
Kirkby-Geddes, E., King, N., \& Bravington, A. (2013). Social capital and community group participation: Examining 'bridging' and 'bonding' in the context of a healthy living centre in the UK. Journal of Community \& Applied Social Psychology, 23(4), 271-285.

Klein, R. J. T., Midgley, G. F., Preston, B. L., Alam, M., Berkhout, F. G. H., Dow, K., \& Shaw, M. R. (2014). Adaptation opportunities, constraints, and limits. In Climate Change 2014: Impacts, Adaptation, and Vulnerability. Part A: Global and sectoral aspects. Contribution of Working Group II to the Fifth Assessment Report of the Intergovernmental Panel on Climate Change (pp. 899-943). Cambridge, United Kingdom and New York, NY, USA: Cambridge University Press.

Kriegler, E., O’Neill, B. C., Hallegatte, S., Kram, T., Lempert, R. J., Moss, R. H., \& Wilbanks, T. (2012). The need for and use of socio-economic scenarios for climate change analysis: A new approach based on shared socio-economic pathways. Global Environmental Change, 22(4), 807-822.

Kunreuther, H., Meyer, R., Zeckhauser, R., Slovic, P., Schwartz, B., Schade, C., ... Hogarth, R. (2002). High stakes decision making: Normative, descriptive and prescriptive considerations. A Journal of Research in Marketing, 13(3), 259-268.

Lado-Sestayo, R., Vivel-Búa, M., \& Otero-González, L. (2017). Drivers and barriers to entry for new hotel start-ups. Tourism Management Perspectives, 23, 87-96.

Lakshmi, S. R., \& Shaji, T. L. (2016). Transformation of coastal settlements due to tourism. Procedia Technology, 24, 1668-1680.

Leary, N., Conde, C., Kulkarni, J., Nyong, A., \& Pulhin, J. (2008). Climate change and vulnerability. London: Earthscan.

Leedy, P. D., \& Ormrod, J. E. (2013). Practical research: planning and design. (J. E. Ormrod, Ed.) (10th ed..). Boston: Boston : Pearson.

Lehmann, P., Brenck, M., Gebhardt, O., Schaller, S., \& Süßbauer, E. (2015). Barriers and opportunities for urban adaptation planning: Analytical framework and evidence from cities in Latin America and Germany. An International Journal Devoted to Scientific, Engineering, Socio-Economic and Policy Responses to Environmental Change, 20(1), 75-97.

Leiper, N. (1995). Tourism management. Collingwood, Vic. : RMIT Press. Retrieved from 
https://trove.nla.gov.au/version/45797868

Leiserowitz, A. A. (2005). American risk perceptions: Is climate change dangerous? Risk Analysis, 25(6), 1433-1442.

Levina, E., \& Tirpak, D. (2006). Adaptation to climate change: Key Terms. Retrieved from www.oecd.org/env/cc

Lewin, A., Weigelt, C., \& Emery, J. (2004). Adaptation and selection in strategy and change: Perspectives on strategic change in organizations. In Handbook of Organizational Change and Innovation (pp. 105-159). London: Oxford University Press.

Lim, B., Spanger-Siegfried, E., Burton, I., Malone, E. L., \& Huq, S. C. N.-Q. 8. C. B. 2005. (2005). Adaptation policy frameworks for climate change: developing strategies, policies, and measures. Cambridge, UK ; New York: Cambridge University Press.

Linnenluecke, M. K., Griffiths, A., \& Mumby, P. J. (2015). Executives' engagement with climate science and perceived need for business adaptation to climate change. Climatic Change, 131(2), 321-333.

Linnenluecke, M. K., Griffiths, A., \& Winn, M. (2012). Extreme weather events and the critical importance of anticipatory adaptation and organizational resilience in responding to impacts. Business Strategy and the Environment, 21(1), 17-32.

Linnerooth-Bayer, J., \& Mechler, R. (2006). Insurance for assisting adaptation to climate change in developing countries: A proposed strategy. Climate Policy, 6(6), 621-636.

Littlejohn, D. (2007). Theoretical perspectives in pedagogical practice: Applying postpositivism and activity theory in today's technologically mediated design education. DDN 702, Perver Baran.

Lockett, S. H., Hatton, J., Turner, R., Stubbins, C., Hodgekins, J., \& Fowler, D. (2012). Using a semi-structured interview to explore imagery experienced during social anxiety for clients with a diagnosis of psychosis: An exploratory study conducted within an early intervention for psychosis service. Behavioural and Cognitive Psychotherapy, 40(1), 55-68.

Longjit, C. (2010). Managing a mature coastal tourism destination: The case of Pattaya, Thailand. the Victoria University of Wellington, Wellington, New Zealand.

Lorenzoni, I., Jordan, A., Hulme, M., Turner, R. K., \& O’Riordan, T. (2000). A co- 
evolutionary approach to climate change impact assessment: Part I. Integrating socioeconomic and climate change scenarios. Global Environmental Change, 10(1), 57-68.

Lorenzoni, I., Nicholson-Cole, S., \& Whitmarsh, L. (2007). Barriers perceived to engaging with climate change among the UK public and their policy implications. Global Environmental Change, 17(3), 445-459.

Lowe, A., Foster, J., \& Winkelman, S. (2009). Asking the climate question: Lessons learned in effective adaptation from urban leaders partners. Center for Clean Air Policy, Washington, DC.

Lozano, R. (2015). A holistic perspective on corporate sustainability drivers. Corporate Social Responsibility and Environmental Management, 22(1), 32-44.

Mack, N., Woodsong, C., MacQueen, K. M., Guest, G., \& Namey, E. (2005). Qualitative research methods: a data collector's field guide. North Carolina: Family Health International.

Magness, D. R., Lovecraft, A. L., \& Morton, J. M. (2012). Factors influencing individual management preferences for facilitating adaptation to climate change within the National Wildlife Refuge System. Wildlife Society Bulletin, 36(3), 457-468.

Mahon, R. (2014). Behavioural links and limits of disaster risk management and climate change adaptation: Demand and supply-side evidence from Caribbean coastal tourism. Lincoln University.

Maiti, S., Jha, S. K., Garai, S., Nag, A., Chakravarty, R., Kadian, K. S., ... Upadhyay, R. C. (2015). Assessment of social vulnerability to climate change in the eastern coast of India. Climatic Change, 131(2), 287-306.

Mannke, F. (2011). Key themes of local adaptation to climate change: Results from mapping community-based initiatives in Africa. In W. Leal Filho (Ed.), Experiences of Climate Change Adaptation in Africa (pp. 17-32). Berlin, Heidelberg: Springer Berlin Heidelberg.

Marin, A. (2010). Riders under storms: Contributions of nomadic herders' observations to analysing climate change in Mongolia. Global Environmental Change, 20(1), 162176.

Marin, A., \& Berkes, F. (2013). Local people's accounts of climate change: To what extent are they influenced by the media? Wiley Interdisciplinary Reviews, 4(1), 1-8. 
Marks, D. (2011). Climate change and Thailand: Impact and response. Contemporary Southeast Asia: A Journal of International and Strategic Affairs, 33(2), 229-258.

Marshall, N A, Marshall, P. A., Tamelander, J., Obura, D., Malleret-King, D., \& Cinner, J. E. (2009). A framework for social adaptation to climate change, 44.

Marshall, Nadine A, Marshall, P. A., Abdulla, A., Rouphael, T., \& Ali, A. (2011). Preparing for climate change: Recognising its early impacts through the perceptions of dive tourists and dive operators in the Egyptian Red Sea. Current Issues in Tourism, 14(6), 507-518.

Masud, M. M., Azam, M. N., Mohiuddin, M., Banna, H., Akhtar, R., Alam, A. S. A. F., \& Begum, H. (2017). Adaptation barriers and strategies towards climate change: Challenges in the agricultural sector. Journal of Cleaner Production, 156, 698-706.

Matasci, C., Kruse, S., Barawid, N., \& Thalmann, P. (2014). Exploring barriers to climate change adaptation in the Swiss tourism sector. Mitigation and Adaptation Strategies for Global Change, 19(8), 1239-1254.

Mazhar, M. U., Bull, R., \& Lemon, M. (2017). Critical success factors for embedding carbon management in organizations: Lessons from the UK higher education sector. Carbon Management, 8(5-6), 379-392.

McClure, L., \& Baker, D. (2018). How do planners deal with barriers to climate change adaptation? A case study in Queensland, Australia. Landscape and Urban Planning, $173,81-88$.

McGregor, S. L., \& Murnane, J. A. (2010). Paradigm, methodology and method: Intellectual integrity in consumer scholarship. International Journal of Consumer Studies, 34(4), 419-427.

McGuiggan, R., \& Lee, G. (2008). Cross-case analysis: An alternative methodology. In ANZMAC 2008 Conference. Retrieved from http://www.duplication.net.au/ANZMAC/ANZMAC Handbook CD.pdf

McNamara, Karen E., Westoby, R., \& Smithers, S. G. (2017). Identification of limits and barriers to climate change adaptation: Case study of two islands in Torres Strait, Australia. Geographical Research, 55(4), 438-455.

McNamara, Karen Elizabeth, \& Gibson, C. (2008). Environmental sustainability in practice? A macro-scale profile of tourist accommodation facilities in Australia's 
coastal zone. Journal of Sustainable Tourism, 16(1), 85-100.

McNeeley, S. M. (2012). Examining barriers and opportunities for sustainable adaptation to climate change in Interior Alaska. Climatic Change, 111(3-4), 835-857.

McSweeney, C., New, M., Lizcano, G., \& Lu, X. (2010). The UNDP climate change country profiles: Improving the accessibility of observed and projected climate information for studies of climate change in developing countries. Bulletin of the American Meteorological Society, 91(2), 157-166.

Measham, T. G., Preston, B. L., Smith, T. F., Brooke, C., Gorddard, R., Withycombe, G., \& Morrison, C. (2011). Adapting to climate change through local municipal planning: Barriers and challenges. Mitigation and Adaptation Strategies for Global Change, 16(8), 889-909.

Meissner, R., Steyn, M., Moyo, E., Shadung, J., Masangane, W., Nohayi, N., \& JacobsMata, I. (2018). South African local government perceptions of the state of water security. Environmental Science \& Policy, 87, 112-127.

Mensah, I. (2006). Environmental management practices among hotels in the greater Accra region. International Journal of Hospitality Management, 25(3), 414-431.

Metcalf, S. J., van Putten, E. I., Frusher, S. D., Tull, M., \& Marshall, N. (2013). Adaptation options for marine industries and coastal communities using community structure and dynamics. Sustainability Science.

Miles, M. B., \& Huberman, A. M. (1994). Qualitative data analysis: An expanded sourcebook. Sage.

Miles, M. B., Huberman, A. M., \& Saldaña, J. (2013). Qualitative data analysis: A methods sourcebook (3rd ed). Thousand Oaks, Califorinia: SAGE Publications, Inc.

MOE. (2016). Analysis of policies to address climate change impacts in Cambodia. Phnom Penh, Cambodia. Retrieved from https://cstn.files.wordpress.com/2009/12/integratedgappolicy-analysis-mar05-en.pdf

MOE \& Mangrove for the Future. (2013). Cambodia National Strategy and Action Plan 2014-2016: Mangroves for the future. Phnom Penh, Cambodia. Retrieved from https://www.mangrovesforthefuture.org/assets/Repository/Documents/CambodiaNSAP-2013-07-04-Webversion.pdf

MOE, \& Danida. (2006, January 10). Water quality in coastal rivers and near shore coastal 
waters of Cambodia, second annual monitoring report. The Ministry of Environment, Cambodia.

MOE, G. \& U. (2015). Vulnerability assessment to climate change in Preah Sihanouk province. Phnom Penh, Cambodia: The Ministry of Environment, Cambodia.

MOE, MEF, \& UNDP. (2011). Human Development Report 2011: Building resilience: The future of rural livelihoods in the face of climate change. Phnom Penh, Cambodia: The Ministry of Environment of Cambodia and UNDP Cambodia. Retrieved from http://hdr.undp.org/sites/default/files/cambodia_2011_nhdr.pdf

Moreno, A., \& Amelung, B. (2009). Climate change and coastal \& marine tourism: Review and analysis. Journal of Coastal Research, SI(56), 1140.

Moreno, A., \& Becken, S. (2009). A climate change vulnerability assessment methodology for coastal tourism. Journal of Sustainable Tourism, 17(4), 473-488.

Moser, S. C., \& Dilling, L. (2004). Making climate HOT. Environment: Science and Policy for Sustainable Development, 46(10), 32-46.

Moser, S. C., \& Ekstrom, J. A. (2010). A framework to diagnose barriers to climate change adaptation. Proceedings of the National Academy of Sciences, 107(51), 22026-22031.

Moser, S. C., Kasperson, R. E., Yohe, G., \& Agyeman, J. (2008). Adaptation to climate change in the Northeast United States: Opportunities, processes, constraints. Mitigation and Adaptation Strategies for Global Change, 13(5-6), 643-659.

Moser, S., \& Ekstrom, J. (2010). Barriers to climate change adaptation: A diagnostic framework. Proceedings of the National Academy of Sciences, 107(51), 22026-22031.

MOT. (2016). Tourism Statistics Report: Year 2016. Phnom Penh, Cambodia. Retrieved from http://www.tourismcambodia.org/images/mot/statistic_reports/tourism_statistics_year _new_2016.pdf

MOT. (2018, May 16). Ministry of Tourism Structure.

Mukheibir, P., \& Ziervogel, G. (2007). Developing a municipal adaptation plan (MAP) for climate change: The city of Cape Town. Environment and Urbanization, 19(1), 143158.

Murthy, I. K., Tiwari, R., \& Ravindranath, N. (2011). Climate change and forests in India: adaptation opportunities and challenges. An International Journal Devoted to 
Scientific, Engineering, Socio-Economic and Policy Responses to Environmental Change, 16(2), 161-175.

Muttarak, R., \& Lutz, W. (2014). Is education a key to reducing vulnerability to natural disasters and hence unavoidable climate change? Ecology and Society, 19(1).

Myers, M. D. (1997). Qualitative research in information systems. MIS Quarterly, 21(2), 241-242.

Nalau, J., Becken, S., Noakes, S., \& Mackey, B. (2017). Mapping tourism stakeholders' weather and climate information-seeking behavior in Fiji. Weather, Climate, and Society, 9(3), 377-391.

Nandy, P., Ahammad, R., Alam, M., \& Islam, A. (2013). Coastal ecosystem based adaptation: Bangladesh experience. In R. Shaw, F. Mallick, \& A. Islam (Eds.), Climate Change Adaptation Actions in Bangladesh (pp. 277-303). Tokyo: Springer Japan.

Narain, U., Margulis, S., \& Essam, T. (2011). Estimating costs of adaptation to climate change. Climate Policy, 11(3), 1001-1019.

NCSC. (2005). Environment and socio-economic report. The Ministry of Environment, Cambodia.

Neuenschwander, L. M., Abbott, A., \& Mobley, A. R. (2012). Assessment of low-Income adults' access to technology: Implications for nutrition education. Journal of Nutrition Education and Behavior, 44(1), 60-65.

Nguyen, Q. A., Miller, F., Bowen, K., \& Tan Sinh, B. (2017). Evaluating capacity for climate change adaptation in the health and water sectors in Vietnam: constraints and opportunities. Climate and Development, 9(3), 258-273.

Nguyen, T. T. D. (2015). Tourism and the environment: The response of privately owned accomodation businesses in Da Nang City, Vietnam. Victoria University of Wellington. Retrieved from http://researcharchive.vuw .ac.nz/handle/10063/4763

Niang-Diop, I., \& Bosch, H. (2005). Formulating an adaptation strategy. In Adaptation policy frameworks for climate change: Developing strategies, policies, and measures. Cambridge, UK ; New York: Cambridge University Press.

Nicholls, R. J., \& Small, C. (2002). Improved estimates of coastal population and exposure to hazards released. Eos, Transactions American Geophysical Union, 83(28), 301. 
Nicholls, S., \& Kang, S. (2012a). Going green: The adoption of environmental initiatives in Michigan's lodging sector. Journal of Sustainable Tourism, 20(7), 953-974.

Nicholls, S., \& Kang, S. (2012b). Green initiatives in the lodging sector: Are properties putting their principles into practice? International Journal of Hospitality Management, 31(2), 609-611.

Nidumolu, U., Lim-Camacho, L., Gaillard, E., Hayman, P., \& Howden, M. (2018). Linking climate forecasts to rural livelihoods: Mapping decisions, information networks and value chains. Weather and Climate Extremes, 1-13.

NIS. (2008). General population census of Cambodia 2008 provisional population totals. The Ministry of Planning, Cambodia. Retrieved from http://www.stat.go.jp/english/info/meetings/cambodia/pdf/pre_rep1.pdf

Njoroge, J. M. (2014). An enhanced framework for regional tourism sustainable adaptation to climate change. Tourism Management Perspectives, 12, 23-30.

Norgaard, K. M. (2006). “We don't really want to know": Environmental justice and socially organized denial of global warming in Norway. Organization \& Environment, 19(3), 347-370.

O'Brien, K. (2009). Climate change and values: Do changing values define the limits to successful adaptation? In Adapting to climate change: thresholds, values, governance. Cambridge: Cambridge University Press.

O'connor, P., \& Frew, A. J. (2002). The future of hotel electronic distribution: expert and industry perspectives. Cornell Hotel and Restaurant Administration Quarterly, 43(3), $33-45$.

Olazabal, M., Galarraga, I., Ford, J., Lesnikowski, A., \& Sainz de Murieta, E. (2017). Towards successful adaptation: A checklist for the development of climate change adaptation plans. Retrieved from http://www.bc3research.org/index.php?option=com_wpapers\&task=showdetails\&Item $\mathrm{id}=279 \&$ idwpaper $=90$

Oliver-Smith, A. (1991). Involuntary resettlement, resistance and political empowerment. Journal of Refugee Studies, 4(2), 132.

Ong, L. T. J., \& Smith, R. A. (2014). Perception and reality of managing sustainable coastal tourism in emerging destinations: The case of Sihanoukville , Cambodia. 
Journal of Sustainable Tourism, 22(2), 256-278.

Orhan, E. (2016). Lessons learned from businesses to ensure community level recovery in a postdisaster period: Case from Adapazari, Turkey. Natural Hazards Review, 17(1), 12.

Page, S. (2009). Tourism management managing for change (3rd ed.). Burlington:

Burlington : Elsevier.

https://doi.org/http://helicon.vuw.ac.nz/login?url=http://www.VUW.eblib.com/EBLW

eb/patron?target=patron\&extendedid=P_535230_0\&

Paré, G. (2004). Investigating information systems with positivist case research.

Communications of the Association for Information Systems, 13(1).

Parry, M. L. (2007). Climate Change 2007: Impacts, adaptation and vulnerability:

contribution of Working Group II to the fourth assessment report of the

Intergovernmental Panel on Climate Change (Vol. 4). Cambridge University Press.

Pasquini, L., Ziervogel, G., Cowling, R. M., \& Shearing, C. (2014). What enables local governments to mainstream climate change adaptation? Lessons learned from two municipal case studies in the Western Cape, South Africa. Climate and Development, $7(1), 1-11$.

Paton, D., Millar, M., \& Johnston, D. (2001). Community resilience to volcanic hazard consequences. Natural Hazards, 24(2), 157-169.

Patt, A. G., \& Weber, E. U. (2014). Perceptions and communication strategies for the many uncertainties relevant for climate policy. Wiley Interdisciplinary Reviews: Climate Change. Hoboken, USA.

Paul, B. K., \& Bhuiyan, R. H. (2010). Urban earthquake hazard: perceived seismic risk and preparedness in Dhaka City, Bangladesh. Disasters, 34(2), 337-359.

Pauw, P., \& Pegels, A. (2013). Private sector engagement in climate change adaptation in least developed countries: An exploration. Climate and Development, 5(4), 1-11.

Pearce, D. G. (2012). Frameworks for tourism research. Wallingford, Oxfordshire: CABI.

Perch-Nielsen, S. L. (2010). The vulnerability of beach tourism to climate change - an index approach. Climatic Change, 100(3-4), 579-606.

Petersen, B., Hall, K. R., Kahl, K., \& Doran, P. J. (2013). Research articles: In their own words: Perceptions of climate change adaptation from the Great Lakes Region's resource management community. Environmental Practice, 15(4), 377-392. 
Peterson, S. (2006). Uncertainty and economic analysis of climate change: A survey of approaches and findings. Environmental Modeling \& Assessment, 11(1), 1-17.

Pham, T. D., Simmons, D. G., \& Spurr, R. (2010). Climate change-induced economic impacts on tourism destinations: The case of Australia. Journal of Sustainable Tourism, 18(3), 449-473.

Phan, T. T. L. (2017). Visitor satisfaction with services for environmental interpretation in Cat Tien National Park, Vietnam. Victoria University of Wellington.

Phirun, N. (2013). Climate change adaptation and livelihoods in inclusive growth: A review of climate change impacts and adaptive capacity in Cambodia. Phnom Penh, Cambodia: CDRI - Cambodia's leading independent development policy research institute. Retrieved from https://www.cdri.org.kh/publication-pageold/pub/wp/wp82e.pdf

Pickard, A. J. (2007). Research methods in information. London, England: Facet Publishing.

Pipithirankarn, M. (n.d.). Predisposing and reinforcing factors influencing intention to buy green product. Retrieved from https://pdfs.semanticscholar.org/752c/daa468de0ca9666f09028d34f22b65bc66bc.pdf

Ponna, P. (2009). Community-based tourism development in Sihanoukville, Cambodia. Prince of Songkla University, Thailand. Retrieved from http://doc2.clib.psu.ac.th/public13/thises/313813.pdf

Poole, P. A. (2009). Politics and society in Southeast Asia. Jefferson, N.C: McFarland \& Co.

Poudel, S. (2013). The influence of the accommodation sector on tourism development and its sustainability: The case study of Strand Camping, Larsmo. Centria University of Applied Sciences.

Pröbstl-Haider, U., \& Haider, W. (2013). Tools for measuring the intention for adapting to climate change by winter tourists: Some thoughts on consumer behavior research and an empirical example. Tourism Review, 68(2), 44-55.

Prutsch, A., Grothmann, T., Schauser, I., Otto, S., \& McCallum, S. (2010). Guiding principles for adaptation to climate change in Europe, 32 .

Rao, P. (2013). Building climate resilience in coastal ecosystems in India: Cases and trends 
in adaptation practices. In Climate Change and Disaster Risk Management (pp. 335349). Springer, Berlin, Heidelberg.

Reser, J., Bradley, G., Glendon, I., Ellul, M., \& Callaghan, R. (2012). Public risk perceptions, understandings, and responses to climate change and natural disasters in Australia and Great Britain. Gold Coast.

Resurreccion, B. P., Sajor, E. E., \& Fajber, E. (2008). Climate change adaptation Southeast Asia: Knowledge gaps and research issues in South East Asia, 84. Retrieved from https://www.unisdr.org/files/8126_ClimateAdaptationSEASept081.pdf

RGC. (2006, June 6). National Adaptation Programme of Action to Climate Change (NAPA). Phnom Penh, Cambodia. The Ministry of Environment. Retrieved from http://unfecc.int/resource/docs/napa/khm01.pdf

RGC. (2010a). Cambodia: Post-Ketsana disaster needs assessment. Phnom Penh, Cambodia: The Royal Government of Cambodia. Retrieved from https://www.gfdrr.org/sites/default/files/GFDRR_Cambodia_PDNA_2010_EN.pdf

RGC. (2010b). Pilot program for climate resilience Royal Government of Cambodia: Proposal for phase 1. Washington DC, the US: Climate investment funds. Phnom Penh, Cambodia. Retrieved from https://wwwcif.climateinvestmentfunds.org/sites/default/files/meetingdocuments/cambodia_phase_1_proposal_jdk_0.pdf

RGC. (2012). Tourism development strategic plan 2012-2020. Retrieved from http://www.tourismcambodia.org/images/mot/legal_documents/tourism_development _stategic_plan_2012_2020_english.pdf

RGC. (2013). Cambodia Climate Change Strategic Plan 2014-2023. Phnom Penh, Cambodia: The Royal Government of Cambodia. Retrieved from http://www.cambodiaip.gov.kh/DocResources/ab9455cf-9eea-4adc-ae9395d149c6d78c_007729c5-60a9-47f0-83ac-7f70420b9a34-en.pdf

Rhee, H. T., \& Yang, S.-B. (2015). Does hotel attribute importance differ by hotel? Focusing on hotel star-classifications and customers' overall ratings. Computers in Human Behavior, 50, 576-587.

Rissik, D., \& Reis, N. (2013). Climate change adaptation good practice - synthesis report: Key lessons from practitioners' experiences. Australia: The Australian Department of 
Industry, Innovation, Climate Change, Science, Research and Tertiary Education.

Rizvi, Ali Raza, Barrow, E., Zapata, F., Cordero, D., Podvin, K., Kutegeka, S., ...

Adhikari, A. (2014). Ecosystem-based adaptation: Building on no-regret adaptation measures. In 20th session of the Conference of the Parties to the UNFCCC and the 10th session of the Conference of the Parties to the Kyoto Protocol. IUCN. Retrieved from

http://cmsdata.iucn.org/downloads/iucn_eba_technical_paper_no_regret_actions_lima _cop_20.pdf

Rizvi, Ali Rizvi, \& Singer, U. (2011). Cambodia coastal situational analysis.

Rowley, T., \& Berman, S. (2000). A brand new brand of corporate social performance. Business \& Society, 39(4), 397-418.

Rudiak-Gould, P. (2013). "We have seen it with our own eyes": Why we disagree about climate change visibility. Weather, Climate, and Society, 5(2), 120-132.

Ryan, A. B. (2006). Post-positivist approaches to research. Researching and Writing Your Thesis: A Guide for Postgraduate Students, 12-26.

Saarinen, J., Hambira, W. L., Atlhopheng, J., \& Manwa, H. (2012). Tourism industry reaction to climate change in Kgalagadi South District, Botswana. Development Southern Africa, 29(2), 273-285.

Sahoo, S., Dhar, A., \& Kar, A. (2016). Environmental vulnerability assessment using Grey Analytic Hierarchy Process based model. Environmental Impact Assessment Review, $56,145-154$.

Saldaña, J. (2009). The coding manual for qualitative researchers. Los Angeles, Calif: Sage.

Saldaña, J. (2013). The coding manual for qualitative researchers (2nd ed.). London: Sage. Samdahl, D. M. (1999). Epistemological and methodological issues in leisure research. Leisure Studies: Prospects for the Twenty-First Century, 119-133.

Sampaio, A. R., Thomas, R., \& Font, X. (2012). Small business management and environmental engagement. Journal of Sustainable Tourism, 20(2), 179-193.

San, V. (2015). Electricity pricing in the residential sector of Cambodia. Phnom Penh, Cambodia: MECON Project. Retrieved from http://www.meconproject.com/wpcontent/uploads/report/[Task 6-Electricity pricing in the residential sector] Cambodia 
country report.pdf

Santos-Lacueva, R., Clavé, S. A., \& Saladié, Ò. (2017). The vulnerability of coastal tourism destinations to climate change: The usefulness of policy analysis. Sustainability, 9(12), 2062.

Sarantakos, S. (1993). Social research. South Melbourne: MacMillan Education Australia. Sarrica, M., \& Wachelke, J. (2010). Peace and war as social representations: A structural exploration with Italian adolescents. Universitas Psychologica, 9(2), 315-330.

Scaglion, R. (2010). Modern crises and traditional strategies: Local ecological knowledge in island Southeast Asia. Human Ecology, 38(1), 175-176.

Scannell, L., \& Gifford, R. (2013). Personally relevant climate change: The role of place attachment and local versus global message framing in engagement. Environment and Behavior, 45(1), 60-85.

Scheyvens, R., \& Momsen, J. (2008). Tourism in small island states: From vulnerability to strengths. Journal of Sustainable Tourism, 16(5), 491-510.

Schipper, E. L. F. (2006). Conceptual history of adaptation in the UNFCCC process. Review of European Community \& International Environmental Law, 15(1), 82-92.

Schnell-Anzola, B., Rowe, M. L., \& LeVine, R. A. (2005). Literacy as a pathway between schooling and health-related communication skills: a study of Venezuelan mothers. International Journal of Educational Development, 25(1), 19-37.

Schott, C., Milfont, T. L., \& Reisinger, A. (2010). Tourism and the implications of climate change: Issues and actions. In Tourism and the implications of climate change: Issues and actions (Vol. 3, pp. 1-24). Bradford: Emerald Group Publishing Limited.

Schröter, D., Polsky, C., \& Patt, A. G. (2005). Assessing vulnerabilities to the effects of global change: an eight step approach. Mitigation and Adaptation Strategies for Global Change, 10(4), 573-595.

Scott, D. (2011). Why sustainable tourism must address climate change. Journal of Sustainable Tourism, 19(1), 17-34.

Scott, D., \& Becken, S. (2010). Adapting to climate change and climate policy: Progress, problems and potentials. Journal of Sustainable Tourism, 18(3), 283-295.

Scott, D., de Freitas, C., \& Matzarakis, A. (2009). Adaptation in the tourism and recreation sector. In Biometeorology for adaptation to climate variability and change (pp. 171- 
194). Springer.

Scott, D., Hall, C. M., \& Gössling, S. (2012). Tourism and climate change : Impacts, adaptation and mitigation. Routledge.

Shah, K. (2011). Strategic organizational drivers of corporate environmental responsibility in the Caribbean hotel industry. Integrating Knowledge and Practice to Advance Human Dignity, 44(4), 321-344.

Shakeela, a, \& Becken, S. (2014). Understanding tourism leaders' perceptions of risks from climate change: An assessment of policy-making processes in the Maldives using the social amplification of risk framework (SARF). Journal of Sustainable Tourism, 23(February 2015), 37-41.

Sharma, B. (2010). Postpositivism. In Encyclopedia of Case Study Research. Thousand Oaks, California, the United States: SAGE Publications, Inc.

Sharma, B., Map, S., (MOT), T. M. of T., Adger, W. N., Brooks, N., Bentham, G., ... Perch-Nielsen, S. L. (2013). Rethinking the role of adaptation in climate policy. Global Environmental Change, 5(2), 62. Contribution of Working Groups I, II and III to the Fifth Assessment Report of the Intergovernmental Panel on Climate Change.

Sharpley, R. (2006). Ecotourism: A consumption perspective. Journal of Ecotourism, 5(12), 7-22.

Shrestha, S. (2014). Introduction. In S. Shrestha (Ed.), Climate change impacts and adaptation in water resources and water use sectors: Case studies from Southeast Asia (pp. 1-7). Springer International Publishing.

Silverman, D. (2010). Doing qualitative research: A practical handbook (3rd ed.). Los Angeles \& London: SAGE.

Simões, E., de Sousa Junior, W. C., de Freitas, D. M., Mills, M., Iwama, A. Y., Gonçalves, I., ... Fidelman, P. (2017). Barriers and opportunities for adapting to climate change on the North Coast of São Paulo, Brazil. Regional Environmental Change, 17(6), 1739-1750.

Simonet, G., \& Fatorić, S. (2016). Does “adaptation to climate change” mean resignation or opportunity? Regional Environmental Change, 16(3), 789-799.

Simpson, M. C., Gössling, S., Scott, D., Hall, C. M., \& Gladin, E. (2008). Climate change adaptation and mitigation in the tourism Sector: Frameworks, tools and practices. 
Tourism. Paris, France.

Smit, B., \& Wandel, J. (2006). Adaptation, adaptive capacity and vulnerability. Global Environmental Change, 16(3), 282-292.

Smithers, J., \& Smit, B. (1997). Human adaptation to climatic variability and change. Global Environmental Change, 7(2), 129-146.

Solar. (2010). Rural women, gender, and climate change: A literature review and invited perspectives on climate change impacts and processes of adaptation in Cambodia. Cambodia: Oxfam America. Retrieved from http://www.rrcap.ait.asia/Publications/LITERATURE_REVIEW__Rural_Women_Gender_and_Climate_Change.pdf

Spence, A., Poortinga, W., \& Pidgeon, N. (2012). The psychological distance of climate change. Risk Analysis, 32(6), 957-972.

Stanny, E., \& Ely, K. (2008). Corporate environmental disclosures about the effects of climate change. Corporate Social Responsibility and Environmental Management, $15(6), 338-348$.

Stein, S., Lauer, Y., \& Kharbili, M. El. (2009, July). Using template analysis as background reading technique for requirements elicitation. Saarbrücken, Germany. Retrieved from https://pdfs.semanticscholar.org/bceb/aa1c8825e40301100941a17c720d229ef5fb.pdf

Strike, V. M., Gao, J., \& Bansal, P. (2006). Being good while being bad: Social responsibility and the international diversification of US firms. Journal of International Business Studies, 37(6), 850.

Strode, D. E. (2012). A theory of coordination in Agile Software Development Projects. The Victoria University of Wellington, Wellington, New Zealand.

Su, Y.-P., Hall, C. M., \& Ozanne, L. (2013). Hospitality industry responses to climate change: A benchmark study of Taiwanese tourist hotels. Asia Pacific Journal of Tourism Research, 18(1-2), 92-107.

Su, Y. P. (2014). The Taiwanese hotel sector's response to climate change: Environmental behaviours and practices. the University of Canterbury, New Zealand. Retrieved from https://ir.canterbury.ac.nz/bitstream/handle/10092/9209/thesis_fulltext.pdf?sequence= 3

Tervo-Kankare, K. (2011). The consideration of climate change at the tourism destination 
level in Finland: Coordinated collaboration or talk about weather? Tourism Planning \& Development, 8(4), 399-414.

The Ministry of Environment, MOE. (2002). Cambodia's initial national communication: Under the United Nations Framework Convention on Climate Change. Phnom Penh, Cambodia.

The Phnom Penh Post. (2018). Sihanoukville tourism First half of 2018 sees 1.3M visitors. The Phnom Penh Post, p. 1.

The Phnom Penh Post. (2019). Chinese own more than 90\% of Sihanoukville business, says report. The Phnom Penh Post, p. 1.

The Post Phnom Penh. (2015, March 15). Cambodian hotel industry shows growth. The

Phnom Penh Post. Retrieved from http://www.phnompenhpost.com/realestate/cambodian-hotel-industry-shows-growth

Theron, P. M. (2015). Coding and data analysis during qualitative empirical research in Practical Theology. In Die Skriflig, 49(3).

Thoeun, H. C. (2015). Observed and projected changes in temperature and rainfall in Cambodia. Weather and Climate Extremes, 7, 61-71.

Thompson, A. r., Clarke, S. a., Newell, R. j., \& Gawkrodger, D. j. (2010). Vitiligo linked to stigmatization in British South Asian women: a qualitative study of the experiences of living with vitiligo. British Journal of Dermatology, 163(3), 481-486.

Tierney, K. J. (2007). Businesses and disasters: Vulnerability, impacts, and recovery (pp. 275-296). https://doi.org/10.1007/978-0-387-32353-4_16

Tilleard, S., \& Ford, J. (2016). Adaptation readiness and adaptive capacity of transboundary river basins. An Interdisciplinary, International Journal Devoted to the Description, Causes and Implications of Climatic Change, 137(3), 575-591.

Tilley, F. (2000). Small firms' environmental ethics: How deep do they go? In Small and Medium-Sized Enterprises and the Environment (pp. 36-48). Sheffield, UK: Taylor \& Francis Group.

Tinat, N. (2011). International organizations and NGOs' perceptions towards the National Tourism Policy and coastal tourism development in Cambodia: The Case of Preah Sihanouk province. The University of Tsukuba, Ibaraki, Japan.

Tobey, J., Rubinoff, P., Robadue, D., Ricci, G., Volk, R., Furlow, J., \& Anderson, G. 
(2010). Practicing coastal adaptation to climate change: Lessons from integrated coastal management. Coastal Management, 38(3), 317-335.

Trenberth KE, Jones PD, Ambenje P, Bojariu R, Easterling D, Klein Tank A, Parker D, Rahimzadeh F, Renwick JA, Rusticucci M, Soden B Z. P. (2007). Observations: surface and atmospheric climate change. In Climate Change 2007: the Physical Science Basis: Contribution of Working Group I to the Fourth Assessment Report of the Intergovernmental Panel on Climate Change (pp. 235-336). Cambridge: Cambridge University Press.

Tribbia, J., \& Moser, S. C. (2008). More than information: what coastal managers need to plan for climate change. Environmental Science \& Policy, 11(4), 315-328.

Trope, Y., \& Liberman, N. (2003). Temporal construal. Psychological Review, 110(3), $403-421$.

Tuan, N. Le. (2016). Tourism distribution in a transitional economy: Hotel ownerships and a distribution channels in Vietnam. The Victoria University of Wellington.

Tuler, S., Agyeman, J., Agyeman, J., da Silva, P. P., LoRusso, K. R., \& Kay, R. (2008). Assessing vulnerabilities: Integrating information about driving forces that affect risks and resilience in fishing communities. Human Ecology Review, 15(2), 171-184.

Tzschentke, N. A., Kirk, D., \& Lynch, P. A. (2008). Going green: Decisional factors in small hospitality operations. International Journal of Hospitality Management, 27(1), 126-133.

Tzschentke, N., Kirk, D., \& Lynch, P. (2008). Ahead of their time? Barriers to action in green tourism firms. The Service Industries Journal, 28(2), 167-178.

UN-Habitat. (2012). Cities and climate change initiatives: Sihanoukville, Cambodia: Climate change vulnerability assessment. Sihanoukville, Cambodia: UN-Habitat.

UN. (2007). Forests and climate Change: Mitigation and adaptation through sustainable forest management. In 60th Annual DPI / NGO Conference (p. 7). England: Forestry Commision England. Retrieved from http://www.un.org/esa/forests/pdf/notes/FCC_60_6092007.pdf

UN. (2017a). List of least developed countries. United Nations Committee for Development Policy and Analysis Division Department of Economic and Social Affairs. 
UN. (2017b). List of least developed countries. United Nations Committee for Development Policy and Analysis Division Department of Economic and Social Affairs. Retrieved from https://www.un.org/development/desa/dpad/wpcontent/uploads/sites/45/publication/ldc_list.pdf

UNEP. (2013a). Vulnerability assessment and adaptation programme for climate change within the coastal zone of Cambodia considering livelihood improvement and ecosystems. Phnom Penh, Cambodia: The United Nations Environmental Programme (UNEP).

UNEP. (2013b). Vulnerability assessment and adaptation programme for climate change within the coastal zone of Cambodia considering livelihood improvement and ecosystems. Phnom Penh, Cambodia: The Ministry of Environment, Global Environment Facility and United Nations Environmental Programme.

UNEP. (2014). Prey Nob polder and coastal area-Climate change adaptation measures and livelihood improvement. Phnom Penh, Cambodia.

UNEP. (2017). Vulnerability assessment and adaptation project for climate change in the coastal zone of Cambodia considering livelihood improvement and ecosystems $(V A A P)$. Sihanoukville, Cambodia. Retrieved from http://wedocs.unep.org/bitstream/handle/20.500.11822/20806/3890_2017_te_unep_ca mbodia_cc_msp_unepspcc_cambodia_vaap.pdf?sequence $=1$

UNFCCC. (2009). Fact sheet: Financing climate change action Investment and financial flows for a strengthened response to climate change. Retrieved from https://unfccc.int/files/press/backgrounders/application/pdf/fact_sheet_financing_clim ate_change.pdf

UNFCCC. (2010). Report of the conference of the parties on its fifteenth session, held in Copenhagen from 7 to 19 December 2009. Copenhagen. Retrieved from https://unfccc.int/resource/docs/2009/cop15/eng/11a01.pdf

Ung, M., Luginaah, I., Chuenpagdee, R., \& Campbell, G. (2015). Perceived self-efficacy and adaptation to climate change in coastal Cambodia. Climate, 4(4), 1.

Ung, M., Luginaah, I., Chuenpagdee, R., \& Campbell, G. (2017). Perceptions of climate change impacts and self-rated health in coastal Cambodia. International Journal of Environmental Studies, 74(2), 325-339. 
UNWTO. (2014). Tourism initiatives in Asia and the Pacific responding to climate change. Madrid, Spain: World Tourism Organization (UNWTO).

UNWTO. (2018a). UNWTO World Tourism Barometer. Madrid. Retrieved from http://cf.cdn.unwto.org/sites/all/files/pdf/unwto_barom18_01_january_excerpt_hr.pdf UNWTO. (2018b). World Tourism Barometer. Retrieved from www.unwto.org

Urwin, K., \& Jordan, A. (2008). Does public policy support or undermine climate change adaptation? Exploring policy interplay across different scales of governance. Global Environmental Change, 18(1), 180-191.

van den Bergh, J., \& Nijkamp, P. (1998). Economic aspects of global change impacts and response strategies in the coastal zone of The Netherlands. Journal of Coastal Conservation, 4(2), 161-168.

Vaughan, C., Dessai, S., Hewitt, C., Baethgen, W., Terra, R., \& Berterretche, M. (2017). Creating an enabling environment for investment in climate services: The case of Uruguay's National Agricultural Information System. Climate Services, 8(C), 62-71.

Veal, A J, \& Darcy, S. (2014). Research methods in sport studies and sport management: A practical guide. Routledge.

Veal, Anthony James. (1997). Research methods for leisure and tourism: A practical guide. (I. of L. \& A. Management, Ed.) (2nd ed.). London: Pitman.

Veal, Anthony James. (2006). Research methods for leisure and tourism: a practical guide. Harlow, England: Prentice Hall/Financial Times.

Vignola, R., Leclerc, G., Morales, M., \& Gonzalez, J. (2017). Leadership for moving the climate change adaptation agenda from planning to action. Current Opinion in Environmental Sustainability, 26-27, 84-89.

Vulturius, G., \& Gerger Swartling, Å. (2015). Overcoming social barriers to learning and engagement with climate change adaptation: Experiences with Swedish forestry stakeholders. Scandinavian Journal of Forest Research, 30(3), 217-225.

Wachinger, G., Renn, O., Begg, C., \& Kuhlicke, C. (2013). The risk perception paradoxImplications for governance and communication of natural hazards. Risk Analysis, 33(6), 1049-1065.

Waddington, K., \& Fletcher, C. (2005). Gossip and emotion in nursing and health-care organizations. Journal of Health Organization and Management, 19(4/5), 378-394. 
Wadgave, U., \& Khairnar, M. R. (2016). Parametric tests for Likert scale: For and against. Asian Journal of Psychiatry.

Walmsley, A. (2012). Climate change mitigation and adaptation in the hospitality industry. In Trends and Issues in Global Tourism 2011 (pp. 77-91). Berlin, Heidelberg: Springer Berlin Heidelberg.

Wan, J.-Z., Wang, C.-J., Qu, H., Liu, R., \& Zhang, Z.-X. (2018). Vulnerability of forest vegetation to anthropogenic climate change in China. Science of The Total Environment, 621, 1633-1641.

Wang, C., Dai, S., \& Xu, H. (2018). Estimating the land opportunism of hotel investment in generating real estate appreciation and firms' market value. Land Use Policy, 77, 752759.

Wang, S., Hurlstone, M. J., Leviston, Z., Walker, I., \& Lawrence, C. (2019). Climate change from a distance: An analysis of construal level and psychological distance from climate change. Frontiers in Psychology, 10.

Warrick, O. C. (2011). Local voices, local choices? Vulnerability to climate change and community-based adaptation in rural Vanuatu. the University of Waikato.

Wasileski, G., Rodríguez, H., \& Diaz, W. (2011). Business closure and relocation: A comparative analysis of the Loma Prieta earthquake and Hurricane Andrew. Disasters, $35(1), 102-129$.

Webb, G. R., Tierney, K. J., \& Dahlhamer, J. M. (2002a). Predicting long-term business recovery from disaster: a comparison of the Loma Prieta earthquake and Hurricane Andrew. Environmental Hazards, 4, 45-58.

Webb, G. R., Tierney, K. J., \& Dahlhamer, J. M. (2002b). Predicting long-term business recovery from disaster: A comparison of the Loma Prieta earthquake and Hurricane Andrew. Global Environmental Change Part B: Environmental Hazards, 4(2-3), 4558.

Wedderburn, S. (2009). Notification of joint Multilateral Development Bank (MDB) programming mission to support Cambodia's preparation towards developing the pilot program for Climate Resilience Strategic Program for Climate Resilience (PPCR). Phnom Penh, Cambodia: The World Bank.

Wei, J., Hansen, A., Zhang, Y., Li, H., Liu, Q., Sun, Y., \& Bi, P. (2014). Perception, 
attitude and behavior in relation to climate change: A survey among CDC health professionals in Shanxi province, China. Environmental Research, 134(C), 301-308.

Wilbanks, T., Romero Lankao, P., Bao, M., Berkhout, F., Cairncross, S., Ceron, J., ... Zapata-Marti, R. (2007). Industry settlement and society. In Climate change 2007: impacts, adaptation and vulnerability: contribution of Working Group II to the fourth assessment report of the Intergovernmental Panel on Climate Change (p. 976). Cambridge, U.K. ; New York: Cambridge University Press.

WMO. (2013). The global climate of 2001-2010-a decade of climate extremes. Geneva. Retrieved from https://library.wmo.int/pmb_ged/wmo_1119_en.pdf

WMO, UNEP, \& WTO. (2008). Climate change and tourism responding to global challenges. Madrid, Spain.

Wolf, J., Lorenzoni, I., Few, R., Abrahmson, V., \& Raine, R. (2009). Conceptual and practical barriers to adaptation: An interdisciplinary analysis of vulnerability and response to heat waves in the UK. In Adapting to climate change: Thresholds, values, governance (1st ed.). Cambridge: Cambridge University Press.

World Bank. (2009). Convenient solutions to an inconvenient truth: Ecosystem-based approaches to climate change. Washington, DC: World Bank.

World Bank. (2011). Climate risk and adaptation country profile: Vulnerability, risk reduction, and adaptation to climate change, Cambodia. Washington, DC. Retrieved from

http://sdwebx.worldbank.org/climateportalb/doc/GFDRRCountryProfiles/wb_gfdrr_cl imate_change_country_profile_for_KHM.pdf

World Bank. (2012). The little green data book. Washington, DC: The World Bank. Retrieved from http://siteresources.worldbank.org/EXTSDNET/Resources/LittleGreen-Data-Book-2012.pdf

World Risk Report. (2012). Focus: Environmental degradation and disasters: World Risk Report 2012. United Nations University (UNU), Institute for Environment and Human Security. Retrieved from http://www.droughtmanagement.info/literature/UNU_world_risk_report_2012_2012.p df

WTTC. (2018a). Travel \& tourism: Economic impact 2018 Cambodia. Retrieved from 
https://www.wttc.org/-/media/files/reports/economic-impact-research/countries2018/cambodia2018.pdf

WTTC. (2018b). World 2018 Annual Research: Key facts. Retrieved from https://www.wttc.org/-/media/files/reports/economic-impact-research/regions2018/world2018.pdf

Xia, H., Quan Vu, H., Lan, Q., Law, R., \& Li, G. (2018). Identifying hotel competitiveness based on hotel feature ratings. Journal of Hospitality Marketing \& Management.

Yin, R. K. (2003). Case study research: Design and methods (3rd ed, Vol. 5). Thousand Oaks, London, New Dellhi: SAGE Publications.

Yin, R. K. (2009). Case study research: Design and methods (4th ed, Vol. 5). Los Angeles, London, New Delhi, Singapore, Washington DC: SAGE Publications.

Yin, R. K. (2014). Case study research: Design and methods (5th ed). Los Angeles: SAGE Publications.

Yoo, G., Kim, A. R., \& Hadi, S. (2014). A methodology to assess environmental vulnerability in a coastal city: Application to Jakarta, Indonesia. Ocean \& Coastal Management, 102, 169-177.

Yusuf, A. A., \& Francisco, H. (2009, November 24). Climate change vulnerability mapping for Southeast Asia. Economy and Environment Program for Southeast Asia (EEPSEA). Retrieved from https://www.idrc.ca/sites/default/files/sp/Documents EN/climate-change-vulnerability-mapping-sa.pdf

Zaval, L., Keenan, E. A., Johnson, E. J., \& Weber, E. U. (2014). How warm days increase belief in global warming. Nature Climate Change, 4(2), 143.

Zhang, H. Q., Ren, L., Shen, H., \& Xiao, Q. (2013). What contributes to the success of Home Inns in china? International Journal of Hospitality Management, 33, 425-434.

Zhang, Y., Lindell, M. K., \& Prater, C. S. (2009). Vulnerability of community businesses to environmental disasters. Disasters, 33(I), 38-57.

Zhou, Y., \& Tol, R. S. J. (2005). Evaluating the costs of desalination and water transport. Water Resources Research, 41(3).

Zou, T., \& Yoshino, K. (2017). Environmental vulnerability evaluation using a spatial principal components approach in the Daxing'anling region, China. Ecological Indicators, 78, 405-415. 
Zuka, S. P. (2014). Prospects and challenges of local community adaptation to climate change in developing countries: The Case study of Malawi. In W. Leal Filho (Ed.), Handbook of Climate Change Adaptation (pp. 1-13). Berlin, Heidelberg: Springer Berlin Heidelberg. 


\section{Appendix A: Interview guide}

\section{An Interview Guide/Design -}

\section{Climate change adaptation in a coastal hotel sector: The case of Sihanoukville,}

Cambodia.

There will be only 1 meeting with each participant of this study. The researcher will digitally record and take notes, as the interview develops. The purpose of the meeting is to address research Q1, Q2, Q3, Q4 \& Q5.

\section{Research aim s/questions:}

The research determines whether SHV's hotel sector adapts to climate change by critically examining the barriers and enabling factors to adaptation addressing four sequential research questions as follows:

The study's overarching topic is divided into five sequential sub-aims:

1. To assess the vulnerability of Sihanoukville's coastal tourism to climate change,

2. To assess the vulnerability of Sihanoukville's hotel sector to climate change,

3. To determine whether/how the hotel sector adapts to climate change impacts,

4. To identify the most significant barriers and enabling factors for adaptation in the context of hotel sector,

5. To critically examine the barriers and enabling factors to adaptation according to the hotel attributes and participants' backgrounds.

\section{Before the interview/conversation}

1) The researcher's self-introduction

2) Explain what the research is about and what it aims to explore with the participant's information.

3) Inform participant that the meeting will require around 1 hour.

4) Participants will be informed that the conversation/interview will be taperecorded, and they will be given a full transcript later.

5) Participant to sign consent form.

6) The researcher will define some key terms such as vulnerability, climate change, climate change adaptation, barriers and enabling factor to CCA etc. The definitions of keys terms are attached in the front each main section in this interview schedule/guide. 


\section{The interview starts}

Before we get started, as mentioned I would like to record this interview, do you mind if I start recording now?

Record

\section{A Checklist of Questions}

\section{ABOUT THE HOTEL}

Firstly, could you give me some of your background, including:

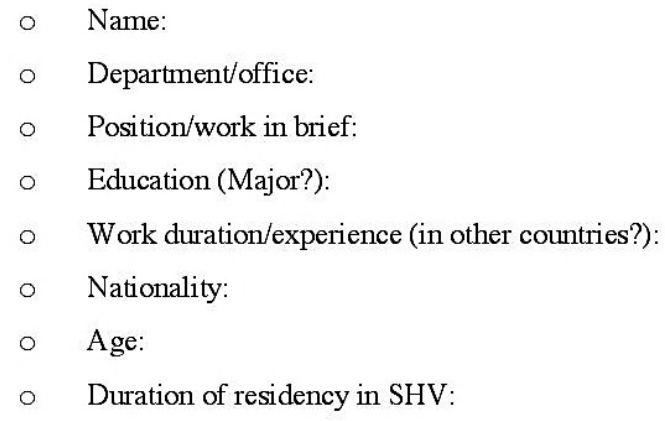

Secondly, could you give me some background on your hotel, including:

- Hotel name:

- Date established (years of trading):

- Quality/Star rating:

○ Size (number of room: and employee):

- Number of rooms:

- Number of employees:

$>$ Full time:

$>$ Part-time:

$>$ Seasonal employees:

- Ownership (Local owned vs. Foreign-owned):

- Business format (owned or leased?): 
- Business location (observed by the researcher):

- Annual room occupancy:

$>$ High season:

$>$ Low season:

- Kind of services provided (restaurant/shop):

- Is your hotel a member of any groups (examples: The Hotel Association of Cambodia, tour network)? 


\section{VULNERABILITY ASSESSMENT}

\section{SIHANOUKVILLE'S COASTAL TOURISM}

\section{Definitions:}

- Climate change: "Climate change in IPCC usage refers to a change in the state of the climate that can be identified (e.g. using statistical tests) by changes in the mean and/or the variability of its properties, and that persists for an extended period, typically decades or longer. It refers to any change in climate over time, whether due to natural variability or as a result of human activity." (IPCC, 2007 p.30).

- Vulnerability: Parry, (2007) in IPCC defines that "vulnerability is the degree to which a system is susceptible to, and unable to cope with, adverse effects of $C C$, including climate variability and extremes. Vulnerability is a function of the character, magnitude, and rate of CC and variation to which a system is exposed, the sensitivity and adaptive capacity of that system." (p.6)

1. Do you believe that Sihanoukville's climate is changing?

$>$ Why is it changing?

\section{SIHANOUKVILLE'S COASTAL TOURISM: ENVIRONMENTAL VULNERABILITY}

2. What environmental vulnerabilities do you see in Sihanoukville? (Or what vulnerabilities of environmental factors caused/brought by climate change in Sihanoukville?)

3. These are some examples of environmental vulnerabilities to climate change impacts. Is Sihanoukville's coastal tourism sector vulnerable to these impacts?

$>$ Can you think of any other examples?

- coastal flooding (as result of sea level rise),

- possible prolong drought (as result of decline in rainfall),

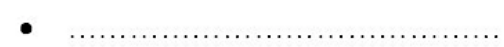

$\bullet$ 
SIHANOUKVILLE'S COASTAL TOURISM: SOCIO-ECONOMIC VULNERABILITY

4. What socio-economic vulnerabilities do you see in Sihanoukville? (Or what vulnerabilities of social and/or economic factors caused/brought by climate change in Sihanoukville?)

5. These are some examples of socio-economic vulnerabilities to climate change impacts. Is Sihanoukville's coastal tourism sector vulnerable to these impacts?

$>$ Can you think of any other examples?

- widespread diseases (as result of increased temperature),

- possible damages of infrastructures (as result of increased storm),

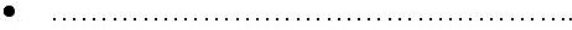

$\bullet$

$\bullet$

6. Do these vulnerabilities (both environmental and socio-economic) influence Sihanoukville's coastal tourism growth? In what ways?

7. In Sihanoukville, how soon do you think climate change impacts will become a pressing issue? (tick all that apply)

\begin{tabular}{|l|l|}
\hline Present & Future \\
\hline$\square$ now (they are already pressing) & $\square$ within the next year \\
\cline { 2 - 2 } & $\square$ within the next 3 years \\
\cline { 2 - 2 } & $\square$ within the next 5 years \\
\cline { 2 - 2 } & $\square$ within the next 10 years \\
\cline { 2 - 2 } & $\square$ more than 20 years from now \\
\hline
\end{tabular}

8. Do you think Sihanoukville's coastal tourism has enough capacity (such as public infrastructures) to respond to the above-mentioned climate vulnerabilities? Why and why not? 


\section{To what extent is Sihanoukville's coastal tourism vulnerable to climate change?}

Why?

$>$ Can you give the extent on this scale?

\begin{tabular}{|c|c|c|c|c|}
\hline Very vulnerable & Vulnerable & Moderately vulnerable & Slightly vulnerable & Not vulnerable \\
\hline 5 & 4 & 3 & 2 & 1 \\
$\square$ & $\square$ & $\square$ & $\square$ & $\square$ \\
\hline
\end{tabular}

\section{SIHANOUKVILLE'S HOTEL}

SIHANOUKVILLE'S HOTEL SECTOR: ENVIRONMENTAL VULNERABILITY

10. What environmental vulnerabilities do you see effecting on your hotel? (Or what vulnerabilities of environmental factors caused/brought by climate change on your hotel?)

11. These are some examples of environmental vulnerabilities to climate change impacts. Is the hotel sector vulnerable to these impacts?

$>$ Can you think of any other examples?

- vulnerable to increased storm surges,

- possible prolong drought (as result of decline in rainfall),

- $\quad$ (.....................................

$\bullet$

$\bullet$

\section{SIHANOUKVILLE'S HOTEL SECTOR: SOCIO-ECONOMIC VULNERABILITY}

12. What socio-economic vulnerabilities do you see effecting on your hotel? (Or what vulnerabilities of social and economic factors caused/brought by climate change on your hotel?)

13. These are some examples of socio-economic vulnerabilities to climate change impacts. Is the hotel sector vulnerable to these impacts?

Can you think of any other examples?

- weakening staff's health (e.g. cold or diarrhea as a result of climate change, too hot or too cold),

- effecting on customers' conveniences,

-

$\bullet$

$\bullet$ 
14. Has your hotel experienced or suffered any loss or damages as a result of any of these climate change impacts?

For example, the hotel-owned facilities such as structures and tourist boats are destroyed or lost during a terrible storm?

15. How worried are you about current climate change on your hotel?

\begin{tabular}{|c|c|c|c|c|}
\hline Very worried & Worried & Moderately worried & Slightly worried & Not worried \\
\hline 5 & 4 & 3 & 2 & 1 \\
$\square$ & $\square$ & $\square$ & $\square$ & $\square$ \\
\hline
\end{tabular}

How about the next 3 or 5 years, are you worried?

\begin{tabular}{|c|c|c|c|c|}
\hline Very worried & Worried & Moderately worried & Slightly worried & Not worried \\
\hline 5 & 4 & 3 & 2 & 1 \\
$\square$ & $\square$ & $\square$ & $\square$ & $\square$ \\
\hline
\end{tabular}

\section{Information}

16. Frequently, the government issues announcements of climate vulnerabilities along the coastal zone. To your knowledge has there been any information provided to your hotel?

17. Where do you receive the information about climate vulnerabilities (Please tick all that apply)?

$\square$ Media (TV, local news...)

$\square$ Local authority

$\square$ The hotel management

$\square$ The Hotel Association of Cambodia

$\square$ Other

$>$ How would you like to receive information on climate change in the future?

18. To what extent is your hotel vulnerable to climate change? (Or how serious a problem do you think climate change is for your hotel?) Why?

$>$ Can you give the extent on this scale?

\begin{tabular}{|c|c|c|c|c|}
\hline Very vulnerable & Vulnerable & Moderately vulnerable & Slightly vulnerable & Not vulnerable \\
\hline 5 & 4 & 3 & 2 & 1 \\
$\square$ & $\square$ & $\square$ & $\square$ & $\square$ \\
\hline
\end{tabular}




\section{CLIMATE CHANGE ADAPTATION}

Definition:

- Climate change adaptation: "adjustment in natural or human systems in response to actual or expected climatic stimuli or their effects, which moderates harms or exploits beneficial opportunities" (Parry, 2007, p.6).

19. From your understanding of the above definition, what is the goal of climate change adaptation (CCA)? Or can you tell what are the benefits of CCA? Or how effective is it?

20. Have you heard of any other coastal adaptations used by other accommodation providers or in other areas in Sihanoukville? What are they?

$>$ What are the initiatives of climate change adaptation (CCA) in Sihanoukville?

21. Do you think there is a need for coastal hotel providers in Sihanoukville to prepare for climate change? Why or why not?

22. Has your hotel adapted to climate change? (Or what actions have you taken or intend to take to tackle the issues of climate change?)

$>$ If yes, in what ways?

- Why did you initiate this adaptation, in response to what?

- Can you tell what are the benefits to your hotel? Or what are new opportunities are being exploited for your hotel?

- Do you plan to initiate other adaptations in the (near) future? In response to what?

$>$ If not, why not? Can you think of any possible adaptations your management team (owner) might be able to do to prepare for/adapt to these impacts that affect your hotel? 


\begin{tabular}{|c|c|c|c|c|}
\hline \multirow{2}{*}{ Type of adaptation } & \multirow{2}{*}{$\begin{array}{l}\text { Examples of climate change } \\
\text { impacts }\end{array}$} & \multirow{2}{*}{ Adaptation in hotel sector } & \multicolumn{2}{|l|}{ Time } \\
\hline & & & Past & Future \\
\hline \multirow{3}{*}{$\begin{array}{l}\text { Technical } \\
\text { This refers to the employment of } \\
\text { innovative technology used to identify } \\
\text { approaches to deal with climate change } \\
\text { and vulnerability }\end{array}$} & $\begin{array}{l}\text { Predicted storm surges, } \\
\text { excessive rainfall and sea } \\
\text { level rise... }\end{array}$ & $\begin{array}{l}\text { - Enable access to early warning equipment } \\
\text { (e.g. radios) }\end{array}$ & & \\
\hline & & 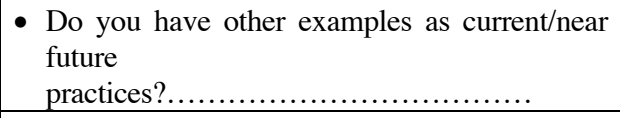 & & \\
\hline & & 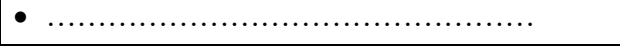 & & \\
\hline \multirow{3}{*}{$\begin{array}{l}\text { Managerial } \\
\text { Tourism businesses, entrepreneurs, and } \\
\text { investors can improve their management } \\
\text { of climate-change by approaching the } \\
\text { problem in a number of ways. These } \\
\text { include vulnerabilities to direct impacts } \\
\text { from climate change }\end{array}$} & $\begin{array}{l}\text { All impacts } \\
\text { extreme weather }\end{array}$ & $\begin{array}{l}\circ \text { Decision to redirect clients away from } \\
\text { impacted areas (to alternative destination) }\end{array}$ & & \\
\hline & & 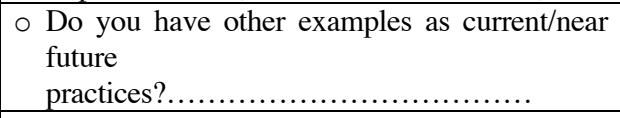 & & \\
\hline & & 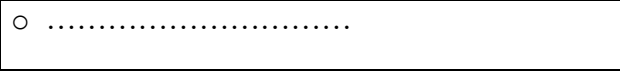 & & \\
\hline \multirow{3}{*}{$\begin{array}{l}\text { Policy } \\
\text { Concerning policy needs, interventions } \\
\text { designed to reduce vulnerability to } \\
\text { climate variability and uncertain } \\
\text { economic conditions in the short-run } \\
\text { and to reduce vulnerability to climate } \\
\text { change and in the longer term. }\end{array}$} & All impacts/storm surges & $\begin{array}{l}\text { - Comply with regulation (e.g. building design } \\
\text { standards for hurricane force winds) }\end{array}$ & & \\
\hline & & 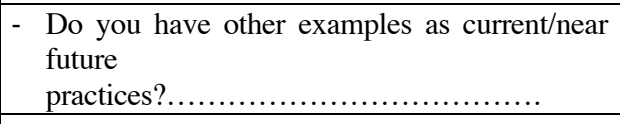 & & \\
\hline & & 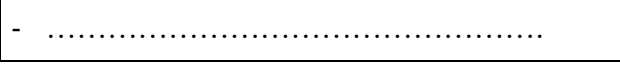 & & \\
\hline $\begin{array}{l}\text { Research } \\
\text { Research and technological innovations }\end{array}$ & $\begin{array}{l}\text { Coastal flooding, coastal } \\
\text { erosion, and storm surges... }\end{array}$ & $\begin{array}{l}\text { - Assess awareness of businesses and staff and } \\
\text { knowledge gaps }\end{array}$ & & \\
\hline
\end{tabular}




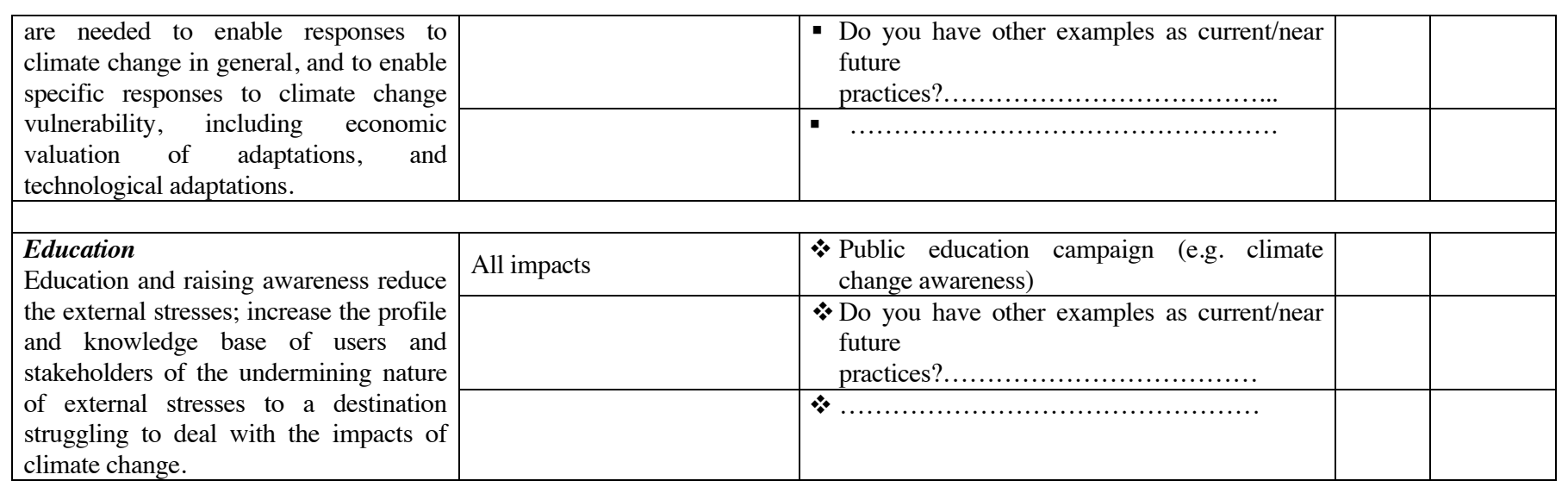


23. In short, do you think your hotel has enough capability (resources) to respond/adapt to current climate or near future vulnerabilities? Why and why not?

\begin{tabular}{|c|c|c|c|}
\hline \multicolumn{2}{|c|}{ Present } & \multicolumn{2}{c|}{ Future } \\
\hline $\begin{array}{c}\square \\
\text { Yes, why? }\end{array}$ & No, why not? & Yes, why? How long in the future? & No, why not? \\
\hline
\end{tabular}

\section{Information}

24. Information of climate vulnerabilities is important for CCA. To adapt to climate change, what information does your hotel need from the Hotel Association of Cambodia (HAC), local authority, NGOs or/and other external parties?

25. In terms of CCA, has your hotel ever received trainings or best practices from other stakeholders such as NGO, local authorities or/and other external parties? 


\section{BARRIERS TO CLIMATE CHANGE ADAPTATION}

Definition:

- Barriers: Huang et al. (2011) refers to a barrier as "... any condition that makes it difficult to achieve progress towards adaptation” (p.185).

26. What barriers to CCA do you see in Sihanoukville's coastal tourism? For example, what has been identified as the difficulty/challenge to adapt to climate change in Sihanoukville's coastal tourism?

27. Based on a thorough review of the existing literature, we provide the following barriers to CCA. These are examples of barriers to CCA applied to hotel sector.

\section{$>$ Can you rate these barriers?}

$>$ Can you think of any other barriers?

\begin{tabular}{|c|c|c|c|c|c|c|c|}
\hline No. & \multicolumn{7}{|c|}{ Barriers to CCA } \\
\hline 1 & \multicolumn{7}{|c|}{$\begin{array}{l}\text { limited resources (e.g. economic limits or budget limits/constraint, lack of technical skills/ } \\
\text { technological limits or human resources) } \\
\text { Or does a lack of resource such as budget technical skills/ technological limits or/and human } \\
\text { resources prevent you from adaptation to climate change? }\end{array}$} \\
\hline \multicolumn{2}{|c|}{$\begin{array}{c}\square \\
5 \\
\text { (very major) }\end{array}$} & $\begin{array}{c}\square \\
4 \\
\text { (major) }\end{array}$ & $\begin{array}{c}\square \\
3 \\
\text { (moderate) }\end{array}$ & $\begin{array}{c}\square \\
2 \\
\text { (small) }\end{array}$ & $\begin{array}{c}\square \\
1 \\
\text { (very small) }\end{array}$ & $\begin{array}{c}\square \\
0 \\
\text { Uninfluential } \\
\text { (Not a barrier) }\end{array}$ & Why? \\
\hline 2 & \multicolumn{7}{|c|}{$\begin{array}{l}\text { limited knowledge/perception of climate change (e.g. limited basic knowledge about causes, } \\
\text { effects and solutions to climate change) }\end{array}$} \\
\hline \multicolumn{2}{|c|}{$\begin{array}{c}\square \\
5 \\
\text { (very major) }\end{array}$} & $\begin{array}{c}\square \\
4 \\
\text { (major) }\end{array}$ & $\begin{array}{c}\square \\
3 \\
\text { (moderate) }\end{array}$ & $\begin{array}{c}\square \\
2 \\
\text { (small) }\end{array}$ & $\begin{array}{c}\square \\
1 \\
\text { (very small) }\end{array}$ & $\begin{array}{c}\square \\
0 \\
\text { Uninfluential } \\
\text { (Not a barrier) }\end{array}$ & Why? \\
\hline 3 & \multicolumn{7}{|c|}{$\begin{array}{l}\text { insufficient engagement and interaction (between three layers: national, provincial and local or } \\
\text { among other stakeholders) }\end{array}$} \\
\hline
\end{tabular}




\begin{tabular}{|c|c|c|c|c|c|c|c|}
\hline \multicolumn{2}{|c|}{$\begin{array}{c}\square \\
5 \\
\text { (very major) }\end{array}$} & $\begin{array}{c}\square \\
4 \\
\text { (major) }\end{array}$ & $\begin{array}{c}\square \\
3 \\
\text { (moderate) }\end{array}$ & $\begin{array}{c}\square \\
2 \\
\text { (small) }\end{array}$ & $\begin{array}{c}\square \\
1 \\
\text { (very small) }\end{array}$ & $\begin{array}{c}\square \\
0 \\
\text { Uninfluential } \\
\text { (Not a barrier) } \\
\end{array}$ & \multirow[t]{2}{*}{ Why? } \\
\hline 4 & \multicolumn{6}{|c|}{ uncertain responsibilities for adaptation } & \\
\hline \multicolumn{2}{|c|}{$\begin{array}{c}\square \\
5 \\
\text { (very major) }\end{array}$} & $\begin{array}{c}\square \\
4 \\
\text { (major) }\end{array}$ & $\begin{array}{c}\square \\
3 \\
\text { (moderate) }\end{array}$ & $\begin{array}{c}\square \\
2 \\
\text { (small) }\end{array}$ & $\begin{array}{c}\square \\
1 \\
\text { (very small) }\end{array}$ & $\begin{array}{c}\square \\
0 \\
\text { Uninfluential } \\
\text { (Not a barrier) }\end{array}$ & Why? \\
\hline 5 & \multicolumn{7}{|c|}{ insufficient information (about climate vulnerabilities, or absence of reliable climate data) } \\
\hline \multicolumn{2}{|c|}{$\begin{array}{c}\square \\
5 \\
\text { (very major) }\end{array}$} & $\begin{array}{c}\square \\
4 \\
\text { (major) }\end{array}$ & $\begin{array}{c}\square \\
3 \\
\text { (moderate) }\end{array}$ & $\begin{array}{c}\square \\
2 \\
\text { (small) }\end{array}$ & $\begin{array}{c}\square \\
1 \\
\text { (very small) }\end{array}$ & $\begin{array}{c}\square \\
0 \\
\text { Uninfluential } \\
\text { (Not a barrier) }\end{array}$ & Why? \\
\hline 6 & \multicolumn{7}{|c|}{$\begin{array}{l}\text { institutional barriers (e.g. lack of institutional flexibility, unclear or contradictory goal within } \\
\text { institution, restriction of individual capacity to adapt etc.), }\end{array}$} \\
\hline \multicolumn{2}{|c|}{$\begin{array}{c}\square \\
5 \\
\text { (very major) }\end{array}$} & $\begin{array}{c}\square \\
4 \\
\text { (major) }\end{array}$ & $\begin{array}{c}\square \\
3 \\
\text { (moderate) }\end{array}$ & $\begin{array}{c}\square \\
2 \\
\text { (small) }\end{array}$ & $\begin{array}{c}\square \\
1 \\
\text { (very small) }\end{array}$ & $\begin{array}{c}\square \\
0 \\
\text { Uninfluential } \\
\text { (Not a barrier) } \\
\end{array}$ & Why? \\
\hline 7 & \multicolumn{6}{|c|}{$\begin{array}{l}\text { regulatory/legal constraints (e.g. policies at national level that limit the ability of local } \\
\text { level to response to climate change), }\end{array}$} & \multirow[b]{2}{*}{ Why? } \\
\hline & $\begin{array}{l}\square \\
5 \\
\text { ery major) }\end{array}$ & $\begin{array}{c}\square \\
4 \\
\text { (major) }\end{array}$ & $\begin{array}{c}\square \\
3 \\
\text { (moderate) }\end{array}$ & $\begin{array}{c}\square \\
2 \\
\text { (small) }\end{array}$ & $\begin{array}{c}\square \\
1 \\
\text { (very small) }\end{array}$ & $\begin{array}{c}\square \\
0 \\
\text { Uninfluential } \\
\text { (Not a barrier) }\end{array}$ & \\
\hline 8 & \multicolumn{7}{|c|}{ low level of leadership and guidance, } \\
\hline & $\begin{array}{l}\square \\
5 \\
\text { ery major) }\end{array}$ & $\begin{array}{c}\square \\
4 \\
\text { (major) }\end{array}$ & $\begin{array}{c}\square \\
3 \\
\text { (moderate) }\end{array}$ & $\begin{array}{c}\square \\
2 \\
\text { (small) }\end{array}$ & $\begin{array}{c}\square \\
1 \\
\text { (very small) }\end{array}$ & $\begin{array}{c}\square \\
0 \\
\text { Uninfluential } \\
\text { (Not a barrier) }\end{array}$ & Why? \\
\hline 9 & & & & & & & \\
\hline
\end{tabular}




\begin{tabular}{|c|c|c|c|c|c|c|}
\hline $\begin{array}{c}\square \\
5 \\
\text { (very major) }\end{array}$ & $\begin{array}{c}\square \\
4 \\
\text { (major) }\end{array}$ & $\begin{array}{c}\square \\
3 \\
\text { (moderate) }\end{array}$ & $\begin{array}{c}\square \\
2 \\
\text { (small) }\end{array}$ & $\begin{array}{c}\square \\
1 \\
\text { (very small) }\end{array}$ & $\begin{array}{c}\square \\
0 \\
\text { Uninfluential } \\
\text { (Not a barrier) }\end{array}$ & Why? \\
\hline \multicolumn{7}{|c|}{ 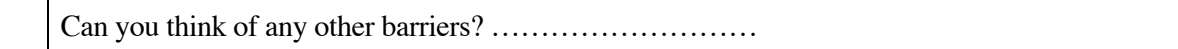 } \\
\hline $\begin{array}{c}\square \\
5 \\
\text { (very major) }\end{array}$ & $\begin{array}{c}\square \\
4 \\
\text { (major) }\end{array}$ & $\begin{array}{c}\square \\
3 \\
\text { (moderate) }\end{array}$ & $\begin{array}{c}\square \\
2 \\
\text { (small) }\end{array}$ & $\begin{array}{c}\square \\
1 \\
\text { (very small) }\end{array}$ & $\begin{array}{c}\square \\
0 \\
\text { Uninfluential } \\
\text { (Not a barrier) }\end{array}$ & Why? \\
\hline \multicolumn{7}{|c|}{ Can you think of any other barriers? ........................ } \\
\hline $\begin{array}{c}\square \\
5 \\
\text { (very } \\
\text { major) }\end{array}$ & $\begin{array}{c}\square \\
4 \\
\text { (major) }\end{array}$ & $\begin{array}{c}\square \\
3 \\
\text { (moderate) }\end{array}$ & $\begin{array}{c}\square \\
2 \\
\text { (small) }\end{array}$ & $\begin{array}{c}\square \\
1 \\
\text { (very small) }\end{array}$ & $\begin{array}{c}\square \\
0 \\
\text { Uninfluential } \\
\text { (Not a barrier) }\end{array}$ & Why? \\
\hline
\end{tabular}

28. Just looking at your hotel, which are the barriers that have occurred within the adaptation processes? (Or why has your hotel not adapted to climate change at all?)

$>$ Why and how have these barriers existed? 


\section{ENABLING FACTORS FOR CLIMATE CHANGE ADAPTATION}

\section{Definition:}

- "Enabling factors are defined as factors that make it possible for individuals to change their behavior or their environment. Enabling factors include resources, conditions of living, societal supports, and skills that facilitate a behavior's occurrence." (Pipithirankarn, undated, p.4).

29. As the key initiatives for CCA in Sihanoukville's tourism, what (process, condition or strategy necessary) you have identified as enabling factors? (Or what has worked really well for CCA in Sihanoukville's tourism?)

30. Based on a thorough review of the existing literature, we provide the following enabling factors for CCA. These are examples of enabling factors for CCA applied to hotel sector.

\section{Can you rate these enabling factors?}

$>$ Can you think of any other enabling factors?

\section{No. Enabling factors for CCA within hotel sector}

sufficient resources (e.g. finance/budget, human resource, and available technology),

1

Or does a sufficiency of resource such as budget technical skills/ technological limits or/and human resource is an enabling factor for adaptation to climate change?

\begin{tabular}{|c|c|c|c|c|c|c|}
\hline $\begin{array}{c}\square \\
5 \\
\text { (Very } \\
\text { important) }\end{array}$ & $\begin{array}{c}\square \\
4 \\
\text { (important) }\end{array}$ & $\begin{array}{c}\square \\
3 \\
\text { (moderately } \\
\text { important) }\end{array}$ & $\begin{array}{c}\square \\
2 \\
\text { (unimportant) }\end{array}$ & $\begin{array}{c}\square \\
1 \\
\text { (very } \\
\text { unimportant) }\end{array}$ & $\begin{array}{c}\square \\
0 \\
\text { Uninfluential } \\
\text { (Not an } \\
\text { enabling } \\
\text { factor) } \\
\end{array}$ & Why? \\
\hline \multicolumn{7}{|c|}{$\begin{array}{l}\text { previous and current adaptation experiences/awareness/knowledge and climate change } \\
\text { education, }\end{array}$} \\
\hline $\begin{array}{c}\square \\
5 \\
\text { (Very } \\
\text { important) }\end{array}$ & $\begin{array}{c}\square \\
4 \\
\text { (important) }\end{array}$ & $\begin{array}{c}\square \\
3 \\
\text { (moderately } \\
\text { important) }\end{array}$ & $\begin{array}{c}\square \\
2 \\
\text { (unimportant) }\end{array}$ & $\begin{array}{c}\square \\
1 \\
\text { (very } \\
\text { unimportant) }\end{array}$ & $\begin{array}{c}\square \\
0 \\
\text { Uninfluential } \\
\text { (Not an } \\
\text { enabling } \\
\text { factor) } \\
\end{array}$ & Why? \\
\hline
\end{tabular}


3 engagement/collaboration/communication with other stakeholders,

\begin{tabular}{|c|c|c|c|c|c|}
\hline $\begin{array}{c}\square \\
5 \\
\text { (Very } \\
\text { important) }\end{array}$ & $\begin{array}{c}\square \\
4 \\
\text { (important) }\end{array}$ & $\begin{array}{c}\square \\
3 \\
\text { (moderately } \\
\text { important) }\end{array}$ & $\begin{array}{c}\square \\
2 \\
\text { (unimportant) }\end{array}$ & $\begin{array}{c}\square \\
1 \\
\text { (very } \\
\text { unimportant) }\end{array}$ & $\begin{array}{c} \\
0 \\
\text { Uninfluential } \\
\text { (Not an } \\
\text { enabling } \\
\text { factor) } \\
\end{array}$ \\
\hline
\end{tabular}

$4 \quad$ individuals' motivation to respond (committed workforce),

\begin{tabular}{|c|c|c|c|c|c|}
\hline$\square$ & $\square$ & $\square$ & $\square$ & $\square$ & $\square$ \\
5 & 4 & 3 & 2 & 1 & 0 \\
$\begin{array}{c}\text { (Very } \\
\text { important) }\end{array}$ & (important) & $\begin{array}{c}\text { (moderately } \\
\text { important) }\end{array}$ & (unimportant) & $\begin{array}{c}\text { (very } \\
\text { unimportant) }\end{array}$ & $\begin{array}{c}\text { Uninfluential } \\
\text { (Not an } \\
\text { enabling } \\
\text { factor) }\end{array}$ \\
\hline
\end{tabular}

5 sufficient information (e.g. about vulnerabilities...)

\begin{tabular}{|c|c|c|c|c|c|}
\hline$\square$ & $\square$ & $\square$ & $\square$ & $\square$ & $\square$ \\
5 & 4 & 3 & 2 & 1 & 0 \\
(Very & (important) & $\begin{array}{c}\text { (moderately } \\
\text { important) }\end{array}$ & (unimportant) & $\begin{array}{c}\text { (very } \\
\text { unimportant) }\end{array}$ & $\begin{array}{c}\text { Uninfluential } \\
\text { (Not an } \\
\text { enabling } \\
\text { factor) }\end{array}$ \\
\hline
\end{tabular}

\begin{tabular}{l|l}
6 & clear adaptation goals, \\
\hline
\end{tabular}

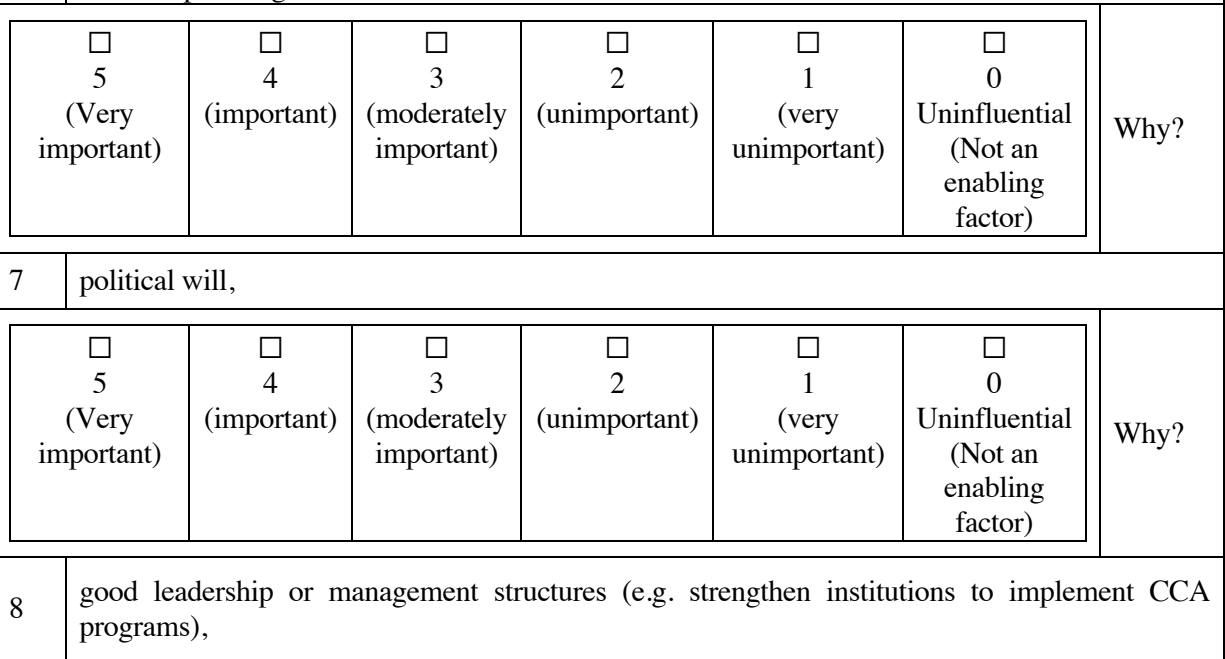




\begin{tabular}{|c|c|c|c|c|c|}
\hline$\square$ & $\square$ & $\square$ & $\square$ & $\square$ & $\square$ \\
5 & 4 & 3 & 2 & 1 & 0 \\
(Very & (important) & $\begin{array}{c}\text { (moderately } \\
\text { important) }\end{array}$ & (unimportant) & $\begin{array}{c}\text { (very } \\
\text { unimportant) }\end{array}$ & $\begin{array}{c}\text { Uninfluential } \\
\text { (Not an } \\
\text { enabling } \\
\text { factor) }\end{array}$ \\
\hline
\end{tabular}

$9 \quad$ convenient location of the hotel,

\begin{tabular}{|c|c|c|c|c|c|}
\hline$\square$ & $\square$ & $\square$ & $\square$ & $\square$ & $\square$ \\
5 & 4 & 3 & 2 & 1 & 0 \\
(Very & (important) & $\begin{array}{c}\text { (moderately } \\
\text { important) }\end{array}$ & & (unimportant) & (very \\
& & & unimportant) & $\begin{array}{c}\text { Uninfluential } \\
\text { (Not an } \\
\text { enabling } \\
\text { factor) }\end{array}$ \\
\hline
\end{tabular}

Can you think of any other enabling factors?

\begin{tabular}{|c|c|c|c|c|c|}
\hline$\square$ & $\square$ & $\square$ & $\square$ & $\square$ & $\square$ \\
5 & 4 & 3 & 2 & 1 & 0 \\
(Very & (important) & $\begin{array}{c}\text { (moderately } \\
\text { important) }\end{array}$ & (unimportant) & $\begin{array}{c}\text { (very } \\
\text { unimportant) }\end{array}$ & $\begin{array}{c}\text { Uninfluential } \\
\text { (Not an } \\
\text { enabling } \\
\text { factor) }\end{array}$ \\
\hline
\end{tabular}

Can you think of any other enabling factors? ....

\begin{tabular}{|c|c|c|c|c|c|}
\hline$\square$ & $\square$ & $\square$ & $\square$ & $\square$ & $\square$ \\
5 & 4 & 3 & 2 & 1 & 0 \\
(Very & (important) & $\begin{array}{c}\text { (moderately } \\
\text { important) }\end{array}$ & (unimportant) & $\begin{array}{c}\text { (very } \\
\text { unimportant) }\end{array}$ & $\begin{array}{c}\text { Uninfluential } \\
\text { (Not an } \\
\text { enabling } \\
\text { factor) }\end{array}$ \\
\hline
\end{tabular}

31. Looking at your hotel, what are the enabling factors in adapting to climate change? [Or what (process, condition or strategy necessary) do you think would make your hotel to prepare for/respond to/adapt to climate change impacts?] 
Any other comments?

Other researcher's notes: 


\section{Appendix B: Participant information sheet}

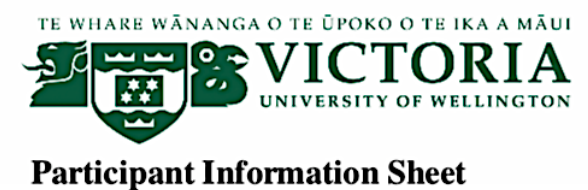

Research Project Title: Climate change adaptation in coastal tourism of developing country: The case of the hotel sector in Sihanoukville, Cambodia.

Researcher: Tinat Nhep, School of Management, Victoria University of Wellington, New Zealand.

I am a Cambodian PhD student at the School of Management, Victoria Business School at Victoria University of Wellington in New Zealand. I am undertaking a research project leading to a thesis. The project involves examining climate change adaptation in the accommodation (hotel) sector in Sihanoukville's coastal tourism. This research project has received approval from the Victoria University of Wellington Human Ethics Committee. If there are any ethics related queries, please contact the HEC Convener, AProf Susan Corbett by email susan.corbett@vuw.ac.nz or telephone +64 44635480.

I would like to invite you to participate in this study and would highly appreciate if you could join this study, as your cooperation will be valuable for my research. However, you can take part in my research quite voluntarily based on your interest. Hence, before making a decision, you should understand what is involved. Therefore, this form provides you with detailed information about the study, which will be discussed with you. This form is also to respect and acknowledge your rights. Once you understand it, you will need to sign the consent form if you wish to participate.

What are the purposes of this study?

As part of my research, I am interested in your views on how Sihanoukville's accommodation (hotel) sector is adapting to climate change. At the same time, your views on barriers and success factors in adapting to climate change will be sought.

What are the possible benefits?

Firstly, it will contribute to the development of a more effectual strategy for CCA towards coastal tourism especially the accommodation (hotel) sector itself so that either vulnerability is minimized or strong adaptation/resilience is understood and built at the destination. Secondly, from a practical point of view, the study will help accommodation sector prepare and manage their adaptation strategies better towards the increasing climate change impacts to benefit their businesses. Finally, the opportunities of CCA and coastal tourism are identified and advised to the accommodation (hotel) sector of coastal tourism destinations.

What will be your roles and responsibilities if you agree to take part in this study?

As a member of the Cambodian tourism industry, especially your involvements in the accommodation sector (hotel \& guesthouse), I hope you will be able to provide me with detailed information about the CCA in the accommodation sector. Your participation and your role in the organization as well as your organization's documents will provide me with the valuable information for the research.

If you agree, you will participate in an individual one-hour interview. With your permission, I will electronically record the interview and a full transcript of the interview will be available. Your participation is voluntary, and you will not be identified personally or professionally in any written report produced as a result of this research, including possible publication in academic conferences and journals.

The interview, which lasts approximately one hour, will be voice recorded for later analysis. If you do not agree to the recording, I will take notes. The interview will take place at your office or another convenient 
location. Apart from the interview, if you have documents either in Khmer or English that would provide insight to this study, I would appreciate being given access (e.g. report on vulnerability to climate change in the accommodation sector).

\section{What will happen to the information you provide?}

The information and documents that you provide will be used for my academic purposes only. The $\mathrm{PhD}$ thesis will be submitted for marking and deposited in the University's Library. It is intended that one or more articles will be submitted for publication in scholarly journals and/ or conference papers. A research summary can be provided to you after the thesis completion.

With your permission, I would like to identify the characteristics of your business in my research such as its ownership, size, type, location etc. (e.g. a locally owned hotel in Sihanoukville), when I include quotes from the interview or information from documents. Any information you provide will be kept confidential to the researcher and my supervisors. The published results will not use your name but a psydoneum will be used instead; other identifying characteristics may be published such as ages and sexes for yourself and quality, size, location, ownership, type ect. for your business. It is also informed that the final thesis will be deposited in the University Library and may be published in conferences and journal articles.

The raw data (interview tapes, transcripts, notes and documents) will be securely stored in a locked cabinet and password protected computer. They will be accessed only by myself and my supervisors. After completion of the thesis, data will be securely stored for three years before being destroyed.

\section{When can you withdraw from the research?}

You can withdraw from the study at any time, up until four weeks after the interview. If you choose to withdraw, any information you have provided will be destroyed or returned to you.

\section{How can you contact me if you have further questions?}

If you have any questions about the research project either now or in the future, please feel welcome to contact me at Tinat.Nhep@vuw.ac.nz or telephone +85512539 390,+64 22398 4388, or you may contact my supervisors, Dr. Christian Schott, at Christian.Schott@ vuw.ac.nz, Tel: +6404 4635719 and A/Prof Mondher Sahli at Mondher.Sahli@vuw.ac.nz, Tel: +64 044635718

Thank you very much for your cooperation.

\section{Researcher: Tinat Nhep \\ PhD Candidate in Tourism Management}

School of Management, Victoria University of Wellington

Room RH914, Rutherford House, 23 Lambton Quay, Pipitea Campus,

Wellington 6140, New Zealand

Phone in New Zealand: +64-4-463 5141

Email: tinat.nhep@vuw.ac.nz

\begin{tabular}{|c|c|c|}
\hline \multicolumn{3}{|l|}{ Supervisors: } \\
\hline Dr. Christian Schott & & Dr. Mondher Salhi \\
\hline Senior Lecturer of Tourism Manage & & Associate Professor in Tourism Management \\
\hline School of & Management & School of Management \\
\hline Victoria University of Wellington & & Victoria University of Wellington \\
\hline Room 924, Rutherford House & & Room 916, Rutherford House \\
\hline 23 Lambton Quay, Pipitea Campus & & 23 Lambton Quay, Pipitea Campus \\
\hline Wellington, New Zealand 6011 & & Wellington, New Zealand 6011 \\
\hline Phone: +64-4-463 5719 & & Phone: +64-4-463 5718 \\
\hline Email: christian.schott@vuw.ac.nz & & Email:mondher.salhi@vuw.ac.nz \\
\hline
\end{tabular}




\section{Appendix C: Participant consent form}

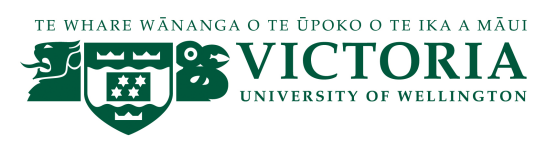

\section{Participant Consent Form}

Research Project Title: Climate change adaptation in coastal tourism in developing countyr: The case of the hotel sector in Sihanoukville, Cambodia

\section{Researcher: Tinat Nhep, School of Management, Victoria University of Wellington, New Zealand}

- I have been given and have understood an explanation of this research project. I have had an opportunity to ask questions and have them answered to my satisfaction.

- I understand that I may withdraw myself (or any information I have provided) from this project, by emailing Tinat.Nhep@vuw.ac.nz within two weeks after receiving a full transcript of the interview.

- I understand that any information I provide will be kept confidential to the researcher and their supervisors. The published results will not use my name but a psydoneum will be used instead; other identifying characteristics may be published such as ages and sexes for myself and quality, size, location, ownership, type ect. for my business. I also understand that the final thesis will be deposited in the University Library and may be published in conferences and journal articles.

- The information will be stored securely and accessible to only the researcher and his supervisors.

- I understand that the data I provide will not be used for any other purpose or released to others.

- I understand that the interview will last around one hour.

- I understand that, if this interview is audio recorded, the recording and transcripts of the interviews will be erased within three years after the conclusion of the project. Furthermore, I will have an opportunity to check the transcripts of the interview.

Please indicate (by ticking the boxes below) which of the following apply:

I agree to this interview being audio recorded.

I would like to receive a full transcript of the interview.

I would like to receive a summary of the results of this research when it is completed.

Signed:

Name of participant:

Date:

Email:

Researcher: $\quad$ Tinat Nhep, Email: Tinat.Nhep@vuw.ac.nz, Tel: +64-4-463 5141

Supervisors: Dr. Christian Schott, Email: Christian.Schott@vuw.ac.nz, Tel: +64-4-463 5719

A/Prof Mondher Sahli, Mondher.Sahli@vuw.ac.nz, Tel: +64-4-463 5718 


\section{Appendix D: Final template}

I. Climate vulnerability assessment in SHV's coastal tourism

1.1. Perceptions if SHV's climate is changing?

1.2. Reason of SHV's climate change

1.3. To what extent is Sihanoukville's coastal tourism vulnerable to climate change?

1.3.1.1. Environmental vulnerability of SHV's coastal tourism

1.3.1.2. Socio-economic vulnerability of SHV's coastal tourism

1.3.1.3. Ecological vulnerability of SHV's coastal tourism

II. Climate vulnerability assessment in SHV's coastal hotel sector

2.1 To what extent is Sihanoukville's coastal tourism vulnerable to climate change?

2.1.1 Environmental vulnerability of SHV's coastal hotel sector

2.1.2 Socio-economic vulnerability of SHV's coastal hotel sector

2.2 Hotel's suffering losses and damages due to climate change

2.3 Medium of receiving or accessing information about climate change in SHV's hotel sector

2.3.1 The extent to which SHV's hotel sector receives climate change information

2.3.2 Sources to access to climate change information in SHV's hotel sector

2.3.3 Recommended ways (to the government and other parties) of providing climate change information to hotel sector

2.3.4 Accuracy of climate change information perceived by SHV's hotel sector

III. Identifications of CCA actions in SHV's hotel sector

3.1. Benefits of CCA perceived by SHV's hotel sector

3.2. Hotel sector's awareness of other CCA initiatives in SHV

3.3. Is there a need for CCA actions in SHV's accommodation sector?

3.4. Has your hotel adapted to climate change?

3.4.1 Technical adaptation

3.4.1.1 Adapting to excessive rainfall 
3.4.1.2 Adapting to rising temperature

3.4.1.3 Adapting to storm

3.4.1.4 Adapting to SLR/beach erosion/storm surge

3.4.1.5 Adapting to prolong drought

3.4.2 Managerial adaptation

3.4.2.1 Adapting to excessive rainfall

3.4.2.2 Adapting to rising temperature

3.4.2.3 Adapting to storm

3.4.2.4 Adapting to SLR/beach erosion/storm surge

3.4.2.5 Adapting to prolong drought

3.4.3 Policy adaptation

3.4.3.1 Adapting to excessive rainfall

3.4.3.2 Adapting to rising temperature

3.4.3.3 Adapting to storm

3.4.3.4 Adapting to SLR/beach erosion/storm surge

3.4.3.5 Adapting to prolong drought

3.4.4 Research adaptation

3.4.4.1 Adapting to excessive rainfall

3.4.4.2 Adapting to rising temperature

3.4.4.3 Adapting to storm

3.4.4.4 Adapting to SLR/beach erosion/storm surge

3.4.4.5 Adapting to prolong drought

3.4.5 Education adaptation

3.4.5.1 Adapting to excessive rainfall

3.4.5.2 Adapting to rising temperature

3.4.5.3 Adapting to storm

3.4.5.4 Adapting to SLR/beach erosion/storm surge

3.4.5.5 Adapting to prolong drought

IV. Barriers and enabling factors to CCA
4.1. Barriers to CCA in SHV's coastal tourism
4.2. Barriers to CCA in SHV's hotel sector 
4.3. Rating (Likert scale) of barriers to CCA in hotel sector

4.4. Enabling factors for CCA in SHV's hotel sector

4.5. Enabling factors for CCA in SHV's hotel sector

4.6. Rating (Likert scale) of enabling factors for CCA in hotel sector 


\section{Appendix E: HEC Approval for interviews}

Dear Tinat Nhep,

Thank you for your application for ethical approval (Climate change adaptation in a coastal hotel sector: The case of Sihanoukville, Cambodia., reference 0000023104), which has now been considered by the Pipitea Sub-Committee of the Human Ethics Committee.

Your application is approved as of today (Saturday, 30 July 2016 at 7:03 PM).

You may wish to check whether there are any new comments on your application. To do this, click on the Comments button (it looks like a speech bubble). If any particular page has comments, they will be marked with a flag on the left hand side of the screen. To access them, navigate to the desired page, and then click on the Page Comments button (it looks like a speech bubble with a page behind it).

Best wishes with the research.

Pipitea Human Ethics Sub-Committee 


\section{Appendix F: Descriptive test/independent sample test}

Appendix F-1

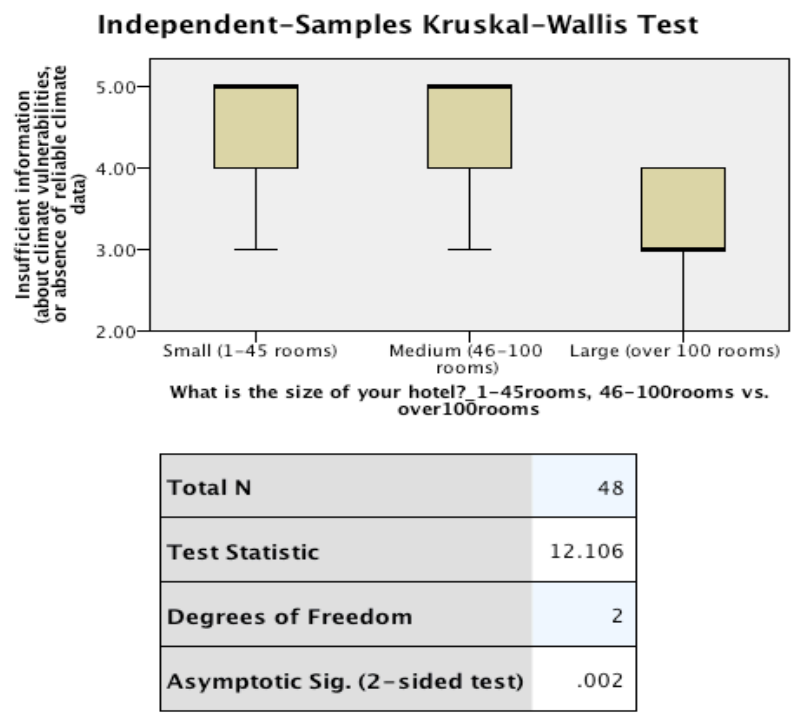

1. The test statistic is adjusted for ties.

Pairwise Comparisons of What is the size of your hotel?_1-45rooms, 46-100rooms vs. over100rooms

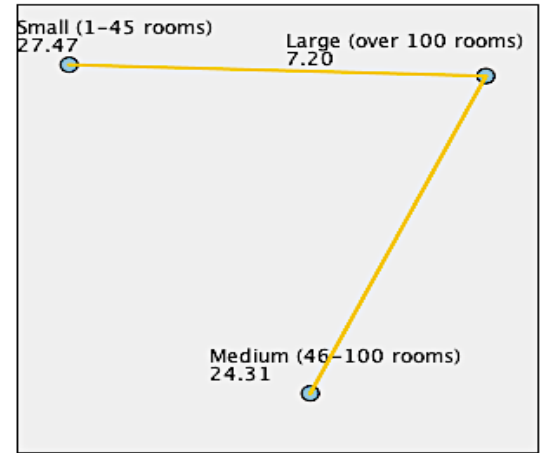

Each node shows the sample average rank of What is the size of your hotel?_1-45rooms, 46-100rooms vs. over100rooms.

\begin{tabular}{|l|rrrrr|}
\hline Sample 1-Sample2 & $\begin{array}{c}\text { Test } \\
\text { Statistic }\end{array}$ & $\begin{array}{c}\text { Std. } \\
\text { Error }\end{array}$ & $\begin{array}{c}\text { Std. Test } \\
\text { Statistic }\end{array}$ & Sig. & Adj.Sig. \\
\hline $\begin{array}{l}\text { Large (over 100 rooms)- } \\
\text { Medium (46-100 rooms) }\end{array}$ & 17.108 & 6.347 & 2.695 & .007 & .021 \\
\hline $\begin{array}{l}\text { Large (over 100 rooms)-Small } \\
\text { (1-45 rooms) }\end{array}$ & 20.267 & 5.826 & 3.479 & .001 & .002 \\
\hline $\begin{array}{l}\text { Medium (46-100 rooms)- } \\
\text { Small (1-45 rooms) }\end{array}$ & 3.159 & 4.005 & .789 & .430 & 1.000 \\
\hline
\end{tabular}

Each row tests the null hypothesis that the Sample 1 and Sample 2 distributions are the

Asymptotic significances (2-sided tests) are displayed. The significance level is .05 . 


\section{Appendix F-2}

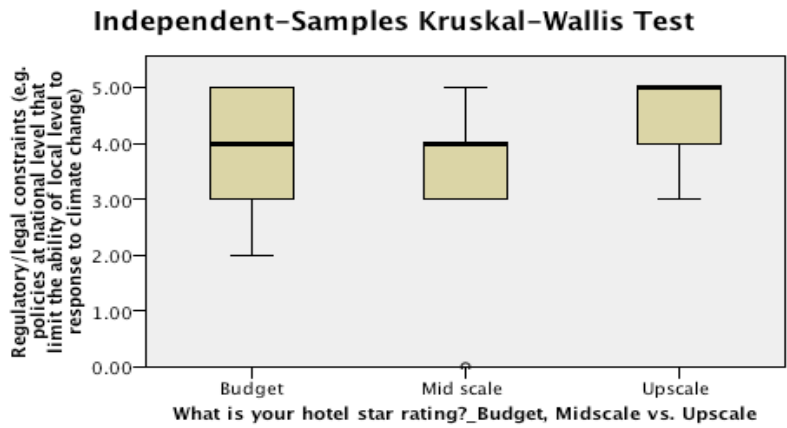

\begin{tabular}{|lr|}
\hline Total N & 48 \\
\hline Test Statistic & 8.003 \\
\hline Degrees of Freedom & 2 \\
\hline Asymptotic Sig. (2-sided test) & .018 \\
\hline
\end{tabular}

1. The test statistic is adjusted for ties.

Pairwise Comparisons of What is your hotel star rating?_Budget, Midscale vs. Upscale

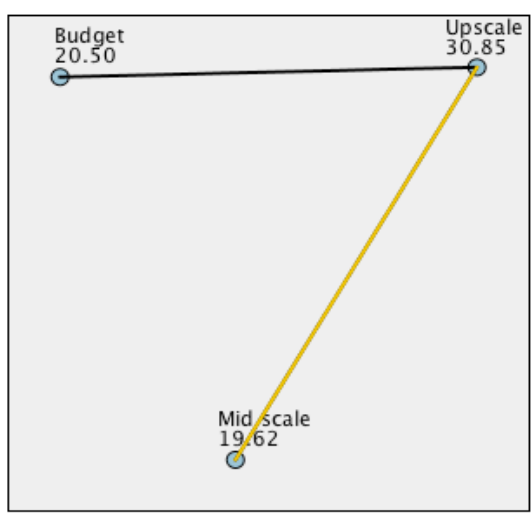

Each node shows the sample average rank of What is your hotel star rating?_Budget, Midscale vs. Upscale.

\begin{tabular}{|lrrrrr|}
\hline Sample1-Sample2 & $\begin{array}{c}\text { Test } \\
\text { Statistic }\end{array}$ & $\begin{array}{c}\text { Std. } \\
\text { Error }\end{array}$ & $\begin{array}{c}\text { Std. Test } \\
\text { Statistic }\end{array}$ & Sig. & Adj.Sig. \\
\hline Mid scale-Budget & .882 & 5.095 & .173 & .863 & 1.000 \\
\hline Mid scale-Upscale & -11.232 & 4.344 & -2.586 & .010 & .029 \\
\hline Budget-Upscale & -10.350 & 4.943 & -2.094 & .036 & .109 \\
\hline
\end{tabular}

Each row tests the null hypothesis that the Sample 1 and Sample 2

distributions are the same.

Asymptotic significances (2-sided tests) are displayed. The significance

level is .05. 


\section{Appendix F-3}

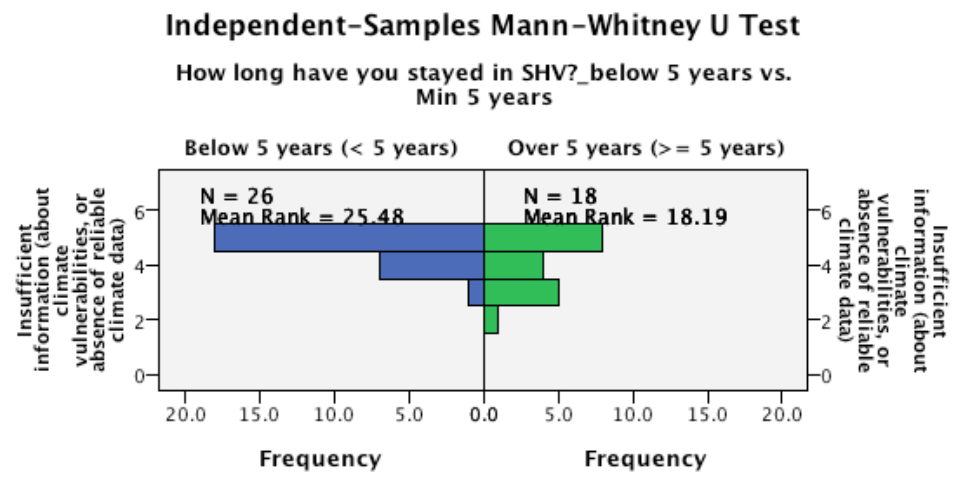

\begin{tabular}{|lr|}
\hline Total N & 44 \\
\hline Mann-Whitney U & 156.500 \\
\hline Wilcoxon W & 327.500 \\
\hline Test Statistic & 156.500 \\
\hline Standard Error & 36.901 \\
\hline Standardized Test Statistic & -2.100 \\
\hline Asymptotic Sig. (2-sided test) & .036 \\
\hline
\end{tabular}




\section{Appendix F-4}

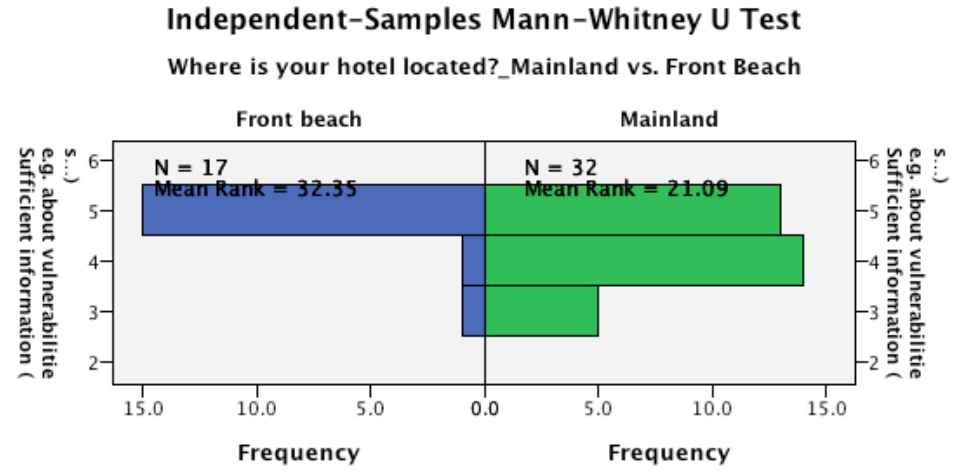

\begin{tabular}{|lr|}
\hline Total N & 49 \\
\hline Mann-Whitney U & 397.000 \\
\hline Wilcoxon W & 550.000 \\
\hline Test Statistic & 397.000 \\
\hline Standard Error & 42.134 \\
\hline Standardized Test Statistic & 2.967 \\
\hline Asymptotic Sig. (2-sided test) & .003 \\
\hline
\end{tabular}




\section{Appendix F-5}

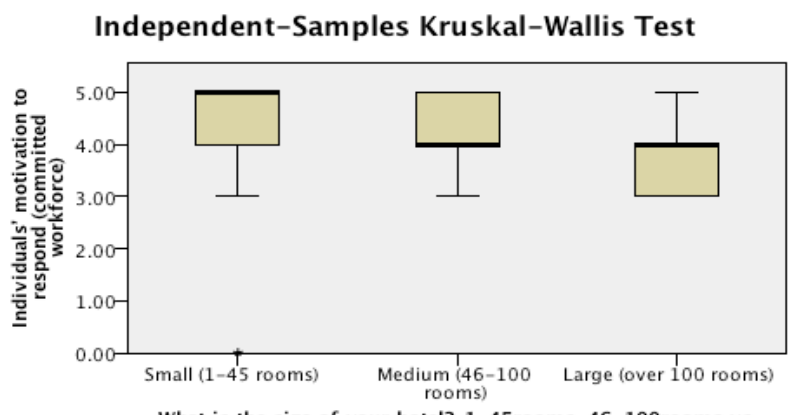

What is the size of your hotel? $1-45$ rooms, $46-100$ rooms vs. over100̄rooms

\begin{tabular}{|lr|}
\hline Total N \\
\hline Test Statistic \\
\hline Degrees of Freedom \\
\hline Asymptotic Sig. (2-sided test). \\
\hline
\end{tabular}

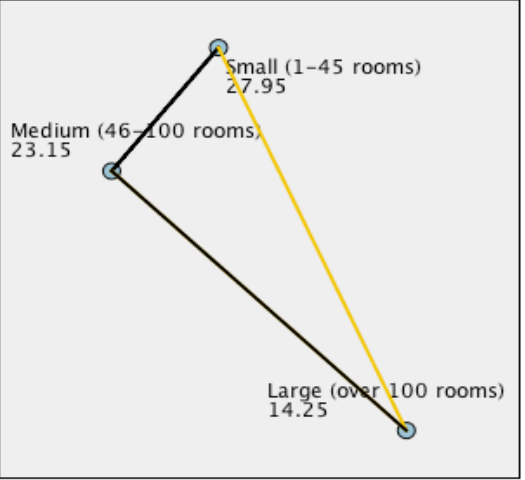

Each node shows the sample average rank of What is the size of your hotel?_1-45rooms, 46-100rooms vs. over100rooms.

\begin{tabular}{|lrrrrr|}
\hline Sample 1-Sample2 & $\begin{array}{c}\text { Test } \\
\text { Statistic }\end{array}$ & $\begin{array}{c}\text { Std. } \\
\text { Error }\end{array}$ & $\begin{array}{c}\text { Std. Test } \\
\text { Statistic }\end{array}$ & Sig. & Adj.Sig. \\
\hline $\begin{array}{l}\text { Large (over 100 rooms)- } \\
\text { Medium (46-100 rooms) }\end{array}$ & 8.904 & 6.244 & 1.426 & .154 & .462 \\
\hline $\begin{array}{l}\text { Large (over 100 rooms)-Small } \\
\text { (1-45 rooms) }\end{array}$ & 13.700 & 5.658 & 2.421 & .015 & .046 \\
\hline $\begin{array}{l}\text { Medium (46-100 rooms)- } \\
\text { Small (1-45 rooms) }\end{array}$ & 4.796 & 4.201 & 1.142 & .254 & .761 \\
\hline
\end{tabular}

Each row tests the null hypothesis that the Sample 1 and Sample 2 distributions are the

Asymptotic significances (2-sided tests) are displayed. The significance level is .05. 


\section{Appendix F-6}

Independent-Samples Mann-Whitney U Test

What is the ownership of your hotel?_Local vs. Foreign

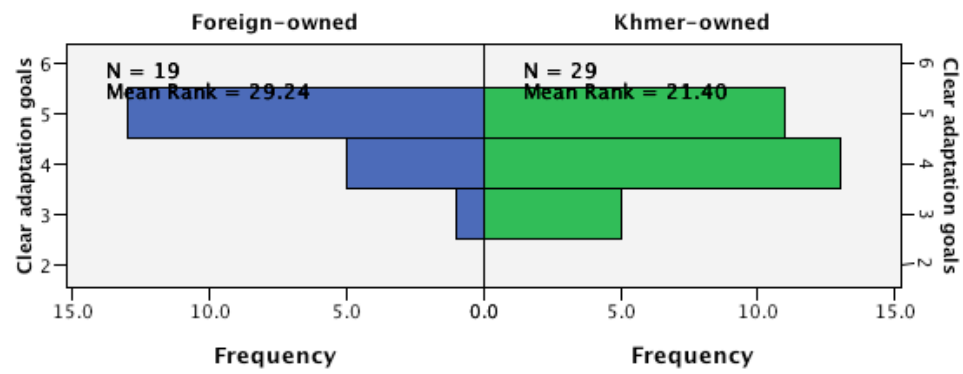

\begin{tabular}{|lr|}
\hline Total N & 48 \\
\hline Mann-Whitney U & 365.500 \\
\hline Wilcoxon W & 555.500 \\
\hline Test Statistic & 365.500 \\
\hline Standard Error & 42.970 \\
\hline Standardized Test Statistic & 2.094 \\
\hline Asymptotic Sig. (2-sided test) & .036 \\
\hline
\end{tabular}




\section{Appendix F-7}

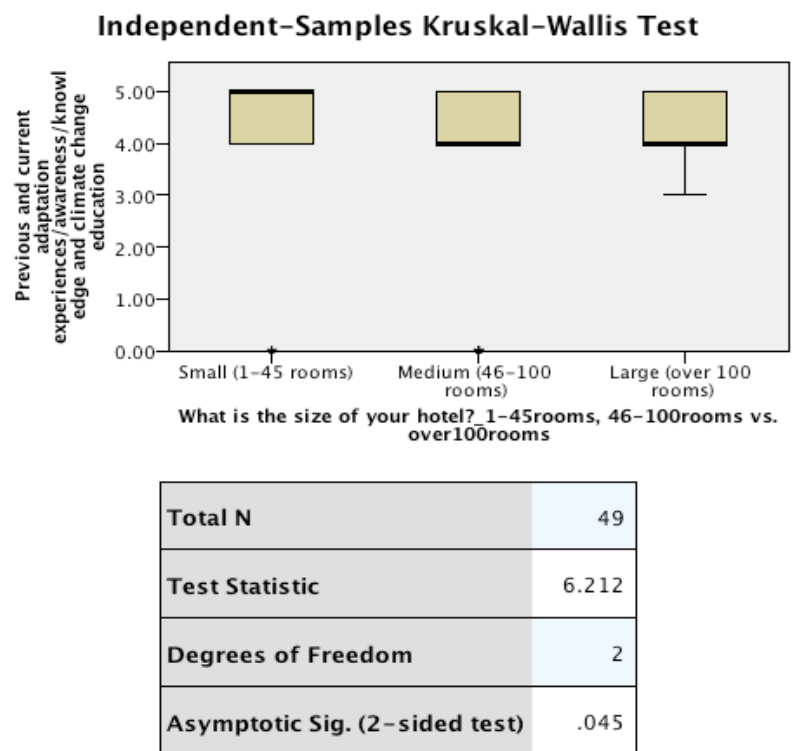

1. The test statistic is adjusted for ties.

Pairwise Comparisons of What is the size of your hotel?_1-45rooms, 46-100rooms vs. over100rooms

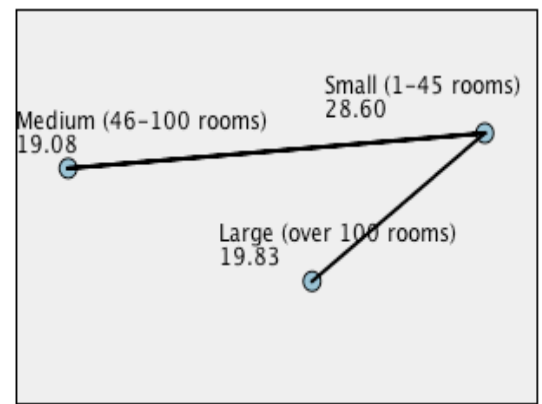

Each node shows the sample average rank of What is the size of your hotel?_1-45rooms, 46-100rooms vs. over100rooms.

\begin{tabular}{|lrrrrr|}
\hline Sample1-Sample2 & $\begin{array}{c}\text { Test } \\
\text { Statistic }\end{array}$ & $\begin{array}{c}\text { Std. } \\
\text { Error }\end{array}$ & $\begin{array}{c}\text { Std. Test } \\
\text { Statistic }\end{array}$ & Sig. & Adj.Sig. \\
\hline $\begin{array}{l}\text { Medium (46-100 rooms)- } \\
\text { Large (over } 100 \text { rooms) }\end{array}$ & -.756 & 6.278 & -.120 & .904 & 1.000 \\
\hline $\begin{array}{l}\text { Medium (46-100 rooms)- } \\
\text { Small (1-45 rooms) }\end{array}$ & 9.523 & 4.223 & 2.255 & .024 & .072 \\
\hline $\begin{array}{l}\text { Large (over } 100 \text { rooms)-Small } \\
\text { (1-45 rooms) }\end{array}$ & 8.767 & 5.688 & 1.541 & .123 & .370 \\
\hline
\end{tabular}

Each row tests the null hypothesis that the Sample 1 and Sample 2 distributions are the same.

Asymptotic significances (2-sided tests) are displayed. The significance level is .05 . 


\section{Appendix F-8}

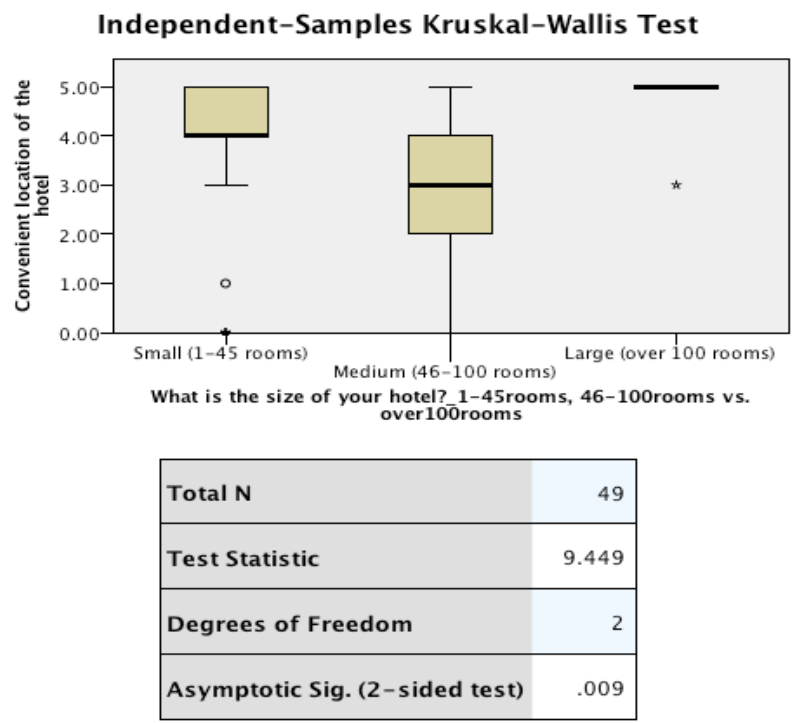

1. The test statistic is adjusted for ties.

Pairwise Comparisons of What is the size of your hotel?_1-45rooms, 46-100rooms vs. over100rooms

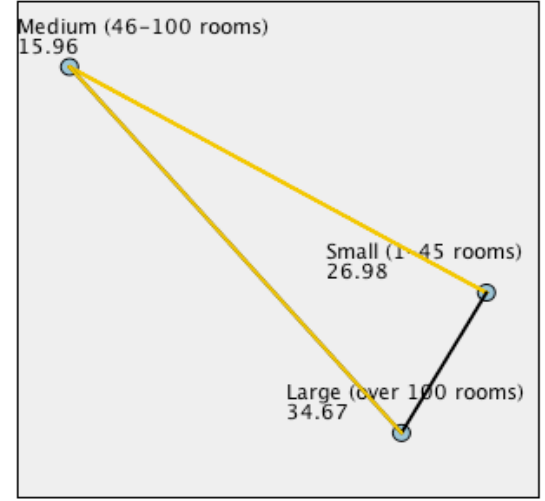

Each node shows the sample average rank of What is the size of your hotel?_1-45rooms, 46-100rooms vs. over100rooms.

\begin{tabular}{|l|ccccr|}
\hline Sample 1-Sample2 & $\begin{array}{c}\text { Test } \\
\text { Statistic }\end{array}$ & $\begin{array}{c}\text { Std. } \\
\text { Error }\end{array}$ & $\begin{array}{c}\text { Std. Test } \\
\text { Statistic }\end{array}$ & Sig. & Adj.Sig. \\
\hline $\begin{array}{l}\text { Medium (46-100 rooms)- } \\
\text { Small (1-45 rooms) }\end{array}$ & 11.022 & 4.507 & 2.446 & .014 & .043 \\
\hline $\begin{array}{l}\text { Medium (46-100 rooms)- } \\
\text { Large (over 100 rooms) }\end{array}$ & -18.705 & 6.699 & -2.792 & .005 & .016 \\
\hline $\begin{array}{l}\text { Small (1-45 rooms)-Large } \\
\text { (over 100 rooms) }\end{array}$ & -7.683 & 6.070 & -1.266 & .206 & .617 \\
\hline
\end{tabular}

Each row tests the null hypothesis that the Sample 1 and Sample 2 distributions are the

Asymptotic significances (2-sided tests) are displayed. The significance level is .05 . 


\section{Appendix F-9}

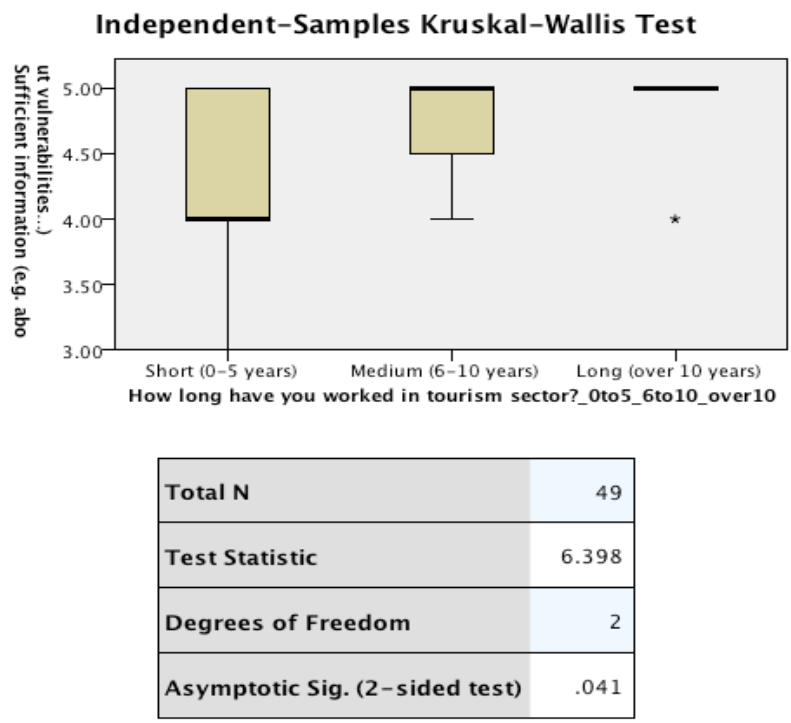

1. The test statistic is adjusted for ties.

Pairwise Comparisons of How long have you worked in tourism sector?_0to5_6to10_over10

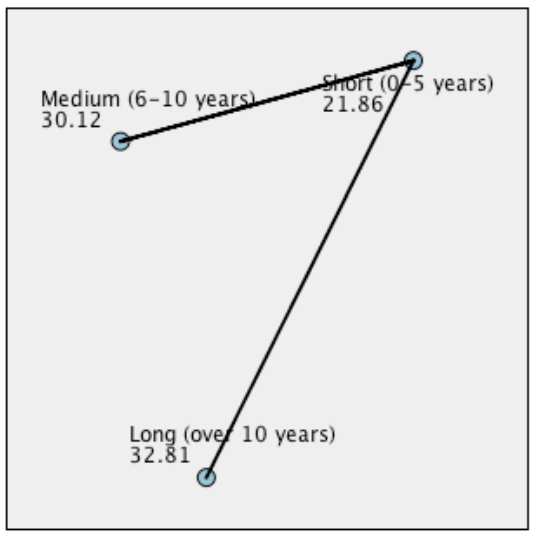

Each node shows the sample average rank of How long have you worked in tourism sector?_0to5_6to10_over10.

\begin{tabular}{|l|ccccc|}
\hline Sample1-Sample2 & $\begin{array}{c}\text { Test } \\
\text { Statistic }\end{array}$ & $\begin{array}{c}\text { Std. } \\
\text { Error }\end{array}$ & $\begin{array}{c}\text { Std. Test } \\
\text { Statistic }\end{array}$ & Sig. & Adj.Sig. \\
\hline $\begin{array}{l}\text { Short (0-5 years)-Medium (6- } \\
\text { 10 years) }\end{array}$ & -8.261 & 4.983 & -1.658 & .097 & .292 \\
\hline $\begin{array}{l}\text { Short (0-5 years)-Long (over } \\
\text { 10 years) }\end{array}$ & -10.949 & 4.983 & -2.197 & .028 & .084 \\
\hline $\begin{array}{l}\text { Medium (6-10 years)-Long } \\
\text { (over 10 years) }\end{array}$ & -2.688 & 6.323 & -.425 & .671 & 1.000 \\
\hline
\end{tabular}

Each row tests the null hypothesis that the Sample 1 and Sample 2 distributions are the same. Asymptotic significances (2-sided tests) are displayed. The significance level is .05 . 
Appendix F-10

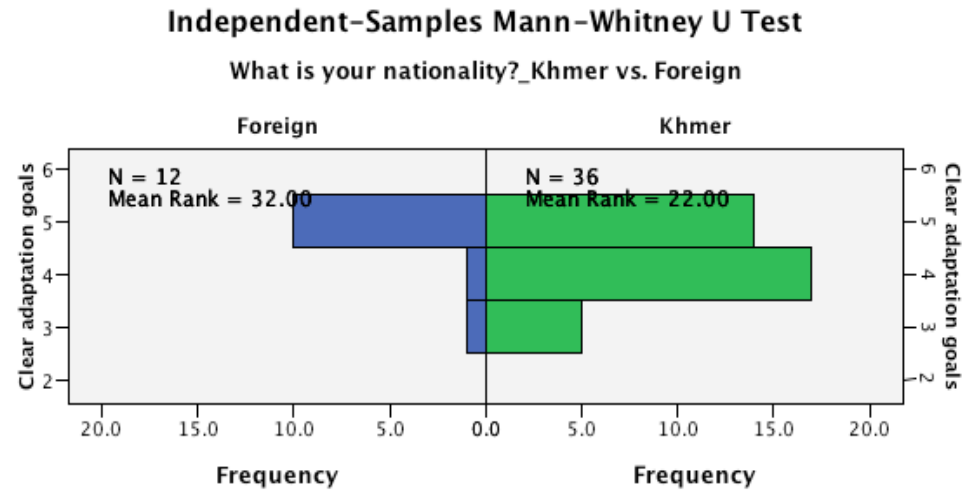

\begin{tabular}{|lr|}
\hline Total N & 48 \\
\hline Mann-Whitney U & 306.000 \\
\hline Wilcoxon W & 384.000 \\
\hline Test Statistic & 306.000 \\
\hline Standard Error & 38.048 \\
\hline Standardized Test Statistic & 2.365 \\
\hline Asymptotic Sig. (2-sided test) & .018 \\
\hline
\end{tabular}




\section{Appendix F-11}

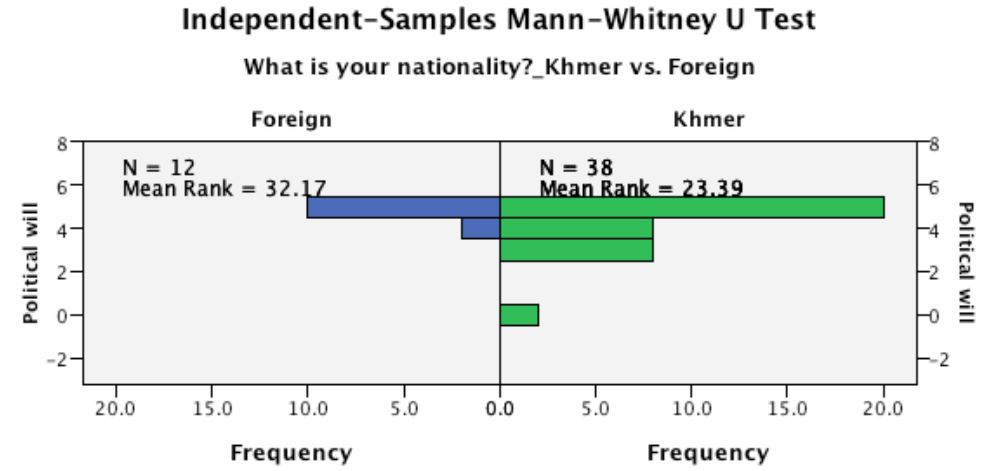

\begin{tabular}{|lr|}
\hline Total N & 50 \\
\hline Mann-Whitney U & 308.000 \\
\hline Wilcoxon W & 386.000 \\
\hline Test Statistic & 308.000 \\
\hline Standard Error & 38.684 \\
\hline Standardized Test Statistic & 2.068 \\
\hline Asymptotic Sig. (2-sided test) & .039 \\
\hline
\end{tabular}


Appendix F-12

Independent-Samples Mann-Whitney U Test How long have you stayed in SHV?_below 5 years vs. Min 5 years

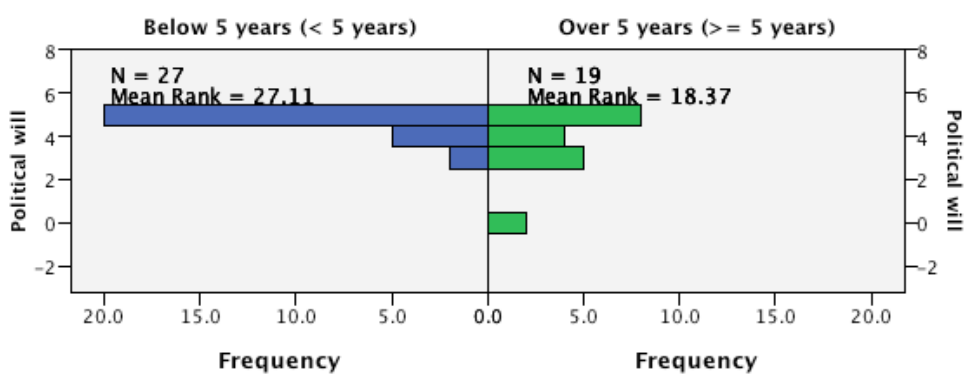

\begin{tabular}{|lr|}
\hline Total N & 46 \\
\hline Mann-Whitney U & 159.000 \\
\hline Wilcoxon W & 349.000 \\
\hline Test Statistic & 159.000 \\
\hline Standard Error & 39.173 \\
\hline Standardized Test Statistic & -2.489 \\
\hline Asymptotic Sig. (2-sided test) & .013 \\
\hline
\end{tabular}




\section{Appendix F-13}

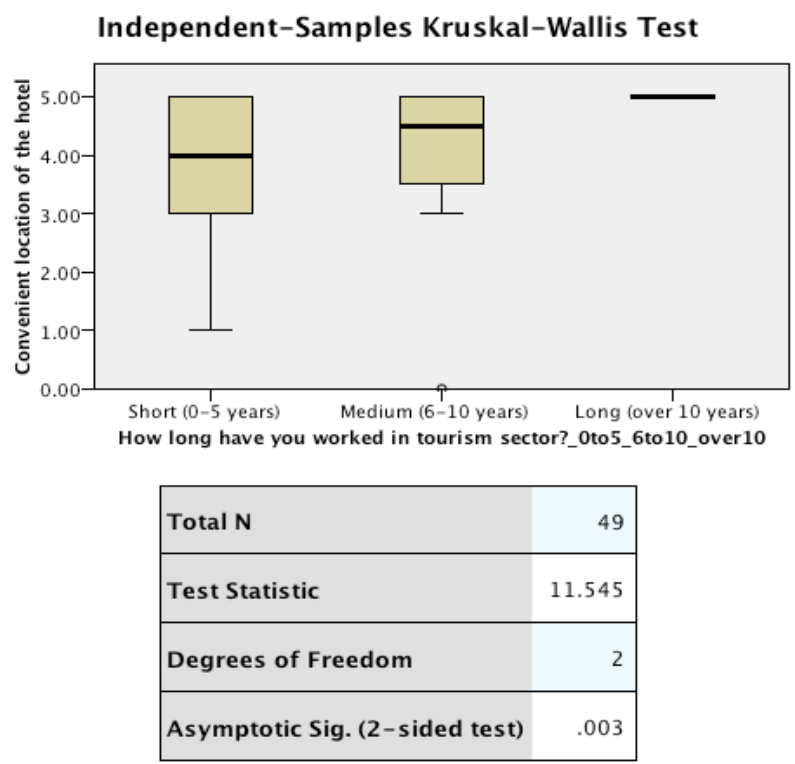

1. The test statistic is adjusted for ties.

Pairwise Comparisons of How long have you worked in tourism sector?_0to5_6to10_over10

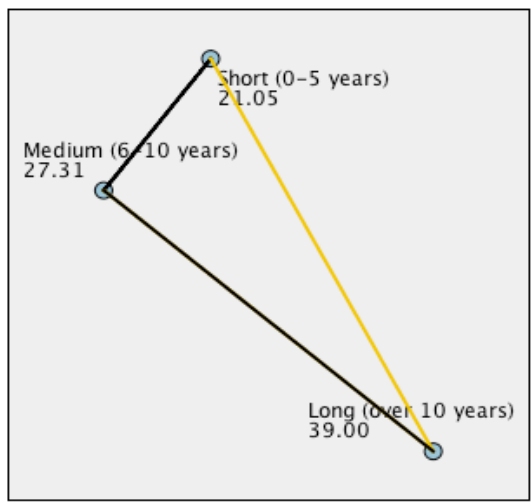

Each node shows the sample average rank of How long have you worked in tourism sector?_0to5_6to10_over10.

\begin{tabular}{|lccccr|}
\hline Sample 1-Sample2 & $\begin{array}{c}\text { Test } \\
\text { Statistic }\end{array}$ & $\begin{array}{c}\text { Std. } \\
\text { Error }\end{array}$ & $\begin{array}{c}\text { Std. Test } \\
\text { Statistic }\end{array}$ & Sig. & Adj.Sig. \\
\hline $\begin{array}{l}\text { Short }(\mathbf{0}-\mathbf{5} \text { years)-Medium (6- } \\
\mathbf{1 0} \text { years) }\end{array}$ & -6.267 & 5.349 & -1.172 & .241 & .724 \\
\hline $\begin{array}{l}\text { Short }(\mathbf{0}-\mathbf{5} \text { years)-Long (over } \\
\mathbf{1 0} \text { years) }\end{array}$ & -17.955 & 5.349 & -3.357 & .001 & .002 \\
\hline $\begin{array}{l}\text { Medium (6-10 years)-Long } \\
\text { (over } \mathbf{1 0} \text { years) }\end{array}$ & -11.688 & 6.786 & -1.722 & .085 & .255 \\
\hline
\end{tabular}

Each row tests the null hypothesis that the Sample 1 and Sample 2 distributions are the same. Asymptotic significances (2-sided tests) are displayed. The significance level is .05 . 


\section{Appendix F-14}

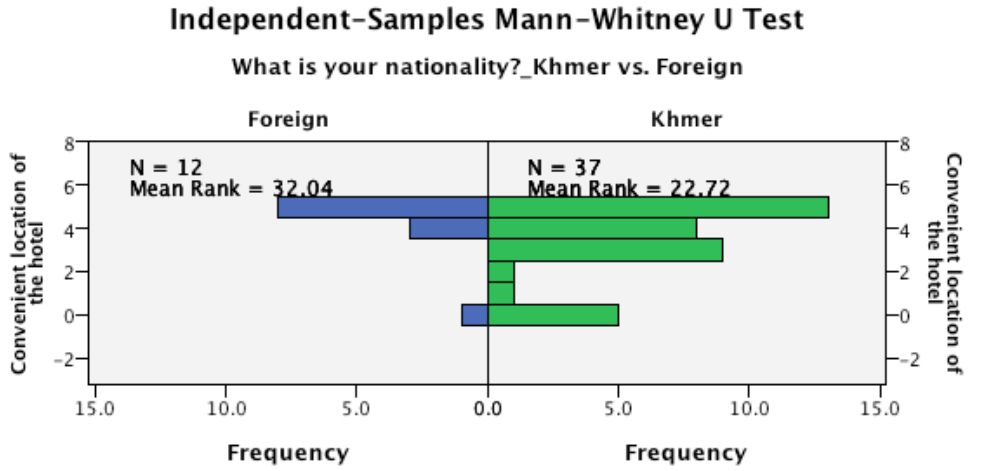

\begin{tabular}{|lr|}
\hline Total N & 49 \\
\hline Mann-Whitney U & 306.500 \\
\hline Wilcoxon W & 384.500 \\
\hline Test Statistic & 306.500 \\
\hline Standard Error & 40.856 \\
\hline Standardized Test Statistic & 2.068 \\
\hline Asymptotic Sig. (2-sided test) & .039 \\
\hline
\end{tabular}

